\title{
Role of genomic variants in the response to biologics targeting common autoimmune disorders
}

by

\author{
Gordana Lenert, PhD
}

The thesis submitted to the Faculty of Graduate and Postdoctoral Affairs in partial fulfillment of the requirements for the degree of

Master of Science

Ottawa-Carleton Joint Program in Bioinformatics

Carleton University

Ottawa, Canada

(C) 2016

Gordana Lenert 


\section{Abstract}

Autoimmune diseases (AID) are common chronic inflammatory conditions initiated by the loss of the immunological tolerance to self-antigens. Chronic immune response and uncontrolled inflammation provoke diverse clinical manifestations, causing impairment of various tissues, organs or organ systems. To avoid disability and death, AID must be managed in clinical practice over long periods with complex and closely controlled medication regimens. The anti-tumor necrosis factor biologics (aTNFs) are targeted therapeutic drugs used for AID management. However, in spite of being very successful therapeutics, aTNFs are not able to induce remission in one third of AID phenotypes.

In our research, we investigated genomic variability of AID phenotypes in order to explain unpredictable lack of response to aTNFs. Our hypothesis is that key genetic factors, responsible for the aTNFs unresponsiveness, are positioned at the crossroads between aTNF therapeutic processes that generate remission and pathogenic or disease processes that lead to AID phenotypes expression.

In order to find these key genetic factors at the intersection of the curative and the disease pathways, we combined genomic variation data collected from publicly available curated AID genome wide association studies (AID GWAS) for each disease. Using collected data, we performed prioritization of genes and other genomic structures, defined the key disease pathways and networks, and related the results with the known data by the bioinformatics approaches. We queried the AID results against known data about the aTNFs interventional pathways. Our findings allowed us to infer potential genetic factors and pathways responsible for the aTNF therapeutic effects in AID. A multitude of publicly available bioinformatics tools and databases allowed us to extract the knowledge, analyse it and provide orthogonal evidence for the results. The results support existence of at least two different sets of pathways responsible for AID pathology, most probably reflecting subtypes of AID. Only one set of pathways might be influenced by aTNFs, offering an explanation why aTNF therapy is not always effective.

Additionally, our results narrow down the complex common genomic variability responsible for aTNF unresponsiveness that could be tested in future. If our results are confirmed by functional assays and/or clinical trials, then the lack of response could be predicted ahead of aTNF therapy, leading to better patient selection and improved prognostic outcomes. The extremely high cost of the standard aTNFs therapy can easily cover the cost of genotyping ahead of medication. 
Table of Contents

ABSTRACT

1. INTRODUCTION

1.1. Motivation

1.2. Autoimmune diseases: burden in society, nature and treatment

1.2.1. Genetics of autoimmune diseases

1.3. Non-responsiveness to anti-TNF therapy

1.3.1. Tumor necrosis factor alpha

1.3.2. Anti-tumor necrosis factor biologics

1.3.3. Therapeutic role of aTNFs 10

1.3.4. Efficacy and safety of aTNFs

1.4. GWAS, variant database, tools, and analyses; genotype-phenotype relationship

1.4.1. Importance of genome wide association studies

1.4.2. GWAS methodology

1.4.2.1. Human Genome Project

1.4.2.2. HapMap Project 16

1.4.2.3. Linkage Disequilibrium 18

1.4.2.4. 1000 Genomes Project 19

1.4.2.5. The Encyclopedia of DNA Elements (ENCODE) project 21

$\begin{array}{ll}\text { 1.4.2.6. Genotyping biochip } & 22\end{array}$

1.4.2.7. Other advancements contributing to GWAS implementation 23

$\begin{array}{ll}\text { 1.4.3. GWAS database } & 25\end{array}$

1.5. Genotype-phenotype relationship in AID 26

1.5.1. From SNPs and genes to molecular networks and pathways 26

1.5.2. Basic concepts of biological networks and pathways 29

$\begin{array}{ll}\text { 1.5.2.1. Biological pathways } & 30\end{array}$

$\begin{array}{ll}\text { 1.5.2.2. Biological networks } & 33\end{array}$

$\begin{array}{lr}\text { 1.6. Biological Ontologies } & 34\end{array}$

$\begin{array}{ll}\text { 1.6.1. Gene Ontology } & 36\end{array}$ 
2.1. Data collection of GWAS AID association SNPs 40

2.1.1. Software tools and databases used for data collection 41

2.1.1.1. NHGRI Catalog $\quad 41$

$\begin{array}{ll}\text { 2.1.1.2. PheGenl } & 41\end{array}$

2.2. SNP-gene analyses: mapping AID SNP to genes and other genomic structures 42

$\begin{array}{ll}\text { 2.2.1. Rationale } & 42\end{array}$

2.2.2. Procedure 43

2.2.3. Software tools and databases $\quad 44$

2.2.3.1. HaploReg $\quad 44$

2.2.3.2. RegulomeDB $\quad 44$

2.2.3.3. dbSNP 45

2.2.4. Evaluation of missense SNPs impact on proteins coded by missSNP AID genes 45

2.2.4.1. Rationale $\quad 45$

2.2.4.2. PolyPhen-2 software tool and procedure $\quad 45$

2.3. Functional analyses of GWAS AID SNPS: gene-pathway prioritization 45

2.3.1. Rationale 46

2.3.2. AID GWAS SNP pathway analysis 46

$\begin{array}{ll}\text { 2.3.2.1. Rationale } & 46\end{array}$

$\begin{array}{ll}\text { 2.3.2.2. Procedure for finding AID SNP pathways } & 47\end{array}$

$\begin{array}{ll}\text { 2.3.2.2.1. Manual search for AID SNP pathways } & 47\end{array}$

$\begin{array}{ll}\text { 2.3.2.2.2. AID SNP pathway enrichment analyses } & 47\end{array}$

$\begin{array}{ll}\text { 2.3.2.2.3. Parsing of the AID SNP pathways } & 47\end{array}$

$\begin{array}{ll}\text { 2.3.3. AID SNP GWAS network analysis } & 48\end{array}$

$\begin{array}{ll}\text { 2.3.3.1. Cytoscape } & 48\end{array}$

$\begin{array}{ll}\text { 2.3.3.1.1. Rationale } & 48\end{array}$

$\begin{array}{ll}\text { 2.3.3.1.2. Procedure } & 48\end{array}$

2.4. Functional analyses of AID GWAS SNP data using Gene Ontology 49

2.4.1. Gene Ontology Enrichment Analysis $\quad 49$

$\begin{array}{lr}\text { 2.4.2. Rationale } & 49\end{array}$ 
2.5. Software tools and databases used in prioritization and GO enrichment analyses 50

$\begin{array}{ll}\text { 2.5.1. STRING } & 50\end{array}$

2.5.1.1. Procedure $\quad 51$

2.5.2. ConsensusPathDB $\quad 51$

2.5.2.1. Procedure $\quad 52$

$\begin{array}{ll}\text { 2.5.3. DiseaseConnectDB } & 52\end{array}$

2.6. Finding interactions of AID GWAS SNPs with non-coding RNAs 53

2.6.1. Rationale $\quad 53$

$\begin{array}{ll}\text { 2.6.2. Procedure } & 53\end{array}$

2.7. Overview of the bioinformatics software tools and databases used in the study 54

$\begin{array}{lr}\text { 3. RESULTS } & 56\end{array}$

3.1. AID-associated single nucleotide polymorphisms (AID SNPs) 56

$\begin{array}{lr}\text { 3.2. Gene candidate identification } & 57\end{array}$

3.2.1. Identification of gene candidate dataset for coding non-synonymous AID SNPs (missense SNP $\begin{array}{ll}\text { gene dataset) } & 57\end{array}$

3.2.1.1. Missense AID SNP dataset evaluation 58

3.2.1.2. Assessment of additional genes in LD with missense SNPs 60

3.2.2. Identification of gene candidate dataset based on non-coding AID GWAS SNPs (ncSNP gene dataset) 61

3.2.2.1. Evaluation of non-coding SNP dataset $\quad 61$

3.2.2.2. Functional assessment of non-coding RNA genes 65

3.2.2.3. Functional assessment of microRNA genes 66

3.3. Functional analyses of GWAS AID SNP gene set: gene-pathway prioritization 66

3.3.1.2. Network analyses using STRING software tools (STRING network analyses) 70

$\begin{array}{ll}\text { 3.3.2. Pathway analyses } & 72\end{array}$

3.3.2.1. Identification of KEGG pathway dataset for the GWAS AID SNP genes 72

3.3.2.1.1. Identification of KEGG pathway dataset for the GWAS AID missSNP genes 73

3.3.2.1.2. Identification of KEGG pathway dataset for the GWAS AID ncSNP genes 73

3.3.2.1.3. Classification of AID SNP KEGG pathway into modules 75 
3.3.2.2. Identification of KEGG pathways of five anti-TNF biologics and TNF

3.3.2.3. Curative pathways: KEGG pathway dataset common to both GWAS AID SNP pathways and TNF signaling pathways 77

3.3.2.4. Pathways enrichment for AID GWAS SNP gene sets by STRING 81

3.3.2.5. Pathways enrichment of AID GWAS SNP genes by ConsensusPathDB 81

3.4. Functional analyses of AID GWAS SNP data using Gene Ontology 83

3.4.2. GO term enrichments by ConsensusPathDB 84

3.5. Disease prioritization 85

3.5.1. Disease Connect DB enrichment for AID SNP dataset 85

3.5.2. STRING disease enrichment for AID GWAS SNP dataset 86

\section{DISCUSSION}

4.1. GWAS AID Associations 89

4.1.1. $P$ value 89

4.1.2. Rare vs. common (and low frequency common) SNPs 91

4.1.3. Genomic context, location, and LD of associated AID SNPS 92

4.1.4. Why there are no AID SNPs located on sex chromosomes? 94

4.1.5. Immunochip and its influence on AID SNPs

4.2. Functional effects of SNP-gene prioritization 95

4.2.1. Functional effects of AID missense SNPs 96

4.2.2. Regulatory effect of AID missense SNPs 98

4.2.3. Functional effects of AID synonymous coding SNPs 98

4.2.4. Functional effect of non-coding AID SNPS 100

4.2.5. Regulatory effect of AID ncSNP associations 100

4.2.6. Genes linked to ncSNPs 102

4.2.7. Relevance of eQTL results linked to AID SNPs 102 4.2.8. MicroRNA genes 103

4.3. Network and pathway analyses 104

4.3.1. Network analyses or gene-network prioritization 104

4.3.2. Pathway analyses or gene-pathway prioritization 107

4.4. Gene Ontology helps clarify the function of GWAS AID SNPS 


\subsection{Tables:}

Table 1. AID-associated SNPs and genes for each disease

Table 2. Missense GWAS AID SNPs characteristics: I part (amino acid change caused by missSNPs, PolyPhen-2 evaluation of functional consequences)

Table 3. Missense GWAS AID SNPs characteristics: II part (analysis of LD blocks by HaploRegv2 and RegulomeDB scoring)

Table 4. Alternative coding genes at the missense AID GWAS SNP loci

Table 5. Characteristics of highest Regulome DB scored GWAS AID ncSNPs

Table 6. Non-coding RNA genes in LD with top intergenic GWAS AID SNPs

Table 7. Characteristics of miRNA in high LD with AID ncSNP dataset

Table 8. AID gene datasets: missSNP genes and ncSNP genes

Table 9. Intersections between missSNP harboring genes/ proteins networks and TNF networks

Table 10. PPI by STRING

Table 11. KEGG pathways for missSNP gene set

Table 12. KEGG pathways of the non-coding SNP gene set

Table 13. Classification of missSNP KEGG pathways

Table 14. Classification of ncSNP KEGG pathways

Table 15. Anti-TNF biologics drug pathways

Table 16. KEGG TNF pathways

Table 17. Relationship between AID SNP pathways and TNF pathways

Table 18. STRING pathways enrichment data for AID GWAS SNP gene/protein datasets

Table 19. ConsensusPathDB: enriched KEGG pathway-based sets for missSNP geneset

Table 20. ConsensusPathDB: enriched KEGG pathway-based sets for allSNP geneset

Table 21. STRING BP, MF and CC GO terms enrichment for AID missSNP, ncSNP and allSNP

Table 22. Enrichment of BP, MF and CC GO terms for missSNP geneset

Table 23. Enrichment of BP, MF and CC GO terms for ncSNP geneset

Table 24. Enrichment of BP, MF and CC GO terms for allSNP geneset

Table 25. Disease Connect DB pathway dataset for AID

Table 26. STRING disease enrichment data for AID GWAS SNP datasets

\subsection{Figures:}

Figure 1. An example of functional evaluation of missense SNP impact by PolyPhen-2

Figure 2a: TNF network

Figure 2b: NFKBIE network

Figure 3a. Image of intersections between missSNP ERAP1 and TNF networks

Figure $3 \mathrm{~b}$. Image of intersections between missSNP NFKBIE and TNF networks 
Figure 4. Union of GWAS AID SNP harboring protein networks

Figure 5. Network union between intersection datasets of missSNPs and TNF networks

Figure 6. Expression pattern of AID missSNP genes

Figure 7. Image of networks constructed by STRING for missSNP, ncSNP and allSNP datasets

7a. missSNP network

7b. ncSNP network

7c. allSNP network

Figure 8. Interconnection between missense SNP harboring gene pathways and TNF

Figure 9. GWAS AID risk genes show high expression in several immune-related human tissues

\subsection{Supplemental Tables:}

Supplemental Table 1. AID GWAS associations retrieved from NHGRI Catalog

Supplemental Table 2. RegulomeDB scoring results for GWAS AID SNPs

Supplemental Table 3. AID GWAS ncSNPs influenced genes and their participating KEGG pathways

Supplemental Table 4. Comparison between all pathways: missSNP gene pathways, ncSNP gene pathways and TNF containing pathways

Supplemental Table 5. ConsensusPathDB: enriched pathway-based sets for missSNP dataset

Supplemental Table 6. ConsensusPathDB: enriched pathway sets for missSNP dataset

Supplemental Table 7. ConsensusPathDB: enriched pathways for ncSNP geneset

Supplemental Table 8. Enriched pathway-based sets for allSNP set

\subsection{Supplemental Figures:}

Supplemental Figure 1. Genomic context distribution of AID GWAS SNPs (pie chart)

Supplemental Figure 2. Location of GWAS AID SNPs on human ideogram

Supplemental Figure 3. Network images for missSNP harboring genes/proteins and TNF 


\section{INTRODUCTION}

\subsection{Motivation}

The aim of our research was to investigate genetic variability of common autoimmune/inflammatory diseases (AID) phenotypes, in order to explain the unpredictable unresponsiveness to the anti-tumor necrosis factor biologics (aTNFs) used as the therapy for AID patients.

The anti-tumor necrosis factor biologics (aTNF) is a relatively new class of bioengineered protein drugs. Approved aTNF biologics have been used broadly and successfully for over a decade in clinical practice for treatment of several AIDs. aTNFs have revolutionized AID treatment as the disease prognoses improved significantly, prompting these biologics to a level of the standard of care for AID (Monaco et al., 2014). However, although the aTNF biologics are designed to induce remission by decreasing symptoms and stopping or sustainably reducing disease progression by directly blocking tumor necrosis factor (TNF) in a body, significant portion of patients do not respond to the aTNF for unknown reasons (Taylor 2003; Monaco et al., 2014). The lack of response has emerged as a significant disadvantage for AID patients and a major issue in the aTNF application. The aTNFs unresponsiveness has been a subject of intensive study in the last few years, but no undisputable basis for it has been found, nor have any clear and definite conclusions been made, even using GWAS methodology (Emery 2012; Umićevic et al., 2013; Marquez et al., 2014).

In order to explain why aTNFs fail to act in the expected way, we sought to identify the key genes potentially responsible for the aTNFs action in AID genotypes and explore the influence of their variability on the response. Our idea is in accordance with the personalized medicine aspiration and the current pharmacogenomics knowledge progress to tailor drug therapy according to individual's genome, simultaneously achieving the best results and avoiding potential harmful side effects.

Following our hypothesis, we sourced existing publicly available databases and we employed existing bioinformatics software tools to research the relation between AIDs pathogenesis and the intervention action of the aTNF biologics. If the genes responsible for the aTNFs intervention in AID phenotypes could be identified and role of their variants entirely understood, then the variants of the responsible genes in individual AID patients could be tested in advance of the aTNFs treatment. We believe that by testing identified variants, it would be possible to identify non-responders, who then would be assigned to alternative therapies. A selective aTNFs application would prevent inevitable side/adverse effects that stem from the aTNF treatment without being balanced with beneficial AID remission effects. In addition, it might be possible to avoid unnecessary expenses associated with the use of the aTNFs, due to the 
very high manufacturing cost and huge demand for the biologics. A similar approach has been implemented in clinical practice for some drugs, where action was found to be highly dependent on variants of the identified genes in the individual genomes (Cavallari et al., 2011). Though still in preliminary phase, this approach represents a foundation of the personalized medicine, where a drug is selected, dosed and applied depending of variants/alleles of the key genes in an intervention pathway of a particular drug.

For the purpose of our research study, we focused only on the AID for which the aTNFs are currently approved as treatment: rheumatoid arthritis (RA) and its juvenile form (JRA), psoriasis (PS), psoriatic arthritis (PsA), ankylosing spondylitis (AS) and inflammatory bowel disease (IBD) including ulcerative colitis (UC) and Crohn disease (CD). We did not include "off label" use of the aTNFs documented in several other AID (uveitis, Bechet), although the convincing data exist about positive outcomes, because the data are not collected in the same manner as for the approved treatments, and the uses are not systematically confirmed to provide unequivocal benefits.

\subsection{Autoimmune diseases: burden in society, nature and treatment}

Human autoimmune diseases (AID) are heterogeneous group of complex chronic inflammatory disorders commonly present in population. AID are primarily initiated by the loss of immunological tolerance to self-antigens (Janeway 2001). The signature characteristic is the persistent and intermittent chronic immune response to own cells, tissues, organs or organ systems. This immune response reflects disbalance in normally tightly controlled immune system homeostasis. All AID feature adaptive immunity against self-antigens (known or yet not defined antigens), and simultaneously activated innate immunity, both intertwined in a specific manner for each disease entity. The self-directed immune system responses display multiple common and specific characteristics among individual AID phenotypes (Cho and Gregersen 2011). The tissues in which the immune response occurs become inadvertently damaged and dysfunctional over time. Reasons for the persistent immune system disbalance and tolerance break down in AID phenotypes are still partly elusive (Smilek and St Clair 2015).

AID usually start years before a clear clinical presentation, and becomes obvious only after multiple autoimmune-response elements converge into pathological and clinical manifestations. AID have different clinical signs and symptoms, and variable clinical course. Tissue lesions in AID could be multiple, but specific for each disease; they often dictate classification into organ-specific or systemic 
AID. Further, AID are classified as seropositive and seronegative depending whether the production of autoantibodies (a humoral part of adaptive immune response type) is associated with the chronic inflammation (Wang et al., 2015).

In addition to very specific characteristics of each disease, dictated by the scope and type of inflammation in organs and tissues, AID phenotypes often share some common epidemiologic, clinical and therapeutical features (Cho and Gregersen 2011; Smilek and St Clair 2015). Recognized shared clinical features of AID phenotypes required similar therapeutic approaches (Cho and Gregersen 2011). This is especially true for AID therapeutic protocols that use the older, mainly empiric drugs with no specific target and with broad effects, discovered quite some time ago on a "trial and error" principle (methotrexate is an example). Invention of such types of drugs is not sought for any more, because better, targeted therapies ask for strictly targeted drugs. However, even now when the targeted therapies are available, many AID still continue to be primarily treated with the non-specific drugs as a first line of a disease control (corticosteroids and chemotherapeutic drugs); the non-targeted, nonspecific drugs have opened up a space for development of new more specific, target-based drugs (like TNFs). Oddly enough, the nonspecific medication historically is a standard for comparison and approval of all other newer drugs for the treatment of autoimmune conditions. Target authentication is necessary for the development of new drugs; it requires deeper knowledge of the AID pathogenic mechanisms than the current one. It means that the development of new, targeted drugs, similar to the aTNFs, demands characterization of the molecules capable of inhibiting specific protein-protein or other interactions within the yet unknown pathways that drive disease activity (Song and Buchwald 2015).

As treatable but incurable diseases, AID cause considerable morbidity, affect quality of life and lead to disability. AID are also associated with reduced life expectancy and significant mortality even when threated (Cho and Gregersen 2011; Smilek and St Clair 2015). They are present not only in older adult population, but also in youth and younger adults at the peak of their reproductive and working years. It has been known for a while that some AID constitute a leading cause of death among young and middle-aged women (Walsh and Rau 2000). Autoimmune diseases collectively are ranked within the top 10 causes of death (Jacobson et al., 1997; Wang et al., 2015). In addition, the chronic nature of these diseases places a significant burden on medical care resources, and increases direct and indirect overall economic costs to the society (Jacobson et al., 1997).

The AID as an aggregate affect between 5-10\% of individuals of European origin according to some current estimations (Cooper, Bynum and Somers 2003; Marson, Housley and Hafler 2015). The prevalence is similar in populations of Asian, African and Caucasian origin, with few exceptions; however, there is an uneven distribution across other less studied populations, or less studied distinct 
diseases (Marson, Housley and Hafler 2015). Incidence rates for individual AID range from less than 5 per 100,000 to more than 500 per 100,000 per year (Cooper and Stroehla 2003). The incidence rate of all AID in the world's population is more than 3\% (Cooper, Bynum and Somers 2009), with a greater ratio of females than males. At least $80 \%$ of all autoimmune diseases occur in women. However, some AID (Crohn disease, Type 1 Diabetes mellitus) are more prevalent in males. AID affect people of all ages, but disease age of onset tends to be AID specific: for example, diabetes T1D is typical for adolescence, multiple sclerosis (MS) for adulthood. AID incidence and prevalence also differ between geographical regions (Wang et al., 2015). Ethnic differences are similarly documented (Wang et al., 2015). Moreover, rates of specific diseases are noticed to change in real time (Ngo, Steyn and McCombe 2014). A comorbidity of multiple autoimmune conditions are recognized in clinical practice (Cho and Gregersen 2011; Cooper and Stroehla 2003). AID clustering is observed both in individuals and families (Cooper and Stroehla 2003; Cárdenas-Roldán et al., 2013). Comorbidity of the autoimmune diseases is important for interpretation and usage of genomics data (Somers et al., 2009).

Currently, it is widely accepted that AID are complex disorders that evolve from interactions between polygenic endogenous risk factors and environmental trigger factors. However, the relations between environmental and genetic factors and the interactions within genetic factors are still mainly incomprehensible (Smilek and St Clair, 2015). Although certain environmental factors that trigger autoimmunity have been recognized (Pollard 2015), the genetic basis is overwhelmingly accepted as a dominant factor in AID susceptibility and in AID development. It is recognized that AID genetic factors are commonly inherited, but an accumulation of rare acquired somatic mutations could not be completely excluded (Banchereau et al., 2013; Marson, Housley and Hafler 2015). It is important to stress that majority of known genetic factors lack comprehensible relations among themselves and disease pathogenesis (Smilek and St Clair, 2015). Consent is that the molecular pathways ruling pathological (or physiological) events are still very poorly characterized in AID, inevitably leading to poor knowledge of disease characteristics and ways to intervene (Califano et al., 2012).

\subsubsection{Genetics of autoimmune diseases}

Strong genetic components to autoimmune disease development have been recognized for almost three decades (Goris and Liston 2012). Identification of genetic factors was achieved by traditional epidemiologic, genetic studies including linkage and candidate-gene association, and by positional cloning studies (Altshuler et al., 2008). They confirmed heritable relations with modest effects and an increased allele sharing between affected siblings, validating the shared genetic risk observed in epidemiological studies (Cho and Gregersen, 2011; Marson, Housley and Hafler 2015). Clustering of autoimmune diseases within families commonly appears (Cooper, Bynum and Somers. 2009). Majority 
of common AID are characterized by sibling recurrence risk of medium values (Vyse and Todd 1996). The studied AID have higher concordance rates of $25 \%-50 \%$ in monozygotic twins (Bogdanos et al., 2012) versus $2 \%-12 \%$ in dizygotic twins; the results are indicative of genetic involvement in inheritance of diseases (Cooper, Bynum and Somers. 2009).

However, all traditional approaches were insufficient in advancing the insight into the complex genetics of common diseases, as only few genes were identified, insufficient to explain causation or inheritance of AID (Goris and Liston 2012). Better understanding of complex AID genotype architecture and genetic inheritance of AIDs phenotypes was boosted only after introduction of a new methodology of genome wide association studies (GWAS) several years ago. For the first time the GWAS methodology, designed as the case-control model, allowed investigation of genotypes of unrelated people with selected pathophenotypes. It does not require the relatives for detection of genetic inheritance factors, but rather uses huge groups of unrelated individuals with preselected AID phenotypes from various human populations (Goris and Liston 2012).

GWAS methodology advanced investigation of complex AID genomic structures by uncovering polymorphism of numerous genetic loci associated with a single disease. Vast majority of the detected polymorphism loci are common single nucleotide polymorphisms (SNPs), the most abundant source of genetic variation in the human genome (ENCODE Consortium Project 2012). The specific alleles or loci, marked by single nucleotide polymorphisms (SNPs), have been shown to confer small risk increments, defining the predisposition for complex AID phenotypes (Cho and Gregersen, 2011). It is believed that AID, like other common diseases, are "formulated" on common gene alleles according to the "common disease, common variant" hypothesis (Reich and Lander 2001; Altshuler, Daly and Lander 2008; Schork et al., 2009). Because of polygenetic nature and consequently polygenic inheritance, AID might be considered common phenotypes in the same way as complex quantitative traits (such as height) in humans (Manolio and Collins 2009).

The abundance of generated GWAS data became quickly a subject of ongoing investigations on correlations between genotypes and phenotypes. Although GWAS hugely advanced understanding of the complexity of common AID diseases, it will take time to comprehend completely disease mechanisms and even more, to use it as a tool for advancement of future treatments and for prediction of health improvement procedures. The new knowledge generated from advanced genomics is still at the beginning of its application potential (Lucas and Lenardo 2015; Green et al., 2011). Predictive values of AID GWAS genetic results have been intensively studied, but they have not materialized yet as expected to a point that an intervention might be in sight. It is possible that the slow progression of the GWAS-based knowledge about mechanisms of AID has a very good reason: according to the newest 
analyses, the heritability of some immune system factors is hard to prove (Brodin et al., 2015). It seems that when it comes to immune system factors and functions, heritability might not go hand in hand with causality. These findings might be interesting if confirmed by other researchers, because the immune system components are directly credited as essential factors for the development of autoimmune diseases.

The GWAS methodology associates well-defined phenotypes with their genotypes; it starts from the preselected clinical phenotypes, and trickles down to identify loci in genomes of selected individuals after scanning them with the biochips. Currently, it is broadly accepted that AID phenotypes are based on genotypes with particular genetic architecture. AID genotypes contain collections of risk factors with modest effects that are commonly present in human populations as allelic variants (usually with frequency $\geq 1 \%$ ). However, what is not clear is how risk gene alleles condition extraordinarily complex combination of genetic and potentially epigenetic factors that predispose for autoimmune diseases (Smilek and St Clair 2015; Farh et al., 2015).

As polygenic diseases, the common AID do not have a single highly penetrable genetic mutation as causative factor, but rather a number of multiple causative mutations or variants of very low penetrance that accumulate to gain a signal comparable to single penetrable mutations signals. Some of the single highly penetrable signals are found in human severe immune deficiencies and because immune deficiencies resemble some components of clinical phenotype in AID, they might be considered in AID phenotype-genotype analyses (Fodil et al., 2016). A very few known rare monogenic autoimmune diseases with Mendelian inheritance patterns within families, might be used to help understand genotype-phenotype interactions (Waterfield and Anderson 2010; Lucas and Lenardo 2015). Even in well-studied monogenic diseases, such as sickle cell anemia caused by single point mutations of the hemoglobin beta-chain, the individual pathophenotypes vary significantly, as the patients have different clinical presentations (Steinberg and Adewoye 2006). The phenotypic complexity is also present in all early age onset monogenic autoimmune diseases (Waterfield and Anderson 2010). The examples show that a genotype with a single penetrable mutated gene does not necessarily yield a single pathophenotype. For that reason, it is not unexpected that the common AID diseases also have extraordinarily complex clinical phenotypes, as measured by the disease symptoms, severity and progression (Cho and Gregersen, 2011).

GWAS results have contributed to several conclusions about the genetic architecture of autoimmune diseases. First, the genetic architecture of polygenic AID is highly complex, opposite to the monogenetic Mendelian autoimmune diseases. Second, combined effects of many variants of genetic factors define susceptibility to autoimmune diseases rather than causality. Third, most of detected genetic factors are 
common in the general population and each of the genetic factors exerts a small effect on risk for developing AID phenotypes (Hindroff et al., 2009; Cho and Gregersen, 2011). It seems that various different combinations of risk alleles are independently able to generate a high level of disease risk. It is unknown which individual loci are necessary and/or sufficient for the development of AID. Furthermore, it is still unclear whether the disease thresholds exists and what they encompass (Goris and Liston 2012). Whether the genetically susceptible individuals will develop an autoimmune disease is unknown, posing currently an unanswered question "if and when" a specific genotype is sufficient for a disease development, and whether an additional trigger must be taken into account to interact with the susceptible genotype for disease expression (Cho and Gregersen, 2011). Future approaches will be necessary to find rare variants that might be unrecognized by the current GWAS methodology, but suspected to contribute to the overall risk. In addition, it will be essential to understand and integrate analyses of epigenetic mechanisms and eQTL data for better insight into the genetic basis of AID (Farh et al., 2015).

The first genes associated with AID heritability and development were the genes of the human leukocyte antigen (HLA) region characterized more than two decades ago; the extremely polymorphic HLA genes differ in allelic frequency between healthy subjects and patients with AID. However, the nature of influence of the HLA polymorphism on the development of AID is not precisely understood even today, although it is confirmed to be substantial with the numerous AID GWAS. It is still a subject of intensive investigation (Fernando et al., 2008; Goris and Liston 2012; Sollid et al., 2014), solely for one reason: complexity. The HLA system (the human version of the major histocompatibility complex, MHC), is an exceptionally complex system, consisting of a dense collection of two classes of genes: Class I with three major and three minor groups of genes, and Class II with nineteen groups of genes. There is an additional group of genes not strictly of HLA nature and function, with exceptional linkage disequilibrium (Class III), and yet unknown number of HLA pseudogenes. All these HLA gene loci are compactly crammed in the region 6p21.3 of the chromosome 6 in humans; the HLA genes are characterized with high linkage equilibrium (Human HLA Database; HLA Gene Family). There are over thousand common alleles for the major HLA genes and above 13,000 alleles for all HLA genes.

The heritability of HLA genes, though of major significance, cannot solely explain heritability of AIDs, because HLA genes participate in a very narrow functionality of antigen presentation and recognition, and individual sustainability towards foreign invasion (Goris and Liston 2012). The process of antigen presentation and recognition represents a core process of the immune system, but is also important for tolerance development within an individual immune system. Although certain alleles are more observed in specific single AID phenotypes, HLA associations do not have predictive values even when their 
heritability has been confirmed (Wang et al., 2015). On the other hand, judging by the disease signs and symptoms, other processes, in addition to the antigen presentation and recognition pathway, must be involved in the development of autoimmune/inflammatory diseases (Cho and Gregersen, 2011).

Before GWAS methodology application, only a few non-HLA risk bearing genes for AID had been identified through candidate gene studies, such as CTLA4 (Ueda et al., 2003) and PTPN22 (Criswell et al., 2005). The GWAS were first to indicate the massive presence of other non-HLA loci associated with AID. It is interesting that well accepted experimental results on genes crucial for autoimmunity in animal models do not overlap with GWAS data. For example, a gene AIRE (autoimmune regulator, a transcription factor) experimentally shown to be a crucial factor for controlling multi-organ autoimmune diseases in experimental animals and human experimental cell models, was only confirmed in RA, and only in patients of Asian ancestry (Terao et al., 2011). Other similar examples have been shown as well. Majority of non-HLA genetic variants are located within non-coding regulatory regions. Recently, to enrich and explain AID GWAS data, fine mapping and QTL expression studies have been performed; they have shown that the variants affect AID by regulating gene expression (Farh et al., 2015).

The AID are also noteworthy diseases from an evolutionary perspective. They have been shown to exhibit a negative effect on the reproductive fitness (Brinkworth and Barreiro 2014). For that reason, AID should have been steadily eliminated from the human population due to the natural selection pressure. There are at least two theoretical explanations why it is not happening (Brinkworth and Barreiro 2014; Raj et al., 2013). The first one stresses that many AIDs occur in the later phases of human life, beyond the reproductive age, due to reduced penetrance of causal genetic mutations. The second, more critical one, points that the persistence of autoimmune conditions might be explained by very essential roles that the implicated immune genes, their products and processes have in immune surveillance and defense. In these cases, the selective pressure is acting to increase host resistance to pathogens and better chances for survival of a population. The genes harboring mutations that qualify as disease AIDs risk factors also might play pivotal advantageous roles in pathogen defense and other life preserving immune functions (e.g. neoplastic surveyance). The same disease risk factors in that case are under very strong natural selection in humans, because they are essential for the survival of the species. Detected risk factors of the immune response are not eliminated from the pool, but propped and enriched (Brinkworth and Barreiro 2014). The results exist to confirm that the risk factors for autoimmunity endure positive pressure due to enhancing certain immune response processes (Raj et al., 2013). Nevertheless, the evolutionary reasons for autoimmune disease persistence in human populations are still not well understood. 


\subsection{Non-responsiveness to anti-TNF therapy}

\subsubsection{Tumor necrosis factor alpha}

The tumor necrosis factor alpha (TNFa or simply TNF) was discovered virtually simultaneously nearly half a century ago, by two groups of researchers from Yale University and University of California at Irvine (Kolb and Granger 1968; Ruddle and Waksman 1968). TNF is a very potent proinflammatory cytokine synthesized by several cell types not limited to the immune system cells. TNF plays role in immune system activation, differentiation, development, regulation of immune signaling, inflammation, survival or apoptosis and autoimmunity (Aggarwal et al., 2012). It is one of the best-studied cytokines, with known characteristics and roles, and established pathways (Janeway 2001).

TNF and Lymphotoxin (LT $\alpha / \beta)$, a molecule very similar to TNF, and their common receptors, all have multiple and important roles in the human immune system. These molecules have been also the subject of study for potential clinical uses (Croft et al., 2013). The essential role of TNF in autoimmunity was revealed after proving its potential to modulate the disease activity in animal models by either increasing or decreasing disease expression (Aggarwal et al., 2012). The first positive effect of TNF blockade was shown in RA in humans, as a proof of concept that its inhibition may ameliorate autoimmunity, revealing TNF as one of the major vulnerable nodes that may be targeted in several AID (Maini et al., 1993).

\subsubsection{Anti-tumor necrosis factor biologics}

The anti-tumor necrosis factor (anti-TNF) inhibitors (or aTNFs) are approved class of biologic drugs targeting TNF-alpha cytokine. They are engineered monoclonal antibodies or fusion proteins that specifically bind TNF (Willrich et al., 2014). The aTNFs are approved for the treatment of several autoimmune /inflammatory diseases, such as rheumatoid arthritis (Singh et al., 2012), ankylosing spondylitis (Maxwell et al., 2015), psoriatic arthritis (Rodgers et al., 2011), psoriasis (Busard et al., 2014), inflammatory bowel disease (Ben-Horin et al., 2014), Crohn disease, CD (Behm and Bickston 2008) and ulcerative colitis (Lawson et al., 2006). Currently, there are five approved TNF inhibitors: (1) infliximab, a chimaeric lgG anti-human monoclonal (Remicade $囚$ ); (2) etanercept, a TNFR2 dimeric fusion protein, with an IgG1 Fc (Enbrel囚); (3) adalimumab, a fully human monoclonal antibody (mAb) (Humira ${ }^{\circledR}$ ); (4) golimumab, a fully human mAb (Simponi®) and (5) certolizumab, a PEGylated Fab fragment (Cimzia $\left.{ }^{\circledR}\right)$. All five aTNF biologics have been approved as therapeutics/medicinal products by the major regulatory agencies, FDA/EMEA/HC/ (North America and Europe) and TGA/PMDA (Australia and Japan). In addition, several generic aTNF biologics are currently in development, or in the submission process (personal communications). According to the proposed new nomenclature, the five 
original aTNFs will be counted as biological originator (bo) aTNF biologics, while biosimilars (bs), after the process of approval, will be named bs aTNF biologics (Smolen et al., 2014).

\subsubsection{Therapeutic role of aTNFs}

The aTNFs therapeutic outcome in AID is considered revolutionary because it provides overwhelmingly positive results. Introduction of aTNF biologics in late 1990s marked a change in the therapeutic approach, as they become the first successful targeted biologics. Before the discovery of aTNF biologics, treatments for the approved AID (and other autoimmune diseases) consisted only of broadspectrum immune modulators or traditional DMARDs (DMARD stands for disease-modifying antirheumatic drugs), which all nonspecifically block immune cell functions: non-steroid antiinflammatory drugs, glucocorticoids, and cytostatics like methotrexate, azathioprine or 6-mercaptopurine and others (Willrich et al., 2015). The advantage of new aTNF biologics, when compared with small molecule drugs, is the result of their dramatically increased target specificity (one that exists for receptors, antibodies), prolonged half-life (proteins) and decreased intrinsic molecular toxicity (naturally occurring molecules) combined (Yin et al., 2015).

The aTNF biologics revised the concept that effective treatments in the autoimmune/inflammatory diseases require broad immune suppression. aTNFs actually challenged the idea that immune pathways are highly redundant and that no single protein targeted therapy might be able to block the inflammation. The aTNFs do work in the complex systems such as AIDs. They proved the concept that upregulation of the immune system, resulting in measurable and damaging chronic inflammation depends on rather fragile, narrow channeled communications between pathways and networks. At least one of communications or pathways integrated into the network of cytokines collapses after neutralization of nodes like TNF. The downregulation of inflammation by aTNFs also supports an idea that a cytokine hierarchy exists and that TNF is most probably a mediator situated near the root of a tree of cytokine networks, negating that the inflammation process is a net of multiple redundant nodes and interactions (Schett et al., 2013). This concept was also supported by the unexpected uniformity of the therapeutic response to TNF inhibition among patients with rheumatoid arthritis, psoriasis, psoriatic arthritis, Crohn disease, ulcerative colitis, ankylosing spondylitis, juvenile arthritis and other less prevalent autoimmune diseases. However, there are exceptions such as multiple sclerosis and SLE, which worsened upon TNF inhibition (Zhu et al., 2010; Probert et al., 2000). Their pathogenesis might be different from aTNF sensitive AID, although they are classified as the same group of diseases, exhibit similar symptoms or signs, and, even more, are treated in a similar fashion. 
Given the profound therapeutic potential of TNF inhibition, it has been suggested that different chronic inflammatory diseases (AID and others), may share common pathophysiology based on the notion that aTNFs are able to disrupt the easily broken obligatory inflammation network. It has been also suggested that even more targeted therapies might follow in future, based on better understanding of aTNFs action in AID (Schett et al., 2013).

\subsubsection{Efficacy and safety of aTNFs}

Current approach to AID treatment focuses on induction of remission and requires chronic management that extremely rarely, if ever, results in a complete cure. aTNFs are given over a long period of time and although very effective, their tapering, interruption and cessation are subjects of current investigations with no consensus. Recent evaluation of the dose reduction, discontinuation or disease activity guided dose tapering of the aTNF agents produced limited conclusions, because of heterogeneity of study data (Malottki et al., 2011).

Unwanted consequences of the aTNFs medication are not trivial, since aTNFs exert characteristics of the immunosuppressive drug class. aTNF adverse events (AE) could be detrimental to effective immune responses and might diminish natural ability of the immune system to fight infections and to resist and control malignant processes. Among AE that might surface during aTNFs therapy, cases of tuberculosis (TB) and lymphomas as the most serious AE occurred in practice (Nanau and Neuman 2014). The reactivation of tuberculosis (TB) has been a recognized risk of aTNF biologics therapy from the beginning (Xie et al., 2014). The current studies are inconclusive regarding the TB incidence rate (British Thoracic Committee 2005; Nanau and Neuman 2014), and not sensitive enough to assess the risk of reactivation of latent TB infection vs. de novo inoculation (Sivamani et al., 2013). The longer-term safety data for the malignancy risk varied across studies, but none reached statistical significance (Singh et al., 2012; Smolen et al., 2013). Also encouraging is the finding that the immune response to the tested vaccines (pneumococcal and influenza vaccines) is not reduced in patients during aTNF therapy (Hua et al., 2014). In addition, aTNFs potentially may cause rewiring of an individual immune system leading to secondary autoimmunity (Singh et al., 2012; Smolen et al., 2013). The use of aTNF biologic drugs has been linked with the paradoxical development of systemic and organ specific autoimmune processes, such as sarcoidosis (a granulomatous disease) and other diseases (Korta et al., 2015). The effects of TNF inhibitors on cardiovascular disease are potentially multifaceted, because these drugs may promote heart failure, but on the other side may also improve risk factors for atherosclerosis (Nguyen and Wu 2014). 
All five aTNF agents are effective in the induction and maintenance of remission; there is no evidence of clinical superiority among them in clinical trials. Nevertheless, aTNF biologics differ in the way they individually are dissolved and administered, by their serum peak and trough levels, in vivo complexes that they can form, and incidence and timing of rare AE (Michaud et al., 2014; Malottki et al., 2011).

The aTNF biologics are not always effective for all patients, and even when effective initially, might lose effectiveness over time. Even in clinical trials, initial failures of aTNF induction therapy occurred in up to $40 \%$ of patients. Like many drugs, aTNFs are less effective in daily clinical practice than in clinical trials, due to the less controlled environment (drug delivery, adherence, other medication, etc.). A proportion of patients with AID that do not respond adequately to treatment with anti-TNF drugs in clinical practice might be even higher (Kamal et al., 2006). The aTNF treatment guidances state that lack of response warrants re-evaluation, as it is considered most probable an intrinsic failure of the treatment (Saag et al., 2008; Smolen et al., 2013). Switching from one aTNF agent to another aTNF after first-line treatment failure may not be a cost-effective treatment strategy (Kamal et al., 2006). However, switching, while controversial, occurs frequently because of patients' comfort and experience (or lack of it) with the aTNF agents. Occasionally, a second agent from the same class may be effective due to individual differences in bioavailability and immunogenicity. The practice is widespread, as the 2006 US survey indicated that $>94 \%$ of rheumatologists had switched patients to another TNF inhibitor, because of lack of efficacy or intolerability of the first agent (Kamal et al., 2006). When more than one TNF inhibitor provides inadequate responses or $A E$, then switching to a completely different class of biological agents with another mode of action (and there are at least four approved non-TNF targeted biologics) may provide a more effective option (Emery 2012). In our opinion, all these data indicate that aTNFs have very narrow if not a single path of action, because if one aTNF agent does not work in a patient, other aTNF agents do not work as well.

Secondary loss of aTNFs response is also a common problem with incidence ranging between $23 \%$ and $46 \%$ at one year after anti-TNF initiation. Immunogenicity of aTNF drugs is one of the mechanisms behind this type of treatment failure (Garcês et al., 2013). Though anti-drug antibodies (ADA) are often presumed as underlying mechanisms for failure of the treatment with a biologic, it is hard to confirm the suspicion by currently available tests. Measurements of aTNF ADA are complicated and expensive and do not always provide an adequate answer that can be used for clinical decisions (van Schouwenburg et al., 2013). In some patients, the formation of anti-TNF ADA might be transient (van Schouwenburg et al., 2013). 


\subsection{GWAS, variant database, tools, and analyses; genotype-phenotype relationship}

\subsubsection{Importance of genome wide association studies}

Genome wide association studies (GWAS) is an advanced bioinformatics methodology that relates human phenotypes to human genotypes. GWAS application associates intrinsic, inborn known common variability of human genomes with preselected phenotypes expressed as complex human traits or common diseases (Hindorff et al., 2009; Schprk et al., 2009). Testing is performed on hundreds of thousands to millions of single nucleotide polymorphisms (SNPs) at a time, in the genomes of thousands of individuals. The GWAS methodology enables researchers to analyse polygenic disease/trait inheritance, for which the classical genetic methodology did not and could not provide an answer.

By late 1990s, the existing genetic mapping of human complex diseases became unpractical and insufficient, because of the very high number of genes suspected to be involved in common diseases. The used human genetic maps, with several hundreds of loci marking human chromosome regions, became obsolete for all complex diseases and traits, except for rare single gene mutation based Mendelian diseases. The genetic markers, labeling DNA polymorphisms detected by positional cloning, were too scarce for studying inheritance of complex diseases and traits in humans. Unsuccessful were the attempts to identify genes for complex diseases with pedigree analyses, as the pedigree studies were only able to identify single highly penetrant genes influencing a range of monogenic diseases/traits, which include a variety of inborn errors of monogenetic genetic diseases such as cystic fibrosis, Duchenne muscular dystrophy, or Huntington disease (Borecki and Province 2008). However, most disease genes were completely unsuspected based on existing knowledge at the time, which made "candidate" genes approach obsolete. In addition, large informative pedigrees, which this type of analyses needs, are very difficult to find and to recruit (Borecki and Province 2008). These analyses are curbed by many limitations: clinical, ethical, and intrinsic, such as locus heterogeneity, incomplete penetrance, and variable expression (Altshuler, Daly and Lander 2008). In the Mendelian diseases, variations in a single gene are both necessary and sufficient to cause a disease. Level of necessity and sufficiency for disease genes in complex diseases and traits are unknown parameters. The known Mendelian disease and genes related to them have been documented extensively in the Mendelian Inheritance in Man or OMIM NCBI database (McKusick, OMIM database).

Alternative approach for disease genes discovery emerged from population genetics and genomics, by means of comparisons of frequencies of genetic variants among affected and unaffected individuals (Risch and Merikangas 1996). Further reasoning led to the so-called "common disease-common variant" (CD-CV) hypothesis: the brilliant idea proposing that common polymorphisms (defined as 
having a minor allele frequency of $>1 \%$ ) might contribute to susceptibility to common diseases (Risch and Merikangas 1996; Lander 1996; Schork et al., 2009).

The GWAS methodology offered a solution for polygenic variant identification in complex common diseases unrestrained by any presumptive hypothesis (Altshuler, Daly and Lander 2008). No previous knowledge of potential gene "candidates" is required for GWAS analyses, making it a method of choice for genetic background in common diseases, since common diseases have been considered for long to arise from the interactions between greatly unknown polygenic risk factors and poorly understood environmental factors (Selmi, Lu and Humble 2012). However, the heritability of common diseases has been firmly established, derived from phenotypic concordance in twins and first-degree family members as well as familial clustering (Czyz et al., 2012).

Before introduction of GWAS, the common diseases heritability in general unrelated population could not be studied. GWAS design follows case-control study design with very large groups of unrelated individuals. Unrelated subjects with common diseases are much easier to recruit; their recruitment location became less important than their willingness and consent to participate in genetics studies. Both are regulated and more demanding than sample collection, mainly because of effortlessness to gather DNA samples for GWAS (Peppercorn 2012). It is important to stress that the CD-CV hypothesis did not assume that all common disease are conditioned only by common causal mutations. Common variants exist in human population because of the nature of human population formation and they are used to detected loci for detailed study of common human diseases or traits. Contrary to the simple understanding that the CD-CV hypothesis means only common variants may cause common diseases, a full spectrum of alleles has been expected always (Lander 1996; Altshuler, Daly and Lander 2008; Schork et al., 2009).

GWAS have been highlighted as a major breakthrough in health research over the last decade (Altshuler, Daly and Lander 2008; Zerhouni and Nabel 2008), not only because of the potential to explain genetic origin and heritability of common diseases, but because the new studies have capacity to provide new feasible therapeutic and prevention approaches. GWAS methodology has currently a central role in human genetics revolution as it has facilitated substantial massive data accumulation for further studies and the advancement of knowledge in medicine and biology (Zerhouni and Nabel 2008).

\subsubsection{GWAS methodology}

GWAS enable advancement towards the fundamental goal to completely catalog genetic polymorphisms of the human genome that cause phenotypic variations and allow characterization of molecular 
mechanisms by which polymorphisms exert their effects (Manolio, Brooks and Collins 2008; Manolio and Collins 2009). GWAS methodology is considered currently the most effective way of mining for variants (Manolio and Collins 2009), because it is purely discovery driven and unbiased towards any particular hypothesis. It became a standard tool for detection of variants, which are deemed inheritable risk factors, responsible for developing of complex diseases. Not only that numerous GWAS examined the human genome to detect risk variants, but they also revealed genetic architecture of human diseases and polygenic traits in healthy people. GWAS genome scanning potential have also facilitated new discoveries in genetic recombination (Hinch et al., 2011; Hindorff, Gillanders and Manolio 2011) and better understanding of natural selection and evolution (Novembre et al., 2008).

GWAS methodology is based on finding small minuscule variations in a form of nucleotide switches that are naturally occurring in human genomes throughout the evolution of the species. Usually any two human genomes differ in one DNA base pair in every thousand base pairs, but the number could be higher (Lander 2011). The single nucleotide polymorphisms (SNPs) are the most common type of DNA variations in the human genome. A typical GWAS may genotype significant number of SNPs, starting from $300 \mathrm{~K}$ to more than million SNPs. It is typically testing the genomes of thousands of individual subjects who are preselected based of their expressed phenotypes (diseases/traits) and assigned into case or control cohorts (Hinds et al., 2005; Altshuler, Daly and Lander 2008). Finding SNPs association is performed by scanning of genome DNA for preselected markers manufactured on biochips, using highly sophisticated detection equipment and procedures. Common genetic variants generally have very small effects that require large sample sizes for detection (Visscher et al., 2012). GWAS methodology must meet stringent levels of statistical significance (e.g. $p<5 \times 10^{-8}$ ), as it has been justified because of multiple hypothesis testing. The association results must be replicated ideally in a separate sample of cases and controls, to minimize the possibility of assay artefacts (Pe'er et al., 2008).

The following projects and innovations allowed the GWAS instituting, as they became the core around which genome-wide association study methodology was built:

\subsubsection{Human Genome Project}

The successful completion of the Human Genome Project (HGP) was an unprecedented scientific achievement; it was the absolute milestone in biology and definitely a turning point in medicine. The HGP has provided an essential foundation for sequencing and analysis of additional human genomes and has laid the foundation for all other discoveries about the human genome. The HGP became an invaluable resource in the search for genes that cause human diseases (monogenic and polygenic). Interestingly enough, the project even started as a motivation to find genes responsible for diabetes, 
many years before publications of the first drafts of the human genome (Venter et al., 2001; Lander et al., 2001).

The HGP established the consensus human reference sequence. It found that the approximately 3.3 billion nucleotides constitute the human genome. Particularly, the human genome seems to encode only 20,000-25,000 protein-coding genes, less than originally estimated (Lander et al., 2001). A very small part of the human genome is determined to be protein-coding sequences, as the total length covered by the coding exons is approximately $34 \mathrm{Mb}$ or $\sim 1.2 \%$ of the genome; the untranslated regions of the transcripts are estimated to cover another $\sim 21 \mathrm{Mb}$ or $\sim 0.7 \%$ of the genome (Lander et al., 2001). The analysis and establishment of the reference sequence was complicated by the presence of polymorphism in the DNA samples, because it was hard to distinguish whether differences between sequence clones reflected errors or polymorphism (Lander et al., 2001).

\subsubsection{HapMap Project}

While the reference sequence constructed by the Human Genome Project is informative about the massive bases in DNA sequence that are invariant across individuals, it did not capture the natural variation of the human genome. The Haplotype Map of the Human Genome Project (HapMap) was founded to focus on DNA sequence differences and to determine common pattern of variation across the human genome (The International HapMap Consortium 2004, 2005). The HapMap Project was launched in 2002, and was a natural extension of the Human Genome Project (The International HapMap Consortium 2003; Lander 2011). The HapMap Project has helped understanding human diversity. It also has helped detect positive natural selection across the human genome (Sabeti et al., and The International HapMap Consortium 2007).

A major motivation for the HapMap was to catalogue and compare variations in various human populations, find their frequencies and associations and make it publicly available for medical research. The idea about helping medical research was based on notion that inherited genetic variation has an important role in the pathogenesis of disease. Interestingly enough, the seminal paper on HapMap Project cited that "the root causes of common human diseases remain largely unknown, preventative measures are generally inadequate, and available treatments are seldom curative" and further stated those reasons as a motivation for the project work (The International HapMap Consortium 2005). The comprehension of genetic background of human diseases has demanded examination of genomes of individuals and comparison of all genetic differences between controls and affected groups. The comparison between groups ultimately could have been accomplished by complete sequencing of the genomes of individuals under study. However, more practically, an alternative comparison between 
existing common variability in human groups, by which the humans differ, is used for finding variation difference in GWAS analysis (Hinds et al., 2005). Today, after facing collection of enormous population variation data and near exhausted analyses of all existing data for some diseases, the search for disease causing variants is back to sequencing methods: a whole-genome sequencing that enables characterization of almost all variants in an individual or, a whole exome sequencing (WES) that provides all variants in protein coding genes.

The HapMap project helped to guide the design and prioritization of SNP genotyping assays for GWAS methodology by documenting the substantial recombination hotspots, structures of linkage disequilibrium and low haplotype diversity linking correlations of SNPs with many of their neighbors (The International HapMap Consortium 2005; Manolio, Brooks and Collins 2008). This feature has been used for finding the relevant SNPs in common diseases.

The HapMap project has achieved a high-density haplotype mapping, first starting with smaller number of population representatives (few representative groups), searching variations in blocks of $500 \mathrm{~kb}$, and later extending it to 100-kilobase regions and larger reference panel (eleven different populations instead of the original four). Advanced phases of the HapMap project encompass variants not only restricted to common SNPs (MAF $\geq 5 \%$ ), but also low-frequency $(0.5 \%<M A F<5 \%)$ variants that account for the vast majority of the heterozygosity in each sample, and a large number of rare $(0.05 \%<$ MAF $<0.5 \%$ ) and private (singletons and MAF <0.05\%) variants (The International HapMap Consortium $2007,2010)$. At that point, the project was also able to detect copy number variations. With the HapMap extensions or phases, a very slight improvement happened for finding common SNPs, but there was greater improvement in genotyping for low frequency and rare SNPs (The International HapMap 3 Consortium 2010). HapMap 3 catalogued both allele frequencies and linkage disequilibrium (LD) between new variants. The vast majority of common SNPs strongly correlate to one or more nearby proxies, which lead to the conclusion that 500,000 SNPs can provide excellent power to test $>90 \%$ of common SNP variation in out-of-Africa populations, with roughly twice that number required in African populations (The International HapMap Consortium 2005).

The HapMap project originally genotyped one million SNPs, but updates over years increased that number to more than 10 million common DNA variants, primarily SNPs, although still in a limited set of DNA samples (The International HapMap 3 Consortium 2010). The vast majority of common variants either is represented in dbSNP database, or is in tight correlation to other SNPs that are represented in dbSNP database (The International HapMap 3 Consortium 2010). 
The HapMap 3 data were obtained with the Affymetrix Human SNP array 6.0 biochip (interrogating 1,852,600 genomic sites) and the Illumina Human1M-single beadchip (interrogating 1,199,187 genomic sites), that were initially applied to 1,486 and 1,284 samples, respectively (The International HapMap 3 Consortium 2010). The HapMap project data constantly gets updates with improvements in sequencing technology, especially for low-frequency variation. We used HapMap 3 SNP data for our research as the most current.

\subsubsection{Linkage Disequilibrium}

Linkage disequilibrium (LD) is defined as a non-random correlation between two loci (two different SNPs or other markers) or allelic association between two alleles. The LD phenomenon is known to exist in the human genome (Gabriel et al., 2002), but vary within human populations. It signifies haplotype maintenance through the evolution. The physical arrangement of SNP alleles along a chromosome is called an "haplotype" (Olivier 2003), and it became an essential characteristics exploited in the GWAS methodology. Properties of LD in the human genome have been studied in detail by HapMap project with the aim to understand both the causes of LD and its application to disease research (Olivier 2003; The International HapMap Consortium 2005).

LD is a consequence of common ancestry and recombination pattern in human genome and is a measure of coinheritance of human haplotypes. LD is defined by parameters providing information about how often two or more loci or alleles are inherited together (D) and how related to each other they are depending on their frequency in the population (r2). In the case of high LD, both measurements are close to 1 (on a scale ranging between $0-1$ ).

The principle of LD at the population level allowed for GWAS methodology, as GWASs are based upon LD. When a sufficient number of SNP markers or tags is available for testing in a genetic study (like it is in GWAS), any common variant, even if it was not assayed directly, should display significant LD with neighboring marker SNPs. A set of evenly spaced SNPs at high resolution across the genome should permit whole-genome association studies. Furthermore, it has become clear that the extent of LD and haplotypes in the human genome is not simply a function of distance between SNPs. The HapMap project found that the LD varies markedly on a scale of 1-100 kb; the LD not only varies in length, but is also often discontinuous and does not proportionally decline with a distance (The International HapMap Consortium 2005). The data reveal that SNPs are typically perfectly correlated to several nearby SNPs, and partially correlated to many others (The International HapMap 3 Consortium 2010). Considering only common SNPs (the original target of study for the HapMap Project) in the population of European ancestry or CEU, one in five SNPs has 20 or more perfect proxies (with perfect LD of 1), and three in 
five SNPs have five or more perfect proxies. In contrast, one in five has no perfect proxies (The International HapMap Consortium 2005; The International HapMap Consortium 2007).

\subsubsection{1000 Genomes Project}

After several years of GWAS, it became clear that a more in-depth look at variation, including rare variation, was necessary to explain additional disease heritability and relationships between phenotype and genotype. The new project, 1000 Genomes Project (1KGP) was designed to provide detailed description of common human genetic variation by applying whole-genome sequencing of healthy people and their progeny belonging to different human populations (The 1000 Genomes Project Consortium 2010). Variations have been obtained by direct sequence comparisons, instead of exploring variations using allele frequencies, by applying a combination of low-coverage whole-genome sequencing, and deep exome sequencing. The 1KGP sequenced 2500 individuals and gathered information on variants in different populations. It provided accurate haplotype information on all forms of human DNA polymorphism in multiple human populations (The 1000 Genomes Project Consortium 2012, 2015). Advances in DNA sequencing technology have enabled the sequencing of individual genomes for the $1000 \mathrm{G}$ project (Bentley et al., 2008).

Coming after massive GWAS implementation, the $1 \mathrm{KGP}$ boosted more detailed and more accurate GWAS data analyses, overcoming limitations of the HapMap project as the only reference for GWAS. The first results from the 1000 Genomes project (the pilot project) were reported in 2010 (The 1000 Genomes Project Consortium 2010). Results of the 1KGP have provided researchers with a population scale map of rare variants to complement and enrich existing knowledge of common variants gained from the HapMap project.

The data from the 1KGP have provided the location, allele frequency, and local haplotype structure of approximately 15 million SNPs (The 1000 Genomes Project Consortium 2012). With the advancement in number of individuals within additional new populations, the 1KGP was able to uncover 84.7 million single nucleotide polymorphisms (SNPs) in addition to other variations of human genomes (insertions and deletions, copy numbers, to name a few), that are far less common in humans (The 1000 Genomes Project Consortium. 2015). The $1 \mathrm{KGP}$, in addition to discovering common variants (MAF $\geq 5 \%$ ), estimated at 8 million, has enabled fine mapping of loci for both low frequency $(0.5 \%<$ MAF $<5 \%)$ estimated at 12 million, and rare (scarcer) variants (MAF < 0.5\%) and private variants, estimated at over 64 million. Regarding population specificities, most common variants are shared across the world, and rarer variants are typically restricted to closely related populations. A major chunk of SNP variability ( $86 \%$ of variants) is restricted to a single continental group. Up to one third of all SNPs for European 
ancestry population (CEU) detected by the 1KGP were novel SNPs (The 1000 Genomes Project Consortium 2010, 2012, 2015).

The project found that the public databases were less complete for coding SNPs at low frequencies, especially for the lower frequency SNPs outside the exons. Of special interest was the finding that detected variations were not evenly distributed across the genome: certain regions, such as the HLA, show very high rates of variation, while others do not (important for common autoimmune/inflammatory diseases).

Although it is difficult to conclude accurately the number of functional SNPs for each individual genome, collectively there were around 10,000 synonymous SNPs and 11,000 nonsynonymous SNPs as reviewed by the project. Approximately one in twenty of nonsynonymous SNPs was found to be damaging SNPs (loss of function (LOF) and damaging function SNPs), which means that an individual might have between from 5 to 250 damaging SNPs per exome, as estimated by extrapolation of the results, apparently depending on the used methodology (The 1000 Genomes Project Consortium 2012, 2015). Overall, an individual genome might have between 3-4 million SNPs, but only 40,000 to 200,000 SNPs $(1-4 \%)$ of them are not common SNPs and have a frequency $<0.5 \%$. The $1 \mathrm{KGP}$ found that a typical genome contains 149-182 sites with protein truncating variants, 10,000 to 12,000 sites with peptide-sequence-altering variants, and 459,000 to 565,000 variant sites overlapping known regulatory regions (untranslated regions (UTRs), promoters, insulators, enhancers, and transcription factor binding sites). It also observed around 2,000 variants per genome associated with complex traits found through genome-wide association studies (GWAS Catalog) and 24-30 variants per genome implicated in rare disease through the Clinical Variation database (ClinVar) (The 1000 Genomes Project Consortium 2015).

Comparison of the two data sets of detected SNPs from the HapMap project and from the 1000 Genomes project revealed that approximately $72 \%$ of HapMap SNPs were also found in $1 \mathrm{KGP}$ data. After filtering out HapMap variants with a MAF of <5\% (separately for each population), 99\% of HapMap SNPs were found in $1 \mathrm{KGP}$ data (Buchanan et al., 2012). However, the rare variants differ more between projects than the common variants. Although the number of $1 \mathrm{KGP}$ variants increased several times, when the recalculation of variants are performed for a particular GWAS, the number of imputed common and intermediate frequency variants increased by $7 \%$, whereas the number of rare variants increased by $>50 \%$. These differences in the detected polymorphism might affect SNP queries or imputation used in GWAS analyses. The number of novel variants is constantly increasing and many believe that the $1 \mathrm{KGP}$ potentially has overshadowed the utility of HapMap (Buchanan et al.; 2012). 
Both the HapMap and 1KGP projects have contributed to identifying linkage disequilibrium patterns and consequently the ability to choose tag SNPs for further GWAS studies. Based on completely different technologies, they also contributed to studying genomic structure, recombination rates, and mutation rates.

In our study, we used data based on the human genome LD results and SNPs data obtained from both HapMap3 project and 1KGP. All GWAS SNPs studied in our research have been confirmed by the 1KGP. For the all GWAS SNPs there was a difference between two sources only in very few SNPs.

\subsubsection{The Encyclopedia of DNA Elements (ENCODE) project}

Once the human genome sequence is established, the next step was the identification and annotation of functional DNA elements in the human genome. The Encyclopedia of DNA Elements (ENCODE) project has been established with the aim to identify and catalog all genomic sequences according to their functions (ENCODE Project Consortium 2004, 2007, 2012). The ENCODE pilot first was tasked with annotating predefined $1 \%$ of the human genome or $30 \mathrm{Mb}$ with functional elements (ENCODE Project Consortium 2004, 2007). The experience and methodology from the pilot project have been consequently extended to rigorous analyses of elements with biological information of the entire genome. ENCODE project is an ongoing collaborative effort that is using different technologies and approaches to identify functional elements (ENCODE Project Consortium 2007, 2012).

The ENCODE has produced high-resolution reproducible maps of DNA segments with biochemical signatures associated with diverse molecular functions (Lander 2011; Ernst et al., 2011; Gerstein et al., 2012). ENCODE project define functional elements as discrete sequence elements that confer biological function. They might be segments that have reproducible biochemical functions (such as binding sites or other chromatin distinctive structures) or serve as templates for defined products (proteins or non-coding RNAs). They are mapped as genes, transcripts, regulatory regions, chromatin sites and methylation patterns. ENCODE project changes the definition of genes to include regions of genome outside of the well-studied protein-coding regions and not only narrow continuous space around protein-coding regions (Rodriguez-Fontenla et al., 2014). Generated detailed maps came from few dozen human cell lines (ENCODE Project Consortium 2011). The ENCODE data in particular enabled assigning various potential biochemical functions for up to $80 \%$ of the genome (Kellis et al., 2014). In contrast to evolutionary and genetic evidence, biochemical data offer clues about both the molecular function of DNA elements and the cell types in which they are active (Kellis et al., 2014). However, the jury is still out regarding the definition of what constitutes biochemical functionality of the huge part of genome, when it is known that only up to $10 \%$ of the human genome is filtered under pressure of the natural 
selection and conservation (Brunet and Doolittle 2014). The findings are not widely accepted because the authors are not predicting how many functional elements such genome might claim (Kellis et al., 2014).

Why is ENCODE important for GWAS? ENCODE data might provide a starting point to study human disease in addition to differentiation and development of particular cells (Ernst et al., 2011; Gerstein et al., 2012; Kellis et al., 2014), as they can be linked with SNP risk factors detected in GWAS. The ENCODE maps might help interpretation of GWAS signals, connecting SNPs with potential mechanisms of gene regulation (Kellis et al., 2014). ENCODE data for a particular region of interest can be retrieved from HaploReg database using search with preset options of LD, type of population, source etc., and from RegulomeDB using its own algorithms for prediction of regulatory significance of elements. Other tools for visualization are available from UCSC Genome Browser (Kent et al., 2002). The browser can be used to show functional features in the region of interest (ENCODE Project Consortium 2010, 2011).

In our study, we used ENCODE data for our research, since the ENCODE project enabled searching for a SNP potential change of a functional element, especially the AID SNPs that reside in the non-genic regions with no clear known functions.

\subsubsection{Genotyping biochip}

Essentially critical for the implementation of GWAS have been the advancements in biochip manufacture technology. Standard chips are structured to detect tag SNPs relatively evenly distributed along the whole genome. The number of SNP tags has constantly increased over time, making standard chips able to provide better resolution suitable for high-density mapping (Eberle et al., 2007). However, in most cases high resolution was not enough to distinguish an association signal due to a direct risk SNP from an indirect association signal due to a LD effect. Standard genotyping chips currently used for GWAS are not well suited for either picking up the remaining common variants that are not yet discovered, or for identifying rare variants, due to the limitations of the technology and because of occurrence of perfect LD (Cortes and Brown 2011).

Other genotyping limitations of the chips in use have been also recognized. The majority of genotyping chips are designed for use in European populations. They might be less informative for other ethnic groups, especially admixtures, particularly if the SNPs are not shared between populations. Another weakness is that many rare variants have yet to be identified and are not represented on the chips (Cortes and Brown 2011; Lee et al., 2014). 
Some newer microarray-based genotyping technologies permit flexibility in choosing the scope and density of SNP markers. The development of custom genotyping chips such as the Immunochip designed for immunogenetics studies, the Metabochip designed for studying metabolic diseases, or a CD chip for cardiovascular disease, has further advanced GWAS research.

Initiated by the Wellcome Trust Case-Control Consortium, the Immunochip was designed by leading investigators covering all of the major autoimmune/ inflammatory diseases (Cortes and Brown 2011). These specific biochips have better resolution in the regions of interest, but on the other side, they might be biased, as they limit a GWAS to the narrower predetermined loci, ones that were selected based on previous knowledge and assumptions coming from it. They potentially may leave undetected loci that have been previously unsuspected loci, undetected loci, loci across the whole genome and loci not related to the previous knowledge (Cortes and Brown 2011). However, at loci with established disease association, the Immunochip contains all known SNPs in the dbSNP database, as well as from the 1000 Genomes project 2010 release (The 1000 Genomes Project Consortium 2010).

Taking into consideration the latest 1KGP results, the current GWAS chips obviously do not identify rare variants very well. Although the companies are now racing to increase rare variant coverage on genotyping chips, it might not be the easiest task, and plain whole genome sequencing (WGS), or even whole exome sequencing (WES), are becoming more popular as a more accurate way to find rare variants. Nevertheless, very high-density chips such as the 5 million SNP chips are existing products in the Illumina pipeline (Cortes and Brown 2011).

With the advancement in human genome knowledge and technology, it is necessary to understand complexity of findings and take into consideration a design of studies and especially interpretation of rare variants results (Lee et al., 2014).

\subsubsection{Other advancements contributing to GWAS implementation}

Additional important advancements that made GWAS possible were technological advancements in computation and informatics, which enabled data storage in various structured biological databases, web-based tools for storing and sharing data and management of unprecedented amount of data for further search and processing. The advancement in construction of databases has been combined with the capability of providing almost endless innovative ways to search for and analyze data (Merelli et al., 2014). These innovative approaches have mined data to provide new results, previously not accessible by any experimental method. The new protocols have become real experiments in the virtual spaces (Agarwal et al., 2014). 
Change of social sharing attitudes, mandatory open access policy for scientific publications, overall public educational advancements, easier information accessibility and exchange, and other changes in social behaviors, all have contributed to a certain extend to broaden ideas of human genomic research. All instances allowed for recruitment of individuals eager (and curious) to provide their DNA specimens necessary for human genome research and GWAS applications (Turner et al., 2011).

Essential to the GWAS implementation is data sharing: fact is that the GWAS research has been critically dependent on data and ideas sharing among various professional and research groups. Exceptional collaborations and huge consortia formations have been necessitated by almost unsurmountable number of human subjects needed for the studies and by complexity in management of generated data.

As GWAS emerged as a powerful unbiased tool for discovering the genetic basis of complex human diseases or traits, and number of SNP markers genotyped by GWAS has dramatically increased, the GWAS statistical design and analysis has been addressed by multiple researchers groups. The goal of statistical improvement has been to identify as many significant features in the genome as possible, while incurring a relatively low proportion of false positive and false negative results (Lee et al., 2014).

The testing of a large number of potential variants in parallel, requires a stringent threshold for significance in order to limit false positives (type 1 errors), meaning that discovery of variants responsible for small effects requires very large sample sizes (Storey and Tibshirani 2003). The large number of correlated markers demanded the multiple hypotheses testing correction (Han et al., 2009). Failure to adjust for multiple testing appropriately might produce excessive false positives or overlook true positive signals. Multiple testing is a challenging issue in genetic association studies not only because of large numbers of markers, but also because many of them exhibit linkage disequilibrium (LD) and are not independent entities (Storey and Tibshirani 2003). The approaches that assume independence may be highly conservative, including Bonferroni correction that is often thought to overestimates the statistical penalty of performing many correlated tests (Gao et al., 2008).

The current concept is to control the false discovery rate (FDR), or the expected proportion of false positives among the rejected hypotheses (Benjamini and Hochberg 1995). The FDR (also called the Bayesian false discovery rate) is a sensible measure of the balance between the number of true positives and false positives in many genomewide studies (Storey and Tibshirani 2003). From a practical viewpoint, the procedure is simple: input the p-values for the genes into an FDR software, get the output of the corresponding q-values (Storey and Tibshirani 2003), and then declare an event significant, if its q-value is less than or equal to 0.05 . This supposedly ensures the FDR to be controlled at $5 \%$ level. 
Novel approaches have been also recently explored, in order to increase the heritability estimation from detected GWAS SNPs and to enhance the detection of newer, rare SNPs that might contribute to explaining heritability without increasing the size of study groups (Yang et al., 2011; Liley and Wallace 2015). Some novel statistical approaches have been proposed recently, but the clear benefits are still unknown; the aim is to extract more SNPs without redoing new studies or increasing the number of subjects, or simply to provide more accurate results (Lee et al., 2014; Lin and Lee 2015). A statistical approach plays a crucial role when it comes to making decisions on cut-off values and exclusion/inclusion of GWAS detected SNPs.

\subsubsection{GWAS database}

As the interpretation of GWAS findings in the context of the disease biology remains challenging, it is essential to make all GWAS data as accessible as possible for the broader scientific community for further analyses. The most complete public database of GWAS data is the NHGRI catalog of GWAS (Welter et al., 2014). The NHGRI Catalog is manually curated and maintained by a professional staff, because the data extraction is an expert activity. The GWAS Catalog data are solely extracted from single studies or meta-analyses, typically reported in peer-reviewed publications (Welter et al., 2014). Usually GWAS data are deposited in the $\mathrm{dbGaP}$ as well, where users must apply for access to individual-level genotype and phenotype data and comply with a data access agreement (Mailman et al., 2007). Deposited GWAS in the NHGRI catalog must conform to the eligibility criteria, including number of SNPs assayed and non-targeted study design (Hindorff, Gillanders and Manolio 2009). The Catalog has been constantly under development. A recent improvement is to structure the trait annotations using ontology principles. All data in the GWAS Catalog will be integrated in an ontology format that can be processed by an ontology reasoner and queried. The schema ontology has been designed to model the key concepts represented in the Catalog: trait, SNP, study, gene, chromosome and relationships that link these concepts (Welter et al., 2014). The availability of mappings between GWAS traits and ontology terms facilitates the integration of the GWAS Catalog with other resources.

GWAS Catalog data can be accessed in few ways. GWAS Catalog data are available through commonly used data portals such as PheGenl tool among others (Mailman et al., 2007). Data are also accessible via the web interface hosted at the NHGRI.

A major challenge for the Catalog future development is the increasing complexity of studies that include ethnicity extraction, search interface to support ontology-driven querying, gene interactions, and accommodation of next generation sequencing technology, as well as improving links to other resources. 


\subsection{Genotype-phenotype relationship in AID}

\subsubsection{From SNPs and genes to molecular networks and pathways}

Genome wide association studies (GWAS) have collected valuable data that provide useful insights into the genetic heritability of many common diseases, including the AID. However, not surprisingly, the plethora of GWAS data has remained unused to its full potential, because the biological mechanisms underlying genotype-phenotype relationships in common diseases remain only partially explained, even for the most studied diseases like T1D (Schork et al., 2009; Carter, Hofree and Ideker 2013). Very few mechanisms by which the common diseases occur are known, leaving majority of GWAS findings outside our useful incorporated knowledge.

Counterintuitively, the GWAS data collected to explain heritability of common diseases, also may help us comprehend underlying biological mechanisms, by finding missing links and putting forward predictions for experimental assessment (Goldstein 2009; Califano et al., 2012). The rationale is that, if a SNP detected by a GWAS is a risk factor for common disease development, than a gene deviated (changed or influenced) by the same SNP is the gene that participates in common disease pathogenesis.

From the very beginning of GWAS data usage, quite odd and unexpected relationships have been discovered for some common diseases. Macular degeneration has been extremely significantly linked to $\mathrm{H}$ factor (CFH gene) of the complement system (Fritsche et al., 2014), creating a connection that was never suspected before. IL28B (renamed IFNL4) gene and its pseudogene condition the chronic viral hepatitis $C$ infection and clearance (Suppiah et al., 2009), but this gene was even unknown before and never discovered to be linked to immune system. However, some crucial genes have been omitted for unknown reasons, such as $\mathrm{C} 4$ and $\mathrm{C} 3$ genes from human complement system not being picked by numerous schizophrenia GWAS until recently, when the genomic variations of nearly 65,000 people were analyzed (Daly et al., 2016), linking them to this common disease.

In addition, because of the knowledge gaps, the overwhelming wealth of common disease genomic data has neither materialized into the development of new therapeutics, not even for the most studied common diseases like diabetes mellitus; nor it helped explain why certain drugs do not help in treatments of some patients (Goldstein 2009; Califano et al., 2012). This application of GWAS data is now in the focus of many pharmaceutical companies, which do not share genomic data with the broad scientific community. Consequently, this lack of knowledge hampers to great extent a goal of 
personalized medicine to assess and use patient's individual genotype to guide clinical care (Fernald et al., 2011).

In common diseases, where the heritability is based on many genes, expressed disease phenotypes are reflection of various pathobiological processes that interact within a complex network; a disease phenotype also progresses over time as gene expression and epigenetic control change (Barabási et al., 2011). The genetic variants (overwhelming majority being SNPs) condition disruptions/modifications of coordinated numerous biological processes within cells and tissues, and establish their transformations into pathobiological processes that result in common diseases phenotypes (Barabási et al., 2011).

In the near future, it is likely that a catalog of virtually all human genomic variations will be produced. However, it is still daunting question how this amazing knowledge might be translated into knowledge about disease mechanisms (Vidal, Cusick and Barabási 2011). There are still major unsurmountable problems regarding how to model human genetic variation impact on formation of pathogenic pathways and networks (Vidal, Cusick and Barabási 2011).

The phenotypic impact of gene variant modifications, including deficiency of any specific gene product caused by SNPs, is not determined solely by the function of a mutated gene, but also by the functions of components with which the gene and/or its product interact (Goldstein DB. 2009). In other words, the impact of any mutation in a gene (SNPs are mutations) is determined by its product network context, making its impact contingent on a very complex modified network that might be changed at multiple points simultaneously (Califano et al., 2012). Even when the interactants are not changed per se, the impact of an original altered gene has multiple outcomes, usually hard to predict and comprehend in advance (Barabási, Gulbahce and Loscalzo 2011).

The potential complexity of the human interactome network is daunting: with 20,000 plus protein-coding genes, encoding yet undefined number of splice variants and post-translationally modified forms of proteins, significant but not yet defined number of functional RNA molecules, and with about a thousand metabolites, the distinct cellular components that serve as the components (nodes) of the interactome easily exceed one hundred thousand (Barabási, Gulbahce and Loscalzo 2011). The number of functionally relevant interactions between the components of such a huge network is expected to be much larger and remains unknown (Venkatesan K et al., 2008).

For the exploration of the complex interplay between human interactome and human diseases, it might be first desirable to comprehend current molecular pathways and phenotypic network maps. The 
protein-protein interaction maps have been constructed with great effort and organized into comprehensive databases, such as MINT, IntAct, BioGRID, HPRD, which are still considered largely incomplete. Metabolic pathways are probably the best identified part of interactome, with KEGG metabolic map collection being the most complete database of human pathways. Mapping of human regulatory networks is in its infancy. RNA networks, encompassing RNA-RNA and RNA-DNA interactions, are still work in progress. However, for some types of RNAs, like microRNAs, interaction data are organized into several comprehensive microRNAs databases with predicted miRNA targets (Barabási, Gulbahce and Loscalzo 2011).

When the human interactome was analyzed as a network by applying network theory rules (Barabási and Albert 1999), the presence of highly connected hubs was determined (Jeong $\mathrm{H}$ et al., 2001). These hubs hold the whole network together and have specific roles in the human interactome. They encompass highly connected proteins that are classified into "party" hubs (that function inside specific cellular processes) and "date" hubs (that link together different specific cellular processes). Evidence from model organisms indicates that hub proteins tend to be encoded by essential genes (Fraser et al., 2002), and that genes encoding hubs are older and evolve more slowly than genes encoding non-hub proteins (Saeed and Deane 2006).

When the position of disease genes in relation to hubs was researched, it was found that essential genes, but not the disease genes, encode hubs (Jeong et al., 2001). From an evolutionary perspective, it is understandable that mutations that disrupt hubs have difficulty being transmitted in the human populations as they negatively influence huge number of biological processes and cannot be kept in the gene pool because of their lethality. Disease genes obviously thrive in the human population, meaning they are transferable because they do not influence survival. Only mutations that functionally impair peripheral genes can persist, forming a pool of mutations conditioning heritable diseases most of which appear in adulthood (Barabási, Gulbahce and Loscalzo 2011). Peripheral genes do not show a tendency to encode hubs and tend to be tissue-specific (Goh et al., 2007). That means most of the common disease genes could be classified as peripheral genes. At the same time, it was discovered that peripheral genes have fewer interactions and might be involved in fewer pathways (Bossi and Lehner 2009). These tissue specific and more recently evolved proteins make fewer interactions than core proteins and participate in fewer pathways. The older a protein is, the more interactions it makes (Bossi and Lehner 2009).

It was also found that newer, peripheral and most likely disease genes interact with hub genes. They interact with hub genes even more often than with each other. Most tissue-specific proteins bind to universally expressed proteins, and function by recruiting or modifying core cellular processes into 
tissue-specific biological processes. On the other hand, most 'housekeeping' proteins that are expressed in all cells also make highly tissue-specific protein interactions; possibly all 'housekeeping' proteins actually have important tissue-specific molecular interactions (Bossi and Lehner 2009).

The hubs making essential genes are proposed to be involved in tissue specific processes as different splicing variants adapted for specific tissue processes. Understanding tissue-specificity is often instrumental to understanding complex diseases, because particular interactions may occur in one type of tissue with participation of expressed, specific and core proteins, while they do not occur in another type of tissue. The same set of genes is present, but they remain inactive in other tissues; when they are not expressed simultaneously, there are no interactions, although theoretically they may occur. In other words, when proteins qualified to interact are not expressed and brought into physical proximity at the same time, they do not necessarily interact in particular tissues even if they belong to hubs (Bossi and Lehner 2009; GTEx Consortium 2015).

This concept of tissue specific and disease genes among them, interacting with modified older core 'housekeeping' genes might help find specific pathways for disease genes and modulate them with applicable drugs in an easier way than projected. It is well recognized that, while the genetic variants that cause hereditary diseases are global (detected in germline) and present in all cells across the entire human body, the diseases are often only manifested in specific tissues. The mechanism for this tissue selectivity and vulnerability at the same time is unknown (Barshir et al., 2014).

\subsubsection{Basic concepts of biological networks and pathways}

Because the biological processes are accomplished via proteins, RNAs and intermediary products, a potential way to explore connections between GWAS results and phenotypes is to identify pathways or networks where these genes execute their roles, and connect their functions with a specific disease. Depending of their expressed variants, proteins behave differently in pathways, or in networks, because protein variants influence the speed and size (quantification) of biological processes. Most likely, the same is true for nucleic acids, where a change in their sequences (such as SNPs) might influence their functional roles (van der Sijde et al., 2014).

Given the complex genetic architecture and synergistic effects among genes, the holistic effect of a network or a pathway is expected to have a larger effect than the sum of the individual effects of each gene in the structure (Khatri, Sirota and Butte 2012). This approach also cuts complexity, increases the power of analyses, and, importantly, allows for easier biological verification (Khatri, Sirota and Butte 2012). Extrapolation from SNPs to pathways or networks via affected gene products (usually, but not 
necessarily proteins), also overcomes the problem of SNP variation in different human populations with the same phenotypic outcome of a specific disease. Various SNPs present in human population groups within the same region might have the same functional consequences. Associated pathways might be more consistently replicated across ethnic groups, compared to the SNP-level association for the same type of disease (Schaid et al., 2012).

Consequently, using the knowledge of predefined canonical pathways (majority are experimentally discovered), the findings of disease-associated pathways can be complementary to the single SNP/single gene analysis for better understanding of the molecular mechanisms in diseases (Khatri, Sirota and Butte 2012). Combining data from GWAS, these SNP-pathway approaches can assess whether groups of genes with related functions are jointly associated with a disease of interest and help generate specific hypotheses for follow-up experimental studies (Carter, Hofree and Ideker 2013; Khatri, Sirota and Butte 2012).

Networks are usually defined as collections of nodes that are joined together by edges; edges could be with or without direction. Biological network models are built from experimental data, but also from queries of the literature databases and public databases of molecular interactions (Carter, Hofree and Ideker 2013). Nodes in the networks might represent various types of molecules, not only proteins, with edges representing relations between nodes not limited to direct molecular interactions (physical or chemical); edges might be defined as other types of relations: co-expression, co-localization, influence on gene regulation, co-regulation by a third molecule, feedback regulations, etc. (Szklarczyk et al., 2015; Carter, Hofree and Ideker 2013).

\subsubsection{Biological pathways}

A biological pathway represents a set of molecules connected with series of actions among molecules that results in an end-product. Its definition has evolved to describe a pathway as a set of interacting genes (or their products) that together performs a very specific biological function. A biological pathway is the strictest definition of a gene set. Relations between members, almost exclusively proteins, are restricted to biochemical reactions or direct physical interactions that build or resolve molecular complexes. Biological pathways are vector-driven structures towards an essential, specific end-point and are finely balanced by quantities and location of every component and reaction, including its regulation or regulation of adjacent pathways (positive or negative feedback). Biological pathways are usually derived from experimental work over time, but with development of bioinformatics software and databases, they might be constructed by imputation and then tested experimentally. 
More recently, four types of pathways have been proposed in attempt to describe the heterogeneity of currently available pathways: molecular, cellular, disease, and intervention pathways (Mooney et al., 2014; Ramanan et al., 2012). Molecular pathways characterize biochemical actions on a molecule or compound; cellular pathways model the regulation of more global cellular processes. It was noted, however, that disease and intervention pathways may simply be collections of genes previously associated with a phenotype, or sometimes combination of basic pathways (molecular or cellular), rather than being based on knowledge of precise biological mechanisms (Ramanan et al., 2012). In addition, concerns about the hierarchical nature of biological pathways have been raised, meaning that some pathways can represent subsets of larger pathway modules. Evidently, the pathways may differ by size (20-200 members), complexity and data sources (Mooney et al., 2014). These differences can have consequences for the interpretation, confidence, and comparability of results from gene set analyses (Khatri, Sirota and Butte 2012).

Pathways are usually much smaller structures than networks, with fewer members and relations than networks, because they are structured usually on experimental data and annotated by a few groups of experts (Barabási, Gulbahce and Loscalzo 2011). Pathways might be defined as a very specific narrowly defined type of a directional network that has a start and an end.

In an ideal case, GWAS candidate loci can be linked to a phenotype using canonical pathways (Carter, Hofree and Ideker 2013; Goldstein 2009). However, in most cases, candidate genes implicated or imputed by GWAS studies are not well characterized and their products have not been included in any known canonical signaling/metabolic pathways. Additionally, a good portion of candidate genes is not qualified beyond its sequence, with no function assigned to it and no annotations. Some candidate genes even have no defined orthologues to provide additional information about their potential function.

Even when they exist, however, canonical pathways are likely to be incomplete and even inaccurate (Carter, Hofree and Ideker 2013; Califano et al., 2012). Some estimates go as far as to claim that many pathways are not known currently and are yet to be discovered (Carter, Hofree and Ideker 2013; Califano et al., 2012), as current data shows that more than $90 \%$ of known proteins are not located in any pathway. Systematic screens of the proteome suggest that canonical pathways capture only a fraction of the true protein-protein interactions that occur within the cell (Guruharsha et al., 2012). Furthermore, biological pathways are often treated in studies that connect pathways with GWAS data as a set of related genes, which jointly perform a biological function, ignoring the specific and directional relationship among the members. This approach of treating a pathway as a network creates partial and vague pathways and not directional pathways, driven in a directional mode with an end-product. 
The linking of GWAS SNP harboring genes within canonical pathways is not only a labor-intensive task; the uncertainty of its execution and outcome is relatively high.

Adding to the problem of defining pathways that hypothetically underlie diseases under GWAS, is the fact that the associated GWAS SNP loci are not always tied with any protein coding genes, even after analyzing for LD (van der Sijde et al., 2014; Hindorff, Gillanders and Manolio 2011). Most of GWASimplicated risk variants reside outside of protein coding genes. These GWAS SNPs influence nonprotein-coding genes or not yet identified genes, as they appear to cluster in stretches of regulatory DNA sequences or regions of DNA with no known formations (Maurano et al., 2012). It has been recently suggested that the majority of the genome is involved in biochemical and regulatory activities of cells, not just the $1 \%-1.5 \%$ of the genome responsible for encoding proteins (ENCODE Project Consortium 2012 ref18). This discovery is augmenting the complexity of currently existing pathways with additional potentially huge number of unknown interactions (Dunham et al., 2012). New classes of molecule such as microRNAs and lincRNAs are increasingly implicated in regulating the activity of protein coding genes (Fernald et al., 2011), but they are not integrated into biochemical or signaling pathways, which at this point are only consisting of protein members. Non-coding genes are currently implemented only in a very few canonical pathways and only in cancers (according to the data available in pathway databases such as KEGG, BioSystems, and NCI Pathways). In cancers, however, the somatic human variability is often different from the heritable variability (SNPs in germline) discovered in common diseases. Hence, even when integrated into a few biological pathways, these non-coding gene variants are not applicable to common diseases. Furthermore, the pathways might be wired in a different way in neoplastic cells compared to non-cancerous cells (ENCODE Project Consortium 2012).

Non-coding genetic alterations, even those affecting non-coding RNA (ncRNA) sequences, are suspected to mediate phenotypic effects primarily by altering the abundance of proteins in the cell and thus perturbing Protein-Protein Interaction (PPI) networks through stoichiometric effects (Esteller 2011; Carter, Hofree and Ideker 2013). Functions of these so-called regulatory SNPs could be very complex and elusive, and involve gene expression regulation through the effect on RNA splicing, transcription factor binding, DNA methylation and miRNA recruitment (Knight 2014; Huang 2015). Using ENCODE up to $80 \%$ of all previously reported associations data have some kind of functional annotations and mainly represent regulatory SNPs (Schaub et al., 2012; Ward and Kellis 2012). For a majority of associations, the SNP whose functional role is most strongly supported by ENCODE data is a SNP in linkage disequilibrium with the reported SNP, not the genotyped SNP reported in the association study (Schaub et al., 2012). However, reported functionalities currently are not incorporated into some structured biological processes, pathways or networks. In addition to regulatory non-coding SNPs, recent advances 
in the theoretical and experimental methods used to study DNA packaging within cells, elucidated the biological function and pathways to which SNPs located within gene deserts can contribute (Schierding et al., 2014).

An investigation of the tissue-specific effects of genetic variants on gene expression uncovered surprisingly complex relationships (Fu et al., 2012; Carter, Hofree and Ideker 2013). Unknown complex relationships between different molecules at this stage of the knowledge are suggesting that network models may be better suited than pathways for dissecting phenotypic consequences of non-coding variants (Ward and Kellis 2012; Kellis et al., 2014).

Canonical pathways are almost always represented as linear chains of events in order to better visualize relationships between molecules and to be able to make predictions for experimental tests in a manageable number of experiments. However, cell regulation is anything but linear; it is instead determined by complex multivariate interactions not amenable to visual interpretation (Califano et al., 2012). Pathways are oversimplified representation of biological processes and are not always able to explain processes (Kellis et al., 2014).

Biological pathways are collected and integrated in databases by initiatives like KEGG (Goto S. et al., 1997), Reactome (Joshi-Tope G et al., 2005) or the user-curated WikiPathways (Pico AR et al., 2008), and many other pathway databases.

\subsubsection{Biological networks}

In contrast to canonical pathways, biological networks represent a view of all connectivity among a large number of genes (Carter, Hofree and Ideker 2013). A biological network typically defines a partnership among molecules, regardless of actual molecular functions its members may perform (Sun 2012; Khatri, Sirota and Butte 2012). Unlike pathways, networks do not explicitly describe a specific biological function or process carried out in a specific biological context. Networks simply aim to describe biological relationships (observed or predicted interactions) between multiple genes or gene products. In general, the heterogeneous evidence is used to build publicly available PPI networks, and they are not centered on biological process (Mooney et al., 2014). Networks are composed mostly of proteins as gene products (e.g., PPI network) and define not only their physical interactions, but also co-

expression, mutual regulation, co-localization, co-mentioning in the same article (after text mining) or biochemical reactions in experimental data etc., as shown in STRING and other databases (Szklarczyk et al., 2015). 
Biological networks represent a collection of all known relationships in the knowledge space and are less stable than the well-curated canonical pathways. They might easily be expanded to contain proteinDNA interactions, or protein-DNA-ncRNA interactions, because these networks are not limited to a functional outcome. The understanding of these biological relationships is steadily growing, hence causing biological networks to expand and change (Sun 2012). However, various biases, including those introduced by measurement technologies, might be carried when the networks are constructed (Khatri, Sirota and Butte 2012).

Genetic variations such as SNPs that are influencing the abundance or activity of individual molecules, consequently affect the interactions and networks in which those molecules participate (Carter, Hofree and Ideker 2013). It has been proposed that to understand genotype-phenotype relationships it will be necessary to quantify the effects of mutations on molecular networks. These changes can have a spectrum of consequences, ranging from completely abrogating protein activity to having no effect at all, to even occasionally enhancing it (Wang et al., 2011; Carter, Hofree and Ideker 2013). A variety of computational strategies has been developed to predict the functional consequences of mutations at the protein level (Jordan et al., 2010; Sunyaev 2012).

Network- and pathway-based methods have been developed to boost the power of the candidate genes identified by GWAS and to bridge the gap between genetic variants and biological phenotypes (Sun 2012). According to some evaluations, these data have enabled a good part of the human genome to get some assignment of biochemical functions, in particular outside of the well-studied protein-coding regions (ENCODE Project Consortium 2012; Kellis et al., 2014).

\subsection{Biological Ontologies}

The term "ontology" has a long history in the philosophy since Aristotle. In the philosophical sense, ontologies are systems of categories that account for a particular way of seeing the world. The goal of building ontology is to construct an exhaustive and definitive classification of all entities (Gruber 2009).

In computer and information science, ontology is a technical term denoting an artifact that is designed for a purpose to enable the modeling of knowledge about some domain (Gruber 2009). Thomas Gruber defined "an ontology as a specification of a conceptualization" and "intended for modeling knowledge about individuals, their attributes, and their relationships to other individuals", and further simplified that "ontologies are what they do: artefacts to help people and their programs communicate, collaborate and 
coordinate" (Gruber 1993). Ontologies simplify unification among programs and serve in the role of data exchange and analyses. They are constructed in a specific domain for the purpose of enabling domain knowledge to be shared and reused. The term 'ontology' is also frequently used to refer simply to controlled terminologies. Controlled terminology defines the common vocabulary in which shared knowledge is represented (Gruber 1993).

New biotechnological discoveries from the last two decades have generated an unprecedented amount of information. At the same time, informatics and information technology became able to manage huge amounts of data and provide tools for their analyses. The amount of information in both bioscience and biomedicine has exponentially increased, especially with the acquired "omics" knowledge (Rubin at al., 2006). Researchers often face problems with extracting the data they need from the large numbers of data sources that are available. It has become a central problem to find ways to readily integrate information and data from biomedical and biological domains, and make it available for searching, sharing, reusing, analyzing, making new extrapolations and generating hypotheses. Difficulties to cope with data have necessitated repositories with the capability to be well maintained and useful (Lazakidou 2010).

According to the latest NAR Database issue, currently there are over 1,500 databases listed in the Molecular Biology Database Collection. All data are typically stored in publicly available databases on the web and often paired with informatics tools for their mining and analyses. In the biomolecular/biomedical domain, experts have been annotating biological entities with controlled vocabularies, introducing terminologies that have allowed common sharing and understanding of biological entities and processes they establish and partake (Lazakidou 2010).

In order to compute the huge factual scientific knowledge, the used semantics has to be precisely defined and standardized (Gruber 1993). To achieve this aim, terminologies and ontologies are used as instruments (Rubin et al., 2006). There are differences between the two formations. Terminologies use standardized and precisely assigned controlled vocabularies as a collection of descriptors for all entities in a domain, and principally represent simple collections of names in a domain, but do not provide relationships between entities. On the other hand, ontologies contain controlled terminologies (controlled vocabularies) plus essential components of the semantic network that encodes relationships between each term of the vocabulary. Ontologies represent networks of controlled vocabulary terms (nodes) and relationships (edges) between the terms. Relationships are mapped in a hierarchal order so that the whole structure becomes an organized "construction". Ontologies represent the powerful instruments for knowledge representation, as they enable formalized knowledge to be structured as required for 
computational processes, so that a machine or a person can explore the web of data (Malladi et al., 2015).

The National Center for Biomedical Ontology (NCBO) became the leading scientific organization for bringing semantic technology to biomedicine (Musen et al., 2012). The NCBO's mandate has been to build a comprehensive library of biomedical ontologies and to create tools and methods allowing researchers to use the ontologies. As a result, the BioPortal resource was created as an open source repository of biomedical ontologies that stores and provides access via the web. BioPortal provides investigators, clinicians, and developers with 'one-stop shopping' to programmatically access biomedical ontologies. BioPortal also supports linkage to data from other biomedical resources. This ontology library is curated and updated by the administrators of BioPortal and the bioinformatics community, which has access to annotations, and mapping of entities (Noy et al., 2009).

The best-known ontology in the biomedical domain is probably the International Classification of Diseases (ICD-11). Main bioscience related ontologies are compiled in the Open Biomedical Ontologies, or OBO Foundry (http://www.obofoundry.org/), and are collaborative projects involving developers of science-based ontologies (Smith et al., 2007). The ontology projects are ruled by the established principles and methodology for ontology development with the aim to construct and maintain a group of connected reference ontologies in the bioscience/biomedical domain (Smith et al., 2007). Many more very useful ontologies have been constructed (Dumontier et al., 2014; Shah, Cole and Musen 2012). Among OBO Foundry ontologies, probably the best-known and most widely used ontology is Gene Ontology.

\subsubsection{Gene Ontology}

The GO project is a community-based ontology that creates evidence-supported annotations to describe biological roles of individual genomic products (e.g. genes, proteins, ncRNAs complexes, etc.) by classifying them using ontology principles. The Gene Ontology development started more than a decade ago focusing on the need to integrate knowledge about genes and their products with consistent annotation (Gene Ontology Consortium 2000). Gene Ontology is managed by the Gene Ontology Consortium (GOC) with more than two hundred collaborators. The GOC has been involved in various projects and collaborations with the goal of expanding and improving the representation of biology (Gene Ontology Consortium 2015). The GO is a dynamic ontology open to new additions and modifications that are inevitably introduced as scientific knowledge expands (Shah, Cole and Musen 2012). 
The GO project has developed three ontologies that describe gene products in terms of their associated characteristics connected with biological processes (BP) they participate in, molecular functions (MF) they execute and cellular components (CC) as sites of action. The GO provides ontology-type organized and controlled vocabulary information for the properties of genes, thus sorting them into three domains. For each of these domains there are distinct terms, which describe gene or gene product properties. The biological process domain provides sets of molecular events with a defined beginning and end. The molecular function domain defines the activities of gene /gene products at the molecular level. The cellular components domain describes the parts of cellular or extracellular environment as a location attached to a gene/gene product.

In addition to maintaining ontology, the project itself has different aspects such as developing the ontology and its tools and maintaining annotations of genes and gene products. GO resources include gene product annotations in the form of comprehensive statements about what gene products do, usually in a species-neutral fashion (Gene Ontology Consortium 2000). The completeness of annotations derived from biomedical literature is uneven for each entity and across the BP, MF and CC ontologies for the same entities. Controlled vocabularies have been structured and maintained and the GO can be queried at different levels. The GO structure, relations, and terminology are modified regularly by GO editors, making it a complex and perpetually changing dynamic organization (Gene Ontology Consortium 2015).

One of the main uses of GO is to perform enrichment analysis on gene sets (Schaid et al., 2012). The Enrichment analysis tool is one of the software tools incorporated into GO, and is simple to use, relatively straightforward and yields easily interpretable information. GO Enrichment analysis software tool provides a mechanism to determine statistically significant functional subgroups within gene groups. Using this software tool is a simple way to gain insight into potential biological significance of a gene set under study. This software tool can determine whether GO terms associated with the particular biological process, molecular function, or cellular component are over-represented in the group of genes deemed significant by the statistical analysis, which allows one to gain insight into the potential biological significance of a gene set under study (Shah, Cole and Musen 2012).

Most recently, the GO descriptors have been tied to biological pathways and diseases. They could be searched using GO tools. Enrichment for BP, MF and CC terms based only on experimental data and not only on available literature (text) is also currently available in the beta version (since May 15th 2015), in addition to complete data from three ontologies (Mi et al., 2013). As proven, the data mining of the GO can be used for the discovery of new biological associations and even new drug targets. 
We used the GO for extracting knowledge about hundreds of SNP bearing/related genes discovered by AID GWAS, including BP, MF, CC, pathway and disease domains. The collected GO descriptors may clarify the potential of these genes to influence AID development and indicate their underlying pathology.

\subsection{Research Approach}

As stated before in the Abstract and Introduction sections of the thesis, the objective of this study is to find the key factors that influence a therapeutic action of the aTNF biologics in AID disease phenotypes (or lack of it).

aTNFs have proven therapeutic (curative) effect on the AIDs, indicating that the same interventional processes may work in several AIDs with different clinical manifestations. Different clinical manifestations stem from disease processes targeting different tissues, but AIDs also have some shared mechanisms as a group of diseases (autoimmune/inflammatory disease have been mostly treated by the similar drugs even before aTNF introduction). If aTNFs, as very specifically targeted drugs, all have positive response in majority of AID patients and are able to induce remission, then some parts of the common pathogenic AID mechanisms must be engaged by aTNFs, resulting in remission. Shared mechanisms between AID disease processes and aTNF interventional processes must overlap to certain extent, and they are responsible for remission upon aTNF therapy. We wanted to find out what particular common set of interactions is responsible for disease modulation. However, if the same set of interactions permissible for aTNF action does not exist in some AID diseased individuals, because it is functionally modified, an AID phenotype cannot be influenced by aTNF drugs, resulting in some degree of non-response.

We investigated the intersection between TNF and AID pathways (between intervention and disease pathways) in order to uncover a common set of interactions that may be responsible for modulating aTNF effect and consequently for variable responses to the aTNF therapy due to variability of the constituents. Our unique research approach integrates the knowledge of the aTNF biologics intervention and the knowledge of AID genotypes-phenotype relationships solely cropped from publicly available GWAS data and other publicly accessible bioinformatics databases. Combining the AID GWAS data with the relevant information from other databases and by using advanced bioinformatics software tools for retrieving and analyzing the data, we aimed to unveil potential network elements, pathways and genes responsible for AID pathogenesis and aTNF response. 
The null hypothesis is that if no such network can be constructed, then there may be other mechanisms responsible for unresponsiveness of AID patients to aTNFs, mechanisms that are not necessarily linked to the natural inheritable human genetic SNP variability.

Our research plan consisted of the several steps that were executed in a sequential order. The first step was to find SNPs associated with the AID disease phenotypes from published GWASs, and then select and retrieve association data. The following step was to analyze the retrieved data using available bioinformatics software tools in order to detect SNP locations and SNP relations with the known genomic structures in the human genome (genomic structured that currently have annotations). In next few steps, depending of the type of SNPs and genes, we defined which genes harbor AID SNPs and explored impact of AID SNPs on characteristics and functions of these genes and gene products. Following the step of SNP-gene prioritization, we further explored potential role of the AID SNP genes/proteins in the known biological processes by several approaches. Using bioinformatics software tools, we completed canonical pathway enrichment analyses, network analyses, GO terms enrichment analysis, and disease enrichment analysis, all of which helped us determine functional properties of AID SNP genes. We then used the acquired knowledge about functional bioprocesses in AID for comparison with known aTNFs intervention actions as drugs. By combining the data from AID SNP functional exploration with the TNF action data, we have determined common pathways and genes we consider the key elements for therapeutic effect of the aTNFs. They are key elements because they determine curative actions of the aTNFs in AID. 


\section{MATERIAL AND METHODS}

This is the graphic presentation of the research procedures (flow) carried out in our research:

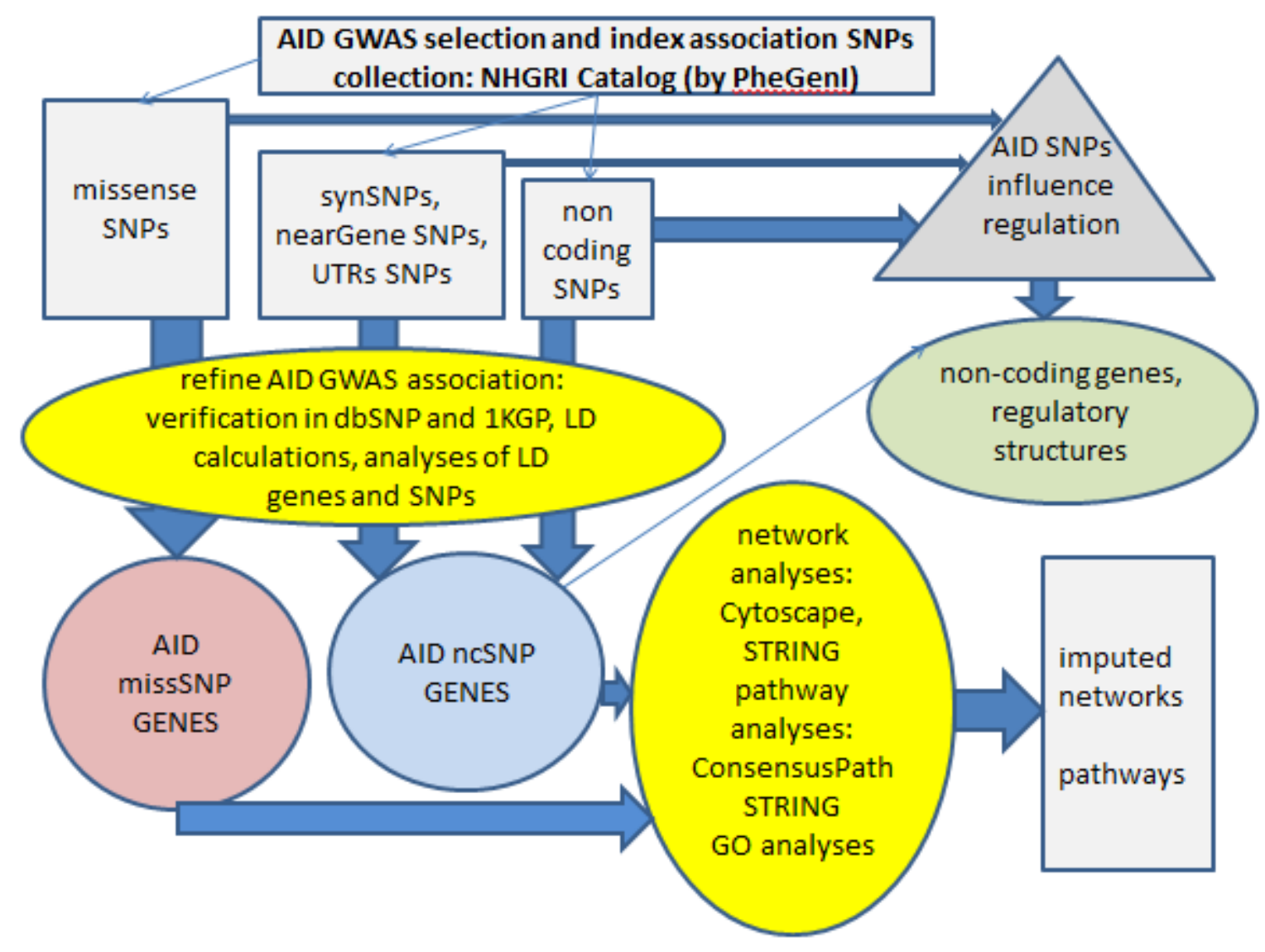

\subsection{Data collection of GWAS AID association SNPs}

The starting point of our research was the collection of publicly available GWAS association data for autoimmune/inflammatory disease phenotypes (AID). All AID GWAS associations were obtained from the NHGRI (National Human Genome Research Institute) GWAS catalog (Hindorff et al., 2009), (http://www.genome.gov/gwastudies). For our study, we selected only AID phenotypes for which the aTNF therapy has been approved: RA, AS, Ps, PsA, CD, IBD and UC.

We used software tool Phenotype-Genotype Integrator (PheGenl), a phenotype-oriented resource for retrieval of data from the NHGRI GWAS catalog, to find all AID SNP associations with the value of $p>$ $1 \times 10^{7}$. We sorted the associations according to their genomic context as intergenic, intron, nearGene-5 or -3 , and coding missense or synonymous SNPs. The selected SNP associations also were labeled with the specific rs identifier, their chromosome location, the closest provisional genes linked to them and PubMed identifiers of the first publications indexed for MEDLINE. Identifier "rs" stands for a reference SNP ID number, a unique identification tag assigned by NCBI after submission and it does not infer position (NCBI resource). 
The associated SNPs reported in AID GWAS are the lead SNPs (often also called tag or signal SNPs). In our research, we analyzed all of them and clearly distinguished whether they are simultaneously functional SNPs. Functional SNPs are defined as SNPs that influence a specific function of a gene or a gene region. Not all lead SNPs are functional SNPs, or vice versa.

\subsubsection{Software tools and databases used for data collection}

For data collection, we used database NHGRI Catalog and PheGenl software tool.

\subsubsection{NHGRI Catalog}

The NHGRI Catalog of GWAS (Welter et al., 2014) provides a publicly available manually curated collection of the published GWASs. It contains at least 100000 single-nucleotide polymorphisms (SNPs) All SNP-trait associations found in GWAS cataloged in it have $p$ value equal or lower than $1 \times 10^{-5}$. NHGRI Catalog data are extracted from the published literature. Data extraction and curation for the GWAS Catalog is an expert activity; each step is performed by scientists supported by a web-based tracking and data entry system, which allows multiple curators to search, annotate, verify and publish the Catalog data. It is updated regularly on a weekly basis. All GWAS in the catalog conform to eligibility criteria, including number of SNPs assayed and non-targeted study design. The number of SNPs extracted and curated per study is not limited to fifty, as it used to be before 2012. For each GWAS, a trait that best represents the phenotype under investigation is assigned by the curators and it does not rely only on author's submission. We used the NHGRI Catalog version that was available and accessible before the end of November 2014.

Until recently, the traits in the GWAS Catalog were available as an unstructured flat list, allowing only querying through direct string matching. To overcome the Catalog's limiting potential, and establish the Catalog as an integral part of a wider network of genomic resources, several ontologies for mapping the Catalog data have been evaluated; this work is still in progress. Recently, schema ontology was designed to model the key concepts represented in the Catalog, containing all the data in the GWAS Catalog in a format that can be processed by an ontology reasoner and queried. It allows for much richer querying than simple string searching. Mapping to ontologies also greatly facilitates data integration across heterogeneous data sources such as data extracted from the scientific literature. A new addition is the GWAS Diagram, a novel interactive dynamic query interface of traits visualized on the human karyotype, linked to literature and other resources.

\subsubsection{PheGenl}

Phenotype-Genotype Integrator (PheGenl) is the software phenotype-oriented resource for retrieval of data from the NHGRI GWAS catalog (Ramos et al., 2013). PheGenl tool amalgamates content from several NIH resources in addition to the data from the NHGRI GWAS studies: dbGaP, which archives the data of studies investigating associations between genotypes and phenotypes; dbSNP, which includes data on SNPs and their frequencies, position, etc; NCBI Gene, which includes gene-specific data, such as nomenclature, chromosomal localization, gene products; and eQTL data from the Genotype-Tissue Expression Project GTEx. 
PheGenl searches items based on MeSH terms (Medical Subject Headings) and retrieves SNP data together with their chromosomal location, gene, SNP ID, and phenotype. Results are provided in a tabulated view and could be downloaded; the results include separate tables with SNPs, genes and associations, a dynamic genomic sequence viewer, and gene expression data if they exist. Data may be sorted according to the source, functional class of SNPs, phenotype, and $p$ values of associations. PheGenl is still under active development and it continues to improve, merging NHGRI genome-wide association study (GWAS) catalog data with several databases housed at the National Center for Biotechnology Information (NCBI), including Gene, dbGaP, OMIM, and dbSNP, all of which we used for our research.

\subsection{SNP-gene analyses: mapping AID SNP to genes and other genomic structures}

\subsubsection{Rationale}

It was necessary first to map GWAS AID SNP data to genes or other genomic structures as a step towards understanding of their impact. The simplest and most used method for linking SNPs to genes is mapping the AID SNPs to the nearest genes or genes within the specified distance; however, it is erroneous not to take into account linkage disequilibrium (LD) patterns that might vary with populations and might provide a possibility of finding regulatory sequences (instead of coding genes) within LD regions.

In our study, we rely on LD regions as more relevant and broader determination of the origin of GWAS signals; we did rely only on proximity between SNPs and genes when we analyzed ncSNPs for ncRNAs in deserted regions, as the LD values for those regions are often vague, unknown or too broad.

We prioritize primarily the coding GWAS AID SNPs (cSNPs) to protein genes, because currently it is only possible to incorporate proteins into pathways or networks. However, we also searched for nonprotein genes in their respective LD. If no genes existed in high or perfect $L D\left(r^{2}=1\right.$ or $\left.r^{2}>0.8\right)$ with a SNP, we extended search into the regions with lower LD (LD with $r^{2}<0.8$ ).

Almost half of the retrieved AIDS GWAS SNPs are physically located in noncoding intergenic regions of human AID genomes. The potential effects of these GWAS ncSNPs might not be executed via proteins (although it is a possibility), but rather via regulatory control over biological processes, because alterations in noncoding structural elements of the genome are linked to regulatory control of biological processes. In the case of ncSNPs, especially ones that are located outside introns or completely outside gene regions, in intergenic regions (once called "gene deserts"), we additionally manually searched for annotated structures in the proximity of each ncSNP on the human genome map, upstream and downstream of each SNP up to $200-500 \mathrm{~Kb}$, independent of the size of its LD region.

Unambiguous assignment of disease causality for sequence variants is often impossible, but using the tools and approach as described, we performed SNP-gene prioritization at the current state of knowledge. 


\subsubsection{Procedure}

Using HaploReg v2 for every tag SNP obtained from the NHGRI catalog, we identified all SNPs in strong LD (perfect or high LD: $r^{2}>0.8$ ), based on 1KG Project data and HapMap European population data, CEU or EUA data. We then searched for all genes in the same high LD region using the same HaploReg tool, correcting if necessary the provisional genes provided in the original GWAS with the newest updated information.

For each AID, we gathered all genes in high LD as potential functional genes influenced by lead SNPS. Genes in LD with any SNP might change with data annotations updates, indicating the importance of the most recent annotations.

In the next steps, all collected genes were evaluated for potential functions using the available data in the Gene NCBI database. Gene roles were detected as described in existing Gene GIFs (gene references into function) data. We used NCBI data primarily, but also Ensembl or Havana project; Havana project often updates annotations outside of the regular releases. Many of the coding genes linked to AID SNPs had no annotations in NCBI databases, nor in other databases. In addition to coding genes, we found that many of the AID SNPs are linked to processed transcripts (mainly various noncoding RNA types) and pseudogenes, some of which might even be processed (translated) (Poliseno et al., 2015). For these structures, we also checked not only NCBI but also other databases such as Ensembl and Havana project (same as for the protein coding genes). Information on ncRNAs are scarce, but even fewer data exist for pseudogenes.

We further examined possible regulatory effects of the AID SNPs (including synonymous cSNPs) with the HaploReg software tool v2 and v4 (Ward and Kellis 2012). Synonymous amino acid substitutions were also evaluated for potential regulatory changes, because, although they do not cause amino acid substitutions, and have neutral effect on protein products, they may influence splicing sites or other regulatory spots within the gene region (Ward and Kellis 2012).

The HaploReg v2 analyses were conducted in two steps. Firstly, we interrogated the lead GWAS SNPs for related high LD sets of SNPs as explained; secondly, we analysed annotations of the expanded SNP sets together with the lead SNP, for their presence within promoters, enhancers, transcription factor binding sites, and expression quantitative trait loci sites. The HaploReg queries data generated by the ENCODE (Encyclopedia of DNA Elements) project (ENCODE 2004; Kellis et al., 2014) for various regulatory elements (e.g., evolutionarily constrained sequences, enhancers, DNAse hypersensitive open chromatin regions, promoters, and 3'- and 5'-untranslated regions).

In addition, we evaluated all sets of SNPs in high LD with the lead GWAS SNPs, by scoring their potential regulatory influence by software tool RegulomeDB (Boyle et al., 2012). We used the 1.1 version of RegulomeDB (Boyle et al., 2012; Xie D. et al., 2013) in order to annotate the AID SNPs with regulatory information.

Scores provided by RegulomeDB for the tested AID SNPs are used to sort all AID SNPs for their relevancy in potential gene regulation. We were able to cover almost all AID SNPS, because currently the RegulomeDB queries are able to identify all common SNPs with the allele frequency $>1 \%$ (from $1 \mathrm{KG}$ project and/or HapMap3). We selected to further analyse only SNPs with the RegulomeDB scores 
of 4 or higher $(3,2$, or 1$)$, because they have higher potential to influence regulatory elements. The RegulomeDB scoring is explained in the Supplemental table 2 in the Results section. Scores are ranked based on potential of a regulatory element to modify multiple functions of the region containing the tested SNP.

As a rule, we checked our results over time of one year at least three times for all resulting data, up to the August 2015; we were able to find new information for a few genes (such as IL6R) that we used in our study.

\subsubsection{Software tools and databases}

\subsubsection{HaploReg}

We used the HaploReg v2/v4 software tool for annotations and prioritization of disease associated risk variants for all SNPs (Ward and Kellis 2012). When a LD threshold is specified (defined with an $r^{2}$ ), results for each variant were shown in a table along with other variants in LD. We tested all noncoding and coding SNPs for their respective LDs with $r^{2}$ values ranging from 1 to 0.2 , and we reviewed and evaluated all potential genes in their respective LD in order to predict a biological relevancy for each SNP variant in publicly available databases. For LD calculations, the 1KHG Pilot project is used for each population that best matches the ancestry of the subjects.

HaploReg provides annotations from two mammalian conservation algorithms, which are designed to detect constrained sequences across mammalian genomes. In addition, HaploReg v2 is displaying change of the motifs caused by every SNP in the selected LD, thus enhancing further speculation about effects of SNPs on regulatory motifs. The HaploReg also displays if some SNPs are bound by proteins, or are DNAse hypersensitive in many cell types and shows enhancers and promoters impacted by each SNP if data are available.

HaploReg v2/v4 is available on http://www.broadinstitute.org/mammals/haploreg/haploreg.php

\subsubsection{RegulomeDB}

The RegulomeDB is a database that annotates SNPs with known and predicted regulatory elements in the intergenic regions of the $H$. sapiens genome. Currently Regulome DB is querying build 141 of $\mathrm{dbSNP}$ and all data at RegulomeDB are mapped to human reference sequence h19. Known and predicted regulatory DNA elements include regions of DNAse hypersensitivity, binding sites of transcription factors, and promoter regions that have been biochemically characterized. Sources of RegulomeDB data include ENCODE project, in addition to NCBI public datasets, and published literature from MEDLINE. RegulomeDB also uses the function information generated by the UCSC Genome Browser (Sayers et al., 2012).

The RegulomeDB server is available at http://www.regulomedb.org, and all ENCODE datasets used in RegulomeDB can be accessed via the ENCODE portal at http://encodeproject.org. 


\subsubsection{3. dbSNP}

The dbSNP database has been established at the National Center for Biotechnology Information (NCBI) to serve as a general catalog of genome variation necessary for association studies with heritable phenotypes, pharmacogenomics, gene mapping, population genetics and evolutionary biology (Sherry et al., 2001). SNPs are the most common genetic variation; they occur roughly every 1200 bp in human chromosomes. The complete contents of dbSNP are available at the public site http://www.ncbi.nlm.nih.gov/snp/.

We used dbSNP 141 build.

\subsubsection{Evaluation of missense SNPs impact on proteins coded by missSNP AID genes}

\subsubsection{Rationale}

The coding sequence variations (cSNPs) resulting in nonsynonymous amino acid substitutions caused by the AID SNPs (missSNPs) were analyzed by PolyPhen software tool in order to evaluate potential influence of the amino acid substitution on its coding protein conformation and subsequently on its function. Mainly v2 was used, but also v4 with updated annotations, because of our repetitive checking for data and results over time of one year (Adzhubei et al., 2010, 2013).

\subsubsection{PolyPhen-2 software tool and procedure}

We used the PolyPhen-2\&4 to evaluate a potential of missSNPs to make a conformation change of respective coding proteins and consequently influence protein functions. The web based software PolyPhen (Polymorphism Phenotyping version 2 and 4) is a software tool, which predicts possible impact of an amino acid substitution on the structure and function of a human protein, employing straightforward physical and comparative considerations.

This was a key evaluation for missSNPs, because it provided us with information about potential change of missSNP harboring protein function that, down the road, might influence pathways and networks. Conformational changes might influence various protein functions such as binding capacity to its interactants (applicable for both pathways and networks) or might induce allosteric modification and impact speed of a pathway, quantity of products and pathway feedback. The detected potential of AID nonsynonymous or missense SNPs to change the coding protein conformation has direct functional consequences on PPI and pathways and networks.

PolyPhen-2 is available on http://genetics.bwh.harvard.edu/pph2/.

\subsection{Functional analyses of GWAS AID SNPs: gene-pathway prioritization}

The GWAS data enables a shift from individual genetic associations to hypotheses about how the effects of multiple genes contribute to disease susceptibility and expression (Hirschhorn 2009). In order to make this shift, functional characteristics of the AID SNPs data must be understood, and for that reason, they were a major part of our study. 


\subsubsection{Rationale}

Functional roles of retrieved AID SNPs could be analyzed in various ways, but the best and most straightforward approach is to find the interactants of the SNP bearing genes/proteins or genomic structures. Potential interactants then might be organized in directional pathways or less organized structures like networks. Most often, the base of the biological functionality of structures influenced by GWAS SNPs consists of proteins interacting with other proteins.

It is important to stress that both pathway and network analyses have foundation in protein-protein interactions (PPI). Very rarely is any other class of macromolecules, except proteins, included in the prediction of formation of networks or pathways for any given gene.

PPI information can be retrieved from a number of online resources. Fewer resources have their focus on PPI prediction, using a variety of algorithms. However, the integration of both known and predicted (imputed) PPI interactions has been achieved currently only by STRING and ConsensusPathDB software tools; both were used in our study as they have some different characteristics and outcomes.

\subsubsection{AID GWAS SNP pathway analysis}

\subsubsection{Rationale}

Biological pathway information helps interpret genomics data and gain a more mechanistic understanding of cellular function once we are able to find biological pathways for the SNP gene set of interest.

If an AID SNP risk factor (GWAS SNPs are considered risk factors for a disease phenotype) is related to a certain pathway, then that particular pathway could be considered engaged in the pathogenesis of an AID. Although small number of genes is currently classified into functional conglomerates known to have a certain biological function, once a gene is annotated for a function or assigned to a well-defined pathway, then the function of that pathway become an element of a disease. The pathway should be counted for analyses in the disease phenotype(s), as it obviously might be changed with an AID SNP risk factor it harbors. However, there is no data or models about the flow of pathways currently, and all attributed changes will have to be tested empirically (observation and experiment) or in predictive models. Our knowledge about pathways is singular, and it does not protrude into multifaceted presentation of an pathway.

Biological pathways are collected in databases like KEGG (Goto et al., 1997), Reactome (Joshi-Tope et al., 2005) or the user-curated WikiPathways (Pico et al., 2008) and many other pathway databases. For our analyses, we almost exclusively used KEGG. Occasionally, we used other pathway databases such as $\mathrm{NCl}$ (http://pid.nci.nih.gov/) and Reactome (http://www.reactome.org/) as a source for pathway data retrieval or crosscheck.

We relied on the KEGG (The Kyoto Encyclopedia of Genes and Genomes) pathway database (Kanehisa et al., 2012, 2014), because it is considered the largest and most complete pathway database and is regularly updated resource. It is also the most connected pathway database, linked to or used by 
many other resources, including NCBI databases, and tools like Cytoscape, STRING, and ConsensusPathDB (all of which we used in our research).

\subsubsection{Procedure for finding AID SNP pathways}

Manually retrieved KEGG pathways and KEGG pathways obtained by pathway enrichment analyses were compared and their potential roles in the pathogenesis of AIDs were evaluated.

\subsubsection{Manual search for AID SNP pathways}

We manually searched and retrieved KEGG pathways for all GWAS SNP genes/proteins and TNF. For each AID we compiled the group of KEGG retrieved pathways guided by the disease SNP harboring genes found for each AID. The AID pathways we consider pathogenic or disease pathways for corresponding AID, because they are believed to be engaged in the AID pathogenic process.

\subsubsection{AID SNP pathway enrichment analyses}

We also sought to identify disease pathways that are shared amongst AID SNP genes (AID disease genes) by performing pathway enrichment analysis using KEGG pathways database. Pathway enrichment analysis (Mooney et al., 2014) is a technique to find biological, functional sense of any gene dataset.

We used two tools to do the pathway enrichment analysis: STRING and ConsensusPathDB that are explained in the section "Databases and software tools" (below).

\subsubsection{Parsing of the AID SNP pathways}

We also wanted to know whether the KEGG pathways, found to home the AID SNP genes, have any common members among them that might be important for the pathogenesis of AID. Finding common members would tell whether KEGG pathways communicate and for that reason influence each other by sharing the same elements. We wanted to find intersections containing subsets of common genes between two disease pathways and explore connections between these common subsets and AID SNP harboring genes. It is intuitive that various pathways could influence each other, especially when they share genes and when they are closely position within the cell loci (membranes, inflammasome, proteasome, etc.).

The only tool that allowed us for this type of analyses was Cytoscape, because both STRING and ConsensusPathDB software do not allow querying for intersections (Su et al., 2014). Cytoscape allows import of major biological pathways from KEGG database (still in development, as it does not contain all KEGG pathways) and then by using the intersecting tool, it finds overlapping sunsets (or common members) between KEGG pathways.

In the process of finding potential AID SNP pathway intersections, we used the CytoKEGG plugin (Bindea et al., 2009) to retrieve known KEGG pathways for TNF and AID SNP GWAS proteins by importing all members of corresponding KEGG pathways. In the second step, we employed tools to intersect two pathways. Thus, we identified common, shared members for each pair of KEGG pathways. 
By comparing the intersecting pathway results, we identified proteins common to the analyzed pathways. Their presence indicates the crosstalk among pathways; pathway crosstalk might have significant influence on conditioning of an AID or a response to aTNF therapy.

\subsubsection{AID SNP GWAS network analysis}

For construction of networks for AID SNP genesets and consequent network analyses, we used Cytoscape software tool. Networks might also be constructed using STRING and ConsensusPathDB tools, but they cannot be dissected, parsed or unionized as with Cytoscape software, nor they can be analysed for mutual members.

\subsubsection{Cytoscape}

\subsection{Rationale}

Cytoscape is one of the most popular open-source software tools for the visual exploration of biomedical networks composed of biomolecules (proteins, genes) and various types of biomolecular interactions among them (Shannon et al., 2003). Cytoscape is a software suite most powerful when used in conjunction with large databases of protein-protein, protein-DNA, and genetic interactions that are increasingly available for humans. It is constantly upgraded as an open source with contributors adding plugins for almost any type of analyses (Saito et al., 2012). Cytoscape provides core functionality to load, visualize, search, filter, and save networks. Many plugins have been contributed by the community to extend Cytoscape functionality and to address specific research needs. The latest generation of Cytoscape (version 3.0 and later) has substantial improvements in function, user interface, and performance (Su et al., 2014).

Cytoscape v3.1.1. is available at http://cytosca-pe.org/download.php, the site hosted at the UCSD (University of California in San Diego).

\subsection{Procedure}

We initialized networks for TNF and each SNP GWAS gene/protein separately. We imported data and limited these networks only to direct interactants. A typical Cytoscape workflow begins by importing interactions (in our case protein-protein, genetic and regulatory interactions) from public databases for all genes under study. First, we performed keyword searching through 'Import' function for each gene using a gene name or gene ID. Public databases of interactions are accessed using plugins (such as AdvancedNetworkMerge plugin we used that are already standard capabilities incorporated into Cytoscape suite). Plugins allow for online data import, network generation and visualization. During the search step, the databases were selected for data import from protein-protein interaction databases such as Biological General Repository for Interaction Datasets (BioGRID), Human Protein Reference Database (HPRD), Molecular Interaction Database (IntAct), STRING etc. (Szklarczyk et al., 2011, 2015; Kerrien et al., 2012). Then we used Cytoscape to perform network partitioning and comparison between networks by generating Venn diagrams (intersections and unions) We queried the networks for their intersections in order to find common, intersecting shared nodes, actually subnetworks that belong to both networks (Saito et al., 2012). The intersections were analyzed for each network. In order to find 
whether any of AID SNP gene nodes interact with each other and in that way discover potential link between AID GWAS genes, we proceeded to construct a union network of the resulting intersecting networks for all available networks. We named this resulting union network the core AID network. The core AID network contained only the GWAS SNP proteins and TNF nodes that are interact among themselves: the nodes linked with the edges indicating a specific type of interaction between each pair of nodes. All other nodes without edges, actually nodes that did not interact among themselves, and did not form a network were eliminated. The edges of all constructed networks signified the interactions between two proteins; they were annotated with details about data source or publications.

\subsection{Functional analyses of AID GWAS SNP data using Gene Ontology}

\subsubsection{Gene Ontology Enrichment Analysis}

An alternative way to find functional characteristics of the AID gene sets is by usage of the Gene Ontology (GO) database, which categorize gene using three hierarchical biological categories: molecular functions, biological processes, and cellular components. The enriched GO terms provide information about potential functionality of the tested gene set that is affected in AID.

We employed GO to perform enrichment analyses on the AID SNP gene sets and find the GO terms that are overrepresented for each gene set under study. If enrichment in any of these GO categories exists, it would help us better understand their function. However, although genes assigned to a particular GO category may be associated with similar functions, this grouping does not indicate known relationships or interactions between the genes in each set, and for that reason is less informative that pathway analyses, but more informative than network analyses, that provides interactions.

\subsubsection{Rationale}

The goal of GO enrichment analysis is to determine which biological processes, molecular function or cellular component terms might be predominantly linked to the affected set of genes in our study (Blake 2013). The simplest approach is to calculate enrichment for each GO term, actually a proportion of genes with certain annotations among the significantly changed genes determined in the AIDS GWAS, when compared to all of the human genes. The set of all genes, or reference set, compasses all known genes in the human genome. An appropriate statistical test must be applied to allow the results to be interpreted as evidence. The analysis process calculates probability as a $p$-value of the occurrence of a term labeled portion among significant set (sample frequency), versus a term labeled portion among reference set (background frequency). P-value is probability of proportion being annotated with the same term for the tested gene set vs. all genes annotated with the same term. Difficulty in determining significance using the calculated $p$-value and a cutoff of 0.05 is that multiple testing increases the likelihood of obtaining what appears to be a statistically significant value by chance. Therefore, a correction must be calculated. However, Bonferroni correction for multiple testing is too restrictive and the false discovery rate (FDR) is a better measure for statistical significance in this case. Calculation of FDR, which provides an estimate of the percentage of false positives among the categories considered enriched at a certain p-value cut-off, allows for a more informed choice. Multiple hypothesis testing is a general problem that is not specific to GO enrichment analyses (Farcomeni 2008). 
Other limitations of GO enrichment analyses are incomplete annotations (at least $20 \%$ of genes do not have GO annotations) and a strong bias towards BP, because GO annotations are not always independent items as conditional for FDR corrections.

\subsubsection{Procedure}

We used both STRING and ConsensusPathDB software tools to perform GO enrichment analyses.

First, we selected the gene sets for analyses, based on the previous step results: missSNPs, ncSNPs and allSNPs. Each set of genes was queried for GO term enrichment separately, and GO terms for biological process (BP), molecular function (MF) and location in cell compartment (CC) were separately searched, retrieved and analysed.

Both Bonferroni and FDR $p$ values were retrieved and presented. We selected the resulting data mainly based on FDR corrections. In some cases, there were no GO term enrichments after applying Bonferroni correction and FDR; in that case, the results were not presented, because they did not have any significance.

\subsection{Software tools and databases used in prioritization and GO enrichment analyses}

\subsubsection{STRING}

The STRING database (Search Tool for the Retrieval of Interacting Genes/Proteins) (http://string-db.org) (Szklarczyk et al., 2011, 2015; Franceschini et al., 2015) is a software tool and database of known and predicted protein interactions. It has been developed and maintained by EMBL (European Molecular Biology Laboratory), SIB (Swiss Institute for Bioinformatics) and CPR (Center for Protein Research). It is dedicated to provide a critical assessment and integration of protein-protein interactions, including direct (physical) as well as indirect (functional) associations. Interactions between proteins help to describe and narrow down a protein's function; more knowledge is available, less difficult is to find a protein's place in the complex systems. STRING provides the most useful networks that integrate all types of interactions: stable physical associations, transient binding, substrate chaining, information transmission and others.

STRING quantitatively integrates interaction data from many sources for a large number of organisms. It incorporates data from genomic information including data encompassing conservation, similarity, data such as protein domains and protein structures, expression data, concurrence and localization data, high-throughput experimental data and textmining from literature: Medline abstracts and open access articles. STRING holds experimental, but also predicted interaction data. Apart from in-house prediction and transfer algorithms, STRING also relies on many fine resources maintained elsewhere. A list of STRING integrated sources is long and rising. Currently STRING has data on over 5 million proteins and more than 200 million interactions stored.

The current version 10.0 of STRING allows for enrichment detection of human disease associations and pathways annotations, which might be statistically enriched in a given network. In addition, it also allows 
enrichment analyses of GO terms enrichment. We used all these features for our analyses: pathway and disease enrichments, and GO term enrichments.

The STRING results can be downloaded or saved as screenshots or tables.

\subsubsection{Procedure}

Starting from the user interface, we have entered the different sets of proteins obtained from the previous steps. Once a protein or set of proteins was identified, we proceeded to the network view, showing nodes as proteins and edges as interaction between them based on evidence. We included all possible evidence available in STRING and we kept adjusted score cutoffs at 0.7 aiming for high confidence results. We did not limit the network size as we wanted to obtain as many interactants and interactions as possible.

Upon switching to advanced mode, we could additionally analyzed enrichment for KEGG pathways and diseases for the given get of AID SNP genes/proteins. The only downside we experienced was limitation of ten or more genes in a set in advanced mode.

We used STRING version 10 for our research. The retrieved data are presented as the screenshots and tables.

\subsubsection{ConsensusPathDB}

ConsensusPathDB is a meta-database that integrates different types of functional interactions from heterogeneous public interaction/pathway resources data resources (Kamburov et al., 2009, 2011). Through the integration of its Release 30, ConsensusPathDB assembles a comprehensive map of human interactions and pathways.

Although ConsensusPathBD has focused primarily on the integration of existing database resources, its schema might be used for additional manual upload of experimental interactions.

The database contains human functional interactions, including gene regulations, physical (proteinprotein and protein-compound) interactions and biochemical (signaling and metabolic) reactions, obtained by integrating such data from source accessible databases including KEGG. Overall, ConsensusPathDB currently contains 41,271 physical entities, 155,432 functional interactions and 2,205 biological pathways in human. It also contains 12,263 unique curated protein complexes (Kamburov et al., 2011, 2013).

The ConsensusPathDB Web interface offers different ways of utilizing these integrated interaction data with tools for visualization, analysis and interpretation. Among other features, the web interface allows for over-representation/enrichment analysis with uploaded identifiers of gene sets. It also contrasts networks based on direct interactions and indirect via second interactants (we used only first interactants). The obtained results may be filtered for cooperation in curated biochemical pathways or co-annotation with Gene Ontology categories. These features we used in our research. 
The ConsensusPathDB can be found on http://consensuspathdb.org/. It stays up-to-date with regular content updates and database releases every 3 months.

\subsubsection{Procedure}

Starting from the ConsensusPathDB user interface for overrepresentation analyses of gene set, we entered the different sets of genes obtained from the previous steps. Once a protein or set of proteins was identified, we filtered results for their participating pathways, and GO terms. We mainly preselected KEGG pathways, but we also analysed Regulome and $\mathrm{NCl}$ pathways data and other pathways if they did not overlap with the KEGG pathways. For GO terms, we filtered results for four different levels of BP, MF and CC GO terms. We selected only data within acceptable statistical range (FDR less than 0.5).

The advantage of ConsensusPathDB is that it has no limits on number of genes in gene sets and present only FDR statistics. For GO term enrichment analyses, ConsensusPathDb allows for filtering parent-child levels of complexity. It also shows protein complexes in addition to pathways and networks for a test gene(s) and occasionally introduces gene-gene and gene-protein, as well as drug-protein interactions as regulatory links. Because this option is still in development, we checked it often, but did not present results in this study.

\subsubsection{DiseaseConnectDB}

The AID pathways were also retrieved and analysed by DiseaseConnectDB software tool. The obtained results were crosschecked with the results of pathways we found based on GWAS SNP data by other methods (previously described). It was used as another tool with different approach that has confirmed our results.

DiseaseConnectDB is a public web database and server based software tool that focuses on the analysis of common molecular mechanisms shared by diseases by integrating comprehensive omics and literature data (Liu et al., 2014). DiseaseConnectDB is currently one of a very few databases that touches on the concepts of disease connections (Rappaport et al., 2013; Peng et al., 2013). It is the most comprehensive resource documenting the shared molecular bases of diseases. The DiseaseConnectDB web server contains 18,707 disease-disease, 660,985 disease-gene, 12,617 druggene and 113,498 drug-disease relations; these data cover 4,791 diseases, 6,215 drugs and 15,182 genes. It has incorporated a large amount of GWAS catalog, gene expression data, microRNA expression data and text-mined knowledge to discover disease-disease connectivity based on molecular mechanisms.

We used DiseaseConnectDB software tool to crosscheck our results on the AID SNP pathways. 


\subsection{Finding interactions of AID GWAS SNPs with non-coding RNAs}

\subsubsection{Rationale}

Functional RNA molecules have recently emerged as important regulators of gene expression. MicroRNAs (miRNAs), together with small interfering RNAs (siRNAs), long noncoding RNAs and other categories of non-coding RNAs, are all in this category that has been intensively researched in the human genome. MicroRNAs (miRNAs) are small evolutionarily conserved regulatory RNAs that modulate mRNA and regulate gene expression at the posttranscriptional or translational level. There is evidence that GWAS SNPs may influence miRNAs structure and function (refs).

Therefore, we sought to find whether any of the AID GWAS SNPs are able to change sequence of miRNAs or interfere with the binding site of any regulatory miRNA in gene regions. If an AID SNP occurs in a miRNA gene, then it could affect its regulatory function and help explain association a SNP role with AID. Additionally, if an AID SNP occurs in the docking region of an AID SNP gene and change the docking sequence for miRNA, that SNP might have impact on regulatory function of a miRNA. Both options were explored using relevant miRNA databases and tools.

\subsubsection{Procedure}

Non-coding RNA (ncRNA) genes were manually searched for around GWAS AID SNPs, up to $200 \mathrm{bp}$ upstream and downstream of each SNP locus starting from the dbSNP, and in the corresponding SNP high LD regions as determined by HaploReg v2 software tool.

For this experiment, we selected the top hits among the GWAS AID SNPs, ones that might be considered to have the highest probability of influencing ncRNAs. That includes the SNPs with the highest $p$ values in the GWAS and the SNPs with the highest scores obtained by RegulomeDB.

In order to find out whether any of the selected AID SNPs interfere with miRNAs, we sought to find the AID SNPs located in miRNA genes. We also examined whether any AID SNPs interferes with known target sequences of miRNAs, where a SNP can change the docking sequence for miRNA in a way that influence its binding.

We searched the microRNA database miRBase for the miRNAs found in high LD with the preselected SNPs. The miRBase (Agarwal et al., 2015) database is a searchable database of published miRNA sequences and annotation and is found at http://www.mirbase.org.

We have also searched miRNA targeting sequences in the regions of the AID SNP genes. We examined all AID SNP gene regions for evidence of co-location of SNPs within its boundaries of AID SNP genes using TargetScan tools. We employed the TargetScan Release 7.0 that can be found at (http://www.targetscan.org/). 


\subsection{Overview of the bioinformatics software tools and databases used in the study}

\begin{tabular}{|c|c|c|}
\hline Tool / Database & Relevance & Version \\
\hline GWAS NHGRI Catalog & $\begin{array}{l}\text { Databases with all GWAS studies; annotated by } \\
\text { professional staff; publicly available }\end{array}$ & 2013-ongoing \\
\hline PheGenl & $\begin{array}{l}\text { Search and retrieve tool for queried disease SNPs from } \\
\text { association studies in the NHGRI Catalog }\end{array}$ & $\begin{array}{l}\text { still in } \\
\text { construction }\end{array}$ \\
\hline HaploReg v2 & $\begin{array}{l}\text { Detection of SNPs in LD with a SNP of interest; } \\
\text { detection of genes in LD with a lead GWAS SNP }\end{array}$ & $\begin{array}{l}\text { Versions } 2 \\
\text { and } 4\end{array}$ \\
\hline RegulomeDB & $\begin{array}{l}\text { Scoring for potential regulatory function of the relevant } \\
\text { GWAS AIDs SNPS }\end{array}$ & Version 1.1 \\
\hline $\mathrm{dbSNP}$ & SNP database; SNP characteristics, location; MAF & Build 141 \\
\hline KEGG database & $\begin{array}{l}\text { Detection of genes of interest; characteristics; known } \\
\text { relations to diseases and their pathways; classification } \\
\text { of pathways and relation to modules }\end{array}$ & $\begin{array}{l}\text { Continuous } \\
\text { release }\end{array}$ \\
\hline Entrez Gene NCBI & $\begin{array}{l}\text { Search for genes and their properties: gene function } \\
\text { and location in the chromosome or region }\end{array}$ & Build 141 \\
\hline $\begin{array}{l}\text { Cytoscape: } \\
\begin{array}{l}\text { Network construction and } \\
\text { analyzer }\end{array} \\
\text { AdvancedNetworkMerge } \\
\text { plugin }\end{array}$ & $\begin{array}{l}\text { Construct networks for genes that are related to the } \\
\text { GWAS AIDs SNPs; analyze the same networks for } \\
\text { unions or intersections }\end{array}$ & v3.1.1 \\
\hline $\begin{array}{l}\text { Cytoscape: } \\
\text { KEGG pathway query tool } \\
\text { and analyzer } \\
\text { CytoKEGG plugin }\end{array}$ & Analyse relationship between KEGG pathways & v3.1.1 \\
\hline
\end{tabular}




\begin{tabular}{|l|l|l|}
\hline STRING & Gene prioritization; pathway enrichment analysis & Version 10 \\
\hline ConsensusPathDB & $\begin{array}{l}\text { Gene prioritization; pathway enrichment and complex } \\
\text { formation analysis }\end{array}$ & Release 30 \\
\hline GO ontology & $\begin{array}{l}\text { Retrieval of GO terms for gene enrichment (BP, MF, } \\
\text { CC and pathways); potential function assignments }\end{array}$ & Version 4.0 \\
\hline DiseaseConnectDB & Retrieval of pathways common to AIDs & Version 1.1 \\
\hline miRBase & $\begin{array}{l}\text { Searching for published miRNA sequences and } \\
\text { annotation }\end{array}$ & Release 21 \\
\hline TargetScan & Predicting targets for microRNAs & Version 7.0 \\
\hline DrugBank & $\begin{array}{l}\text { Characteristics of TNF biologics; interactants, } \\
\text { inhibitors, targets }\end{array}$ & Version 4.3 \\
\hline PharmaGKB & Characteristics of TNF biologics & Version 4.0 \\
\hline
\end{tabular}




\section{RESULTS}

\subsection{AID-associated single nucleotide polymorphisms (AID SNPs)}

We retrieved 383 SNP-AID associations from the NHGRI GWAS Catalog collection by the end of November 2014 (Table 1, Supplemental Table 1 and Supplemental Figure 1). These AID SNPs were associated with 356 unique genes. Some AID associated SNPs are shared by two or more genes, thus resulting in a many to one SNP-gene association. The AID SNPs were dispersed throughout all somatic chromosomes; sex chromosomes ( $x$ and $y$ ) did not contain any AID SNPs (Ideogram, Supplemental Figure 2.).

Genomic context for each retrieved SNP is provided in the Supplemental Table 1; the genomic context distribution of the SNP associations is provided in the Table 1, alongside with the numbers of affected genes for each AID, and in the chart (Supplemental Figure 1.).

For each association the SNP identification number (rs), location on a chromosome and a source of information are given in the Supplemental Table 1. More than half of all AID SNPs are intergenic, $30 \%$ are intronic and only 7,5\% are missense SNPs; 3\% are UTR-3 and UTR-5 SNPs, 5\% are nearGene-5 SNPs and $1 \%$ are nearGene-3 SNPs; coding synonymous SNPs represent only $2,5 \%$ and there are only 2 frameshift SNP among retrieved GWAS associations.

Table 1. AID-associated SNPs and genes for each disease

\begin{tabular}{|l|c|c|c|c|c|c|}
\hline \multicolumn{4}{|l|}{ Number of } \\
\hline $\begin{array}{l}\text { Autoimmune/inflammatory } \\
\text { diseases (AID) }\end{array}$ & SNPs & Genes & $\begin{array}{c}\text { miss } \\
\text { SNPs }\end{array}$ & $\begin{array}{c}\text { nearGene } \\
\text { SNPs }\end{array}$ & $\begin{array}{c}\text { intronic } \\
\text { SNPs }\end{array}$ & $\begin{array}{c}\text { intergenic } \\
\text { SNPs }\end{array}$ \\
\hline Rheumatoid Arthritis (RA) & 102 & 73 & 4 & 6 & 34 & 58 \\
\hline Arthritis, Psoriatic (PSA) & 5 & 8 & 1 & 1 & 0 & 3 \\
\hline Psoriasis (PS) & 35 & 39 & 5 & 1 & 14 & 15 \\
\hline Crohn Disease (CD) & 96 & 120 & 7 & 5 & 37 & 47 \\
\hline $\begin{array}{l}\text { Inflammatory Bowel Diseases } \\
\text { (IBD) }\end{array}$ & 21 & 15 & 1 & 0 & 13 & 7 \\
\hline
\end{tabular}




\begin{tabular}{|l|c|c|c|c|c|c|}
\hline Spondylitis, Ankylosing (AS) & 26 & 35 & 2 & 1 & 8 & 15 \\
\hline Ulcerative Colitis (UC) & 98 & 52 & 7 & 9 & 21 & 61 \\
\hline
\end{tabular}

AID associated GWAS SNPS: number of SNP for each AID organized according to their context and number of genes influenced by the same SNPS as provided in the NHGRI Catalog on November 2015.

\subsection{Gene candidate identification}

We performed SNP-gene prioritization experiments to assign both coding and non-coding SNPs to their most probably associated genes.

We first identified and verified all AID SNP variants using the dbSNP database. All AID SNPs have been recognized in the 1000 Genome project and HapMap3. We manually verified the genes assigned to the AID SNPs using the dbSNP database and HaploReg v2 software. We found that several genes assigned originally to the AID SNPs were not exactly located in the SNP regions according to the newest information from HapMap3 and $1000 \mathrm{G}$ project. We used further the updated, corrected version of genes associated to the AID SNPs.

The AID SNPs from HLA regions were excluded due to the intrinsic high variability of HLA regions crammed on the chromosome $6 p$ 31.21. section and in perfect LDs $\left(r^{2}=1\right)$ over the long stretches of chromosome in the same region.

\subsubsection{Identification of gene candidate dataset for coding non-synonymous AID SNPs (missense SNP gene dataset)}

We identified 23 missense SNPs among significant $\left(p<1 \times 10^{-7}\right.$ ) retrieved AID SNPs (Table 2). All missense AID SNPs have been detected in the 1000 Genome Project and HapMap3. The minor allele frequency count (MAF) is given for each SNP. It shows all missense AID SNPs are common in the human population, at least in the population of European origin (usually labeled as CEU or EUR), for which the majority of AID GWASs had been performed.

We find that only 3 out of 23 missense SNPs were present as the solely risk variants associated with a particular AID. The majority of missSNPs were risk variants for multiple AIDs under study. The same missSNPs were also the risk factors for other immune/inflammatory diseases such as SLE or T1D (as found in the NHGRI catalog database) (Table 2). 
This finding suggests the pleiotropic relations among missSNPs, their genes and AIDs.

\subsubsection{Missense AID SNP dataset evaluation}

The characteristics of GWAS AID missSNP are provided in the Table 2 and 3. Each missense AID SNP (missSNP) is labeled with rs number, minor allele frequency count (MAF), presence in the 1000 Genome Project, and the type of amino acid alteration caused by a nucleotide switch. Each missSNP the associated gene is given labeled with corresponding official symbol and NCBI gene identifier. Also presented is the AID in which the missSNP is found alongside with other autoimmune diseases (other than AID). Relationship of the missSNPs with other SNPs in the same gene is commented (if they are known in the NCBI databases).

\section{Table 2. Missense GWAS AID SNPs characteristics: part I} (amino acid changes caused by missSNPs, PolyPhen-2 evaluation of functional consequences)

We sought to predict the functional impact of the AID missense SNPs on proteins coded by the genes harboring these missSNPs. We used the PolyPhen-2 to predict their functional impact, and the results of the experiments are presented in Table 2. Results of amino acid change evaluation are given as "damaging" if the amino acid change is highly probable to alter protein conformation to the point of damaging its function, or "benign" if the conformational alteration is not damaging for protein function based on current knowledge. Roughly at minimum, one third of the missense SNPs (8 out of 23) were evaluated as damaging for the function of corresponding proteins coded by the genes harboring the missSNPs.

An illustration of the obtained results for evaluation is provided in the Figure 1, where for instance, missSNP rs8192591 is predicted to be damaging (Figure 1). Each missSNP evaluation has been repeated at least several times.

\section{Figure 1. An example of functional evaluation of missense SNP impact by PolyPhen-2}

To assess the potential of the missense SNPs to modify gene regulation and expression of the genes in the affected regions, we analyzed the high LD haploblocks for each missSNP using RegulomeDB before August 2015 (Table 3).

Six SNPs (out of 23 missense SNPs) with the highest scores (1a-3b) were found. The scores suggest that rs2476601, rs4077515, rs3764147, rs3184504, rs20541, and rs2305480 may significantly modify regulatory function of their corresponding DNA segments in high LD. The rest of the missSNPs were 
scored 4-6 (or no data existed at the time these analyses were carried out), indicating that they were less likely or not at all able to influence regulatory functions.

The majority of missSNPs (14 out of 23) belongs to the regions that are highly conserved across the mammals, even conserved in the vertebrates (Table 3). The majority of missense SNPs variants are able to modify several regulatory DNA elements including regions of DNAase hypersensitivity, promoter and enhancer histone marks, transcription factors binding sites and promoter regions.

Among the observed motif changes, there is an affected NF-kB motif in the region for ERAP1 gene, while the TRAF3IP2 gene region contains a STAT motif changed due to the presence of rs33980500 variant. Only 2 missSNPs were not evaluated as expression quantitative trait loci (eQTL), while the majority had multiple eQTL results. Almost each missSNP has several regulatory markers (Table 3 ).

\section{Table 3. Missense GWAS AID SNPs characteristics: II part} (analyses of LD blocks by HaploReg v2 and RegulomeDB scoring)

Further, we analysed LD regions around the missSNPs for other SNPs and genes. Potentially, other genes might also be reflected or represented by the tagged missSNPs on used GWAS chip platforms. Alternatively, other SNPs in high LD with the tagged missSNPs might also be functional SNPs and acting in accordance with the tagged missSNPs, but were not detected on the used GWAS platforms because the used chip platforms did not recognized them.

We found that nearly half of all missSNPs (11 out of 23 missSNPs) were in high LD with additional SNPs in the same gene, and at least nine missSNPs were in high LD with SNPs belonging to other genes (Table 3). This suggests that high or perfect LD missSNP blocks show existence of additional SNPs and alternative genes for majority of AID missSNPs.

We were able to distinguish between lead (tagged) and functional SNPs among the set of 23 missense SNPs based on orthogonal evidence. The missSNPs belonging to MST1, MICA, YDCJ and GSMBD genes were not functional SNPs. Several other missense SNPs, all assessed as functional, had additional functional SNPs in very high LD, including SNPs within the boundaries of another gene. This was case for CARD9, SNAPC4, NFKBIE, EGFL8, GPSM3, IL6R, IL17REL, and IL7R genes; these SNPs might have additional functional consequences.

We found that some lead missSNPs were in high LD with another missense SNP in the same gene. These other SNPs were not recognized by the used GWAS platforms (Table 3). However, their existence might influence and change functional characteristics of missSNPs and genes they harbor, but 
it is not possible to evaluate them together, because there is no adequate tool for collective evaluation of SNPs at the same time.

For example, the NFKBIE gene has a missense SNP rs2233434 detected as a lead, in perfect LD $\left(r^{2}=1\right)$ with another NFKBIE missense SNP rs2233433. Their independent damaging potential on protein sequence and regulatory function potential is not high (scored 4) when calculated for each variant separately based on the PolyPhen-2 and RegulomeDB tools. However, they might be more significant variants when both are present simultaneously (both are common SNPs, both are missense SNPs and both are in perfect LD, meaning inherited simultaneously).

Similarly, GSDMB was found to have two missense SNPs also in high LD. One missense SNP (rs2305480 discovered by the AID GWAS) was potentially damaging for GSDMB protein's conformation and function, and the other was not; however, both GSDMB missSNPs have high scored potential for gene expression modification (1f score), meaning they both may have a strong effect when present.

There is no software tool currently that evaluate simultaneously two or more SNPs, even missSNPs that change amino acid sequence, nor to evaluate changed DNA sequence as for synonymous coding SNPs, because it would require structure-function knowledge that does not exist yet.

\subsubsection{Assessment of additional genes in LD with missense SNPs}

Nine missense SNPs (out of 23 missSNPs) had alternative functional genes in high LD $\left(r^{2}>0.8\right)$. We assessed these additional genes for potential roles in AIDs, because the lead missSNPs might reflect their influence on AID as well.

We examined these additional genes in the context of their function within the immune system, presence in the immune system pathways and association with other autoimmune/inflammatory diseases. The gene evaluation and selection process based on these criteria and its results are presented in the Table 4.

\section{Table 4. Alternative coding genes at the missense AID GWAS SNP loci}

Only the alternative gene UBE2L3 appears to have sufficient support for an AID-related role. UBE2L3 gene encodes a member of the E2 ubiquitin-conjugating enzyme family. This enzyme is demonstrated to participate in the ubiquitination of p53, c-Fos, and the NF-kB precursor p105 in vitro. The modification of proteins with ubiquitin is an important cellular mechanism for targeting abnormal or short-lived proteins for degradation. 
For all other high LD genes, based on our criteria we cannot argue that the alternative genes are substantial genes. However, because the data for the analysed genes is so scarce in all current gene databases, we could not select more genes with certainty. However, it is obvious from the results provided in the Table 4 that even the selected genes are selected based on partially convincing criteria, because the alternative is simply even less known (example is NOTCH gene vs EGFL8 gene, or ATXN2 gene etc.). The selection is made solely based on currently available data, which may change.

Nearly all AID missSNP exhibited at least one of the characteristics with profound functional consequences, so they might be considered functional on several levels: influencing coding genes, influencing other genes in LD, or disturbing regulatory function in their regions.

\subsubsection{Identification of gene candidate dataset based on non-coding AID GWAS SNPs (ncSNP gene dataset)}

The majority of AID GWAS SNPs appear to be located within intronic or intergenic regions (Supplemental Table 1 and Supplemental Figure 1). This makes it challenging to assign functional roles to these variants and explain their disease causation. Based on available data, we examined non-coding AID SNPs functional relevance by searching for genes (coding or non-coding) and regulatory elements associated with them.

\subsubsection{Evaluation of non-coding SNP dataset}

The non-coding AID SNPs with the $p$ value smaller than $p<1 \times 10^{-7}$ value were retrieved from the AID GWASs (Supplemental Table 2).

To help separate potential functional non-coding variants from the large pool of the AID ncSNPs, we used Regulome DB and HaploReg-v2, along with data from the HaploReg v2, NCBI dbSNP and the EntrezGene NCBI databases.

We thoroughly searched the ncSNP LD blocks identified by HaploReg v2 for genes, checking the regions not only in a perfect LD, but also regions with medium stringency of linkage disequilibrium (0.8$\left.0.6<r^{2}>0.4\right)$.

To test for regulatory functional potential of ncSNPs, we assessed chromatin status of the ncSNP LD blocks for potential for regulatory function by the Regulome DB. The RegulomeDB scoring data for all ncSNPs are presented in the Supplemental Table 2:

\section{Supplemental Table 2. RegulomeDB scoring results for GWAS AID SNPS}


Almost $25 \%$ of all AID ncSNPs obtained the highest scores (1f-3b). This indicates their significant capacity to influence gene regulation. The ncSNPs that scored 4-6 were evaluated as less likely to modify gene regulation and were not further examined.

The high-scored SNPs were further analyzed and their characteristics are presented in the Table 5, classified by the associated AID:

\section{Table 5. Characteristics of highest Regulome DB scored GWAS AID ncSNPs}

Almost all high-scored ncSNPs were associated with at least one coding gene, non-coding RNA genes (ncRNAs) or pseudogenes. Only six out of more than fifty highly scored ncSNPs were located in the regions with no known genes. Consequently, the majority of highly scored ncSNP have some kind of potential functional influence in the AID's genes.

The genes in LD presented in the Table 5 somewhat differ from the genes reported in the original GWAS studies for the same ncSNPs. We believe that our selection is more accurate, because we identified these genes with more precision, using updated, richer and more precise annotations from the Human 1000 Genome Project data and HapMap3 data, than ones existed at the time of a GWAS publication.

For example, a risk variant for IBD is the high scoring ncSNP rs8049439, and it is associated with ATXN2L (a gene that encodes a protein of unknown function) and with TUFM (a gene that encodes a protein that participates in protein translation in mitochondria). Neither has any known links to immune system functions. In addition, this ncSNP is in high LD with an microRNA, also with no data about its relevancy in the immune system.

The majority of the dozen highly scored ncSNPs UC risk variants are linked to immune system functions. One of them, rs3024505, linked to IL10 gene, and is also a risk variant linked to multiple autoimmune/ inflammatory diseases.

We have found that ncSNP rs9263739, scored 1f, was in a perfect LD with the two missense SNPs in the region of the Psoriasis susceptibility 1 candidate 1, 2 and 3 (PSORS1 C1, C2 and C3) genes: two protein-coding genes, $\mathrm{C} 1$ and $\mathrm{C} 2$, and an ncRNA gene, C3. This SNP was originally detected as a very high risk SNP for UC ( $p$ value $=4.000 \times 10^{-67}$ ), located in the region within the gene CCHCR1. Our results negate involvement of CCHCR1 gene. According to our analyses, rs9263739 signals PSORS1 candidate genes $\mathrm{C} 1$ and $\mathrm{C} 2$, actually their two missSNPs, one in each gene region. Both missSNPs are evaluated by PolyPhen-2 to be benign (results not shown). PSORS1 candidate genes are also found to 
be associated with psoriasis in the GWASs containing risk intronic SNPs for C1 and C2 (Table 1). They are recognized by OMIM as risk genes for psoriasis, but not for UC. We have no explanation why CCHCR1 gene is still recognized as a UC linked risk gene instead of PSORS1 C1 and C2 genes, except that they are all situated on chromosome 6, a chromosome notoriously difficult to resolve because of many shorter haploblocks and strong LDs along it.

The same rs9263739 SNP is as an example of a lead vs a functional SNP: it is a lead SNP, but it is not a functional SNP, because it is in perfect LD with two other missense SNPs of PSORS1C1 gene; missense SNPs are by definition functional SNPs. Interesting enough, none of these two missSNPs was among the detected GWAS AID missSNPs. Both exist in 1000G Project.

Another example of clarification of non-coding SNP location was the rs9858542 variant, assigned to gene BSN. However, this SNP is in high LD with already known missense SNP rs3197999, which belongs to MST1 gene. For that reason, it is a lead but not a functional SNP for this region; we already analyzed the functional SNP missSNP rs3197999 of MST1 gene and found it not functionally damaging for the MST1 protein (Table 2).

For rs1297265, we were not able to find any known genes in LD, so we were not able to confirm its links with NRIP1 or CYCSP42 genes. The same was true for rs3806308, similarly with no genes in LD. Our SNP-gene prioritization analyses of ten highly scored RA ncSNPs showed interesting results. Several high scoring ncSNPs have links to genes exclusively associated with immune system functions, like CD40, IRF5, RBPJ, CCR6 and CTLA4, but others have links to genes with no specific role within the immune system, like TNPO3, encoding a tRNA import factor, APOM encoding apolipoprotein M, PDE2A a phosphodiesterase or NCOA5, nuclear receptor coactivator. The latter have roles that are more general in the biosystem.

For example, rs805297 is associated with CSNK2B: this gene encodes a casein kinase, a ubiquitous protein kinase, which regulates metabolic pathways, signal transduction, transcription, translation, and replication, while its beta subunit serves regulatory functions. CSNK2B has no exclusive role in the immune system as it participates in ribosome biogenesis in eukaryotes, tight and adherens junction formation. However, as such it is involved in the immune system signaling: Toll-like receptor signaling pathway, BCR signaling pathway and NF-kappaB signaling pathway, and also in infection diseases (as per KEGG and NCBI Gene databases).

We found that other genes associated with ncSNPs have no known function, although their association with AIDs might be explained with their physical proximity to immune genes or location. For examples, 
gene PRRC2A has no known function, but it is localized in the vicinity of the genes for TNF alpha and TNF beta. Gene LY6 encodes lymphocyte antigen 6 complex, also with no known function, but experimentally detected in lymphocytes.

Among 12 high-scored ncSNPs associated with CD, majority is linked to the genes with no immune functions. SMAD3 gene encodes one of the SMAD proteins that act as signal transducers and transcriptional modulators and mediate multiple signaling; including signaling in cancer; however, it does not have any specific function in the immune system. HORMAD2 also has no known function. CCDC88B encoded coil-coil containing protein may be involved in linking organelles to microtubules. Genes SLC22A4 and SLC22A4 belong to solute carrier family 22 of organic cation transporters, which are critical for elimination of many endogenous small organic cations. None has a specific links to the immune system.

Psoriasis was linked to 15 high-scored ncSNPs, with the striking incidence that majority of them are located on the chromosome 6 (C2, SKIV2L, NOTCH4, GPSM3, WASF5, RNF5, DDR1, PBX2, TCF19). Many of these highly scored ncSNP genes have roles in the immune system. Almost half of ncSNPs belong to the HLA complex genes. Other associated genes are predominantly located very close to HLA complex, like genes DDR1 and MUC22, but have no assigned function.

Gene C2 functions in the immune system as one of key component of humoral immunity. It is connected with some autoimmune phenomena.

SKIV2L encodes an endogenous antiviral helicase that participates in RNA degradation and acts in an early phase of immune response.

WASF5P is pseudogene and belongs to the family of genes encoding Wiskott-Aldrich syndrome (WAS) proteins. Wiskott-Aldrich syndrome is a very well recognized disease of the immune system, an immune deficiency. WASF5P pseudogene, which apparently might not be transcribed nor translated, resembles WASF3 protein that is involved in the transmission of signals to the actin cytoskeleton and in the Fc gamma receptor mediated phagocytosis.

PSORS1C1, 2 and 3 genes are dominantly present among highly scored ncSNPs associated with psoriasis, as three loci out of 15 analyzed ncSNPs are linked with the complex. The PSORS1C complex is also connected with several diseases including AIDs; PsA is also linked to the PSORS1C complex, but so far, the $p$ values of associated SNPs were higher than our chosen threshold and for that reason not included in the study (data not shown). 
The ncSNP gene dataset, obtained after ncSNP-gene prioritization analyses, consisted of several completely new genes, but also genes previously determined in the missSNPs dataset (such as NOTCH, MST1 and UBE2L3). Prior to determining the final ncSNP dataset, we eliminated the HLA genes due to their complexity and extremely high LD (same as for the missSNPs dataset) and already detected missense SNP harboring genes. We also did not take into consideration pseudogenes, as they are not usually translated genes and have no information regarding potential function in the immune system (or any other process).

We used the final set of ncSNP gene in further functional analyses, in parallel with missSNP gene dataset.

\subsubsection{Functional assessment of non-coding RNA genes}

We extended our search for non-coding RNA genes in the neighborhoods of the top AID GWAS SNPS. We selected sixty top AID GWAS SNPS, ten for each AID, solely based on their $p$ values as the most influential and relevant risk SNPs. The regions around these SNPs were analyzed for ncRNA genes in a similar fashion as previously done. We used HaploReg v2 to determine high or perfect LD blocks, Regulome DB to score their regulatory function potential and NCBI dbSNP to find annotations and provide map enabling manual search for non-coding genes. Results of the analyses are presented in the Table 6:

\section{Table 6. Non-coding RNA genes in LD with top intergenic GWAS AID SNPS}

Most ncSNPs have RNA genes in high LD, even several different types of ncRNA genes, including micro RNAs (miRNAs) and pseudogenes.

A subset of 8 SNPs with the lowest $p$ values (8 out of 53 analyzed), were also high RegulomeDB scoring SNPs, thus indicating their very high potential to significantly change gene expression of the targeted genes, or to influence regulatory functions of the region (data collected on Feb 3rd 2015.).

Amongst these eight high-scored ncSNPs, only two loci did not have any ncRNA genes in LD. More than a half of loci contained miRNA genes, and often had two or more different kinds of ncRNA genes in the same region, in addition to miRNAs. 


\subsubsection{Functional assessment of microRNA genes}

A set of twelve miRNAs genes in LD with the top ncSNPs were further examined to determine their functional relationship with AID. The results of miRNAs analyses were presented in the Table 9, divided in three sections: $a, b$ and $c$.

\section{Table 7. Characteristics of miRNA in high LD with AID ncSNP dataset}

\section{Section $7 a$}

The majority of the miRNAs were already known human miRNAs with their specific targets (Table 7a). However, none of miRNAs targeted the AID genes.

\section{Section $7 b$}

We also retrieved all miRNA that target 3'-UTRs of the AID genes (Table 7b). None of the twelve miRNAs in LD with the top AID ncSNPs were overlapping with the miRNA detected in the UTR-3' regions of the genes harboring missense AID SNPs.

\section{Section $7 c$}

A few ncSNPs were located in the 3'-UTR- loci of the targeted genes (Table 7c). To check whether we missed any other miRNA that might be altered by AID SNPs, we retrieved all SNPs that belong to 3'UTR regions, irrespectively of genes they belong. Then we inspected these regions for miRNAs. Repeated comparisons between two sources did not reveal any overlapping data among miRNA targeting AID SNP genes and miRNA linked to AID ncSNPs.

\subsection{Functional analyses of GWAS AID SNP gene set: gene-pathway prioritization}

All functional analyses were performed based on the knowledge that majority of genomic components or genes must be incorporated into some type of functional units or biological processes.

After the SNP-gene prioritization analyses, we were able to define the final datasets of the AID SNP harboring and SNP influenced genes (Table 8).

\section{Table 8. AID gene datasets: missSNP genes and ncSNP genes}

The SNP datasets contained only coding genes, as the existing tools for functional analyses can only connect coding genes with pathways and networks. We kept the gene sets separately as two sets, 
missSNP gene set and ncSNP gene set, because missSNP gene set was determined with much more certainty than the ncSNP gene set. We did not want to test all genes together as pooling might undermine efficacy of finding the various enrichments (according to our results shown below), although there is no reason to make one set.

We performed the following functional analyses of the GWAS candidate AID SNPS: gene-network prioritization and gene-pathway analyses, GO term enrichment analyses (for BP, MF and CC) and disease-pathway enrichment analyses. Except for GO enrichments, all used software tools were based on known or imputed protein-protein interactions (PPI). We used several bioinformatics tools for the functional analyses: Cytoscape, ConsensusPathDB and STRING.

\subsubsection{Network analyses}

\subsubsection{Network analyses using Cytoscape software tools}

The networks for each missense SNP harboring gene and TNF were constructed using Cytoscape (Supplemental Figure 3.). The structured SNP networks consisted only of primary (or first) known or predicted interactants for each protein of interest. The protein-protein interaction (PPI) networks of the missense SNP genes and TNF PPI networks are shown in Figure 2. There were no data available (at the time) to construct the networks for several proteins encoded by GSDMB, IL17REL and IL7 missense SNP harboring genes, and for that reason they had to be omitted from the analyses.

\section{Supplemental Figure 3. Network images for missSNP harboring genes/proteins and TNF}

The constructed networks consisted of known or predicted interactants (gene/protein) indicated by the nodes: a cloud of nodes around a central node representing SNP harboring gene/protein or TNF; the nodes were connected by edges representing detected biological interactions between two nodes based on predictive methods (as provided in Methods). The edges signify biological interactions independently of a method by which an interaction was established: experimental biochemical results, text mining data, expression patterns, co-expression and co-localization data, and imputed interactions based on similarity of genes or proteins. In addition to human gene/protein networks, the corresponding networks in mouse, rat (red and white networks in the Supplemental Figure 3.), and occasionally in other organisms, if data were available and in case the orthologue annotations of the same proteins were known in these species and interactions between them were identified.

The biggest network was created for TNF gene: it included over 1500 nodes representing interacting proteins (primary interactants) connected with edges (Figure 2a). The networks constructed for the 
missSNP gene/protein contained much smaller number of the nodes; they varied from several nodes to several dozen of nodes. All nodes were connected with edges representing biological interactions between pairs of nodes, a central node and first interactants' nodes. An example of missSNP harboring gene such as NFKBIE gene/protein is presented in the Figure 2b.

\section{Figure 2a: TNF network}

\section{Figure 2b: NFKBIE network}

We queried the missSNP gene networks to find common, intersecting set of interacting proteins (overlapping members between two networks) among the GWAS AID SNP harboring protein networks, as well as between each GWAS AID SNP harboring gene/protein network and the TNF network. The number of common interactants in the intersections between the constructed networks varied greatly, from no interactants to a very few interactants, up to a dozen or more of common interactants in some intersections of the paired networks (empty intersections are not presented).

\section{Figure 3a. Image of intersection between missSNP ERAP1 and TNF networks}

\section{Figure 3b. Image of intersection between missSNP NFKBIE and TNF networks}

The resulting intersections were not structured in the form of networks; instead, they were presented as collections of unconnected nodes. There were no edges that would indicate any type of biological interactions among these nodes, except for a very few edges detected only in several instances. In case that common intersecting set of genes existed in other species, they were also collected and presented. Next, we examined interactions among SNP harboring gene/protein networks by taking the union of networks. The resulting network is shown in Figure 4.

\section{Figure 4. Union of GWAS AID SNP harboring protein networks}

The union visualization shows how the missSNP genes in AIDs have a potential to interact with each other via direct known or unknown, imputed neighbors (or indirect) intermediators. In the union only the first interactants were preselected and presented; yet the union is a very complex structure, but still a single structure. There are no separate networks, as all networks are interconnected. The union network is suggestive of a space for interactive communications among the SNP harboring proteins that might be important in AIDs pathology.

The number of intersecting nodes representing common members among SNP networks and between SNP networks and TNF network is shown in the Table 9 for easier view and comprehension. 
These results indicate how connected the paired genes/proteins are: the greater the number of interactants, the better connected the two genes may be.

As the Table 9 shows, the intersections of the networks belonging to the missSNP genes/proteins and TNF network have a significant number of common interactants. The very existence of the common members, especially in such high numbers, could not have been detected without this computational approach (they are all first interactants filtered by the Cytoscape tool). Here, we show for the first time that majority of the SNP gene/protein networks do have a number of common intersecting members. Especially rich intersection datasets were obtained for the TNF network intersecting with TYK2, TNFAIP3 and NOD2 gene/protein networks. TNF and IL7R also have several common members, including TNF (not shown in the Table 9). The common intersecting members show how relatively central the TNF gene is in relation to the AID SNP harboring genes, because it is almost always present in every intersection, or at least, one of its receptors (TNF receptors) is present.

In addition, there were very rich intersections containing couple of dozens of genes between TYK2, PTPN22, IL23R, CARD9 and TNFAIP3 networks. Since the majority of these genes/proteins are functionally damaged by the very same AID missSNPs (called risk variants for AIDs), their networks might have a ripple effect on all other networks because of their changed protein functions. Only RTKN2 and LACC1 SNP harboring genes/protein networks did not have any common interactants with TNF or other missSNP networks. This finding could have reflected simply a lack of knowledge about their roles and their poor annotation in the databases (which are evident), and not necessarily the lack of potential interactants. However, they might also serve as a negative control for the Cytoscape process.

It was noticeable that some intersections contained the same gene subsets repeatedly. The repetitive common members in the intersection subsets indicated subnetworks, with not yet well defined common functionalities, like unknown pathways, or parts of unknown pathways (because if they were defined, they would appear as nodes with edges, or networks).

We further explored a possibility that all intersecting sets, obtained from querying missSNP harboring gene/protein networks and TNF network, had some subnetwork in common. We searched for the common interactants among all missSNP networks and TNF network by a process of unification of their intersections. When each SNP harboring gene/protein network was queried for unifying set of genes by the Cytoscape tools, the following result was obtained (Figure 5):

Figure 5. Network union between intersection datasets of missSNPs and TNF networks 
As a result of unification of the missSNP genes/proteins and TNF networks intersections, we found a very simple network of several nodes with edges. This small union network consisted of the nodes connected with edges representing only TNF and eight missSNP harboring genes: CARD9, IL23R, SH2B3, NOD2, TNFAIP3, TYK2, NFKBIE and MICA. The connecting edges in this union network were based on predictive text mining developed by capturing knowledge from the published literature. These results indicate that, although the direct interactions between missSNP harboring genes/proteins and TNF have not been recognized, and interconnecting paths were not (yet) discovered, the full network could be constructed on imputed data. While the edges have not exposed any experimental data (as they were imputed results based on text mining), nor any particular publication confirming interconnection between GWAS missSNP genes/proteins and TNF was disclosed, the resulting network made a rational base for elucidating the AID GWAS SNP roles. This union network implied a potential for anti-TNF action via these edges, and suggested the factual base for a search of the key gene/protein players responsible for anti-TNF drug response in AIDs.

When we retrieved data about expression levels of genes with missSNPs, or AID risk genes, the obtained expression pattern indicated their high expression in several immune-related human tissues and cells. The same genes were also expressed in several adult tissues, but also in fetal tissues. Few genes, like MICA and IL13 did not have noticeable expression in any tested tissue; others, like IL6R and NOD2 were expressed very scarcely. Expression pattern is very important, because obviously, only genes that are expressed simultaneously may interact and form networks or pathways.

\section{Figure 6. Expression pattern of AID missSNP genes}

The Cytoscape network analyses enabled inclusion of various gene data, more inclusive than other tools or databases. At the same time, that is exactly why network analyses by Cytoscape have the capability to make imputations based on broad data sources. Tools that are more conservative and databases like STRING, ConsensusPathDB and KEGG cannot execute the same analyses and none of the Cytoscape results can be found by using these tools.

\subsubsection{Network analyses using STRING software tools (STRING network analyses)}

We also analysed networks of GWAS AID SNP harboring genes and TNF constructed by STRING software tool. Though the STRING tool does not have the same potential for intersecting various networks as Cytoscape does, it allows for evaluation of significance of protein-protein interactions (PPI) for the analysed set of proteins. 
The STRING network images in the Figures 7a, 7b, and 7c present missSNP, ncSNP and allSNP network respectively. All networks were constructed with the high confidence (index of 0.9) and were significantly enriched in protein-protein interactions (PPI) with $p$ values lower than $1 \times 10^{-4}$. The enrichment level $p$ values are shown separately in the Table 10. Most of the interactions were identified with the highest confidence from text mining data, but also from biochemical experimental data and coexpression data.

\section{Figure 7. Image of networks constructed by STRING for missSNP, ncSNP and allSNP datasets}

\section{7a. missSNP network}

\section{7b. ncSNP network}

\section{7c. allSNP network}

The edges among missSNP harboring proteins represented textmined connections, but they were also enforced by biochemical and coexpression experimental data. The edges indicated only 3 interaction among the missSNP set, between TYK2 and IL23R and IL6R. NOD2 and ATG16L1 were linked by edges indicating textmining and coexpression connections. However, TNF was not detected to interact with any of missSNP proteins using STRING. In case of the ncSNP set, eight interactions were observed with the highest confidence (index of 0.9). They also represented text mining data, mainly enforced with experimental data. IRF1 and IRF5 had edges detected by three methods: textmining, coexpression and presence in curated databases. The two chemokines' receptors CCR6 and CXCR1, had additional binding detected in co-precipitation biochemical experiments. The rest of edges between protein pairs represented textmining data and experimental data. TNF was linked with three proteins, CD40, TRAF1 and IL10, the later being only text mining based link. For the compiled set of allSNP dataset that contains missSNP set and ncSNP set together, several new links were detected among genes/protein nodes, which were not recognized before in the missSNP set, nor in the ncSNP set. In the allSNP set several nodes were connected with edges representing experimental data for following pairs: IL10 and IL6R, PTPN22 and CTLA 4, IL10 and TYK2, NOTCH4 and RBPJ. The links based on textmining, co-expression and experimental results were detected between TNFAIP3, TRAF1, CD40 and TNF, connecting for the first time missSNP proteins TNFAIP3, and IL6R, IL23R and TYK2 on one side and NOD2 and ATG16L1 on other side. Only when the allSNP dataset was tested by STRING, we were able to confirm partially the results detected by Cytoscape network analyses.

These three genesets were further tested for PPI enrichment (Table 10). STRING results indicated that the all three sets were highly enriched for PPI, as the number of detected interactions (edges) outnumbered number of expected interactions, even by several orders in case of the missSNP set. 


\begin{tabular}{|l|c|c|c|}
\hline & missSNPs set & ncSNPs set & all SNPs set \\
\hline p value: & $9.1 \mathrm{e}-4$ & $6.3 \mathrm{e}-4$ & $1.8 \mathrm{e}-7$ \\
\hline interactions observed: & 3 & 8 & 16 \\
\hline Interactions expected: & 0 & 2 & 3 \\
\hline number of proteins: & 24 & 40 & 61 \\
\hline
\end{tabular}

The fact that ncSNP set was enriched for PPI, opposes a possibility that the set was not enough coherent. When two sets had been analyzed together, the allSNP set was also highly enriched for PPI, exceeding the sum of PPI for both sets. This finding indicates that ncSNP set brought some PPI enrichment to complement the missSNP set, confirming that our selection of genes performed at the level of SNP-gene prioritization for ncSNPs was the right one.

Network analyses by STRING have shown that the SNP sets are enriched for PPI. It also indicated several links among SNP harboring/influenced genes as well as with TNF.

\subsubsection{Pathway analyses}

\subsubsection{Identification of KEGG pathway dataset for the GWAS AID SNP genes}

To clarify and confirm the encouraging findings from the Cytoscape network analyses of AID SNP genes, as well as some STRING data, which found common interactants between several AID GWAS missSNP genes and TNF, we further searched the KEGG database for all pathways that might home (contain) the selected coding genes harboring AID SNPs or being influenced by AID SNPs. After we found KEGG pathways (AID SNP pathways), we analysed their intersections for the common genes/proteins between two KEGG pathways. 


\subsection{Identification of KEGG pathway dataset for the GWAS AID missSNP genes}

More than 30 KEGG pathways that contain GWAS AID missense SNP harboring genes/proteins have been identified and retrieved for analyses (Table 11). The retrieved KEGG pathways (missSNP KEGG pathways were only selected for the experiment) are associated with one or several AIDs via the inflicted genes. Some were even common to other immune system diseases.

Seven of the missSNP harboring genes (out of 23 missSNP genes) did not have currently any annotated pathway in the KEGG database (nor in any other pathway database including $\mathrm{NCl}$, Reactome, InAct, etc.; data not presented). In addition, the same genes lacking pathways, were often poorly annotated elsewhere (NCBI Entrez Gene or European Ensemble databases or Havana Project), and even their basic functions were almost always unknown.

\section{Table 11. KEGG pathways for the genes harboring missense SNPs}

There was no obvious relationship between the number of pathways in which the missSNP gene participate and the number of associated diseases. Much "wired" genes, like TYK2 (non-receptor tyrosine-protein kinase), participate in numerous pathways and are associated with several AIDs. Others, like FCGR2A (receptor for Fc fragment of IgG) or NFKBIE (NF-kappa-B inhibitor epsilon), are also very "wired" by their presence in several pathways, but are associated with only one or two AID diseases. In addition, several missSNP genes had only one participating pathway, like PTPN22 (protein tyrosine phosphatase, non-receptor type 22 (lymphoid)), but that single pathway is present in almost all AID phenotypes (and in other immune/inflammatory diseases, like SLE or T1D).

\subsection{Identification of KEGG pathway dataset for the GWAS AID ncSNP genes}

The ncSNP genes dataset was also tested for participation in KEGG pathways after exclusion of HLA genes, RNA genes and pseudogenes (Supplemental Table 3 and Table 12). The RNA genes were excluded because they lack any established involvement in the known KEGG pathways as stated before. Pseudogenes such as ncSNP genes USP81P or WASF5P are generally not translated, even not transcribed and their role in pathways, although suspected, is far from being clear. However, they continue to be very intriguing participants, as they might have roles in the immune system. The HLA genes were excluded because of the complexity of their genetic structure. Although the HLA participating pathways are well known, they were not included in our study, because they all function only in one biological process, antigen peptides presentation, and for that reason have significant, but limited consequence on pathogenesis of AID. 
When it comes to existing annotations of genes, the same that was valid for the missSNP genes, was valid for the ncSNPs genes: generally their annotations are very poor. The functional properties are even less known for ncSNP genes than for missSNP genes, and they are less integrated into the KEGG pathways than the missSNP genes (Table 12).

We evaluated ncSNP genes not only for their potential to participate in the KEGG pathways, but also for their functions related to associations with other immune genes, or their relevance in the AIDs and/or other inflammatory/ immune related diseases (Supplemental Table 3).

These additional data for all ncSNP genes were collected from NCBI Entrez Gene, KEGG and the NHGRI Catalog. Only genes that currently have any rationale backed by existing data and by the established criteria for implication of a role in the immune system network were taken into consideration for gene-pathway prioritization and they were considered ncSNP gene set (Supplemental Table 3).

\section{Supplemental Table 3. AID GWAS ncSNPs influenced genes and their participating KEGG pathways}

Significant part of ncSNP genes (the genes we found to be influenced by or aligned with ncSNPs) had no known function. However, some of the ncSNP genes do participate in the KEGG pathways.

Majority of these ncSNP associated KEGG pathways have been already presented among the missSNP gene set pathways (and not only because of scale relationship between genes and pathways, since as many as several dozens of genes/proteins might participate in only one pathway). Other ncSNP pathways were not previously found among missSNP gene dataset of pathways (Table 12).

\section{Table 12. KEGG pathways of the genes harboring non-coding SNPs}

The ncSNP KEGG pathways, not previously detected in missSNP gene KEGG set pathways, are Intestinal immune network for IgA production and Toll-like receptor signaling pathway, both linked to CD40 gene. Other pathways linked to CD40 gene, a gene known to be involved in the immune system, are $\mathrm{T}$ cell receptor signaling pathway and Jak-STAT signaling pathway. These pathways are the key signaling pathways already detected amongst missSNP KEGG pathways.

Our finding reflects a concept that it might be insufficient to take into consideration only missSNP variants from AID GWAS in order to find underlying mechanisms. The detected missSNPs and ncSNPs genes KEGG pathways (we named them all AID SNP KEGG pathways) might be all relevant for SNP harboring genes functioning; we defined all of them as potentially pathological pathways for the AID diseases under study. 


\subsection{Classification of AID SNP KEGG pathway into modules}

We further classified the AID SNP genes containing pathways according to the pathway KEGG classification as shown in the Tables 13 and 14. The pathway classification is important step, because it enhanced our understanding of more advanced modules in which the pathways are thought to operate. However, the modules classification is still a work in progress and many pathways are still only partially classified into the modules (according to the KEGG database).

\section{Table 13. Classification of missSNP KEGG pathways}

\section{Table 14. Classification of ncSNP KEGG pathways}

More than half of the detected pathways represent description of pathogenesis of human diseases, either systemic or infectious diseases (Tables 13 and 14). These disease pathways are very complex pathways consisting of assemblies or pathways, and they usually encompass several basic signaling and developmental or system bound pathways within each disease pathway.

Apart from these disease pathways, there is much smaller number of basic or core KEGG pathways (Table 13 and 14). We found only 21 basic pathways among the missSNP KEGG pathways and 15 ncSNP KEGG pathways. We consider all listed basic pathways, both missSNP KEGG and ncSNP KEGG to be pathogenic pathways in AIDs. Among these basic pathways, only 6-7 pathways belong to the signaling pathways. Interesting enough, majority of GWAS AID SNP impacted genes belong to these pathways: more than twelve SNP genes participate in only 5 core pathways (data not shown).

Since our research was aimed to find common, intersecting genes between curative and pathogenic pathways, only the SNP KEGG pathways present at the same time in the dataset of TNF pathways were further taken into consideration.

In order to find the overlapping, common pathways, we had first to define TNF pathways, which aTNF drugs use once administered into human body.

\subsubsection{Identification of KEGG pathways of five anti-TNF biologics and TNF}

In order to find potential overlapping of pathways between AID SNP gene pathways (defined as pathological AID pathways) and anti-TNF biologics (curative) pathways, the next step was to identify the pathways that are engaged by the five anti-TNF biologics drugs used for AID therapy. The pathways of 
five anti-TNF biologics are annotated in the KEGG pathway database, retrieved and presented in the Table 15:

\section{Table 15. Anti-TNF biologics drug pathways}

Only four pathways were shared between all five drugs/biologics (highlighted) and a few others are common to the majority of aTNF biologics. The reason for different usage of the pathways by the aTNF biologics is unclear, as four out of five drugs have relatively similar targets, and only slightly different protein structures (data from Drug Bank). The differences in the existing pathway usage for aTNF drugs a provided in the Table 15., do not coincide with the differences in the molecular structure of the five aTNF biologics and cannot be explained other than the incomplete data.

The aTNF biologics have more than 12 targeted proteins other than TNF (TNF is a biointeractor with the bioactive role) with no known pharmacological action: all subunits of the complement component 1(C1 q, r, and s), Fc receptors with high or low affinity (Fc receptors) and Prostaglandin G/H synthase 2 (data collected from Drug Bank).

The reported narrow pathway usage found in the KEGG databases for aTNFs does not reflect their targets. We considered that it would be erroneous to use only the reported anti-TNF pathways, and neglect all known TNF pathways through which the aTNF biologics might act as well. We concluded that it was reasonable to assume that all currently known TNF pathways have been influenced with the aTNF biologics therapy, and not only a few annotated. Table 16. provides all known canonical TNF pathways (as retrieved from KEGG database). They all are able to propagate anti-TNF drugs' action and for that reason, we considered all TNF pathways important for the research:

\section{Table 16. KEGG TNF pathways}

We analysed the position of TNF in every pathway and made conclusions regarding whether the TNF is a signaling molecule in a pathway, or it is a product of a pathway. This is an important difference, because the direct effect of aTNFs as inhibiting molecules is binding to TNF and inhibiting TNF action in the intercellular space. Many of these KEGG TNF pathways only claim that the TNF is its participant, but do not provide clear role assigned to TNF as its member (Table 16.). Among the pathways, only few pathways have TNF as a signaling, trigger molecule (12 pathways out of 45 TNF pathways), or as an end-product ( 9 pathways out of 45 TNF pathways), and two pathways have TNF both as a signaling molecule, and as its product. More data would be needed to provide clearer role of TNF. However, all listed TNF pathways from KEGG database were further used to search for overlapping pathways 
between GWAS AID SNP pathways and TNF pathways, although our analysis suggested that not all pathways might contribute equally to the TNF role overall.

\subsubsection{Curative pathways: KEGG pathway dataset common to both GWAS AID SNP pathways and TNF signaling pathways}

Not all SNP gene pathways were influenced by TNF participation; only ones influenced by TNF were considered further for analyses. We cross-examined both sets of pathways and detected GWAS SNP KEGG pathways that are shared with known TNF pathways. The ncSNP gene pathways and missSNP gene pathways were separately analyzed for common members with TNF pathways and the resulting subsets of overlapping pathways are presented in the Supplemental Table 4 and Supplemental Table 9 (color coded for easier identification).

Supplemental Table 4. Comparison between all pathways: missSNP gene pathways, ncSNP gene pathways and TNF containing pathways

We found that three pathogenic AID SNP pathways, common to both missSNP and ncSNP pathways, did not have any members common to TNF signaling pathways: two important signaling pathways, Notch signaling pathway and Jak-STAT signaling pathway, and one immune system pathway, Intestinal immune network for IgA production. Consequently, these three pathogenic pathways were not considered further as potential curative (intervention) pathways, because simply they could not be influenced by anti-TNF therapy (Table 17). Similarly, B cell receptor signaling pathway, a very important missSNP KEGG pathogenic pathway, is not influenced with TNF at any point (Table 17).

Only pathways that are common to the group of TNF pathways and the group of SNP gene pathways we consider being important paths for the anti-TNF biologics action (intervention capable). We named these KEGG pathways potentially curative AID pathways and presented them in the Table 17 , together with pathways that cannot support TNF action because they do not contain it, but may still influence pathogenesis of the AID.

\section{Table 17. Relationship between AID SNP pathways and TNF pathways}

All three pathways selected for ncSNP genes associations have weaker links than missSNP harboring genes. MAPK signaling pathway was extracted based on gene GNA12, a gene that has very few annotations and is not highly probable to participate in the pathways. The same is valid for gene SMAD3 that led us to TGF-beta signaling pathway. Genes CD40 and IRF1, IRF5 are linked with Toll-like 
receptor signaling pathway and are well-annotated genes, but the pathway is very complex and insufficiently linked to TNF (TNF is only a product of this pathway: Table 16).

Hematopoietic cell lineage pathway has very uncertain role because it is a very complex pathway, a collection of huge number of proteins and role of IL13 gene liking AID SNPS to it is unclear. Adipocytokine signaling pathway, linked to NFKBIE gene, is not clearly annotated as linked to immune system, and its potential role is clearer in T1D, than in any of AID under study.

We wanted to confirm these results from the KEGG database using Cytoscape tools. In order to find intersecting members (genes/proteins) of the selected SNP AID pathways and TNF pathways, we used the Cytoscape to query intersections of these pathways retrieved from KEGG database. For this interrogation, SNP and TNF KEGG pathways were queried among themselves in pairs. The following results were found in the Supplemental Table 5.

\section{Supplemental Table 5. Subset of pathways common to AID missSNP pathways and TNF} signaling pathways: intersections analysed by Cytoscape

As concluded from this experiment, only few SNP AID pathways contain TNF signaling pathway members in the intersecting gene sets. The data confirmed previous results shown in the Table 17 for majority of the TNF-SNP common pathways.

It is very important to stress that anti-TNF blockers (anti-TNF biologics) do not influence the SNP AID pathways that did not contain the intersecting set.

For example, the NOTCH signaling pathway is completely independent from TNF signaling and from all other SNP-gene-harboring pathways. Previously we discovered NOTCH pathway as a potential pathogenic AID pathway; it turns to be one of SNP pathways not able to "succumb" to TNF modification, not able to interfere with TNF signaling. For that reason, we think that anti-TNF biologics are not able to modify the NOTCH pathway.

However, TNF could influence Osteoclast differentiation pathway, at least a mayor part of this pathway. TNF also could influence B cell receptor signaling and Ubiquitin mediated proteolysis according to Cytoscape analyses, contradictory to our earlier results for these pathways obtained from KEGG database.

Several other listed pathways could be linked via TNF and "talk" among themselves only because of the presence of TNF gene: NOD like signaling, Antigen processing and presentation, and Cytokine-cytokine 
receptor interactions (actually not a real pathway, but more collections of receptor-ligand complexes). These three pathways are examples of the pathways influenced by TNF. They could be modified by anti-TNF biologics.

The Jak-STAT pathway could influence only cytokines and cytokines receptor containing pathways; there are quite a number of missSNP genes in this group, among cytokines and cytokines receptors (IL6R, IL13, IL7R, II17REL, etc.). However, Jak-STAT pathway had no influence on TCR and BCR signaling, nor on the Antigen processing and presentation, IgA production in intestines, or on NFkB signaling pathway. The TGF-beta signaling and MAPK signaling pathways, found in the ncSNP pathway group, also had no common members with Jak-STAT pathway (data not shown in the tables).

In conclusion, the results in the Table 17 and Supplemental table 5 showed separation between at least two groups (if not more) of AID GWAS pathways that could communicate intra-group, among themselves, but not inter-groups, between the two groups. If we consider the AID GWAS SNP gene pathways potentially pathological pathways, then these two pathway groups (or more) obviously have consequences on the therapy with anti-TNF biologics. The image illustrating at least two separate groups of pathways was shown in the Figure 8.

In this illustration, missense SNP genes are shown as the nodes connected with the edges representing KEGG pathways. Two distinct groups of SNP harboring genes are clearly separated as they do communicate intra groups via different pathways.

Several missSNPs harboring genes (SH2B3, NOTCH4, LACC1, MST1, MICA, and ATG16L1) do not belong to any of the two well-defined groups of pathways. NOTCH4 participates only in its own pathway, NOTCH signaling, which is completely separated from any other pathway. The similar case is with $\mathrm{SH} 2 \mathrm{~B} 3$ that only belongs in the Neurotrophin signaling pathway, exclusively occurring in neurons.

\section{Figure 8. Interconnection between missense SNP harboring gene pathways and TNF}

ATG16L1 only belongs to the pathway for Regulation of autophagy, which has no tissue restrictions, but on the distal end, this pathway interacts with Apoptosis and Antigen processing and presentation pathways. Both pathways have no connections with Jak-STAT pathway or the group of pathways linked to Jak-STAT pathways. Genes LACC1, MST1 and MICA do not have any known KEGG pathway (or any other pathway from other databases; results not presented).

PTPN22 and ERAP1 are part of Antigen processing and presentation pathway. This pathway is influenced directly by TNF, so both genes are connected with TNF signaling pathways. 


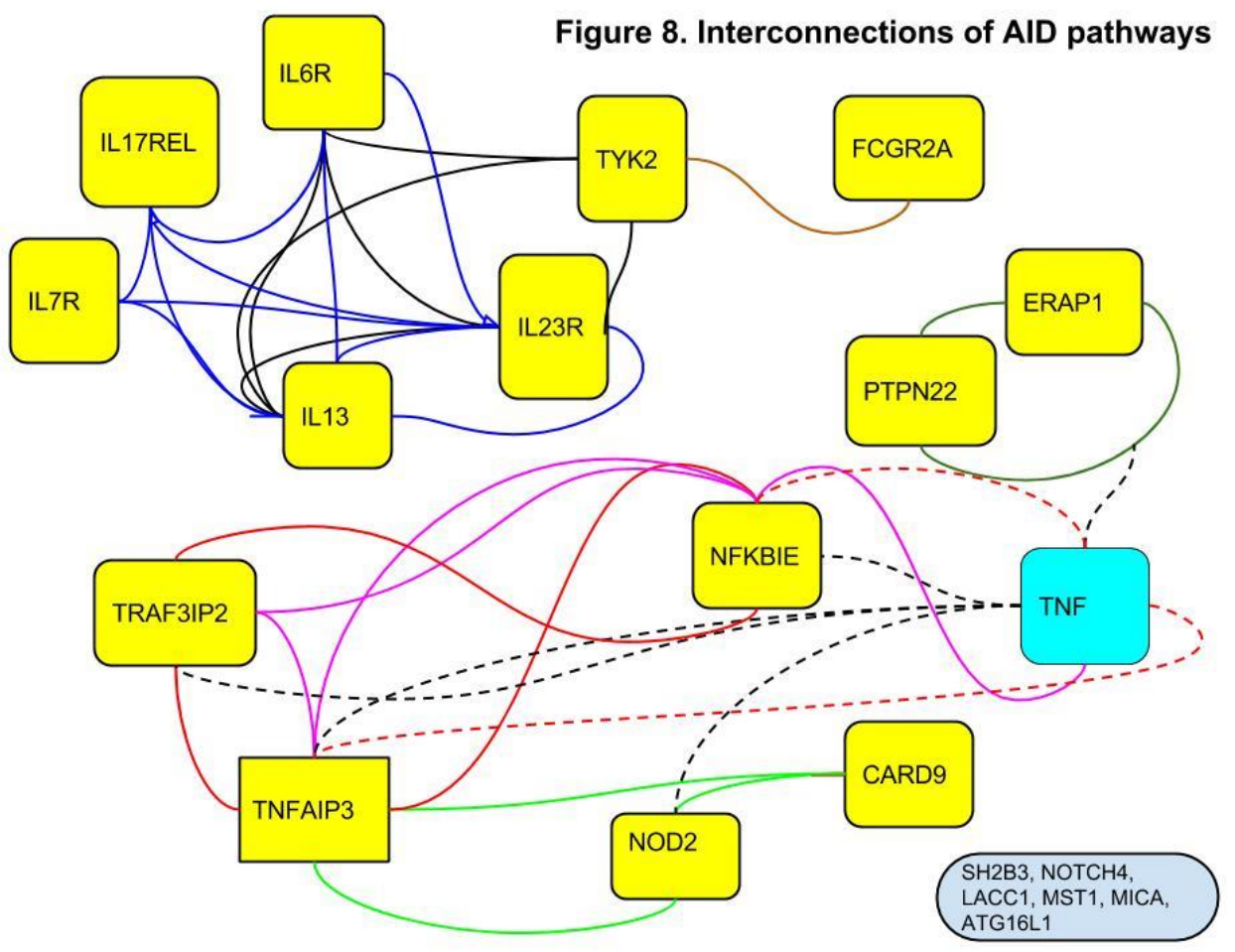

Legend: connecting lines between genes denote KEGG pathways:

red: NFkB signaling

black: Jak_STAT signaling

blue: Cytokine-Cytokine R

green: NOD-like signaling

brown: Osteoclast differentiation

pink: TNF signaling

dark green: Antigen Proc\&Presen

black dotted: TNF signaling

red dotted: $T$ cell $R$ signaling

Color of gene's cell:

yellow background:

gene is connected to the selected KEGG pathways

blue background:

gene is not connected to the selected KEGG pathways

The Apoptosis pathway is influenced by TNF and NFkB pathway (which is also influenced by TNF). It has several SNP gene members, but has no connections with Jak-STAT pathway.

All pathways with TNF influenced members could be clearly modified by anti-TNF biologics, while others could not. The obtained results are manually extracted first and then confirmed by Cytoscape software tools.

However, for additional confirmation (since we had a few discrepancies, or were not able to test the difference between Cytoscape data and manual KEGG retrieval), we also tested the AID SNP influenced genes and TNF gene pathways with additional bioinformatics tools: STRING and ConsensusPathDB, using gene-pathway prioritization tools based on PPI. The obtained results of genepathway prioritization are presented in the Tables 19, 20 and 21. 


\subsubsection{Pathways enrichment for AID GWAS SNP gene sets by STRING}

The STRING bioinformatics tool was employed to perform pathways enrichment analyses of the AID GWAS SNP affected genes. Three genes sets were separately analyzed and compared: missSNP gene set, ncSNP gene set and the set consisted of all GWAS SNP genes.

All three sets, (missSNP set, ncSNP set and allSNP set), analyzed for STRING pathways enrichment were determined earlier (Table 8). The scoring level of confidence for pathway enrichment was set at 0.7 or high for all attributes. The STRING retrieved the following pathways:

\section{Table 18. STRING pathways enrichment data for AID GWAS SNP gene/protein datasets}

Four core and two disease pathways were enriched for missSNP gene set. However, ncSNP gene set has shown only one pathway, which is more collection of cytokines and their receptors than a directional pathway.

The allSNP set included wider list of KEGG pathways enrichment, than the missSNP set. It showed that the ncSNP set, although was enriched only in one pathway, contributed to the enrichment of its KEGG pathways when assembled together with the missSNP set. The number of genes participating in the enriched KEGG pathways had increased, and new KEGG pathways were added to the list of pathways enriched for all AID SNPs. This might bring us to the similar conclusion as before, that although the ncSNP set was not enriched per se, when added to the missSNP set they represented a unified set of genes/proteins enriched for specific biological functions. All STRING pathway data were considered after the correction for false discovery rate (FDR) as pathogenic pathways for AIDs.

\subsubsection{Pathways enrichment of AID GWAS SNP genes by ConsensusPathDB}

Using ConsensusPathDB the analyses for over-representation of pathways were performed for the same sets of AID GWAS SNPs: missSNP, ncSNP and allSNP sets.

\section{Supplemental Table 6. ConsensusPathDB: enriched pathway sets for missSNP dataset}

The missSNP set retrieved 78 enriched pathways from the several pathway databases that serve as source databases for ConsensusPathDB software (Supplemental Table 6). This set appeared in several new pathways not obtained previously from STRING enrichment results, but were recognized before in the data obtained in the Cytoscape analyses. New pathways were NFkB signaling, TNF signaling and Toll like receptors signaling pathways. ConsensusPathDB also retrieved several other pathways that did 
not exist in the KEGG, such as IL2, IL4, IL6 and IL23 signaling pathways, though they could be considered as the parts of the more integrated KEGG pathway for cytokines and cytokine receptors.

When the same set of missSNPs was tested for overrepresentation only in KEGG pathway database, only 23 pathways from KEGG were detected after correction for FDR (Table 19).

\section{Table 19. ConsensusPathDB: enriched KEGG pathway-based sets for missSNP geneset}

The number of pathways was much higher, indicated better retrieval enrichment of the used tool. ConsensusPathDB was able even in KEGG database to retrieve more pathways than STRING, where only five KEGG pathways were detected.

Pathway enrichment analyses retrieved 26 pathways for the ncSNP set from the several pathway databases used by ConsensusPathDB retrieval tools (Supplemental Table 7).This set showed new pathways, not obtained previously from STRING for the same ncSNP set: T cell receptor signaling and Chemokine signaling pathways; all these pathways came from the KEGG database.

\section{Supplemental Table 7. ConsensusPathDB: enriched pathway sets for ncSNP geneset}

Interestingly enough, the ncSNP set pathway enrichment was not indicative of immune system pathways, because only couple of pathways were linked to the immune system, like Cytokine-Cytokine Receptor and $\mathrm{T}$ cell signaling pathways (pathways that did not show up in the pathway enrichment analyses for missSNP set).

The allSNP set was tested separately (Supplemental Table 8) for pathway enrichment from all pathway databases sources and from KEGG database (Table 20).

\section{Supplemental Table 8. ConsensusPathDB: enriched pathway sets for allSNP geneset}

More than $70 \%$ of the allSNP set genes were present at least in one pathway. Huge number of enriched pathway sets was retrieved (85), but most of them were overlapping. The set with the most members was Jak-STAT set and Cytokine-cytokine receptor interactions set with 6-8 members. However, when only KEGG pathways were selected, all seven core pathways were retrieved, in addition to Cytokinecytokine receptor interactions and Chemokine signaling (both are complex ligand-receptor type of interactions) (Table 20.).

Table 20. ConsensusPathDB: enriched KEGG pathway-based sets for allSNP geneset 
The set of seven core KEGG pathways was the same retrieved by the Cytoscape tools. Two additional pathways, Toll-like receptor signaling pathway and Chemokine signaling pathway, were additionally discovered as enriched pathways for the allSNP dataset. This result also confirms our previously shown result after manually searching KEGG pathway database.

This way, we confirmed with other independent software tools the same findings presented in the Figure 6.

\subsection{Functional analyses of AID GWAS SNP data using Gene Ontology}

In order to understand potential function attributes and connection between AID SNPs and disease mechanisms, we applied GO term enrichment analyses. Gene ontology terms enrichment analyses are independent of PPI and allow extracting knowledge about biological processes (BP), molecular function (MF) and cellular (CC) activity location for the genes of interest. GO term enrichment analyses complement our search for pathways and networks of the proteins that harbor AID SNPs or proteins/genes that are influenced by AID SNPs.

We used STRING and ConsensusPathDB tools to retrieve and analyze GO term set enriched for BP, MF and CC ontology terms assigned to SNP genes. The results are presented in the Tables 21-24. Only the significantly ranked GO terms for the sets are provided, based on $p$ and $q$ values. The numbers and percentages of SNP proteins in the analyzed sets are given, along with the total number of known proteins in a group. We used both STRING and ConsensusPathDB because they provided slightly different data resulting from different algorithm applications.

\subsubsection{GO term enrichments by STRING}

The STRING enrichment analyses were limited by the number of members in the analytical sets, because the sets had to contain at least ten members in order to be evaluated by STRING. It worked well for the three AID SNPs sets, but finer parsing down to subsets was not possible.

Table 21. STRING BP, MF and CC GO terms enrichment for AID misSSNP, nCSNP and allSNP genesets

GO annotations for biological process (BP) indicate that all three sets exhibited GO term enrichment. The GO terms indicated that the sets were enriched for immune system and its function, development and regulation. The allSNP set was the richest in the BP terms as expected (Table 21). However, very 
few annotation terms or none existed for terms delineating the molecular function (MF) or cell component (CC) spaces (Table 21).

The most probable reason for these results was that GO terms are biased towards BP terms as MF and CC terms were poorly annotated for the sets (or in general). From the BP terms, it was clear that the SNP sets indicated engagement in the immune system, immune system development, its function and control. Poorly annotated terms for MF and CC were not sufficient to make any conclusions, neither about specific functions nor about location of the analyzed SNP sets. It was known from other data that most of proteins were membrane receptors, lymphokines, enzymes or translation factors, but that could not be confirmed by their enrichment in GO terms.

For that reason, we also employed ConsensusPathDB and its tools that did not have the limitations for enrichment analyses; in addition, the ConsensusPathDB has a richer base of pathways' search space.

\subsubsection{GO term enrichments by ConsensusPathDB}

Using ConsensusPathDB, the analyses for over-representation of $\mathrm{GO}$ terms were performed for the same sets of AID GWAS SNPs: missSNP, ncSNP and allSNP sets (Tables 22-24).

\section{Table 22. Enrichment of BP, MF and CC GO terms for missSNP geneset}

GO BP term enrichment indicated involvement in the immune system functions, activation of its components often in response to stress (the most numerous terms) and the development and regulation of the immune system. These findings were in line with previously obtained GO terms from STRING, but they were better classified for GO levels and provided clearer answer to biological processes for missSNP set. Regarding MF and CC, the missSNP set was enriched for terms indicating protein binding, to the less extent antigen binding, and its location was tied to cellular membranes.

More detailed analyses of MF terms enriched for missSNP dataset indicated that the protein binding function was linked to terms describing receptor-ligand binding for cytokines or growth factors facilitated by CARD domain of the interacting proteins. They also included antigen binding function, which is the one of the core of immune functions (Table 22).

Further deeper search for CC terms only returned location descriptions linked to cell membrane, cell surface or its periphery. Higher-level CC terms, like cell organelles, were not enriched for the missSNP set (Table 22). 
However, again we have seen the bias towards GO BP annotations vs CC or MF annotations in ConsensusPathDB similar to STRING, except the later categories were less rich and less detailed. It was not clear whether it was caused by the nature of SNP sets or general deficiencies of annotations of GO CC and MF spaces.

The ncSNP set retrieved fewer results, but still was able to indicated clearer picture for their functional engagement like immune response and signaling (Table 23):

\section{Table 23. Enrichment of BP, MF and CC GO terms for ncSNP geneset}

GO enriched term sets for ncSNP dataset pointed towards immune system involvement and its regulation and response. They also included terms describing signal transduction and cell migration. Cell component localization was the same as for the missSNP set, but the MF terms indicated signal transduction, protein binding and transcription factor binding for specific DNA sequences.

When we tested the allSNP set for GO term enrichment, we obtain very similar results regarding immune system processes. The most numerous enriched ontology terms indicated immune response and stimulus response, signal transduction, regulation and development. At the MF level, terms were enriched for protein binding and signal transducing. At the level CC enrichment of location terms argued for cell membranes at the periphery of cells as a place of activity of the majority of proteins harboring/influenced by the AID SNPs (Table 23).

\section{Table 24. Enrichment of BP, MF and CC GO terms for allSNP geneset}

Almost all biological process terms fitted into known immune system processes (Table 24). The MF terms were connected with protein binding, transcription factors binding and signal transduction, all in line with roles playing in the immune system (or any other system for that matter) (Table 24). The CC terms for allSNP set did not differ from the CC terms for the missSNP and ncSNP sets (Table 24).

\subsection{Disease prioritization}

\subsubsection{Disease Connect DB enrichment for AID SNP dataset}

We employed the DiseaseConnectDB bioinformatics database and tools to search from an opposite side of our already presented approach and to double check whether this relatively new tool would confirm our results using other tools (ref). 
When the AID diseases were searched for enrichment in the pathway set employing DisseaseConnectDB software tool, we obtained the results very similar about the pathways engaged in the AID diseases to what the STRING and ConsensusPathDB tools were able to provide (Table 25). As shown in the table, all pathways found to be active in AID diseases, indicate pleiotropy on pathways level. We already have shown pleiotropy at the gene level using different bioinformatics tools (Table 3).

\section{Table 25. Disease Connect DB pathway dataset for AID}

Out of more than 40 pathways linked to the AIDs by DiseaseConnectDB tools, we selected 20 pathways based on their presence in two or more AID diseases. We believe that even pathways that are currently detected to be present in only two AIDs, mainly CD and RA, will be found more often in three other AIDs once more meta-GWAS are performed and more data is collected for these diseases.

Among the associated pathways, only seven pathways are real core signaling pathways, transducing signals from/to environment and cells/tissues. These pathways are a part of general environmental information processing or signals transduction within the immune organismal system. There are three core-signaling pathways, and four immune systems signaling pathways.

The other $2 / 3$ of overlapping pathways between AIDs belong to human diseases modules, diseases like metabolic, immune or infectious diseases caused by parasites, viruses or bacteria. The DiseaseConnectDB discovered pathways for the AID SNPS, belong to the category of human diseases identical to the pathways prioritized for the same AID SNP genes manually using KEGG database, or by STRING or ConsensusPathDB. They are complex pathways that consist of several core pathways, including signaling pathways and immune system pathways among them.

\subsubsection{STRING disease enrichment for AID GWAS SNP dataset}

We wanted also to compare these pathways-diseases prioritization results with the disease enrichment data recently available in STRING software system.

\section{Table 26. STRING disease enrichment data for AID GWAS SNP genesets}

Analysis using STRING tool for disease enrichment has shown that GWAS SNP genes were significantly enriched (after FDR correction) for the almost all of the seven autoimmune/ inflammatory diseases under study except ulcerative colitis, or all AIDs except psoriasis.

After checking for the complete list of diseases available in STRING database, we realized that UC was not listed in the STRING database. The reason is not clear, but IBD and Crohn disease, are sometimes 
considered two separate diseases within UC umbrella; both IBD and CD are on the top of the list of diseases in which the allSNP set is enriched. Again, the ncSNP set did not show enrichment for any diseases, but also had no negative effect on the list of enriched diseases for missSNP set that was identical to allSNP set; the ncSNP gene set was neutral (data not shown in the Table 26). 


\section{DISCUSSION}

There is a strong consensus that genome-wide association studies (GWAS) have allowed acquiring enormous knowledge about genetics of human autoimmune diseases (AID), probably more than any other scientific approach before (Zerhouni and Nabel 2008; Visscher et al., 2012). The agreement comes even from the scientists who deny CD-CV postulate (McClellan and King 2010). The AID GWAS have characterized hundreds of genetic associations as potential risk factors for autoimmune disease development and inheritance. In addition to identification of the inheritance and risk genome structures, the GWAS acquired knowledge has a potential to clarify, even discover novel disease mechanisms by defining the operational AID biological pathways; it also has a potential to pave a road to development of new drugs for management of autoimmune diseases.

Starting from GWAS data, we launched on a task to find why the already approved aTNF therapy for several AID does not work in a good portion of AID patients. One possibility, apart from all others (natural antibody inhibition, acquired immunization to aTNFs, imbalance between pharmacokinetic parameters, under-dosing, etc.), is that the genetic makeup is accountable for the unresponsiveness. There is a possibility that patients who respond and who do not respond to aTNFs, differ in a couple or dozens of gene alleles that are key to aTNFs response. Variability of those gene alleles could be tested ahead of the aTNF therapy and used to make prediction about the responsiveness. Similar situation has been discovered already for several drugs, where an advance allele gene testing helps making suitable therapy choices (Limdi and Veenstra 2008). This thinking represents a base of pharmacogenomics and it is in line with its goals: taking into consideration genome characteristics of an individual, provide the optimal medication that will not trigger negative adverse events, but deliver a cure.

Our rationale was that, by using AID GWAS-generated knowledge, we could define genes and consequently, more important, the pathways engaged in the AID pathogenesis and aTNFs response. We assumed that the pathological AID pathways are all pathways discovered starting from AID GWAS SNPs. We reasoned that a crossroad between the pathways engaged in aTNFs action (which are known), and the pathways responsible for diseases activity (unknown but believed to exist), would point towards genes/pathways responsible for the aTNFs response in AID. Overlapping between the AID pathological pathways and aTNF interventional pathways could define a subset of curative pathways, which should contain key structures responsible for both activities: aTNF actions driving to remission or AID natural disease progression. A successful aTNF therapy induces a remission in AID patients, a condition in which we do not expect the disease pathological pathways to be any more active: a 
successful aTNF therapy shuts down the pathological pathways; but when aTNF therapy is unsuccessful, the pathological pathways stay operational and disease remains active.

Incoming new studies, if funded, should study further the polymorphism of these newly defined key genes or pathways in individuals in order to confirm their different functionality in cells and tissues of responders and non-responders. Furthermore, those genes could be studied by combining the information in AID patients' health records with detailed dense genotyping or better, narrow scoped sequencing of the regions, whole exomes (WES) or genomes (WGS) by the next generation sequencing methods (Kiezun et al., 2012).

\subsection{GWAS AID Associations}

In order to provide insights into the AID disease biology, we encountered several challenges in the interpretation of GWAS association. They had to be first resolved, before we could identify candidate gene sets based on currently available AID GWAS data.

\subsection{1. $P$ value}

The common practice has been to consider only GWAS associations with statistical significance labeled with $p$ values equal or smaller than $p<5 \times 10^{-8}$ (Manolio et al., 2009). Lower levels of significance may be used, with $p$ values of $p<1 \times 10^{-5}$, even $p<1 \times 10^{-4}$, in order to include more associations and still obtain meaningful results in SNP-gene prioritizations. However, such high $p$ values, used as a cut-off $p$ values, might condition retrieval of many false positive associations. Too many false positives might "dilute" SNPgene prioritization results and result in no enrichment in parameters that define gene set functions. No enrichment might originate from the fact that selected genes have no common function. No enrichment might result from the ratio between genes with coherent functions vs. genes with incoherent functions; in that case, the last group obscure the first group in the same gene set. On the other side, if the cut-off value is too low $\left(p<5 \times 10^{-8}\right)$ or more, many legitimate associations are lost and with them a part of real biological functions (Visscher et al., 2012). Some researchers argue that even associations with $p=5 \times 10^{-}$

${ }^{3}$ are meaningful (Visscher et al., 2012). After testing a few sets for results, we decided to crop all AID associations with $\mathrm{p}$ values equal or smaller than $1 \times 10^{-7}$. However, in our case, very few AID associations had $p$ value of $1 \times 10^{-7}$, as the majority of AID SNPs had much lower $p$ values (Supplemental Table 1). The selection of SNP association cut-off $p$ value was especially important, because some AID have few GWAS associations, yet we wanted to include associations for every AID in which aTNF therapy has been applied (an example is AS with very few associations found so far). However, unless other relevant orthogonal data supported a SNP with a $\mathrm{p}$ value of $1 \times 10^{-7}$, or higher, we avoided using it for gene or 
pathway prioritization analyses. NHGRI Catalog collects all association with $\mathrm{p}$ values equal or lower than $p<1 \times 10^{-5}$ as real and justifiable associations, keeping as many associations as possible.

We retrieved associations for each AID under study and treated them as a pool of data of equal value, not giving more weight to a disease with more GWAS SNP or lower $p$ values. We were not interested in differences between AIDs, because for our purpose, the bigger pool of SNPs provided larger searching space for SNP-gene-pathway or network prioritization, since we have been interested in finding common ground why aTNF works/or not in all of selected diseases.

The AID associations used for our research mainly encompass common SNPs with high frequency (MAF $\geq 5 \%$ ), but also common SNPs with low frequency $(0.5 \%<M A F<5 \%)$. We sourced associations only from the NHGRI Catalog, because NHGRI associations are curated (see Methods), while associations from other sources are not. The associations taken directly from publications suffer from lack of the necessary review and selection criteria. We did not use very low MAF SNPs $(0.1 \%<$ MAF < $0.5 \%$ ), nor we used SNPs available from sources other than NHGRI Catalog. We did not collect nor analysed rare SNPS, because they are not in the Catalog, as they are not intentionally recognized by GWAS methodology. However, choosing lower cut off for $p$ values does not necessarily mean elimination of rare variant signals, because there is always a possibility those SNPs with lower $p$ values do reflect rare mutations, possible even several rare mutations grouped in a narrow gene segment. Rare variants are especially indistinguishable if they are in very high or perfect $L D$, as it is the case for HLA complex.

We also did not use combination of significant SNP associations within a gene in a broad sense in order to obtain lower $p$ values, because combined SNPs could not be analyzed by the software tools we used. If one gene region has two or even several SNPs associated with an AID, it is logical to assume that the gene has more weight overall as a risk factor for the AID. The situation is even more complicated when two SNPs are in high or perfect LD, as they cannot be analyzed for their combined influence, which obviously exists (our examples are two missSNPs in NFKBIE gene in perfect LD). Again, there is no available software that could analyze these cases.

Some recent data claims that pooling GWAS studies with a significant pleiotropy between diseases, allows for a less stringent new statistical method. It enhances discovery of new SNPs from already published studies, which might explain additional hereditary component, by increasing $\mathrm{p}$ values to $10^{-5}$ or even less (Lilley and Wallace 2015). It is important to keep in mind that genes might be incorrectly included in gene sets not only because of $p$ values, but also because of erroneously assigned functions by poor annotations or lack of it. 


\subsubsection{Rare vs. common (and low frequency common) SNPs}

Several common AID missSNP risk factors in our study are not exactly common SNPs by the strict definition, as their minor allele frequency (MAF) values are lower than 5\%. However, they are considered common SNPs with lower frequency $(0.5 \%<\mathrm{MAF}<5 \%)$ in European population, and not rare variants $(\mathrm{MAF}<0.1 \%)$. The examples of common SNPs with lower MAF are the missSNPs in TYK2 and IL23R genes, which we found to be associated with multiple AID (Table 2). Both missSNP are situated within highly conserved regions, typical for common variants.

The rare risk variants (MAF<0.1\%), otherwise undetectable by GWAS, might still be responsible for an association signal in AID detected by GWAS, because rare SNPs might be in high LD with lead SNPS (Beaudoin M, et al., 2013). When analyzing SNPs in high LD with a lead SNP, rare variants could be among them as well, because rare SNPs are present in the dbSNP and therefore used by software tools like the HaploReg. Although we did not collect and analysed rare SNPs, rare SNPs might signal via common SNPs in GWAS that we analyzed, as it was impossible to eliminate rare SNPs. The status of rare SNPs in common disease is still an issue on which the opinions are sharply split (Schork et al., 2009; McClellen and King 2010; Visscher et al., 2012; Hunt et al., 2013; Lee et al., 2014). However, recently it was shown that rare coding-region variants at known AID loci play negligible role in common autoimmune disease susceptibility (Hunt et al., 2013.). With the improved detailed annotations of SNPs from HapMap3 and 1000G projects, more low frequency common SNPs have been linked to GWAS associations. Currently, up to $60 \%$ of known SNPs in the human genome are variants with an allele frequency $<5 \%$. Low frequency common variants of moderate effects are likely to play an important role in uncovering complex disease/trait heritability that has not been explained so far by high frequency common variants. However, low frequency variants are often population specific: e.g., majority variants are population specific between European Americans and African Americans (Tennessen et al., 2012). Factors such as rapid population growth and weak purifying selection have allowed ancestral populations to accumulate an excess of low frequency variants across the human genomes (Tennessen et al., 2012).

The CD-CV hypothesis vs. the CD-RV hypothesis discords argue that the genetic variability in the human population with considerable frequency and low penetrance contributes to genetic susceptibility to common diseases vs. that the rare genetic variability with high penetrance is the major contributor to genetic susceptibility to common diseases. The current debate is about the frequency of diseasecausing alleles. The CD-CV is accepted by many as an explanation of the risk factors for common diseases (Reich and Lander 2001), in spite of widely accepted realization that common risk GWAS variants have failed to explain the vast majority of genetic heritability for any common human disease 
(Manolio et al., 2009; Visscher et al., 2012). They are defending the CD-CV theory with arguments that only common variants add up when the extent of common diseases in humans is calculated (Schork et al., 2009). They claim that the statistics used in GWAS is depressing heritability, and are proposing improvements in statistical approach able to explain a large proportion of heritability, claiming that the rest of unexplained heritability is contributed by non-variant causes (Visscher et al., 2012; Moser et al., 2015).

Other side argue that rare variants (defined by sequencing techniques as a $M A F<0.1 \%$ ), are the only alleles that may contribute to complex diseases (McClellan and King 2010). Rare SNPs cannot be neglected because the average ratio of rare to common alleles is $6: 1$, indicating overwhelming majority of rare genomic variants in the human genome (Tennessen et al., 2012). Since rare genetic variants are predicted to outnumber common variants, it is important to keep in mind they still might provide lead SNP signals observed by GWAS, especially when the lead SNP has no functional effect on disease risk as judged by the orthogonal evidence. The influence of rare variants could be resolved only with sequencing of the whole genomes or exomes, or smaller regions. However, the same daunting problem of finding which rare variants are disease-causing mutations will stay problematic as before; sequencing does not avoid a trap of multiple measuring (McClellan and King 2010; Kiezun et al., 2012).

The nature of the genetic contribution on individual susceptibility to common complex diseases is still unresolved. Much more data will be needed for any argument to prevail. Whether the causing variants are rare or common, nevertheless, they must influence biological processes by modulating them. Knowledge about how mutations disrupt or modulate key biological pathways and networks underlying autoimmune diseases is essential for understanding the biology of diseases and potential drug intervention. The GWAS data have led to that goal if not completely, then at minimum partially, as one way of acquiring necessary knowledge about polymorphism of pathways and potential drug targets.

\subsubsection{Genomic context, location, and LD of associated AID SNPs}

The second problem we had to resolve was the exact genomic context of the AID SNPs retrieved associations. Occasionally, we had to resolve contradictory information on few locations assigned for a SNP, using the newest data from $1000 \mathrm{G}$ project and latest version of HaploReg3.

Before assigning a genomic context of all retrieved associations, we checked each SNP location in the $\mathrm{dbSNP}$ database. Knowing the exact location of an associated SNP and the most recent version of gene or SNP location in the NCBI databases, did help further analysis such as impact of amino-acid change, but it did not necessarily resolve a cause of association signals. Causal variant is an unknown variant 
that has direct or indirect functional consequences on disease risk, even if there are no tools to prove causality. Even when the exact location of a SNP was resolved, and its genomic context was defined, the most probable cause of the association signals still had to be analysed with available tools and annotations.

The associated loci (GWAS tag SNPs) usually signal variable size regions, which contain multiple candidate genes for disease susceptibility in LD. They do not provide direct, restricted information about an exact location. This is conditioned by the human genome architecture and nature of inheritance. Limitations of GWAS biochip design, including the 200K SNP Immunochip used for the majority of AID GWAS, also contribute to this locus uncertainty. Since LD varies along the chromosome, a size of an LD segment had to be assigned for each SNP. We used HaploReg-v2 and -v4 software to determine LD for each AID SNP based on the newest updates in annotations.

When the associated lead SNPs are nonsynonymous, missense SNPs, they are by definition considered functional SNPs. However, that does not mean they are the causal SNPs for disease risk factors. It has to be confirmed whether a missSNP is responsible for a functional change in a coded protein, which further has a potential to contribute disease biology. If there is other missense SNPs in the same high LD gene region not tagged by the GWAS, it additionally complicated the conclusion. The outcome is vague, because the tagged missSNP and not tagged missSNP (if not both, when their MAFs are high) can influence the protein function in different ways (Farh et al., 2015). For many SNPs, we still do not know whether they actually have a direct functional effect, even when they belong to the "obvious" candidates, which alter the amino acid sequence of the coded proteins. In addition, a cause of an association signal with a missense SNP might be any SNP in LD, not necessarily other missense SNP in the same gene or a gene in high LD. A lead missense SNP could be in high LD with SNPs in other genes, being it missense SNPs or not, if they are causal variants. Only functional experimental results or prediction models may resolve this issue, but the prediction models are still too complicated (Visscher et al., 2012) and experiments are always expensive.

In our study, we have examples of all above discussed situations (Table 2 and Table 4). We resolved all of them using orthogonal data from NCBI and KEGG databases as presented in the Results. We restricted our choice of the SNPs for gene prioritization, expression regulation, and network and pathway analyses to the very high or perfect LD SNPs, newest version of annotations in NCBI and KEGG databases and by using the most updated Cytoscape, HaploReg and RegulomeDB software tools. However, again, only functional experiments will be able to resolve our predictions. 


\subsubsection{Why there are no AID SNPs located on sex chromosomes?}

We did not detect any GWAS AID SNPs on sex chromosomes with the conditions we used for their retrieval from the NHGRI catalog, although AID are promising case studies for investigating the role of $X$ chromosome in disease. Most AID have higher prevalence in females and loci on $\mathrm{X}$ chromosome have been suggested before as potential risk factors for autoimmune diseases (Ober et al., 2008). Several AID are so prevalent in females that they might be considered sexual dimorphic complex traits (Chang et al., 2014). Although AID have been extensively studied by GWAS, very few of these GWAS have studied the contribution of $X$ and, combined, have provided little evidence for its role in determining disease susceptibility. Only $15 \times$ chromosome associations out of the 2,800 significant associations were reported by GWAS in nearly 300 diseases/traits, making barely a half percent of all considered SNPs (Hindorff et al., 2013; Wise et al., 2013). GWAS either omitted $X$ chromosome sequence or erroneously linked the data (Wise et al., 2013). However, the chromosome $X$ constitutes $5 \%$ of the nuclear genome and underlies almost $10 \%$ of Mendelian disorders registered in Online Mendelian Inheritance in Man (OMIM) (Amberger et al., 2009).

Specific attention has been paid to this sex chromosome exclusion problem very recently, by introducing software for carrying out X-wide association studies, which will help finding more AID SNP associations. Several $X$ linked genes were found that influence immune response pathways, and particularly may explain additional heritability of several autoimmune diseases (Chang et al., 2014).

\subsubsection{Immunochip and its influence on AID SNPs}

Majority of GWAS studies regarding autoimmune/inflammatory diseases have been performed using Immunochip 200K or its upgrades (Illumina Inc., San Diego, CA). The Immunochip was introduced by the Wellcome Trust Case Control Consortium (WTCCC) as a powerful tool for immunogenetics gene mapping with preselected tags, believed at that time to include all parental SNP that may influence immune system function. The Immunochip is a custom-made Illumina array containing nearly 200,000 SNPs mapping to 186 loci previously genetically known to be associated with 12 autoimmune diseases preselected by the WTCCC (WTCCC 2007). Its cost was much lower than comparables, allowing advantageous usage of the same technique across the board.

There are several limitations of the biochip used for majority of AIS GWAS studies. One of the major is that Immunochip is not unbiased biochip platform, because the SNPs are preselected based on the previous knowledge about the immune diseases (both knowledge about and scope of immune diseases have advanced a lot since then). Although the GWAS methodology is unbiased, the tag SNPs selection 
is not, and may limit or distort the GWAS outcomes. Furthermore, the selected tag Immunochip SNPS do not cover regions with no previous associations ever found, nor do they cover the whole genome. Immunochip is not able to unearth SNPs with unsuspected links to common immune diseases, which is an undisputed possibility supported by surprising GWAS results (Yu et al., 2011). Immunochip is not covering the whole human exome or genome, not even the reference DNA sequence that is skewed towards European populations. Additional weakness of the Immunochip is that it is biased towards SNPs found in populations of European ancestry (EUR) not necessarily present in other populations. However, newer variants of Immunochip have been changed by including SNPs from other populations, but that cannot twist existing AID GWAS, since in all meta-analyses only SNPs that exist in all studies may be taken into consideration. It is also insufficiently designed for HLA region complexity, although HLA genes undoubtedly make a base of several important immune recognition and response processes.

The selected AID GWAS studies were performed by Immunochip (actually by several generations of immune-biochips). They mainly refer to the EUR populations (to the best of our knowledge) and for that reason, our all analyses using HaploReg have been always referred to the same population. However, differences in interpretation may always be a source of unintentional errors. Especially difficult is to restrict North American studies with significant underlying admixture populations, knowing in particular that LDs in African population are different (much shorter regions) from ASN and EUR populations (Hinch 2011).

In addition, the 200K SNP Immunochip contains a substantial proportion of lower common MAF polymorphic variants. They are responsible for uncertainty of identifying variants that tag regions of variable sizes containing multiple candidate genes for disease susceptibility. The lower MAF strongly associated variants SNPs tend to be in LD with the causal variant, rather than have a biological function themselves (Trynka et al., 2011). Newer upgrades of Immunochip are enriched with SNPs from 1000KG project and HapMap3, producing better outcomes (Miller 2013). Even improved chip designs with up to one million or more SNPs might suffer from the same problem, insufficiently reflecting the complex architecture of the human genome. It is not clear if the randomly chosen tag SNPs, evenly distributed along the chromosomes, would help better detection of relevant SNPs.

\subsection{Functional effects of SNP-gene prioritization}

Accurate functional annotation of SNP variants to the proper regions of the genome is crucial for understanding the biological significances of GWAS results for the underlying mechanisms of associated diseases (Ward and Kellis 2012). Once we selected variants, removed questionable or high $p$ value and eliminated duplicates, the next step was to find if the selected AID SNPs were connected to known 
structures with defined functional role, including genes in high LD. All retrieved AID SNPs were tested for their potential functional and regulatory roles or influence on regulatory processes. For it, we used the publicly available functional annotation software: PolyPhen, HaploReg and Regulome DB.

\subsubsection{Functional effects of AID missense SNPs}

We found 23 coding SNPs in our study (Tables 2 and 3). Coding variants could contribute to altered gene functions in different ways, depending on the characteristics of variants: non-synonymous variants cause amino acid sequence change in the corresponding coded protein and might induce protein function changes depending of the missSNP location; synonymous variants (cd-syn) may alter the translation efficiency (Kubo et al., 2007).

Functional effects of the missSNPs were studied using PolyPhen-v2 software tool to evaluate effect of the amino acid switches on the function of corresponding proteins. Problem often was that not all missSNPs ware completely and correctly catalogued for their functional evaluation by position, identifier, splicing etc. An incorrect position of a SNP may be triggered by different versions (hypothetical or recently corrected versions) of the coding protein, especially for less annotated proteins, or by difference in its splicing pattern. In addition, PolyPhen software often does not agree with the position or content of amino acid change from the databases; identifier (rs) numbers sometimes are mismatched as well. In search of an acceptable (most accurate) position of missSNP, several databases had to be consulted before the result was obtained. If a missSNP could not be recognized by PolyPhen software tool, it was not evaluated for its functional effect. We had a couple of missSNPs that could not be evaluated by an earlier version of PolyPhen-v2 we used; however, their function was resolved only recently by the upgraded PolyPhen version and with additional help of HaploReg-v4 (examples are missSNPs within boundaries of genes NOD2, IL7R and IL17REL).

Currently there is a debate what represents a modification of a protein conformation caused by an amino acid change. Because proteins are very complex structures, it is possible that the level of change for one domain of protein does not necessarily pertain for the other domain. Less annotated proteins (many SNP harboring proteins/genes have no known function or no annotation, or even no orthologs) do not have well defined active sites, and it is improbable that without that specific knowledge any tool can estimate valid level of functional distortion produced by an amino acid switch. If the active binding site is not defined, (it is usually defined by the known protein function), then it is less probable that the right evaluation of amino acid change could have been achieved. In addition, allosteric modifications occur independently of change in active sites, and influence protein function by changing its normal 
interactions. Many proteins have known active sites, but their regulatory sites are still vague (Zhang et al., 2012).

Even less obvious is how any of the amino acid changes in proteins influence pathways concerning upstream or downstream events, and regulation of potential positive or negative feedback loops. The proteins harboring damaging missSNP might have modulated translation. If they are still functional with the changed conformation, and continue to interact with other molecules, they must disturb dynamics of pathways or networks they participate, or complexes they form. That means what is evaluated as a damaging missSNP effect is damaging for a protein function with certainty on one level, but other levels might be also affected, yet "invisible" for current evaluation capability. For that reason, the nondamaging effects of amino acid switches have to be taken with caution, especially for proteins with no known function (Teng et al., 2009). Since the knowledge about functions of a good part of AID missSNP harboring proteins is currently incomplete, all the prediction results we obtained have to be taken only conditionally.

Majority of the AID missSNPs are situated within much conserved regions of DNA, indicating that they are structures inherited and preserved for often-unknown reasons, and not eliminated as mutations from the population. The difference between missSNP in the conserved and non-conserved regions of DNA is not fully understood either, although some evolutionary explanations might be foreseen (Stefl et al., 2013).

The strongest obstacle for the evaluation of missense SNP functional effect is whether the variant is only a tag (lead) SNP or a functional causal SNP in the assigned gene, or both. Missense SNPs are by definition evaluated as functional, causal SNPs. We found that is questionable rule because if a protein has additional missSNP in the same gene, they cannot be simultaneously evaluated for a global combined functional effect: we came across such examples within NFKBIE, GSDMB and TYK2 genes (Table 3). Double missense SNPs or "double hit" may be only evaluated by PolyPhen-2 tool as two separate events. Especially questionable is evaluation of two missense SNPs in high or perfect LD that are obviously inherited together and cannot be considered independent events. We detected this situation with gene NFKBIE: rs2233434, evaluated as benign, is in perfect LD with another NFKBIE missSNP in perfect LD and 56 nucleotides apart, undetected by any GWAS, but evaluated as damaging by PolyPhen-v2. Both missSNPs are located in the conserved region, and non-damaging missSNP has more than a dozen eQTL results linked with it (while damaging missSNP has none!) as evaluated by the HaploReg. Additional problem with rs2233434 is that it is in high LD with other missSNP in conserved 
region of another gene (HaploReg-v4.1 data). The case is not limited to one population, as EUR and ASN both exert the same missSNPs.

Multiple transcript variants that are encoding different isoforms represent additional complexity when analyzing coding SNPs, synonymous or nonsynonymous. No bioinformatics tool yet is available to present or predict the complexity, especially when it is well known that only very few proteins do not have splicing variants: it is recognized the splicing variants present a base of gene complexity of the human genome. To illustrate how complex the relations between coding SNPs are, here is an example we dealt with: rs27434 is the synonymous SNP of ERAP1 genes, but there is no data on its function within ERAP1, except that is located in the highly conserved region. In LD with this cd-synSNP is another missSNP rs27044 with a damaging effect as evaluated by PolyPhen v2. In addition, rs27434 is in perfect LD with yet another synonymous SNP of ERAP1 gene, not detected by GWAS. In this and similar cases, we analyzed further only ERAP1 as a gene and its participation in networks or pathways, without knowledge on how various SNP influence on ERAP1 protein conformation and quantifying interactions. However, less frequent are cases where missSNP "improve" protein function (Stefl et al., 2013).

\subsubsection{Regulatory effect of AID missense SNPs}

All genes harboring missSNPs except IL6R and NOD2 have shown multiple expression quantitative trait loci (eQTLs) results obtained in multiple cell lines and tissues, adding additional weight to these missense variants. Change in the eQTL loci results in modifications of level of expression of the corresponding gene transcripts, changing mRNA and protein quantity in tissues or serum (Soubrier 2013).

In addition to change in protein binding sites, multiple regulatory characteristic have been recognized for missense SNPs, such as histone marks, DNAse binding sites and motif change. They all indicate that the missense SNPs detected by AID GWAS represent polymorphisms that influence regulatory elements with functional consequences on autoimmune diseases that are not easily understood based on available data and tools at this time.

\subsubsection{Functional effects of AID synonymous coding SNPs}

We evaluated a few synonymous AID SNPs (Supplemental table 2), focusing only on cd-synSNPs that have a significant potential to change regulation of gene expression. Because several cd-synSNPs belong to the genes that already contain missSNPs, we did not analyze them separately, but as a part of 
the same gene (example is ERAP1). We did not have any nonsense SNPs causing stop codon that would result in aborted or partial proteins usually with no function (Ward and Kellis, 2012).

There were only nine GWAS AID synonymous SNPs (cds-syn SNPs) (Supplemental table 2). We did not have any nonsense SNPs causing stop codon that would result in aborted or partial proteins usually with no function (Ward and Kellis, 2012). We were focusing only on cd-synSNPs that have a significant potential to change regulation of gene expression.

All synonymous SNPs are evaluated by RegulomeDB for regulatory influence. Few are found in the same genes that we already included in the functional SNP gene set, the genes like NOTCH4, NOD2, CARD9 and PADI4. Only four synSNPs were scored with the highest scores.

One of these cds-syn SNP (rs1142287) belongs to the coding sequence of gene SCAMP3; it is found as a risk SNP for Crohn disease. The SCAMP3 encoded protein is an integral membrane protein; it functions as a carrier to the cell surface in post-Golgi recycling pathways and trafficking in endosomal pathways. The second cds-syn SNP (rs9858542) is found in BSN gene, but its encoded protein is very specifically expressed only in the presynaptic cytoskeleton. In addition, rs9858542 is in perfect LD with two missSNPs; one missSNP belongs to BSN gene and other one belongs to MST1 gene, the gene already on the list of significantly associated AID missSNPs. No annotation exists to explain link between BSN protein and two AID (CD and UC). The third cds-syn SNP was rs495337 found in SPATA2 gene; it is evaluated as very low impact SNP by RegulomeDB. The SPATA2 gene has no known function. There is only one study with orthologs in zebrafish, which suggests SPATA2 has a function in proliferation of beta cells in pancreas (Maran et al., 2009). The fourth synonymous SNP rs2240335 belongs to gene PADI4 and does not have any other functional SNPs in high LD. This gene is a member of a gene family that encodes enzymes responsible for the conversion of arginine residues to citrulline residues, a process very important for cutrulintaion of antibodies in RA. This gene may play a role in granulocyte and macrophage development leading to inflammation and immune response. For that reasons, we consider rs2240335 an interesting result, because PADI4 was long been on the list of AID risk factor genes.

The cd-synSNP rs3810936 belongs to TNFSF15, and has low impact, if any, on regulation. However, this gene encodes a cytokine of the tumor necrosis factor (TNF) ligand family; it can activate NF-kB pathway, but is not expressed in either B or T cells. TNFSF15 does not act in any of the known TNF pathways. We could not find any data supporting a potential role for this synSNP. 


\subsubsection{Functional effect of non-coding AID SNPs}

Similarly to the coding SNPs, the AID non-coding SNPs (ncSNPs) could also be lead SNPs in GWAS for genes in their LD, both for coding genes or non-coding genes. Alternatively, ncSNPs might represent solely changed regulatory elements that have an effect on gene expression of cis genes. Focusing exclusively on exome and SNPs solely influencing exome, such as missense coding SNPs or synonymous coding SNPs, is an extremely serious limitation in complex trait genetics (even an error). Noncoding genetic variations play essential roles in complex traits and complex diseases, roles larger than in Mendelian genetics or in somatic cancer genetics (Kiezun et al., 2012).

We retrieved and analysed the AID SNPs in untranslated regions: UTRs, both 5' and 3' ends, and nearGene regions and in intronic regions. Variants in the 5'-UTRs may influence the promoter activity of a gene, whereas the variants in the $3^{\prime}-$ UTRs may change the mRNA degradation rate mediated by microRNAs and RNA-binding proteins (Frazer et al., 2009). We did not specifically detect any AID SNP with a location at splice junction sites. Variants located at splice junctions may alter the splicing patterns of genes (Faustino and Cooper, 2003). Because there are no precise tools that would assign functions to ncSNPs, we consider them only as a part of genes they are located in a broader sense.

SNP variants of pseudogenes are difficult to understand, especially when it is known that pseudogenes may have a huge repercussion on the immune response (Pink et al., 2011). Among our analysed AID SNPS, we have an example of AID SNPs located in the pseudogene WASF5P (Chr.6) (Table 5). WASF5P is pseudogene of the gene WASF3 (Chr.13) that belongs to the family of genes encoding Wiskott-Aldrich syndrome (WAS) proteins (NCBI Gene). Wiskott-Aldrich syndrome is a serious disease of the immune system, an immune deficiency (Massaad et al., 2013). This gene family encodes the multiprotein complex that connects kinases and actin and serves to transduce signals that involve changes in cell shape, motility or function. However, WASF5P has no known function (Kurisu and Takenawa 2009).

\subsubsection{Regulatory effect of AID ncSNP associations}

ncSNPs might modify expression level of the corresponding gene transcripts, changing mRNA and protein quantity in tissues or serum (Soubrier 2013). Potential for regulatory effect of all retrieved AID ncSNPs was assessed for each variant using RegulomeDB software tool that provides scores based on available data, mainly from ENCODE project. We selected ncSNPs with highest RegulomeDB scores (from 1 to 3 ) as the ncSNPs with the highest potential to influence gene expression. We did not study 
ncSNPs with lower scores not only because they are less likely to exert their influence but also because of their sheer number (Suppl. Table 1).

More than $35 \%$ of numerous ncSNP loci had very high RegulomeDB score (Table 6), reflecting elements with strong regulatory role like eQTL. Usually ncSNPs were annotated with regulatory elements including histone marks, DNAse sites, protein binding sites and motifs that have been changed, in addition of numerous eQTL results in several cell lines and tissues. However, it is very difficult to understand what the meaning of all these associations is, except that they do exist and for a reason must be taken into consideration. Again, no publicly available software tool is able to concatenate them into biological processes or pathways, or linked them to the known complexes. They are evaluated as the regulatory elements associated with AID, but everything in between is still kept in dark (Edwards et al., 2013). ncSNPs do not provide explanation for inheritance, nor did they fit into any mechanism for AID development. Even if we accept that they represent regulatory sequences (based on ENCODE data), there are no annotations for their possible influence on gene expression through transcriptional, posttranscriptional, and posttranslational mechanisms; no immune pathways are annotated for regulatory elements so that we could use comparison between normal (wild type) and variant (changed) regulatory elements. Only few papers have addressed this issue, referring that changed regulatory motifs could have specific functional consequences. Consensus among researchers is that experimental approaches are necessary for confirming mechanistic relevance of regulatory elements, and future functional experiments will resolve problems and predictions unveiled by the AID GWAS data (Tak and Farnham 2015).

However, we found one good example how regulatory impact of ncSNPs might be connected with the nearest genes or a gene within boundaries they locate. However, ncSNPs might also influence distant genes, keeping in mind that a LD region could be discrete and not necessarily linear. Such example is UC SNP variant rs9263739 (with $p=5 \times 10^{-67}$ ) located in the intron of gene CCHCR1. This ncSNP it is in perfect LD with two missSNPs in the coding regions of other two genes, PSORIS1C1 and PSORIS1C2 genes. The two missSNPs are detected in several AID (psoriasis, SLE, MS etc.) as risk factors, for both EUR and ASN populations. Because the CCHCR1 gene has no known function, we made a choice to evaluate this signal as coming from two missSNPs on two genes in its perfect LD. PSORIS1C1 and PSORIS1C2 genes are among the first genes linked to psoriasis by linkage studies and evaluated in OMIM as the strongest risk factors. However, no data existed for UC.

Other AID ncSNPs located in the 3' and 5' regulatory regions, were also very often found together with intronic or missSNPs in the same gene region (Supplemental Table 1). We did not analyse them separately, although ncSNPs located in the 3' and 5' regulatory regions are considered variants with 
proven influence on transcription of corresponding genes (Okada et al., 2014). We included them directly in the list of ncSNP coding gene set, and did not analyse their potential regulatory function.

\subsubsection{Genes linked to ncSNPs}

Not all tag ncSNPs could be linked to regulatory effects. They might simply reflect genes in their vicinity. We searched for the potential coding genes located in the high LD with ncSNPs as lead SNPs, using HaploReg software. Almost $90 \%$ of all highly scored ncSNP have several coding or non-coding genes in their corresponding high LD (r2> 0.8). We detected 39 coding genes in high LD with ncSNPs based on the current annotations (Table 6 and 8). This set of genes, named ncSNP gene set, we further used for gene-pathway prioritization analysis (Table 5).

We also probed the lead ncSNPs for non-coding genes in high LD $\left(r^{2}>0.8\right)$, which we could not be put in any pathways or networks prioritization probes. As our results show, an unexpected abundance of noncoding genes emerged, including micro RNAs (miRNA), long noncoding intergenic RNAs (lincRNA), small nuclear RNAs (snRNA), and other uncharacterized RNAs. Only one quarter of analysed loci in LD with ncSNPs did not have any ncRNA genes or annotations data did not exist at the time (Table 6 and Table 7). Often more than one RNA genes were detected in LD for each analysed ncSNP.

Non-coding genes in high LD with ncSNPs were further analysed for their function. However, none of RNA genes could be explained for their role, because all of them, with no exceptions, were very poorly annotated. Obtained RNA genes could not be analysed as a separate gene set for their functional properties either, because there are no databases or tools (yet) that incorporate RNA genes into functional pathways (except occasionally for micro RNAs).

\subsubsection{Relevance of eQTL results linked to AID SNPs}

Levels of gene expression are highly heritable (Morley et al., 2004), and they are defined by eQTLs. We have detected many eQTLs for the AID SNP associations (Supplemental table 3). However, it is not clear how disease risk variants influence eQTLs. It is obvious from our results that a good number of AID SNP variants might change eQTLs in their corresponding regions. The majority of identified eQTL are cis-acting or local eQTL (Cheung and Spielman 2009), arbitrarily defined as regulation of genes within $1 \mathrm{Mb}$, but genetic variants can also affect the expression of genes that reside further away or are on different chromosomes, distal or trans-eQTL (Westra et al., 2013). Furthermore, the target genes of eQTL associations could be coding genes or noncoding RNAs (Kumar et al., 2013). We have found all classes of genes or regulatory elements in the vicinity of the analyzed GWAS AID SNPs. There are no software tools for searching distant genes. 


\subsubsection{MicroRNA genes}

Among the detected ncRNA genes connected with the AID ncSNPs, we specifically analysed miRNAs because of their well-known role in gene regulation (Ventriglia et al., 2015). Several computational tools could provide information about miRNA targets. Micro RNAs are quite conserved structures that typically regulate gene expression through binding to $3^{\prime}$ UTRs of targeted mRNAs to direct their posttranscriptional repression (Bartel 2009). Predicting potential target genes is the major challenge in exploring miRNA function, given that a single miRNA can potentially regulate hundreds of different genes. However, using TargetScan computational tool for target prediction, we have found only a few targets for the detected miRNAs. We detected 12 miRNAs in high LD to ncSNPs. Majority of these miRNAs have known targets as found employing TargetScan (Table 7a and 7b). However, we did not find among them any miRNAs to target GWAS AID SNP coding genes, nor we could confirm that they regulate any risk factors for AID, leaving a space for further exploration of additional genes linked to AID that are influenced by the detected miRNAs.

We also searched whether any of miRNA are influenced by SNPs in 3'-UTR of genes known to be linked to AID. We were not able to find any miRNA in the associations we have selected based on used $p$ values (Table 7c). Evidence exists that miRNAs modulate immune cell function and that level of miRNAs changes in immune cells in T1D, as that the dysregulation in immune cells can lead to immune pathology at least in T1D; however, that knowledge is still not integrated with pathways or other networks (Ventriglia G et al., 2015). The future innovative approaches are necessary for confirming not only miRNAs mechanistic relevance in AID, but also the role of so many other RNA genes of various types connected with AID SNP associations that we detected.

\subsubsection{SNP function analyses and human orthologs}

Comparison of orthologs to human SNPs and genes have been often used for clarification or to gain new knowledge about diseases/traits and SNPs. Although model organisms are used frequently for human disease studies, the phenotypic relevance of model organism genes that are orthologous to human disease genes remains unclear and questionable (Lehner 2013). It is impossible to accurately predict phenotypic variation based on genetic variants in orthologs. Mutations in an orthologous pair of genes do not always exhibit similar phenotypes in different species. Tested SNPs in mice were not able to produce the same disease that occurs in humans. Obviously, using orthologs in model organisms could not unequivocally prove that a SNP is the direct cause of any given association. Using orthologs, even when they resemble greatly the human counterparts (as they do in a sense of a function), their 
regulation, pathways and expression patterns may differ significantly. Most of the newly selected gene targets and drug targets developed from animal models have failed in humans (Wang et al., 2015).

\subsection{Network and pathway analyses}

In order to find a set of genes underlying the AID phenotypes, annotations for AID gene sets have to be clustered and compared to identify groups of genes that act similarly and thus may be a part of the same pathway or network. This is achieved by gene-network and gene-pathway prioritization analyses.

We defined separately the missSNP and the ncSNP sets for easier prioritization (Table 8). We could not use less than 10 members in any group of genes for prioritization, because ten or more genes are needed for the computational tools we have used. For that reason, we could not take only a subset of a few genes for gene-pathway prioritization, even when we had an argument for it.

\subsubsection{Network analyses or gene-network prioritization}

Using Cytoscape software tools, we were able to find a network that consisted of eight missSNP harboring proteins and TNF (nodes) connected by edges representing any type of relations. This was a positive result because it indicated existence of biological relations between TNF and NFKBIE, TYK2, TNFAIP3, NOD2, CARD9, MICA, SH2B3 and IL23R proteins. These links have not being registered by any other used method (Figure 5), nor other SNP-harboring gene nodes tested in a same way showed any edges between themselves, indicating lack of relations. The inferred functional connections between eight missSNP genes and TNF nodes are based on integration of huge number of data gathered from various sources and databases, all of which the Cytoscape tools compiles for network analyses. All edges between the nodes are of inferred nature, deduced from text mining data and structural similarities between proteins with experimentally detected interactions (some in model organisms). However, no experimental data or publications have been indicated, so we could not refer to any publications or experimental data. That is exactly the value of the network analyses by Cytoscape: its capability to make imputations based on broad data sources. The Cytoscape visualization of the networks and their interactions is suggestive of a space of the interactive communications among the SNP harboring proteins, the communications that might be important in AID pathology.

Another significant result obtained from the analyses with Cytoscape tools was the indications that all networks constructed for the missSNP proteins and TNF are integrated into one single complex conglomerate of networks, confirming that the interactions do exist between them, directly across the 
first interactants they share. If they were not a part of one unifying network, we would obtain two or more unconnected independent network structures using the same tools (Figure 4).

STRING software when used to perform network analyses, was not able to confirm the Cytoscape results (Figures $7 \mathrm{a}, \mathrm{b}$ and $\mathrm{c}$ ). STRING constructed networks for missSNP dataset, ncSNP dataset and allSNP dataset (a third dataset compiling both sets of SNPs together). All three sets are highly enriched for PPI (Table 10), confirming that they are a coherent gene/protein set with potential common biological function(s). However, in the STRING networks, we did not find edges between TNF and missSNP genes/proteins; STRING detected edges between TNF and CD40, TRAF1 and IL10, and constructed a much smaller, partial network between TNF and these three ncSNP genes/proteins with edges that represent mainly textmining, similarity or co-expression links. The STRING networks could not be parsed in the way Cytoscape allows network parsing, so intersections or unions could not be studied by STRING.

Regarding the widely accepted theory that essential genes have more PPI partners than nonessential genes (Jeong et al., 2001), it is important to emphasize that TNF has the largest network with over 1500 genes/proteins, all first interactants (as detected by Cytoscape). This huge number of direct interactants might qualify TNF as an essential hub protein. Among the disease genes detected by GWAS (disease genes are the genes in SNP gene sets), almost all missSNP genes have significant number of interactants (10-100), all except LACC1 and RTNK2 genes that have none (Table 9). Few missSNP genes have less PPI than others (less than 50 edges), but still enough not to consider them low impact genes. However, they are not hub genes either, based on the number of interactions they can make and their expression patterns (Goh KI et al., 2007). From the expression pattern of AID missSNP genes (Figure 6), it was clear they are present in immune cells, but also in fetal tissues and several adult tissues, so they are not limited to only one type of cells or tissues (hub genes are by definition expressed in many tissues most of the time). Expression patterns of the missSNP genes are important, because if the proteins are not expressed together in time and space, they cannot obviously interact directly, assemble pathways or cooperate in any type of networks.

Within the human disease network (HDN), immunological diseases are enriched with nonessential disease genes (Goh et al., 2007; Seong KH et al., 2015). For example, PTPN22, FCGR2A and NOD2 genes are considered disease nonessential genes, although we found a huge number of interaction they make in networks. Some other highly wired genes like TNFAIP3, TYK2, SH2B3 and CARD9 are not included in the HDN list of classified genes. For the gene classification of the HDN, the major criterion is disease mortally, and the AID we studied, although have high mortality unless treated, are not 
considered as such (Crohn disease, psoriasis, SLE, etc.). However, T1D is on the list of diseases with essential disease genes.

The major downside of the HDN theory is that it only takes into account OMIM based diseases and their genes. The majority of the GWAS AID genes are not found in OMIM, because the OMIM data cover only genes that were discovered in pedigree studies based on linkage analyses of monogenetic diseases, and do not cover diseases with unknown or complex genetic background. OMIM contains 2430 noncoding SNPs $(0.0001 \%$ of all human SNPs) and 5327 coding genes ( $0.01 \%$ of all -100 -fold enrichment) out of known $\sim 432$ thousand coding SNPs in the current built of human dbSNP database (OMIM McKusick; Bromberg 2013). For that reason, the HDN contains insufficient number of disease genes and diseases. Interestingly enough, the classification of cancer genes in HDN is dubious (to say the least), because they are considered hub and essential genes, but they are still very locally expressed. In addition, there are many erroneous conclusions such as that TNFRSF1A gene is considered an essential gene for familial periodic fever disease (which is not a deadly disease and is classified into Immune system diseases). On the other hand, TNFRSF1A gene participates in human pathways majority of which we found to be crucial for AID, yet none of the missSNP AID genes is considered essential (Seong et al., 2015).

Essential human genes are likely to encode hub proteins and are expressed widely in most tissues (Goh et al., 2007). This suggests that the vast majority of disease genes are nonessential, as they show no tendency to encode hub proteins. Their expression pattern (mainly in specific tissues), indicates that they are localized in the functional periphery of the network. However, recently, when the analysis was expanded to examine the PPIs of human disease genes, it was found that essential disease genes have more PPI partners than non-essential disease genes (Seong et al., 2015). Comparison of the AID missSNP genes with results of this study is leaving unclear their status. In our opinion, AID missSNP might not be essential genes, but they are definitively high-wired genes and collectively, and their variants might be able to influence many biological processes. The examples are missSNP genes TYK2, NFKBIE and several others. Both NFKBIE and TYK2 are extremely important key enzymes in two nonoverlapping essential signaling pathways, NF-kB and Jak-STAT signaling pathways that are operational in many cells and tissues, and not limited to lymphoid cells. Major role of NFKBIE gene encoded protein is inhibiting NF-kappa-B by complexing with it and trapping it in the cytoplasm. The TYK2 protein associates with the cytoplasmic domain of type I and type II cytokine receptors and promulgate cytokine signals by phosphorylating receptor subunits. It is also associated with both the type I and type III interferon signaling pathways (NCBI Gene database). 


\subsubsection{Pathway analyses or gene-pathway prioritization}

It is rarely the case that one gene is responsible for one function, contrary to the classical view. Rather, an assembly of genes constitutes a functional module or a molecular pathway. By definition, a molecular pathway leads to some specific end-point in cellular functionality via a series of interactions between molecules in the cell. Furthermore, it is an oversimplification to view a single pathway as a discrete and independent entity. Pathways act on defined sites overlapping with other pathways compiled in modules.

In order to connect SNP data and disease phenotypes, it is essential to sort SNPs influenced genes into functional pathways and then link them to diseases (Bromberg 2013). Biologically this process makes sense, because if diseases result from pathways' breakdown, then disabling any of the pathway components can produce similar phenotypes, and genes responsible for similar diseases often participate in the same interaction networks or pathways. Some newer proposals of disease classification go so far to propose that a disease soon will be considered a collection of mischief pathways. Accordingly, the drugs (probably a cocktail of drugs) will be targeting damaged, altered pathways, and not only certain gene product, usually an enzyme. It would be important to distinguish between neutral SNP changes from pathogenic SNP changes not only on the protein level, but also on pathway level. Not every SNP, even missense SNP will change a protein towards malfunctioning. However, even less is known about what changes are needed to for a pathway to accumulate dysfunctional members, in order to go over its "tipping point", and become a disease pathway. No tool is currently available to provide this type of information yet.

We have used gene-gene (protein-protein) interaction and pathway information to prioritize candidate genes and find pathways in which AID SNPs function, but we are not able to distinguish whether such pathway is damaged or not by the presence of such variant gene/protein.

The Cytoscape encouraging results prompted us to search for connections between missSNP harboring proteins and canonical pathways. We conducted molecular pathway enrichment analysis using KEGG databases as a main pathway information resource, but we also compared the KEGG pathways with pathways from other sources, like Regulome, INC, Wikipathways, etc. of it was necessary. The goal of pathway-level methods is to determine if the gene sets, found by genetic associations from a GWAS, are enriched for any canonical pathway (gene-pathway prioritization). Extrapolation of the results would be then that a particular pathway might be a disease-causing pathway.

Majority of the AID missSNP genes participate at least in one KEGG canonical pathway (Table 13). The same ratio was detected for ncSNP gene set when manually searched for pathways in KEGG database 
(Table 14). Significant number of KEGG pathways overlap between two sets of genes. Interestingly, when the SNP gene KEGG pathways were analysed for a type of pathway, two distinctive groups of pathways have emerged, signaling pathways and diseases pathways. We consider only signaling pathways the core pathways; disease pathways are rather collections of core pathways. Some pathways are collections of interactions with ligands and receptors (like networks), and illustrate a disease or network of the cytokines and the cytokine receptors. They are not pathways where the members are interacting in an directional way up to the end-point resulting molecules. We considered only core pathways that are harboring SNP genes for further analyses.

The SNP gene core pathways or signaling pathways were interrogated against TNF signaling pathways in order to find overlapping pathways. The TNF signaling pathways have been obtained also from KEGG pathway database. We consider them the biological vehicles for allocation of aTNF biologics actions, because aTNF biologics inhibit TNF wherever it acts.

After manual comparison of all SNP gene pathways and TNF pathways (Supplemental table 4), a much smaller set of nine intersecting core pathways was obtained (Supplemental table 5, Table 17). We consider these selected SNP gene/TNF pathways as a subset of pathways responsible for disease development (pathological pathways), but at the same time these pathways are also responsible for the propagation of aTNFs actions. The set of pathways is responsible for remission of AID, because they are a playfield where aTNF drugs interfere with autoimmune/inflammatory disease pathways, and regulated them down to remission status by reverting them into normal functioning pathways. We consider these pathways curative pathways. Consequently, other AID pathways, except the curative pathways, cannot be regulated by the aTNF biologics.

However, the selected pathways are not all equally influenced by TNF. For some, TNF is on a proximal side as it acts as a signal or trigger molecule and for others, TNF is on a distal side, being triggered by the pathway or is synthesized as an end-product of the pathway activation (Table 16). Toll-like receptor signaling pathway is not regulated by TNF, as TNF is not a signaling molecule in the pathway; TNF is synthesized as a consequence of activation of the toll receptors with their ligands. Toll-like receptor signaling pathway is also influenced with Jak-STAT system, the system that TNF have no common genes with it (Table 17).

Similarly, the NOD-like receptor signaling pathway is not triggered or controlled by TNF, but it does not have the Jak-STAT complex either, unlike the Toll-like receptor signaling pathway. 
In the Adipocytokine signaling pathway, TNF is a major signaling molecule. However, this is a pathway connected only with the endocrine system and it is not an immune system pathway. It explains why the proinflammatory cytokine TNF has been implicated as a link between obesity and insulin resistance. It was not confirmed that Adipocytokine signaling pathway has any role in AID under study.

TNF is also very vaguely connected with Fc epsilon RI signaling pathway; it is not a signaling or control molecule, and it is only proposed to be synthesized after activation of this pathway. The member of this pathway, gene FCGR2A, is harboring missSNP detected by AID GWAS.

TNF is a product of T cell signaling pathway, but it does not influence B cell signaling pathway.

TNF is a signal of NF-kB pathway but is also augmented by it and serve as a positive feedback loop for NF-kB pathway. TNF does not influence Ubiquitin mediated proteolysis, the pathways that is directly connected by NFKBIE and NF-kB signaling pathway. Ubiquitin mediated proteolysis, a very important pathway for regulation of protein degradation, as it is directly linked with NFKBIE and its complex that undergoes ubiquitination as a regulation process. Any SNP variant in NFKBIE has a potential to change its binding to complex further degraded by Ubiquitin mediated proteolysis. Ubiquitin mediated proteolysis also has as a member the GWAS AID SNP linked gene/protein UBE2L3, so it might be one of the pathogenic AID pathways because it contains a risk factor for AID and it degrades the NFKBIE complex and release a transcription factor that travels into a nucleus.

Another interesting pathway/process is Apoptosis pathway, which is triggered by TNF, and might be considered in this group of the curative pathways, but none of AID SNPs is connected with Apoptosis. We are suggesting that at least NFKBIE gene should be included, because it is involved in the NF-kB pathway, but it is not yet annotated as such (and for that reason, we were not able to retrieve Apoptosis as a pathway for NFKBIE from KEGG, and other tools based on KEGG like Cytoscape). However, it is well accepted that apoptosis is regulated, more precisely inhibited, by the NF-kB pathway and the apoptotic process is stopped and reverted into a survival mode when NF-kB pathway is activated in a cell. In addition, both counterparts of NFKBIE, NFKBIA and NFKBIB are members of NF-kB pathway and Apoptosis. It would be interesting to see in future how missSNP in NFKBIE participate in the process: whether missSNP augments the apoptosis or it enhances inhibition of the apoptosis. Jak-STAT signaling pathway has no role in apoptosis.

As a result of the orthogonal evidence, we found that at least two sets of pathogenic AID pathways exist. Only one set of pathways is controlled by TNF and only that set of pathways can be modified by aTNF biologics (Figure 6). The second set of pathogenic pathways includes Jak-STAT signaling pathway and 
Jak-STAT complex dependent signaling pathways such as the cytokines' pathways that solely employ Jak-STAT complex to propagate signals from various cytokines (TYK2 belong to JAK family of tyrosine protein kinases).

NOD-like signaling pathway has TNF as its product that augments further inflammation, together with IL6 cytokine.

Other pathways that harbor missSNP genes do not have any overlapping with two sets of pathways and act independently of TNF and Jak-STAT signaling (for example Notch signaling pathway). Another example is Regulation of autophagy, a potential GWAS AID SNP pathway with no connection with TNF.

The finding of at least two independent sets of pathways explains unresponsiveness to aTNF drugs. The pathways that are not influenced by TNF cannot be controlled by its inhibitors. It also explains how and why some of AID are better controlled, (but not cured or reversed) with a relatively new class of drugs, Xeljanz (Tofacitinib citrate). XELJANZ is a prescription drug approved by the FDA in 2012, and acts as a Janus kinase (JAK) inhibitor.

Some AID SNP pathways are influenced by both TNF and Jak-STAT complex: Osteoclast differentiation pathway is influenced by TNF as a signaling ligand, but is controlled by Jak-STAT signaling partially, dough only partially.

Disease pathways do not belong to the core signaling pathways, but their contributions to AID disease development are undisputable. They are pathological pathways, but are not controlled by aTNF drugs. Their existence is an additional burden for the AID therapy and potential cure.

We also prioritized gene-pathway using STRING (Table 18) and ConsensusPathDB (Table 19 and 20).

STRING enrichment in pathways for the missSNP gene set revealed only three signaling pathways, all three included in the set of pathways we discovered manually searching KEGG database. When the allSNP gene set was analysed, it showed additional two pathways, one of them not discovered before (Chemokine signaling pathway) in addition to already detected $\mathrm{T}$ cell receptor signaling pathway. We selected the STRING pathways after correction for FDR $(<0.05)$.

When we used ConsensusPathDB for pathway enrichment analyses, 76 pathways were retrieved with 17 missSNP genes (out of 23) present at least in one pathway. Many of these pathways, coming from several databases in addition to KEGG, such as Reactome, Wikipathways, Signalink, PID, BioCarta, etc. are repetition of the same KEGG pathways. However, a few new pathways were retrieved as well: NF- 
kB signaling, TNF signaling, Toll-like receptors signaling and Cytokine-cytokine receptor signaling, which are contained completely in the same KEGG collection of pathways.

Pathway enrichment by ConsensusPathDB for the ncSNP gene set has not detected any new pathways that were not previously detected for the missSNP genes set. It is interesting that similar portion of ncSNP genes (70\%) participate in various pathways. Pathway enrichment for the all SNP genes did not improved with the number of genes, leaving the same portion of the SNP genes detected to participate in pathways $(70 \%)$. We found that ConsensusPathDB pathway enrichment analyses provided valuable results and was easy to use. We did analyses separately enrichment for complex formation of SNP genes (new ConsensusPathDB tool), but the results for complex formation enrichment were inconsistent and difficult for interpretation, and for that reason we did not include them in our research.

The ConsensusPathDB results for AID SNP gene-pathway prioritization indicates that close to 1100 genes are engaged in the pathways in which AID GWAS SNP may operate. A bit over $70 \%$ of AID SNPS have influence on nine KEGG pathways. The rest of $30 \%$ of genes, which are harboring AID SNPS or are influenced by AID SNPs, cannot be assigned to any pathways, which still make a big portion of unknown pathways. It would be hard completely to understand the genomic variation that is represented by GWAS SNPs. For that reason, better annotation of all components must be accomplished before making any scientific conclusions about influence of the genomic variation on the phenotypic expression of autoimmune/inflammatory diseases.

However, identical results obtained using several different approaches are good indication that what is shown by our study is a true reflection of the relationship between variants and AID.

The SNP-gene-pathway prioritization analyses showed that the genes and other structures harboring mutations that qualify as AID risk factors also play pivotal roles in the pathogen defense and other life preserving immune functions (e.g. neoplastic surveyance). It might be a reason why common risk loci are so numerous and are not purified from human genomes. They are intertwining with other genes responsible for controlling infections or neoplastic formation.

\subsection{Gene Ontology helps clarify the function of GWAS AID SNPs}

In order to uncover the potential biological rationale between AID SNPs and disease mechanisms, we applied GO term enrichment/ or overrepresentation analyses. GO term enrichment analyses are 
independent from all other used approaches. GO terms are differently structured, but the source of information is based on the same scientific facts.

Enrichments of the GO terms for the biological process (BP), molecular function (MF) and cellular components (CC) of SNP gene sets have been performed by STRING (Table 21) and ConsensusPathDB tools (Tables 22, 23 and 24). We used both Bonferroni correction for multiple testing and FDR for comparison, as the Bonferroni correction is considered too strict for GO term enrichments.

The GO enrichments have provided information on functional activity and its location of AID SNP gene sets. They indicate that AID SNP gene sets are functioning in biological processes mainly connected with the functions of immune system and their regulation. Interactions are realized through the proteinprotein domains' interactions and are located on the various cell membranes, including cell surface membrane.

It is interesting that ncSNP gene set is enriched with MF term for sequence-specific DNA binding transcription factor activity, which is not found among missSNP gene set. All GO terms are consistent with previously obtained results, because no new or unforeseen process has been discovered.

More than $95 \%$ of missSNP gene set and $87 \%$ ncSNP gene sets have been classified or recognized by GO terms, which represent a significant result, in comparison to $70 \%$ of gene classification by gene or pathways prioritization methods, or even less when the AID SNPs are manually searched.

There is a huge disbalance between numbers of BP GO terms compared with MF and CC terms. It seems that it is more a systematic problem of GO terms than a bias towards BO terms caused by our selection of genes.

\subsubsection{GO disease terms enrichment for AID GWAS SNP gene sets}

At the end of our research, we wanted to validate the obtained results and we chose to test what enrichment in diseases, if any, could be obtained by enrichment of GO terms for diseases (Table 26). We used STRING to find the disease GO terms and were positively surprised when all SNP gene set returned enrichment in all autoimmune/inflammatory diseases we started with. Other less specific GO disease terms also have been enriched for, all in the higher category of disease classification.

In the next step, we wanted to test whether we would obtain to find the same pathways for the AID, using relatively new software tool DiseaseConnectDB, which connects diseases with pathways. 
The retrieved pathways have shown the same pattern as obtained by using other tools (Table 25). We organized them using the same method for pathway selection as before (core vs. complex disease pathways) and obtained the pathway set enriched in AID. The set is completely overlapping with the pathway set results presented in the Table 17.

When the pathways found by DiseaseConnectDB tools are compared with TNF pathways (Table 17), the result shows that all signaling pathways, except Jak-STAT pathway, are intercalated with TNF action either on a receiving end or a dispatching end. It confirms the previous finding on shared pathways using other software tools in our study.

Independent of tools or databases used for analytical purposes, the results have shown that the AID GWAS SNPs participate in at least two groups of pathways and the number of core pathways is relatively small. Although we were not able to pick up any particular gene responsible for variable response/ no response of AID patient population to the anti-TNF biologics, we were able to find a very few pathways with not more than 100 genes that carry response to anti-TNF drugs.

\subsection{Pleiotropy at the gene and disease level}

We have the several autoimmune /inflammatory diseases under study, linked for the same missense SNPs, obviously targeting the same genes. In addition, other immune system related disease (and not only the AID under study) also demonstrated the same missense SNPs or variants in the same genes (Table 1 and 2).

We detected more than dozen shared SNP associations detected by GWAS for AID (Table 2). As the SNP-gene prioritization analyses show, many shared genes or shared risk factors followed the basic commonality among SNPs. The same phenomenon continued later when we analyzed the pathways detected for the SNP harboring or influenced genes. This was not surprising finding, because the very idea that different AID can be and are treated with the same therapy has in its base realization that the same pathways are operative in all these AID and for that reason the aTNF biologics act as successful therapy in these diseases. Common SNPs seems to be confirmation of what was already pragmatically discovered: AID diseases share pathological pathways, the pathological pathways are similarly distorted by their protein members that are as proteins damaged by the mutations in their coding genes. What is new is that the coding variants are consequence of common SNP variants that have been detected by GWAS. The variants are not just rare mutations, but most probably variant "load" that exist in human populations, which under certain circumstances become active risk factors. The phenomenon is called 
pleiotropy, and it obviously exists at the several levels: SNP variants, genes, pathways, pathway or network modules and diseases.

Allelic pleiotropy, where one genetic variant influences several distinct phenotypes, is increasingly recognized as a common phenomenon from GWAS findings, especially for immune-mediated diseases (Solovieff et al., 2013).

Furthermore, it was found that the same gene, with the different SNPs, is associated with the multiple diseases. However, it was impossible to evaluate whether the other SNPs contribute or not to the same functional effect as the missense SNPs on the same gene, even when they are missSNPs as well. We have similar case for the gene encoding IL7R (Table 2). Allelic pleiotropy is not only linked to missense SNPs, as non-coding SNPs are also shared among common diseases.

There is no bioinformatics tools currently that can compare influence of the different GWAS variants on the function of the same protein, gene, or DNA regulatory structure. It would be very important issue, as the existing data show completely different influence of the same variants in GWAS studies: the same SNP is protective in one disease and detrimental in another disease (Cho and Gregersen 2011; Wang et al., 2015). No explanation is provided, but it might be connected with other participants of the pathways or networks they are engaged in.

Investigation of pleiotropic effects could better inform on disease biology and predict potential adverse events of derived targets (Hebbring et al., 2014). The leading signals of association might be the exact same tag SNPs, but whether these GWAS association signals in AID refer to the same or different distinct causal variants or causal genes, remains unclear (Diogo et al., 2014).

The network analyses research has found that if a gene has more connections in the cellular network (not necessarily only PPI, but also co-expression, co-localization, co-regulation interactions) or if the gene is a hub gene, then its perturbation tends to result in the disconnection of multiple cellular functions, which leads to disease pleiotropy (Bromberg 2013).

An additional approach to investigate pleiotropy comprehensively is through genotype data linked to clinical data derived from electronic medical records. This unbiased approach, called phenome-wide association study (PheWAS), allows for genotypes of interest to be tested for association to hundreds of clinically relevant phenotypes (Hebbring et al., 2014).

The pleiotropy of SNP-gene and pathways is obvious at the disease symptoms level, so that some current studies even suggest a reclassification of diseases according to the pathways, which underlie a 
disease. The diseases might be considered as perturbations of pathways or pathway/network modules that have reflected in signs and symptoms of the diseases. This new approach might also have a positive effect on therapy and discovery of new treatments because new drugs would be correcting not only function of one gene or its product (for example an enzyme), but they would be able to modulate a whole pathway, reestablishing its normal activity (Costapas and Hafler 2013). 


\section{CONCLUSION}

In our research, no individual single gene was discovered to be responsible for therapeutic effect of the aTNFs, as it might be expected if compared with some reports on other (simple) drugs. Instead, the four major canonical signaling pathways have been detected as the most probable carriers of the response to aTNFs, making the difference between achieving remission in some AID patients and lack of therapeutic response in others. They are NF-kB signaling pathway, Antigen processing and presentation pathway, $T$ cell receptor signaling pathway and TNF signaling pathway. These four pathways are structured and affected by the hundreds of genes. The genes influenced by the risk GWAS SNP variants associated with the AID phenotypes are among these genes. The variants of these genes/proteins may modulate these pathways in many yet unknown ways. Based on the common knowledge that proteins overwhelmingly are functionally damaged by missSNP, the AID missSNPs most probably can only provoke disregulation and disturb the four pathways' flow negatively, conditioning disease development on one side and permitting intervention by aTNFs on the other side. All other non-missense risk SNPs also carry potential to contribute to the processes, but at the level that cannot be evaluated at this time.

Our results indicate that the AID pathways, ones that are responsible for pathogenesis of the diseases, belong to at least two groups of pathways. Two separate groups of pathogenic disease pathways may be redundant in the pathogenesis of certain AID. One group of pathway(s) may be more active in a subset of individual AID patients based on their particular risk SNPs, while the second group of pathway(s) may be active in others, thus explaining the lack of aTNF clinical response in that subgroup of AID patients. Balance between pathogenic pathways in each subject dictates the response to aTNF.

The detected regulatory elements and non-coding genes that we found to be relevant for AID pathogenesis, could not be fitted into any pathways, because there are no bioinformatics tools to incorporate regulatory elements and non-coding gene products into existing canonical biological processes. Finding ways to incorporate them into functional biological processes represents a future next step in understanding of their biology.

By comprehending the study problem, finding ways to answer it and solving methodological problems along the way, we gained the comprehensive methodological knowledge how to reveal the biological sense behind the GWAS AID SNPs. This comprehensive knowledge could be beneficial for analyses of complex pathogenesis of other common diseases as well as potential intervention pathways that should be targeted by drugs (new drugs or existing repurposed drugs). As the result of our research, new 
problems are detected, opening avenues worth further investigation, and leaving space for deepening our understanding of the complex diseases such as AID.

Gene Ontology emerged as a very helpful approach and an easy to use organization structure that might directly provide enrichment of descriptive data for genes of interest. All GO aspects are useful for analyzing underling biology of genes with GWAS detected variability in human genomes. However, GO is currently biased towards rich domain of biological processes, leaving poorly described molecular functions and especially cellular compartmentalization, and even less integrated pathways or diseases descriptors. Other similar ontologies of non-gene genetic elements are needed to help solve complex questions like one we dealt with in our research.

Based on our experience in application of bioinformatics software tools, we concluded that the existing bioinformatics tools and databases might provide only partial answers. Mutual obstacles are lack of complete annotations in the current public databases and insufficient integration of tools, as they are still not matching the complexity of the biological problems.

Our research highlighted the state of the current annotations in public bioinformatics databases, which need much more information and refinement in order to produce relevant conclusions for clinical problems similar the one we investigated. All databases suffer from inconsistency and incompleteness. It is almost disappointing how huge the portion of genes is with no or very scarce annotations. Little is known about non-coding genes, and almost nothing about pseudogenes; participation of non-coding genes in the biological processes is almost completely a void space.

There is also a great need for new, improved and enriched bioinformatics software tools; especially tools that are able to explore simultaneous influence of SNPs and genes, as well as to analyse potential polymorphism of pathways directly based on SNP polymorphism. It is also obvious that many tools suffer from oversimplification. Existing software tools, although exceptionally operative for the purpose they are invented, have very limited capacity for complex research needed in biology and medicine. 


\section{REFERENCES}

1000 Genomes Project Consortium, Abecasis GR, Altshuler D, Auton A, Brooks LD, Durbin RM, Gibbs RA, Hurles ME, McVean GA. A map of human genome variation from population-scale sequencing. Nature. 2010;467:1061-1073.

1000 Genomes Project Consortium, Abecasis GR, Auton A, Brooks LD, DePristo MA, Durbin RM, Handsaker RE, Kang HM, Marth GT, McVean GA. An integrated map of genetic variation from 1,092 human genomes. Nature. 2012;491(7422):56-65.

1000 Genomes Project Consortium, Auton A, Brooks LD, Durbin RM, Garrison EP, Kang HM, Korbel JO, Marchini JL, McCarthy S,McVean GA, Abecasis GR. A global reference for human genetic variation. Nature. 2015 Oct 1;526(7571):68-74. doi: 10.1038/nature15393.

Adzhubei IA, Schmidt S, Peshkin L, Ramensky VE, Gerasimova A, Bork P, Kondrashov AS, Sunyaev SR. A method and server for predicting damaging missense mutations. Nat Methods 7(4):248-249 (2010).

Adzhubei I, Jordan DM, Sunyaev SR. Predicting Functional Effect of Human Missense Mutations Using PolyPhen-2. Current protocols in human genetics / editorial board, Jonathan L Haines et al., 2013; 07:Unit7.20. doi:10.1002/0471142905.hg0720s76.

Agarwal V, Bell GW, Nam JW, Bartel DP. Predicting effective microRNA target sites in mammalian mRNAs. Elife. 2015;4. doi: 10.7554/eLife.05005.

Agarwal P, Owzar K. Next Generation Distributed Computing for Cancer Research. Cancer Informatics. 2014;13(Suppl 7):97-109

Aggarwal BB, Gupta SC, Kim JH. Historical perspectives on tumor necrosis factor and its superfamily: 25 years later, a golden journey. Blood. 2012;119(3):651-665.

Altshuler D, Daly MJ, Lander ES. Genetic mapping in human disease. Science.2008;322 (5903): 881-8.

Amberger J, Bocchini CA, Scott AF, Hamosh A. McKusick's Online Mendelian Inheritance in Man (OMIM). Nucleic Acids Research 2009; 37:D793-796.

Ashburner M, Ball CA, Blake JA, Botstein D, Butler H, Cherry JM, Davis AP, Dolinski K, Dwight SS, Eppig JT, et al., Gene ontology: tool for the unification of biology. The Gene Ontology Consortium. Nat.Genet.2000;25:25-29.

Banchereau R, Cepika A-M, Pascual V. Systems Approaches to Human Autoimmune Diseases. Current opinion in immunology. 2013;25(5):598-605.

Barabási AL, Albert R. Emergence of Scaling in Random Networks. Science.1999;286:509-512.

Barabási AL, Gulbahce N, Loscalzo J. Network medicine: a network-based approach to human disease. Nat Rev Genet. 2011;12:56-68.

Barshir R, Shwartz O, Smoly IY Yeger-Lotem E. Comparative analysis of human tissue interactomes reveals factors leading to tissue-specific manifestation of hereditary diseases. PLoS Comput Biol. 2014;10(6):e1003632.

Bartel DP. MicroRNAs: target recognition and regulatory functions. Cell. 2009;136:215-233. 
Beaudoin M, Goyette P, Boucher G, Lo KS, Rivas MA, Stevens C, et al. Deep resequencing of GWAS loci identifies rare variants in CARD9, IL23R and RNF186 that are associated with ulcerative colitis. PLoS Genet. 2013;9: e1003723.

Behm BW and Bickston SJ.Tumor necrosis factor-alpha antibody for maintenance of remission in Crohn's disease. Cochrane Database Syst Rev. 2008;(1):CD006893.

Ben-Horin S, Kopylov U, Chowers Y. Optimizing anti-TNF treatments in inflammatory bowel disease. Autoimmun Rev. 2014;13(1):24-30.

Benjamini $\mathrm{Y}$, Hochberg $\mathrm{Y}$. Controlling the false discovery rate - a practical and powerful approach to multiple testing. J Roy Stat Soc (B) 1995;57:289-300.

Bentley DR, Balasubramanian S, Swerdlow HP, Smith GP, Milton J, Brown CG, et al. Accurate whole human genome sequencing using reversible terminator chemistry. Nature.2008;456:53-9.

Bindea $G$, Mlecnik $B$, Hackl $H$, Charoentong $P$, Tosolini $M$, Kirilovsky $A$, Fridman WH, Pagès F, Trajanoski Z, Galon J. ClueGO: a Cytoscape plug-in to decipher functionally grouped gene ontology and pathway annotation networks. Bioinformatics. 2009; 25(8):1091-3.

Blake JA. Ten Tips for Using the Gene Ontology. PLoS Comput Biol 2013;9(11): e1003343.

Bogdanos DP, Smyk DS, Rigopoulou El, Mytilinaiou MG, Heneghan MA, Selmi C, Gershwin ME. Twin studies in autoimmune disease:genetics, gender and environment. J Autoimmun.2012;38(2-3):J156-69.

Borecki IB, Province MA. Genetic and genomic discovery using family studies. Circulation. 2008;118 (10):1057-63.

Bossi A, Lehner B. Tissue specificity and the human protein interaction network. Mol Syst Biol. 2009; $5: 260$.

Boyle AP, Hong EL, Hariharan M, Cheng Y, Schaub MA, Kasowski M, Karczewski KJ, Park J, Hitz BC, Weng S, Cherry JM, Snyder M. Annotation of functional variation in personal genomes using RegulomeDB. Genome Res. 2012;22(9):1790-7.

Brinkworth JF and Barreiro LB. The contribution of natural selection to present-day susceptibility to chronic inflammatory and autoimmune disease. Curr Opin Immunol. 2014;31:66-78.

British Thoracic Society Standards of Care Committee. BTS recommendations for assessing risk and for managing Mycobacterium tuberculosis infection and disease in patients due to start anti-TNF-alpha treatment. Thorax. 2005;60(10):800-5.

Brodin P, Jojic V, Gao T, et al., Variation in the human immune system is largely driven by non-heritable influences. Cell. 2015;160(0):37-47. doi:10.1016/j.cell.2014.12.020.

Bromberg Y Chapter 15: Disease Gene Prioritization. PLoS Comput Biol 2013;9(4): e1002902.

Brunet TDP and Doolittle WF. Getting "function" right. Proc Natl Acad Sci USA. 2014; 111(33): E3365.

Buchanan CC, Torstenson ES, Bush WS, Ritchie MD. A comparison of cataloged variation between International HapMap Consortium and 1000 Genomes Project data. J Am Med Inform Assoc. 2012;19(2):289-94. 
Busard C, Zweegers J, Limpens J, Langendam M, Spuls PI. Combined use of systemic agents for psoriasis: a systematic review. JAMA Dermatol. 2014;150(11):1213-20.

Califano A, Butte AJ, Friend S, Ideker T, Schadt E. Leveraging models of cell regulation and GWAS data in integrative network-based association studies. Nat Genet. 2012; 44(8): 841-847.

Cárdenas-Roldán J, Rojas-Villarraga A, Anaya JM. How do autoimmune diseases cluster in families? A systematic review and meta-analysis. BMC Med. 2013;11:73.

Carter H, Hofree M, Ideker T. Genotype to phenotype via network analysis. Curr Opin Genet Dev. 2013;23(6):611-21.

Cavallari LH, Shin J, Perera MA. Role of pharmacogenomics in the management of traditional and novel oral anticoagulants. Pharmacotherapy. 2011;31:1192-207.

Chang D, Gao F, Slavney A, Ma L, Waldman YY, Sams AJ, Billing-Ross P, Madar A, Spritz R, Keinan A. Accounting for eXentricities: analysis of the $X$ chromosome in GWAS reveals $X$-linked genes implicated in autoimmune diseases. PLoSOne. 2014;9(12):e113684. eCollection 2014.

Chen GK, Jorgenson E, and Witte JS. An empirical evaluation of the common disease-common variant hypothesis. BMC Proc. 2007; 1(Suppl 1): S5.

Cheung V.G., Spielman R.S. Genetics of human gene expression: mapping DNA variants that influence gene expression. Nat. Rev. Genet. 2009;10:595-604.

Cho JH, Gregersen PK. Genomics and the Multifactorial Nature of Human Autoimmune Disease. N Engl J Med 2011; 365:1612-1623.

Cooper GS, Bynum ML, Somers EC. Recent insights in the epidemiology of autoimmune diseases: improved prevalence estimates and understanding of clustering of diseases. J Autoimmun. 2009;33(34):197-207.

Cooper GS and Stroehla BC. The epidemiology of autoimmune diseases. Autoimmunity Reviews. 2003;2 (3):119-125.

Cortes A, Brown MA. Promise and pitfalls of the Immunochip. Arthritis Res Ther. 2011;13(1):101.

Cotsapas C, Hafler DA. Immune-mediated disease genetics: the shared basis of pathogenesis. Trends Immunol. 2013 Jan;34(1):22-6.

Crisswell L.A., Pfeiffer KA, Lum, RFB, et al., Analysis of families in the Multiple Autoimmune Disease Genetics Consortium (MADGC) Collection: the PTPN22 620W allele associates with multiple autoimmune phenotypes. Am J Hum Genet. 2005;76: 561-571.

Croft M, Benedict CA, Ware CF. Clinical targeting of the TNF and TNFR superfamilies. Nat Rev Drug Discov. 2013;12(2):147-168.

Czyz W, Morahan JM, Ebers GC, Ramagopalan SV. Genetic, environmental and stochastic factors in monozygotic twin discordance with a focus on epigenetic differences. BMC Med. 2012;10:93.

Davis MM. A prescription for human immunology. Immunity. 2008;29:835-8. 
Daly MJ, Carroll MC, Stevens B, McCarroll SA. Schizophrenia Working Group of the Psychiatric Genomics Consortium. Schizophrenia risk from complex variation of complement component 4. Nature. 2016;530 (7589):177-83.

Diogo D, Okada Y, Plenge RM. Genome-wide association studies to advance our understanding of critical cell types and pathways in rheumatoid arthritis: recent findings and challenges. Curr Opin Rheumatol. 2014;26(1):85-92.

Dumontier M, Baker CJ, Baran J, Callahan A, Chepelev L, Cruz-Toledo J, Del Rio NR et al. The Semanticscience Integrated Ontology (SIO) for biomedical research and knowledge discovery. J Biomed Semantics. 2014;5(1):14.

Dunham I, Kundaje A, Aldred SF, Collins PJ, Davis CA, Doyle F, Epstein CB, Frietze S, Harrow J, Kaul R, et al., An integrated encyclopedia of DNA elements in the human genome. Nature. 2012;489:57-74.

Eberle MA, Ng PC, Kuhn K, Zhou L, Peiffer DA, Galver L, Viaud-Martinez KA, Lawley CT, Gunderson $\mathrm{KL}$, Shen R, Murray SS. Power to detect risk alleles using genome-wide tag SNP panels. PLoS Genet. 2007;3(10):1827-37.

Edwards SL, SL, Beesley J, French JD, and Dunning AM. Beyond GWASs: Illuminating the Dark Road from Association to Function Am J Hum Genet. 2013;93(5): 779-797.

Emery $\mathrm{P}$. Optimizing outcomes in patients with rheumatoid arthritis and an inadequate response to antiTNF treatment. Rheumatology (Oxford). 2012;51 Suppl 5:v22-30.

ENCODE Project Consortium. The ENCODE (ENCyclopedia Of DNA Elements) Project. Science.2004; $306: 636-640$.

ENCODE Project Consortium. Identification and analysis of functional elements in $1 \%$ of the human genome by the ENCODE pilot project. Nature. 2007; 447(7146): 799-816.

ENCODE Project Consortium. An integrated encyclopedia of DNA elements in the human genome. Nature. 2012;489(7414):57-74.

Ernst J, Kheradpour P, Mikkelsen TS, Shoresh N, Ward LD, Epstein CB, Zhang X, Wang L, Issner R, Coyne M, Ku M, Durham T,Kellis M, Bernstein BE. Mapping and analysis of chromatin state dynamics in nine human cell types. Nature. 2011;473(7345):43-49.

Esteller M. Non-coding RNAs in human disease. Nat Rev Genet. 2011;12:861-874.

Farcomeni A. A review of modern multiple hypothesis testing, with particular attention to the false discovery proportion. Stat Methods Med Res. 2008;17:347-388.

Farh KKH, Marson A, Zhu J, Kleinewietfeld M, Housley WJ, Beik S, et al., Genetic and epigenetic fine mapping of causal autoimmune disease variants. Nature. 2015;518:337-43.

Faustino NA, Cooper TA. Pre-mRNA splicing and human disease. Genes Dev 2003;17: 419-437.

Fernald GH, Capriotti E, Daneshjou R, Karczewski KJ, Altman RB. Bioinformatics challenges for personalized medicine. Bioinformatics. 2011;27(13):1741-8.

Fernando MM, Stevens CR, Walsh EC, De Jager PL, Goyette P, Plenge RM, Vyse TJ, Rioux JD. Defining the role of the $\mathrm{MHC}$ in autoimmunity: a review and pooled analysis. PLoS Genet. 2008;4(4):e1000024. 
Fodil N, Langlais D, Gros P. Primary Immunodeficiencies and Inflammatory Disease: A Growing Genetic Intersection. Trends Immunol. 2016;37(2):126-40.

Franceschini A, Szklarczyk D, Frankild S, Kuhn M, Simonovic M, Roth A, Lin J, Minguez P, Bork P, von Mering C, Jensen LJ. STRING v9.1: protein-protein interaction networks, with increased coverage and integration. Nucleic Acids Res. 2013;41(Database issue):D808-15.

Fraser HB, Hirsh AE, Steinmetz LM, Scharfe C, Feldman MW. Evolutionary rate in the protein interaction network. Science 2002;296(5568):750-2.

Frazer KA, Murray SS, Schork NJ, Topol EJ. Human genetic variation and its contribution to complex traits. Nat Rev Genet 2009; 10: 241-251.

Fritsche LG, Fariss RN, Stambolian D, Abecasis GR, Curcio CA, Swaroop A. Age-Related Macular Degeneration: Genetics and Biology Coming Together. Annual review of genomics and human genetics. 2014;15:151-171. doi:10.1146/annurev-genom-090413-025610.

Fu J, Wolfs MG, Deelen P, Westra HJ, Fehrmann RS, Te Meerman GJ, Buurman WA, Rensen SS, Groen HJ, Weersma RK, et al., Unraveling the regulatory mechanisms underlying tissue-dependent genetic variation of gene expression. PLoS Genet. 2012;8:e1002431.

Gabriel SB, Schaffner SF, Nguyen H, Moore JM, Roy J, Blumenstiel B, et al., The structure of haplotype blocks in the human genome. Science. 2002;296:2225-2229.

Gao X, Starmer J, Martin ER. A multiple testing correction method for genetic association studies using correlated single nucleotide polymorphisms. Genet Epidemiol. 2008;32(4):361-9.

Garcês S, Demengeot J, Benito-Garcia E. The immunogenicity of anti-TNF therapy in immune-mediated inflammatory diseases: a systematic review of the literature with a meta-analysis. Ann Rheum Dis. 2013;72(12):1947-55.

Gene Ontology Consortium (over 160 collaborators). Gene ontology: tool for the unification of biology. Nat Genet. 2000;25(1):25-9.

Gene Ontology Consortium. Gene Ontology Consortium: going forward. Nucleic Acids Res. 2015;43 (Database issue):D1049-56.

Gerstein MB, Kundaje A, Hariharan M, Landt SG, Yan KK, Cheng C, et al. Architecture of the human regulatory network derived from ENCODE data. Nature. 2012; 489 (7414):91-100.

Goh KI, Cusick ME, Valle D, Childs B, Vidal M, Barabási AL. The human disease network. Proc Natl Acad Sci USA. 2007;104(21):8685-90.

Goldstein DB. Common genetic variation and human traits. N Engl J Med. 2009;360 (17): 1696-8.

Goris A, Liston A. The immunogenetic architecture of autoimmune disease. Cold Spring Harb Perspect Biol. 2012; 1;4(3).pii: a007260.

Goto S, Bono H, Ogata H, Fujibuchi W, Nishioka T, Sato K, Kanehisa M. Organizing and computing metabolic pathway data in terms of binary relations. Pac Symp Biocomput. 1997:175-186.

Green ED, Guyer MS, Manolio TA, Peterson JL. National Human Genome Research Institute. Charting a course for genomic medicine from base pairs to bedside. Nature.2011;470 (7333): 204-13. 
Gruber TR. Ontology in The Encyclopedia of Database Systems. Ling L and Tamer Özsu (Eds.), Springer-Verlag, 2009.

Gruber TR. A Translation Approach to Portable Ontology Specifications. Knowledge Acquisition 1993;5 (2):199-220.

GTEx Consortium. The Genotype-Tissue Expression (GTEx) pilot analysis: multi tissue gene regulation in humans. Science 2015; 348: 648-660.

Guruharsha KG, Kankel MW, Artavanis-Tsakonas S. The Notch signalling system: recent insights into the complexity of a conserved pathway. Nat Rev Genet. 2012; 13(9):654-66.

Han B, Kang HM, Eskin E. Rapid and accurate multiple testing correction and power estimation for millions of correlated markers. PLoS Genet. 2009;4:e1000456.

Hebbring SJ. The challenges, advantages and future of phenome-wide association studies. Immunology. 2014;141: 157-165.

Hinch A.G., Tandon A., Patterson N., Song Y., Rohland N., Palmer C.D., Chen G.K., Wang K., Buxbaum S.G., Akylbekova E.L. The landscape of recombination in African Americans. Nature. 2011;476: 170175.

Hindorff LA, Sethupathy P, Junkins HA, Ramos EM, Mehta JP, Collins FS, Manolio TA. Potential etiologic and functional implications of genome-wide association loci for human diseases and traits. Proc Natl Acad Sci USA. 2009;106(23):9362-7.

Hindorff LA, Gillanders EM, Manolio TA. Genetic architecture of cancer and other complex diseases: lessons learned and future directions. Carcinogenesis. 2011;32:945-954.

Hindorff LA, MacArthur J, Morales J, Junkins HA, Hall PN, et al. (2013) A Catalog of Published Genomewide Association Studies. Available: http://www.genome.gov/gwastudies/.

Hinds DA, Stuve LL, Nilsen GB, Halperin E, Eskin E, Ballinger DG, et al., Whole-genome patterns of common DNA variation in three human populations. Science. 2005; 307: 1072-1079.

Hirschhorn JN. Genomewide association studies: illuminating biologic pathways. N Engl J Med.2009; 360 (17):1699-701.

Hua C, Barnetche T, Combe B, Morel J. Effect of methotrexate, anti-tumor necrosis factor $\alpha$, and rituximab on the immune response to influenza and pneumococcal vaccines in patients with rheumatoid: a systematic review and meta-analysis. Arthritis Care Res. 2014;66(7):1016-26.

Huang Q. Genetic study of complex diseases in the post-GWAS era. J Genet Genomics. 2015;42(3): 87-98.

Hunt KA, Mistry V, Bockett NA, Ahmad T, Ban M, Barker JN, Barrett JC, Blackburn H, Brand O, et al., Burren O, Capon F. Negligible impact of rare autoimmune-locus coding-region variants on missing heritability. Nature. 2013;498:232-235.

International HapMap Consortium.The International HapMapProject.Nature.2003;426(6968):789-96.

International Human Genome Sequencing Consortium. Finishing the euchromatic sequence of the human genome. Nature. 2004;431:931-945. 
International HapMap Consortium. A haplotype map of the human genome. Nature. 2005;437 (7063): 1299-320.

International HapMap Consortium. A second generation human haplotype map of over 3.1 million SNPs. Nature. 2007;449(7164):851-61.

International HapMap Consortium. Sabeti PC, Varilly P, Fry B, et al., Genome-wide detection and characterization of positive selection in human populations. Nature. 2007;449(7164):913-918.

International HapMap 3 Consortium, Altshuler DM, Gibbs RA, Peltonen L, et al., Integrating common and rare genetic variation in diverse human populations. Nature.2010;467(7311):52-58.

ENCODE Project Consortium. A User's Guide to the Encyclopedia of DNA Elements (ENCODE). Becker PB, ed. PLoS Biology. 2011;9(4):e1001046.

ENCODE Project Consortium (plus 596 collaborators). An Integrated Encyclopedia of DNA Elements in the Human Genome. Nature. 2012;489 (7414):57-74.

Janeway CJ Jr, Paul Travers P, Walport M and Shlomchik MJ. The Immune System in Health and Disease. Chapters 11,12 and 13. In "Immunobiology" ed. Janeway CJ Jr; 5th Edition New York: Garland Science; 2001.

Jacobson DL, Gange SJ, Rose NR, Graham NM. Epidemiology and estimated population burden of selected autoimmune diseases in the United States. Clin Immunol Immunopathol. 1997;84(3):223-43.

Jeong H, Mason SP, Barabási A-L, Oltvai ZN. Lethality and centrality in protein networks. Nature. 2001; 411: 41-42.

Jordan DM, Ramensky VE, Sunyaev SR. Human allelic variation: perspective from protein function, structure, and evolution. Curr Opin Struct Biol. 2010;20:342-350.

Joshi-Tope G, Gillespie M, Vastrik I, D'Eustachio P, Schmidt E, de Bono B, Jassal B, Gopinath GR, Wu GR, Matthews L, Lewis S, Birney E, Stein L. Reactome: a knowledgebase of biological pathways. Nucleic Acids Res. 2005;33 (Database issue):D428-32.

Kamal KM, Madhavan SS, Hornsby JA, Miller LA, Kavookjian J, Scott V. Use of tumor necrosis factor inhibitors in rheumatoid arthritis: a national survey of practicing United States rheumatologists. Joint Bone Spine 2006;73:718-24.

Kamburov A, Wierling $\mathrm{C}$, Lehrach $\mathrm{H}$, Herwig R. ConsensusPathDB: a database for integrating human functional interaction networks. Nucleic Acids Res. 2009;37: D623-D628.

Kamburov A, Pentchev K, Galicka H, Wierling C, Lehrach H, Herwig R. ConsensusPathDB: toward a more complete picture of cell biology. Nucleic Acids Res. 2011;39 (Database issue):D712-7.

Kamburov A, Stelzl U, Lehrach H, Herwig R. The ConsensusPathDB interaction database: 2013 update. Nucleic Acids Res. 2013;41(Database issue):D793-800.

Kanehisa M, Goto S, Sato Y, Furumichi M, Tanabe M. KEGG for integration and interpretation of largescale molecular data sets. Nucleic Acids Res. 2012;40(Database issue):D109-14.

Kanehisa M, Goto S, Sato Y, Kawashima F, Furumichi M, Tanabe M. Data, information, knowledge and principle: back to metabolism in KEGG. Nucleic Acids Res. 2014;42: D199-D205. 
Kellis M, Wold B, Snyder MP, Bernstein BE, et al., (over 20 authors). Defining functional DNA elements in the human genome. Proc Natl Acad Sci USA. 2014;111(17):6131-8.

Kent WJ, Sugnet CW, Furey TS, Roskin KM, Pringle TH, Zahler AM, Haussler D. The human genome browser at UCSC. Genome Res. 2002 Jun;12(6):996-1006.

Kiezun A, Garimella K, Do R, Stitziel NO, Neale BM, McLaren PJ, Gupta N, Sklar P, Sullivan PF, Moran JL, Hultman CM, Lichtenstein P, Magnusson P, Lehner T, Shugart YY, Price AL, de Bakker PI, Purcell SM, Sunyaev SR. Exome sequencing and the genetic basis of complex traits. Nat Genet. 2012;44(6):623-630.

Kerrien S, Aranda B, Breuza L, Bridge A, Broackes-Carter F, et al. The IntAct molecular interaction database in 2012. Nucleic Acids Res. 2012;40:D841-D846.

Khatri P, Sirota M, Butte AJ. Ten years of pathway analysis: current approaches and outstanding challenges. PLoS Comput Biol. 2012;8(2):e1002375

Knight JC. Approaches for establishing the function of regulatory genetic variants involved in disease. Genome Med. 2014; 6(10): 92-4.

Kolb WR, Granger GA. Lymphocyte in vitro cytotoxicity: characterization of human lymphotoxin. Proc Natl Acad Sci U S A. 1968 Dec; 61(4): 1250-1255.

Korta DZ, Ochieng P, Fishman D, Katz SE. Pulmonary sarcoidosis and latent tuberculosis in a patient with psoriasis treated with adalimumab. Dermatol Online J. 2015;21(1).

Kubo M, Hata J, Ninomiya T, Matsuda K, Yonemoto K, Nakano T, Matsushita T, et al. A nonsynonymous SNP in PRKCH (protein kinase $\mathrm{C}$ eta) increases the risk of cerebral infarction. Nat Genet 2007;39:212-217.

Kumar V., Westra H.J., Karjalainen J., Zhernakova D.V., Esko T., Hrdlickova B., Almeida R., Zhernakova A., Reinmaa E., Võsa U. Human disease-associated genetic variation impacts large intergenic non-coding RNA expression. PLoS Genet. 2013;9:e1003201.

Kurisu S, Takenawa T. The WASP and WAVE family proteins.Genome Biology. 2009;10(6):226.

Lander ES. The new genomics: global views of biology. Science. 1996;274(5287):536-9.

Lander ES, Linton LM, Birren B, Nusbaum C, Zody MC, Baldwin J, Devon K, Dewar K, et al. Initial sequencing and analysis of the human genome. Nature. 2001;409:860-921.

Lander ES. Initial impact of the sequencing of the human genome. Nature. 2011;470 (7333):187-97.

Lander paper in New Engl J on intervention?

Lawson MM, Thomas AG, Akobeng AK. Tumour necrosis factor alpha blocking agents for induction of remission in ulcerative colitis. Cochrane Database Syst Rev. 2006; (3):CD005112.

Lazakidou, Athina. Web-Based Applications in Healthcare and Biomedicine. Ed: Lazakidou, A; Springer 2010; 7:143-156.

Lee S, Gonçalo R. Abecasis GR, Boehnke M, and Lin X. Rare-Variant Association Analysis: Study Designs and Statistical Tests. Am J Hum Genet. 2014; 95(1): 5-23. 
Lehner B. Genotype to phenotype: lessons from model organisms for human genetics. Nat Rev Genet. 2013;14: 168-178.

Lilley J, Wallace C. A pleiotropy-informed Bayesian false discovery rate adapted to a shared control design finds new disease associations from GWAS summary statistics. PLoS Genet. 2015;11(2):e1004926. eCollection 2015.

Lin YT and Lee WC. Importance of presenting the variability of the false discovery rate control. BMC Genet. 2015;16:97.

Liu CC, Tseng YT, Li W, Wu CY, Mayzus I, Rzhetsky A, Sun F, Waterman M, ChenJ, Chaudhary PM, Loscalzo J,Crandall E, Zhou XJ. DiseaseConnect: a comprehensive web server for mechanismbased disease-disease connections. Nucleic Acids Res. 2014;42 (Web Server issue):W137-46.

Lucas CL, Lenardo MJ. Identifying genetic determinants of autoimmunity and immune dysregulation. Curr Opin Immunol. 2015;37:28-33.

Limdi N, Veenstra D. Warfarin Pharmacogenetics. Pharmacotherapy. 2008;28(9):1084-1097.

Mailman MD, Feolo M, Jin Y, Kimura M, Tryka K, Bagoutdinov R, Hao L, Kiang A, Paschall J, Phan L, et al., The NCBI dbGaP database of genotypes and phenotypes. Nat. Genet. 2007; 39:1181-1186.

Maini RN, Brennan FM, Williams R, Chu CQ, Cope AP, Gibbons D, Elliott M, Feldmann M. TNF-alpha in rheumatoid arthritis and prospects of anti-TNF therapy. Clin Exp Rheumatol. 1993;11 Suppl 8:S173-5.

Malladi VS, Erickson DT, Podduturi NR, et al. Ontology application and use at the ENCODE DCC. Database: The Journal of Biological Databases and Curation. 2015;2015: bav010.

Malottki K, Barton P, Tsourapas A, Uthman AO, Liu Z, Routh K, et al., Adalimumab, etanercept, infliximab, rituximab and abatacept for the treatment of rheumatoid arthritis after the failure of a tumour necrosis factor inhibitor: a systematic review and economic evaluation. Health Technol Assess. 2011;15 (14):271-278.

Manolio TA, Brooks LD, Collins FS. A HapMap harvest of insights into the genetics of common disease.J Clin Invest. 2008 May;118(5):1590-605.

Manolio TA, Collins FS, Cox NJ, Goldstein DB, Hindorff LA, Hunter DJ, et al., Finding the missing heritability of complex diseases. Nature. 2009; 461:747-753.

Manolio TA, Collins FS. The HapMap and genome-wide association studies in diagnosis and therapy. Annu Rev Med. 2009;60:443-56.

Maran C, Tassone E, Masola V, Onisto M. The Story of SPATA2 (Spermatogenesis-Associated Protein 2): From Sertoli Cells to Pancreatic Beta-Cells. Curr Genomics. 2009; 10(5):361-3.

Marson A, Housley WJ, Hafler DA. Genetic basis of autoimmunity. J Clin Invest. 2015; 125 (6):2234-41.

Marquez A, Ferreiro-Iglesias A, Dávila-Fajardo CL, et al. Lack of validation of genetic variants associated with anti-tumor necrosis factor therapy response in rheumatoid arthritis: a genome-wide association study replication and meta-analysis. Arthritis Research \& Therapy. 2014;16(2):R66.

Massaad MJ, Ramesh N, Geha RS. Wiskott-Aldrich syndrome: a comprehensive review. Ann N Y Acad Sci. 2013;1285:26-43. 
Maurano MT, Humbert R, Rynes E, Thurman RE, Haugen E, Wang H, Reynolds AP, Sandstrom R, Qu $\mathrm{H}$, Brody J, et al., Systematic localization of common disease-associated variation in regulatory DNA. Science. 2012;337:1190-1195.

Maxwell LJ, Zochling J, Boonen A, Singh JA, Veras MM, Tanjong Ghogomu E, Benkhalti Jandu M, Tugwell P, Wells GA. TNF-alpha inhibitors for ankylosing spondylitis. Cochrane Database Syst Rev. 2015;4:CD005468.

McClellan J and King MC. Genetic heterogeneity in human disease. Cell. 2010;141(2):210-217.

McKusick V.A. Nathans Institute of Genetic Medicine, Johns Hopkins University and National Center for Biotechnology Information, National Library of Medicine. Online Mendelian Inheritance in Man, OMIM. Available at: http://www.ncbi.nlm.nih.gov/omim

Mi H, Muruganujan A, Casagrande JT, Thomas PD. Large-scale gene function analysis with the PANTHER classification system. Nat Protoc. 2013;8(8):1551-66.

Michaud TL, Rho YH, Shamliyan T, Kuntz KM, Choi HK. The comparative safety of tumor necrosis factor inhibitors in rheumatoid arthritis: a meta-analysis update of 44 trials. Am J Med. 2014;127 (12):1208-32.

Miller FW, Cooper RG, Vencovsky J, et al., Genome-wide Association Study of Dermatomyositis Reveals Genetic Overlap with other Autoimmune Disorders. Arthritis and rheumatism. 2013;65(12):3239-3247.

Merelli I, Pérez-Sánchez H, Gesing S, D’Agostino D. Managing, Analysing, and Integrating Big Data in Medical Bioinformatics: Open Problems and Future Perspectives. BioMed Research International. 2014: $13 ; 4023$.

Monaco C, Nanchahal J, Taylor P, Feldmann M. Anti-TNF therapy: past, present and future. International Immunology. 2015;27(1):55-62.

Mooney MA, Nigg JT, McWeeney SK, Wilmot B. Functional and genomic context in pathway analysis of GWAS data. Trends Genet. 2014;30(9):390-400.

Morley M., Molony C.M., Weber T.M., Devlin J.L., Ewens K.G., Spielman R.S., Cheung V.G. Genetic analysis of genome-wide variation in human gene expression. Nature. 2004; 430: 743-747.

Moser G, Lee SH, Hayes BJ, Goddard ME, Wray NR, Visscher PM. Simultaneous discovery, estimation and prediction analysis of complex traits using a bayesian mixture model. PLoS Genet. 2015 Apr 7;11(4):e1004969.eCollection 2015.

Musen MA, Noy NF, Shah NH, Whetzel PL, Chute CG, Story MA, Smith B; NCBO team. The National Center for Biomedical Ontology. J Am Med Inform Assoc. 2012;19 (2):190-5.

Nanau RM, Neuman MG. Safety of anti-tumor necrosis factor therapies in arthritis patients. J Pharm Pharm Sci. 2014;17(3):324-61.

Ngo ST, Steyn FJ, McCombe PA. Gender differences in autoimmune disease. Front Neuroendocrinol. 2014;35(3):347-69.

Nguyen $\mathrm{T}$ and $\mathrm{Wu}$ JJ. Relationship between tumor necrosis factor- $\alpha$ inhibitors and cardiovascular disease in psoriasis: a review. Perm J. 2014 ;18(1):49-54. 
Novembre J., Johnson T., Bryc K., Kutalik Z., Boyko A.R., Auton A., et al. Genes mirror geography within Europe. Nature. 2008;456:98-101.

Noy NF, Shah NH, Whetzel PL, Dai B, Dorf M, Griffith N, Jonquet C, Rubin DL, Storey MA, Chute CG, Musen MA. BioPortal: ontologies and integrated data resources at the click of a mouse. Nucleic Acids Res. 2009 37(Web Server issue):W170-3.

Nucleic Acids Research Database issue. 2015;43 (Database issue):D1-5. The 2015 Nucleic Acids Research Database Issue and molecular biology database collection.

Ober C, Loisel Da, Gilad Y. Sex-specific genetic architecture of human disease. Nat Rev Genetics 2008;9:911-922.

Okada Y, Wu D, Trynka G, Raj T, Terao C, Ikari K, et al. Genetics of rheumatoid arthritis contributes to biology and drug discovery. Nature. 2014;506(7488):376-81.

Olivier M. A haplotype map of the human genome. Physiol Genomics. 2003;13(1):3-9.

Pe'er I, Yelensky R, Altshuler D, Daly MJ. Estimation of the multiple testing burden for genomewide association studies of nearly all common variants. Genet. Epidemiol. 2008;32, 381-385.

Peng K., Xu W., Zheng J., Huang K., Wang H., Tong J., Lin Z., Liu J., Cheng W., Fu D., et al. The Disease and Gene Annotations (DGA): an annotation resource for human disease. Nucleic Acids Res. 2013;41:D553-560.

Peppercorn J, Shapira I, Deshields T, et al. Ethical aspects of participation in the database of genotypes and phenotypes of the National Center for Biotechnology Information: the Cancer and Leukemia Group B Experience. Cancer. 2012;118 (20): 5060-8.

Pico AR, Kelder T, van lersel MP, Hanspers K, Conklin BR, Evelo C. WikiPathways: pathway editing for the people. PLoS Biol. 2008;6(7):e184.

Pink RC, Wicks K, Caley DP, Punch EK, Jacobs L, Francisco Carter DR. Pseudogenes: Pseudofunctional or key regulators in health and disease? RNA. 2011;17(5):792-798.

Poliseno L, Marranci A, Pandolfi PP. Pseudogenes in Human Cancer. Front Med. 2015;2:68.eCollection 2015.

Pollard KM. Environment, autoantibodies, and autoimmunity. Front Immunol. 2015 Feb 11;6:60. doi: 10.3389/fimmu.2015.00060. eCollection 2015.

Probert L, Eugster HP, Akassoglou K, Bauer J, Frei K, Lassmann H, Fontana A. TNFR1 signalling is critical for the development of demyelination and the limitation of T-cell responses during immunemediated CNS disease. Brain. 2000;123 (10):2005-19.

Ramanan VK, Shen L, Moore JH, Saykin AJ. Pathway analysis of genomic data: concepts, methods, and prospects for future development. Trends Genet. 2012;28(7):323-32.

Raj T, Kuchroo M, Replogle JM, Raychaudhuri S, Stranger BE, De Jager PL. Common risk alleles for inflammatory diseases are targets of recent positive selection. Am J Hum Genet. 2013;92(4):517-29.

Ramos EM, Hoffman D, Junkins HA, Maglott D, Phan L, Sherry ST, Feolo M, Hindorff LA. PhenotypeGenotype Integrator (PheGenl): synthesizing genome-wide association study (GWAS) data with existing genomic resources. Eur. J. Hum. Genet. 2013;22:144-147. 
Rappaport N, Nativ N, Stelzer G, Twik M, Guan-Golan Y, Stein TI, Bahir I, Belinky F, Morrey CP, Safran M, Lancet D. MalaCards: an integrated compendium for diseases and their annotation. Database (Oxford) 2013:bat018.

Reich D, Lander ES. On the allelic spectrum of human disease. Trends Genet. 2001;17: 502-510.

Risch N, Merikangas K. The future of genetic studies of complex human diseases. Science.1996; 273:1516-7.

Rodgers M, Epstein D, Bojke L, Yang H, et al., Etanercept, infliximab and adalimumab for the treatment of psoriatic arthritis: a systematic review and economic evaluation. Health Technol Assess. 2011;15(10):i-xxi, 1-29.

Rodriguez-Fontenla C, Calaza M, Gonzalez A. Genetic distance as an alternative to physical distance for definition of gene units in association studies. BMC Genomics. 2014;15(1):408.

Ruddle $\mathrm{NH}$, Waksman $\mathrm{BH}$. Cytotoxicity mediated by soluble antigen and lymphocytes in delayed hypersensitivity. J Exp Med. 1968; 128(6): 1267-1279.

Rubin DL, Lewis SE, Mungall CJ, Misra S, Westerfield M, Ashburner M, Sim I, Chute CG, Solbrig H, Storey MA, Smith B, Day-Richter J, Noy NF, Musen MA. National Center for Biomedical Ontology: advancing biomedicine through structured organization of scientific knowledge. OMICS. 2006; 10 (2):185-98.

Saeed $\mathrm{R}$ and Deane CM. Protein-protein interactions, evolutionary rate, abundance and age. BMC Bioinformatics 2006;7:128.

Saito R, Smoot ME, Ono K, Ruscheinski J, Wang P-L, Lotia S, Pico AR, Bader GD, Ideker T. A travel guide to Cytoscape plugins. NatureMethods 2012; 9(11):1069-1076.

Sayers EW, Barrett T, Benson DA, Bolton E, Bryant SH, Canese K, et al. Database resources of the National Center for Biotechnology Information. Nucleic Acids Res 2012;40: D13-D25.

Schaid DJ, Sinnwell JP, Jenkins GD, McDonnell SK, Ingle JN, Kubo M, et al. Using the gene ontology to scan multilevel gene sets for associations in genome wide association studies. Genet Epidemiol. 2012;36(1):3-16.

Schaub MA, Boyle AP, Kundaje A, Batzoglou S, Snyder M. Linking disease associations with regulatory information in the human genome. Genome Res. 2012;22(9):1748-59.

Schett G, Elewaut D, Mclnnes IB, Dayer JM, Neurath MF. How cytokine networks fuel inflammation: Toward a cytokine-based disease taxonomy. Nat Med. 2013;19(7):822-4.

Schierding W, Cutfield WS, O'Sullivan JM. The missing story behind Genome Wide Association Studies: single nucleotide polymorphisms in gene deserts have a story to tell. Front Genet. 2014;5:39.

Schork NJ, Murray SS, Frazer KA, Topol EJ. Common vs. Rare Allele Hypotheses for Complex Diseases. Cur Opin Gen Dev. 2009;19(3):212-219. doi:10.1016/j.gde.2009.04.010.

Selmi C1, Lu Q, Humble MC.J. Heritability versus the role of the environment in autoimmunity. Autoimmun. 2012;39(4):249-52.

Seong KH, Kim I, Hwang J, and Kim S. Network Modules of the Cross-Species Genotype-Phenotype Map Reflect the Clinical Severity of Human Diseases. PLoS One. 2015; 10(8): e0136300. 
Shah NH, Cole T, and Musen MA. Chapter 9: Analyses Using Disease Ontologies. PLoS Comput Biol. 2012; 8(12): e1002827.

Shannon P, Markiel A, Ozier O, Baliga NS, Wang JT, Ramage D, Amin N, Schwikowski B, Ideker T. Cytoscape: a software environment for integrated models of biomolecular interaction networks. Genome Res. 2003;13 (11):2498-2504.

Sherry ST, Ward MH, Kholodov M, Baker J, Phan L, Smigielski EM, Sirotkin K. dbSNP: the NCBI database of genetic variation. Nucleic Acids Res. 2001; 29(1): 308-311.

Singh J, Furst D, Bharat A, et al. Update 2012 of the American College of Rheumatology recommendations for the use of disease-modifying antirheumatic drugs and biologic agents in the treatment of rheumatoid arthritis. Arthritis Care Res 2012;64:625-39.

Sivamani RK, Goodarzi H, Garcia MS, et al. Biologic therapies in the treatment of psoriasis: a comprehensive evidence-based basic science and clinical review and a practical guide to tuberculosis monitoring. Clin Rev Allergy Immunol. 2013;44(2):121-40.

Smilek, DE and St Clair, EW. Solving the puzzle of autoimmunity: critical questions. F1000Prime Rep. 2015;7:17. eCollection 2015.

Smith B, Ashburner M, Rosse C, Bard J, Bug W, Ceusters W, Goldberg LJ, et al. The OBO Foundry: coordinated evolution of ontologies to support biomedical data integration. Nat Biotechnology 2007;25(11):1251-5.

Smolen JS, van der Heijde D, Machold KP, et al. Proposal for a new nomenclature of disease-modifying antirheumatic drugs. Ann Rheum Dis 2014;73:3-5.

Smolen JS, Landewé R, Breedveld FC, Buch M, Burmester G, Dougados M, et al. EULAR recommendations for the management of rheumatoid arthritis with synthetic and biological diseasemodifying antirheumatic drugs: 2013 update. Ann Rheum Dis. 2014; 73(3):492-509.

Solovieff N, Cotsapas C, Lee PH, Purcell SM, Smoller JW. Pleiotropy in complex traits: challenges and strategies. Nat Rev Genet. 2013;14: 483-495.

Sollid LM, Pos W, Wucherpfennig KW. Molecular Mechanisms for Contribution of MHC Molecules to Autoimmune Diseases. Current opinion in immunology. 2014;0:24-30.

Somers EC, Thomas SL, Smeeth L, Hall AJ. Are individuals with an autoimmune disease at higher risk of a second autoimmune disorder? Am J.Epidemiol. 2009;169:749-55.

Song Y, Buchwald P. TNF Superfamily Protein-Protein Interactions: Feasibility of Small-Molecule Modulation. Curr Drug Targets. 2015;16(4):393-408.

Steinberg $\mathrm{MH}$ and Adewoye $\mathrm{AH}$. Modifier genes and sickle cell anemia. Curr Opin Hematol. 2006;13 (3):131-6.

Stefl S, Nishi H, Petukh M, Panchenko AR, Alexov E. Molecular mechanisms of disease-causing missense mutations. J Mol Biol. 2013;425(21):3919-3936.

Storey JD and Tibshirani R. Statistical significance for genomewide studies. Proc Natl Acad Sci USA. 2003;100:9440-9445.

Su G, Morris JH, Demchak B, Bader GD. Biological network exploration with Cytoscape 3. Curr Protoc Bioinformatics. 2014;47:8.13.1-8.13.24. 
Soubrier F. From an ACE polymorphism to genome-wide searches for eQTL. J Clin Invest. 2013;123 (1):111-112.

Sun YV. Integration of biological networks and pathways with genetic association studies. Hum Genet. 2012;131(10):1677-86.

Sunyaev SR. Inferring causality and functional significance of human coding DNA variants. Hum Mol Genet.2012;21:R10-R17.

Suppiah V, Moldovan M, Ahlenstiel G, Berg T, Weltman M, Abate ML, Bassendine M, et al. IL28B is associated with response to chronic hepatitis $C$ interferon-alpha and ribavirin therapy. Nat Genet. 2009;41(10):1100-4.

Szklarczyk D, Franceschini A, Kuhn M, Simonovic M, Roth A, Minguez P, Doerks T, et al. The STRING database in 2011: functional interaction networks of proteins, globally integrated and scored. Nucleic Acids Res. 2011;39 (Database issue):D561-D568.

Szklarczyk D, Franceschini A, Wyder S, Forslund K, Heller D, Huerta-Cepas J, Simonovic M, Roth A, et al. STRING v10: protein-protein interaction networks, integrated over the tree of life. Nucleic Acids Res. 2015 Jan;43 (Database issue): D447-D452.

Tak YG, Farnham PJ. Making sense of GWAS: using epigenomics and genome engineering to understand the functional relevance of SNPs in non-coding regions of the human genome. Epigenetics Chromatin. 2015;8:57.

Taylor PC. Anti-TNFalpha therapy for rheumatoid arthritis: an update. Intern Med.2003; 42(1):15-20.

Teng S, Madej T, Panchenko A, Alexov E. Modeling Effects of Human Single Nucleotide Polymorphisms on Protein-Protein Interactions. Biophysical Journal. 2009;96(6):2178-2188.

Tennessen JA, Bigham AW, O'Connor TD, Fu W, Kenny EE, et al. Evolution and Functional Impact of Rare Coding Variation from Deep Sequencing of Human Exomes. Science. 2012;337: 64-69.

Terao C, Yamada R, Ohmura K, et al. The human AIRE gene at chromosome 21q22 is a genetic determinant for the predisposition to rheumatoid arthritis in Japanese population. Hum Mol Genet. 2011;20 (13):2680-5.

Trynka G, Hunt KA, Bockett NA, Romanos J, Mistry V, Szperl A, Bakker SF, et al. Dense genotyping identifies and localizes multiple common and rare variant association signals in celiac disease. Nat Genet. 2011;43(12):1193-201.

Turner CF, Pan H, Silk GW, Ardini MA, Bakalov V, Bryant S, Cantor S, Chang KY, et al. The NIDDK Central Repository at 8 years: ambition, revision, use and impact. Database (Oxford). 2011:bar043.

Ueda H, Howson JM, Esposito L, Heward J, Snook H, Chamberlain G, et al. Association of the T-cell regulatory gene CTLA4 with susceptibility to autoimmune disease. Nature 2003;423: 506-511.

Umićevic MM, Cui J, Vermeulen SH, Stahl EA, Toonen EJ, Makkinje RR, Lee AT, et al. Genomewide association analysis of anti-TNF drug response in patients with rheumatoid arthritis. Ann Rheum Dis. 2013 Aug;72(8):1375-81.

van der Sijde MR, Ng A, Fu J. Systems genetics: From GWAS to disease pathways. Biochim Biophys Acta. 2014;1842(10):1903-1909. 
van Schouwenburg PA, Krieckaert CL, Rispens T, Aarden L, Wolbink GJ, Wouters D. Long-term measurement of anti-adalimumab using $\mathrm{pH}$-shift-anti-idiotype antigen binding test shows predictive value and transient antibody formation. Ann Rheum Dis. 2013;72(10):1680-6.

Venkatesan K, Rual JF, Vazquez A, Stelzl U, Lemmens I, Hirozane-Kishikawa T, et al. An empirical framework for binary interactome mapping. Nature Methods. 2008;6:83-90.

Venter JC, Adams MD, Myers EW, Li PW, Mural RJ, Sutton GG, et al. The sequence of the human genome. Science. 2001;291:1304-1351.

Ventriglia G, Nigi L, Sebastiani G, Dotta F. MicroRNAs: Novel Players in the Dialogue between Pancreatic Islets and Immune System in Autoimmune Diabetes. Biomed Res Int. 2015;2015:749734.

Vidal M, Cusick ME, Barabási AL. Interactome networks and human disease. Cell. 2011;144(6):986-98.

Visscher PM, Brown M, McCarthy MI, Yang J. Five years of GWAS discovery. Am J Hum Genet. The American Society of Human Genetics. 2012;90: 7-24.

Vyse TJ, Todd JA. Genetic analysis of autoimmune disease. Cell. 1996; 85: 311-318

Walsh SJ, Rau LM. Autoimmune diseases: a leading cause of death among young and middle-aged women in the United States. Am J Public Health. 2000;90(9):1463-6.

Wang L, Liu H, Jiao Y, Wang E, Clark SH, Postlethwaite AE, Gu W, Chen H. et al. Differences between Mice and Humans in Regulation and the Molecular Network of Collagen, Type III, Alpha-1 at the Gene Expression Level: Obstacles that Translational Research Must Overcome. Int J Mol Sci. 2015; 16 (7):15031-56.

Wang X, Gulbahce N, Yu H. Network-based methods for human disease gene prediction. Brief Funct Genomics. 2011;10:280-293.

Wang L, Wang FS, Gershwin ME. Human autoimmune diseases: a comprehensive update. J Intern Med. 2015;278(4):369-95.

Ward LD, Kellis M. Interpreting noncoding genetic variation in complex traits and human disease. Nat Biotechnol 2012;30:1095-1106.

Ward LD, Kellis M. HaploReg: a resource for exploring chromatin states, conservation, and regulatory motif alterations within sets of genetically linked variants. Nucleic Acids Res. 2012;40:D930-D934.

Waterfield $\mathrm{M}$ and Anderson MS. Clues to immune tolerance: the monogenic autoimmune syndromes. Ann N Y Acad Sci. 2010;1214:138-55.

The Wellcome Trust Case Control Consortium. Genome-wide association study of 14,000 cases of seven common diseases and 3,000 shared controls. Nature. 2007;447(7145):661-678.

Welter D, MacArthur J, Morales J, Burdett T, Hall P, Junkins H, et al. The NHGRI GWAS Catalog: a curated resource of SNP-trait associations. Nucleic Acids Res. 2014; 42(Database issue):D1001-6.

Westra HJ, Peters MJ, Esko T, Yaghootkar H, Schurmann C, Kettunen J, et al. Systematic identification of trans eQTLs as putative drivers of known disease associations. Nat. Genet. 2013;45:1238-1243.

Willrich MA, Murray DL, Snyder MR. Tumor necrosis factor inhibitors: clinical utility in autoimmune diseases. Transl Res. 2015;165(2):270-82. 
Wise AL, Gyi L, Manolio TA. eXclusion: toward integrating the $X$ chromosome in genome-wide association analyses. Am J Hum Genet. 2013;92(5):643-7.

Xie D, Boyle AP, Wu L, Zhai J, Kawli T, Snyder M. Dynamic trans-acting factor co-localization in human cells. Cell. 2013;155(3):713-24.

Xie X, Li F, Chen JW, Wang J. Risk of tuberculosis infection in anti-TNF-a biological therapy: from bench to bedside. J Microbiol Immunol Infect. 2014;47(4):268-74.

Yang J, Manolio TA, Pasquale LR, Boerwinkle E, Caporaso N, Cunningham JM, et al. Genome partitioning of genetic variation for complex traits using common SNPs. Nat Genet. 2011; 43(6): 519525.

Yin L, Chen X, Vicini P, Rup B, Hickling TP. Therapeutic outcomes, assessments, risk factors and mitigation efforts of immunogenicity of therapeutic protein products. Cell Immunol. 2015;295(2):118-126.

Zhang Z, Miteva MA, Wang L, Alexov E. Analyzing Effects of Naturally Occurring Missense Mutations. Comput Math Method in Medicine. 2012;2012:805827.

Zerhouni EA, Nabel EG. Protecting aggregate genomic data. Science.2008;322(5898):44.

Zhu LJ, Yang X, Yu XQ. Anti-TNF-a Therapies in Systemic Lupus Erythematosus. J Biomed Biotech. 2010;2010:465898. doi:10.1155/2010/465898.

\section{Websites:}

ACR aTNF data from ACR website:

http://www.rheumatology.org/l-Am-A/Patient-Caregiver/Treatments/Anti-TNF

ConsensusPathDB: http://consensuspathdb.org/

Cytoscape v3.1.1. : http://cytosca-pe.org/download.php

Drug Bank: http://www.drugbank.ca

ENCODE portal: http://encodeproject.org.

ENSEMBL portal: www.ensembl.org/Homo_sapiens/Info/Indexl

FDA aTNF data on FDA website:

http://www.fda.gov/drugs/drugsafety/postmarketdrugsafetyinformationforpatientsandproviders/ucm10934 0.htm

HAVANA project: http://vega.sanger.ac.uk/index.html

HLA Gene Family: http://ghr.nlm.nih.gov/geneFamily/hla 
Human HLA Database: http://www.ebi.ac.uk/ipd/imgt/hla/stats.html

Human Proteome Map: http://www.humanproteomemap.org

miRBase: http://www.mirbase.org/

NCBI portal: http://www.ncbi.nlm.nih.gov/

NCl: http://pid.nci.nih.gov/

OMIM portal: http://www.ncbi.nlm.nih.gov/omim

PharmaGKB: https://www.pharmgkb.org/

PolyPhen-2: http://genetics.bwh.harvard.edu/pph2/

PubMed: http://www.ncbi.nlm.nih.gov/pubmed

Reactome: http://www.reactome.org/

STRING: http://string-db.org

TargetScan: http://www.targetscan.org/ 


\section{TABLES AND FIGURES}


Table 2. Missense GWAS AID SNPs characteristics: I part

(amino acid change caused by missSNPs; PolyPhen-2 evaluation of functional consequences; MAF)

\begin{tabular}{|c|c|c|c|c|c|c|c|c|c|c|}
\hline No. & rs & Context & Gene name & Gene ID & $\begin{array}{c}\text { Functional impact } \\
\text { (PolyPhen-2) }\end{array}$ & $\begin{array}{c}\text { 1KG } \\
\text { Project } \\
\text { presence }\end{array}$ & $\begin{array}{l}\text { MAF (minor } \\
\text { allele count) }\end{array}$ & AA change & Disease & Comment \\
\hline 1 & rs2476601 & missense & PTPN22 & 26191 & benign & $1000 \mathrm{G}$ & $A=0.0274 / 137$ & $\mathrm{~V}[\mathrm{Val}] \Rightarrow \mathrm{A}[\mathrm{Ala}]$ & RA, CD (T1D) & same rs \\
\hline 2 & rs4077515 & missense & CARD9 & 64170 & benign & $1000 \mathrm{G}$ & $\mathrm{T}=0.367 / 799$ & $\mathrm{~S}[\mathrm{Ser}] \Rightarrow \mathrm{N}[$ Asn $]$ & CD, UC, AS & $\begin{array}{l}\text { same rs (has } \\
\text { a near-gene } \\
\text { SNP ) }\end{array}$ \\
\hline 3 & rs30187 & missense & ERAP1 & 51752 & damaging & $1000 \mathrm{G}$ & $\mathrm{T}=0.411 / 896$ & $\mathrm{~K}$ [Lys] $\Rightarrow \mathrm{R}[$ Arg] & AS, Ps & $\begin{array}{l}\text { same rs (has } \\
\text { an intron rs) }\end{array}$ \\
\hline 4 & rs11209026 & missense & IL23R & 149233 & damaging & $1000 \mathrm{G}$ & $A=0.033 / 72$ & $\mathrm{R}[\mathrm{Arg}] \Rightarrow \mathrm{Q}[\mathrm{GIn}]$ & $\begin{array}{l}\text { CD, AS, Ps, IBD, UC (asthma, } \\
\text { T1D) }\end{array}$ & same rs \\
\hline 5 & rs33980500 & missense & TRAF3IP2 & 10758 & damaging & $1000 \mathrm{G}$ & $\mathrm{T}=0.082 / 178$ & $\mathrm{D}[\mathrm{Asp}] \Rightarrow \mathrm{N}[\mathrm{Asn}]$ & PsA,Ps, CD & same rs \\
\hline 6 & rs2233434 & missense & NFKBIE & 4794 & benign & $1000 \mathrm{G}$ & $\mathrm{G}=0.074 / 161$ & $\vee$ [Val] $\Rightarrow$ A [Ala] & RA & - \\
\hline 7 & rs3197999 & missense & MST1 & 4485 & benign & $1000 \mathrm{G}$ & $A=0.215 / 469$ & $\mathrm{R}[\mathrm{Arg}] \Rightarrow \mathrm{C}$ [Cys] & $\mathrm{CD}, \mathrm{UC}$ & same rs \\
\hline 8 & rs2241880 & missense & ATG16L1 & 55054 & benign & $1000 \mathrm{G}$ & $\mathrm{G}=0.389 / 847$ & $\mathrm{~T}[\mathrm{Thr}] \Rightarrow \mathrm{A}[\mathrm{Ala}]$ & CD, UC & same rs \\
\hline 9 & rs3764147 & missense & LACC1 & 144811 & benign & $1000 \mathrm{G}$ & $\mathrm{G}=0.303 / 659$ & $\mathrm{I}[\mathrm{lle}] \Rightarrow \mathrm{V}[\mathrm{Val}]$ & CD (leprosy) & same rs \\
\hline 10 & rs12720356 & missense & TYK2 & 7297 & damaging & $1000 \mathrm{G}$ & $C=0.045 / 99$ & $\mathrm{I}[\mathrm{lle}] \Rightarrow \mathrm{S}[\mathrm{Ser}]$ & $\begin{array}{l}\text { CD, Ps, PsA, UC, IBD, RA } \\
\text { (T1D,T2D) }\end{array}$ & same rs \\
\hline 11 & rs3184504 & missense & $\mathrm{SH} 2 \mathrm{~B} 3$ & 10019 & benign & $1000 \mathrm{G}$ & $\mathrm{T}=0.1474 / 738$ & $\mathrm{~W}[\mathrm{Trp}] \Rightarrow \mathrm{R}[\mathrm{Arg}]$ & $\begin{array}{l}\text { RA (T1D, hypotyroidism, celiac } \\
\text { disease) }\end{array}$ & same rs \\
\hline 12 & rs2230926 & missense & TNFAIP3 & 7128 & benign & $1000 \mathrm{G}$ & $G=0.124 / 270$ & $\mathrm{~F}[\mathrm{Phe}] \Rightarrow \mathrm{C}[$ Cys $]$ & RA (SLE, PS, UC, celiac d.), PsA & $\begin{array}{l}\text { same rs (has } \\
\text { intron rs) }\end{array}$ \\
\hline 13 & rs2066847 & frameshift & NOD2 & 64127 & damaging & $1000 \mathrm{G}$ & $C=0.008 / 17$ & $L[$ Leu $] \Rightarrow P[$ Pro $]$ & $\mathrm{CD}, \mathrm{IBD}, \mathrm{Ps}$ & same rs \\
\hline 14 & rs20541 & missense & IL13 & 3596 & benign & $1000 \mathrm{G}$ & $A=0.268 / 584$ & $\mathrm{Q}[\mathrm{GIn}] \Rightarrow \mathrm{R}[\mathrm{Arg}]$ & Ps (lgE) & same rs \\
\hline 15 & rs2228145 & missense & IL6R & 3570 & benign & $1000 \mathrm{G}$ & $C=0.319 / 695$ & $\mathrm{D}[\mathrm{Asp}] \Rightarrow \mathrm{A}[\mathrm{Ala}]$ & RA (asthma, CRP, etc) & same rs \\
\hline 16 & rs1063635 & missense & MICA & 100507436 & no data & no & no data & $\mathrm{R}[\mathrm{Arg}] \Rightarrow \mathrm{Q}[\mathrm{GIn}]$ & RA, Ps & only rs \\
\hline 17 & rs3125734 & missense & RTKN2 & 219790 & benign & $1000 \mathrm{G}$ & $\mathrm{T}=0.351 / 764$ & $\mathrm{H}[\mathrm{His}] \Rightarrow \mathrm{R}[\mathrm{Arg}]$ & RA & only rs \\
\hline 18 & rs2298428 & missense & YDJC & 150223 & damaging & $1000 \mathrm{G}$ & $\mathrm{T}=0.226 / 492$ & $\mathrm{~A}[\mathrm{Ala}] \Rightarrow \mathrm{T}[\mathrm{Thr}]$ & RA, CD (Celiac) & same rs \\
\hline 19 & rs8192591 & missense & $\mathrm{NOTCH} 4$ & 4855 & damaging & $1000 G$ & $\mathrm{~T}=0.0312 / 156$ & $\mathrm{G}[\mathrm{Gly}] \Rightarrow \mathrm{S}[\mathrm{Ser}]$ & $\begin{array}{l}\text { Ps (RA, scleroderma, SLE, T1D, } \\
\text { T2D, MS, asthma, schizophrenia) }\end{array}$ & $\begin{array}{l}\text { not same rs } \\
\text { ( } 3 \text { miss and } \\
\text { syn rss in } \\
\text { high LD) } \\
\end{array}$ \\
\hline 20 & rs1801274 & missense & FCGR2A & 2212 & benign & $1000 \mathrm{G}$ & $\mathrm{G}=0.430 / 937$ & $\mathrm{H}[\mathrm{His}] \Rightarrow \mathrm{R}[\mathrm{Arg}]$ & UC (mucokutaneous disease) & same rs \\
\hline 21 & rs2305480 & missense & GSDMB & 55876 & damaging & $1000 \mathrm{G}$ & $A=0.2867 / 1436$ & $\mathrm{P}[\mathrm{Pro}] \Rightarrow \mathrm{S}[\mathrm{Ser}]$ & $\begin{array}{l}\text { UC (asthma same; RA, T1D, CD } \\
\text { different rs) }\end{array}$ & not same rs \\
\hline 22 & rs5771069 & missense & IL17REL & 400935 & benign & 1000G & $\mathrm{G}=0.2208 / 1106$ & $L[$ Leu $] \Rightarrow$ P [Pro] & UC & - \\
\hline 23 & rs3194051 & missense & IL7R & 3575 & benign & $1000 \mathrm{G}$ & $G=0.2208 / 1106$ & $\mathrm{I}[\mathrm{lle}] \Rightarrow \mathrm{V}[\mathrm{Val}]$ & UC (T1D, MS) & different miss rs \\
\hline
\end{tabular}

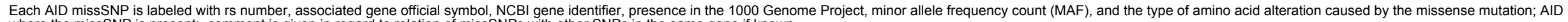
where the missSNP is present; comment is given in regard to relation of missSNPs with other SNP's in the same gene if known.

Yellow field: the genes with damaging missSNP

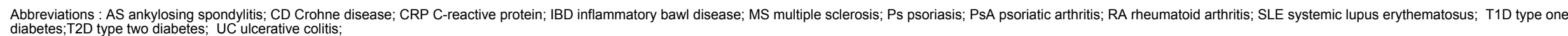
NFKBIE has an intronic 2b SNP rs3799963 in perfect LD with 2 missense SNPs rs2233434 and rs2233433

YDJC miss SNP rs 2298428 is in perfect LD with 3'UTR of UBE2L3 double hit rs7445 and rs7444, both in conserved regions; UBE2L3 could be also a functional gene 
Table 3. Missense GWAS AID SNPs characteristics: II part

analysis of LD blocks by HaploReg v2 and RegulomeDB scoring

\begin{tabular}{|c|c|c|c|c|c|c|c|c|c|c|c|c|c|c|c|}
\hline No & rs & $\begin{array}{l}\text { Gene } \\
\text { name }\end{array}$ & Gene ID & $\begin{array}{l}\text { Variants in LD with the } \\
\text { lead SNP }\end{array}$ & Genes in LD & $\begin{array}{l}\text { Conserved } \\
\text { region }\end{array}$ & $\begin{array}{c}\text { Promoter } \\
\text { histone } \\
\text { marks }\end{array}$ & $\begin{array}{c}\text { Enhanser } \\
\text { histone } \\
\text { marks }\end{array}$ & DNase & $\begin{array}{c}\text { Proteins } \\
\text { bound }\end{array}$ & $\begin{array}{l}\text { eQTL } \\
\text { tissues }\end{array}$ & $\begin{array}{l}\text { Motifs } \\
\text { changed }\end{array}$ & \begin{tabular}{|c|} 
dbSNP \\
functional \\
annotation
\end{tabular} & $\begin{array}{l}\text { Regulome } \\
\text { DB score }\end{array}$ & $\begin{array}{c}\text { Comment about } \\
\text { lead vS } \\
\text { functional SNP }\end{array}$ \\
\hline 1 & rs2476601 & PTPN22 & 26191 & $\begin{array}{l}\text { no other genes; lead SNP is functinal, } \\
\text { well annotated and scored high }\end{array}$ & PTPN22 & yes & & HMEC & 7 cell types & STAT3 & $\begin{array}{c}13 \text { eQTL } \\
\text { results }\end{array}$ & Ets, NERF1a, PU.1 & missense & $2 b$ & Lead and Functional SNP \\
\hline 2 & rs4077515 & CARD9 & 64170 & $\begin{array}{l}\text { two genes with two functional 1f } \\
\text { SNPs in LD: CARD9 missSNP } \\
\text { scored 1f, synSNP in LD; SNAPC4 } \\
\text { has a cons missSNP scored 1f and } \\
\text { several synSNPs, some conserv.; } \\
\text { lead SNP is functinal but other } \\
\text { functional SNPs might exist. }\end{array}$ & $\begin{array}{l}\text { CARD9, } \\
\text { SNAPC4 }\end{array}$ & yes & & $\begin{array}{c}\text { HSMM, K562, } \\
\text { NHLF }\end{array}$ & & & $\begin{array}{c}34 \text { eQTL } \\
\text { results }\end{array}$ & & missense & 1f & $\begin{array}{l}\text { Lead and Functional SNP; } \\
\text { other functional SNPS } \\
\text { may exist tin CARD9 and } \\
\text { in SNAPC4 }\end{array}$ \\
\hline 3 & rs30187 & ERAP1 & 51752 & $\begin{array}{l}\text { no other genes in LD; syn rs within the } \\
\text { same gene. }\end{array}$ & ERAP1 & yes & & & 17 cell types & & $\begin{array}{c}15 \text { eQTL } \\
\text { results }\end{array}$ & $\begin{array}{l}\text { NF-kappaB, } \\
\text { Zbtb3 }\end{array}$ & missense & 5 & Lead and Functional SNP \\
\hline 4 & rs11209026 & IL23R & 149233 & $\begin{array}{l}\text { no other genes in LD; intronic rss in LD } \\
\text { less annoted; lead SNP is functional. }\end{array}$ & IL23R & yes & & & & & $\begin{array}{l}5 \text { eQTL } \\
\text { results }\end{array}$ & GR & missense & 5 & Lead and Functional SNP \\
\hline 5 & rs33980500 & TRAF3IP2 & 10758 & $\begin{array}{l}\text { no other genes in LD; lead SNP is } \\
\text { functional. }\end{array}$ & $\begin{array}{c}\text { TRAF3IP2, } \\
\text { TRAF3IP2-AS1 }\end{array}$ & yes & & 7 cell types & 36 cell types & & $\begin{array}{l}6 \text { eQTL } \\
\text { results }\end{array}$ & $\begin{array}{l}\text { BCL,FXR, Maf, } \\
\text { Poulf1, STAT }\end{array}$ & missense & 4 & Lead and Functional SNP \\
\hline 6 & rs 2233434 & NFKBIE & 4794 & $\begin{array}{l}2 \text { miss SNPs within NFKBIE in perfect LD, } \\
\text { both scored } 4 \text {; several genes in LD: } \\
\text { TCTE1, AARS2 with syn scored less than } \\
5 \text {. }\end{array}$ & $\begin{array}{c}\text { NFKBIE, } \\
\text { TCTE1, AARS2 }\end{array}$ & no & 8 cell types & Huvec & 6 cell types & $\begin{array}{l}\text { CTCF, } \\
\text { ZNF263 }\end{array}$ & $\begin{array}{c}17 \text { eQTL } \\
\text { results }\end{array}$ & & missense & 4 & $\begin{array}{l}\text { Lead and Functional SNP; } \\
\text { double hit }\end{array}$ \\
\hline 7 & rs3197999 & MST1 & 4485 & $\begin{array}{l}\text { in LD with APEH with syn cons SNP; BSN } \\
\text { with miss and syn rSs, scored less than 5; } \\
\text { potentally lead SNP is not a functional } \\
\text { SNP }\end{array}$ & $\begin{array}{c}\text { MST1, APEH, } \\
\text { BSN }\end{array}$ & yes & & & & & $\begin{array}{c}24 \text { eQTL } \\
\text { results }\end{array}$ & $\begin{array}{l}\text { SMC3, TCF } 12, \\
\text { ZBRK1 }\end{array}$ & missense & no data & $\begin{array}{l}\text { Lead SNP most probably } \\
\text { not Functional SNP }\end{array}$ \\
\hline 8 & rs2241880 & ATG16L1 & 55054 & $\begin{array}{l}\text { no other gene in LD; no other miss SNP; } \\
\text { in perfect LD with cons intronic SNP of } \\
\text { SCARNAS, with no scoring data. }\end{array}$ & ATG16L1 & no & & & & & $\begin{array}{l}6 \text { eQTL } \\
\text { results }\end{array}$ & NRSF & missense & no data & Lead and Functional SNP \\
\hline 9 & rs3764147 & LACC1 & 144811 & $\begin{array}{l}\text { no other genes; } \text { high scoring } 1 \text { f very likely } \\
\text { to affect binding and expression. }\end{array}$ & LACC1 & yes & & HSMM & 15 cell types & & $\begin{array}{l}9 \text { eQTL } \\
\text { results }\end{array}$ & $\begin{array}{l}\text { ATF3, GATA, } \\
\text { Hand1, Mrg, } \\
\text { smad3 }\end{array}$ & missense & $1 \mathrm{f}$ & Lead and Functional SNP \\
\hline 10 & rs12720356 & TYK2 & 7297 & $\begin{array}{l}\text { no other genes; it is the only missSNP in } \\
\text { TYK2 gene }\end{array}$ & TYK2 & yes & & & $\begin{array}{l}\text { 8988T, } \\
\text { Chorion }\end{array}$ & & \begin{tabular}{|c|}
27 eQTL \\
results
\end{tabular} & & missense & 5 & Lead and Functional SNP \\
\hline 11 & rs3184504 & SH2B3 & 10019 & $\begin{array}{l}\text { ATXN2 gene in LD with several intronic } \\
\text { SNPs scored low; lead SNP is a functional } \\
\text { SNP. }\end{array}$ & SH2B3, ATXN2 & yes & & $\begin{array}{l}\text { Huvec, } \\
\text { GM12878 }\end{array}$ & & & $\begin{array}{l}15 \text { eQTL } \\
\text { results }\end{array}$ & HES1, Mtf1 & missense & $3 a$ & Lead and Functional SNP \\
\hline 12 & rs2230926 & TNFAIP3 & 7128 & $\begin{array}{l}\text { no other genes; lead SNP is a functional } \\
\text { SNP }\end{array}$ & TNFAIP3 & yes & & M12878 & NHEK & & $\begin{array}{l}2 \text { eQTL } \\
\text { results }\end{array}$ & $\begin{array}{c}\text { GR, } \operatorname{Sin} 3 A k- \\
20\end{array} \mid$ & missense & 4 & Lead and Functional SNP \\
\hline 13 & rs2066847 & NOD2 & 64127 & $\begin{array}{l}\text { no other genes in LD; lead SNP is a } \\
\text { functional SNP }\end{array}$ & NOD2 & no & & & & & & $\underset{\substack{\text { BDP1, SIX5, SRF, } \\
\text { p300 }}}{\mid}$ & frameshift & 5 & Lead and Functional SNP \\
\hline
\end{tabular}




\begin{tabular}{|c|c|c|c|c|c|c|c|c|c|c|c|c|c|c|c|}
\hline 14 & rs20541 & IL13 & 3596 & $\begin{array}{l}\text { no other genes in LD; likely lead SNP is a } \\
\text { functional SNP; no scoring data for other } \\
\text { IL13 SNPS }\end{array}$ & IL13 & no & & M12878 & & & $\begin{array}{l}8 \text { eQTL } \\
\text { results }\end{array}$ & RXRA & missense & $3 a$ & Lead and Functional SNP \\
\hline 15 & rs2228145 & IL6R & 3570 & $\begin{array}{l}\text { no other genes in LD; two other intronic } \\
\text { SNPS scored } 2 \mathrm{~b} \text { likely affecting binding; } \\
\text { lead SNP is not the only functional SNP. }\end{array}$ & IL6R & yes & & $\begin{array}{l}\text { HMEC, } \\
\text { GM12878 }\end{array}$ & & & & & missense & 5 & \begin{tabular}{|} 
Lead SNP might not be \\
the only functional SNP
\end{tabular} \\
\hline 16 & rs1063635 & MICA & 100507436 & $\begin{array}{l}\text { no other genes in LD; no other SNPs. } \\
\text { Lead SNP scored 6; poor annotaion. }\end{array}$ & MICA & no & & & & & \begin{tabular}{|c|}
13 eQTL \\
results
\end{tabular} & $\sin 3 A k-20$ & missense & 6 & $\begin{array}{l}\text { Lead SNP might not be } \\
\text { Functional SNP }\end{array}$ \\
\hline 17 & rs3125734 & RTKN2 & 219790 & $\begin{array}{l}\text { no other genes in LD; no other annotated } \\
\text { SNPs. }\end{array}$ & RTKN2 & yes & & & & & $\begin{array}{l}2 \text { eQTL } \\
\text { results }\end{array}$ & Maf & missense & 5 & Lead and Functional SNP \\
\hline 18 & rs2298428 & YDJC & 150223 & $\begin{array}{l}\text { lead SNP scored 4; UBE2L3 in LD, has } \\
\text { intronic SNPS, no miss SNPS; UBE2L3 3'- } \\
\text { UTR NNPS scored 2b and 3a both cons; no } \\
\text { QTL in LD. }\end{array}$ & YDJC, UBE2L3 & yes & 4 cell types & & $\begin{array}{l}\text { NB4, SK-N- } \\
\text { SH RA }\end{array}$ & $\mid \begin{array}{l}\text { POL2, SIN3 } \\
\text { AK20, CHD2, } \\
\text { HEY1, SMC3 }\end{array}$ & $\begin{array}{c}18 \text { eQTL } \\
\text { results }\end{array}$ & $\begin{array}{c}\text { Iff, KAP1, Nanog, } \\
\text { PRDM1, Pax-5, } \\
\text { TATA }\end{array}$ & missense & 4 & $\begin{array}{l}\text { Lead and but not } \\
\text { Functional SNP UBE2L3 } \\
\text { gene is more important } \\
\text { functionally with high } \\
\text { scored SNPS. }\end{array}$ \\
\hline 19 & rs8192591 & NOTCH4 & 4855 & 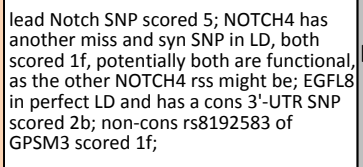 & $\begin{array}{c}\text { NOTCH4, } \\
\text { EGFL8, GPSM3 } \\
\text { (GPSM3 in } \\
\text { lower LD than } \\
\text { EGFL8) }\end{array}$ & no & & & & & $\begin{array}{c}15 \text { eQTL } \\
\text { results }\end{array}$ & BCL, NRSF & missense & 5 & $\begin{array}{l}\text { Lead missSNP might not } \\
\text { be the only functional } \\
\text { SNP; more NOTCH4 } \\
\text { functional SNPS may exist } \\
\text { in LD; EGFL } 3 \text { 'UTR SNP } \\
\text { might be functional. }\end{array}$ \\
\hline 20 & rs1801274 & FCGR2A & 2212 & $\begin{array}{l}\text { no other genes in LD; several intronic, } \\
\text { low scored. }\end{array}$ & FCGR2A & no & & & & & $\begin{array}{c}11 \text { eQTL } \\
\text { results }\end{array}$ & & missense & no data & Lead and Functional SNP \\
\hline 21 & rs 2305480 & GSDMB & 55876 & $\begin{array}{l}\text { GSDMB has second missSNP ih perfect } \\
\text { LD, both scored 1f; ZPBP2 in LD with one } \\
\text { miss and several cons intronic SNPS (one } \\
\text { is 1f). }\end{array}$ & GSDMB, ZPBP2 & no & & & HepG2 & & \begin{tabular}{|l}
$23 \mathrm{eQTL}$ \\
results
\end{tabular} & BAF155, TAL1 & missense & If & $\begin{array}{l}\text { Lead and functional SNP; } \\
\text { two functional miss SNPS } \\
\text { exist; double hit. }\end{array}$ \\
\hline 22 & rs5771069 & IL17REL & 400935 & $\begin{array}{l}\text { PIM3 gene in LD with no miss or cons rs } \\
\text { and all scored low; this lead SNP is scored } \\
5 \text { and might not be the only functional } \\
\text { SNP. }\end{array}$ & IL17REL, PIM3 & no & & $\mathrm{H} 1$ & $\begin{array}{l}\text { LNCaP, GM } \\
12892 \\
\text { Osteobl }\end{array}$ & & $\begin{array}{c}17 \text { eQTL } \\
\text { results }\end{array}$ & $\begin{array}{c}\text { BCL,Egr-1, } \\
\text { Nanog, PRDM1, } \\
\text { Pu.1,SAT, TATA, } \\
\text { YY1 }\end{array}$ & missense & 5 & $\begin{array}{l}\text { Lead and functional SNP, } \\
\text { potentally not the only } \\
\text { one }\end{array}$ \\
\hline 23 & rs3194051 & IL17R & 3575 & $\begin{array}{l}\text { no other missense SNP in LD, all SNPS } \\
\text { scored low, } 5 \text { or less. CAPSL in LD (no } \\
\text { function). }\end{array}$ & IL7R, CAPSL & no & & $\begin{array}{l}\text { GM12878, } \\
\text { Huvec }\end{array}$ & $\begin{array}{l}\text { Adult CD4, } \\
\text { Tho, NHDF- } \\
\text { Ad }\end{array}$ & & $\begin{array}{l}9 \text { eQTL } \\
\text { results }\end{array}$ & & missense & 5 & Lead and Functional SNP \\
\hline
\end{tabular}

Note: potentally damaging SNPs are shaded yellow

NFKBIE has intronic 2b SNP rs3799963 (not from any GWAS) in a perfect LD with 2 missense SNPs rs2233434 and rs2233433

Missense AID SNPs: each SNP is labeled with its rs identifier, and associated genes; whether the SNP is in high LD with other genes and SNPs,

weather it is nested in conserved regions, or if it has any chromatin marks and functional annotations, and its RegulomeDB score. Also provided

results of our evaluation based on all data if the misSSNP is a functional in addition to being a lead GWAS SNP. 
Table 4. Alternative coding genes at the missense AID GWAS SNP loci

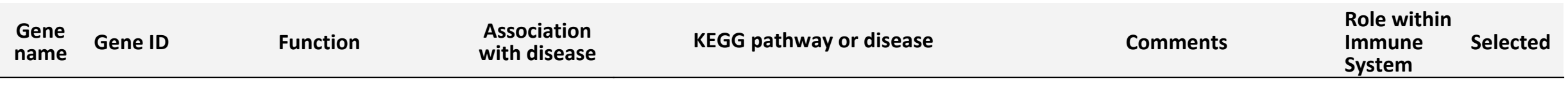

\begin{tabular}{|c|c|c|c|c|c|c|c|c|}
\hline \multicolumn{9}{|c|}{ NOTCH4, EGFL8, GPSM3 } \\
\hline EGFL8 & 80864 & unknown & $\begin{array}{l}\text { Macular } \\
\text { degeneration }\end{array}$ & none & & & unknown & \\
\hline GPSM3 & 63940 & unknown & Ps, SLE & none & & & unknown & \\
\hline $\mathrm{NOTCH} 4$ & 4855 & $\begin{array}{l}\text { functions as a receptor for } \\
\text { membrane bound ligands }\end{array}$ & $\begin{array}{l}\text { RA, Ps, T1D, MS, } \\
\text { macular } \\
\text { degeneration, } \\
\text { asthma, } \\
\text { sceroderma } \\
\text { systemic, etc. }\end{array}$ & $\begin{array}{l}\text { hsa04320 } \\
\text { hsa04330 } \\
\text { hsa04919 } \\
\text { hsa05206 }\end{array}$ & $\begin{array}{l}\text { Dorso-ventral axis formation } \\
\text { Notch signaling pathway } \\
\text { Thyroid hormone signaling pathway } \\
\text { MicroRNAs in cancer }\end{array}$ & $\begin{array}{l}\text { Notch family are Type } 1 \text { transmembrane } \\
\text { proteins. The Notch signaling network is } \\
\text { an evolutionarily conserved intercellular } \\
\text { signaling pathway which regulates } \\
\text { interactions between physically adjacent } \\
\text { cells. }\end{array}$ & no & $\mathbf{x}$ \\
\hline \multicolumn{9}{|c|}{ SH2B3, ATXN2 } \\
\hline SH2B3 & 10019 & $\begin{array}{l}\text { an intracellular adaptor protein } \\
\text { involved in a range of signaling } \\
\text { activities by growth factor and } \\
\text { cytokine receptors; regulates B } \\
\text { lymphopoiesis, megakaryopoiesis, } \\
\text { and expansion of hematopoietic } \\
\text { stem cells by constraining cytokine } \\
\text { signals }\end{array}$ & $\begin{array}{l}\text { T1D, RA, Coronary } \\
\text { Artery Disease, } \\
\text { Hypothyroidism, } \\
\text { etc. }\end{array}$ & hsa04722 & Neurotrophin signaling pathway & $\begin{array}{l}\text { Sh2b3 plays a regulatory role in the } \\
\text { expansion of DCs and might influence } \\
\text { inflammatory immune responses in } \\
\text { peripheral lymphoid tissues. JAK2 is an } \\
\text { established SH2B3 target }\end{array}$ & yes & $\mathbf{x}$ \\
\hline
\end{tabular}




\begin{tabular}{|c|c|c|c|c|c|c|c|c|}
\hline ATXN2 & 6311 & $\begin{array}{l}\text { unknown, might participates in the } \\
\text { regulation of RNA metabolism }\end{array}$ & $\begin{array}{l}\text { Celiac disease, } \\
\text { autoimmune } \\
\text { diseases, etc. }\end{array}$ & H00063 & Spinocerebellar ataxia (SCA) & & no & \\
\hline \multicolumn{9}{|c|}{ YDJC, UBE2L3 } \\
\hline YDJC & 150223 & unknown, but widely present & $\begin{array}{l}\mathrm{CD} \text {, Celiac disease, } \\
\text { autoimmune } \\
\text { diseases }\end{array}$ & none & & & no & \\
\hline UBE2L3 & 7332 & $\begin{array}{l}\text { participant in ubiquitination, } \\
\text { an important cellular } \\
\text { mechanism for targeting } \\
\text { abnormal or short-lived } \\
\text { proteins for degradation; } \\
\text { widely present. }\end{array}$ & $\mathrm{CD}, \mathrm{SLE}$ & hsa04120 & Ubiquitin mediated proteolysis & $\begin{array}{l}\text { This gene encodes a member of the E2 } \\
\text { ubiquitin-conjugating enzyme family; } \\
\text { demonstrated to participate in the } \\
\text { ubiquitination of p53, c-Fos, and the NF- } \\
\text { kB precursor p105 in vitro. }\end{array}$ & no & $\mathbf{x}$ \\
\hline \multicolumn{9}{|c|}{ GSDMB, ZPBP2 } \\
\hline GSDMB & 55876 & $\begin{array}{l}\text { implicated in the regulation of } \\
\text { apoptosis in epithelial cells, } \\
\text { and is linked to cancer }\end{array}$ & $\begin{array}{l}\text { f UC, T1D, RA, CD, } \\
\text { asthma, }\end{array}$ & none & & $\begin{array}{l}\text { a member of the gasdermin-domain } \\
\text { containing protein family }\end{array}$ & no & $\mathbf{x}$ \\
\hline ZPBP2 & 124626 & zona pellucida binding protein 2 & UC, CD, RA, T1D & none & & no annotaion & no & \\
\hline \multicolumn{9}{|c|}{ IL17REL, PIM3 } \\
\hline IL17REL & 132014 & $\begin{array}{l}\text { member of IL-17 receptors; signal } \\
\text { transduction pathway mediated by } \\
\text { IL-17 is still poorly defined; IL-17 } \\
\text { receptors activate noncanonical } \\
\text { signal transduction pathways }\end{array}$ & $\begin{array}{l}\text { UC, also Ps, RA, } \\
\text { uveitis, etc. }\end{array}$ & ko04060 & $\begin{array}{l}\text { Cytokine-cytokine receptor } \\
\text { interaction }\end{array}$ & $\begin{array}{l}\text { Activity of this protein is important in the } \\
\text { immune response to bacterial and fungal } \\
\text { pathogens. Encoded protein signals to } \\
\text { downstream components of the MAPK } \\
\text { pathway, signals through Act1; activates } \\
\text { the classical NF-KB pathway. There are at } \\
\text { least } 5 \text { IL-17R proteins }\end{array}$ & yes & $\mathbf{x}$ \\
\hline
\end{tabular}




\begin{tabular}{|c|c|c|c|c|c|c|c|c|}
\hline PIM3 & 415116 & $\begin{array}{l}\text { Pim-3 proto-oncogene, } \\
\text { serine/threonine kinase }\end{array}$ & none & none & & no annotaion & unknown & \\
\hline \multicolumn{9}{|c|}{ IL7R, CAPSL } \\
\hline CAPSL & 133690 & calcyphosine-like & T1D, Liver Cirrhosis & none & & unknown & no & \\
\hline IL7R & 3575 & $\begin{array}{l}\text { protein encoded by this gene is a } \\
\text { receptor for interleukine } 7 \text {; shown } \\
\text { to play a critical role in the } V(D) J \\
\text { recombination during lymphocyte } \\
\text { development }\end{array}$ & $\begin{array}{l}\text { UC, T1D, MS, } \\
\text { neoplasms }\end{array}$ & $\begin{array}{l}\text { hsa04060 } \\
\text { hsa04068 } \\
\text { hsa04151 } \\
\text { hsa04630 } \\
\text { hsa04640 } \\
\text { hsa05340 }\end{array}$ & $\begin{array}{l}\text { Cytokine-cytokineR interaction } \\
\text { FoxO signaling pathway } \\
\text { PI3K-Akt signaling pathway } \\
\text { Jak-STAT signaling pathway } \\
\text { Hematopoietic cell lineage } \\
\text { Primary immunodeficiency }\end{array}$ & $\begin{array}{l}\text { control the accessibility of the TCR } \\
\text { gamma locus by STAT5 and histone } \\
\text { acetylation; also blocking apoptosis is an } \\
\text { essential function of this protein during } \\
\text { differentiation and activation of T } \\
\text { lymphocytes; may be associated with the } \\
\text { pathogenesis of the severe combined } \\
\text { immunodeficiency (SCID). }\end{array}$ & yes & $\mathbf{x}$ \\
\hline \multicolumn{9}{|c|}{ MST1, APEH, BSN } \\
\hline MST1 & 4485 & $\begin{array}{l}\text { encoded protein contains four } \\
\text { kringle domains and a serine } \\
\text { protease domain, similar to that } \\
\text { found in hepatic growth factor, } \\
\text { but no proteolytic activity }\end{array}$ & $U C, C D$ & none & & $\begin{array}{l}\text { similar to macrophage-stimulating } 1 \\
\text { receptor }\end{array}$ & no & $\mathbf{x}$ \\
\hline APEH & 327 & $\begin{array}{l}\text { encodes the enzyme acylpeptide } \\
\text { hydrolase, which catalyzes the } \\
\text { hydrolysis of the terminal } \\
\text { acetylated amino acid } \\
\text { preferentially from small } \\
\text { acetylated peptides. }\end{array}$ & UC & none & & acylaminoacyl-peptide hydrolase & no & \\
\hline
\end{tabular}




\begin{tabular}{|c|c|c|c|c|c|c|c|c|}
\hline BSN & 8927 & $\begin{array}{l}\text { gene is expressed primarily in } \\
\text { neurons in the brain; encodes a } \\
\text { scaffolding protein involved in } \\
\text { organizing the presynaptic } \\
\text { cytoskeleton }\end{array}$ & $U C, C D$ & none & & $\begin{array}{l}\text { bassoon presynaptic cytomatrix } \\
\text { protein }\end{array}$ & no & \\
\hline \multicolumn{9}{|c|}{ NFKBIE, TCTE1, AARS2 } \\
\hline NFKBIE & 4794 & $\begin{array}{l}\text { The protein encoded by this } \\
\text { gene binds to components of } \\
\text { NF-kappa-B, trapping the } \\
\text { complex in the cytoplasm and } \\
\text { preventing it from activating } \\
\text { genes in the nucleus. }\end{array}$ & RA & yes & many KEGG pathways & $\begin{array}{l}\text { Phosphorylation of the encoded } \\
\text { protein targets it for destruction by } \\
\text { the ubiquitin pathway, which } \\
\text { activates NF-kappa-B by making it } \\
\text { available to translocate to the } \\
\text { nucleus }\end{array}$ & & $\mathbf{x}$ \\
\hline TCTE1 & 202500 & t-complex-associated-testis-expr & none & none & & no annotaion & & \\
\hline AARS2 & 57505 & $\begin{array}{l}\text { the encoded protein is a } \\
\text { mitochondrial enzyme that } \\
\text { specifically aminoacylates alanyl- } \\
\text { tRNA }\end{array}$ & $\mathrm{MI}$ & none & & $\begin{array}{l}\text { Mutations in this gene are a cause of } \\
\text { combined oxidative phosphorylation } \\
\text { deficiency } 8 \text {. }\end{array}$ & & \\
\hline \multicolumn{9}{|c|}{ CARD9, SNAPC4 } \\
\hline CARD9 & 64170 & $\begin{array}{l}\text { this gene is a member of the } \\
\text { CARD protein family, which is } \\
\text { defined by the presence of a } \\
\text { characteristic } \\
\text { caspase-associated recruitment } \\
\text { domain (CARD) }\end{array}$ & UC, CD, AS & $\begin{array}{l}\text { hsa04621 } \\
\text { hsa05152 }\end{array}$ & $\begin{array}{l}\text { NOD-like receptor signaling } \\
\text { pathway } \\
\text { Tuberculosis }\end{array}$ & $\begin{array}{l}\text { This protein was identified by its } \\
\text { selective association with the CARD } \\
\text { domain of BCL10, a postive regulator } \\
\text { of apoptosis and NF-kappaB } \\
\text { activation, }\end{array}$ & & $\mathbf{x}$ \\
\hline SNAPC4 & 6621 & $\begin{array}{l}\text { snRNA-activating protein } \\
\text { complex subunit } 4\end{array}$ & none & none & & $\begin{array}{l}\text { Transcription factors: potental role } \\
\text { in relation to GWAS SNP genes } \\
\text { does not exist }\end{array}$ & & \\
\hline
\end{tabular}

Coding genes associated with missense AID SNPs: name and ID of coding genes, function if known, association with diseases, participation in canonical KEGG pathways and role in the immune system. 


\section{Table 5. Characteristics of highest Regulome DB scored GWAS AID ncSNPs}

\section{No AID db SNP ID Score Context Gene name $\underset{\text { value }}{\text { GWAS }} \mathrm{p}$ Source PubMed}

\section{Genes in high LD $(>0.8)$}

Source PubMed

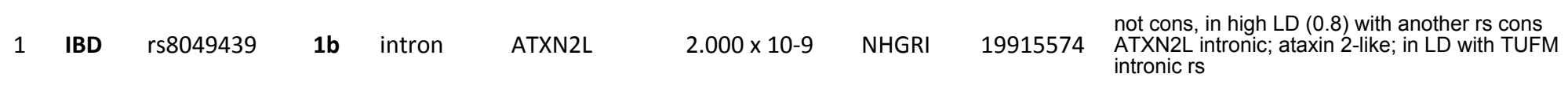

TUFM in high LD (translation elongation factor)

ATXN2L

microRNA 4721 in high LD

\begin{tabular}{|c|c|c|c|c|c|c|c|c|c|c|}
\hline 2 & UC & rs798502 & 1b & intron & GNA12 & $3.000 \times 10-15$ & NHGRI & 21297633 & $\begin{array}{l}\text { intronic non cons SNP is in LD }(0.8) \text { with cons rss } \\
\text { in GNA12 }\end{array}$ & GNA12 \\
\hline 3 & & rs11676348 & 1b & intergenic & $\begin{array}{l}\text { CXCR2, } \\
\text { CXCR1 }\end{array}$ & $1.000 \times 10-10$ & NHGRI & 21297633 & $\begin{array}{l}\text { in LD with CXCR2 gene; in high LD with CXCR2 } \\
\text { syn cons SNP; in LD with intragenic cons CXCR1 } \\
\text { rs that is site for STAT3 binding }\end{array}$ & $\begin{array}{l}\text { CXCR1, CXCR2 (chemokine } \\
\text { (C-X-C motif) receptor } 1 \text { and 2) }\end{array}$ \\
\hline 4 & & rs9263739 & $1 f$ & intron & CCHCR1 & $4.000 \times 10-67$ & NHGRI & 19915573 & $\begin{array}{l}\text { not cons; in total LD (1) with } 2 \text { missense SNPs of } \\
\text { PSORS1C1 gene; in high LD for PSORC1C2 and } \\
\text { C3 (psoriasis susceptibility } 1 \text { candidate } 2 \text { and } 3 \text { ) }\end{array}$ & $\begin{array}{l}\text { PSORS1C1 (Gene ID: 170679), } 2 \\
\text { (Gene ID: 170680) and 3 } \\
\text { (PSORS1C3 GenelD: } \\
\text { 100130889 is ncRNA gene) }\end{array}$ \\
\hline 5 & & rs4728142 & $1 f$ & intergenic & KCP, IRF5 & $2.000 \times 10-8$ & NHGRI & 21297633 & in LD with IRF5 intronicSNPs & IRF5 \\
\hline 6 & & rs9858542 & $1 f$ & intron & BSN & $7.000 \times 10-9$ & NHGRI & 19915572 & $\begin{array}{l}\text { in perfect LD with miss, syn, and 3'UTR BSN and } \\
\text { miss MST1 }\end{array}$ & MST1 \\
\hline 7 & & rs10781499 & $1 f$ & intron & CARD9 & $3.000 \times 10-19$ & NHGRI & 21297633 & $\begin{array}{l}\text { in high LD }(0.98) \text { with missense SNP in CARD9 } \\
\text { already accounted for; in high LD with cons miss } \\
\text { SNP within SNAPC4 }\end{array}$ & CARD9 \\
\hline 8 & & rs734999 & $1 f$ & intergenic & $\begin{array}{l}\text { TNFRSF14, } \\
\text { C1orf93 }\end{array}$ & $3.000 \times 10-9$ & NHGRI & 21297633 & $\begin{array}{l}\text { in LD with miss SNP in TNFRSF14 a member of } \\
\text { the TNFreceptor superfamily }\end{array}$ & TNFRSF14 \\
\hline 9 & & rs8067378 & $1 f$ & intergenic & $\begin{array}{l}\text { ZPBP2, } \\
\text { GSDMB }\end{array}$ & $1.000 \times 10-7$ & NHGRI & 20228799 & $\begin{array}{l}\text { in high or perfect LD with a cons miss SNPs of } \\
\text { ZPBP } 2 \text { and } 2 \text { miss SNPs of GSDMB; both genes } \\
\text { have no known function }\end{array}$ & ZPBP2 and GSDMB \\
\hline 10 & & rs907611 & $2 a$ & nearGene-5 & LSP1 & $1.000 \times 10-10$ & NHGRI & 21297633 & in perfect LD with 5'-UTR of LSP1 & LPS1 \\
\hline 11 & & rs1297265 & $2 a$ & intergenic & $\begin{array}{l}\text { NRIP1, } \\
\text { CYCSP42 }\end{array}$ & $7.000 \times 10-13$ & NHGRI & 21297633 & $\begin{array}{l}\text { not cons, no genes in LD; only several cons } \\
\text { regions in LD. }\end{array}$ & none \\
\hline
\end{tabular}




\begin{tabular}{|c|c|c|c|c|c|c|c|c|c|c|}
\hline 12 & & rs3024505 & $2 \mathbf{b}$ & intergenic & $\begin{array}{l}\text { RPS14P1, } \\
\text { IL10 }\end{array}$ & $6.000 \times 10-17$ & NHGRI & 21297633 & $\begin{array}{l}\text { 3' of IL10; this rs also linked with T1D, IBD, CD } \\
\text { and UC by PhenGenl }\end{array}$ & IL10 \\
\hline 13 & & rs3806308 & $2 \mathbf{b}$ & near gene- 5 & RNF186 & $7.000 \times 10-9$ & NHGRI & 19122664 & $\begin{array}{l}\text { not cons, one of several UC linked rs totally } \\
\text { intergenic, no genes in LD }\end{array}$ & none \\
\hline 14 & & rs3024493 & $2 b$ & intron & IL10 & $1.000 \times 10-12$ & NHGRI & 20228798 & not cons; on other genes in LD & IL10 \\
\hline 15 & & rs6451493 & $3 \mathbf{a}$ & intergenic & $\begin{array}{l}\text { DAB2, } \\
\text { PTGER4 }\end{array}$ & $3.000 \times 10-9$ & NHGRI & 21297633 & no coding genes in LD; a novel miRNA in high LD & none \\
\hline 16 & RA & rs660895 & 1f & intergenic & $\begin{array}{l}\text { HLA-DRB1, } \\
\text { HLA-DQA1 }\end{array}$ & $1.000 \times 10-108$ & NHGRI & 17804836 & $\begin{array}{l}\text { no cons (HLA complex); no other genes; no } \\
\text { ncRNA }\end{array}$ & HLA \\
\hline 17 & & rs3093023 & 1f & intron & CCR6 & $2.000 \times 10-11$ & NHGRI & 20453842 & $\begin{array}{l}\text { not cons; in high LD with many SNPs intronic to } \\
\text { chemokine (C-C motif) receptor } 6 \text { with OMIM for } \\
\text { RA; participate in C-C pathways; no ncRNa }\end{array}$ & CCR6 \\
\hline 18 & & rs805297 & 1f & nearGene-5 & APOM & $3.000 \times 10-10$ & NHGRI & 21844665 & $\begin{array}{l}\text { no cons, LD high with } 3 \text { SNPs in cons regions of } 3 \\
\text { genes BAG6 intronic rs, PRRC2A syn rs, } \\
\text { CSNK2B 3' UTR cons rs; and two intronic rs in } \\
\text { two genes LY6G5B, LY6G6F. Note: BAG4 or } \\
\text { SODD athanogene is tumor necrosis factor } \\
\text { receptor superfamily member 1A (TNFRSF1A ID } \\
7132 \text { ) inhibitor/mpdulator) }\end{array}$ & $\begin{array}{l}\text { BAG6 is BCL2-associated } \\
\text { athanogene } \\
\text { PRRC2A } \\
\text { LY6 cluster } \\
\text { APOM } \\
\text { CSNK2B }\end{array}$ \\
\hline 19 & & rs3781913 & 1f & intron & PDE2A & $6.000 \times 10-10$ & NHGRI & 22446963 & $\begin{array}{l}\text { not cons, intronic in LD with cons SNP in the } \\
\text { same gene (phosphodiesterase 2A, } \\
\text { cGMP-stimulated) and two nc RNA }\end{array}$ & $\begin{array}{c}\text { microRNA 139; Gene ID: } 406931 \\
\text { PDE2A } \\
\text { ncRNA uncharacterized } \\
\text { LOC102724448 }\end{array}$ \\
\hline 20 & & rs4810485 & 1f & intron & CD40 & $3.000 \times 10-9$ & NHGRI & 20453842 & $\begin{array}{l}\text { not cons; in perfect LD with cons } 5 \text { '-UTR rs and a } \\
\text { cons near Gene rs in CD40 (TNF receptor } \\
\text { superfamily member } 5 \text { ) and intronic rs of NCOA5, } \\
\text { nuclear receptor coactivator } 5\end{array}$ & $\begin{array}{l}\text { NCOA5 } \\
\text { CD40 }\end{array}$ \\
\hline 21 & & rs881375 & $2 b$ & intergenic & $\begin{array}{l}\text { PHF19, } \\
\text { TRAF1 }\end{array}$ & $4.000 \times 10-8$ & NHGRI & 19503088 & $\begin{array}{l}\text { highly cons, 3' nearGene of TRAF1; in perfect } \\
\text { LD with many intronic, syn and 3'-UTR of TRAF1 } \\
\text { (some are cons) }\end{array}$ & TRAF1 \\
\hline 22 & & rs874040 & $2 \mathbf{b}$ & intergenic & $\begin{array}{l}\text { C4orf52, } \\
\text { RBPJ }\end{array}$ & $1.000 \times 10-16$ & NHGRI & 20453842 & $\begin{array}{l}\text { recombination signal binding protein for } \\
\text { immunoglobulin kappa J region; Gene ID: } 3516 \text {. } \\
\text { The protein encoded by this gene is a } \\
\text { transcriptional regulator important in the Notch } \\
\text { signaling pathway. }\end{array}$ & RBPJ \\
\hline
\end{tabular}


thyrotropin-releasing hormone degrading

enzyme; an extracellular peptida other genes or SNPs in LD

THRADE

not cons; 3' of TNOP3 (transportin 3, tRNA import factor is a nuclear import receptor for

rs3783637 2b intron GCH1

20453842

GTP cyclohydrolase I; noother SNPs in LD

$\mathrm{GCH} 1$

$1.000 \times 10-8 \quad \mathrm{NHGRI}$

2045384

non cons, in LD with many rs in 3' nearGene of

rs12831974

2b intron THRADE

$6.000 \times 10-7$

NHGRI

21452313

thyrotropin-releasing hormone degrading enzyme;

THRADE

rs16906916

3a intergenic

SV2B,

$5.000 \times 10-7$

NHGRI an extracel

22491018 Novel lincRNA; SLCO3A1 in very low LD

none

\begin{tabular}{|c|c|c|c|c|c|c|c|c|c|}
\hline 32 & CD & rs181359 & $1 f$ & intron & UBE2L3 & $5.000 \times 10-16$ & NHGRI & 21102463 & $\begin{array}{l}\text { not cons; in perfect LD with intronic SNPs of } \\
\text { UBE2L3 only }\end{array}$ \\
\hline 3 & & rs2549794 & $1 f$ & intron & ERAP2 & $1.000 \times 10-10$ & NHGRI & 21102463 & not cons; in perfect LD with intronic ERAP2 \\
\hline 34 & & rs6596075 & $1 f$ & intergenic & $\begin{array}{l}\text { SLC22A5, } \\
\text { C5orf56 }\end{array}$ & $3.000 \times 10-7$ & NHGRI & 17554300 & $\begin{array}{l}\text { not cons; in LD with cons } 33^{\prime}-\text { UTR of SLC22A5 and } \\
\text { few intronic SNP of the same gene }\end{array}$ \\
\hline 3 & & rs694739 & $1 f$ & intergenic & $\begin{array}{l}\text { PRDX5, } \\
\text { CCDC88B }\end{array}$ & $6.000 \times 10-10$ & NHGRI & 21102463 & $\begin{array}{l}\text { not cons; in strong LD with miss, 5'-UTR and syn } \\
\text { of gene CCDC88B; }\end{array}$ \\
\hline 6 & & rs713875 & $1 f$ & intergenic & $\begin{array}{l}\text { RPS3AP51, LIF } \\
22\end{array}$ & $7.000 \times 10-12$ & NHGRI & 21102463 & $\begin{array}{l}\text { not cons; in LD with HORMAD2 intergenic SNPs; } \\
\text { novel antisense RP3-438O4.4; not in LD with } \\
\text { ribosomal protein S3a pseudogene } 51\end{array}$ \\
\hline 7 & & rs9258260 & $1 f$ & intergenic & $\begin{array}{l}\text { IFITM4P, } \\
3.8-1.5\end{array}$ & $2.000 \times 10-10$ & NHGRI & 22412388 & $\begin{array}{l}\text { not cons;intergenic, no functional genes in LD } \\
\text { except interferon induced transmembrane protein } \\
4 \text { pseudogene; non-coding RNAs (ncRNAs); HLA- } \\
\text { F antisense RNA } 1\end{array}$ \\
\hline 38 & & rs9858542 & $1 f$ & intron & BSN & $7.000 \times 10-9$ & NHGRI & 19915572 & $\begin{array}{l}\text { in perfect LD with miss, syn, and } 3 \text { 'UTR BSN and } \\
\text { miss MST1 }\end{array}$ \\
\hline
\end{tabular}

UBE2L3

ERAP2

SLC22A5

CCDC88B

HORMAD2

none

MST1 
$2.000 \times 10-18 \quad$ NHGRI

18587394

not conservative; in high LD with miss SNP in SLC22A4 (6583); in LD with cons SNP in 5'UTR of IRF1 gene.

41

rs426383

rs4409764

rs3024505

$2 b$

2b

intergenic

TNFSF15

RPS14P1,

IL10

GOT1,

NKX2-3
$3.000 \times 10-10 \quad \mathrm{NHGRI}$

$2.000 \times 10-14 \quad$ NHGRI

$2.000 \times 10-20 \quad N H G R$ not cons, no LD for cons SNPs

highly conservative rs in the region of $3^{\prime}$ ncRNA in perfect LD with syn SNP of NKX2-3, NK2

SLC22A4: solute carrier family 22 organic cation transporter

RF1: interferon regulatory factor 1 a transcription factor

two ncRNAs: uncharacterized LOC102723741; uncharacterized LOC101927732

TNFSF15

IL10

NKX2-3

homeobox 3 transcription factor and a few intronic long intergenic non-protein coding RNA 1475

\section{Ps \&}

\section{PsA}

rs9267673 1f intron

ZBTB12,
C2 $2.300 \times 10-33$
$\mathrm{dbGaP}$
C2 (complement component, an serum glycoprotein of the classical pathway).

SKIV2L: superkiller viralicidic activity 2 -like

PSORS1C1 psoriasis susceptibility 1 candidate 1 is the closeset functional gene but in a very weak LD $(r 2<0.2)$

RNA, U6 small nuclear 1133 pseudogene

HCG22 HLA complex group 22 (ID: 285834)

$\mathrm{NOTCH} 4$

syn non cons SNP of G-protein signaling NOTCH4

GPSM3 
$1 f$ intergenic

WASF5P,

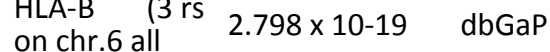

scored 1f)

rs2395471

$\begin{array}{cccc}\text { rs3094187 } & \text { 1f } & \text { UTR-5 } & \text { TCF19 } \\ \text { rs8365 } & \text { 1f } & \text { UTR-3 } & \text { RNF5 } \\ \text { rs176095 } & \text { 1f } & \text { nearGene-5 } & \text { PBX2 }\end{array}$

rs1265086

\section{1f}

nearGene-3 CCHCR1

54

rs3131043

\section{1f}

rs3130573

$1 f$ intron

PSORS1C

MUC21,
HCG22

$2.993 \times 10-11$

$8.836 \times 10-18$

$\mathrm{dbGaP}$

$3.725 \times 10-13$

$1.094 \times 10-17 \quad \mathrm{dbGaP}$

$2.183 \times 10-12 \quad \mathrm{dbGaP}$

$2.689 \times 10-11 \quad d b G a P$

$3.454 \times 10-12$

$\mathrm{dbGaP}$

$1.814 \times 10-1$

$\mathrm{dbGaP}$

$6.212 \times 10-15 \quad \mathrm{dbGaP}$

dbGaP

NHGRI 1f intron

$\mathrm{dbGaP}$

20953189

phs000019

rs not cons in 5
in LD up to 0.2

phs000019 TCF19, transcription factor 19

phs000019 ring finger protein 5, E3 ubiquitin protein ligase phs000019 G-protein signaling modulator 3; high LD with pre-

not cons; in total LD (1) with 2 missense SNPs of PSORS1C1 gene; in high LD for PSORC1C2 and $\mathrm{C} 3$ (psoriasis susceptibility 1 candidate 2 and 3 )

discoidin domain receptor tyrosine kinase 1 ;

involved in the regulation of cell growth,

differentiation and metabolism. in high LD with

miss SNP of VARS2, valyl-tRNA synthetase 2 ,

mitochondrial

HLA complex group 20 (non-protein coding); has

two SNPs scored $2 \mathrm{~b}$ within gene LINC00243, lon

phs000019 intergenic non-protein coding RNA 243; same

location has several SNPs all conecte with HCG20

phs000019 no other SNPs in LD

phs000019 in LD with MUC22, no SNPs, no function; HLA
complex close but not in perfect LD

no genes in LD
WASF5P

HLA-B

HLA-C

TCF19

RNF5

PBX2

PSORS1C1 (Gene ID: 170679), 2 (Gene ID: 170680) and 3 (PSORS1C3 GenelD: 100130889 is ncRNA gene)

DDR1, VARS2

HCG20 and LINC00243

PSORS1C1

MUC22 HCG22, HLA complex

rs13210693

2a intergenic

FLJ37396

$9.000 \times 10-7$

NHGRI

22138694

no genes in LD; several cons missSNPs in

none 
Table 6. Non-coding RNA genes in LD with top intergenic GWAS AID SNPs

\begin{tabular}{|c|c|c|c|c|c|c|c|c|c|c|c|c|}
\hline No & rs \# & Context & $\begin{array}{l}\text { Gene closeset } \\
\text { to SNP } \\
\text { location }\end{array}$ & Cons & Genes in LD (by HaploReg v2) & $\begin{array}{l}\text { No of } \\
\text { ncRNA } \\
\text { genes }\end{array}$ & ncRNA Gene ID & Name of ncRNA genes & $\begin{array}{l}\text { miRNA } \\
\text { gene } \\
\text { present } \\
\text { in a SNP } \\
\text { region }\end{array}$ & \begin{tabular}{|l} 
any \\
other \\
type of \\
RNA \\
gene is \\
present
\end{tabular} & $\begin{array}{l}\text { no } \\
\text { ncRNA } \\
\text { gene } \\
\text { present }\end{array}$ & $\begin{array}{l}\text { Regulome } \\
\text { DB score for } \\
\text { the rs * }\end{array}$ \\
\hline 1 & rs6457620 & intergenic & $\begin{array}{l}\text { HLA-DQB1, } \\
\text { HLA-DQA2 }\end{array}$ & no & $\begin{array}{c}23 \mathrm{~kb}-25 \mathrm{~kb} 5^{\prime} \text { of HLA-DQB1; } 21 \mathrm{~kb} 5^{\prime} \\
\text { of XXbac-BPG254F23.7 }\end{array}$ & 1 & 100616218 & microRNA 3135b & yes & & & $1 f$ \\
\hline 2 & rs9268853 & intergenic & $\begin{array}{l}\text { HLA-DRB9, } \\
\text { HLA-DRB5 }\end{array}$ & no & $16 \mathrm{~kb}-28 \mathrm{~kb} 3$ ' of HLA-DRA & 0 & & & & & none & \\
\hline 3 & rs660895 & intergenic & $\begin{array}{l}\text { HLA-DRB1, } \\
\text { HLA-DQA1 }\end{array}$ & no & 19kb-14kb 5' of HLA-DQA1 & 1 & 100616218 & microRNA 3135b & yes & & & $1 f$ \\
\hline 4 & rs6457617 & intergenic & $\begin{array}{l}\text { HLA-DQB1, } \\
\text { HLA-DQA2 }\end{array}$ & no & $\begin{array}{c}23 \mathrm{~kb}-25 \mathrm{~kb} 5 \text { ' of HLA-DQB1; } 25 \mathrm{~kb}- \\
21 \mathrm{~kb} 5 \text { ' of XXbac-BPG254F23.7 }\end{array}$ & 1 & 100616218 & microRNA 3135b & yes & & & \\
\hline 5 & rs13192471 & intergenic & $\begin{array}{l}\text { HLA-DQB1, } \\
\text { HLA-DQA2 }\end{array}$ & no & $\begin{array}{c}21 \mathrm{~kb}-12 \mathrm{~kb} 5 \text { ' of XXbac- } \\
\text { BPG254F23.7 }\end{array}$ & 1 & 100616218 & microRNA 3135b & yes & & & \\
\hline 6 & rs9272219 & intergenic & $\begin{array}{l}\text { HLA-DRB1, } \\
\text { HLA-DQA1 }\end{array}$ & no & HLA-DQA1 & 1 & 100616218 & microRNA 3135b & yes & & & $1 f$ \\
\hline 7 & rs6679677 & intergenic & RSBN1, PHTF1 & no & $\begin{array}{c}\text { PTPN22 in high LD with miss SNP, } \\
\text { 3' of RP11-129J12.2 }\end{array}$ & 1 & 101927324 & $\begin{array}{l}\text { LINC01475, long intergenic } \\
\text { non-protein coding RNA } \\
1475\end{array}$ & & yes & & \\
\hline 8 & rs9296015 & intergenic & $\begin{array}{l}\text { NOTCH4, } \\
\text { C6orf10 }\end{array}$ & no & $\begin{array}{c}2.1 \mathrm{~kb}-4.8 \mathrm{~kb} \text { 3' of XXbac- } \\
\text { BPG154L12.4; 9.1kb-1.9kb 5' of } \\
\text { XXbac-BPG154L12.4; }\end{array}$ & 1 & 102466190 & microRNA 6721 & yes & & & \\
\hline 9 & rs615672 & intergenic & $\begin{array}{l}\text { HLA-DRB1, } \\
\text { HLA-DQA1 }\end{array}$ & no & $16 \mathrm{~kb}-19 \mathrm{~kb} 5$ ' of HLA-DRB1 & 0 & & & & & none & \\
\hline 10 & rs11742570 & intergenic & DAB2, PTGER4 & medium & $2.8 \mathrm{~kb}-120 \mathrm{~kb} 3^{\prime}$ of $\mathrm{AC} 108105.1$ & 2 & 619564 & $\begin{array}{l}\text { SNORD72 small nucleolar } \\
\text { RNA, C/D box72 }\end{array}$ & & yes & & \\
\hline
\end{tabular}




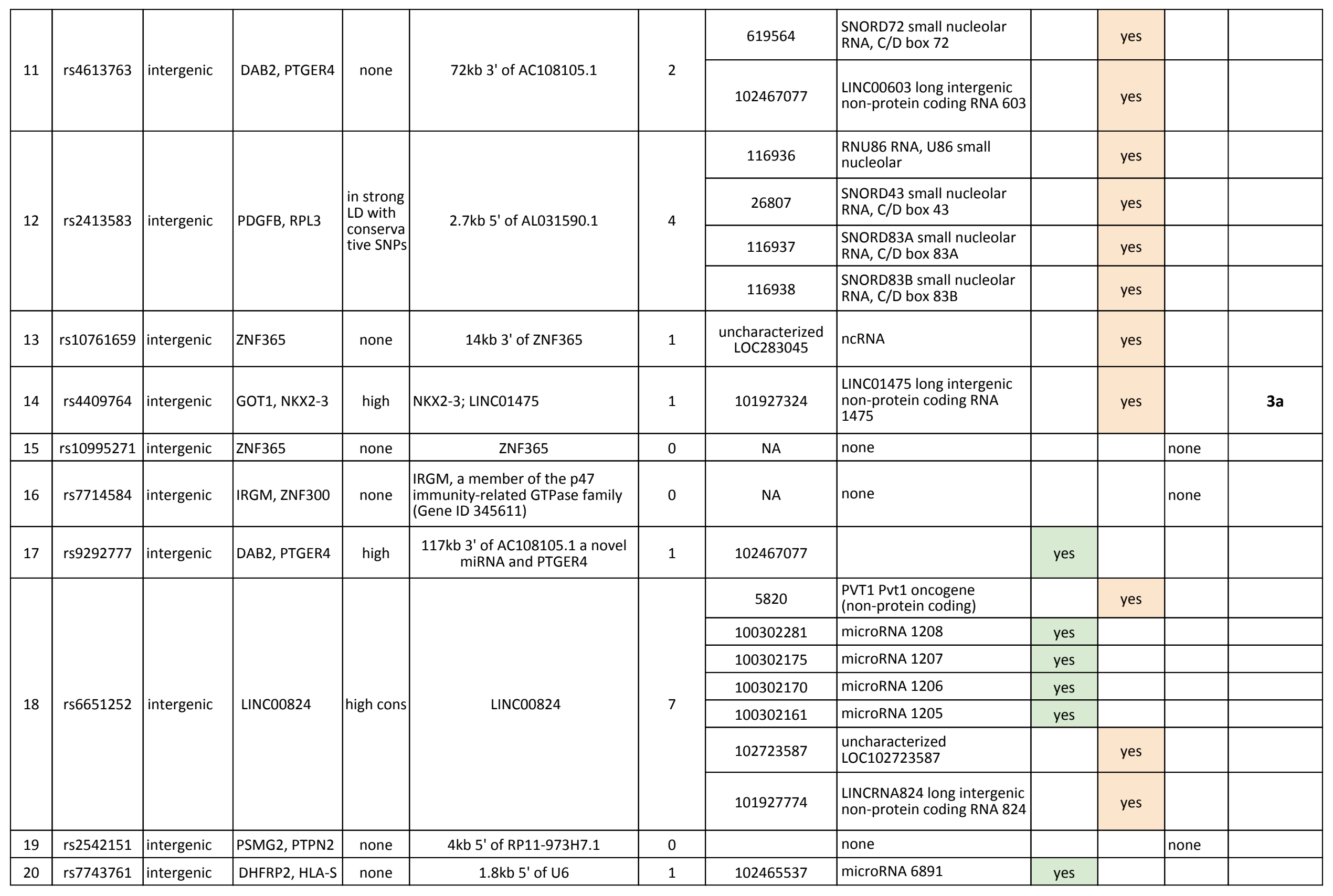




\begin{tabular}{|c|c|c|c|c|c|c|c|c|c|c|c|c|}
\hline 21 & rs4349859 & intergenic & HLA-S, MICA & none & $1.8 \mathrm{~kb} 5^{\prime}$ of MICA & 1 & 101929111 & $\begin{array}{l}\text { long intergenic non-protein } \\
\text { coding RNA } 1149\end{array}$ & & yes & & \\
\hline 22 & rs2242944 & intergenic & $\begin{array}{l}\text { FL445139, RPL2 } \\
\text { 3AP12 }\end{array}$ & none & $64 \mathrm{~kb} 5$ ' of AF064858.10 & 1 & 101928435 & $\begin{array}{l}\text { LOC101928435, ncRNA } \\
\text { uncharactarized }\end{array}$ & & yes & & $2 b$ \\
\hline 23 & rs10865331 & intergenic & \begin{tabular}{|l} 
B3GNT2, TMEM \\
17
\end{tabular} & none & $59 \mathrm{~kb} 3$ ' of snoU13 and B3GNT2 & 1 & 100847087 & microRNA 5192 & yes & yes & & \\
\hline 24 & rs11616188 & intergenic & LTBR, RPL31P10 & none & $\begin{array}{l}\text { LTBR and } 328 \mathrm{bp} 5 \text { ' of RP1- } \\
\text { 102E24.8 a novel linc RNA }\end{array}$ & 1 & $\begin{array}{l}\text { RP1-102E24.8 (Vega } \\
\text { gene) }\end{array}$ & linc RNA & & yes & & \\
\hline 26 & rs11249215 & intergenic & RUNX3, SYF2 & none & $\begin{array}{c}\text { within RP11-84D1.2 (Vega gene), a } \\
\text { novel linc RNA }\end{array}$ & 1 & 100616365 & microRNA 4425 & yes & & & \\
\hline 27 & rs4552569 & intergenic & $\begin{array}{l}\text { RPL13AP14, } \\
\text { EDIL3 }\end{array}$ & none & $63 \mathrm{~kb} 3$ ' of EDIL3 & 0 & & & & & none & \\
\hline 28 & rs6556416 & intergenic & IL12B, ADRA1B & none & $\begin{array}{c}\text { 29kb 3' of AC008697.1, a novel } \\
\text { lincRNA }\end{array}$ & 1 & 100873889 & $\begin{array}{l}\text { RNA, U4atac small nuclear 2, } \\
\text { pseudogene }\end{array}$ & & yes & & \\
\hline 30 & rs10484554 & intergenic & WASF5P, HLA-B & no & $\begin{array}{l}\text { HLA-C, a novel lincRNA, WASF5P, } \\
\text { HLA-B and USP8P1 }\end{array}$ & 1 & 10246553 & microRNA 6891 & yes & yes & & \\
\hline 31 & rs9468933 & intergenic & WASF5P, HLA-B & no & $\begin{array}{l}\text { HLA-C, a novel lincRNA, WASF5P, } \\
\text { HLA-B and USP8P1 }\end{array}$ & 1 & 10246553 & microRNA 6891 & yes & yes & & \\
\hline 32 & rs2894207 & intergenic & WASF5P, HLA-B & no & $\begin{array}{l}\text { HLA-C, a novel lincRNA, WASF5P, } \\
\text { HLA-B and USP8P1 }\end{array}$ & 1 & 10246553 & microRNA 6891 & yes & yes & & \\
\hline 33 & rs9380237 & intergenic & WASF5P, HLA-B & no & $\begin{array}{l}\text { HLA-C, a novel lincRNA, WASF5P, } \\
\text { HLA-B and USP8P1 }\end{array}$ & 1 & 10246553 & microRNA 6891 & yes & yes & & \\
\hline 34 & rs2524163 & intergenic & WASF5P, HLA-B & no & $\begin{array}{l}\text { HLA-C, a novel lincRNA, WASF5P, } \\
\text { HLA-B and USP8P1 }\end{array}$ & 1 & 10246553 & microRNA 6891 & yes & yes & & \\
\hline 35 & rs2243868 & intergenic & WASF5P, HLA-B & no & $\begin{array}{l}\text { HLA-C, a novel lincRNA, WASF5P, } \\
\text { HLA-B and USP8P1 }\end{array}$ & 1 & 10246553 & microRNA 6891 & yes & yes & & $1 f$ \\
\hline 36 & rs2853923 & intergenic & WASF5P, HLA-B & no & $\begin{array}{l}\text { HLA-C, a novel lincRNA, WASF5P, } \\
\text { HLA-B and USP8P1 }\end{array}$ & 1 & 10246553 & microRNA 6891 & yes & yes & & \\
\hline 37 & rs9380240 & intergenic & WASF5P, HLA-B & no & $\begin{array}{l}\text { HLA-C, a novel lincRNA, WASF5P, } \\
\text { HLA-B and USP8P1 }\end{array}$ & 1 & 10246553 & microRNA 6891 & yes & yes & & \\
\hline 39 & rs6426833 & intergenic & RNF186, OTUD3 & no & $\begin{array}{l}\text { RNF186 and 26kb 3' of RP11- } \\
\text { 91K11.2 Novel antisense; TMCO4 }\end{array}$ & 0 & & & & & none & 2c \\
\hline 40 & rs10758669 & intergenic & RCL1, JAK2 & no & $3.4 \mathrm{~kb} 5^{\prime}$ of JAK2 & 1 & 406894 & microRNA 101-2 & yes & & & \\
\hline
\end{tabular}




\begin{tabular}{|c|c|c|c|c|c|c|c|c|c|c|c|c|}
\hline 41 & rs9268877 & intergenic & $\begin{array}{l}\text { HLA-DRB9, HLA- } \\
\text { DRB5 }\end{array}$ & no & $18 \mathrm{~kb} 3^{\prime}$ of HLA-DRA & 0 & & & & & none & \\
\hline 42 & rs2836878 & intergenic & $\begin{array}{l}\text { FU45139, RPL2 } \\
\text { 3AP12 }\end{array}$ & high & $\begin{array}{c}\text { PSMG1 and } 64 \mathrm{~kb} 5 \text { ' of } \\
\text { AF064858.10 a novel linc RNA }\end{array}$ & 0 & $\begin{array}{l}\text { AF064858.10 (Vega } \\
\text { gene) }\end{array}$ & a novel linc RNA & & yes & & \\
\hline 43 & rs2395185 & intergenic & $\begin{array}{l}\text { HLA-DRB9, HLA- } \\
\text { DRB5 }\end{array}$ & no & $20 \mathrm{~kb} 3$ ' of HLA-DRA & 0 & & & & & none & \\
\hline 44 & rs6871626 & intergenic & IL12B, ADRA1B & no & $37 \mathrm{~kb} 3^{\prime}$ of AC008697.1 & 1 & 285627 & uncharacterized LOC285627 & & yes & & \\
\hline 45 & rs6584283 & intergenic & GOT1, NKX2-3 & medium & RP11-129J12.2, a novel linc RNA & 1 & 101927324 & $\begin{array}{l}\text { LINC1475, long intergenic } \\
\text { non-protein coding RNA } \\
1475\end{array}$ & & yes & & \\
\hline \multirow{3}{*}{46} & \multirow{3}{*}{ rs6017342 } & \multirow{3}{*}{ intergenic } & \multirow{3}{*}{$\begin{array}{c}\text { HNF4A, RPL37A } \\
\text { P1 }\end{array}$} & \multirow{3}{*}{ no } & \multirow{3}{*}{$5 \mathrm{~kb} 3$ ' of HNF4A } & \multirow{3}{*}{3} & 100500813 & microRNA 3646 & yes & & & \\
\hline & & & & & & & 101927219 & $\begin{array}{l}\text { HNF4A-AS1, HNF4A } \\
\text { antisense RNA } 1\end{array}$ & & yes & & \\
\hline & & & & & & & 101927242 & $\begin{array}{l}\text { LINC01430, long intergenic } \\
\text { non-protein coding RNA } \\
1430\end{array}$ & & yes & & \\
\hline 47 & rs2006996 & intergenic & $\begin{array}{l}\text { TNFSF15, TNFSF } \\
8\end{array}$ & no & $24 \mathrm{~kb} 5$ ' of TNFSF15 & 0 & \multicolumn{2}{|c|}{ no LD with any other gene } & & & none & \\
\hline 48 & rs2836878 & intergenic & $\begin{array}{l}\text { FL445139, RPL2 } \\
\text { 3AP12 }\end{array}$ & no & $\begin{array}{c}\text { PSMG1 and } 64 \mathrm{~kb} 5^{\prime} \text { of } \\
\text { AF064858.10 }\end{array}$ & 1 & 102724740 & $\begin{array}{l}\text { LOC102724740, ncRNA } \\
\text { uncharacterized } \\
\text { LOC102724740 }\end{array}$ & & yes & & \\
\hline 49 & rs9271366 & intergenic & $\begin{array}{l}\text { HLA-DRB1, HLA- } \\
\text { DQA1 }\end{array}$ & no & 9.1kb 5' of HLA-DQA1 & 1 & 100616218 & microRNA 3135b & yes & & & \\
\hline 50 & rs10500264 & intergenic & $\begin{array}{l}\text { SLC7A10, CEBP } \\
\text { A }\end{array}$ & no & $34 \mathrm{~kb} 5$ ' of SLC7A10 & 1 & 80054 & $\begin{array}{l}\text { CEBPA-AS1, CEBPA antisense } \\
\text { RNA } 1\end{array}$ & & yes & & \\
\hline 51 & rs11209032 & intergenic & IL23R, IL12RB2 & no & IL23R, and $6.7 \mathrm{~kb} 5$ ' of U4atac & 1 & 102724481 & $\begin{array}{l}\text { RNA, U4atac small nuclear 4, } \\
\text { pseudogene }\end{array}$ & & yes & & \\
\hline 52 & rs477515 & intergenic & $\begin{array}{l}\text { HLA-DRB1, HLA- } \\
\text { DQA1 }\end{array}$ & no & $12 \mathrm{~kb} 5^{\prime}$ of HLA-DRB1 & 1 & 100616218 & microRNA 3135b & yes & & & \\
\hline 53 & rs8054797 & intergenic & BRD7, NKD1 & no & $\begin{array}{c}\text { BRD7 and 5.6kb 3' of RP11- } \\
21 \mathrm{~B} 23.2\end{array}$ & 1 & 102465462 & microRNA 6771 & yes & yes & & \\
\hline
\end{tabular}

* If no score is given, the score was more than $3: 4,5,6$ or no data existed at the time of data collection

ncSNP and ncRNAs: official rs identifier for each ncSNP, genomic context, originally assigned gene with official gene symbols, weather it is nested in conserved regions, name of genes found in LD, NCBI gene identifier and number of RNA genes, whether miRNA or other

type of RNA genes are present or not, and RegulomeDB score for each SNP 
Table 7a. miRNA genes linked with AID GWAS ncSNPs found in TargetScanHumanDB

\begin{tabular}{|c|c|c|c|c|}
\hline No & $\begin{array}{c}\text { miRNA in high } \\
\text { LD with GWAS } \\
\text { AID SNPS } \\
\end{array}$ & $\begin{array}{l}\text { Found among } \\
\text { TargetScan } \\
\text { Human DB }\end{array}$ & Number of targeted conseved sites & $\begin{array}{l}\text { Targeting } \\
\text { AID SNP } \\
\text { genes }\end{array}$ \\
\hline 1 & MIR101-2 & yes & $\begin{array}{c}803 \text { transcripts with conserved sites, with a } \\
\text { total of } 915 \text { conserved sites and } 337 \text { poorly } \\
\text { conserved sites. }\end{array}$ & no \\
\hline 2 & MIR1205 & yes & $\begin{array}{l}426 \text { transcripts with conserved sites, with a } \\
\text { total of } 448 \text { conserved sites and } 329 \text { poorly } \\
\text { conserved sites. }\end{array}$ & no \\
\hline 3 & MIR1206 & yes & $\begin{array}{c}215 \text { transcripts with conserved sites, with a } \\
\text { total of } 225 \text { conserved sites and } 93 \text { poorly } \\
\text { conserved sites. }\end{array}$ & no \\
\hline 4 & MIR1207 & no & & no \\
\hline 5 & MIR1208 & yes & $\begin{array}{l}322 \text { transcripts with conserved sites, with a } \\
\text { total of } 337 \text { conserved sites and } 146 \text { poorly } \\
\text { conserved sites. }\end{array}$ & no \\
\hline 6 & MIR3135B & yes & $\begin{array}{c}63 \text { transcripts with conserved sites, with a } \\
\text { total of } 63 \text { conserved sites and } 10 \text { poorly } \\
\text { conserved sites. }\end{array}$ & no \\
\hline 7 & MIR3646 & yes & & no \\
\hline 8 & MIR4425 & yes & $\begin{array}{c}1141 \text { transcripts with conserved sites, with a } \\
\text { total of } 1351 \text { conserved sites and } 1387 \text { poorly } \\
\text { conserved sites. }\end{array}$ & no \\
\hline 9 & MIR5192 & no & & no \\
\hline 10 & MIR6721 & no & & no \\
\hline 11 & MIR6771 & no & & no \\
\hline 12 & MIR6891 & no & & no \\
\hline
\end{tabular}

Official name of miRNA genes, whether it was found in the miRNA database, number of miRNA targets and whether it targets any AID SNP harboring genes

Table 7b. Micro RNAs targeting 3'-UTR of the AID GWAS SNP genes

\begin{tabular}{|c|c|c|c|}
\hline No & Gene name & Name & $\begin{array}{l}\text { Conservation level } \\
\text { of miRNA family }\end{array}$ \\
\hline 1 & PTPN22 & hsa-miR-3646 & conserved \\
\hline 2 & CARD9 & none & \\
\hline \multirow{2}{*}{3} & \multirow{2}{*}{ ERAP1 } & has-miR-143 & conserved \\
\hline & & hsa-miR-4770 & conserved \\
\hline 4 & IL23R & hsa-miR-4729 & conserved \\
\hline \multirow{2}{*}{5} & \multirow{2}{*}{ TRAF3IP2 } & hsa-miR-4795 & conserved \\
\hline & & hsa-miR-548n & conserved \\
\hline \multirow{8}{*}{6} & \multirow{8}{*}{ NFKBIE } & hsa-miR-2115 & conserved \\
\hline & & hsa-miR-1184 & conserved \\
\hline & & hsa-miR-4667-3p & conserved \\
\hline & & hsa-miR-3180-5p & conserved \\
\hline & & hsa-miR-1264 & conserved \\
\hline & & hsa-miR-3611 & conserved \\
\hline & & hsa-miR-2355-5p & conserved \\
\hline & & hsa-miR-3591-5p & conserved \\
\hline \multirow{4}{*}{7} & \multirow{4}{*}{ MST1 } & hsa-miR-654-3p & conserved \\
\hline & & hsa-miR-3916 & conserved \\
\hline & & hsa-miR-3125 & conserved \\
\hline & & hsa-miR-583 & conserved \\
\hline 8 & ATG16L1 & hsa-miR-142-3p & conserved \\
\hline 9 & LACC1 & no data & \\
\hline \multirow[t]{2}{*}{10} & \multirow[t]{2}{*}{ TYK2 } & miR-124ab & $\begin{array}{l}\text { broadly conserved } \\
\text { among vertebrates }\end{array}$ \\
\hline & & hsa-miR-506 & conserved \\
\hline \multirow{2}{*}{11} & \multirow{2}{*}{ SH2B3 } & hsa-miR-181abcd & conserved \\
\hline & & hsa-miR-4262 & conserved \\
\hline
\end{tabular}




\begin{tabular}{|c|c|c|c|}
\hline \multirow{3}{*}{12} & \multirow{3}{*}{ TNFAIP3 } & miR-23 & $\begin{array}{l}\text { broadly conserved } \\
\text { among vertebrates }\end{array}$ \\
\hline & & miR-125 & $\begin{array}{l}\text { broadly conserved } \\
\text { among vertebrates }\end{array}$ \\
\hline & & miR-29 & $\begin{array}{l}\text { broadly conserved } \\
\text { among vertebrates }\end{array}$ \\
\hline \multirow{2}{*}{13} & \multirow{2}{*}{ NOD2 } & miR-122 & $\begin{array}{l}\text { broadly conserved } \\
\text { among vertebrates }\end{array}$ \\
\hline & & miR-30 & $\begin{array}{l}\text { broadly conserved } \\
\text { among vertebrates }\end{array}$ \\
\hline 14 & IL13 & has-miR-155 & conserved \\
\hline \multirow{2}{*}{15} & \multirow{2}{*}{ IL6R } & has-miR-9 & conserved \\
\hline & & has-miR-124 & conserved \\
\hline 16 & MICA & none & \\
\hline \multirow{2}{*}{17} & \multirow{2}{*}{ RTKN2 } & hsa-miR-204 & conserved \\
\hline & & hsa-miR-211 & conserved \\
\hline \multirow{2}{*}{18} & \multirow{2}{*}{ YDJC } & hsa-miR-1184 & conserved \\
\hline & & hsa-miR-4477a & conserved \\
\hline \multirow{5}{*}{19} & \multirow{5}{*}{ NOTCH4 } & hsa-miR-3945 & conserved \\
\hline & & hsa-miR-4265 & conserved \\
\hline & & hsa-miR-4322 & conserved \\
\hline & & hsa-miR-4296 & conserved \\
\hline & & hsa-miR-607 & conserved \\
\hline \multirow{4}{*}{20} & \multirow{4}{*}{ FCGR2A } & hsa-miR-4490 & conserved \\
\hline & & hsa-miR-3691-5p & conserved \\
\hline & & hsa-miR-4752 & conserved \\
\hline & & hsa-miR-3911 & conserved \\
\hline 21 & TNF & miR-19 & $\begin{array}{l}\text { broadly conserved } \\
\text { among vertebrates }\end{array}$ \\
\hline
\end{tabular}

Name of the missSNP harboring gene which 3'-UTR was inspected for miRNA, official name of miRNA genes in their 3'-UTR regions, and miRNAs level of conservation

Table 7c. GWAS AID SNPs found in the UTR-3' regions of targeted genes

(found by PhenGenl, analized by HaploReg and RegulomeDB)

\begin{tabular}{|c|c|c|c|c|c|c|c|c|c|c|}
\hline No & AID & rs & Context & Gene & Loca & & P-value & Source & ncRNA in LD & $\begin{array}{l}\text { Regulome } \\
\text { DB score }\end{array}$ \\
\hline 1 & Ps & rs2395029 & UTR-3 & HCP5 & 6 & :31,431,780 & $2.000 \times 10-26$ & NHGRI & lincRNA 1149 & $2 \mathrm{~b}$ \\
\hline 2 & Ps & rs8365 & UTR-3 & RNF5 & 6 & : $32,148,403$ & $1.094 \times 10-17$ & $\mathrm{dbGaP}$ & none & If \\
\hline 3 & Ps & rs2240803 & UTR-3 & DPCR1 & 6 & $: 30,920,957$ & $8.193 \times 10-13$ & $\mathrm{dbGaP}$ & $\begin{array}{l}\text { novel misc RNA, } Y \\
\text { RNA, ncRNA }\end{array}$ & 5 \\
\hline 4 & IBD & rs10889677 & UTR-3 & IL23R & 1 & $: 67,725,120$ & $9.037 \times 10-11$ & $\mathrm{dbGaP}$ & none & no data \\
\hline 5 & UC & rs2297441 & UTR-3 & RTEL1 & 20 & $: 62,327,582$ & $2.000 \times 10-10$ & NHGRI & none & 4 \\
\hline 6 & UC & rs10889677 & UTR-3 & IL23R & 1 & $: 67,725,120$ & $1.000 \times 10-8$ & NHGRI & none & no data \\
\hline 7 & $C D$ & rs504963 & UTR-3 & FUT2 & 19 & $: 49,208,865$ & $2.000 \times 10-8$ & NHGRI & $\begin{array}{l}\text { none, in LD with } \\
\text { nonsence SNP }\end{array}$ & 5 \\
\hline 8 & RA & rs1329568 & UTR-3 & LOC100130458 & 9 & $: 37,037,976$ & $8.000 \times 10-7$ & NHGRI & none & 4 \\
\hline
\end{tabular}

Name of the missSNP harboring gene which 3'-UTR was inspected for miRNA, official name of miRNA genes in their 3'-UTR regions, and miRNAs level of conservation 
Table 8. Genes in missSNP gene set and ncSNP gene set

\begin{tabular}{|c|c|}
\hline missSNP set & ncSNP set \\
\hline PTPN22 & ANKRD55 \\
\hline CARD9 & APOM \\
\hline ERAP1 & ATXN2L \\
\hline IL23R & BAG6 \\
\hline TRAF3IP2 & $\mathrm{C} 2$ \\
\hline NFKBIE & CCR6 \\
\hline MST1 & CD40 \\
\hline ATG16L1 & CTLA4 \\
\hline LACC1 & CXCR1 \& 2 \\
\hline TYK2 & CCDC88B \\
\hline SH2B3 & DDR1 \\
\hline TNFAIP3 & ERAP2 \\
\hline NOD2 & GNA12 \\
\hline IL13 & GPSM3 \\
\hline IL6R & HORMAD2 \\
\hline MICA & IL10 \\
\hline RTKN2 & IRF1 \\
\hline YDJC & IRF5 \\
\hline NOTCH4 & LPS1 \\
\hline FCGR2A & NKX2-3 \\
\hline GSDMB & PBX2 \\
\hline IL17REL & PDE2A \\
\hline IL7R & PSORS1C1 \\
\hline UBE2L3 & PSORS1C2 \\
\hline & RBPJ \\
\hline & RNF5 \\
\hline & SKIV2L \\
\hline & SLC22A4 \\
\hline & SLC22A5 \\
\hline & SMAD3 \\
\hline & SNAPC4 \\
\hline & TCF19 \\
\hline & TNFRSF14 \\
\hline & TNFSF15 \\
\hline & TNPO3 \\
\hline & TRAF1 \\
\hline & TRHDE \\
\hline & TUMF \\
\hline & UBE2L3 \\
\hline & USP8P1 \\
\hline & ZPBP2 \\
\hline
\end{tabular}


Table 9. Intersections between missSNP harboring genes/ proteins networks and TNF network

Number of intersecting nodes for each pair of genes, followed by gene names (in no specific order)

\begin{tabular}{|c|c|c|c|c|c|c|c|c|c|c|c|c|c|c|c|}
\hline $\begin{array}{l}\text { Genes with missense GWAS } \\
\text { AID SNPs }\end{array}$ & \multirow{2}{*}{\begin{tabular}{|l|} 
PTPN22 \\
$150+$ \\
\end{tabular}} & \multirow{2}{*}{\begin{tabular}{|l} 
ERAP1 \\
$50+$
\end{tabular}} & \multirow{2}{*}{\begin{tabular}{|l|} 
TYK2 \\
$250+$
\end{tabular}} & \multirow{2}{*}{\begin{tabular}{|l} 
CARD9 \\
$100+$
\end{tabular}} & \multirow{2}{*}{\begin{tabular}{|l|} 
IL23R \\
$130+$
\end{tabular}} & \multirow{2}{*}{\begin{tabular}{|l} 
TRAF3IP2 \\
90
\end{tabular}} & \multirow{2}{*}{\begin{tabular}{|l} 
NFKBIE \\
$50+$
\end{tabular}} & \multirow{2}{*}{\begin{tabular}{|l} 
ATG16L1 \\
$100+$
\end{tabular}} & \multirow{2}{*}{\begin{tabular}{|l} 
RTKN2 \\
3
\end{tabular}} & \multirow{2}{*}{\begin{tabular}{|l} 
TNFAIP3 \\
$200+$
\end{tabular}} & \multirow{2}{*}{\begin{tabular}{|l} 
SH2B3 \\
$120+$
\end{tabular}} & \multirow{2}{*}{\begin{tabular}{|l|} 
MST1 \\
$100+$
\end{tabular}} & \multirow{2}{*}{$\begin{array}{l}\text { LACC1 } \\
0\end{array}$} & \multirow{2}{*}{\begin{tabular}{|l} 
NOD2 \\
$150+$
\end{tabular}} & \multirow{2}{*}{\begin{tabular}{|l|} 
MICA \\
$150+$
\end{tabular}} \\
\hline $\begin{array}{l}\text { No of nodes for } \\
\text { each gene }\end{array}$ & & & & & & & & & & & & & & & \\
\hline TNF & 58 & 17 & $100+$ & $50+$ & 75 & 34 & 25 & 32 & 0 & $200+$ & 23 & 27 & 0 & $80+$ & 36 \\
\hline $1500+$ & $\begin{array}{l}\text { INFG CARD9 } \\
\text { TNFAIP3 } \\
\text { HLA-B IL23R }\end{array}$ & \begin{tabular}{|l|} 
CARD9 IL6R IL23R \\
HLA-B TNFRSF1A \\
TNFAIP3 INFG ....
\end{tabular} & & $\begin{array}{l}\text { IL23R NFKB } \\
\text { IL1B TRL2,9 } \\
\text { NOD2 } \\
\text { TRAF2 } \\
\text { STAT3 } \\
\text { CARD9 ... }\end{array}$ & \begin{tabular}{|l|} 
IL23R \\
TNFAIP3 \\
CARD9 IL23A \\
INFG HLA-B \\
$\cdots .$.
\end{tabular} & & & $\begin{array}{l}\text { IL23R NOD2 } \\
\text { CARD9 ... }\end{array}$ & & & \begin{tabular}{|l} 
JAK2 TNFAIP2 \\
CTLA4 ZAP70
\end{tabular} & & & & $\begin{array}{ll}\text { HLA } & \text { KLRK1 } \\
\cdots\end{array}$ \\
\hline \multirow[t]{2}{*}{ PTPN22 } & & 8 & 22 & 19 & 45 & 5 & 2 & 18 & 0 & 44 & 30 & 13 & 0 & 31 & 18 \\
\hline & & \begin{tabular}{|l} 
TNFAIPS IL23R \\
TNIP1 HLA-C \\
FCRL3 HLA-B \\
CARD9 INFG
\end{tabular} & & & & $\begin{array}{l}\text { HLA-C TNFSF13B } \\
\text { DEFB4A CD40 } \\
\text { TRAF1 }\end{array}$ & $\begin{array}{l}\text { TNIP2 } \\
\text { TRAF1 }\end{array}$ & $\begin{array}{l}\text { NOD2 CARD9 } \\
\text { IL23R } \\
\text { TNFSF15 ... }\end{array}$ & & & & $\begin{array}{l}\text { NOD2 IL23R } \\
\text { CARD9 } \\
\text { ATG16L1 ... }\end{array}$ & & & \\
\hline \multirow[t]{2}{*}{ ERAP1 } & & & 5 & 5 & 18 & 6 & 2 & 3 & 0 & 12 & 1 & 2 & 0 & 8 & 5 \\
\hline & & & $\begin{array}{l}\text { INFG IL6R } \\
\text { IL23R IL23A } \\
\text { IL28RA }\end{array}$ & $\begin{array}{l}\text { CARD9 } \\
\text { IL23R IL23A } \\
\text { ERAP1 } \\
\text { SNAPC4 }\end{array}$ & $\begin{array}{l}\text { SNAPC4 INFG } \\
\text { HLA-C TNIP1 } \\
\text { IL23R CARD9 } \\
\text { ERAP1 } \\
\text { TNFAIP3 HLA- } \\
\text { B IL23A PHB2 } \\
\text { FCRL3 ... }\end{array}$ & $\begin{array}{l}\text { TNFRSF1A HLA-C } \\
\text { ERAP1 TRAFAIP2 } \\
\text { IL28RA BCL2A1 } \\
\text { ST20 }\end{array}$ & TNIP1 IL1A & $\begin{array}{l}\text { IL23R IL23A } \\
\text { CARD9 }\end{array}$ & & \begin{tabular}{|l|} 
ERAP1 TNFAIP3 \\
HLA-C TNIP1 \\
BCL2A1 ST20 \\
TNFRS1A IL23A \\
IL1A PPP1R15A \\
IL23R IL1R2
\end{tabular} & TNFAIP3 & $\begin{array}{l}\text { CARD9 } \\
\text { IL23R }\end{array}$ & & $\begin{array}{l}\text { HLA-C IL23A } \\
\text { IL23R CARD9 } \\
\text { MRAP } \\
\text { TNFAIP3 IFNG } \\
\text { IL1A }\end{array}$ & \begin{tabular}{|l|} 
CD8A HLA-B \\
HLA-C MRAP \\
KLRC2 KLRC3
\end{tabular} \\
\hline TYK2 & & & & 21 & $50+$ & 9 & 7 & 12 & 0 & 29 & 15 & 4 & 0 & 30 & 12 \\
\hline & & & & \begin{tabular}{|l} 
\\
PTPN2 TNF \\
IL6 CCR6 \\
IL12B TLR3 \\
FAM92B \\
IL1B STAT3 \\
TRF5 \\
TFKBIA \\
IL17A \\
NFKB2 \\
NFKB1 \\
NF23A \\
IL23R...
\end{tabular} & \begin{tabular}{|l|} 
STAT3 IL12B \\
IL23R TNF \\
PTPN2 IL1R1 \\
JAK1 IL12A \\
CCR6 JAK2 \\
STAT1 IL6 \\
NFKBIA ...
\end{tabular} & $\begin{array}{l}\text { NFKB1 TRAF6 } \\
\text { FAM48A IL17A } \\
\text { CD40 TNFRSF13C } \\
\text { TNFSF13B }\end{array}$ & \begin{tabular}{|l|} 
TNF NFKB2 \\
RELA NFKB1 \\
CXCL10 \\
FAM48A \\
BTRC
\end{tabular} & \begin{tabular}{|l} 
IL1B IL18 \\
CCR6 STAT3 \\
IL12B JAK2 \\
PPPNN2 IL23R \\
IL23A ...
\end{tabular} & & \begin{tabular}{|l} 
TRAF6 RELA \\
CTLA4 IL23R \\
TNF NFKB2 \\
NFKB1 STAT4 \\
IL23R IL23A \\
TNFSF13B \\
NFKBIA IL12B \\
MYD88...
\end{tabular} & $\begin{array}{l}\text { LCK TNF IL12A } \\
\text { CTLA4... }\end{array}$ & $\begin{array}{l}\text { IL12B IRF5 } \\
\text { CXCL10 } \\
\text { IL23R }\end{array}$ & & $\begin{array}{l}\text { TNF STAT3 } \\
\text { IL23R ... }\end{array}$ & $\begin{array}{l}\text { NCR1 JAK2 } \\
\text { IL15 II2 STAT3 } \\
\text { IL IL } 24 \text { TNF } \\
\text { NFKB1 ... }\end{array}$ \\
\hline
\end{tabular}




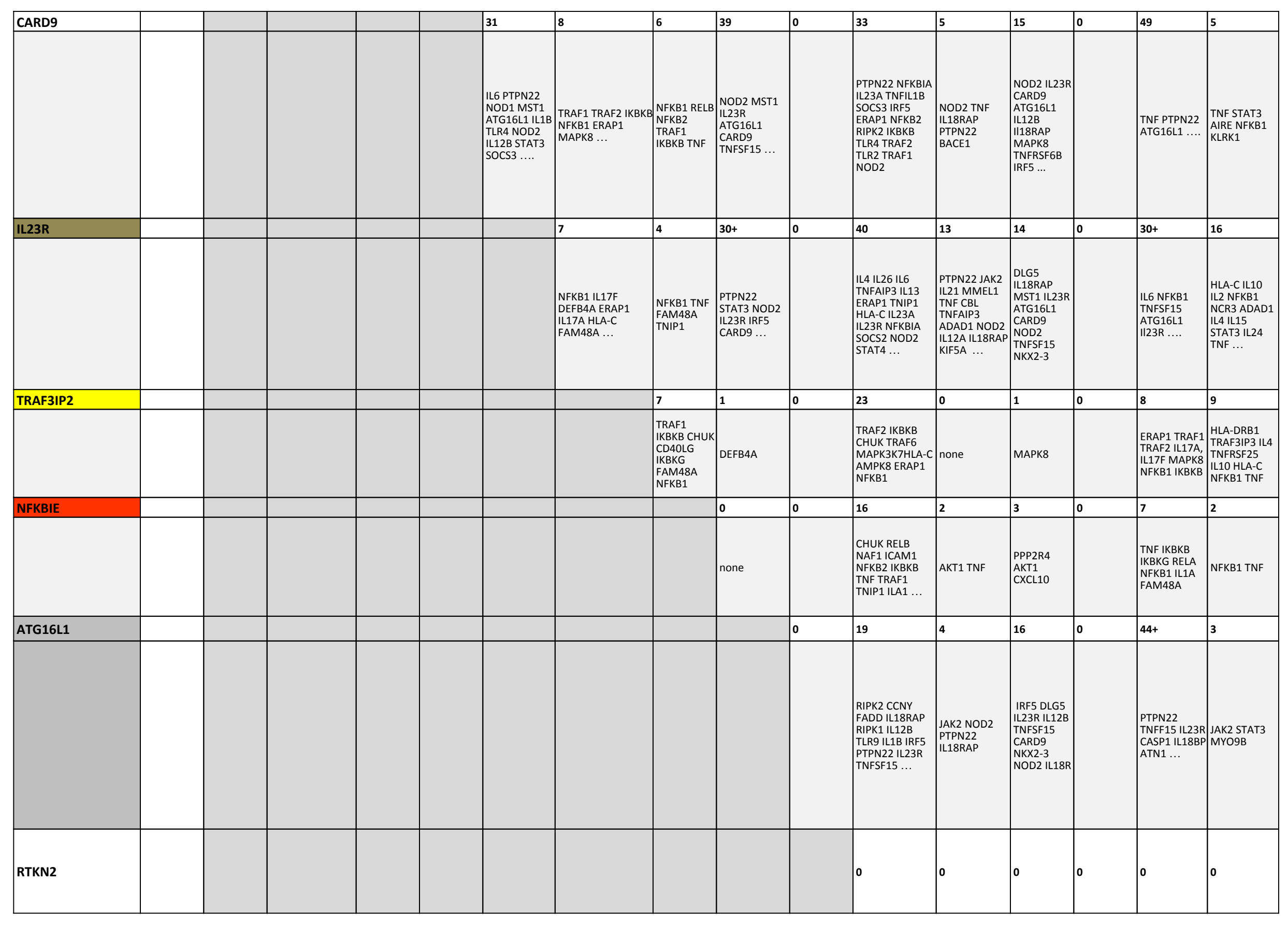




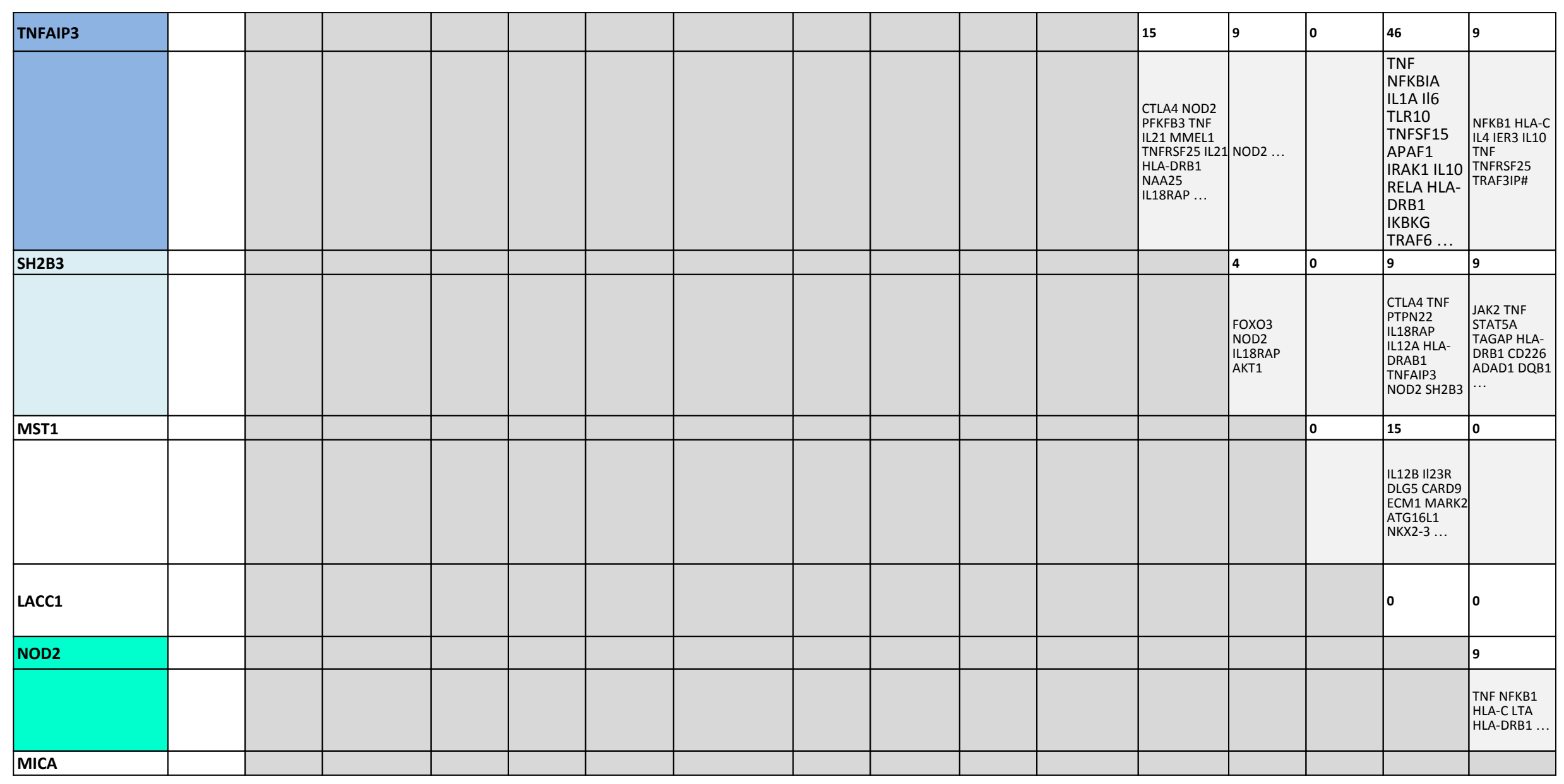

Note:

- missSNP harboring gene/proteins and TNF intersections are described with the number of intersecting nodes for each pair of networks, followed by symbols of some of the genes in the intersections in no specific order

- TNF and IL7R have many common mamabers and TNF among them (not shown in the table) 
Table 11. KEGG pathways for the genes harboring missense SNPs

\begin{tabular}{|c|c|c|c|}
\hline Gene ID & Pathway ID & Pathway name & GWAS related AID \\
\hline PTPN22 & hsa04612 & Antigen processing and presentation & RA, CD (T1D, vitiligo) \\
\hline CARD9 & $\begin{array}{l}\text { hsa04621 } \\
\text { hsa05152 }\end{array}$ & $\begin{array}{l}\text { NOD-like receptor signaling pathway } \\
\text { Tuberculosis }\end{array}$ & $\mathrm{CD}, \mathrm{UC}, \mathrm{AS}$ \\
\hline ERAP1 & hsa04612 & Antigen processing and presentation & AS, Ps \\
\hline IL23R & $\begin{array}{l}\text { hsa04060 } \\
\text { hsa04630 } \\
\text { hsa05321 }\end{array}$ & $\begin{array}{l}\text { Cytokine-cytokine receptor interaction } \\
\text { Jak-STAT signaling pathway } \\
\text { Inflammatory bowel disease (IBD) }\end{array}$ & $\begin{array}{l}\mathrm{CD}, \mathrm{AS}, \mathrm{Ps}, \mathrm{IBD}, \mathrm{UC} \\
\text { (Behcet syndrome, } \\
\text { leprosy, asthma, T1D) }\end{array}$ \\
\hline TRAF3IP2 & $\begin{array}{l}\text { hsa04668 } \\
\text { hsa04064 } \\
\text { hsa05160 } \\
\text { hsa05168 } \\
\text { hsa05169 }\end{array}$ & $\begin{array}{l}\text { TNF signaling pathway } \\
\text { NF-kappa B signaling pathway } \\
\text { Hepatitis C } \\
\text { Herpes simplex infection } \\
\text { Epstein-Barr virus infection }\end{array}$ & PsA,Ps, CD (T1D, T2D) \\
\hline NFKBIE & $\begin{array}{l}\text { hsa04660 } \\
\text { hsa04662 } \\
\text { hsa04064 } \\
\text { hsa04920 } \\
\text { hsa05169 }\end{array}$ & $\begin{array}{l}\text { T cell receptor signaling pathway } \\
\text { B cell receptor signaling pathway } \\
\text { NF-kappa B signaling pathway } \\
\text { Adipocytokine signaling pathway } \\
\text { Epstein-Barr virus infection }\end{array}$ & RA \\
\hline MST1 & none & & $C D, U C$ \\
\hline ATG16L1 & hsa04140 & Regulation of autophagy & $C D, U C$ \\
\hline LACC1 & none & & CD (leprosy) \\
\hline TYK2 & $\begin{array}{l}\text { hsa04380 } \\
\text { hsa04630 } \\
\text { hsa05145 } \\
\text { hsa05160 } \\
\text { hsa05162 } \\
\text { hsa05164 } \\
\text { hsa05168 } \\
\text { hsa05169 }\end{array}$ & $\begin{array}{l}\text { Osteoclast differentiation } \\
\text { Jak-STAT signaling pathway } \\
\text { Toxoplasmosis } \\
\text { Hepatitis C } \\
\text { Measles } \\
\text { Influenza A } \\
\text { Herpes simplex infection } \\
\text { Epstein-Barr virus infection }\end{array}$ & $\begin{array}{l}\text { CD, Ps, PsA, UC, IBD, RA } \\
\text { (T1D,T2D) }\end{array}$ \\
\hline $\mathrm{SH} 2 \mathrm{~B} 3$ & hsa04722 & Neurotrophin signaling pathway & RA (T1D, hypotyroidism) \\
\hline TNFAIP3 & $\begin{array}{l}\text { hsa04064 } \\
\text { hsa04621 } \\
\text { hsa04668 } \\
\text { hsa05162 } \\
\text { hsa05169 }\end{array}$ & $\begin{array}{l}\text { NF-kappa B signaling pathway } \\
\text { NOD-like receptor signaling pathway } \\
\text { TNF signaling pathway } \\
\text { Measles } \\
\text { Epstein-Barr virus infection }\end{array}$ & $\begin{array}{l}\text { RA, PsA, Ps, UC (SLE, } \\
\text { celiac disease) }\end{array}$ \\
\hline NOD2 & hsa04621 & NOD-like receptor signaling pathway & $C D, I B D, P s$ \\
\hline
\end{tabular}


hsa04668

hsa05131

hsa05152

hsa05321

IL13

IL6R

hsa04060

hsa04630

hsa04664

hsa05162

hsa05310

hsa05321

hsa04060

hsa04151

hsa04630

hsa04640

hsa04932

MICA

none

RTKN2

none

YDJC

none

NOTCH4

hsa04330

FCGR2A

hsa04145

hsa04380

hsa04611

hsa04666

hsa05140

hsa05150

hsa05152

hsa05322

GSDMB

none

IL17REL

hsa04060

IL7R
TNF signaling pathway

Shigellosis

Tuberculosis

Inflammatory bowel disease (IBD)

Cytokine-cytokine receptor interaction

Ps (asthma)

Jak-STAT signaling pathway

Fc epsilon RI signaling pathway

Measles

Asthma

Inflammatory bowel disease (IBD)

Cytokine-cytokine receptor interaction

RA (asthma, T1D)

PI3K-Akt signaling pathway

Jak-STAT signaling pathway

Hematopoietic cell lineage

Non-alcoholic fatty liver disease (NAFLD)
RA, Ps

RA

RA, CD (celiac disease)

Notch signaling pathway

UC, RA, CD (asthma, T1D)
Phagosome

Osteoclast differentiation

Platelet activation

Fc gamma R-mediated phagocytosis

Leishmaniasis

Staphylococcus aureus infection

Tuberculosis

Systemic lupus erythematosus

Cytokine-cytokine receptor interaction

UC, Ps, PsA

Cytokine-cytokine receptor interaction UC (T1D)

PI3K-Akt signaling pathway

Jak-STAT signaling pathway

Hematopoietic cell lineage

Primary immunodeficiency

Ps ( SLE, T1D, T2D, MS, SS, asthma)

UC (mucokutaneous disease)

hsa04060

hsa04151

hsa04630

hsa04640

hsa05340

Provided are missSNP harboring genes, labeled with gene symbols, KEGG pathways IDs and association with AIDs 


\section{Table 12. KEGG pathways of the genes harboring non-coding SNPs}

Gene ID

C2

CCR6

CD40

CTLA4

CXCR1\&2
Pathway ID Pathway name

hsa04610

hsa05133

hsa05150

hsa05322

hsa04060

hsa04062

hsa04060

hsa04064

hsa04514

hsa04620

hsa04672

hsa05144

hsa05145

hsa05166

hsa05169

hsa05202

hsa05310

hsa05320

hsa05322

hsa05330

hsa05340

hsa05416

diseases

hsa04514

hsa04660

hsa05320

hsa05323

$\mathrm{H} 00081$

$\mathrm{H} 00082$

$\mathrm{H} 00083$

$\mathrm{H} 00408$

hsa04060

hsa04062

hsa04144

hsa05120

Pertussis

Malaria

Toxoplasmosis

HTLV-I infection

Asthma

Allograft rejection

Viral myocarditis

Graves' disease

Allograft rejection
Complement and coagulation cascades

Staphylococcus aureus infection

Systemic lupus erythematosus

Cytokine-cytokine receptor interaction

RA

Chemokine signaling pathway

Cytokine-cytokine receptor interaction

RA

NF-kappa B signaling pathway (noncanonical)

Cell adhesion molecules (CAMs)

Toll-like receptor signaling pathway

Intestinal immune network for IgA production

Epstein-Barr virus infection

Transcriptional misregulation in cancer

Autoimmune thyroid disease

Systemic lupus erythematosus

Primary immunodeficiency

Immunodef, Hyper IgM syndrom

Cell adhesion molecules (CAMs)

RA

T cell receptor signaling pathway

Autoimmune thyroid disease

Rheumatoid arthritis

Hashimoto's thyroiditis

Type I diabetes mellitus

Cytokine-cytokine receptor interaction

UC

\section{A}

GWAS

related

AID

PS, PsA 
Cytokine-cytokine receptor interaction

UC, CD

hsa04068

FoxO signaling pathway

hsa04630

Jak-STAT signaling pathway

hsa04660

$T$ cell receptor signaling pathway

hsa04672

hsa05133

Intestinal immune network for IgA production

hsa05140

Pertussis

hsa05142

Leishmaniasis

hsa05143

Chagas disease (American trypanosomiasis)

hsa05144

African trypanosomiasis

hsa05145

Malaria

hsa05146

Toxoplasmosis

hsa05150

Amoebiasis

hsa05152

Staphylococcus aureus infection

hsa05169

hsa05310

Tuberculosis

hsa05320

Epstein-Barr virus infection

hsa05321

Asthma

hsa05322

Autoimmune thyroid disease

hsa05330

Inflammatory bowel disease (IBD)

Systemic lupus erythematosus

Allograft rejection

IRF1

hsa04917

Prolactin signaling pathway

RA

hsa05133

Pertussis

hsa05160 Hepatitis C (engaged Toll-like R)

hsa04620 Toll-like receptor signaling pathway

IRF5

hsa04620

Toll-like receptor signaling pathway

UC

CXCR1\&2

hsa04060

Cytokine-cytokine receptor interaction

UC

hsa04062

Chemokine signaling pathway

hsa04144

Endocytosis

hsa05120

Epithelial cell signaling in Helicobacter pylori infection

LSP1

hsa05152

Tuberculosis

UC

RBPJ

hsa04330

Notch signaling pathway

RA

hsa05169 Epstein-Barr virus infection

hsa05203 Viral carcinogenesis

TNFSF15

hsa04060

Cytokine-cytokine receptor interaction

$C D$

H00286

Crohn's disease

TNFRSF14

hsa04060

Cytokine-cytokine receptor interaction

UC 
hsa05168 Herpes simplex infection

TRAF1

hsa04064 NF-kappa B signaling pathway

RA

hsa04668 TNF signaling pathway

hsa05168 Herpes simplex infection

hsa05169 Epstein-Barr virus infection

hsa05200 Pathways in cancer

hsa05202 Transcriptional misregulation in cancer

hsa05203 Viral carcinogenesis

hsa05222 Small cell lung cancer

RBPJ

hsa04330 Notch signaling pathway

RA

hsa05169 Epstein-Barr virus infection

hsa05203 Viral carcinogenesis 
Table 13. Classification of AID missSNP KEGG pathways

\begin{tabular}{|c|c|c|c|}
\hline No & $\begin{array}{l}\text { Human KEGG } \\
\text { pathways ID }\end{array}$ & Pathway name & Cathegory of pathways: \\
\hline 1 & hsa04060 & Cytokine-cytokine receptor interaction & Signaling molecules and interaction; Environmental Information Processing \\
\hline 2 & hsa04064 & NF-kappa B signaling pathway & Signal transduction; Environmental Information Processing \\
\hline 3 & hsa04066 & HIF-1 signaling pathway & Signal transduction; Environmental Information Processing \\
\hline 4 & hsa04140 & Regulation of autophagy & Transport and catabolism; Cellular Processes \\
\hline 5 & hsa04145 & Phagosome & Transport and catabolism; Cellular Processes \\
\hline 6 & hsa04151 & PI3K-Akt signaling pathway & Signal transduction; Environmental Information Processing \\
\hline 7 & hsa04330 & Notch signaling pathway & Signal transduction; Environmental Information Processing \\
\hline 8 & hsa04380 & Osteoclast differentiation & Development; Organismal Systems \\
\hline 9 & hsa04611 & Platelet activation & Immune system; Organismal Systems \\
\hline 10 & hsa04612 & Antigen processing and presentation & Immune system; Organismal Systems \\
\hline 11 & hsa04621 & NOD-like receptor signaling pathway & Immune system; Organismal Systems \\
\hline 12 & hsa04630 & Jak-STAT signaling pathway & Signal transduction; Environmental Information Processing \\
\hline 13 & hsa04640 & Hematopoietic cell lineage & Immune system; Organismal Systems \\
\hline 14 & hsa04660 & T cell receptor signaling pathway & Immune system; Organismal Systems \\
\hline 15 & hsa04662 & B cell receptor signaling pathway & Immune system; Organismal Systems \\
\hline 16 & hsa04664 & Fc epsilon RI signaling pathway & Immune system; Organismal Systems \\
\hline 17 & hsa04666 & Fc gamma R-mediated phagocytosis & Immune system; Organismal Systems \\
\hline 18 & hsa04668 & TNF signaling pathway & Signal transduction; Environmental Information Processing \\
\hline 19 & hsa04722 & Neurotrophin signaling pathway & Nervous system; Organismal Systems \\
\hline 20 & hsa04919 & Thyroid hormone signaling pathway & Endocrine system; Organismal Systems \\
\hline 21 & hsa04920 & Adipocytokine signaling pathway & Endocrine system; Organismal Systems \\
\hline 22 & hsa04932 & Non-alcoholic fatty liver disease (NAFLD) & Endocrine and metabolic diseases; Human Diseases \\
\hline 23 & hsa05131 & Shigellosis & Infectious diseases: Bacterial \\
\hline 24 & hsa05140 & Leishmaniasis & Infectious diseases: Parasitic \\
\hline 25 & hsa05145 & Toxoplasmosis & Infectious diseases: Parasitic \\
\hline 26 & hsa05150 & Staphylococcus aureus infection & Infectious diseases: Bacterial \\
\hline 27 & hsa05152 & Tuberculosis & Infectious diseases: Bacterial \\
\hline 28 & hsa05160 & Hepatitis C & Infectious diseases: Viral \\
\hline 29 & hsa05162 & Measles & Infectious diseases: Viral \\
\hline 30 & hsa05164 & Influenza A & Infectious diseases: Viral \\
\hline 31 & hsa05168 & Herpes simplex infection & Infectious diseases: Viral \\
\hline 32 & hsa05169 & Epstein-Barr virus infection & Infectious diseases: Viral \\
\hline 33 & hsa05206 & MicroRNAs in cancer & Cancers: Human Diseases \\
\hline 34 & hsa05310 & Asthma & Immune diseases Human Diseases \\
\hline 35 & hsa05321 & Inflammatory bowel disease (IBD) & Immune diseases Human Diseases \\
\hline 36 & hsa05340 & Primary immunodeficiency & Immune diseases Human Diseases \\
\hline 37 & hsa05322 & Systemic lupus erythematosus & Immune diseases; Human Diseases \\
\hline 38 & H01109 & Chronic Mucocutaneous Candidiasis (CMC) & Immune diseases; Human Diseases \\
\hline
\end{tabular}


Table 14. Classification of KEGG pathways in which ncSNP genes participate

No

Human KEGG pathways ID

hsa04060

hsa04062

hsa04064

hsa04068

hsa04144

hsa04330

hsa04514

hsa04610

hsa04612

hsa04620

hsa04630

hsa04660

hsa04668

hsa04672

hsa04917

hsa05120

hsa05133

hsa05133

hsa05140

hsa05142

hsa05143

hsa05144

hsa05145

hsa05146

hsa05150

hsa05152

hsa05160

hsa05166

hsa05168

hsa05169

hsa05200

hsa05202

hsa05203

hsa05222

hsa05310

hsa05320

hsa05321

hsa05322

hsa05323

hsa05330

hsa05340

hsa05416

H00081

H00082

H00083

H00286

H00408
Pathway name

Cytokine-cytokine receptor interaction

Chemokine signaling pathway

NF-kappa B signaling pathway

FoxO signaling pathway

Endocytosis

Notch signaling pathway

Cell adhesion molecules (CAMs)

Complement and coagulation cascades

Antigen processing and presentation

Toll-like receptor signaling pathway

Jak-STAT signaling pathway

$T$ cell receptor signaling pathway

TNF signaling pathway

Intestinal immune network for IgA production

Prolactin signaling pathway

Epithelial cell signaling in Helicobacter pylori infection

Pertussis

Pertussis

Leishmaniasis

Chagas disease (American trypanosomiasis)

African trypanosomiasis

Malaria

Toxoplasmosis

Amoebiasis

Staphylococcus aureus infection

Tuberculosis

Hepatitis C (engaged Toll-like R)

HTLV-I infection

Herpes simplex infection

Epstein-Barr virus infection

Pathways in cancer

Transcriptional misregulation in cancer

Viral carcinogenesis

Small cell lung cancer

Asthma

Autoimmune thyroid disease

Inflammatory bowel disease (IBD)

Systemic lupus erythematosus

Rheumatoid arthritis

Allograft rejection

Primary immunodeficiency

Viral myocarditis

Hashimoto's thyroiditis

Graves' disease

Allograft rejection

Crohn's disease

Type I diabetes mellitus

\section{Cathegory of pathways:}

Signaling molecules and interaction; Environmental Information Processing Signaling molecules and interaction; Environmental Information Processing Signal transduction; Environmental Information Processing Signal transduction; Environmental Information Processing Cellular Processes; Transport and catabolism

Signal transduction; Environmental Information Processing

Signaling molecules and interaction; Environmental Information Processing Immune system; Organismal Systems Immune system; Organismal Systems Immune system; Organismal Systems

Signal transduction; Environmental Information Processing Immune system; Organismal System

Signal transduction; Environmental Information Processing

Immune system; Organismal Systems

Nervous system; Organismal Systems

Infectious diseases: Bacterial

Infectious diseases: Bacterial

Infectious diseases: Bacterial

Infectious diseases: Parasitic

Infectious diseases: Parasitic

Infectious diseases: Parasitic

Infectious diseases: Parasitic

Infectious diseases: Parasitic

Infectious diseases: Parasitic

Infectious diseases: Bacterial

Infectious diseases: Bacterial

Infectious diseases: Viral

Infectious diseases: Viral

Infectious diseases: Viral

Infectious diseases: Viral

Cancers: Human Diseases

Cancers: Human Diseases

Cancers: Human Diseases

Cancers: Human Diseases

Immune diseases; Human Diseases

Immune diseases; Human Diseases

Immune diseases; Human Diseases

Immune diseases; Human Diseases Immune diseases; Human Disease Immune diseases; Human Diseases Immune diseases; Human Diseases Cardiovascular diseases; Human Diseases

Immune diseases; Human Diseases Immune diseases; Human Diseases Immune diseases; Human Diseases Immune diseases; Human Diseases Immune diseases; Human Diseases 
Table 15. Comparison of five anti-TNF biologic drugs by their known pathways

\begin{tabular}{|c|c|c|c|c|c|}
\hline \multirow[t]{4}{*}{ all } & \multicolumn{5}{|c|}{ USP drug classification [BR:br08302] } \\
\hline & \multicolumn{5}{|l|}{ Immunological Agents } \\
\hline & \multicolumn{5}{|l|}{ Immune Suppressants } \\
\hline & \multicolumn{5}{|c|}{ Tumor Necrosis Factor (TNF) Blockers } \\
\hline drug & $\begin{array}{l}\text { ENBREL (Immunex } \\
\text { Corporation) }\end{array}$ & $\begin{array}{l}\text { REMICADE (Janssen } \\
\text { Biotech) }\end{array}$ & HUMIRA (AbbVie) & $\begin{array}{l}\text { SIMPONI ARIA (Janssen } \\
\text { Biotech) }\end{array}$ & $\begin{array}{l}\text { CIMZIA } \\
\text { (UCB);Certolizumab } \\
\text { pegol (genetical } \\
\text { recombination) (JAN); }\end{array}$ \\
\hline class & $\begin{array}{l}\text { recombinant fusion } \\
\text { protein }\end{array}$ & monoclonal antibody & monoclonal antibody & monoclonal antibody & $\begin{array}{l}\text { humanized Fab, } \\
\text { pegylated }\end{array}$ \\
\hline \multirow[t]{2}{*}{ target } & TNF-alpha (hsa:7124) & TNF-alpha (hsa:7124) & TNF-alpha (hsa:7124) & TNF-alpha (hsa:7124) & TNF-alpha (hsa:7124) \\
\hline & $\begin{array}{l}\text { TNF-beta (LT-alpha) } \\
\text { (hsa:4049) }\end{array}$ & & & & \\
\hline \multicolumn{6}{|c|}{ pathways } \\
\hline & $\begin{array}{l}\text { hsa04010 MAPK } \\
\text { signaling pathway }\end{array}$ & $\begin{array}{l}\text { hsa04010 MAPK } \\
\text { signaling pathway }\end{array}$ & $\begin{array}{l}\text { hsa04010 MAPK } \\
\text { signaling pathway }\end{array}$ & $\begin{array}{l}\text { hsa04010 MAPK signaling } \\
\text { pathway }\end{array}$ & $\begin{array}{l}\text { hsa04010 MAPK } \\
\text { signaling pathway }\end{array}$ \\
\hline & $\begin{array}{l}\text { hsa04060 Cytokine- } \\
\text { cytokine receptor } \\
\text { interaction }\end{array}$ & $\begin{array}{l}\text { hsa04060 Cytokine- } \\
\text { cytokine receptor } \\
\text { interaction }\end{array}$ & $\begin{array}{l}\text { hsa04060 Cytokine- } \\
\text { cytokine receptor } \\
\text { interaction }\end{array}$ & $\begin{array}{l}\text { hsa04060 Cytokine- } \\
\text { cytokine receptor } \\
\text { interaction }\end{array}$ & $\begin{array}{l}\text { hsa04060 Cytokine- } \\
\text { cytokine receptor } \\
\text { interaction }\end{array}$ \\
\hline & hsa04210 Apoptosis & hsa04210 Apoptosis & hsa04210 Apoptosis & & hsa04210 Apoptosis \\
\hline & $\begin{array}{l}\text { hsa04350 TGF-beta } \\
\text { signaling pathway }\end{array}$ & $\begin{array}{l}\text { hsa04350 TGF-beta } \\
\text { signaling pathway }\end{array}$ & $\begin{array}{l}\text { hsa04350 TGF-beta } \\
\text { signaling pathway }\end{array}$ & $\begin{array}{l}\text { hsa04350 TGF-beta } \\
\text { signaling pathway }\end{array}$ & $\begin{array}{l}\text { hsa04350 TGF-beta } \\
\text { signaling pathway }\end{array}$ \\
\hline & $\begin{array}{l}\text { hsa04380 Osteoclast } \\
\text { differentiation }\end{array}$ & $\begin{array}{l}\text { hsa04380 Osteoclast } \\
\text { differentiation }\end{array}$ & & & \\
\hline & $\begin{array}{l}\text { hsa04612 Antigen } \\
\text { processing and } \\
\text { presentation }\end{array}$ & $\begin{array}{l}\text { hsa04612 Antigen } \\
\text { processing and } \\
\text { presentation }\end{array}$ & $\begin{array}{l}\text { hsa04612 Antigen } \\
\text { processing and } \\
\text { presentation }\end{array}$ & & $\begin{array}{l}\text { hsa04612 Antigen } \\
\text { processing and } \\
\text { presentation }\end{array}$ \\
\hline & $\begin{array}{l}\text { hsa04920 Adipocytokir } \\
\text { e signaling pathway }\end{array}$ & $\begin{array}{l}\text { hsa04920 Adipocytokin } \\
\text { e signaling pathway }\end{array}$ & & $\begin{array}{l}\text { hsa04920 Adipocytokine } \\
\text { signaling pathway }\end{array}$ & $\begin{array}{l}\text { hsa04920 Adipocytokin } \\
\text { e signaling pathway }\end{array}$ \\
\hline & $\begin{array}{l}\text { hsa05323 Rheumatoid } \\
\text { arthritis }\end{array}$ & $\begin{array}{l}\text { hsa05323 Rheumatoid } \\
\text { arthritis }\end{array}$ & $\begin{array}{l}\text { hsa05323 Rheumatoid } \\
\text { arthritis }\end{array}$ & $\begin{array}{l}\text { hsa05323 Rheumatoid } \\
\text { arthritis }\end{array}$ & $\begin{array}{l}\text { hsa05323 Rheumatoid } \\
\text { arthritis }\end{array}$ \\
\hline & & & $\begin{array}{l}\text { hsa04650 Natural killer } \\
\text { cell mediated } \\
\text { cytotoxicity }\end{array}$ & & $\begin{array}{l}\text { hsa04650 Natural killer } \\
\text { cell mediated } \\
\text { cytotoxicity }\end{array}$ \\
\hline & & & & $\begin{array}{l}\text { hsa04664 Fc epsilon RI } \\
\text { signaling pathway }\end{array}$ & \\
\hline & & & & hsa05310 Asthma & \\
\hline & & & & & $\begin{array}{l}\text { hsa04620 Toll-like } \\
\text { receptor signaling } \\
\text { pathway }\end{array}$ \\
\hline activity & $\begin{array}{l}\text { To decrease signs and } \\
\text { symptoms of } \\
\text { rheumatoid arthritis } \\
\text { [DS:H00287 H00288 } \\
\text { H00630] }\end{array}$ & not defined & $\begin{array}{l}\text { Treatment of } \\
\text { rheumatoid arthritis } \\
\text { and other chronic } \\
\text { inflammatory disease }\end{array}$ & $\begin{array}{l}\text { Treatment of } \\
\text { inflammatory disorders, } \\
\text { rheumatoid arthritis, } \\
\text { uveitis, asthma and } \\
\text { Crohn's disease }\end{array}$ & $\begin{array}{l}\text { Treatment of } \\
\text { rheumatoid arthritis } \\
\text { and inflammatory } \\
\text { bowel disease, } \\
\text { specifically Crohn's } \\
\text { disease }\end{array}$ \\
\hline
\end{tabular}

USP classification; name of biologics and manufacturer; class and targets of biologics; detected pathways; action of each biologics. 
Table 16. TNF human pathways from KEGG

Hits 45 from KEGG database

\section{KEGG PATHWAY that have TNF as an participant}

hsa04010

MAPK signaling pathway - Homo sapiens (human)

hsa04060

Cytokine-cytokine receptor interaction - Homo sapiens (human)

hsa04064

hsa04150

hsa04210

hsa04350

hsa04380

hsa04612

hsa04620

hsa04621

hsa04622

hsa04640

hsa04650

hsa04660

hsa04664

hsa04668

hsa04920

hsa04930

hsa04932

hsa04940

hsa05010

hsa05014

hsa05133

hsa05134

hsa05140

hsa05142

hsa05143

hsa05144

hsa05145

hsa05146

hsa05152

hsa05160

hsa05161

hsa05164

hsa05166

hsa05168

hsa05205

hsa05310

hsa05321

hsa05322

hsa05323

hsa05330

hsa05332

hsa05410

hsa05414

NF-kappa B signaling pathway - Homo sapiens (human)

mTOR signaling pathway - Homo sapiens (human)

Apoptosis - Homo sapiens (human)

TGF-beta signaling pathway - Homo sapiens (human)

Osteoclast differentiation - Homo sapiens (human)

Antigen processing and presentation - Homo sapiens (human)

Toll-like receptor signaling pathway - Homo sapiens (human)

NOD-like receptor signaling pathway - Homo sapiens (human)

RIG-I-like receptor signaling pathway - Homo sapiens (human)

Hematopoietic cell lineage - Homo sapiens (human)

Natural killer cell mediated cytotoxicity - Homo sapiens (human)

T cell receptor signaling pathway - Homo sapiens (human)

Fc epsilon RI signaling pathway - Homo sapiens (human)

TNF signaling pathway - Homo sapiens (human)

Adipocytokine signaling pathway - Homo sapiens (human)

Type II diabetes mellitus - Homo sapiens (human)

Non-alcoholic fatty liver disease (NAFLD) - Homo sapiens (human)

Type I diabetes mellitus - Homo sapiens (human)

Alzheimer's disease - Homo sapiens (human)

Amyotrophic lateral sclerosis (ALS) - Homo sapiens (human)

Pertussis - Homo sapiens (human)

Legionellosis - Homo sapiens (human)

Leishmaniasis - Homo sapiens (human)

Chagas disease (American trypanosomiasis) - Homo sapiens (human)

African trypanosomiasis - Homo sapiens (human)

Malaria - Homo sapiens (human)

Toxoplasmosis - Homo sapiens (human)

Amoebiasis - Homo sapiens (human)

Tuberculosis - Homo sapiens (human)

Hepatitis C - Homo sapiens (human)

Hepatitis B - Homo sapiens (human)

Influenza A - Homo sapiens (human)

HTLV-I infection - Homo sapiens (human)

Herpes simplex infection - Homo sapiens (human)

Proteoglycans in cancer - Homo sapiens (human)

Asthma - Homo sapiens (human)

Inflammatory bowel disease (IBD) - Homo sapiens (human)

Systemic lupus erythematosus - Homo sapiens (human)

Rheumatoid arthritis - Homo sapiens (human)

Allograft rejection - Homo sapiens (human)

Graft-versus-host disease - Homo sapiens (human)

Hypertrophic cardiomyopathy (HCM) - Homo sapiens (human)

Dilated cardiomyopathy - Homo sapiens (human)
Is TNF a product or a signal in the pathway?

signal (only partially, no Jak-STAT)) collection of proteins signal

only probable signal

signal

signal

signal

signal

product

product

product

signal

product

product

product

signal

signal (other half is Jak-STAT

dipendant)

signal

signal and product

signal and product

na
na
na
na
na
na
na
na
na
na
signal
signal
product
product
signal
product
na
na
na
na
na
na
na
na
na

KEGG pathway ID and name; position of TNF ( as a signal or a product) in a pathway where data are available. na: does not apply 


\section{Table 17.}

\section{Relationship between AIDSNP pathways and TNF pathways}

\section{Pathways common to all AID SNP pathways and TNF pathways}

$\begin{array}{ccl}\text { No. } & \text { KEGG pathway ID } & \text { KEGG pathway name } \\ 1 & \text { hsa04010 } & \text { MAPK signaling pathway } \\ 2 & \text { hsa04060 } & \text { Cytokine-cytokine receptor interaction } \\ 3 & \text { hsa04064 } & \text { NF-kappa B signaling pathway } \\ 4 & \text { hsa04210 } & \text { Apoptosis (survival mode by NFkB pathway) } \\ 5 & \text { hsa04350 } & \text { TGF-beta signaling pathway } \\ 6 & \text { hsa04380 } & \text { Osteoclast differentiation } \\ 7 & \text { hsa04612 } & \text { Antigen processing and presentation } \\ 8 & \text { hsa04620 } & \text { Toll-like receptor signaling pathway } \\ 9 & \text { hsa04621 } & \text { NOD-like receptor signaling pathway } \\ 10 & \text { hsa04640 } & \text { Hematopoietic cell lineage } \\ 11 & \text { hsa04660 } & \text { T cell receptor signaling pathway } \\ 12 & \text { hsa04664 } & \text { Fc epsilon RI signaling pathway } \\ 13 & \text { hsa04668 } & \text { TNF signaling pathway } \\ 14 & \text { hsa04920 } & \text { Adipocytokine signaling pathway }\end{array}$

\section{Pathways common to AID SNP pathways but not TNF pathways}

\section{No. KEGG pathway ID KEGG pathway name}

$\begin{array}{ll}1 & \text { hsa04120 } \\ 2 & \text { hsa04630 } \\ 3 & \text { hsa04330 } \\ 4 & \text { hsa04672 } \\ 5 & \text { hsa04662 }\end{array}$

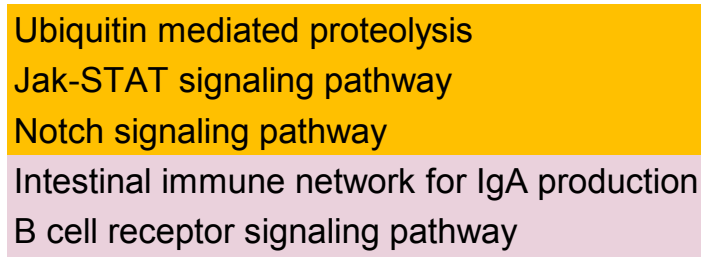

Note:

pathways common to ncSNP and TNF pathways

pathways common to missSNP, ncSNP and TNF pathways

pathways common to missSNP and TNF pathways missSNP pathways or ncSNP pathways, but not TNF

pathways common to missSNP pathways and ncSNP pathways, but not TNF 
Table 18. STRING pathways enrichment data for AID GWAS SNP genesets

KEGG ID Term name of the KEGG pathway $\begin{aligned} & \text { Number } \\ & \text { Of Genes p-value } \quad p-v a l u e \_f d r \quad p-v a l u e \_b o n f e r r o n i\end{aligned}$

\begin{tabular}{|c|c|c|c|c|c|}
\hline \multicolumn{6}{|c|}{ missSNPs set } \\
\hline hsa04630 & Jak-STAT signaling pathway & 5 & $3.25 \mathrm{E}-07$ & $7.70 \mathrm{E}-05$ & 7.70E-05 \\
\hline hsa04621 & NOD-like receptor signaling pathway & 3 & 2.17E-05 & $2.57 \mathrm{E}-03$ & $5.14 \mathrm{E}-03$ \\
\hline hsa04060 & Cytokine-cytokine receptor interaction & 4 & $1.08 \mathrm{E}-04$ & $8.51 \mathrm{E}-03$ & $2.55 \mathrm{E}-02$ \\
\hline hsa04380 & Osteoclast differentiation & 3 & 2.16E-04 & $1.02 \mathrm{E}-02$ & $5.12 \mathrm{E}-02$ \\
\hline hsa05162 & Measles & 3 & 2.67E-04 & $1.58 \mathrm{E}-02$ & $6.33 \mathrm{E}-02$ \\
\hline hsa05152 & Tuberculosis & 3 & $6.16 \mathrm{E}-04$ & $2.92 \mathrm{E}-02$ & $1.46 \mathrm{E}-01$ \\
\hline \multicolumn{6}{|c|}{ ncSNPs set } \\
\hline hsa04060 & Cytokine-cytokine receptor interaction & 4 & $1.54 \mathrm{E}-04$ & $3.64 \mathrm{E}-02$ & $3.64 \mathrm{E}-02$ \\
\hline \multicolumn{6}{|c|}{ all SNPs set } \\
\hline hsa04060 & Cytokine-cytokine receptor interaction & 8 & 4.22E-08 & $1.00 \mathrm{E}-05$ & 1.00E-05 \\
\hline hsa04630 & Jak-STAT signaling pathway & 6 & $5.83 \mathrm{E}-07$ & $6.91 \mathrm{E}-05$ & $1.38 \mathrm{E}-04$ \\
\hline hsa04621 & NOD-like receptor signaling pathway & 4 & 4.73E-06 & $3.74 \mathrm{E}-04$ & $1.12 \mathrm{E}-03$ \\
\hline hsa05162 & Measles & 4 & 1.31E-04 & $7.79 \mathrm{E}-03$ & $3.11 \mathrm{E}-02$ \\
\hline hsa05120 & Epithelial cell signaling in $\mathrm{H}$. pylori infect & 3 & 2.99E-04 & $1.33 \mathrm{E}-02$ & 7.07E-02 \\
\hline hsa05140 & Leishmaniasis & 3 & 3.87E-04 & $1.33 \mathrm{E}-02$ & $9.18 \mathrm{E}-02$ \\
\hline hsa05152 & Tuberculosis & 4 & $3.92 \mathrm{E}-04$ & $1.33 \mathrm{E}-02$ & 9.29E-02 \\
\hline hsa04062 & Chemokine signaling pathway & 4 & 4.74E-04 & $1.41 \mathrm{E}-02$ & $1.12 \mathrm{E}-01$ \\
\hline hsa04660 & T cell receptor signaling pathway & 3 & $1.21 \mathrm{E}-03$ & 3.19E-02 & 2.87E-01 \\
\hline hsa05310 & Asthma & 2 & $1.64 \mathrm{E}-03$ & $3.88 \mathrm{E}-02$ & $3.88 \mathrm{E}-01$ \\
\hline hsa04380 & Osteoclast differentiation & 3 & $1.82 \mathrm{E}-03$ & $3.92 \mathrm{E}-02$ & 4.31E-01 \\
\hline hsa05160 & Hepatitis C & 3 & 2.19E-03 & 4.07E-02 & 5.18E-01 \\
\hline hsa05145 & Toxoplasmosis & 3 & 2.23E-03 & 4.07E-02 & 5.29E-01 \\
\hline
\end{tabular}


Table 19. Enriched KEGG pathway-based set for missSNP geneset by ConsensusPathDB

uploaded list:

22

mapped entities:

20

enriched pathway-based sets:

23

18 genes $(90.0 \%)$ from the input list are present in at least one pathway

pathway name

NOD-like receptor signaling pathway - Homo sapiens (human)

Inflammatory bowel disease (IBD) - Homo sapiens (human)

Jak-STAT signaling pathway - Homo sapiens (human)

TNF signaling pathway - Homo sapiens (human)

Osteoclast differentiation - Homo sapiens (human)

Cytokine-cytokine receptor interaction - Homo sapiens (human)

Measles - Homo sapiens (human)

Tuberculosis - Homo sapiens (human)

Leishmaniasis - Homo sapiens (human)

Hematopoietic cell lineage - Homo sapiens (human)

NF-kappa B signaling pathway - Homo sapiens (human)

Toxoplasmosis - Homo sapiens (human)

Asthma - Homo sapiens (human)

Hepatitis C - Homo sapiens (human)

Influenza A - Homo sapiens (human)

Herpes simplex infection - Homo sapiens (human)

Legionellosis - Homo sapiens (human)

Epstein-Barr virus infection - Homo sapiens (human)

Shigellosis - Homo sapiens (human)

Fc epsilon RI signaling pathway - Homo sapiens (human)

RIG-I-like receptor signaling pathway - Homo sapiens (human)

Adipocytokine signaling pathway - Homo sapiens (human)

Apoptosis - Homo sapiens (human)

$\begin{array}{ccccc}\text { set size } & \begin{array}{c}\text { candidates } \\ \text { contained }\end{array} & \text { p-value } & \text { q-value } & \begin{array}{c}\text { pathway } \\ \text { source }\end{array} \\ 57 & 5(8.8 \%) & 2.82 E-08 & 9.30 E-07 & \text { KEGG } \\ 67 & 4(6.3 \%) & 3.06 E-06 & 3.61 E-05 & \text { KEGG } \\ 156 & 5(3.4 \%) & 3.28 E-06 & 3.61 E-05 & \text { KEGG } \\ 110 & 4(3.7 \%) & 2.72 E-05 & 0.000224 & \text { KEGG } \\ 131 & 4(3.3 \%) & 4.10 E-05 & 0.000229 & \text { KEGG } \\ 265 & 5(2.0 \%) & 4.16 E-05 & 0.000229 & \text { KEGG } \\ 134 & 4(3.1 \%) & 5.43 E-05 & 0.000256 & \text { KEGG } \\ 179 & 4(2.3 \%) & 0.000165 & 0.00068 & \text { KEGG } \\ 74 & 3(4.2 \%) & 0.000217 & 0.000797 & \text { KEGG } \\ 87 & 3(3.7 \%) & 0.000319 & 0.00105 & \text { KEGG } \\ 91 & 3(3.4 \%) & 0.000393 & 0.00118 & \text { KEGG } \\ 120 & 3(2.6 \%) & 0.00086 & 0.00237 & \text { KEGG } \\ 32 & 2(6.7 \%) & 0.00112 & 0.00283 & \text { KEGG } \\ 133 & 3(2.3 \%) & 0.0012 & 0.00283 & \text { KEGG } \\ 177 & 3(1.8 \%) & 0.0026 & 0.00572 & \text { KEGG } \\ 186 & 3(1.7 \%) & 0.00306 & 0.00632 & \text { KEGG } \\ 55 & 2(3.7 \%) & 0.00358 & 0.00696 & \text { KEGG } \\ 202 & 3(1.5 \%) & 0.00407 & 0.00746 & \text { KEGG } \\ 61 & 2(3.3 \%) & 0.00455 & 0.00791 & \text { KEGG } \\ 70 & 2(2.9 \%) & 0.00563 & 0.00868 & \text { KEGG } \\ 70 & 2(2.9 \%) & 0.00579 & 0.00868 & \text { KEGG } \\ 70 & 2(2.9 \%) & 0.00579 & 0.00868 & \text { KEGG } \\ 86 & 2(2.5 \%) & 0.0079 & 0.0113 & \text { KEGG }\end{array}$

Number of queried genes entered for enrichment analyses and number of genes mapped in pathways; number of enriched pathway sets; pathways names with the number of genes in each pathway and percentage of queried genes for each pathway along with number of genes in a gene set for each pathway; $p$ values, after fdr and Bonferroni corrections. 
Table 20. Enriched KEGG pathway-based sets for allSNP set by ConsensusPathDB uploaded list:

56

mapped entities:

enriched pathway-based sets:
54

27

39 genes (72.2\%) from the input list are present in at least one pathway.

pathway name

Cytokine-cytokine receptor interaction - Homo sapiens (human)

Epstein-Barr virus infection - Homo sapiens (human)

Inflammatory bowel disease (IBD) - Homo sapiens (human)

Jak-STAT signaling pathway - Homo sapiens (human)

Autoimmune thyroid disease - Homo sapiens (human)

NOD-like receptor signaling pathway - Homo sapiens (human)

Asthma - Homo sapiens (human)

NF-kappa B signaling pathway - Homo sapiens (human)

Allograft rejection - Homo sapiens (human)

Herpes simplex infection - Homo sapiens (human)

TNF signaling pathway - Homo sapiens (human)

Toxoplasmosis - Homo sapiens (human)

Staphylococcus aureus infection - Homo sapiens (human)

Measles - Homo sapiens (human)

Systemic lupus erythematosus - Homo sapiens (human)

Epithelial cell signaling in Helicobacter pylori infection - Homo sapiens (human)

Leishmaniasis - Homo sapiens (human)

Pertussis - Homo sapiens (human)

Tuberculosis - Homo sapiens (human)

Chemokine signaling pathway - Homo sapiens (human)

T cell receptor signaling pathway - Homo sapiens (human)

Chagas disease (American trypanosomiasis) - Homo sapiens (human)

Viral carcinogenesis - Homo sapiens (human)

Toll-like receptor signaling pathway - Homo sapiens (human)

Endocytosis - Homo sapiens (human)

Primary immunodeficiency - Homo sapiens (human)

Osteoclast differentiation - Homo sapiens (human)

$\begin{array}{lllll}\text { set size } & \begin{array}{l}\text { candidates } \\ \text { contained }\end{array} & \text { p-value } & \text { q-value } & \begin{array}{c}\text { pathway } \\ \text { source }\end{array} \\ 265 & 11(4.4 \%) & 7.32 \mathrm{E}-10 & 3.58 \mathrm{E}-08 & \text { KEGG } \\ 202 & 8(4.0 \%) & 4.27 \mathrm{E}-07 & 1.05 \mathrm{E}-05 & \text { KEGG } \\ 67 & 5(7.9 \%) & 2.88 \mathrm{E}-06 & 4.70 \mathrm{E}-05 & \text { KEGG } \\ 156 & 6(4.1 \%) & 1.31 \mathrm{E}-05 & 0.000161 & \text { KEGG } \\ 54 & 4(7.7 \%) & 3.50 \mathrm{E}-05 & 0.000343 & \text { KEGG } \\ 57 & 4(7.0 \%) & 5.05 \mathrm{E}-05 & 0.000412 & \text { KEGG } \\ 32 & 3(10.0 \%) & 0.000166 & 0.00117 & \text { KEGG } \\ 91 & 4(4.5 \%) & 0.000275 & 0.00168 & \text { KEGG } \\ 39 & 3(8.1 \%) & 0.000313 & 0.0017 & \text { KEGG } \\ 186 & 5(2.8 \%) & 0.000436 & 0.00214 & \text { KEGG } \\ 110 & 4(3.7 \%) & 0.000621 & 0.00277 & \text { KEGG } \\ 120 & 4(3.5 \%) & 0.00076 & 0.0031 & \text { KEGG } \\ 57 & 3(5.7 \%) & 0.000907 & 0.00342 & \text { KEGG } \\ 134 & 4(3.1 \%) & 0.0012 & 0.0042 & \text { KEGG } \\ 136 & 4(3.0 \%) & 0.00131 & 0.00427 & \text { KEGG } \\ 68 & 3(4.5 \%) & 0.00179 & 0.00548 & \text { KEGG } \\ 74 & 3(4.2 \%) & 0.0022 & 0.00635 & \text { KEGG } \\ 75 & 3(4.0 \%) & 0.00247 & 0.00673 & \text { KEGG } \\ 179 & 4(2.3 \%) & 0.00341 & 0.00879 & \text { KEGG } \\ 189 & 4(2.2 \%) & 0.00417 & 0.0102 & \text { KEGG } \\ 104 & 3(3.0 \%) & 0.00572 & 0.013 & \text { KEGG } \\ 104 & 3(2.9 \%) & 0.00604 & 0.013 & \text { KEGG } \\ 206 & 4(2.0 \%) & 0.00622 & 0.013 & \text { KEGG } \\ 106 & 3(2.9 \%) & 0.00637 & 0.013 & \text { KEGG } \\ 213 & 4(1.9 \%) & 0.00677 & 0.0133 & \text { KEGG } \\ 36 & 2(5.7 \%) & 0.00705 & 0.0133 & \text { KEGG } \\ 131 & 3(2.5 \%) & 0.00941 & 0.0171 & \text { KEGG }\end{array}$

Number of queried genes entered for enrichment analyses and number of genes mapped in pathways; number of enriched pathway sets; pathways names with the number of genes in each pathway and percentage of queried genes for each pathway along with number of genes in a gene set for each pathway; $p$ values, after fdr and Bonferroni corrections. 
Table 21. GO term BP, MF and CC annotation enrichment for AID SNP sets

(yellow highlited are significant terms based on $\mathrm{p}$ values for Bonferroni or FDR)

$\begin{array}{ll}\text { missSNP set } & \text { BP terms } \\ \text { GO_id } & \\ \text { GO:0002376 } & \text { immune system process } \\ \text { GO:0009617 } & \text { response to bacterium } \\ \text { GO:0002684 } & \text { positive regulation of immune system process } \\ \text { GO:0001818 } & \text { negative regulation of cytokine production } \\ \text { GO:0002682 } & \text { regulation of immune system process } \\ \text { GO:0006955 } & \text { immune response } \\ \text { GO:0051707 } & \text { response to other organism } \\ \text { GO:0043207 } & \text { response to external biotic stimulus } \\ \text { GO:0009607 } & \text { response to biotic stimulus } \\ \text { GO:0045088 } & \text { regulation of innate immune response } \\ \text { GO:0070423 } & \text { nucleotide-binding oligomerization domain containing signaling pa } \\ \text { GO:0034136 } & \text { negative regulation of toll-like receptor } 2 \text { signaling pathway } \\ \text { GO:0050776 } & \text { regulation of immune response } \\ \text { GO:0002753 } & \text { cytoplasmic pattern recognition receptor signaling pathway } \\ \text { GO:0002822 } & \text { regulation of adaptive immune response based on somatic recom } \\ \text { GO:0002697 } & \text { regulation of immune effector process } \\ \text { GO:0050731 } & \text { positive regulation of peptidyl-tyrosine phosphorylation } \\ \text { GO:0002819 } & \text { regulation of adaptive immune response }\end{array}$

ncSNP set

GO_id

GO:0002520

GO:0002407

GO:0036336

GO:0038112

GO:0001775

GO:0045321

GO:0030097

GO:0048534

GO:0002521

GO:0007221

GO:0046649

GO:0032655

GO:0043117

GO:0030098

GO:0009611

GO:0043112

GO:0071350

GO:0050900

GO:0030183

allSNP set

GO_id

GO:0006955

GO:0002520

GO:0009617

GO:0002376

GO:0032495

GO:0043207

GO:0051707

GO:0009605

GO:0032655

GO:0045321

immune system development

dendritic cell chemotaxis

dendritic cell migration

interleukin-8-mediated signaling pathway

cell activation

leukocyte activation

hemopoiesis

hematopoietic or lymphoid organ development

leukocyte differentiation

positive regulation of transcription of Notch receptor target

lymphocyte activation

regulation of interleukin-12 production

positive regulation of vascular permeability

lymphocyte differentiation

response to wounding

receptor metabolic process

cellular response to interleukin-15

leukocyte migration

$B$ cell differentiation

immune response

immune system development

response to bacterium

immune system process

response to muramyl dipeptide

response to external biotic stimulus

response to other organism

response to external stimulus

regulation of interleukin-12 production

leukocyte activation

$\begin{array}{ccrr}\begin{array}{c}\text { No of } \\ \text { genes }\end{array} & \text { p-value } & \text { p-value_fdr } & \text { p-value_bonferroni } \\ 12 & 1.73 E-08 & 2.18 \mathrm{E}-04 & 2.18 \mathrm{E}-04 \\ 7 & 4.61 \mathrm{E}-08 & 2.91 \mathrm{E}-04 & 5.81 \mathrm{E}-04 \\ 8 & 1.26 \mathrm{E}-07 & 5.28 \mathrm{E}-04 & 1.58 \mathrm{E}-03 \\ 5 & 2.24 \mathrm{E}-07 & 7.07 \mathrm{E}-04 & 2.83 \mathrm{E}-03 \\ 9 & 3.05 \mathrm{E}-07 & 7.70 \mathrm{E}-04 & 3.85 \mathrm{E}-03 \\ 9 & 8.23 \mathrm{E}-07 & 1.33 \mathrm{E}-03 & 1.04 \mathrm{E}-02 \\ 7 & 8.43 \mathrm{E}-07 & 1.33 \mathrm{E}-03 & 1.06 \mathrm{E}-02 \\ 7 & 8.43 \mathrm{E}-07 & 1.33 \mathrm{E}-03 & 1.06 \mathrm{E}-02 \\ 7 & 1.17 \mathrm{E}-06 & 1.64 \mathrm{E}-03 & 1.48 \mathrm{E}-02 \\ 5 & 2.23 \mathrm{E}-06 & 2.81 \mathrm{E}-03 & 2.81 \mathrm{E}-02 \\ 3 & 2.61 \mathrm{E}-06 & 2.87 \mathrm{E}-03 & 3.29 \mathrm{E}-02 \\ 2 & 2.73 \mathrm{E}-06 & 2.87 \mathrm{E}-03 & 3.44 \mathrm{E}-02 \\ 7 & 3.92 \mathrm{E}-06 & 3.81 \mathrm{E}-03 & 4.95 \mathrm{E}-02 \\ 3 & 4.75 \mathrm{E}-06 & 3.97 \mathrm{E}-03 & 5.98 \mathrm{E}-02 \\ 4 & 4.87 \mathrm{E}-06 & 3.97 \mathrm{E}-03 & 6.14 \mathrm{E}-02 \\ 5 & 5.03 \mathrm{E}-06 & 3.97 \mathrm{E}-03 & 6.35 \mathrm{E}-02 \\ 4 & 6.12 \mathrm{E}-06 & 4.42 \mathrm{E}-03 & 7.72 \mathrm{E}-02 \\ 4 & 6.32 \mathrm{E}-06 & 4.42 \mathrm{E}-03 & 7.96 \mathrm{E}-02\end{array}$

No of

$\begin{array}{ccrr}\text { genes } & \text { p-value } & \text { p-value_fdr } & p \text {-value_bonferroni } \\ 7 & 3.13 \mathrm{E}-07 & 2.48 \mathrm{E}-03 & 3.94 \mathrm{E}-03 \\ 3 & 4.80 \mathrm{E}-07 & 2.48 \mathrm{E}-03 & 6.05 \mathrm{E}-03\end{array}$

$\begin{array}{llll}7 & 3.13 \mathrm{E}-07 & 2.48 \mathrm{E}-03 & 3.94 \mathrm{E}-03 \\ 3 & 4.80 \mathrm{E}-07 & 2.48 \mathrm{E}-03 & 6.05 \mathrm{E}-03 \\ 3 & 5.90 \mathrm{E}-07 & 2.48 \mathrm{E}-03 & 7.44 \mathrm{E}-03 \\ 2 & 1.09 \mathrm{E}-06 & 2.59 \mathrm{E}-03 & 1.37 \mathrm{E}-02 \\ 7 & 1.16 \mathrm{E}-06 & 2.59 \mathrm{E}-03 & 1.46 \mathrm{E}-02 \\ 6 & 1.23 \mathrm{E}-06 & 2.59 \mathrm{E}-03 & 1.55 \mathrm{E}-02 \\ 6 & 2.60 \mathrm{E}-06 & 4.69 \mathrm{E}-03 & 3.28 \mathrm{E}-02 \\ 6 & 4.58 \mathrm{E}-06 & 6.51 \mathrm{E}-03 & 5.77 \mathrm{E}-02 \\ 5 & 4.65 \mathrm{E}-06 & 6.51 \mathrm{E}-03 & 5.86 \mathrm{E}-02 \\ 2 & 6.52 \mathrm{E}-06 & 8.22 \mathrm{E}-03 & 8.22 \mathrm{E}-02 \\ 5 & 9.86 \mathrm{E}-06 & 1.13 \mathrm{E}-02 & 1.24 \mathrm{E}-01 \\ 3 & 1.19 \mathrm{E}-05 & 1.25 \mathrm{E}-02 & 1.50 \mathrm{E}-01 \\ 2 & 3.04 \mathrm{E}-05 & 2.79 \mathrm{E}-02 & 3.83 \mathrm{E}-01 \\ 4 & 3.10 \mathrm{E}-05 & 2.79 \mathrm{E}-02 & 3.90 \mathrm{E}-01 \\ 7 & 3.71 \mathrm{E}-05 & 3.12 \mathrm{E}-02 & 4.68 \mathrm{E}-01 \\ 3 & 4.45 \mathrm{E}-05 & 3.50 \mathrm{E}-02 & 5.60 \mathrm{E}-01 \\ 2 & 4.87 \mathrm{E}-05 & 3.61 \mathrm{E}-02 & 6.14 \mathrm{E}-01 \\ 4 & 6.74 \mathrm{E}-05 & 4.71 \mathrm{E}-02 & 8.50 \mathrm{E}-01 \\ 3 & 7.10 \mathrm{E}-05 & 4.71 \mathrm{E}-02 & 8.95 \mathrm{E}-01\end{array}$

No of

genes $p$-value $p$-value_fdr p-value_bonferroni

$17 \quad 4.64 \mathrm{E}-11 \quad 5.85 \mathrm{E}-07 \quad 5.85 \mathrm{E}-07$

$\begin{array}{cccc}17 & 4.64 \mathrm{E}-11 & 5.85 \mathrm{E}-07 & 5.85 \mathrm{E}-07 \\ 11 & 9.88 \mathrm{E}-10 & 6.22 \mathrm{E}-06 & 1.24 \mathrm{E}-05 \\ 10 & 2.76 \mathrm{E}-09 & 8.91 \mathrm{E}-06 & 3.48 \mathrm{E}-05 \\ 18 & 2.83 \mathrm{E}-09 & 8.91 \mathrm{E}-06 & 3.56 \mathrm{E}-05 \\ 4 & 4.54 \mathrm{E}-09 & 1.14 \mathrm{E}-05 & 5.72 \mathrm{E}-05 \\ 11 & 1.28 \mathrm{E}-08 & 2.26 \mathrm{E}-05 & 1.62 \mathrm{E}-04 \\ 11 & 1.28 \mathrm{E}-08 & 2.26 \mathrm{E}-05 & 1.62 \mathrm{E}-04 \\ 16 & 1.44 \mathrm{E}-08 & 2.26 \mathrm{E}-05 & 1.81 \mathrm{E}-04 \\ 5 & 2.04 \mathrm{E}-08 & 2.44 \mathrm{E}-05 & 2.57 \mathrm{E}-04 \\ 9 & 2.06 \mathrm{E}-08 & 2.44 \mathrm{E}-05 & 2.60 \mathrm{E}-04\end{array}$


GO:0009607

GO:0030097

GO:0002682

GO:0001775

GO:0098542

GO:0048534

GO:0050777

GO:0009611

GO:0002819

GO:0031347

GO:0070423

GO:0002237

GO:0001819

GO:0002684

GO:0002753

GO:0050864

GO:0071350

GO:0070663

GO:0042742

GO:0046649

GO:0050776

GO:0001817

GO:0002252

GO:0035872

GO:0070672

GO:0051249

GO:0042127

GO:0002407

GO:0002822

GO:0043331

GO:0038112

GO:0036336

GO:0032496

GO:0006954

GO:0045088

GO:0002521

GO:0006950

GO:0001818

GO:0034136

GO:0002697

GO:0032735

GO:0006952

GO:0050670

GO:0032944

GO:0032675

GO:0007221

GO:0002677

GO:0030098

GO:0030595

GO:0050869

GO:0032720

GO:0080134

GO:0045087

GO:0042110

GO:0070431

GO:0034135

GO:0032479

GO:0014070

GO:0071219

GO:2000026

GO:0043330 response to biotic stimulus

hemopoiesis

regulation of immune system process

cell activation

defense response to other organism

hematopoietic or lymphoid organ development

negative regulation of immune response

response to wounding

regulation of adaptive immune response

regulation of defense response

nucleotide-binding oligomerization domain containing signaling pa

response to molecule of bacterial origin

positive regulation of cytokine production

positive regulation of immune system process

cytoplasmic pattern recognition receptor signaling pathway

regulation of $B$ cell activation

cellular response to interleukin-15

regulation of leukocyte proliferation

defense response to bacterium

lymphocyte activation

regulation of immune response

regulation of cytokine production

immune effector process

nucleotide-binding domain, leucine rich repeat containing recepto

response to interleukin-15

regulation of lymphocyte activation

regulation of cell proliferation

dendritic cell chemotaxis

regulation of adaptive immune response based on somatic recom

response to dsRNA

interleukin-8-mediated signaling pathway

dendritic cell migration

response to lipopolysaccharide

inflammatory response

regulation of innate immune response

leukocyte differentiation

response to stress

negative regulation of cytokine production

negative regulation of toll-like receptor 2 signaling pathway

regulation of immune effector process

positive regulation of interleukin-12 production

defense response

regulation of lymphocyte proliferation

regulation of mononuclear cell proliferation

regulation of interleukin- 6 production

positive regulation of transcription of Notch receptor target

negative regulation of chronic inflammatory response

lymphocyte differentiation

leukocyte chemotaxis

negative regulation of $B$ cell activation

negative regulation of tumor necrosis factor production

regulation of response to stress

innate immune response

T cell activation

nucleotide-binding oligomerization domain containing 2 signaling

regulation of toll-like receptor 2 signaling pathway

regulation of type I interferon production

response to organic cyclic compound

cellular response to molecule of bacterial origin

regulation of multicellular organismal development

response to exogenous dsRNA
$11 \quad 2.13 \mathrm{E}-08 \quad 2.44 \mathrm{E}-05 \quad 2.68 \mathrm{E}-04$

$9 \quad 6.24 \mathrm{E}-08 \quad 6.56 \mathrm{E}-05 \quad 7.87 \mathrm{E}-04$

$13 \quad 8.95 \mathrm{E}-08 \quad 8.68 \mathrm{E}-05 \quad 1.13 \mathrm{E}-03$

$10 \quad 9.77 \mathrm{E}-08 \quad 8.80 \mathrm{E}-05 \quad 1.23 \mathrm{E}-03$

$8 \quad 1.27 \mathrm{E}-07 \quad 1.07 \mathrm{E}-04 \quad 1.60 \mathrm{E}-03$

$9 \quad 1.44 \mathrm{E}-07 \quad 1.13 \mathrm{E}-04 \quad 1.81 \mathrm{E}-03$

$5 \quad 1.77 \mathrm{E}-07 \quad 1.23 \mathrm{E}-04 \quad 2.23 \mathrm{E}-03$

$12 \quad 1.85 \mathrm{E}-07 \quad 1.23 \mathrm{E}-04 \quad 2.33 \mathrm{E}-03$

$6 \quad 1.85 \mathrm{E}-07 \quad 1.23 \mathrm{E}-04 \quad 2.34 \mathrm{E}-03$

$9 \quad 2.54 \mathrm{E}-07 \quad 1.60 \mathrm{E}-04 \quad 3.21 \mathrm{E}-03$

$4 \quad 2.75 \mathrm{E}-07 \quad 1.65 \mathrm{E}-04 \quad 3.46 \mathrm{E}-03$

$7 \quad 3.16 \mathrm{E}-07 \quad 1.81 \mathrm{E}-04 \quad 3.98 \mathrm{E}-03$

$7 \quad 4.59 \mathrm{E}-07 \quad 2.52 \mathrm{E}-04 \quad 5.79 \mathrm{E}-03$

$10 \quad 6.07 \mathrm{E}-07 \quad 3.11 \mathrm{E}-04 \quad 7.65 \mathrm{E}-03$

$4 \quad 6.17 \mathrm{E}-07 \quad 3.11 \mathrm{E}-04 \quad 7.78 \mathrm{E}-03$

$5 \quad 8.77 \mathrm{E}-07 \quad 4.13 \mathrm{E}-04 \quad 1.11 \mathrm{E}-02$

$3 \quad 8.86 \mathrm{E}-07 \quad 4.13 \mathrm{E}-04 \quad 1.12 \mathrm{E}-02$

$6 \quad 9.40 \mathrm{E}-07 \quad 4.23 \mathrm{E}-04 \quad 1.18 \mathrm{E}-02$

$6 \quad 1.01 \mathrm{E}-06 \quad 4.38 \mathrm{E}-04 \quad 1.27 \mathrm{E}-02$

$7 \quad 1.26 \mathrm{E}-06 \quad 5.29 \mathrm{E}-04 \quad 1.59 \mathrm{E}-02$

$10 \quad 1.31 \mathrm{E}-06 \quad 5.32 \mathrm{E}-04 \quad 1.65 \mathrm{E}-02$

$8 \quad 1.64 \mathrm{E}-06 \quad 6.46 \mathrm{E}-04 \quad 2.07 \mathrm{E}-02$

$8 \quad 1.82 \mathrm{E}-06 \quad 6.96 \mathrm{E}-04 \quad 2.30 \mathrm{E}-02$

$4 \quad 1.95 \mathrm{E}-06 \quad 7.23 \mathrm{E}-04 \quad 2.46 \mathrm{E}-02$

$3 \quad 2.10 \mathrm{E}-06 \quad 7.57 \mathrm{E}-04 \quad 2.65 \mathrm{E}-02$

$7 \quad 2.38 \mathrm{E}-06 \quad 8.32 \mathrm{E}-04 \quad 3.00 \mathrm{E}-02$

$12 \quad 2.94 \mathrm{E}-06 \quad 1.00 \mathrm{E}-03 \quad 3.71 \mathrm{E}-02$

$3 \quad 3.33 \mathrm{E}-06 \quad 1.11 \mathrm{E}-03 \quad 4.20 \mathrm{E}-02$

$5 \quad 3.75 \mathrm{E}-06 \quad 1.19 \mathrm{E}-03 \quad 4.72 \mathrm{E}-02$

$4 \quad 3.79 \mathrm{E}-06 \quad 1.19 \mathrm{E}-03 \quad 4.78 \mathrm{E}-02$

$2 \quad 3.90 \mathrm{E}-06 \quad 1.20 \mathrm{E}-03 \quad 4.92 \mathrm{E}-02$

$3 \quad 4.10 \mathrm{E}-06 \quad 1.23 \mathrm{E}-03 \quad 5.17 \mathrm{E}-02$

$6 \quad 4.33 \mathrm{E}-06 \quad 1.27 \mathrm{E}-03 \quad 5.45 \mathrm{E}-02$

$7 \quad 5.42 \mathrm{E}-06 \quad 1.55 \mathrm{E}-03 \quad 6.83 \mathrm{E}-02$

$6 \quad 5.61 \mathrm{E}-06 \quad 1.57 \mathrm{E}-03 \quad 7.07 \mathrm{E}-02$

$6 \quad 7.72 \mathrm{E}-06 \quad 2.12 \mathrm{E}-03 \quad 9.74 \mathrm{E}-02$

$17 \quad 7.99 \mathrm{E}-06 \quad 2.14 \mathrm{E}-03 \quad 1.01 \mathrm{E}-01$

$5 \quad 9.26 \mathrm{E}-06 \quad 2.43 \mathrm{E}-03 \quad 1.17 \mathrm{E}-01$

$2 \quad 1.17 \mathrm{E}-05 \quad 3.01 \mathrm{E}-03 \quad 1.47 \mathrm{E}-01$

$6 \quad 1.45 \mathrm{E}-05 \quad 3.62 \mathrm{E}-03 \quad 1.83 \mathrm{E}-01$

$3 \quad 1.46 \mathrm{E}-05 \quad 3.62 \mathrm{E}-03 \quad 1.85 \mathrm{E}-01$

$11 \quad 1.54 \mathrm{E}-05 \quad 3.72 \mathrm{E}-03 \quad 1.94 \mathrm{E}-01$

$5 \quad 1.65 \mathrm{E}-05 \quad 3.93 \mathrm{E}-03 \quad 2.08 \mathrm{E}-01$

$5 \quad 1.75 \mathrm{E}-05 \quad 4.08 \mathrm{E}-03 \quad 2.21 \mathrm{E}-01$

$4 \quad 1.78 \mathrm{E}-05 \quad 4.08 \mathrm{E}-03 \quad 2.24 \mathrm{E}-01$

$2 \quad 2.34 \mathrm{E}-05 \quad 5.08 \mathrm{E}-03 \quad 2.94 \mathrm{E}-01$

$2 \quad 2.34 \mathrm{E}-05 \quad 5.08 \mathrm{E}-03 \quad 2.94 \mathrm{E}-01$

$5 \quad 2.34 \mathrm{E}-05 \quad 5.08 \mathrm{E}-03 \quad 2.95 \mathrm{E}-01$

$4 \quad 2.39 \mathrm{E}-05 \quad 5.10 \mathrm{E}-03 \quad 3.01 \mathrm{E}-01$

$3 \quad 2.63 \mathrm{E}-05 \quad 5.52 \mathrm{E}-03 \quad 3.31 \mathrm{E}-01$

$3 \quad 2.91 \mathrm{E}-05 \quad 6.02 \mathrm{E}-03 \quad 3.67 \mathrm{E}-01$

$9 \quad 3.20 \mathrm{E}-05 \quad 6.39 \mathrm{E}-03 \quad 4.03 \mathrm{E}-01$

$9 \quad 3.20 \mathrm{E}-05 \quad 6.39 \mathrm{E}-03 \quad 4.03 \mathrm{E}-01$

$5 \quad 3.67 \mathrm{E}-05 \quad 7.23 \mathrm{E}-03 \quad 4.63 \mathrm{E}-01$

$2 \quad 3.89 \mathrm{E}-05 \quad 7.43 \mathrm{E}-03 \quad 4.90 \mathrm{E}-01$

$2 \quad 3.89 \mathrm{E}-05 \quad 7.43 \mathrm{E}-03 \quad 4.90 \mathrm{E}-01$

$4 \quad 4.04 \mathrm{E}-05 \quad 7.60 \mathrm{E}-03 \quad 5.09 \mathrm{E}-01$

$8 \quad 4.26 \mathrm{E}-05 \quad 7.90 \mathrm{E}-03 \quad 5.37 \mathrm{E}-01$

$4 \quad 5.74 \mathrm{E}-05 \quad 1.04 \mathrm{E}-02 \quad 7.23 \mathrm{E}-01$

$10 \quad 5.76 \mathrm{E}-05 \quad 1.04 \mathrm{E}-02 \quad 7.27 \mathrm{E}-01$

$3 \quad 5.99 \mathrm{E}-05 \quad 1.05 \mathrm{E}-02 \quad 7.55 \mathrm{E}-01$ 
GO:0050900

GO:0002757

GO:1901698

GO:0048584

GO:0002694

GO:0050920

GO:0060326

GO:0032663

GO:0002706

GO:0042531

GO:0002377

GO:0010536

GO:0032817

GO:0051251

GO:0051240

GO:0042113

GO:0048583

GO:0050863

GO:0050865

GO:0071216

GO:0050793

GO:0043117

GO:0010533

GO:0050731

GO:0042509

GO:0002696

GO:0032703

GO:0002676

GO:0046427

GO:0050830

GO:0050867

GO:0002703

GO:0030888

GO:0051607

GO:0032494

GO:0032740

GO:0070664

GO:0002710

GO:0032695

GO:0002862

GO:0071345

GO:0033993

GO:0035556

GO:0032088

GO:0002688

GO:0050795

GO:0010243

GO:0002764

GO:0043112

GO:0042534

GO:0052200

GO:0052173

GO:0075136

GO:0071260

GO:2000106

GO:0002709

GO:0032647

GO:0030522

GO:0050730

GO:0032103

GO:0048660 leukocyte migration

immune response-activating signal transduction

response to nitrogen compound

positive regulation of response to stimulus

regulation of leukocyte activation

regulation of chemotaxis

cell chemotaxis

regulation of interleukin-2 production

regulation of lymphocyte mediated immunity

positive regulation of tyrosine phosphorylation of STAT protein

immunoglobulin production

positive regulation of activation of Janus kinase activity

regulation of natural killer cell proliferation

positive regulation of lymphocyte activation

positive regulation of multicellular organismal process

B cell activation

regulation of response to stimulus

regulation of $\mathrm{T}$ cell activation

regulation of cell activation

cellular response to biotic stimulus

regulation of developmental process

positive regulation of vascular permeability

regulation of activation of Janus kinase activity

positive regulation of peptidyl-tyrosine phosphorylation

regulation of tyrosine phosphorylation of STAT protein

positive regulation of leukocyte activation

negative regulation of interleukin- 2 production

regulation of chronic inflammatory response

positive regulation of JAK-STAT cascade

defense response to Gram-positive bacterium

positive regulation of cell activation

regulation of leukocyte mediated immunity

regulation of $B$ cell proliferation

defense response to virus

response to peptidoglycan

positive regulation of interleukin-17 production

negative regulation of leukocyte proliferation

negative regulation of $T$ cell mediated immunity

negative regulation of interleukin-12 production

negative regulation of inflammatory response to antigenic stimulu

cellular response to cytokine stimulus

response to lipid

intracellular signal transduction

negative regulation of NF-kappaB transcription factor activity

regulation of leukocyte chemotaxis

regulation of behavior

response to organonitrogen compound

immune response-regulating signaling pathway

receptor metabolic process

regulation of tumor necrosis factor biosynthetic process

response to host defenses

response to defenses of other organism involved in symbiotic inter

response to host

cellular response to mechanical stimulus

regulation of leukocyte apoptotic process

regulation of $T$ cell mediated immunity

regulation of interferon-alpha production

intracellular receptor signaling pathway

regulation of peptidyl-tyrosine phosphorylation

positive regulation of response to external stimulus

regulation of smooth muscle cell proliferation
6.06E-05

1.05E-02

7.64E-01

7.67E-01

7.81E-01

7.91E-01

$8.18 \mathrm{E}-01$

8.67E-01

8.67E-01

8.81E-01

9.30E-01

$1.00 \mathrm{E}+00$

$1.00 \mathrm{E}+00$

$1.00 \mathrm{E}+00$

$1.00 \mathrm{E}+00$

$1.00 \mathrm{E}+00$

$1.00 \mathrm{E}+00$

$1.00 \mathrm{E}+00$

$1.00 \mathrm{E}+00$

$1.00 \mathrm{E}+00$

$1.00 \mathrm{E}+00$

$1.00 \mathrm{E}+00$

$1.00 \mathrm{E}+00$

$1.00 \mathrm{E}+00$

$1.00 \mathrm{E}+00$

$1.00 \mathrm{E}+00$

$1.00 \mathrm{E}+00$

$1.00 \mathrm{E}+00$

$1.00 \mathrm{E}+00$

$1.00 \mathrm{E}+00$

$1.00 \mathrm{E}+00$

$1.00 \mathrm{E}+00$

$1.00 \mathrm{E}+00$

$1.00 \mathrm{E}+00$

$1.00 \mathrm{E}+00$

$1.00 \mathrm{E}+00$

$1.00 \mathrm{E}+00$

$1.00 \mathrm{E}+00$

$1.00 \mathrm{E}+00$

$1.00 \mathrm{E}+00$

$1.00 \mathrm{E}+00$

$1.00 \mathrm{E}+00$

$1.00 \mathrm{E}+00$

$1.00 \mathrm{E}+00$

$1.00 \mathrm{E}+00$

$1.00 \mathrm{E}+00$

$1.00 \mathrm{E}+00$

$1.00 \mathrm{E}+00$

$1.00 \mathrm{E}+00$

$1.00 \mathrm{E}+00$

$1.00 \mathrm{E}+00$

$1.00 \mathrm{E}+00$

$1.00 \mathrm{E}+00$

$1.00 \mathrm{E}+00$

$1.00 \mathrm{E}+00$

$1.00 \mathrm{E}+00$

$1.00 \mathrm{E}+00$

$1.00 \mathrm{E}+00$

$1.00 \mathrm{E}+00$

$1.00 \mathrm{E}+00$

$1.00 \mathrm{E}+00$

$1.00 \mathrm{E}+00$

$1.00 \mathrm{E}+00$ 
GO:0002700 GO:0050870

GO:0030889

GO:0033033

GO:0048535

GO:0046425

GO:0050778

GO:0032680

GO:0032649

GO:0032660

GO:0002823

GO:0030183

GO:0002707

GO:0044130

GO:0065008

GO:0051239

GO:0060337

GO:0050728

GO:0071357

GO:0042176

GO:0034340

GO:0071496

GO:0002820

GO:0034097

GO:0032101

GO:0051241

missSNP set

GO_id

GO:0005126

GO:0005138

GO:0050700

ncSNP set

allSNP set

GO:0005126

GO:0004918

GO:0005138

GO:0070851

GO:0050700

missSNP set

GO_id

GO:0009986 regulation of production of molecular mediator of immune respon positive regulation of $T$ cell activation

negative regulation of $B$ cell proliferation

negative regulation of myeloid cell apoptotic process

lymph node development

regulation of JAK-STAT cascade

positive regulation of immune response

regulation of tumor necrosis factor production

regulation of interferon-gamma production

regulation of interleukin-17 production

negative regulation of adaptive immune response based on somati

$B$ cell differentiation

negative regulation of lymphocyte mediated immunity

negative regulation of growth of symbiont in host

regulation of biological quality

regulation of multicellular organismal process

type I interferon signaling pathway

negative regulation of inflammatory response

cellular response to type I interferon

regulation of protein catabolic process

response to type I interferon

cellular response to external stimulus

negative regulation of adaptive immune response

response to cytokine

regulation of response to external stimulus

negative regulation of multicellular organismal process

\section{MF terms}

cytokine receptor binding

interleukin-6 receptor binding

CARD domain binding

none

\section{cytokine receptor binding}

interleukin-8 receptor activity

interleukin- 6 receptor binding

growth factor receptor binding

CARD domain binding

CC terms

ncSNP set

allSNP set

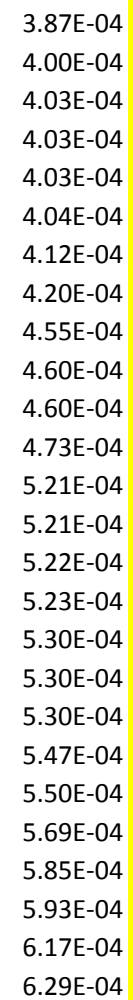

$1.00 \mathrm{E}+00$

$1.00 \mathrm{E}+00$

$1.00 \mathrm{E}+00$

$1.00 \mathrm{E}+00$

$1.00 \mathrm{E}+00$

$1.00 \mathrm{E}+00$

$1.00 \mathrm{E}+00$

$1.00 \mathrm{E}+00$

$1.00 \mathrm{E}+00$

$1.00 \mathrm{E}+00$

$1.00 \mathrm{E}+00$

$1.00 \mathrm{E}+00$

$1.00 \mathrm{E}+00$

$1.00 \mathrm{E}+00$

$1.00 \mathrm{E}+00$

$1.00 \mathrm{E}+00$

$1.00 \mathrm{E}+00$

$1.00 \mathrm{E}+00$

$1.00 \mathrm{E}+00$

$1.00 \mathrm{E}+00$

$1.00 \mathrm{E}+00$

$1.00 \mathrm{E}+00$

$1.00 \mathrm{E}+00$

$1.00 \mathrm{E}+00$

$1.00 \mathrm{E}+00$

No of

genes $p$-value $p$-value_fdr $p$-value_bonferroni

\begin{tabular}{ll|l|l|}
5 & $6.69 \mathrm{E}-07$ & $2.57 \mathrm{E}-03$ & $2.57 \mathrm{E}-03$ \\
2 & $9.09 \mathrm{E}-06$ & $1.74 \mathrm{E}-02$ & $3.49 \mathrm{E}-02$ \\
2 & $2.54 \mathrm{E}-05$ & $3.25 \mathrm{E}-02$ & $9.75 \mathrm{E}-02$
\end{tabular}


Table 22. GO terms enrichemnt for missSNP geneset by ConsensusPathDB

Enrichement of GO BP terms for missSNP gene set

\begin{tabular}{|c|c|c|c|c|c|}
\hline \\
\hline \multicolumn{6}{|c|}{$\begin{array}{ll}\text { uploaded list: } & 23 \\
\text { mapped entities: } & 22\end{array}$} \\
\hline enriched gene ontology-based sets: & 2 & & & & \\
\hline \multicolumn{6}{|c|}{21 genes $(95.5 \%)$ from the input list are present in at least one GO category. } \\
\hline gene ontology term & $\begin{array}{l}\text { category, } \\
\text { level }\end{array}$ & set size & $\begin{array}{l}\text { candidates } \\
\text { contained }\end{array}$ & p-value & q-value \\
\hline GO:0006955 immune response & BP 2 & 1457 & $13(0.9 \%)$ & $4.43 \mathrm{E}-10$ & 2.26E-08 \\
\hline GO:0002252 immune effector process & BP 2 & 631 & $9(1.5 \%)$ & 1.19E-08 & 3.04E-07 \\
\hline GO:0051707 response to other organism & BP 2 & 744 & $8(1.1 \%)$ & 8.18E-07 & 1.39E-05 \\
\hline GO:0009607 response to biotic stimulus & BP 2 & 776 & $8(1.1 \%)$ & 1.13E-06 & 1.45E-05 \\
\hline GO:0045321 leukocyte activation & BP 2 & 673 & $7(1.1 \%)$ & $5.82 E-06$ & 5.94E-05 \\
\hline GO:0001913 T cell mediated cytotoxicity & BP 2 & 37 & $3(8.6 \%)$ & 8.68E-06 & 7.38E-05 \\
\hline $\begin{array}{l}\text { GO:0002440 production of molecular mediator of immune } \\
\text { response }\end{array}$ & BP 2 & 130 & $4(3.2 \%)$ & $1.24 \mathrm{E}-05$ & $9.02 E-05$ \\
\hline GO:0002520 immune system development & BP 2 & 786 & $7(0.9 \%)$ & $1.65 \mathrm{E}-05$ & 0.000105 \\
\hline GO:0006950 response to stress & BP 2 & 3557 & $13(0.4 \%)$ & 2.03E-05 & 0.000115 \\
\hline GO:0001909 leukocyte mediated cytotoxicity & BP 2 & 78 & $3(3.9 \%)$ & 9.04E-05 & 0.000454 \\
\hline GO:0008283 cell proliferation & BP 2 & 1861 & $9(0.5 \%)$ & $9.78 \mathrm{E}-05$ & 0.000454 \\
\hline GO:0002253 activation of immune response & BP 2 & 438 & $5(1.2 \%)$ & 0.000108 & 0.000458 \\
\hline GO:0051716 cellular response to stimulus & BP 2 & 6497 & $16(0.3 \%)$ & 0.000134 & 0.000527 \\
\hline GO:0009605 response to external stimulus & BP 2 & 2113 & $9(0.4 \%)$ & 0.000258 & 0.000938 \\
\hline GO:0044700 single organism signaling & BP 2 & 5897 & $14(0.2 \%)$ & 0.00102 & 0.00339 \\
\hline GO:0098602 single organism cell adhesion & BP 2 & 721 & $5(0.7 \%)$ & 0.00106 & 0.00339 \\
\hline GO:0019882 antigen processing and presentation & BP 2 & 233 & $3(1.3 \%)$ & 0.00235 & 0.00706 \\
\hline GO:0044767 single-organism developmental process & BP 2 & 5402 & $12(0.2 \%)$ & 0.00696 & 0.0197 \\
\hline GO:0044763 single-organism cellular process & BP 2 & 11949 & $19(0.2 \%)$ & 0.00814 & 0.0219 \\
\hline
\end{tabular}

Enrichement of GO MF terms for missSNP set

\begin{tabular}{|c|c|c|c|c|c|}
\hline $\begin{array}{l}\text { uploaded list: } \\
\text { mapped entities: } \\
\text { enriched gene ontology-based sets: } \\
21 \text { genes }(95.5 \%) \text { from the input list are prese }\end{array}$ & $\begin{array}{r}23 \\
22 \\
8 \\
\text { ne GO cates }\end{array}$ & & & & \\
\hline gene ontology term & $\begin{array}{l}\text { category, } \\
\text { level }\end{array}$ & set size & $\begin{array}{c}\text { candidates } \\
\text { contained }\end{array}$ & p-value & q-value \\
\hline GO:0005126 cytokine receptor binding & MF 4 & 257 & $5(2.0 \%)$ & 8.01E-06 & 0.000112 \\
\hline GO:0050700 CARD domain binding & MF 4 & 8 & $2(25.0 \%)$ & 3.60E-05 & 0.000252 \\
\hline GO:0004896 cytokine receptor activity & MF 4 & 90 & $3(3.8 \%)$ & 0.000105 & 0.000492 \\
\hline GO:0070851 growth factor receptor binding & MF 4 & 122 & $3(2.5 \%)$ & 0.000333 & 0.00116 \\
\hline GO:0005515 protein binding & MF 2 & 8713 & $17(0.2 \%)$ & 0.00169 & 0.0203 \\
\hline GO:0005102 receptor binding & MF 3 & 1369 & $6(0.5 \%)$ & 0.00331 & 0.0238 \\
\hline GO:0019955 cytokine binding & MF 3 & 80 & $2(2.7 \%)$ & 0.0034 & 0.0238 \\
\hline GO:0003823 antigen binding & MF 2 & 108 & $2(2.0 \%)$ & 0.0062 & 0.0372 \\
\hline
\end{tabular}

Enrichement of GO CC terms for missSNP set

uploaded list: $\quad 23$

mapped entities:

enriched gene ontology-based sets:

5

21 genes (95.5\%) from the input list are present in at least one GO category.

\section{gene ontology term}

GO:0009986 cell surface

GO:0044459 plasma membrane part

GO:0098552 side of membrane

GO:0005886 plasma membrane

GO:0071944 cell periphery

$\begin{array}{cc}\begin{array}{c}\text { category, } \\ \text { level }\end{array} & \text { set size } \\ \text { CC } 2 & 690 \\ \text { CC } 2 & 2279 \\ \text { CC } 2 & 307 \\ \text { CC } 2 & 4776 \\ \text { CC } 2 & 4870\end{array}$

p-value q-value contained

$5(0.8 \%)$

$8(0.4 \%)$

$3(1.0 \%)$

$11(0.2 \%)$

$11(0.2 \%)$

$\begin{array}{rr}0.0008 & 0.0176 \\ 0.00206 & 0.0226 \\ 0.00433 & 0.0317 \\ 0.00765 & 0.0396 \\ 0.00899 & 0.0396\end{array}$




\section{Table 23. Enrichment of BP, MF and CC GO terms for ncSNP geneset}

uploaded list:

mapped entities:

enriched gene ontology-based sets:
23

23

36

20 genes $(87.0 \%)$ from the input list are present in at least one GO category.

\begin{tabular}{|c|c|c|c|c|c|c|}
\hline \multicolumn{2}{|c|}{ gene ontology term } & \multirow{2}{*}{$\begin{array}{c}\text { category, } \\
\text { level } \\
\text { BP } 2\end{array}$} & \multirow{2}{*}{$\begin{array}{c}\text { set size } \\
1457\end{array}$} & \multirow{2}{*}{$\begin{array}{c}\text { candidates } \\
\text { contained } \\
10(0.7 \%)\end{array}$} & \multirow{2}{*}{$\begin{array}{l}\text { p-value } \\
7.49 \mathrm{E}-07\end{array}$} & q-value \\
\hline GO:0006955 & immune response & & & & & $3.82 \mathrm{E}-05$ \\
\hline GO:0045321 & leukocyte activation & BP 2 & 673 & $7(1.1 \%)$ & 4.00E-06 & 0.000102 \\
\hline GO:0002520 & immune system development & BP 2 & 786 & $7(0.9 \%)$ & 1.14E-05 & 0.000195 \\
\hline GO:0050900 & leukocyte migration & BP 2 & 321 & $5(1.6 \%)$ & $1.76 \mathrm{E}-05$ & 0.000225 \\
\hline GO:0009605 & response to external stimulus & BP 2 & 2113 & $10(0.5 \%)$ & $2.24 \mathrm{E}-05$ & 0.000229 \\
\hline GO:0006950 & response to stress & BP 2 & 3557 & $12(0.3 \%)$ & 6.64E-05 & 0.000564 \\
\hline GO:0042221 & response to chemical & BP 2 & 3872 & $12(0.3 \%)$ & 0.00015 & 0.000873 \\
\hline GO:0048870 & cell motility & BP 2 & 1175 & $7(0.6 \%)$ & 0.000154 & 0.000873 \\
\hline GO:0051674 & localization of cell & BP 2 & 1175 & $7(0.6 \%)$ & 0.000154 & 0.000873 \\
\hline GO:0008283 & cell proliferation & BP 2 & 1861 & $8(0.4 \%)$ & 0.000425 & 0.00195 \\
\hline GO:0044765 & single-organism transport & BP 2 & 3605 & $11(0.3 \%)$ & 0.000456 & 0.00195 \\
\hline GO:0002252 & immune effector process & BP 2 & 631 & $5(0.8 \%)$ & 0.000458 & 0.00195 \\
\hline GO:0042330 & taxis & BP 2 & 692 & $5(0.7 \%)$ & 0.000682 & 0.00261 \\
\hline GO:1902578 & single-organism localization & BP 2 & 3791 & $11(0.3 \%)$ & 0.000716 & 0.00261 \\
\hline GO:0098602 & single organism cell adhesion & BP 2 & 721 & $5(0.7 \%)$ & 0.000837 & 0.00284 \\
\hline GO:0065008 & regulation of biological quality & BP 2 & 3239 & $10(0.3 \%)$ & 0.000892 & 0.00284 \\
\hline GO:0051707 & response to other organism & BP 2 & 744 & $5(0.7 \%)$ & 0.000956 & 0.00287 \\
\hline GO:0009607 & response to biotic stimulus & BP 2 & 776 & $5(0.7 \%)$ & 0.00116 & 0.00329 \\
\hline GO:0051234 & establishment of localization & BP 2 & 4397 & $11(0.3 \%)$ & 0.00263 & 0.00705 \\
\hline GO:0001776 & leukocyte homeostasis & BP 2 & 75 & $2(2.8 \%)$ & 0.00285 & 0.00726 \\
\hline GO:0050789 & regulation of biological process & BP 2 & 10286 & $17(0.2 \%)$ & 0.00571 & 0.0131 \\
\hline GO:0051716 & cellular response to stimulus & BP 2 & 6497 & $13(0.2 \%)$ & 0.00582 & 0.0131 \\
\hline $\begin{array}{l}\text { GO:0044707 } \\
\text { process }\end{array}$ & single-multicellular organism & BP 2 & 6462 & $13(0.2 \%)$ & 0.0059 & 0.0131 \\
\hline GO:0044459 & plasma membrane part & CC 2 & 2279 & $7(0.3 \%)$ & 0.00672 & 0.128 \\
\hline GO:0044700 & single organism signaling & BP 2 & 5897 & $12(0.2 \%)$ & 0.0082 & 0.0174 \\
\hline GO:0033036 & macromolecule localization & BP 2 & 2313 & $7(0.3 \%)$ & 0.00877 & 0.0179 \\
\hline GO:0002253 & activation of immune response & BP 2 & 438 & $3(0.7 \%)$ & 0.0111 & 0.0218 \\
\hline GO:0016265 & death & BP 2 & 1899 & $6(0.3 \%)$ & 0.0126 & 0.0238 \\
\hline GO:0007155 & cell adhesion & BP 2 & 1375 & $5(0.4 \%)$ & 0.0138 & 0.0251 \\
\hline GO:0009719 & response to endogenous stimulus & BP 2 & 1409 & $5(0.4 \%)$ & 0.0144 & 0.0254 \\
\hline GO:0004871 & signal transducer activity & MF 2 & 1687 & $5(0.3 \%)$ & 0.0263 & 0.162 \\
\hline $\begin{array}{l}\text { GO:0003700 } \\
\text { transcription } f\end{array}$ & $\begin{array}{l}\text { sequence-specific DNA binding } \\
\text { actor activity }\end{array}$ & MF 2 & 1090 & $4(0.4 \%)$ & 0.0279 & 0.162 \\
\hline GO:0005515 & protein binding & MF 2 & 8713 & $14(0.2 \%)$ & 0.0346 & 0.162 \\
\hline $\begin{array}{l}\text { GO:0044767 } \\
\text { process }\end{array}$ & single-organism developmental & BP 2 & 5402 & $10(0.2 \%)$ & 0.0405 & 0.0689 \\
\hline GO:0009653 & anatomical structure morphogenesis & BP 2 & 2484 & $6(0.2 \%)$ & 0.0435 & 0.0715 \\
\hline GO:0005886 & plasma membrane & $\mathrm{CC} 2$ & 4776 & $9(0.2 \%)$ & 0.0466 & 0.332 \\
\hline
\end{tabular}




\section{Table 24. Enrichment of BP, MF and CC GO terms for allSNP geneset}

Enriched gene ontology-based sets for allSNP set for BP GO

\begin{tabular}{ll}
\hline uploaded list: & 56 \\
mapped entities: & 54 \\
enriched gene ontology-based sets: & 44
\end{tabular}

41 genes $(91.1 \%)$ from the input list are present in at least one GO category.

gene ontology term

GO:0006955 immune response

GO:0002252 immune effector process

GO:0045321 leukocyte activation

GO:0002520 immune system development

GO:0051707 response to other organism

GO:0006950 response to stress

GO:0009607 response to biotic stimulus

GO:0009605 response to external stimulus

GO:0008283 cell proliferation

GO:0098602 single organism cell adhesion

GO:0051716 cellular response to stimulus

GO:0002253 activation of immune response

GO:0050900 leukocyte migration

GO:0002440 production of molecular mediator of immune

GO:0044700 single organism signaling

GO:0048870 cell motility

GO:0051674 localization of cell

GO:0044459 plasma membrane part

GO:0042221 response to chemical

GO:0001913 T cell mediated cytotoxicity

GO:0097278 complement-dependent cytotoxicity

GO:0044707 single-multicellular organism process

GO:0005515 protein binding

GO:0050789 regulation of biological process

GO:0065008 regulation of biological quality

GO:0001776 leukocyte homeostasis

GO:0016265 death

GO:0009986 cell surface

GO:0007155 cell adhesion

GO:0001909 leukocyte mediated cytotoxicity

GO:0042330 taxis

GO:0044767 single-organism developmental process

GO:0044110 growth involved in symbiotic interaction

GO:0033036 macromolecule localization

GO:0005886 plasma membrane

GO:0071944 cell periphery

GO:0009719 response to endogenous stimulus

GO:0044763 single-organism cellular process

GO:0044765 single-organism transport

\begin{tabular}{ll} 
category, & set size \\
level & \\
BP 2 & 1457 \\
BP 2 & 631 \\
BP 2 & 673 \\
BP 2 & 786 \\
BP 2 & 744 \\
BP 2 & 3557 \\
BP 2 & 776 \\
BP 2 & 2113 \\
BP 2 & 1861 \\
BP 2 & 721 \\
BP 2 & 6497 \\
BP 2 & 438 \\
BP 2 & 321 \\
BP 2 & 130 \\
BP 2 & 5897 \\
BP 2 & 1175 \\
BP 2 & 1175 \\
CC 2 & 2279 \\
BP 2 & 3872 \\
BP 2 & 37 \\
BP 2 & 6 \\
BP 2 & 6462 \\
MF 2 & 8713 \\
BP 2 & 10286 \\
BP 2 & 3239 \\
BP 2 & 75 \\
BP 2 & 1899 \\
CC 2 & 690 \\
BP 2 & 1375 \\
BP 2 & 78 \\
BP 2 & 692 \\
BP 2 & 5402 \\
BP 2 & 21 \\
BP 2 & 2313 \\
CC 2 & 4776 \\
CC 2 & 4870 \\
BP 2 & 1409 \\
\hline BP 2 & 11949 \\
3605 \\
\hline
\end{tabular}

q-value

9.33E-14

1.12E-09

1.61E-09

9.82E-09

4.59E-08

4.59E-08

5.55E-08

1.66E-07

9.64E-07

$1.68 \mathrm{E}-05$

1.69E-05

2.22E-05

2.49E-05

4.03E-05

0.000111

0.000125

0.000125

0.0013

0.000202

0.000214

0.000225

0.000531

0.00357

0.000581

0.000723

0.00145

0.00145

0.00927

0.00152

0.00152

0.00153

0.00186

0.00206

0.0022

0.0108

0.0108

0.00542

0.00542

0.00542 


\begin{tabular}{|c|c|c|c|c|c|c|}
\hline GO:0051234 & establishment of localization & BP 2 & 4397 & $18(0.4 \%)$ & 0.0037 & 0.00634 \\
\hline GO:0098552 & side of membrane & $\mathrm{CC} 2$ & 307 & $4(1.4 \%)$ & 0.00405 & 0.0235 \\
\hline GO:0048856 & anatomical structure development & BP 2 & 4828 & $19(0.4 \%)$ & 0.00419 & 0.00698 \\
\hline GO:1902578 & single-organism localization & BP 2 & 3791 & $16(0.4 \%)$ & 0.00517 & 0.00838 \\
\hline GO:0004871 & signal transducer activity & MF 2 & 1687 & $9(0.6 \%)$ & 0.00805 & 0.0685 \\
\hline
\end{tabular}

\section{Enriched gene ontology-based sets for allSNP set for MF GO}

uploaded list: $\quad 56$

mapped entities: $\quad 54$

enriched gene ontology-based sets: $\quad 12$

41 genes $(91.1 \%)$ from the input list are present in at least one GO category.

\begin{tabular}{|c|c|c|c|c|c|c|}
\hline \multicolumn{2}{|c|}{ gene ontology term } & $\begin{array}{l}\text { category, } \\
\text { level }\end{array}$ & & $\begin{array}{l}\text { candidates } \\
\text { contained }\end{array}$ & & \multirow{2}{*}{$\begin{array}{l}\text { q-value } \\
\text { 7.98E-07 }\end{array}$} \\
\hline GO:0004896 & cytokine receptor activity & MF 4 & 90 & $6(7.5 \%)$ & $2.49 \mathrm{E}-08$ & \\
\hline GO:0005126 & cytokine receptor binding & MF 4 & 257 & $7(2.8 \%)$ & 1.29E-06 & $2.06 \mathrm{E}-05$ \\
\hline GO:0019955 & cytokine binding & MF 3 & 80 & $4(5.3 \%)$ & $2.48 \mathrm{E}-05$ & 0.000546 \\
\hline GO:0050700 & CARD domain binding & MF 4 & 8 & $2(25.0 \%)$ & 0.00014 & 0.00117 \\
\hline GO:0070851 & growth factor receptor binding & MF 4 & 122 & $4(3.4 \%)$ & 0.000146 & 0.00117 \\
\hline GO:0005515 & protein binding & MF 2 & 8713 & $31(0.4 \%)$ & 0.00021 & 0.00357 \\
\hline GO:0019956 & chemokine binding & MF 4 & 13 & $2(16.7 \%)$ & 0.000328 & 0.0021 \\
\hline GO:0008528 & G-protein coupled peptide recept & MF 4 & 121 & $3(2.9 \%)$ & 0.00174 & 0.00839 \\
\hline GO:0001653 & peptide receptor activity & MF 4 & 123 & $3(2.8 \%)$ & 0.00183 & 0.00839 \\
\hline GO:0005102 & receptor binding & MF 3 & 1369 & $9(0.7 \%)$ & 0.00257 & 0.0282 \\
\hline GO:0004871 & signal transducer activity & MF 2 & 1687 & $9(0.6 \%)$ & 0.00805 & 0.0685 \\
\hline GO:0019904 & protein domain specific binding & MF 3 & 586 & $5(0.9 \%)$ & 0.00893 & 0.065 \\
\hline
\end{tabular}

Enriched gene ontology-based sets for allSNP set for CC GO

uploaded list: $\quad 56$

mapped entities: $\quad 54$

enriched gene ontology-based sets: $\quad 7$

41 genes $(91.1 \%)$ from the input list are present in at least one GO category.

gene ontology term

GO:0044459 plasma membrane part

GO:0009986 cell surface

GO:0005886 plasma membrane

GO:0071944 cell periphery

GO:0005887 integral component of plasma me CC 4

GO:0031226 intrinsic component of plasma me CC 3

GO:0098552 side of membrane

$\begin{array}{lc}\begin{array}{l}\text { category, } \\ \text { level }\end{array} & \text { set size } \\ \text { CC } 2 & 2279 \\ \text { CC } 2 & 690 \\ \text { CC } 2 & 4776 \\ \text { CC } 2 & 4870 \\ \text { CC } 4 & 1342 \\ \text { CC } 3 & 1396 \\ \text { CC } 2 & 307\end{array}$

$\begin{array}{rrr}\begin{array}{r}\text { candidates } \\ \text { contained }\end{array} & \text { p-value } & \text { q-value } \\ 15(0.7 \%) & 4.47 \mathrm{E}-05 & 0.0013 \\ 7(1.1 \%) & 0.00064 & 0.00927 \\ 20(0.4 \%) & 0.00114 & 0.0108 \\ 20(0.4 \%) & 0.00149 & 0.0108 \\ 9(0.7 \%) & 0.00172 & 0.031 \\ 9(0.7 \%) & 0.00229 & 0.0481 \\ 4(1.4 \%) & 0.00405 & 0.0235\end{array}$


Table 25.

DiseaseConnectDB KEGG pathway dataset for AID: pleiotropy at the pathway-disease level

\begin{tabular}{|c|c|c|c|c|}
\hline \multicolumn{2}{|c|}{ Pathway map/module } & Pathway name & Pathway ID & AID Diseases \\
\hline \multirow{3}{*}{\begin{tabular}{|l|} 
Environmental \\
Information \\
Processing
\end{tabular}} & Signal molecules & Cytokine-cytokine receptor interaction & hsa04060 & RA CD AS \\
\hline & Signal transduction & NF-kappa B signaling pathway & hsa04064 & RA CD PS \\
\hline & Signal transduction & Jak-STAT signaling pathway & hsa04630 & RA CD PS \\
\hline \multirow{4}{*}{$\begin{array}{l}\text { Organismal } \\
\text { Systems }\end{array}$} & Immune system & Antigen processing and presentation & hsa04612 & RA CD \\
\hline & Immune system & NOD-like receptor signaling pathway & hsa04621 & CD PS \\
\hline & Immune system & T cell receptor signaling pathway & hsa04660 & RA CD \\
\hline & Immune system & Intestinal immune network for IgA production & hsa04672 & RA CD \\
\hline \multicolumn{5}{|c|}{ Human Diseases } \\
\hline & $\begin{array}{l}\text { Endocrine and } \\
\text { metabolic diseases }\end{array}$ & Type I diabetes mellitus & hsa04940 & CD PS \\
\hline & \multirow[t]{6}{*}{ Immune diseases } & Asthma & hsa05310 & RA CD \\
\hline & & Autoimmune thyroid disease & hsa05320 & RA CD \\
\hline & & Systemic lupus erythematosus & hsa05322 & RA CD \\
\hline & & Rheumatoid arthritis & hsa05323 & RA CD \\
\hline & & Graft-versus-host disease & hsa05332 & RA CD \\
\hline & & Allograft rejection & hsa05330 & RA CD PsA \\
\hline & \multicolumn{4}{|l|}{ Infectious diseases } \\
\hline & \multirow[t]{3}{*}{\begin{tabular}{|l|} 
Infectious diseases: \\
Parasitic
\end{tabular}} & Malaria & hsa05144 & RA CD \\
\hline & & Toxoplasmosis & hsa05145 & RA CD PS \\
\hline & & Amoebiasis & hsa05146 & RA CD AS \\
\hline & \multirow[t]{2}{*}{$\begin{array}{l}\text { Infectious diseases: } \\
\text { Bacterial }\end{array}$} & Staphylococcus aureus infection & hsa05150 & RA CD \\
\hline & & Tuberculosis & hsa05152 & RA CD \\
\hline & \multirow[t]{4}{*}{$\begin{array}{l}\text { Infectious diseases: } \\
\text { Viral }\end{array}$} & Measles & hsa05162 & RA PS \\
\hline & & Influenza A & hsa05164 & RA CD PS \\
\hline & & HTLV-I infection & hsa05166 & RA CD \\
\hline & & Herpes simplex infection & hsa05168 & RA CD PsA PS \\
\hline
\end{tabular}

Source is Disease ConnectDB; by each AID:

https://docs.google.com/spreadsheets/d/1kMUw7xJHXjYXrXgIWsCWDQ6qcrTIY8L1QSHY3BJIJ9k/edit\#gid=2121775356 


\section{Table 26. STRING disease enrichment data for AID GWAS SNP harboring geneset}

\begin{tabular}{llcccc} 
GO_id & \multicolumn{1}{c}{ Term } & $\begin{array}{c}\text { No of } \\
\text { Genes }\end{array}$ & p-value & p-value_fdr & $\begin{array}{c}\text { p-value_- } \\
\text { bonferroni }\end{array}$ \\
DOID:8778 & Crohn's disease & & & & \\
DOID:0050589 & Inflammatory bowel disease & 3 & $3.23 \mathrm{E}-09$ & $6.20 \mathrm{E}-06$ & $1.49 \mathrm{E}-05$ \\
DOID:3342 & Bone inflammation disease & 3 & $3.23 \mathrm{E}-09$ & $6.20 \mathrm{E}-06$ & $1.49 \mathrm{E}-05$ \\
DOID:9008 & Psoriatic arthritis & 4 & $4.04 \mathrm{E}-09$ & $6.20 \mathrm{E}-06$ & $1.86 \mathrm{E}-05$ \\
DOID:225 & Syndrome & 3 & $4.52 \mathrm{E}-08$ & $5.19 \mathrm{E}-05$ & $2.08 \mathrm{E}-04$ \\
DOID:5295 & Intestinal disease & 3 & $9.66 \mathrm{E}-08$ & $8.89 \mathrm{E}-05$ & $4.45 \mathrm{E}-04$ \\
DOID:4 & Disease & 3 & $6.54 \mathrm{E}-07$ & $4.87 \mathrm{E}-04$ & $3.01 \mathrm{E}-03$ \\
DOID:0080001 & Bone disease & 11 & $7.41 \mathrm{E}-07$ & $4.87 \mathrm{E}-04$ & $3.41 \mathrm{E}-03$ \\
DOID:7147 & Ankylosing spondylitis & 4 & $1.31 \mathrm{E}-06$ & $7.55 \mathrm{E}-04$ & $6.04 \mathrm{E}-03$ \\
DOID:7 & Disease of anatomical entity & 2 & $5.46 \mathrm{E}-06$ & $2.79 \mathrm{E}-03$ & $2.51 \mathrm{E}-02$ \\
DOID:77 & Gastrointestinal system disease & 8 & $7.07 \mathrm{E}-06$ & $3.25 \mathrm{E}-03$ & $3.25 \mathrm{E}-02$ \\
DOID:17 & Musculoskeletal system disease & 4 & $8.42 \mathrm{E}-06$ & $3.52 \mathrm{E}-03$ & $3.87 \mathrm{E}-02$ \\
DOID:676 & Juvenile rheumatoid arthritis & 2 & $5.64 \mathrm{E}-05$ & $2.16 \mathrm{E}-02$ & $2.60 \mathrm{E}-01$ \\
DOID:7148 & Rheumatoid arthritis & 2 & $9.49 \mathrm{E}-05$ & $3.12 \mathrm{E}-02$ & $4.37 \mathrm{E}-01$ \\
DOID:848 & Arthritis & 2 & $1.23 \mathrm{E}-04$ & $3.77 \mathrm{E}-02$ & $5.65 \mathrm{E}-01$
\end{tabular}

Diseases IDs and names are given, in bold are emphasized AIDs; number of genes in a gene set for each disease; $p$ values, after fdr and Bonferroni corrections. 
Figure 1. An example of functional evaluation of missense SNP impact by PolyPhen-2

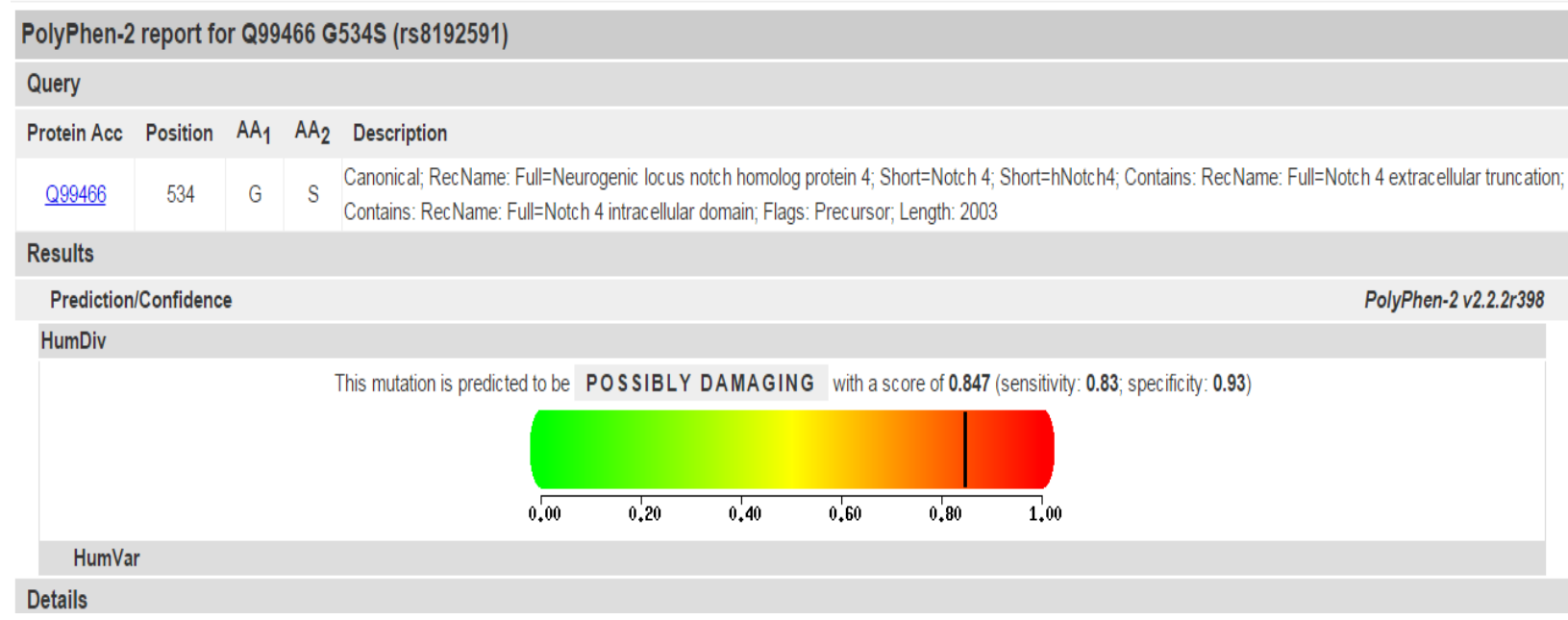

Prediction of conformational alteration caused by an amino acid change: the resulting report for rs8192591 is presented with a score on the scale from zero to one and color coded green to red for a level of damage. Specificity and sensitivity of evaluation for each test is also provided by PolyPhen-2. 
Figure 2a: TNF network
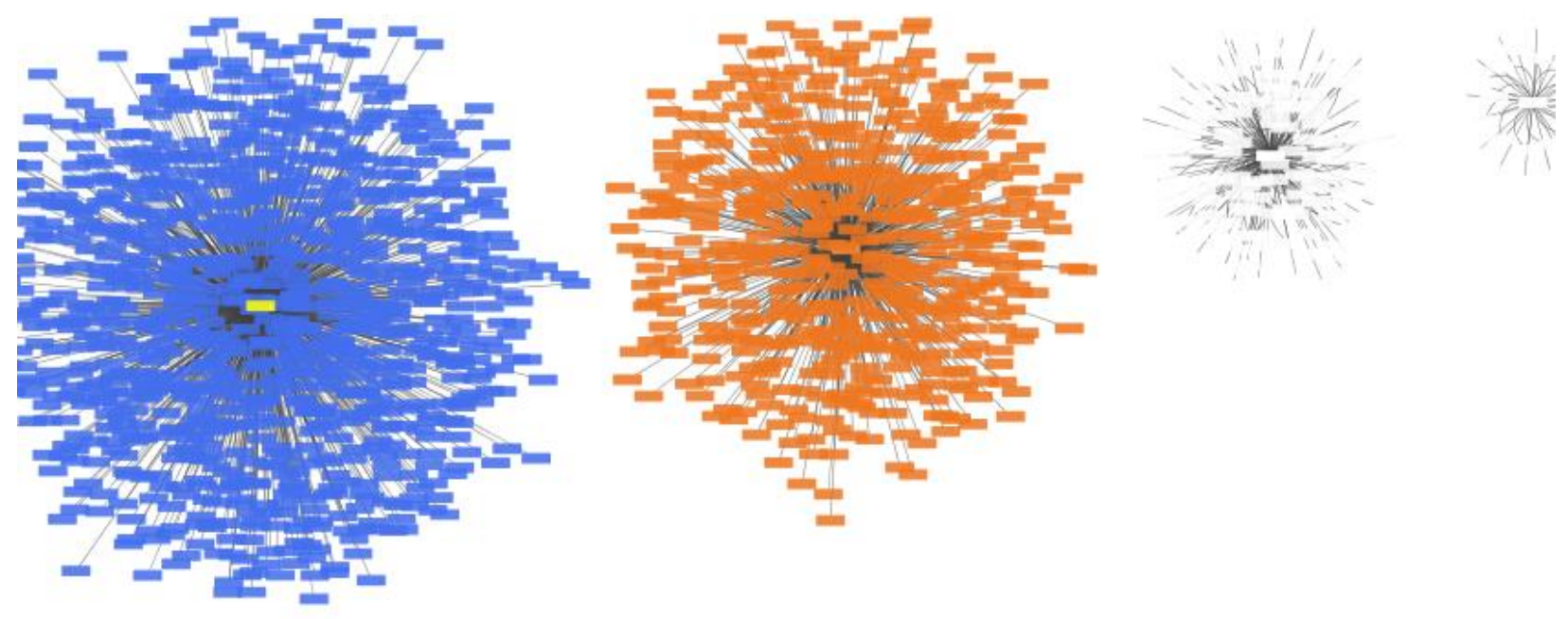

Figure 2b: NFKBIE network
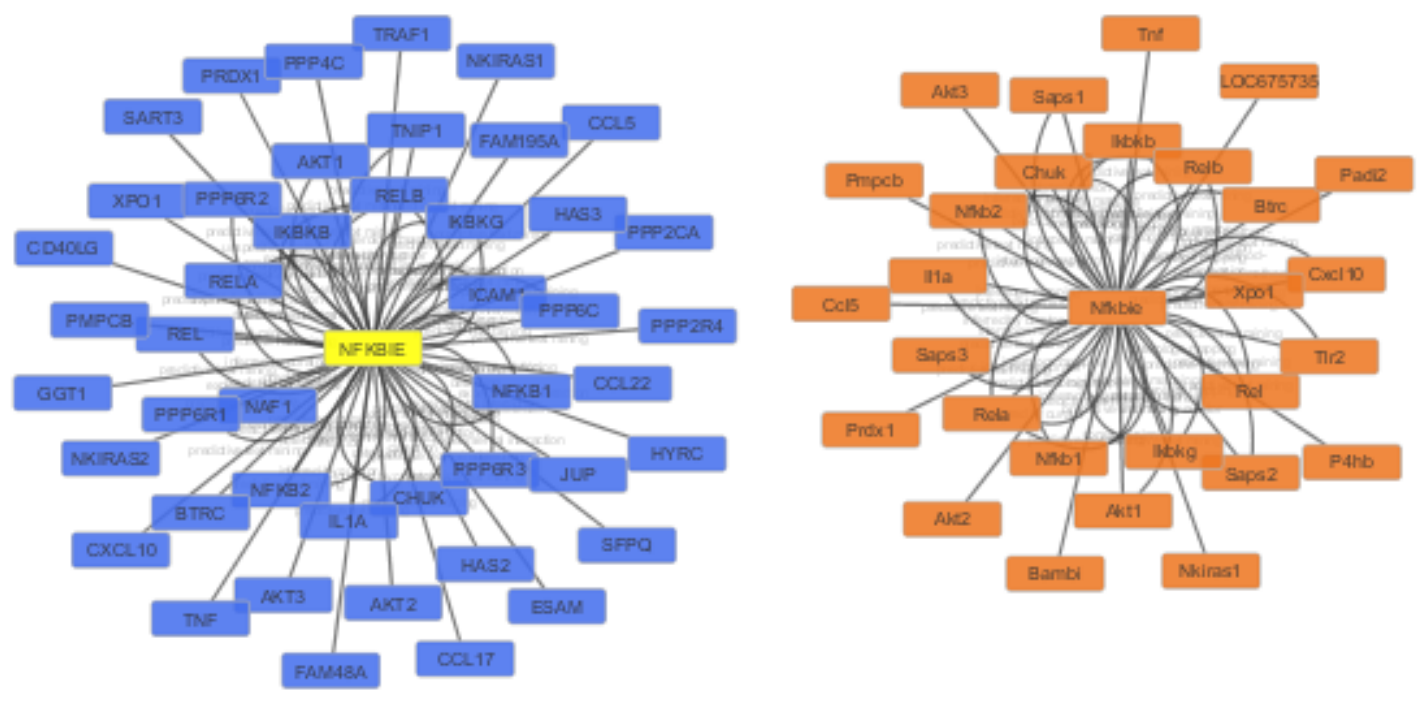

Networks for SNP genes and TNF are presented: color coded for a species (human is blue, red is murine network, white is for rat); nodes represent proteins; edges represent any type of interactions between two proteins. 
Figure 3a. Image of intersection between ERAP1 and TNF networks (nodes represent proteins that are common to both networks)

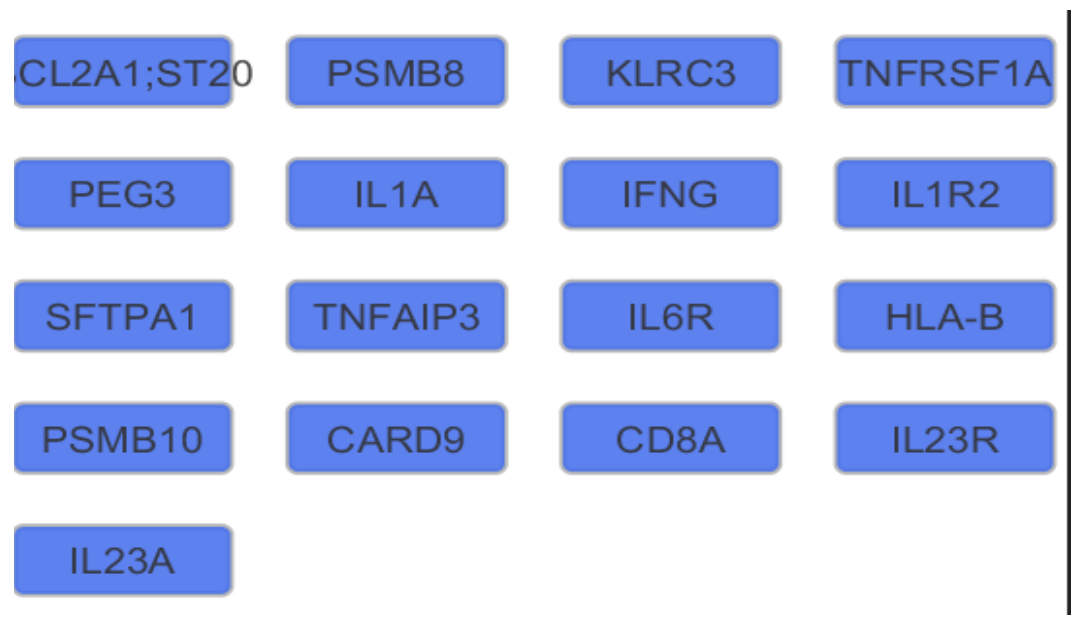

Figure 3b. Image of intersection between missSNP NFKBIE and TNF networks

(nodes represent proteins that are common to both networks)

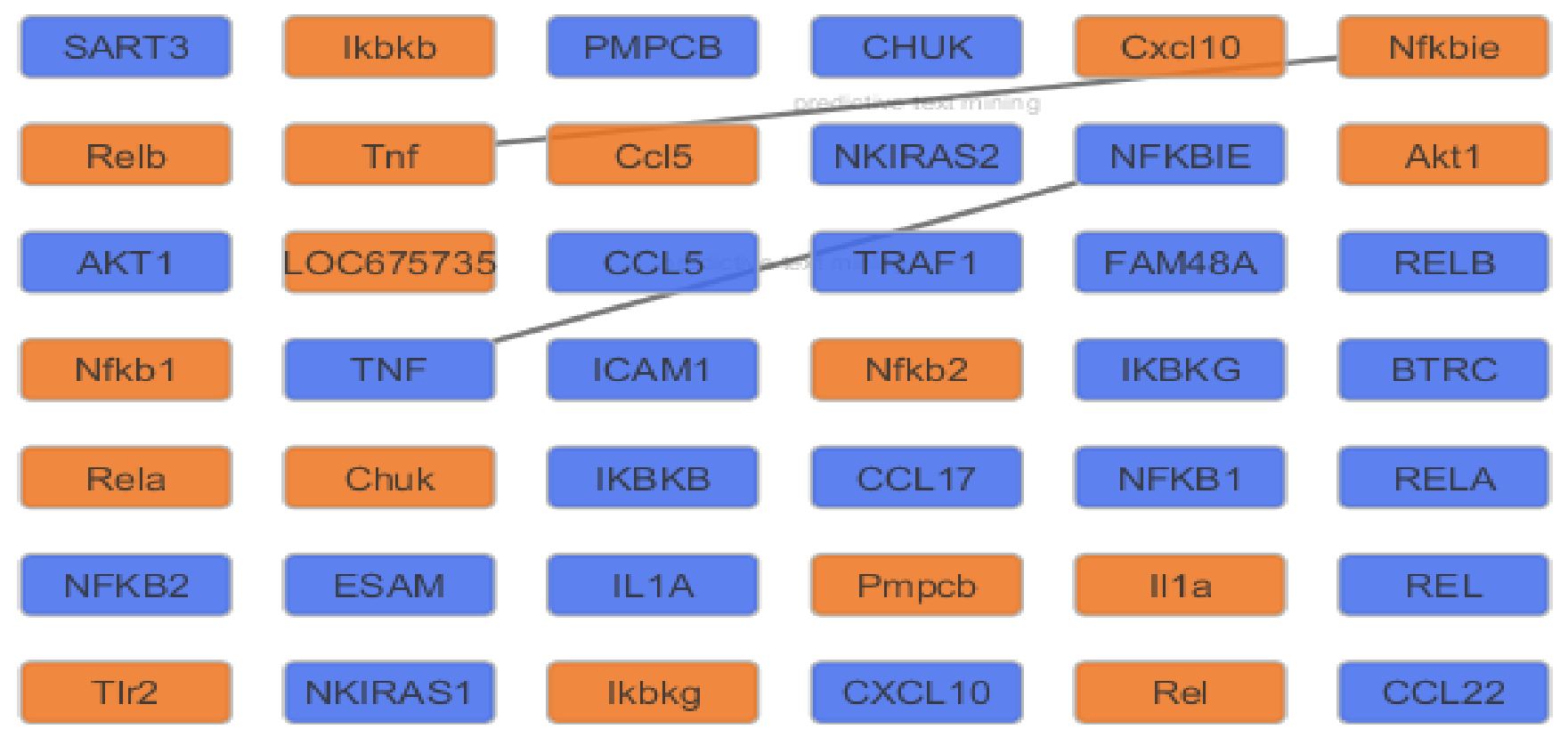

Nodes labeled with gene symbols; color-coded for each species: blue for human, and red and white for mouse and rat respectively. 
Figure 4.

\section{Union of GWAS AID SNP harboring protein networks}

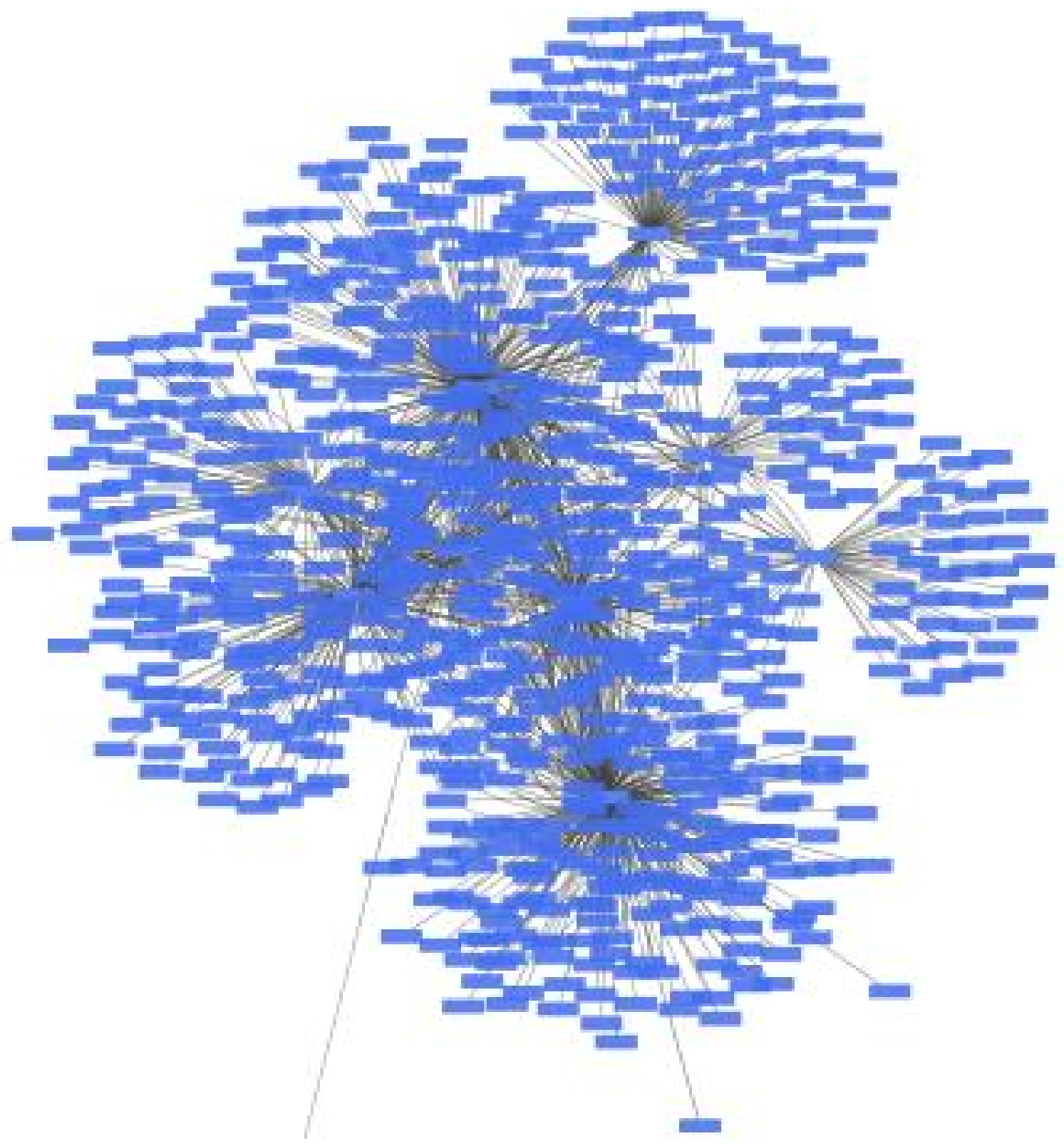

Nodes labeled with official gene symbols; edges represent links between pairs of nodes; clouds are color-coded blue for human. 


\section{Figure 5.}

\section{Network union between intersection datasets of all missSNPs and TNF networks}

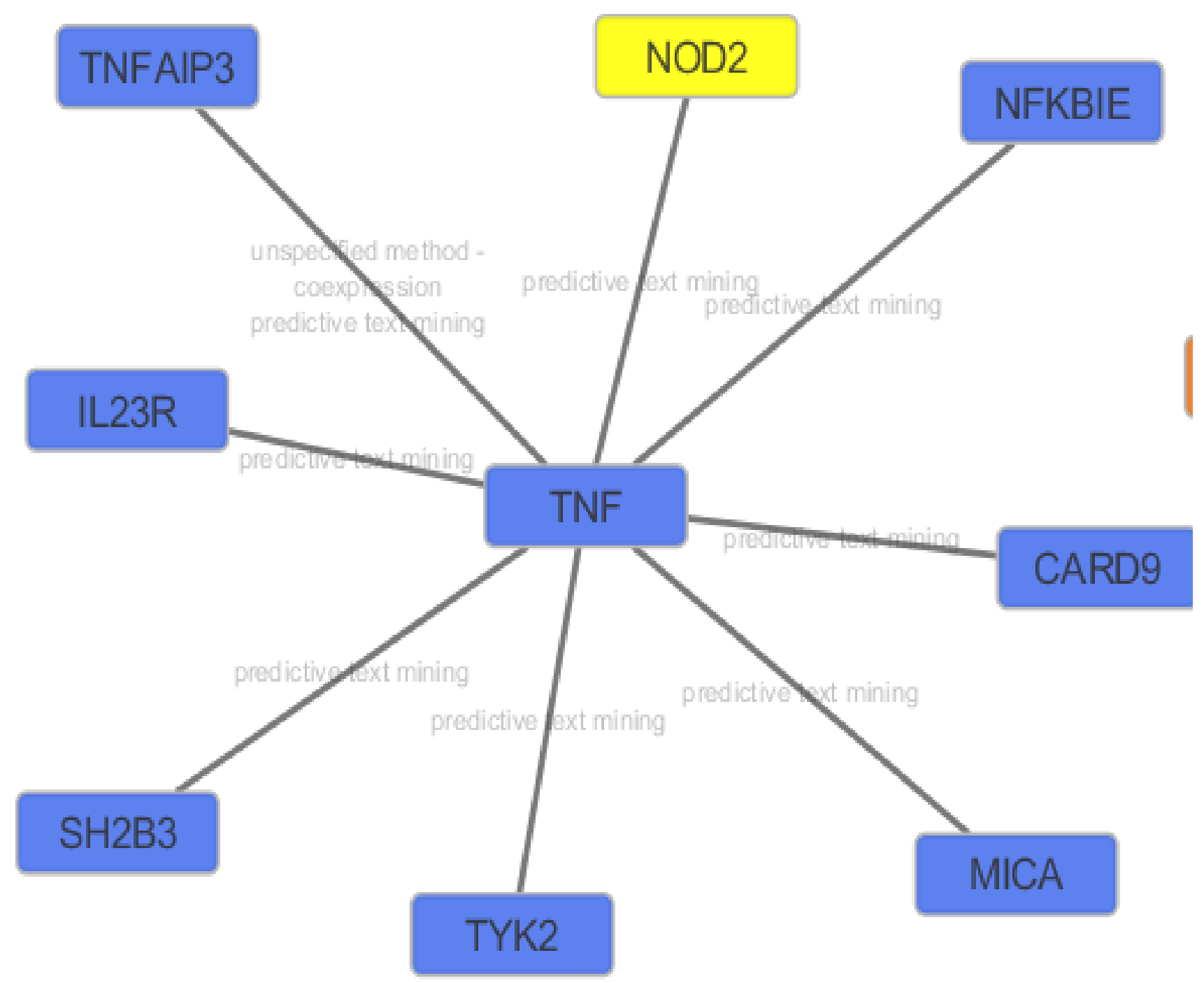

Resulting network consists of the nodes labeled with official human gene symbols and edges represent links between nodes (proteins) that were detected by different methods such as predictive textmining and coexpression. 
Figure 6.

GWAS AID missSNP harboring genes show high expression in several immune-related human tissues and cells

colored squares indicate intensity of expression for each gene and tissues/cells
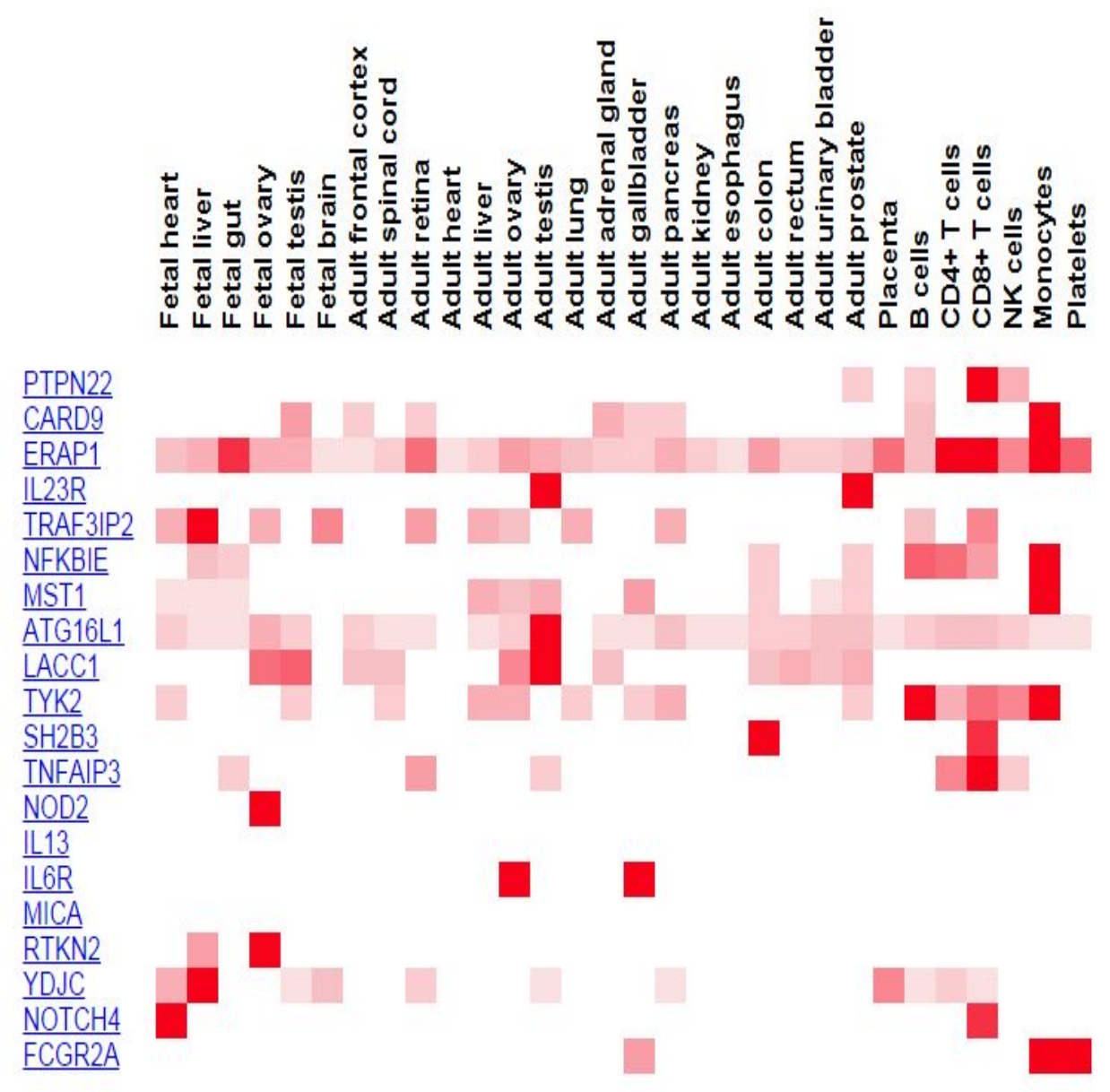
Figure 7. Image of networks constructed by STRING for missSNP, ncSNP and allSNP datasets

7a) missSNP

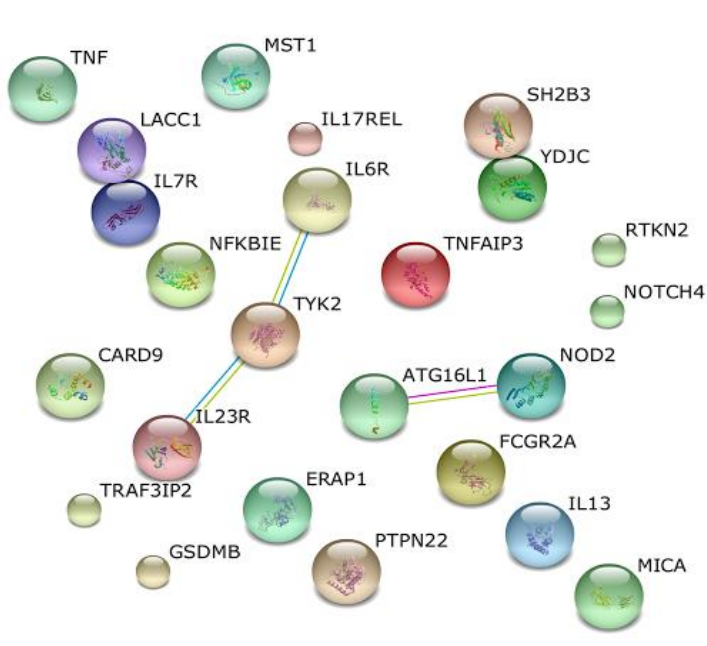

7b) ncSNP

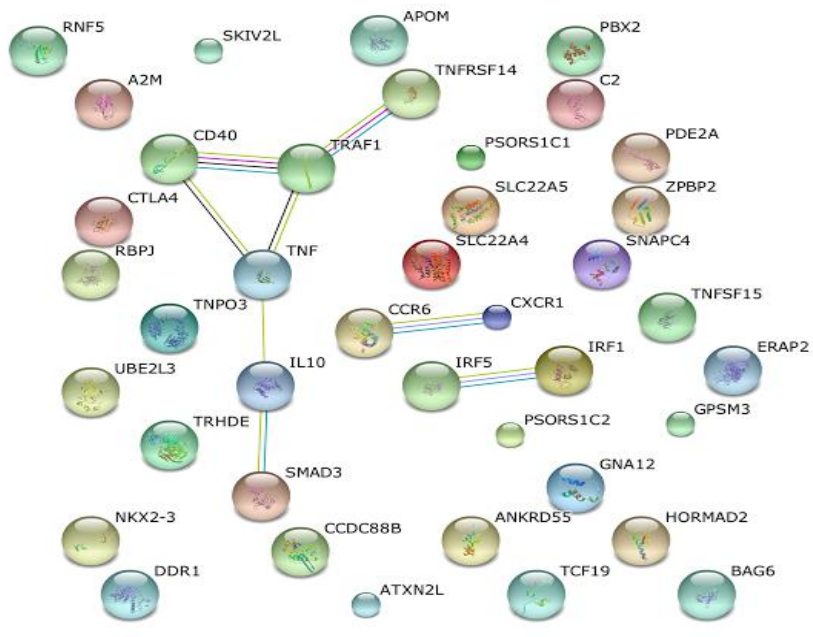

7c) allSNP

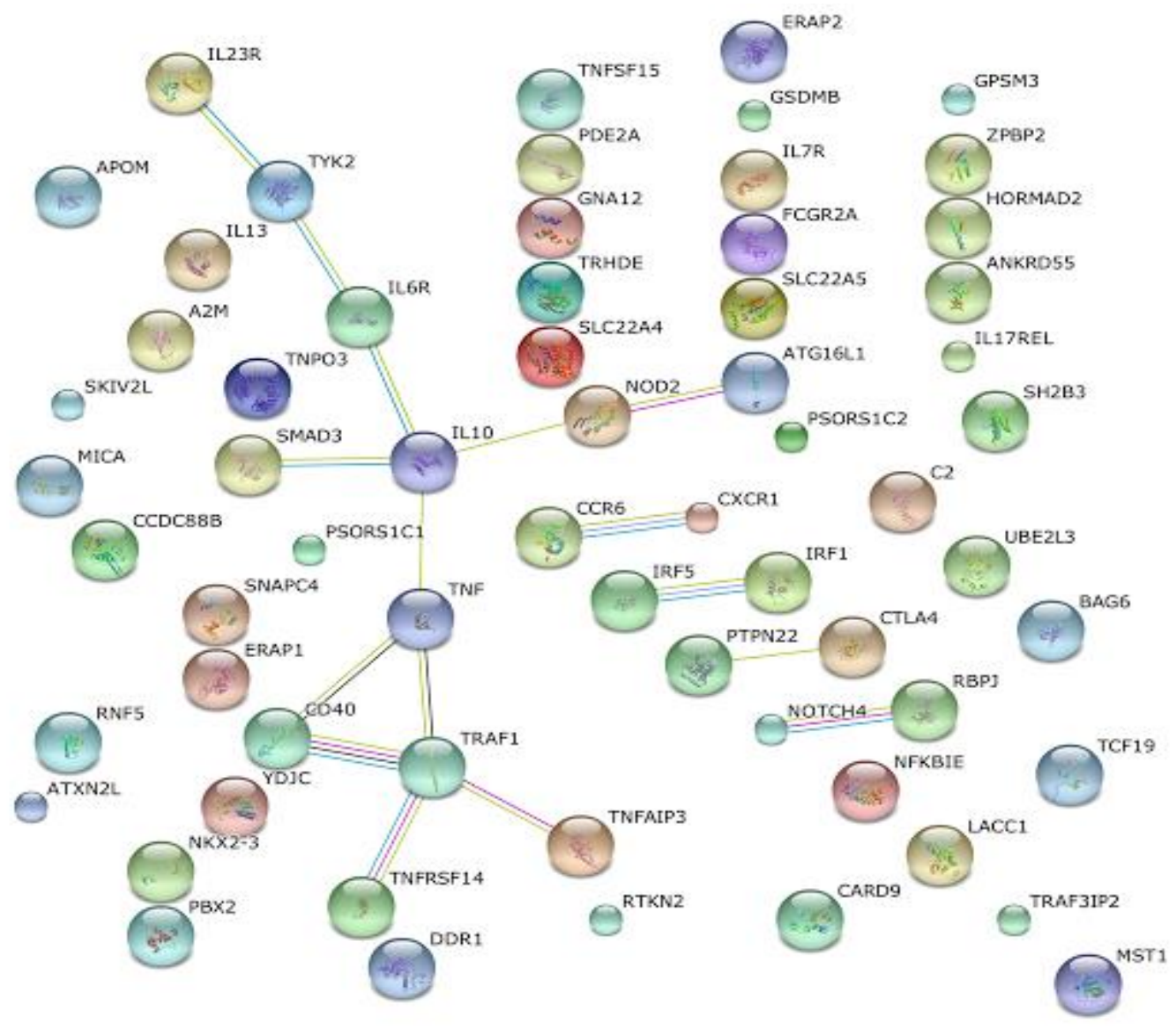




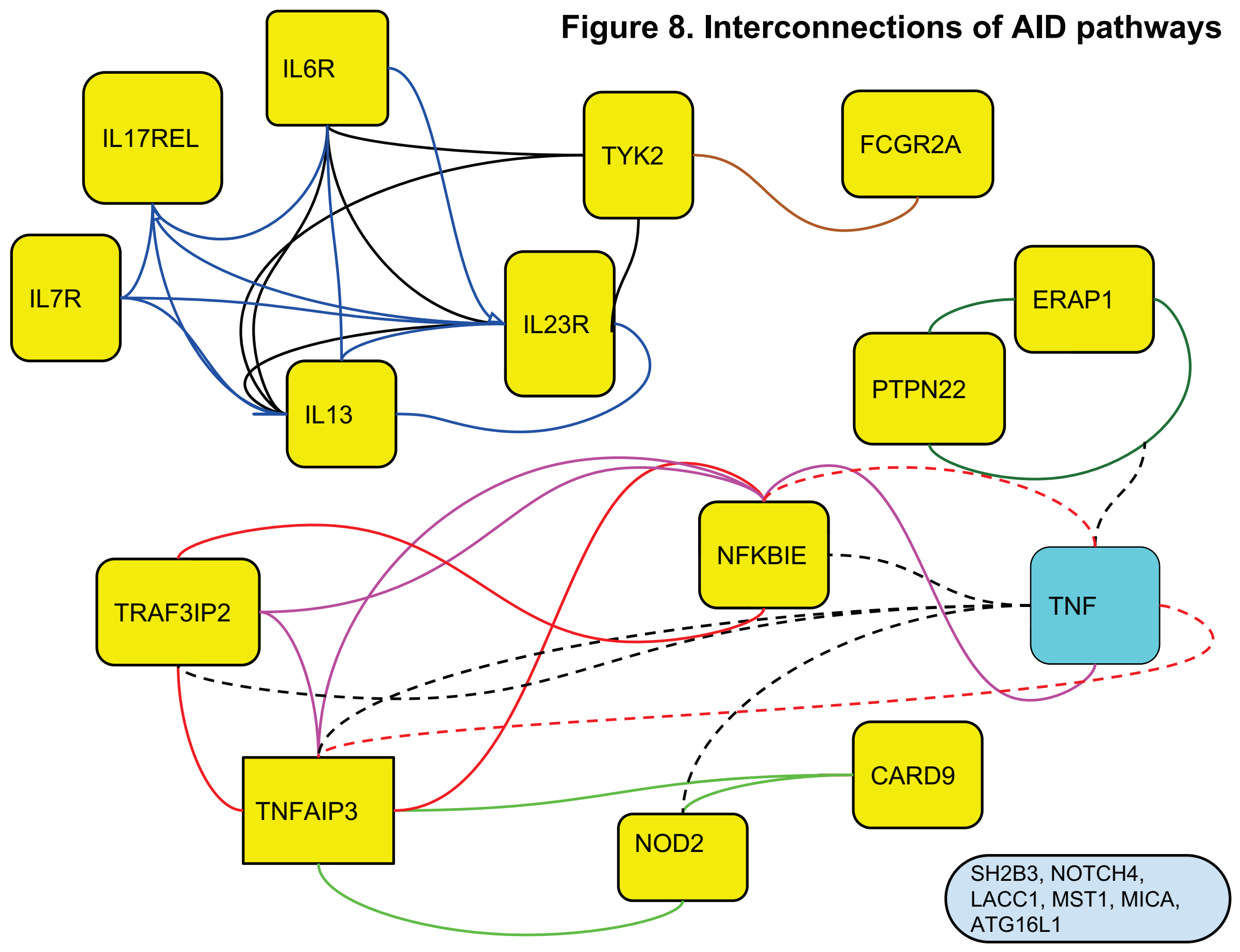

Legend: connecting lines between genes denote KEGG pathways:

red: NFkB signaling

black: Jak_STAT signaling

blue: Cytokine-Cytokine $\mathrm{R}$

green: NOD-like signaling

brown: Osteoclast differentiation

pink: TNF signaling

dark green: Antigen Proc\&Presen

black dotted: TNF signaling

red dotted: $T$ cell $R$ signaling

\section{Color of gene's cell:}

yellow background:

gene is connected to the selected KEGG pathways

blue background:

gene is not connected to the selected KEGG pathways 


\section{Supplemental Table 1. AID GWAS associations retrieved from NHGRI Catalog}

at the end if November 201

Arthritis, Psoriatic PsA

\begin{tabular}{|c|c|c|c|c|c|c|c|c|}
\hline$\#$ & rs \# & Context & Gene & Location & P-value & $\begin{array}{l}\text { RegulomeDB } \\
\text { score }\end{array}$ & Source & PubMed \\
\hline 1 & rs13191343 & nearGene-5 & HLA-C & $6: 31,241,109$ & $2.000 \times 10-72$ & $4,5,6$ & NHGRI & 20953186 \\
\hline 2 & rs33980500 & missense & TRAF3IP2 & $6: 111,913,262$ & $1.000 \times 10-20$ & $"$ & NHGRI & 20953186 \\
\hline 3 & rs12188300 & intergenic & IL12B, ADRA1B & $5: 158,829,527$ & $7.000 \times 10-17$ & $"$ & NHGRI & 20953186 \\
\hline 4 & rs13017599 & intergenic & REL, RPS12P3 & $2: 61,164,331$ & $1.000 \times 10-8$ & $"$ & NHGRI & 22170493 \\
\hline 5 & rs702873 & intergenic & RPL21P33, REL & $2: 61,081,542$ & $2.000 \times 10-7$ & $"$ & NHGRI & 22170493 \\
\hline
\end{tabular}

Arthritis, Rheumatoid RA

\begin{tabular}{|c|c|c|c|c|c|c|c|c|}
\hline \# & rs \# & Context & Gene & Location & P-value & $\begin{array}{l}\text { RegulomeDB } \\
\text { score }\end{array}$ & Source & PubMed \\
\hline 1 & rs4750316 & UTR-3 & DKFZp667F0711 & $10: 6,393,260$ & $2.000 \times 10-7$ & $*$ & NHGRI & 20453842 \\
\hline 2 & rs4750316 & UTR-3 & DKFZp667F0711 & $10: 6,393,260$ & $4.000 \times 10-7$ & $*$ & NHGRI & 18794853 \\
\hline 3 & rs1329568 & UTR-3 & LOC100130458 & $9: 37,037,976$ & $8.000 \times 10-7$ & $*$ & NHGRI & 22491018 \\
\hline 4 & rs805297 & nearGene-5 & APOM & $6: 31,622,606$ & $3.000 \times 10-10$ & $1 f$ & NHGRI & 21844665 \\
\hline 5 & rs2812378 & nearGene-5 & CCL21 & $9: 34,710,260$ & $3.000 \times 10-8$ & $*$ & NHGRI & 18794853 \\
\hline 6 & rs2841277 & nearGene-5 & PLD4 & $14: 105,391,005$ & $2.000 \times 10-14$ & $*$ & NHGRI & 22446963 \\
\hline 7 & rs6496667 & nearGene-5 & ZNF774 & $15: 90,893,668$ & $1.000 \times 10-7$ & $*$ & NHGRI & 22446963 \\
\hline 8 & rs3087243 & nearGene-3 & CTLA4 & 2 : 204,738,919 & $1.000 \times 10-8$ & $3 a$ & NHGRI & 20453842 \\
\hline 9 & rs10488631 & nearGene-3 & TNPO3 & $7: 128,594,183$ & $4.000 \times 10-11$ & $3 a$ & NHGRI & 20453842 \\
\hline 10 & rs2233434 & missense & NFKBIE & $6: 44,232,920$ & $6.000 \times 10-19$ & $*$ & NHGRI & 22446963 \\
\hline 11 & rs2476601 & missense & PTPN22 & $1: 114,377,568$ & $9.000 \times 10-74$ & $2 b$ & NHGRI & 20453842 \\
\hline 12 & rs2476601 & missense & PTPN22 & $1: 114,377,568$ & $2.000 \times 10-21$ & $2 b$ & NHGRI & 19503088 \\
\hline 13 & rs2476601 & missense & PTPN22 & $1: 114,377,568$ & $2.000 \times 10-11$ & $2 b$ & NHGRI & 17804836 \\
\hline 14 & rs3184504 & missense & $\mathrm{SH} 2 \mathrm{~B} 3$ & $12: 111,884,608$ & $6.000 \times 10-7$ & $*$ & NHGRI & 20453842 \\
\hline 15 & rs2230926 & missense & TNFAIP3 & $6: 138,196,066$ & $2.000 \times 10-7$ & $*$ & NHGRI & 20453841 \\
\hline 16 & rs2075876 & intron & AIRE & $21: 45,709,153$ & $4.000 \times 10-9$ & $*$ & NHGRI & 21505073 \\
\hline 18 & rs6859219 & intron & ANKRD55 & $5: 55,438,580$ & $1.000 \times 10-11$ & $2 b$ & NHGRI & 20453842 \\
\hline 19 & rs2867461 & intron & ANXA3 & $4: 79,513,215$ & $1.000 \times 10-12$ & $*$ & NHGRI & 22446963 \\
\hline 21 & rs10821944 & intron & ARID5B & $10: 63,785,089$ & $6.000 \times 10-18$ & $*$ & NHGRI & 22446963 \\
\hline 23 & rs26232 & intron & C5orf30 & $5: 102,596,720$ & $4.000 \times 10-8$ & $2 b$ & NHGRI & 20453842 \\
\hline 24 & rs6910071 & intron & C6orf10 & $6: 32,282,854$ & $1.000 \times 10-299$ & $*$ & NHGRI & 20453842 \\
\hline 25 & rs2395148 & intron & C6orf10 & $6: 32,321,554$ & $2.000 \times 10-10$ & $*$ & NHGRI & 18576341 \\
\hline 26 & rs3093024 & intron & CCR6 & $6: 167,532,793$ & $8.000 \times 10-19$ & $*$ & NHGRI & 20453841 \\
\hline 27 & rs3093023 & intron & CCR6 & $6: 167,534,290$ & $2.000 \times 10-11$ & $1 f$ & NHGRI & 20453842 \\
\hline 28 & rs840016 & intron & CD247 & $1: 167,408,670$ & $2.000 \times 10-7$ & $*$ & NHGRI & 20453842 \\
\hline 29 & rs4810485 & intron & CD40 & $20: 44,747,947$ & $3.000 \times 10-9$ & $1 f$ & NHGRI & 20453842 \\
\hline 30 & rs4810485 & intron & CD40 & $20: 44,747,947$ & $8.000 \times 10-9$ & $1 f$ & NHGRI & 18794853 \\
\hline 31 & rs42041 & intron & CDK6 & $7: 92,246,744$ & $4.000 \times 10-7$ & $*$ & NHGRI & 18794853 \\
\hline 32 & rs4942242 & intron & ENOX1 & $13: 44,217,064$ & $2.000 \times 10-7$ & $*$ & NHGRI & 22491018 \\
\hline 33 & rs1914816 & intron & ETFA & $15: 76,546,933$ & $7.000 \times 10-7$ & $*$ & NHGRI & 22491018 \\
\hline 34 & rs13315591 & intron & FAM107A & $3: 58,556,841$ & $5.000 \times 10-8$ & $*$ & NHGRI & 20453842 \\
\hline 35 & rs7940423 & intron & GALNTL4 & $11: 11,504,228$ & $1.000 \times 10-7$ & $*$ & NHGRI & 22491018 \\
\hline 36 & rs3783637 & intron & $\mathrm{GCH} 1$ & $14: 55,348,118$ & $2.000 \times 10-7$ & $2 b$ & NHGRI & 22446963 \\
\hline 37 & rs706778 & intron & IL2RA & $10: 6,098,949$ & $1.000 \times 10-11$ & $*$ & NHGRI & 20453842 \\
\hline 38 & rs13119723 & intron & KIAA1109 & $4: 123,218,313$ & $7.000 \times 10-7$ & $*$ & NHGRI & 20453842 \\
\hline 39 & rs1678542 & intron & KIF5A & $12: 57,968,715$ & $9.000 \times 10-8$ & $*$ & NHGRI & 18794853 \\
\hline 40 & rs13393173 & intron & LASS6 & 2 : 169,389,091 & $4.000 \times 10-7$ & $*$ & NHGRI & 18615156 \\
\hline 41 & rs17118552 & intron & MDGA2 & $14: 47,874,557$ & $2.000 \times 10-7$ & $*$ & NHGRI & 22491018 \\
\hline 42 & rs3890745 & intron & MMEL1 & $1: 2,553,624$ & $1.000 \times 10-7$ & $*$ & NHGRI & 18794853 \\
\hline 43 & rs3890745 & intron & MMEL1 & $1: 2,553,624$ & $4.000 \times 10-7$ & $*$ & NHGRI & 20453842 \\
\hline 44 & rs7046653 & intron & MOBKL2B & $9: 27,490,967$ & $5.000 \times 10-7$ & $*$ & NHGRI & 18615156 \\
\hline 45 & rs6500395 & intron & N4BP1 & $16: 48,621,402$ & $6.000 \times 10-7$ & $*$ & NHGRI & 22491018 \\
\hline
\end{tabular}




\begin{tabular}{|c|c|c|c|c|c|c|c|c|}
\hline 46 & rs3781913 & intron & PDE2A & $11: 72,373,496$ & $6.000 \times 10-10$ & $1 f$ & NHGRI & 22446963 \\
\hline 47 & rs2075876 & intron & PFKL & $21: 45,709,153$ & $4.000 \times 10-9$ & $*$ & NHGRI & 21505073 \\
\hline 48 & rs6026990 & intron & PHACTR3 & $20: 58,177,615$ & $6.000 \times 10-7$ & $*$ & NHGRI & 22491018 \\
\hline 49 & rs854555 & intron & PON1 & $7: 94,930,391$ & $2.000 \times 10-7$ & $*$ & NHGRI & 18615156 \\
\hline 50 & rs7404928 & intron & PRKCB & $16: 23,888,840$ & $4.000 \times 10-7$ & $*$ & NHGRI & 22446963 \\
\hline 51 & rs1957895 & intron & PRKCH & $14: 61,908,332$ & $4.000 \times 10-7$ & $*$ & NHGRI & 22446963 \\
\hline 52 & rs12901682 & intron & PSMA4 & $15: 78,833,223$ & $4.000 \times 10-8$ & $*$ & NHGRI & 22491018 \\
\hline 53 & rs2847297 & intron & PTPN2 & $18: 12,797,694$ & $2.000 \times 10-8$ & $*$ & NHGRI & 22446963 \\
\hline 54 & rs13137105 & intron & RCHY1 & $4: 76,416,387$ & $9.000 \times 10-7$ & $*$ & NHGRI & 22491018 \\
\hline 55 & rs13031237 & intron & REL & $2: 61,136,129$ & $8.000 \times 10-7$ & $*$ & NHGRI & 20453842 \\
\hline 56 & rs16977065 & intron & RIT2 & $18: 40,437,651$ & $1.000 \times 10-7$ & $*$ & NHGRI & 22491018 \\
\hline 57 & rs1809529 & intron & SLC6A11 & $3: 10,877,611$ & $3.000 \times 10-7$ & $*$ & NHGRI & 22491018 \\
\hline 58 & rs934734 & intron & SPRED2 & $2: 65,595,586$ & $5.000 \times 10-10$ & $*$ & NHGRI & 20453842 \\
\hline 59 & rs11121380 & intron & SPSB1 & $1: 9,408,959$ & $5.000 \times 10-8$ & $*$ & NHGRI & 22491018 \\
\hline 60 & rs7574865 & intron & STAT4 & 2 : 191,964,633 & $3.000 \times 10-7$ & $*$ & NHGRI & 20453842 \\
\hline 61 & rs7574865 & intron & STAT4 & 2 : 191,964,633 & $2.000 \times 10-7$ & $*$ & NHGRI & 20453841 \\
\hline 62 & rs3761847 & intron & TRAF1 & $9: 123,690,239$ & $4.000 \times 10-14$ & $*$ & NHGRI & 17804836 \\
\hline 63 & rs3761847 & intron & TRAF1 & $9: 123,690,239$ & $2.000 \times 10-7$ & $*$ & NHGRI & 20453842 \\
\hline 64 & rs12831974 & intron & TRHDE & $12: 72,724,034$ & $6.000 \times 10-7$ & $2 b$ & NHGRI & 21452313 \\
\hline 65 & rs11203203 & intron & UBASH3A & $21: 43,836,186$ & $4.000 \times 10-7$ & $*$ & NHGRI & 20453842 \\
\hline 66 & rs12046117 & intron & VTCN1 & $1: 117,751,365$ & $1.000 \times 10-7$ & $*$ & NHGRI & 19116933 \\
\hline 67 & rs1543922 & intron & ZNF175 & $19: 52,084,836$ & $3.000 \times 10-7$ & $*$ & NHGRI & 22491018 \\
\hline 68 & rs2240335 & cds-synon & PADI4 & $1: 17,674,537$ & $2.000 \times 10-8$ & $*$ & NHGRI & 21452313 \\
\hline 69 & rs2240335 & cds-synon & PADI4 & $1: 17,674,537$ & $2.000 \times 10-8$ & $*$ & NHGRI & 21505073 \\
\hline 70 & rs11676922 & intergenic & AFF3, LONRF2 & $2: 100,806,940$ & $1.000 \times 10-14$ & $*$ & NHGRI & 20453842 \\
\hline 71 & rs10865035 & intergenic & AFF3, LONRF2 & $2: 100,835,734$ & $2.000 \times 10-7$ & $*$ & NHGRI & 20453842 \\
\hline 72 & rs1898036 & intergenic & ATPBD4, COX6CP4 & $15: 36,349,846$ & $2.000 \times 10-7$ & $*$ & NHGRI & 22491018 \\
\hline 73 & rs11900673 & intergenic & B3GNT2, TMEM17 & $2: 62,452,661$ & $1.000 \times 10-8$ & $*$ & NHGRI & 22446963 \\
\hline 74 & rs2002842 & intergenic & BDP1P, SALL3 & $18: 76,409,597$ & $6.000 \times 10-7$ & $*$ & NHGRI & 18668548 \\
\hline 75 & rs11051970 & intergenic & BICD1, FGD4 & $12: 32,537,488$ & $1.000 \times 10-7$ & $*$ & NHGRI & 22491018 \\
\hline 76 & rs12565755 & intergenic & C1orf87, NFIA & $1: 61,041,875$ & $5.000 \times 10-8$ & $*$ & NHGRI & 22491018 \\
\hline 77 & rs874040 & intergenic & C4orf52, RBPJ & $4: 26,108,197$ & $1.000 \times 10-16$ & $2 b$ & NHGRI & 20453842 \\
\hline 78 & rs951005 & intergenic & C9orf144B, C9orf144 & $9: 34,743,681$ & $4.000 \times 10-10$ & $*$ & NHGRI & 20453842 \\
\hline 79 & rs11937061 & intergenic & CCNG2, CXCL13 & $4: 78,136,933$ & $2.000 \times 10-7$ & $*$ & NHGRI & 22491018 \\
\hline 80 & rs657075 & intergenic & CSF2, P4HA2 & $5: 131,430,118$ & $3.000 \times 10-10$ & $*$ & NHGRI & 22446963 \\
\hline 81 & rs6138150 & intergenic & CST2, CST5 & $20: 23,847,009$ & $3.000 \times 10-7$ & $*$ & NHGRI & 18615156 \\
\hline 82 & rs1273516 & intergenic & CYP4F22, RPL23AP2 & $19: 15,677,710$ & $9.000 \times 10-7$ & $*$ & NHGRI & 22491018 \\
\hline 83 & rs2837960 & intergenic & DSCAM, C21orf130 & $21: 42,511,918$ & $2.000 \times 10-7$ & $*$ & NHGRI & 17554300 \\
\hline 84 & rs6138892 & intergenic & EBF4, RPL19P1 & $20: 2,755,488$ & $3.000 \times 10-7$ & $*$ & NHGRI & 22491018 \\
\hline 85 & rs4937362 & intergenic & ETS1, FLI1 & $11: 128,492,739$ & $8.000 \times 10-7$ & $*$ & NHGRI & 22446963 \\
\hline 86 & rs2736340 & intergenic & FAM167A, BLK & $8: 11,343,973$ & $6.000 \times 10-9$ & $*$ & NHGRI & 19503088 \\
\hline 87 & rs9604529 & intergenic & FLJ44054 & $13: 114,622,597$ & $7.000 \times 10-7$ & $*$ & NHGRI & 22491018 \\
\hline 88 & rs16938910 & intergenic & GDAP1, PCBP2P2 & $8: 75,373,948$ & $4.000 \times 10-7$ & $*$ & NHGRI & 22491018 \\
\hline 89 & rs12109285 & intergenic & GUSBP1, CDH12 & $5: 21,749,348$ & $1.000 \times 10-7$ & $*$ & NHGRI & 22491018 \\
\hline 90 & rs1610677 & intergenic & HCP5P12, HLA-G & $6: 29,789,171$ & $4.000 \times 10-15$ & $*$ & NHGRI & 21653640 \\
\hline 91 & rs6457620 & intergenic & HLA-DQB1, HLA-DQA & $6: 32,663,999$ & $4.000 \times 10-186$ & $*$ & NHGRI & 18794853 \\
\hline 92 & rs6457617 & intergenic & HLA-DQB1, HLA-DQA & $6: 32,663,851$ & $5.000 \times 10-75$ & $*$ & NHGRI & 17554300 \\
\hline 93 & rs13192471 & intergenic & HLA-DQB1, HLA-DQA & $6: 32,671,103$ & $2.000 \times 10-58$ & $*$ & NHGRI & 20453841 \\
\hline 94 & rs7765379 & intergenic & HLA-DQB1, HLA-DQA & $6: 32,680,928$ & $5.000 \times 10-23$ & $*$ & NHGRI & 21452313 \\
\hline 95 & rs6457617 & intergenic & HLA-DQB1, HLA-DQA & $6: 32,663,851$ & $1.000 \times 10-9$ & $*$ & NHGRI & 18668548 \\
\hline 96 & rs660895 & intergenic & HLA-DRB1, HLA-DQA & $6: 32,577,380$ & $1.000 \times 10-108$ & If & NHGRI & 17804836 \\
\hline 97 & rs9272219 & intergenic & HLA-DRB1, HLA-DQA & $6: 32,602,269$ & $1.000 \times 10-45$ & $*$ & NHGRI & 21653640 \\
\hline 98 & rs615672 & intergenic & HLA-DRB1, HLA-DQA & $6: 32,574,171$ & $8.000 \times 10-27$ & $*$ & NHGRI & 17554300 \\
\hline 99 & rs9268853 & intergenic & HLA-DRB9, HLA-DRB5 & $6: 32,429,643$ & $5.000 \times 10-109$ & $*$ & NHGRI & 21653640 \\
\hline 100 & rs6028945 & intergenic & HSPE1P1, MAFB & $20: 38,820,805$ & $2.000 \times 10-7$ & $*$ & NHGRI & 18615156 \\
\hline 101 & rs743777 & intergenic & IL2RB, C1QTNF6 & $22: 37,551,607$ & $1.000 \times 10-7$ & $*$ & NHGRI & 17554300 \\
\hline 102 & rs743777 & intergenic & IL2RB, C1QTNF6 & $22: 37,551,607$ & $2.000 \times 10-7$ & $*$ & NHGRI & 21653640 \\
\hline 103 & rs10488631 & intergenic & IRF5, TNPO3 & $7: 128,594,183$ & $4.000 \times 10-11$ & $3 a$ & NHGRI & 20453842 \\
\hline 104 & rs2280381 & intergenic & IRF8, FOXF1 & $16: 86,018,633$ & $2.000 \times 10-7$ & $*$ & NHGRI & 22446963 \\
\hline
\end{tabular}


105 rs7155603

106 rs231735

107 rs983332

108 rs6774280

109 rs9296015

110 rs1406428

111 rs6920220

112 rs10499194

113 rs6920220

114 rs6920220

115 rs1329568

116 rs16906916

117 rs881375

118 rs12131057

119 rs10945919

120 rs13017599

121 rs12529514

122 rs437943

123 rs17374222

124 rs11761231

125 rs6679677

126 rs6679677

127 rs72991

128 rs7164176

129 rs800586

130 rs2872507 intergenic intergenic intergenic intergenic intergenic intergenic intergenic intergenic intergenic intergenic intergenic intergenic intergenic intergenic intergenic intergenic intergenic intergenic intergenic intergenic intergenic intergenic intergenic intergenic intergenic intergenic

$\begin{array}{lrll}\text { JDP2, BATF } & 14: 75,960,536 & 1.000 \times 10-7 \\ \text { KRT18P39, CTLA4 } & 2: 204,693,876 & 6.000 \times 10-9 \\ \text { LMO4, RPL36AP10 } & 1: 88,132,380 & 5.000 \times 10-7 \\ \text { MRPS35P1, MRPS36 } & 3: 6,255,997 & 9.000 \times 10-7 \\ \text { NOTCH4, C6orf10 } & 6: 32,218,989 & 2.000 \times 10-38 \\ \text { NRXN1, CRYGGP } & 2: 51,736,994 & 2.000 \times 10-7 \\ \text { OLIG3, TNFAIP3 } & 6: 138,006,504 & 9.000 \times 10-13 \\ \text { OLIG3, TNFAIP3 } & 6: 138,002,637 & 1.000 \times 10-9 \\ \text { OLIG3, TNFAIP3 } & 6: 138,006,504 & 2.000 \times 10-9 \\ \text { OLIG3, TNFAIP3 } & 6: 138,006,504 & 1.000 \times 10-7 \\ \text { PAX5, RPL32P21 } & 9: 37,037,976 & 8.000 \times 10-7 \\ \text { PCDH15, GAPDHP21 } & 10: 56,848,985 & 8.000 \times 10-7 \\ \text { PHF19, TRAF1 } & 9: 123,652,898 & 4.000 \times 10-8 \\ \text { POU3F1, RRAGC } & 1: 38,624,129 & 4.000 \times 10-7 \\ \text { QKI, C6orf118 } & 6: 164,186,677 & 3.000 \times 10-7 \\ \text { REL, RPS12P3 } & 2: 61,164,331 & 2.000 \times 10-12 \\ \text { RNF182, CD83 } & 6: 14,096,658 & 2.000 \times 10-8 \\ \text { RPL31P31, ARAP2 } & 4: 35,372,098 & 4.000 \times 10-7 \\ \text { RPLP1, GEMIN8P1 } & 15: 69,995,344 & 2.000 \times 10-7 \\ \text { RPS14P10, RPS15AP2 } & 7: 131,370,039 & 4.000 \times 10-7 \\ \text { RPS2P14, RSBN1 } & 1: 114,303,808 & 6.000 \times 10-42 \\ \text { RPS2P14, RSBN1 } & 1: 114,303,808 & 6.000 \times 10-25 \\ \text { SC5DL, SORL1 } & 11: 121,243,716 & 5.000 \times 10-7 \\ \text { SV2B, TRNAY16P } & 15: 92,211,774 & 5.000 \times 10-7 \\ \text { TRPS1, EIF3H } & 8: 116,813,905 & 2.000 \times 10-7 \\ \text { ZPBP2, GSDMB } & 17: 38,040,763 & 9.000 \times 10-7\end{array}$

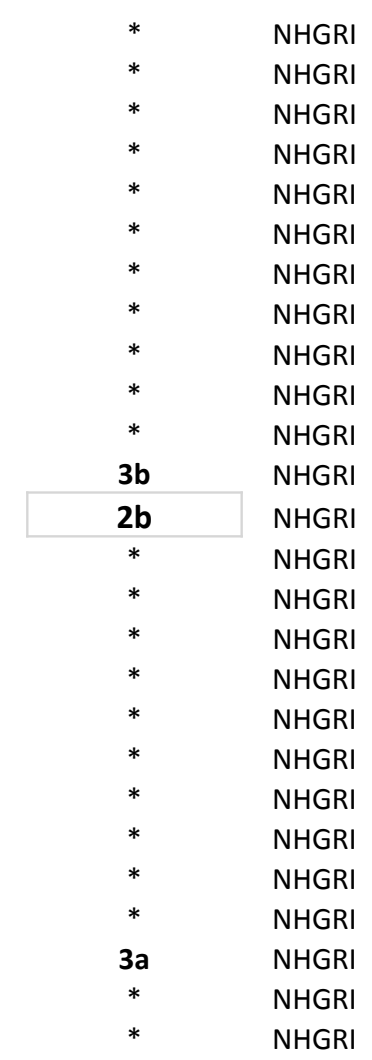

20453842

19503088

18615156

22491018

21505073

22491018

20453842

17982456

18794853

17982456

22491018

22491018

19503088

20453842

18615156

19503088

22446963

18615156

20453842

17554300

18794853

17554300

22491018

22491018

22491018

20453842

\section{Ankylosing Spondylitis AS}

\begin{tabular}{|c|c|c|c|c|c|c|c|c|c|}
\hline$\#$ & rs \# & Context & Gene & & Location & P-value & $\begin{array}{l}\text { RegulomeDB } \\
\text { score }\end{array}$ & Source & PubMed \\
\hline 1 & rs10781500 & nearGene-5 & CARD9 & 9 & : 139,269,338 & $1.000 \times 10-7$ & $*$ & NHGRI & 21743469 \\
\hline 2 & rs30187 & missense & ERAP1 & 5 & : 96,124,330 & $2.000 \times 10-27$ & $*$ & NHGRI & 21743469 \\
\hline 3 & rs11209026 & missense & IL23R & 1 & $: 67,705,958$ & $2.000 \times 10-17$ & $*$ & NHGRI & 21743469 \\
\hline 4 & rs11209026 & missense & IL23R & 1 & : 67,705,958 & $9.000 \times 10-14$ & $*$ & NHGRI & 20062062 \\
\hline 5 & rs17095830 & intron & ANO6 & 12 & $: 45,774,908$ & $2.000 \times 10-8$ & $*$ & NHGRI & 22138694 \\
\hline 6 & rs4389526 & intron & ANTXR2 & 4 & : 80,946,475 & $9.000 \times 10-8$ & $*$ & NHGRI & 21743469 \\
\hline 7 & rs4333130 & intron & ANTXR2 & 4 & : 80,949,829 & $9.000 \times 10-8$ & $*$ & NHGRI & 20062062 \\
\hline 8 & rs1326986 & intron & C10orf112 & 10 & : 19,929,513 & $4.000 \times 10-7$ & $*$ & NHGRI & 20062062 \\
\hline 9 & rs2075726 & intron & CSF2RB & 22 & : 37,310,046 & $9.000 \times 10-7$ & $*$ & NHGRI & 22138694 \\
\hline 10 & rs13210693 & intron & FLJ37396 & 6 & : 109,598,964 & $9.000 \times 10-7$ & $*$ & NHGRI & 22138694 \\
\hline 11 & rs2297909 & intron & KIF21B & 1 & : 200,960,307 & $5.000 \times 10-12$ & $*$ & NHGRI & 21743469 \\
\hline 12 & rs27434 & cds-synon & ERAP1 & 5 & : 96,129,512 & $5.000 \times 10-12$ & $*$ & NHGRI & 20062062 \\
\hline 13 & rs10865331 & intergenic & B3GNT2, TMEM17 & 2 & $: 62,551,472$ & $7.000 \times 10-34$ & $*$ & NHGRI & 21743469 \\
\hline 14 & rs10865331 & intergenic & B3GNT2, TMEM17 & 2 & $: 62,551,472$ & $2.000 \times 10-19$ & $*$ & NHGRI & 20062062 \\
\hline 15 & rs10440635 & intergenic & DAB2, PTGER4 & 5 & $: 40,490,790$ & $3.000 \times 10-7$ & $*$ & NHGRI & 21743469 \\
\hline 16 & rs7743761 & intergenic & DHFRP2, HLA-S & 6 & $: 31,336,100$ & $5.000 \times 10-304$ & $*$ & NHGRI & 20062062 \\
\hline 17 & rs13210693 & intergenic & FLJ37396, CCDC162 & 6 & : 109,598,964 & $9.000 \times 10-7$ & $*$ & NHGRI & 22138694 \\
\hline 18 & rs2242944 & intergenic & FLJ45139, RPL23AP1 & 21 & $: 40,465,178$ & $8.000 \times 10-20$ & $*$ & NHGRI & 20062062 \\
\hline 19 & rs378108 & intergenic & FL45139, RPL23AP1 & 21 & $: 40,469,520$ & $2.000 \times 10-11$ & $*$ & NHGRI & 21743469 \\
\hline 20 & rs4349859 & intergenic & HLA-S, MICA & 6 & $: 31,365,787$ & $1.000 \times 10-200$ & $*$ & NHGRI & 21743469 \\
\hline 21 & rs6556416 & intergenic & IL12B, ADRA1B & 5 & : 158,818,745 & $2.000 \times 10-8$ & $*$ & NHGRI & 21743469 \\
\hline 22 & rs2310173 & intergenic & IL1R2, IL1R1 & 2 & $: 102,663,628$ & $5.000 \times 10-7$ & $*$ & NHGRI & 20062062 \\
\hline 23 & rs8070463 & intergenic & KPNB1, TBKBP1 & 17 & $: 45,768,836$ & $5.000 \times 10-8$ & $*$ & NHGRI & 21743469 \\
\hline 24 & rs11616188 & intergenic & LTBR, RPL31P10 & 12 & $: 6,502,742$ & $4.000 \times 10-12$ & $*$ & NHGRI & 21743469 \\
\hline 25 & rs12146962 & intergenic & MTCO1P2, NPAS3 & 14 & : 33,381,098 & $9.000 \times 10-7$ & $*$ & NHGRI & 22138694 \\
\hline 26 & rs4552569 & intergenic & RPL13AP14, EDIL3 & 5 & : 83,173,593 & $9.000 \times 10-10$ & $*$ & NHGRI & 22138694 \\
\hline 27 & rs11249215 & intergenic & RUNX3, SYF2 & 1 & : 25,297,184 & $9.000 \times 10-11$ & $*$ & NHGRI & 21743469 \\
\hline 28 & rs1018326 & intergenic & UBE2E3, ITGA4 & 2 & $: 182,007,800$ & $2.000 \times 10-7$ & $*$ & NHGRI & 20062062 \\
\hline
\end{tabular}


Crohn Disease, CD

\begin{tabular}{|c|c|c|c|c|c|c|c|c|c|}
\hline$\#$ & rs \# & Context & Gene & & Location & P-value & $\begin{array}{l}\text { RegulomeDB } \\
\text { score }\end{array}$ & Source & PubMed \\
\hline 1 & rs504963 & UTR-3 & FUT2 & 19 & : 49,208,865 & $2.000 \times 10-8$ & $*$ & NHGRI & 20570966 \\
\hline 2 & rs10210302 & nearGene-5 & ATG16L1 & 2 & $: 234,158,839$ & $5.000 \times 10-14$ & $*$ & NHGRI & 17554300 \\
\hline 3 & rs12677663 & nearGene-5 & C8orf84 & 8 & : 74,007,347 & $2.000 \times 10-8$ & $*$ & NHGRI & 22412388 \\
\hline 4 & rs11190140 & nearGene-5 & NKX2-3 & 10 & : 101,291,593 & $3.000 \times 10-16$ & $*$ & NHGRI & 18587394 \\
\hline 5 & rs11190141 & nearGene-5 & NKX2-3 & 10 & : 101,292,390 & $5.000 \times 10-7$ & $*$ & NHGRI & 22412388 \\
\hline 6 & rs11574514 & nearGene-5 & PSMB10 & 16 & $:$ 67,971,380 & $2.000 \times 10-7$ & $*$ & NHGRI & 22412388 \\
\hline 7 & rs2241880 & missense & ATG16L1 & 2 & $: 234,183,368$ & $1.000 \times 10-13$ & $*$ & NHGRI & 17435756 \\
\hline 8 & rs2241880 & missense & ATG16L1 & 2 & $: 234,183,368$ & $1.000 \times 10-12$ & $*$ & NHGRI & 22412388 \\
\hline 9 & rs2241880 & missense & ATG16L1 & 2 & $: 234,183,368$ & $3.000 \times 10-7$ & $*$ & NHGRI & 20570966 \\
\hline 10 & rs3764147 & missense & C13orf31 (LACC1) & 13 & : 44,457,925 & $2.000 \times 10-13$ & $1 f$ & NHGRI & 18587394 \\
\hline 11 & rs4077515 & missense & CARD9 & 9 & : 139,266,496 & $1.000 \times 10-36$ & $1 f$ & NHGRI & 21102463 \\
\hline 12 & rs11209026 & missense & IL23R & 1 & $: 67,705,958$ & $1.000 \times 10-64$ & $*$ & NHGRI & 21102463 \\
\hline 13 & rs11209026 & missense & IL23R & 1 & : 67,705,958 & $4.000 \times 10-21$ & $*$ & NHGRI & 22293688 \\
\hline 14 & rs11209026 & missense & IL23R & 1 & $: 67,705,958$ & $1.000 \times 10-18$ & $*$ & NHGRI & 22412388 \\
\hline 15 & rs11209026 & missense & IL23R & 1 & $: 67,705,958$ & $2.000 \times 10-18$ & $*$ & NHGRI & 17447842 \\
\hline 16 & rs3197999 & missense & MST1 & 3 & : 49,721,532 & $6.000 \times 10-17$ & $*$ & NHGRI & 21102463 \\
\hline 17 & rs3197999 & missense & MST1 & 3 & : 49,721,532 & $1.000 \times 10-12$ & $*$ & NHGRI & 18587394 \\
\hline 18 & rs2476601 & missense & PTPN22 & 1 & $: 114,377,568$ & $1.000 \times 10-8$ & $2 b$ & NHGRI & 18587394 \\
\hline 19 & rs12720356 & missense & TYK2 & 19 & : 10,469,975 & $1.000 \times 10-12$ & $*$ & NHGRI & 21102463 \\
\hline 20 & rs3792109 & intron & ATG16L1 & 2 & : 234,184,417 & $7.000 \times 10-41$ & no data & NHGRI & 21102463 \\
\hline 21 & rs3828309 & intron & ATG16L1 & 2 & $: 234,180,410$ & $2.000 \times 10-32$ & 4 & NHGRI & 18587394 \\
\hline 22 & rs1847472 & intron & $\mathrm{BACH} 2$ & 6 & : 90,973,159 & $5.000 \times 10-9$ & $*$ & NHGRI & 21102463 \\
\hline 23 & rs 102275 & intron & C11orf10 & 11 & $: 61,557,803$ & $2.000 \times 10-11$ & $*$ & NHGRI & 21102463 \\
\hline 24 & rs12521868 & intron & C5orf56 & 5 & $: 131,784,393$ & $1.000 \times 10-20$ & $*$ & NHGRI & 21102463 \\
\hline 25 & rs2188962 & intron & C5orf56 & 5 & $: 131,770,805$ & $2.000 \times 10-18$ & $2 a$ & NHGRI & 18587394 \\
\hline 26 & rs2188962 & intron & C5orf56 & 5 & $: 131,770,805$ & $1.000 \times 10-7$ & $*$ & NHGRI & 20570966 \\
\hline 27 & rs6908425 & intron & CDKAL1 & 6 & : 20,728,731 & $9.000 \times 10-10$ & $*$ & NHGRI & 18587394 \\
\hline 28 & rs151181 & intron & CLN3 & 16 & : 28,490,517 & $2.000 \times 10-11$ & $*$ & NHGRI & 21102463 \\
\hline 29 & rs1998598 & intron & DENND1B & 1 & $: 197,727,642$ & $9.000 \times 10-9$ & $*$ & NHGRI & 21102463 \\
\hline 30 & rs13428812 & intron & DNMT3A & 2 & $: 25,492,467$ & $9.000 \times 10-10$ & $*$ & NHGRI & 21102463 \\
\hline 31 & rs2549794 & intron & ERAP2 & 5 & : 96,244,549 & $1.000 \times 10-10$ & $1 f$ & NHGRI & 21102463 \\
\hline 32 & rs2301436 & intron & FGFR1OP & 6 & $: 167,437,988$ & $1.000 \times 10-12$ & $*$ & NHGRI & 18587394 \\
\hline 33 & rs2301436 & intron & FGFR1OP & 6 & : 167,437,988 & $6.000 \times 10-8$ & $*$ & NHGRI & 20570966 \\
\hline 34 & rs780093 & intron & GCKR & 2 & $: 27,742,603$ & $5.000 \times 10-11$ & $*$ & NHGRI & 21102463 \\
\hline 35 & rs8005161 & intron & GPR65 & 14 & $: 88,472,595$ & $4.000 \times 10-18$ & $*$ & NHGRI & 21102463 \\
\hline 36 & rs2058660 & intron & IL18RAP & 2 & $: 103,054,449$ & $2.000 \times 10-12$ & $*$ & NHGRI & 21102463 \\
\hline 37 & rs11465804 & intron & IL23R & 1 & $: 67,702,526$ & $7.000 \times 10-63$ & $*$ & NHGRI & 18587394 \\
\hline 38 & rs7517847 & intron & IL23R & 1 & : 67,681,669 & $3.000 \times 10-12$ & $*$ & NHGRI & 17435756 \\
\hline 39 & rs11805303 & intron & IL23R & 1 & $: 67,675,516$ & $6.000 \times 10-12$ & $*$ & NHGRI & 17554300 \\
\hline 40 & rs11465804 & intron & IL23R & 1 & $: 67,702,526$ & $1.000 \times 10-6$ & $*$ & NHGRI & 20570966 \\
\hline 41 & rs12722489 & intron & IL2RA & 10 & : 6,102,012 & $3.000 \times 10-9$ & $*$ & NHGRI & 21102463 \\
\hline 42 & rs2274910 & intron & ITLN1 & 1 & $: 160,852,046$ & $1.000 \times 10-9$ & $*$ & NHGRI & 18587394 \\
\hline 43 & rs1793004 & intron & NELL1 & 11 & : 20,698,929 & $3.000 \times 10-6$ & $*$ & NHGRI & 17684544 \\
\hline 44 & rs2076756 & intron & NOD2 & 16 & : 50,756,881 & $4.000 \times 10-69$ & 5 & NHGRI & 21102463 \\
\hline 45 & rs2076756 & intron & NOD2 & 16 & : 50,756,881 & $1.000 \times 10-37$ & $*$ & NHGRI & 22412388 \\
\hline 46 & rs2076756 & intron & NOD2 & 16 & $: 50,756,881$ & $1.000 \times 10-21$ & $*$ & NHGRI & 17684544 \\
\hline 47 & rs5743289 & intron & NOD2 & 16 & $: 50,756,774$ & $6.000 \times 10-17$ & $*$ & NHGRI & 17804789 \\
\hline 48 & rs2076756 & intron & NOD2 & 16 & : 50,756,881 & $7.000 \times 10-14$ & $*$ & NHGRI & 17435756 \\
\hline 49 & rs17221417 & intron & NOD2 & 16 & : 50,739,582 & $4.000 \times 10-11$ & $*$ & NHGRI & 17554300 \\
\hline 50 & rs5743289 & intron & NOD2 & 16 & $: 50,756,774$ & $1.000 \times 10-7$ & $*$ & NHGRI & 17447842 \\
\hline 51 & rs2797685 & intron & PER3 & 1 & : 7,879,063 & $7.000 \times 10-9$ & $*$ & NHGRI & 21102463 \\
\hline 52 & rs6738825 & intron & PLCL1 & 2 & $: 198,896,895$ & $4.000 \times 10-9$ & $*$ & NHGRI & 21102463 \\
\hline 53 & rs13003464 & intron & PUS10 & 2 & : 61,186,829 & $5.000 \times 10-9$ & $*$ & NHGRI & 22412388 \\
\hline
\end{tabular}




\begin{tabular}{|c|c|c|c|c|c|c|c|c|}
\hline 54 & rs10181042 & intron & PUS10 & $2: 61,224,259$ & $7.000 \times 10-9$ & $*$ & NHGRI & 21102463 \\
\hline 55 & rs17309827 & intron & SLC22A23 & $6: 3,433,318$ & $7.000 \times 10-9$ & $*$ & NHGRI & 21102463 \\
\hline 56 & rs17293632 & intron & SMAD3 & $15: 67,442,596$ & $3.000 \times 10-19$ & $2 a$ & NHGRI & 21102463 \\
\hline 57 & rs7423615 & intron & SP140 & $2: 231,116,874$ & $3.000 \times 10-13$ & $*$ & NHGRI & 21102463 \\
\hline 58 & rs744166 & intron & STAT3 & $17: 40,514,201$ & $7.000 \times 10-12$ & $*$ & NHGRI & 18587394 \\
\hline 59 & rs10495903 & intron & THADA & $2: 43,806,918$ & $2.000 \times 10-14$ & $*$ & NHGRI & 21102463 \\
\hline 60 & rs4263839 & intron & TNFSF15 & $9: 117,566,440$ & $3.000 \times 10-10$ & $2 b$ & NHGRI & 18587394 \\
\hline 61 & rs181359 & intron & UBE2L3 & $22: 21,928,641$ & $5.000 \times 10-16$ & $1 f$ & NHGRI & 21102463 \\
\hline 62 & rs4809330 & intron & ZGPAT & $20: 62,349,586$ & $3.000 \times 10-15$ & $*$ & NHGRI & 21102463 \\
\hline 63 & rs 1250550 & intron & ZMIZ1 & $10: 81,060,317$ & $1.000 \times 10-30$ & $*$ & NHGRI & 21102463 \\
\hline 64 & rs7076156 & intron & ZNF365 & $10: 64,415,184$ & $7.000 \times 10-9$ & $*$ & NHGRI & 22412388 \\
\hline 65 & rs2066847 & frameshift & NOD2 & $16: 50,763,778$ & $3.000 \times 10-24$ & $*$ & NHGRI & 18587394 \\
\hline 66 & rs2066847 & frameshift & NOD2 & $16: 50,763,778$ & $2.000 \times 10-15$ & $*$ & NHGRI & 20570966 \\
\hline 67 & rs9858542 & cds-synon & BSN & $3: 49,701,983$ & $4.000 \times 10-8$ & $1 f$ & NHGRI & 17554300 \\
\hline 68 & rs9858542 & cds-synon & BSN & $3: 49,701,983$ & $5.000 \times 10-8$ & If & NHGRI & 17554261 \\
\hline 69 & rs1142287 & cds-synon & SCAMP3 & $1: 155,230,131$ & $2.000 \times 10-13$ & $*$ & NHGRI & 21102463 \\
\hline 70 & rs3810936 & cds-synon & TNFSF15 & $9: 117,552,885$ & $1.000 \times 10-15$ & $*$ & NHGRI & 21102463 \\
\hline 71 & rs6545946 & intergenic & B3GNT2, TMEM17 & $2: 62,713,533$ & $7.000 \times 10-9$ & $*$ & NHGRI & 22412388 \\
\hline 72 & rs359457 & intergenic & BOD1, CPEB4 & $5: 173,279,842$ & $3.000 \times 10-12$ & $*$ & NHGRI & 21102463 \\
\hline 73 & rs1398024 & intergenic & C10orf67, OTUD1 & $10: 23,665,438$ & $4.000 \times 10-7$ & $*$ & NHGRI & 18723019 \\
\hline 74 & rs7927894 & intergenic & C11orf30, LRRC32 & $11: 76,301,316$ & $1.000 \times 10-9$ & $*$ & NHGRI & 18587394 \\
\hline 75 & rs11584383 & intergenic & C1orf81, KIF21B & $1: 200,935,866$ & $1.000 \times 10-11$ & $*$ & NHGRI & 18587394 \\
\hline 76 & rs762421 & intergenic & C21orf33, ICOSLG & $21: 45,615,561$ & $1.000 \times 10-9$ & $*$ & NHGRI & 18587394 \\
\hline 77 & rs13361189 & intergenic & C5orf62, IRGM & $5: 150,223,387$ & $2.000 \times 10-10$ & $*$ & NHGRI & 17554261 \\
\hline 78 & rs1456893 & intergenic & C7orf72, IKZF1 & $7: 50,269,672$ & $5.000 \times 10-9$ & $*$ & NHGRI & 18587394 \\
\hline 79 & rs3091315 & intergenic & $\mathrm{CCL} 2, \mathrm{CCL} 7$ & $17: 32,593,665$ & $2.000 \times 10-13$ & $*$ & NHGRI & 21102463 \\
\hline 80 & rs3091316 & intergenic & $\mathrm{CCL} 2, \mathrm{CCL} 7$ & $17: 32,593,974$ & $4.000 \times 10-8$ & $*$ & NHGRI & 22412388 \\
\hline 81 & rs7807268 & intergenic & CNTNAP2, RPL32P17 & $7: 148,258,048$ & $4.000 \times 10-7$ & $*$ & NHGRI & 17554300 \\
\hline 82 & rs11742570 & intergenic & DAB2, PTGER4 & $5: 40,410,584$ & $7.000 \times 10-36$ & $*$ & NHGRI & 21102463 \\
\hline 83 & rs4613763 & intergenic & DAB2, PTGER4 & $5: 40,392,728$ & $7.000 \times 10-27$ & $*$ & NHGRI & 18587394 \\
\hline 84 & rs9292777 & intergenic & DAB2, PTGER4 & $5: 40,437,948$ & $3.000 \times 10-18$ & $*$ & NHGRI & 17554261 \\
\hline 85 & rs17234657 & intergenic & DAB2, PTGER4 & $5: 40,401,509$ & $2.000 \times 10-12$ & $*$ & NHGRI & 17554300 \\
\hline 86 & rs1373692 & intergenic & DAB2, PTGER4 & $5: 40,431,183$ & $2.000 \times 10-12$ & $*$ & NHGRI & 17447842 \\
\hline 87 & rs9292777 & intergenic & DAB2, PTGER4 & $5: 40,437,948$ & $2.000 \times 10-11$ & $*$ & NHGRI & 22412388 \\
\hline 88 & rs1992660 & intergenic & DAB2, PTGER4 & $5: 40,415,067$ & $4.000 \times 10-7$ & $*$ & NHGRI & 17684544 \\
\hline 89 & rs2062305 & intergenic & FABP3P2, TNFSF11 & $13: 43,052,880$ & $5.000 \times 10-10$ & $*$ & NHGRI & 21102463 \\
\hline 90 & rs10801047 & intergenic & FAM5C, RGS18 & $1: 191,559,356$ & $3.000 \times 10-8$ & $*$ & NHGRI & 17554261 \\
\hline 91 & rs9286879 & intergenic & FASLG, TNFSF18 & $1: 172,862,234$ & $2.000 \times 10-9$ & $*$ & NHGRI & 18587394 \\
\hline 92 & rs12035082 & intergenic & FASLG, TNFSF18 & $1: 172,898,377$ & $2.000 \times 10-7$ & $*$ & NHGRI & 17554261 \\
\hline 93 & rs2836754 & intergenic & FLJ45139, RPL23AP1 & $21: 40,291,740$ & $5.000 \times 10-7$ & $*$ & NHGRI & 17554261 \\
\hline 94 & rs281379 & intergenic & FUT2, MAMSTR & $19: 49,214,274$ & $7.000 \times 10-12$ & $*$ & NHGRI & 21102463 \\
\hline 95 & rs4409764 & intergenic & GOT1, NKX2-3 & $10: 101,284,237$ & $2.000 \times 10-20$ & $3 a$ & NHGRI & 21102463 \\
\hline 96 & rs10883365 & intergenic & GOT1, NKX2-3 & $10: 101,287,764$ & $4.000 \times 10-10$ & $*$ & NHGRI & 17554261 \\
\hline 97 & rs10883365 & intergenic & GOT1, NKX2-3 & $10: 101,287,764$ & $6.000 \times 10-8$ & $*$ & NHGRI & 17554300 \\
\hline 98 & rs9469220 & intergenic & HLA-DQB1, HLA-DQA & $6: 32,658,310$ & $2.000 \times 10-6$ & $1 f$ & NHGRI & 17554300 \\
\hline 99 & rs9258260 & intergenic & IFITM4P, 3.8-1.5 & $6: 29,723,161$ & $2.000 \times 10-10$ & $1 f$ & NHGRI & 22412388 \\
\hline 100 & rs10045431 & intergenic & IL12B, ADRA1B & $5: 158,814,533$ & $4.000 \times 10-13$ & $*$ & NHGRI & 18587394 \\
\hline 101 & rs10045431 & intergenic & IL12B, ADRA1B & $5: 158,814,533$ & $7.000 \times 10-8$ & $*$ & NHGRI & 20570966 \\
\hline 102 & rs6887695 & intergenic & IL12B, ADRA1B & $5: 158,822,645$ & $9.000 \times 10-7$ & $*$ & NHGRI & 17554261 \\
\hline 103 & rs3091338 & intergenic & IL3, CSF2 & $5: 131,402,738$ & $4.000 \times 10-8$ & $*$ & NHGRI & 22412388 \\
\hline 104 & rs7714584 & intergenic & IRGM, ZNF300 & $5: 150,270,420$ & $8.000 \times 10-19$ & $*$ & NHGRI & 21102463 \\
\hline 105 & rs11747270 & intergenic & IRGM, ZNF300 & $5: 150,258,867$ & $3.000 \times 10-16$ & $*$ & NHGRI & 18587394 \\
\hline 106 & rs1000113 & intergenic & IRGM, ZNF300 & $5: 150,240,076$ & $3.000 \times 10-7$ & $*$ & NHGRI & 17554300 \\
\hline 107 & rs6601764 & intergenic & KLF6, AKR1E2 & $10: 3,862,542$ & $9.000 \times 10-7$ & $*$ & NHGRI & 17554300 \\
\hline 108 & rs11167764 & intergenic & MRPL11P2, NDFIP1 & $5: 141,479,065$ & $2.000 \times 10-9$ & $*$ & NHGRI & 21102463 \\
\hline 109 & rs1819658 & intergenic & MRPS35P3, IPMK & $10: 59,913,151$ & $9.000 \times 10-17$ & $*$ & NHGRI & 21102463 \\
\hline 110 & rs1736135 & intergenic & NRIP1, CYCSP42 & $21: 16,805,220$ & $7.000 \times 10-9$ & $*$ & NHGRI & 18587394 \\
\hline 111 & rs2413583 & intergenic & PDGFB, RPL3 & $22: 39,659,773$ & $1.000 \times 10-26$ & $*$ & NHGRI & 21102463 \\
\hline 112 & rs694739 & intergenic & PRDX5, CCDC88B & $11: 64,097,233$ & $6.000 \times 10-10$ & $1 f$ & NHGRI & 21102463 \\
\hline
\end{tabular}




$\begin{array}{lll}113 & \text { rs2542151 } & \text { intergenic } \\ 114 & \text { rs2542151 } & \text { intergenic } \\ 115 & \text { rs2542151 } & \text { intergenic } \\ 116 & \text { rs6651252 } & \text { intergenic } \\ 117 & \text { rs10758669 } & \text { intergenic } \\ 118 & \text { rs4902642 } & \text { intergenic } \\ 119 & \text { rs11175593 } & \text { intergenic } \\ 120 & \text { rs7746082 } & \text { intergenic } \\ 121 & \text { rs17582416 } & \text { intergenic } \\ 122 & \text { rs3024505 } & \text { intergenic } \\ 123 & \text { rs713875 } & \text { intergenic } \\ 124 & \text { rs13073817 } & \text { intergenic } \\ 125 & \text { rs6596075 } & \text { intergenic } \\ 126 & \text { rs11229030 } & \text { intergenic } \\ 127 & \text { rs736289 } & \text { intergenic } \\ 128 & \text { rs7705924 } & \text { intergenic } \\ 129 & \text { rs212388 } & \text { intergenic } \\ 130 & \text { rs10734105 } & \text { intergenic } \\ 131 & \text { rs7702331 } & \text { intergenic } \\ 132 & \text { rs1551398 } & \text { intergenic } \\ 133 & \text { rs1906493 } & \text { intergenic } \\ 134 & \text { rs10761659 } & \text { intergenic } \\ 135 & \text { rs10995271 } & \text { intergenic } \\ 136 & \text { rs224136 } & \text { intergenic } \\ 137 & \text { rs10761659 } & \text { intergenic } \\ 138 & \text { rs2872507 } & \text { intergenic }\end{array}$

$\begin{array}{lrll}\text { PSMG2, PTPN2 } & 18: 12,779,947 & 5.000 \times 10-17 \\ \text { PSMG2, PTPN2 } & 18: 12,779,947 & 3.000 \times 10-8 \\ \text { PSMG2, PTPN2 } & 18: 12,779,947 & 2.000 \times 10-7 \\ \text { PVT1, GSDMC } & 8: 129,567,181 & 4.000 \times 10-18 \\ \text { RCL1, JAK2 } & 9: 4,981,602 & 3.000 \times 10-9 \\ \text { RPL12P7, ZFP36L1 } & 14: 69,210,199 & 2.000 \times 10-10 \\ \text { RPL30P13, LRRK2 } & 12: 40,601,940 & 3.000 \times 10-10 \\ \text { RPL35P3, PRDM1 } & 6: 106,435,269 & 2.000 \times 10-10 \\ \text { RPS12P16, CUL2 } & 10: 35,287,650 & 2.000 \times 10-9 \\ \text { RPS14P1, IL10 } & 1: 206,939,904 & 2.000 \times 10-14 \\ \text { RPS3AP51, LIF } & 22: 30,592,487 & 7.000 \times 10-12 \\ \text { SATB1, KCNH8 } & 3: 18,706,858 & 7.000 \times 10-9 \\ \text { SLC22A5, C5Orf56 } & 5: 131,742,228 & 3.000 \times 10-7 \\ \text { SLC43A3, RTN4RL2 } & 11: 57,203,009 & 8.000 \times 10-9 \\ \text { SLC7A10, CEBPA } & 19: 33,757,062 & 9.000 \times 10-9 \\ \text { SLCO6A1, PAM } & 5: 101,946,798 & 2.000 \times 10-8 \\ \text { TAGAP, FNDC1 } & 6: 159,490,436 & 2.000 \times 10-11 \\ \text { TCERG1L, FLJ46300 } & 10: 133,172,119 & 3.000 \times 10-8 \\ \text { TMEM174, FOXD1 } & 5: 72,551,134 & 6.000 \times 10-12 \\ \text { TRIB1, FAM84B } & 8: 126,540,051 & 5.000 \times 10-9 \\ \text { TRIB1, FAM84B } & 8: 127,092,882 & 3.000 \times 10-7 \\ \text { ZNF365, ALDH7A1P4 } & 10: 64,445,564 & 4.000 \times 10-22 \\ \text { ZNF365, ALDH7A1P4 } & 10: 64,438,486 & 4.000 \times 10-20 \\ \text { ZNF365, ALDH7A1P4 } & 10: 64,470,675 & 1.000 \times 10-10 \\ \text { ZNF365, ALDH7A1P4 } & 10: 64,445,564 & 2.000 \times 10-7 \\ \text { ZPBP2, GSDMB } & 17: 38,040,763 & 5.000 \times 10-9\end{array}$

$\begin{array}{ccc}* & \text { NHGRI } & 18587394 \\ * & \text { NHGRI } & 17554261 \\ * & \text { NHGRI } & 17554300 \\ * & \text { NHGRI } & 21102463 \\ * & \text { NHGRI } & 18587394 \\ * & \text { NHGRI } & 21102463 \\ * & \text { NHGRI } & 18587394 \\ * & \text { NHGRI } & 18587394 \\ * & \text { NHGRI } & 18587394 \\ \text { 2b } & \text { NHGRI } & 21102463 \\ \mathbf{1 f} & \text { NHGRI } & 21102463 \\ * & \text { NHGRI } & 21102463 \\ \mathbf{1 f} & \text { NHGRI } & 17554300 \\ * & \text { NHGRI } & 22412388 \\ * & \text { NHGRI } & 21102463 \\ * & \text { NHGRI } & 22412388 \\ * & \text { NHGRI } & 21102463 \\ * & \text { NHGRI } & 22412388 \\ * & \text { NHGRI } & 21102463 \\ * & \text { NHGRI } & 18587394 \\ * & \text { NHGRI } & 22412388 \\ * & \text { NHGRI } & 21102463 \\ * & \text { NHGRI } & 18587394 \\ * & \text { NHGRI } & 17435756 \\ * & \text { NHGRI } & 17554300 \\ * & \text { NHGRI } & 18587394\end{array}$

Inflammatory Bowel Diseases, IBD

\begin{tabular}{|c|c|c|c|c|c|c|c|c|}
\hline$\#$ & rs \# & Context & Gene & Location & P-value & $\begin{array}{l}\text { RegulomeDB } \\
\text { score }\end{array}$ & Source & Study \\
\hline 1 & rs10889677 & UTR-3 & IL23R & $1: 67,725,120$ & $9.037 \times 10-11$ & $*$ & $\mathrm{dbGaP}$ & phs000130 \\
\hline 2 & rs11209026 & missense & IL23R & $1: 67,705,958$ & $4.000 \times 10-11$ & $*$ & NHGRI & \\
\hline 3 & rs11209026 & missense & IL23R & $1: 67,705,958$ & $4.592 \times 10-11$ & $*$ & $\mathrm{dbGaP}$ & phs000130 \\
\hline 4 & rs11209026 & missense & IL23R & $1: 67,705,958$ & $7.000 \times 10-11$ & $*$ & NHGRI & \\
\hline 5 & rs2315008 & intron & ZGPAT & $20: 62,343,956$ & $9.000 \times 10-15$ & $*$ & NHGRI & \\
\hline 6 & rs2076756 & intron & NOD2 & $16: 50,756,881$ & $1.262 \times 10-14$ & $*$ & $\mathrm{dbGaP}$ & phs000130 \\
\hline 7 & rs7517847 & intron & IL23R & $1: 67,681,669$ & $2.991 \times 10-13$ & $*$ & $\mathrm{dbGaP}$ & phs000130 \\
\hline 8 & rs7517847 & intron & IL23R & $1: 67,681,669$ & $4.000 \times 10-13$ & $*$ & NHGRI & \\
\hline 9 & rs1343151 & intron & IL23R & $1: 67,719,129$ & $1.628 \times 10-11$ & $*$ & $\mathrm{dbGaP}$ & phs000130 \\
\hline 10 & rs10489629 & intron & IL23R & $1: 67,688,349$ & $6.790 \times 10-11$ & $*$ & $\mathrm{dbGaP}$ & phs 000130 \\
\hline 11 & rs2201841 & intron & IL23R & $1: 67,694,202$ & $3.574 \times 10-10$ & $*$ & $\mathrm{dbGaP}$ & phs000130 \\
\hline 12 & rs11465804 & intron & IL23R & $1: 67,702,526$ & $3.737 \times 10-10$ & $*$ & $\mathrm{dbGaP}$ & phs000130 \\
\hline 13 & rs5743289 & intron & NOD2 & $16: 50,756,774$ & $4.000 \times 10-10$ & $*$ & NHGRI & \\
\hline 14 & rs2076756 & intron & NOD2 & $16: 50,756,881$ & $5.000 \times 10-10$ & $*$ & NHGRI & \\
\hline 15 & rs1004819 & intron & IL23R & $1: 67,670,213$ & $1.504 \times 10-9$ & $*$ & $\mathrm{dbGaP}$ & phs000130 \\
\hline 16 & rs8049439 & intron & ATXN2L & $16: 28,837,515$ & $2.000 \times 10-9$ & $1 b$ & NHGRI & \\
\hline 17 & rs2412973 & intron & HORMAD2 & $22: 30,529,631$ & $2.000 \times 10-9$ & $*$ & NHGRI & \\
\hline 18 & rs1250550 & intron & ZMIZ1 & $10: 81,060,317$ & $6.000 \times 10-9$ & $*$ & NHGRI & \\
\hline 19 & rs2066843 & cds-synon & NOD2 & $16: 50,745,199$ & $7.869 \times 10-13$ & $*$ & $\mathrm{dbGaP}$ & phs000130 \\
\hline 20 & rs9271366 & intergenic & HLA-DRB1, HLA-DQA & $6: 32,586,854$ & $2.000 \times 10-70$ & $*$ & NHGRI & \\
\hline 21 & rs9271366 & intergenic & HLA-DRB1, HLA-DQA & $6: 32,586,854$ & $3.000 \times 10-31$ & $*$ & NHGRI & \\
\hline 22 & rs2006996 & intergenic & TNFSF15, TNFSF8 & $9: 117,592,638$ & $4.000 \times 10-16$ & $*$ & NHGRI & \\
\hline 23 & rs2006996 & intergenic & TNFSF15, TNFSF8 & $9: 117,592,638$ & $4.000 \times 10-13$ & $*$ & NHGRI & \\
\hline 24 & rs2836878 & intergenic & FLJ45139, RPL23AP1 & $21: 40,465,534$ & $4.000 \times 10-12$ & $*$ & NHGRI & \\
\hline 25 & rs9271366 & intergenic & HLA-DRB1, HLA-DQA & $6: 32,586,854$ & $8.000 \times 10-11$ & $*$ & NHGRI & \\
\hline 26 & rs10500264 & intergenic & SLC7A10, CEBPA & $19: 33,750,314$ & $4.000 \times 10-10$ & $*$ & NHGRI & \\
\hline 27 & rs11209032 & intergenic & IL23R, IL12RB2 & $1: 67,740,092$ & $8.645 \times 10-10$ & $*$ & $\mathrm{dbGaP}$ & phs000130 \\
\hline 28 & rs477515 & intergenic & HLA-DRB1, HLA-DQA & $6: 32,569,691$ & $1.000 \times 10-8$ & $*$ & NHGRI & \\
\hline
\end{tabular}


Psoriasis, PS

\begin{tabular}{|c|c|c|c|c|c|c|c|c|c|}
\hline$\#$ & rs \# & Context & Gene & & Location & P-value & $\begin{array}{l}\text { RegulomeDB } \\
\text { score }\end{array}$ & Source & Study \\
\hline 1 & rs8192583 & UTR-5 & GPSM3 & & : 32,163,274 & $2.250 \times 10-20$ & 1f & $\mathrm{dbGaP}$ & phs000019 \\
\hline 2 & rs3094187 & UTR-5 & TCF19 & 6 & : 31,126,944 & $3.725 \times 10-13$ & 1f & $\mathrm{dbGaP}$ & phs000019 \\
\hline 3 & rs2240803 & UTR-3 & DPCR1 & 6 & : 30,920,957 & $8.193 \times 10-13$ & * & $\mathrm{dbGaP}$ & phs000019 \\
\hline 4 & rs2395029 & UTR-3 & HCP5 & 6 & : 31,431,780 & $2.000 \times 10-26$ & $*$ & NHGRI & \\
\hline 5 & rs8365 & UTR-3 & RNF5 & 6 & : 32,148,403 & $1.094 \times 10-17$ & If & $\mathrm{dbGaP}$ & phs000019 \\
\hline 6 & rs3130453 & STOP-GAIN & CCHCR1 & 6 & : 31,124,849 & $2.057 \times 10-12$ & * & $\mathrm{dbGaP}$ & phs000019 \\
\hline 7 & rs3132965 & nearGene-5 & AGPAT1 & 6 & : 32,146,997 & $8.389 \times 10-16$ & * & $\mathrm{dbGaP}$ & phs000019 \\
\hline 8 & rs3094187 & nearGene-5 & CCHCR1 & 6 & : 31,126,944 & $3.725 \times 10-13$ & $*$ & $\mathrm{dbGaP}$ & phs000019 \\
\hline 9 & rs 7773175 & nearGene-5 & HLA-C & 6 & : 31,240,959 & $4.772 \times 10-30$ & * & $\mathrm{dbGaP}$ & phs000019 \\
\hline 10 & rs2249742 & nearGene-5 & HLA-C & 6 & : 31,240,721 & $6.346 \times 10-18$ & 1f & $\mathrm{dbGaP}$ & phs000019 \\
\hline 11 & rs2395471 & nearGene-5 & HLA-C & 6 & $: 31,240,692$ & $8.836 \times 10-18$ & If & $\mathrm{dbGaP}$ & phs000019 \\
\hline 12 & rs2249741 & nearGene-5 & HLA-C & & : 31,240,712 & $3.340 \times 10-12$ & * & $\mathrm{dbGaP}$ & phs000019 \\
\hline 13 & rs2524082 & nearGene-5 & HLA-C & 6 & $: 31,241,761$ & $2.215 \times 10-11$ & $*$ & $\mathrm{dbGaP}$ & phs000019 \\
\hline 14 & rs9267502 & nearGene-5 & LST1 & 6 & : 31,553,194 & $3.786 \times 10-19$ & * & $\mathrm{dbGaP}$ & phs000019 \\
\hline 15 & rs2734573 & nearGene-5 & MCCD1 & 6 & : 31,494,738 & $1.499 \times 10-12$ & * & $\mathrm{dbGaP}$ & phs000019 \\
\hline 16 & rs176095 & nearGene-5 & PBX2 & 6 & : 32,158,319 & $2.183 \times 10-12$ & If & $\mathrm{dbGaP}$ & phs000019 \\
\hline 17 & rs3130453 & nearGene-5 & TCF19 & 6 & $: 31,124,849$ & $2.057 \times 10-12$ & * & $\mathrm{dbGaP}$ & phs000019 \\
\hline 18 & rs2021723 & nearGene-5 & TRIM40 & 6 & : 30,103,923 & $8.690 \times 10-15$ & * & $\mathrm{dbGaP}$ & phs000019 \\
\hline 19 & rs8365 & nearGene-3 & AGER & 6 & $: 32,148,403$ & $1.094 \times 10-17$ & * & $\mathrm{dbGaP}$ & phs000019 \\
\hline 20 & rs1265086 & nearGene-3 & CCHCR1 & 6 & $: 31,109,882$ & $2.689 \times 10-11$ & 1f & $\mathrm{dbGaP}$ & phs000019 \\
\hline 21 & rs9468843 & nearGene-3 & DDR1 & 6 & : 30,867,958 & $9.009 \times 10-14$ & $*$ & $\mathrm{dbGaP}$ & phs000019 \\
\hline 22 & rs176095 & nearGene-3 & GPSM3 & 6 & $: 32,158,319$ & $2.183 \times 10-12$ & * & $\mathrm{dbGaP}$ & phs000019 \\
\hline 23 & rs2853950 & nearGene-3 & HLA-C & 6 & : 31,236,175 & $1.756 \times 10-14$ & $*$ & $\mathrm{dbGaP}$ & phs000019 \\
\hline 24 & rs11575907 & missense & HLA-DOB & 6 & : 32,782,112 & $5.960 \times 10-19$ & * & $\mathrm{dbGaP}$ & phs000019 \\
\hline 25 & rs20541 & missense & IL13 & 5 & : 131,995,964 & $5.000 \times 10-15$ & * & NHGRI & \\
\hline 26 & rs11209026 & missense & IL23R & 1 & $: 67,705,958$ & $7.000 \times 10-7$ & * & NHGRI & \\
\hline 27 & rs8192591 & missense & NOTCH4 & 6 & $: 32,185,796$ & $3.403 \times 10-24$ & $*$ & $\mathrm{dbGaP}$ & phs000019 \\
\hline 28 & rs33980500 & missense & TRAF3IP2 & 6 & $: 111,913,262$ & $1.000 \times 10-16$ & * & NHGRI & \\
\hline 29 & rs 12720356 & missense & TYK2 & 19 & : 10,469,975 & $4.000 \times 10-11$ & * & NHGRI & \\
\hline 30 & rs2295663 & intron & BAT5 & 6 & $: 31,669,295$ & $8.396 \times 10-24$ & * & $\mathrm{dbGaP}$ & phs000019 \\
\hline 31 & rs9267673 & intron & $\mathrm{C} 2$ & 6 & $: 31,883,679$ & $2.300 \times 10-33$ & 1f & $\mathrm{dbGaP}$ & phs000019 \\
\hline 32 & rs6906662 & intron & C6orf10 & 6 & : 32,266,506 & $2.109 \times 10-14$ & * & $\mathrm{dbGaP}$ & phs000019 \\
\hline 33 & rs27524 & intron & CAST & 5 & : 96,101,944 & $3.000 \times 10-11$ & * & NHGRI & \\
\hline 34 & rs1265078 & intron & CCHCR1 & 6 & $: 31,112,602$ & $2.039 \times 10-21$ & * & $\mathrm{dbGaP}$ & phs000019 \\
\hline 35 & rs7993214 & intron & COG6 & 13 & $: 40,350,912$ & $2.000 \times 10-6$ & * & NHGRI & \\
\hline 36 & rs2239518 & intron & DDR1 & 6 & $: 30,865,725$ & $3.454 \times 10-12$ & $2 b$ & $\mathrm{dbGaP}$ & phs000019 \\
\hline 37 & rs27524 & intron & ERAP1 & 5 & : 96,101,944 & $3.000 \times 10-11$ & * & NHGRI & \\
\hline 38 & rs10782001 & intron & FBXL19 & 16 & : 30,942,625 & $9.000 \times 10-10$ & $*$ & NHGRI & \\
\hline 39 & rs3213094 & intron & IL12B & 5 & : 158,750,769 & $3.000 \times 10-26$ & $*$ & NHGRI & \\
\hline 40 & rs3213094 & intron & IL12B & 5 & : 158,750,769 & $5.000 \times 10-11$ & $*$ & NHGRI & \\
\hline 41 & rs2201841 & intron & IL23R & 1 & $: 67,694,202$ & $3.000 \times 10-8$ & $*$ & NHGRI & \\
\hline 42 & rs17716942 & intron & KCNH7 & 2 & $: 163,260,691$ & $1.000 \times 10-13$ & * & NHGRI & \\
\hline 43 & rs 12586317 & intron & KIAA0391 & 14 & $: 35,682,172$ & $2.000 \times 10-8$ & $*$ & NHGRI & \\
\hline 44 & rs9295938 & intron & MUC21 & 6 & : 30,953,105 & $9.163 \times 10-18$ & * & $\mathrm{dbGaP}$ & phs000019 \\
\hline 45 & rs 4795067 & intron & NOS2 & 17 & $: 26,106,675$ & $4.000 \times 10-11$ & $*$ & NHGRI & \\
\hline 46 & rs3823418 & intron & PSORS1C1 & 6 & : 31,100,942 & $1.169 \times 10-33$ & $*$ & $\mathrm{dbGaP}$ & phs000019 \\
\hline 47 & rs3094205 & intron & PSORS1C1 & 6 & $: 31,091,862$ & $1.733 \times 10-15$ & $*$ & $\mathrm{dbGaP}$ & phs000019 \\
\hline 48 & rs3130573 & intron & PSORS1C1 & 6 & : 31,106,268 & $6.212 \times 10-15$ & If & $\mathrm{dbGaP}$ & phs000019 \\
\hline 49 & rs3130573 & intron & PSORS1C2 & 6 & $: 31,106,268$ & $6.212 \times 10-15$ & $*$ & $\mathrm{dbGaP}$ & phs000019 \\
\hline 50 & rs240993 & intron & REV3L & 6 & $: 111,673,714$ & $5.000 \times 10-20$ & * & NHGRI & \\
\hline 51 & rs3132965 & intron & RNF5 & 6 & $: 32,146,997$ & $8.389 \times 10-16$ & $*$ & $\mathrm{dbGaP}$ & phs000019 \\
\hline 52 & rs2066808 & intron & STAT2 & 12 & : 56,737,973 & $1.000 \times 10-9$ & * & NHGRI & \\
\hline 53 & rs2066808 & intron & STAT2 & 12 & : 56,737,973 & $2.000 \times 10-7$ & * & NHGRI & \\
\hline 54 & rs3093662 & intron & TNF & 6 & : 31,544,189 & $4.752 \times 10-21$ & * & $\mathrm{dbGaP}$ & phs000019 \\
\hline
\end{tabular}




\begin{tabular}{|c|c|c|c|c|c|}
\hline 55 & rs610604 & intron & TNFAIP3 & $6: 138,199,417$ & $9.000 \times 10-12$ \\
\hline 56 & rs610604 & intron & TNFAIP3 & $6: 138,199,417$ & $7.000 \times 10-7$ \\
\hline 57 & rs2077580 & intron & TNXB & $6: 32,020,844$ & $7.445 \times 10-32$ \\
\hline 58 & rs2107195 & intron & TRIM15 & $6: 30,137,866$ & $1.675 \times 10-17$ \\
\hline 59 & rs1076160 & intron & TSC1 & $9: 135,776,034$ & $6.000 \times 10-7$ \\
\hline 60 & rs280519 & intron & TYK2 & $19: 10,472,933$ & $4.000 \times 10-9$ \\
\hline 61 & rs8192583 & intron & NOTCH4 & $6: 32,163,274$ & $2.250 \times 10-20$ \\
\hline 62 & rs443198 & cds-synon & NOTCH4 & $6: 32,190,406$ & $1.526 \times 10-11$ \\
\hline 63 & rs495337 & cds-synon & SPATA2 & $20: 48,522,330$ & $1.000 \times 10-8$ \\
\hline 64 & rs495337 & cds-synon & SPATA2 & $20: 48,522,330$ & $2.000 \times 10-7$ \\
\hline 65 & rs7762370 & intergenic & BTNL2, HLA-DRA & $6: 32,400,190$ & $5.130 \times 10-19$ \\
\hline 66 & rs9501624 & intergenic & BTNL2, HLA-DRA & $6: 32,399,286$ & $2.357 \times 10-12$ \\
\hline 67 & rs1975974 & intergenic & C17orf51, UBBP4 & $17: 21,707,060$ & $1.000 \times 10-7$ \\
\hline 68 & rs3130955 & intergenic & HCG22, C6orf15 & $6: 31,054,511$ & $1.312 \times 10-20$ \\
\hline 69 & rs2844627 & intergenic & HCG27, HLA-C & $6: 31,229,462$ & $2.307 \times 10-36$ \\
\hline 70 & rs2394895 & intergenic & HCG27, HLA-C & $6: 31,206,979$ & $2.593 \times 10-29$ \\
\hline 71 & rs3130517 & intergenic & HCG27, HLA-C & $6: 31,190,303$ & $1.430 \times 10-28$ \\
\hline 72 & rs3130713 & intergenic & HCG27, HLA-C & $6: 31,205,617$ & $9.808 \times 10-28$ \\
\hline 73 & rs3130467 & intergenic & HCG27, HLA-C & $6: 31,187,075$ & $1.339 \times 10-27$ \\
\hline 74 & rs9263967 & intergenic & HCG27, HLA-C & $6: 31,186,245$ & $7.296 \times 10-15$ \\
\hline 75 & rs3130685 & intergenic & HCG27, HLA-C & $6: 31,206,206$ & $8.583 \times 10-14$ \\
\hline 76 & rs3130425 & intergenic & HCG27, HLA-C & $6: 31,218,327$ & $1.808 \times 10-12$ \\
\hline 77 & rs3095250 & intergenic & HCG27, HLA-C & $6: 31,208,340$ & $2.892 \times 10-12$ \\
\hline 78 & rs3132496 & intergenic & HCG27, HLA-C & $6: 31,208,610$ & $2.898 \times 10-12$ \\
\hline 79 & rs3132486 & intergenic & HLA-C, RPL3P2 & $6: 31,243,170$ & $6.660 \times 10-13$ \\
\hline 80 & rs3132485 & intergenic & HLA-C, RPL3P2 & $6: 31,243,389$ & $2.623 \times 10-12$ \\
\hline 81 & rs2647087 & intergenic & HLA-DQB1, HLA-DQA & $6: 32,681,049$ & $1.865 \times 10-16$ \\
\hline 82 & rs2858333 & intergenic & HLA-DQB1, HLA-DQA & $6: 32,681,085$ & $8.551 \times 10-16$ \\
\hline 83 & rs2856726 & intergenic & HLA-DQB1, HLA-DQA & $6: 32,666,721$ & $5.086 \times 10-12$ \\
\hline 84 & rs12203586 & intergenic & HLA-DQB1, HLA-DQA & $6: 32,679,591$ & $5.743 \times 10-12$ \\
\hline 85 & rs9268853 & intergenic & HLA-DRB9, HLA-DRB5 & $6: 32,429,643$ & $8.870 \times 10-13$ \\
\hline 86 & rs10484552 & intergenic & HLA-E, GNL1 & $6: 30,484,036$ & $7.487 \times 10-19$ \\
\hline 87 & rs13437088 & intergenic & HLA-S, MICA & $6: 31,355,119$ & $2.539 \times 10-13$ \\
\hline 88 & rs10947208 & intergenic & HLA-S, MICA & $6: 31,361,837$ & $2.182 \times 10-12$ \\
\hline 89 & rs7756521 & intergenic & IER3, DDR1 & $6: 30,848,253$ & $7.581 \times 10-19$ \\
\hline 90 & rs4711229 & intergenic & IER3, DDR1 & $6: 30,756,744$ & $5.431 \times 10-14$ \\
\hline 91 & rs9295917 & intergenic & IER3, DDR1 & $6: 30,769,772$ & $5.091 \times 10-13$ \\
\hline 92 & rs4713380 & intergenic & IER3, DDR1 & $6: 30,785,273$ & $6.508 \times 10-13$ \\
\hline 93 & rs12526186 & intergenic & IER3, DDR1 & $6: 30,736,151$ & $1.272 \times 10-12$ \\
\hline 94 & rs13198118 & intergenic & IER3, DDR1 & $6: 30,770,732$ & $2.074 \times 10-12$ \\
\hline 95 & rs7749924 & intergenic & IER3, DDR1 & $6: 30,797,991$ & $2.249 \times 10-12$ \\
\hline 96 & rs9295924 & intergenic & IER3, DDR1 & $6: 30,782,361$ & $2.685 \times 10-12$ \\
\hline 97 & rs3131043 & intergenic & IER3, DDR1 & $6: 30,758,466$ & $1.814 \times 10-11$ \\
\hline 98 & rs2546890 & intergenic & IL12B, ADRA1B & $5: 158,759,900$ & $1.000 \times 10-20$ \\
\hline 99 & rs4649203 & intergenic & IL28RA, GRHL3 & $1: 24,519,920$ & 7.000 x 10-8 \\
\hline 100 & rs4085613 & intergenic & LCE3E, LCE3D & $1: 152,550,018$ & $7.000 \times 10-30$ \\
\hline 101 & rs4112788 & intergenic & LCE3E, LCE3D & $1: 152,551,276$ & $3.000 \times 10-10$ \\
\hline 102 & rs9501106 & intergenic & MICA, HLA-X & $6: 31,388,109$ & $3.527 \times 10-16$ \\
\hline 103 & rs9295993 & intergenic & MICA, HLA-X & $6: 31,388,595$ & $1.344 \times 10-15$ \\
\hline 104 & rs9266844 & intergenic & MICA, HLA-X & $6: 31,384,331$ & $1.022 \times 10-11$ \\
\hline 105 & rs7772549 & intergenic & MICA, HLA-X & $6: 31,407,643$ & $1.032 \times 10-11$ \\
\hline 106 & rs9266846 & intergenic & MICA, HLA-X & $6: 31,384,889$ & $2.441 \times 10-11$ \\
\hline 107 & rs17476793 & intergenic & MICC, SUCLA2P1 & $6: 30,410,988$ & $1.261 \times 10-17$ \\
\hline 108 & rs13191258 & intergenic & MUC21, HCG22 & $6: 30,978,717$ & $3.951 \times 10-33$ \\
\hline 109 & rs2844645 & intergenic & MUC21, HCG22 & $6: 31,015,182$ & $7.292 \times 10-19$ \\
\hline 110 & rs9366764 & intergenic & MUC21, HCG22 & $6: 30,979,793$ & $8.095 \times 10-18$ \\
\hline 111 & rs9394031 & intergenic & MUC21, HCG22 & $6: 30,991,643$ & $8.254 \times 10-18$ \\
\hline 112 & rs3871466 & intergenic & MUC21, HCG22 & $6: 30,983,683$ & $7.904 \times 10-17$ \\
\hline 113 & rs2523870 & intergenic & MUC21, HCG22 & $6: 31,014,116$ & $2.725 \times 10-12$ \\
\hline
\end{tabular}

\begin{tabular}{|c|c|c|}
\hline$*$ & NHGRI & \\
\hline$*$ & NHGRI & \\
\hline$*$ & $\mathrm{dbGaP}$ & phs000019 \\
\hline$*$ & $\mathrm{dbGaP}$ & phs000019 \\
\hline$*$ & NHGRI & \\
\hline$*$ & NHGRI & \\
\hline$*$ & $\mathrm{dbGaP}$ & phs000019 \\
\hline $1 f$ & $\mathrm{dbGaP}$ & phs000019 \\
\hline$*$ & NHGRI & \\
\hline$*$ & NHGRI & \\
\hline$*$ & $\mathrm{dbGaP}$ & phs000019 \\
\hline$*$ & $\mathrm{dbGaP}$ & phs000019 \\
\hline$*$ & NHGRI & \\
\hline $1 f$ & $\mathrm{dbGaP}$ & phs000019 \\
\hline$*$ & $\mathrm{dbGaP}$ & phs000019 \\
\hline$*$ & $\mathrm{dbGaP}$ & phs000019 \\
\hline$*$ & $\mathrm{dbGaP}$ & phs000019 \\
\hline$*$ & $\mathrm{dbGaP}$ & phs000019 \\
\hline$*$ & $\mathrm{dbGaP}$ & phs000019 \\
\hline$*$ & $\mathrm{dbGaP}$ & phs000019 \\
\hline$*$ & $\mathrm{dbGaP}$ & phs000019 \\
\hline$*$ & $\mathrm{dbGaP}$ & phs000019 \\
\hline$*$ & $\mathrm{dbGaP}$ & phs000019 \\
\hline$*$ & $\mathrm{dbGaP}$ & phs000019 \\
\hline$*$ & $\mathrm{dbGaP}$ & phs000019 \\
\hline$*$ & $\mathrm{dbGaP}$ & phs000019 \\
\hline$*$ & $\mathrm{dbGaP}$ & phs000019 \\
\hline$*$ & $\mathrm{dbGaP}$ & phs000019 \\
\hline$*$ & $\mathrm{dbGaP}$ & phs000019 \\
\hline $1 f$ & $\mathrm{dbGaP}$ & phs000019 \\
\hline$*$ & $\mathrm{dbGaP}$ & phs000019 \\
\hline$*$ & $\mathrm{dbGaP}$ & phs000019 \\
\hline$*$ & $\mathrm{dbGaP}$ & phs000019 \\
\hline$*$ & $\mathrm{dbGaP}$ & phs000019 \\
\hline$*$ & $\mathrm{dbGaP}$ & phs000019 \\
\hline$*$ & $\mathrm{dbGaP}$ & phs000019 \\
\hline$*$ & $\mathrm{dbGaP}$ & phs000019 \\
\hline $2 b$ & $\mathrm{dbGaP}$ & phs000019 \\
\hline$*$ & $\mathrm{dbGaP}$ & phs000019 \\
\hline $2 b$ & $\mathrm{dbGaP}$ & phs000019 \\
\hline$*$ & $\mathrm{dbGaP}$ & phs000019 \\
\hline$*$ & $\mathrm{dbGaP}$ & phs000019 \\
\hline If & $\mathrm{dbGaP}$ & phs000019 \\
\hline$*$ & NHGRI & \\
\hline$*$ & NHGRI & \\
\hline$*$ & NHGRI & \\
\hline$*$ & NHGRI & \\
\hline$*$ & $\mathrm{dbGaP}$ & phs000019 \\
\hline$*$ & $\mathrm{dbGaP}$ & phs000019 \\
\hline$*$ & $\mathrm{dbGaP}$ & phs000019 \\
\hline$*$ & $\mathrm{dbGaP}$ & phs000019 \\
\hline$*$ & $\mathrm{dbGaP}$ & phs000019 \\
\hline$*$ & $\mathrm{dbGaP}$ & phs000019 \\
\hline$*$ & $\mathrm{dbGaP}$ & phs000019 \\
\hline$*$ & $\mathrm{dbGaP}$ & phs000019 \\
\hline$*$ & $\mathrm{dbGaP}$ & phs000019 \\
\hline$*$ & $\mathrm{dbGaP}$ & phs000019 \\
\hline$*$ & $\mathrm{dbGaP}$ & phs000019 \\
\hline$*$ & $\mathrm{dbGaP}$ & phs000019 \\
\hline
\end{tabular}




$\begin{array}{lll}114 & \text { rs2517552 } & \text { intergenic } \\ 115 & \text { rs2894176 } & \text { intergenic } \\ 116 & \text { rs2517527 } & \text { intergenic } \\ 117 & \text { rs9262492 } & \text { intergenic } \\ 118 & \text { rs6916062 } & \text { intergenic } \\ 119 & \text { rs9267463 } & \text { intergenic } \\ 120 & \text { rs9267464 } & \text { intergenic } \\ 121 & \text { rs2734573 } & \text { intergenic } \\ 122 & \text { rs8016947 } & \text { intergenic } \\ 123 & \text { rs702873 } & \text { intergenic } \\ 124 & \text { rs842636 } & \text { intergenic } \\ 125 & \text { rs12191877 } & \text { intergenic } \\ 126 & \text { rs12191877 } & \text { intergenic } \\ 127 & \text { rs12191877 } & \text { intergenic } \\ 128 & \text { rs12580100 } & \text { intergenic } \\ 129 & \text { rs6809854 } & \text { intergenic } \\ 130 & \text { rs1008953 } & \text { intergenic } \\ 131 & \text { rs17728338 } & \text { intergenic } \\ 132 & \text { rs1015465 } & \text { intergenic } \\ 133 & \text { rs2082412 } & \text { intergenic } \\ 134 & \text { rs10484554 } & \text { intergenic } \\ 135 & \text { rs9468933 } & \text { intergenic } \\ 136 & \text { rs10484554 } & \text { intergenic } \\ 137 & \text { rs2894207 } & \text { intergenic } \\ 138 & \text { rs9380237 } & \text { intergenic } \\ 139 & \text { rs2524163 } & \text { intergenic } \\ 140 & \text { rs2243868 } & \text { intergenic } \\ 141 & \text { rs2853923 } & \text { intergenic } \\ 142 & \text { rs9380240 } & \text { intergenic } \\ 143 & \text { rs2442719 } & \text { intergenic } \\ 144 & \text { rs3873386 } & \text { intergenic } \\ 145 & \text { rs2156875 } & \text { intergenic } \\ 146 & \text { rs9468937 } & \text { intergenic } \\ 147 & \text { rs3134792 } & \text { intergenic } \\ 148 & \text { rs9267673 } & \text { intergenic } \\ & & \end{array}$

$\begin{array}{lrl}\text { MUC21, HCG22 } & 6: 31,007,590 & 8.159 \times 10-12 \\ \text { MUC21, HCG22 } & 6: 30,986,038 & 9.713 \times 10-12 \\ \text { MUC21, HCG22 } & 6: 31,021,547 & 1.831 \times 10-11 \\ \text { MUC21, HCG22 } & 6: 30,986,015 & 2.993 \times 10-11 \\ \text { NOTCH4, C6orf10 } & 6: 32,219,041 & 2.524 \times 10-22 \\ \text { PPIAP9, RPL15P4 } & 6: 31,490,480 & 1.104 \times 10-23 \\ \text { PPIAP9, RPL15P4 } & 6: 31,490,646 & 1.806 \times 10-22 \\ \text { PPIAP9, RPL15P4 } & 6: 31,494,738 & 1.499 \times 10-12 \\ \text { PSMA6, RPLP0P3 } & 14: 35,832,666 & 2.000 \times 10-11 \\ \text { RPL21P33, REL } & 2: 61,081,542 & 4.000 \times 10-9 \\ \text { RPL21P33, REL } & 2: 61,091,950 & 6.000 \times 10-6 \\ \text { RPL3P2, WASF5P } & 6: 31,252,925 & 1.000 \times 10-100 \\ \text { RPL3P2, WASF5P } & 6: 31,252,925 & 2.024 \times 10-51 \\ \text { RPL3P2, WASF5P } & 6: 31,252,925 & 4.000 \times 10-32 \\ \text { RPS26, ERBB3 } & 12: 56,439,209 & 1.000 \times 10-7 \\ \text { SATB1, KCNH8 } & 3: 18,784,423 & 1.000 \times 10-7 \\ \text { SDC4, SYS1 } & 20: 43,980,726 & 1.000 \times 10-7 \\ \text { TNIP1, ANXA6 } & 5: 150,478,318 & 1.000 \times 10-20 \\ \text { TRIM31, TRIM40 } & 6: 30,086,340 & 3.685 \times 10-15 \\ \text { UBLCP1, IL12B } & 5: 158,717,789 & 2.000 \times 10-28 \\ \text { WASF5P, HLA-B } & 6: 31,274,555 & 4.000 \times 10-214 \\ \text { WASF5P, HLA-B } & 6: 31,265,057 & 1.621 \times 10-46 \\ \text { WASF5P, HLA-B } & 6: 31,274,555 & 2.000 \times 10-39 \\ \text { WASF5P, HLA-B } & 6: 31,263,751 & 3.766 \times 10-38 \\ \text { WASF5P, HLA-B } & 6: 31,264,392 & 2.576 \times 10-28 \\ \text { WASF5P, HLA-B } & 6: 31,259,579 & 5.329 \times 10-20 \\ \text { WASF5P, HLA-B } & 6: 31,261,276 & 2.798 \times 10-19 \\ \text { WASF5P, HLA-B } & 6: 31,265,737 & 2.701 \times 10-15 \\ \text { WASF5P, HLA-B } & 6: 31,268,832 & 4.412 \times 10-14 \\ \text { WASF5P, HLA-B } & 6: 31,320,538 & 2.809 \times 10-13 \\ \text { WASF5P, HLA-B } & 6: 31,273,745 & 1.173 \times 10-12 \\ \text { WASF5P, HLA-B } & 6: 31,317,347 & 3.707 \times 10-12 \\ \text { WASF5P, HLA-B } & 6: 31,270,118 & 4.828 \times 10-12 \\ \text { WASF5P, HLA-B } & 6: 31,312,326 & 1.000 \times 10-9 \\ \text { ZBTB12, C2 } & 6: 31,883,679 & 2.300 \times 10-33\end{array}$

$\begin{array}{ccc}* & \text { dbGaP } & \text { phs000019 } \\ * & \text { dbGaP } & \text { phs000019 } \\ * & \text { dbGaP } & \text { phs000019 } \\ \text { 2b } & \text { dbGaP } & \text { phs000019 } \\ * & \text { dbGaP } & \text { phs000019 } \\ * & \text { dbGaP } & \text { phs000019 } \\ * & \text { dbGaP } & \text { phs000019 } \\ * & \text { dbGaP } & \text { phs000019 } \\ * & \text { NHGRI } & \\ * & \text { NHGRI } & \\ * & \text { NHGRI } & \\ * & \text { NHGRI } & \\ * & \text { dbGaP } & \text { phs000019 } \\ * & \text { NHGRI } & \\ \text { 3a } & \text { NHGRI } & \\ * & \text { NHGRI } & \\ * & \text { NHGRI } & \\ * & \text { NHGRI } & \\ * & \text { dbGaP } & \text { phs000019 } \\ * & \text { NHGRI } & \\ * & \text { NHGRI } & \\ * & \text { dbGaP } & \text { phs000019 } \\ * & \text { NHGRI } & \\ * & \text { dbGaP } & \text { phs000019 } \\ * & \text { dbGaP } & \text { phs000019 } \\ * & \text { dbGaP } & \text { phs000019 } \\ * & \text { dbGaP } & \text { phs000019 } \\ * & \text { dbGaP } & \text { phs000019 } \\ * & \text { dbGaP } & \text { phs000019 } \\ * & \text { dbGaP } & \text { phs000019 } \\ * & \text { dbGaP } & \text { phs000019 } \\ * & \text { dbGaP } & \text { phs000019 } \\ * & \text { phs000019 }\end{array}$

Ulcerative Colitis, UC

\begin{tabular}{|c|c|c|c|c|c|c|c|c|}
\hline$\#$ & rs \# & Context & Gene & Location & P-value & $\begin{array}{l}\text { RegulomeDB } \\
\text { score }\end{array}$ & Source & PubMed \\
\hline 1 & rs10889677 & UTR-3 & IL23R & $1: 67,725,120$ & $1.000 \times 10-8$ & no data & NHGRI & 19122664 \\
\hline 2 & rs2297441 & UTR-3 & RTEL1 & $20: 62,327,582$ & $2.000 \times 10-10$ & 4 & NHGRI & 21297633 \\
\hline 3 & rs10781500 & nearGene-5 & CARD9 & $9: 139,269,338$ & $7.000 \times 10-6$ & no data & NHGRI & 19915572 \\
\hline 4 & rs678170 & nearGene-5 & FAM55A & $11: 114,431,956$ & $5.000 \times 10-14$ & 5 & NHGRI & 21297633 \\
\hline 5 & rs907611 & nearGene-5 & LSP1 & $11: 1,874,072$ & $1.000 \times 10-10$ & $2 a$ & NHGRI & 21297633 \\
\hline 6 & rs11190140 & nearGene-5 & NKX2-3 & $10: 101,291,593$ & $1.000 \times 10-8$ & 5 & NHGRI & 20228799 \\
\hline 7 & rs3806308 & nearGene-5 & RNF186 & $1: 20,142,866$ & $7.000 \times 10-9$ & $2 b$ & NHGRI & 19122664 \\
\hline 8 & rs2297441 & nearGene-5 & TNFRSF6B & $20: 62,327,582$ & $2.000 \times 10-10$ & 4 & NHGRI & 21297633 \\
\hline 9 & rs1317209 & nearGene-3 & RNF186 & $1: 20,140,036$ & $2.000 \times 10-10$ & 5 & NHGRI & 20228799 \\
\hline 10 & rs4077515 & missense & CARD9 & $9: 139,266,496$ & $5.000 \times 10-8$ & $1 f$ & NHGRI & 20228799 \\
\hline 11 & rs1801274 & missense & FCGR2A & $1: 161,479,745$ & $2.000 \times 10-20$ & $*$ & NHGRI & 21297633 \\
\hline 12 & rs1801274 & missense & FCGR2A & $1: 161,479,745$ & $2.000 \times 10-12$ & $*$ & NHGRI & 19915573 \\
\hline 13 & rs2305480 & missense & GSDMB & $17: 38,062,196$ & $3.000 \times 10-8$ & $*$ & NHGRI & 20228799 \\
\hline 14 & rs5771069 & missense & IL17REL & $22: 50,435,480$ & $4.000 \times 10-8$ & $*$ & NHGRI & 20228798 \\
\hline 15 & rs5771069 & missense & IL17REL & $22: 50,435,480$ & $2.000 \times 10-7$ & $*$ & NHGRI & 21297633 \\
\hline 16 & rs11209026 & missense & IL23R & $1: 67,705,958$ & $5.000 \times 10-28$ & $*$ & NHGRI & 21297633 \\
\hline 17 & rs11209026 & missense & IL23R & $1: 67,705,958$ & $3.000 \times 10-10$ & $*$ & NHGRI & 19915572 \\
\hline 18 & rs11209026 & missense & IL23R & $1: 67,705,958$ & $1.000 \times 10-8$ & $*$ & NHGRI & 19122664 \\
\hline 19 & rs3194051 & missense & IL7R & $5: 35,876,274$ & $4.000 \times 10-8$ & $*$ & NHGRI & 21297633 \\
\hline
\end{tabular}




\begin{tabular}{|c|c|c|c|c|c|}
\hline 20 & rs3197999 & missense & MST1 & $3: 49,721,532$ & $4.000 \times 10-9$ \\
\hline 21 & rs17388568 & intron & ADAD1 & $4: 123,329,362$ & $9.000 \times 10-7$ \\
\hline 22 & rs9822268 & intron & APEH & $3: 49,719,729$ & $2.000 \times 10-17$ \\
\hline 23 & rs7554511 & intron & C1orf106 & $1: 200,877,562$ & $2.000 \times 10-13$ \\
\hline 24 & rs7554511 & intron & C1orf106 & $1: 200,877,562$ & $1.000 \times 10-7$ \\
\hline 25 & rs9263739 & intron & CCHCR1 & $6: 31,111,356$ & $4.000 \times 10-67$ \\
\hline 26 & rs12261843 & intron & CCNY & $10: 35,554,054$ & $7.000 \times 10-10$ \\
\hline 27 & rs4781011 & intron & CIITA & $16: 10,975,311$ & $3.000 \times 10-7$ \\
\hline 28 & rs267939 & intron & DAP & $5: 10,752,315$ & $6.000 \times 10-12$ \\
\hline 29 & rs798502 & intron & GNA12 & $7: 2,789,880$ & $3.000 \times 10-15$ \\
\hline 30 & rs3024493 & intron & IL10 & $1: 206,943,968$ & $1.000 \times 10-12$ \\
\hline 31 & rs3024493 & intron & IL10 & $1: 206,943,968$ & $8.000 \times 10-8$ \\
\hline 32 & rs2201841 & intron & IL23R & $1: 67,694,202$ & $1.000 \times 10-13$ \\
\hline 33 & rs2870946 & intron & IL26 & $12: 68,596,661$ & $5.000 \times 10-7$ \\
\hline 34 & rs2158836 & intron & LAMB1 & $7: 107,580,839$ & $7.000 \times 10-6$ \\
\hline 35 & rs4654925 & intron & OTUD3 & $1: 20,227,723$ & $9.000 \times 10-22$ \\
\hline 36 & rs35675666 & intron & PARK7 & $1: 8,021,973$ & $5.000 \times 10-9$ \\
\hline 37 & rs7608910 & intron & PUS10 & $2: 61,204,856$ & $2.000 \times 10-14$ \\
\hline 38 & rs13003464 & intron & PUS10 & $2: 61,186,829$ & $7.000 \times 10-9$ \\
\hline 39 & rs1992950 & intron & SATB2 & $2: 200,290,359$ & $5.000 \times 10-7$ \\
\hline 40 & rs4246905 & intron & TNFSF15 & $9: 117,553,249$ & $6.000 \times 10-12$ \\
\hline 41 & rs1728785 & intron & ZFP90 & $16: 68,591,230$ & $3.000 \times 10-8$ \\
\hline 42 & rs9858542 & cds-synon & BSN & $3: 49,701,983$ & $7.000 \times 10-9$ \\
\hline 43 & rs9268480 & cds-synon & BTNL2 & $6: 32,363,844$ & $3.000 \times 10-7$ \\
\hline 44 & rs10781499 & cds-synon & CARD9 & $9: 139,266,405$ & $3.000 \times 10-19$ \\
\hline 45 & rs2155219 & intergenic & C11orf30, LRRC32 & 11 : 76,299,194 & $5.000 \times 10-16$ \\
\hline 46 & rs10800309 & intergenic & C1orf192, FCGR2A & $1: 161,472,158$ & $3.000 \times 10-9$ \\
\hline 47 & rs11584383 & intergenic & C1orf81, KIF21B & $1: 200,935,866$ & $2.000 \times 10-7$ \\
\hline 48 & rs2838519 & intergenic & C21orf33, ICOSLG & $21: 45,615,023$ & $6.000 \times 10-11$ \\
\hline 49 & rs941823 & intergenic & COG6, FOXO1 & $13: 41,013,977$ & $4.000 \times 10-12$ \\
\hline 50 & rs9548988 & intergenic & COG6, FOXO1 & $13: 40,505,510$ & $3.000 \times 10-7$ \\
\hline 51 & rs11676348 & intergenic & CXCR2, CXCR1 & $2: 219,010,146$ & $1.000 \times 10-10$ \\
\hline 52 & rs6451493 & intergenic & DAB2, PTGER4 & $5: 40,410,935$ & $3.000 \times 10-9$ \\
\hline 53 & rs2836878 & intergenic & FLJ45139, RPL23AP12 & $21: 40,465,534$ & $2.000 \times 10-22$ \\
\hline 54 & rs6584283 & intergenic & GOT1, NKX2-3 & $10: 101,290,301$ & $8.000 \times 10-21$ \\
\hline 55 & rs6584283 & intergenic & GOT1, NKX2-3 & $10: 101,290,301$ & $2.000 \times 10-7$ \\
\hline 56 & rs6584283 & intergenic & GOT1, NKX2-3 & $10: 101,290,301$ & $2.000 \times 10-6$ \\
\hline 57 & rs17085007 & intergenic & GPR12, RPS20P32 & $13: 27,531,267$ & $1.000 \times 10-16$ \\
\hline 58 & rs17085007 & intergenic & GPR12, RPS20P32 & $13: 27,531,267$ & $7.000 \times 10-8$ \\
\hline 59 & rs4676406 & intergenic & GPR35, AQP12B & $2: 241,579,108$ & $8.000 \times 10-11$ \\
\hline 60 & rs9268853 & intergenic & HLA-DRB9, HLA-DRB5 & $6: 32,429,643$ & $1.000 \times 10-55$ \\
\hline 61 & rs9268877 & intergenic & HLA-DRB9, HLA-DRB5 & $6: 32,431,147$ & $4.000 \times 10-23$ \\
\hline 62 & rs2395185 & intergenic & HLA-DRB9, HLA-DRB5 & $6: 32,433,167$ & $5.000 \times 10-22$ \\
\hline 63 & rs9268877 & intergenic & HLA-DRB9, HLA-DRB5 & $6: 32,431,147$ & $6.000 \times 10-18$ \\
\hline 64 & rs2395185 & intergenic & HLA-DRB9, HLA-DRB5 & $6: 32,433,167$ & $1.000 \times 10-16$ \\
\hline 65 & rs9268923 & intergenic & HLA-DRB9, HLA-DRB5 & $6: 32,432,835$ & $4.000 \times 10-15$ \\
\hline 66 & rs6017342 & intergenic & HNF4A, RPL37AP1 & $20: 43,065,028$ & $1.000 \times 10-20$ \\
\hline 67 & rs6017342 & intergenic & HNF4A, RPL37AP1 & $20: 43,065,028$ & $9.000 \times 10-17$ \\
\hline 68 & rs6871626 & intergenic & IL12B, ADRA1B & $5: 158,826,792$ & $1.000 \times 10-21$ \\
\hline 69 & rs2310173 & intergenic & IL1R2, IL1R1 & $2: 102,663,628$ & $3.000 \times 10-12$ \\
\hline 70 & rs10975003 & intergenic & INSL6, INSL4 & $9: 5,213,687$ & $1.000 \times 10-6$ \\
\hline 71 & rs16940202 & intergenic & IRF8, FOXF1 & $16: 86,014,241$ & $6.000 \times 10-19$ \\
\hline 72 & rs4728142 & intergenic & KCP, IRF5 & $7: 128,573,967$ & $2.000 \times 10-8$ \\
\hline 73 & rs1297265 & intergenic & NRIP1, CYCSP42 & $21: 16,817,051$ & $7.000 \times 10-13$ \\
\hline 74 & rs1736135 & intergenic & NRIP1, CYCSP42 & $21: 16,805,220$ & $2.000 \times 10-7$ \\
\hline 75 & rs6920220 & intergenic & OLIG3, TNFAIP3 & $6: 138,006,504$ & $8.000 \times 10-17$ \\
\hline 76 & rs254560 & intergenic & PITX1, H2AFY & $5: 134,443,606$ & $1.000 \times 10-9$ \\
\hline 77 & rs10758669 & intergenic & RCL1, JAK2 & $9: 4,981,602$ & $2.000 \times 10-25$ \\
\hline 78 & rs10758669 & intergenic & RCL1, JAK2 & $9: 4,981,602$ & $1.000 \times 10-6$ \\
\hline
\end{tabular}

\begin{tabular}{|c|c|c|}
\hline$*$ & NHGRI & 20228799 \\
\hline$*$ & NHGRI & 21297633 \\
\hline$*$ & NHGRI & 21297633 \\
\hline$*$ & NHGRI & 21297633 \\
\hline$*$ & NHGRI & 19915572 \\
\hline$*$ & NHGRI & 19915573 \\
\hline$*$ & NHGRI & 21297633 \\
\hline $2 b$ & NHGRI & 20228799 \\
\hline$*$ & NHGRI & 21297633 \\
\hline $1 b$ & NHGRI & 21297633 \\
\hline $2 b$ & NHGRI & 20228798 \\
\hline $2 b$ & NHGRI & 19915572 \\
\hline$*$ & NHGRI & 20228799 \\
\hline$*$ & NHGRI & 19122664 \\
\hline$*$ & NHGRI & 19122664 \\
\hline$*$ & NHGRI & 20228798 \\
\hline$*$ & NHGRI & 21297633 \\
\hline$*$ & NHGRI & 21297633 \\
\hline$*$ & NHGRI & 20228799 \\
\hline$*$ & NHGRI & 20228799 \\
\hline$*$ & NHGRI & 21297633 \\
\hline$*$ & NHGRI & 19915572 \\
\hline $1 f$ & NHGRI & 19915572 \\
\hline$*$ & NHGRI & 19915573 \\
\hline $1 f$ & NHGRI & 21297633 \\
\hline$*$ & NHGRI & 21297633 \\
\hline$*$ & NHGRI & 20228799 \\
\hline$*$ & NHGRI & 20228799 \\
\hline$*$ & NHGRI & 21297633 \\
\hline$*$ & NHGRI & 21297633 \\
\hline$*$ & NHGRI & 19915572 \\
\hline $1 f$ & NHGRI & 21297633 \\
\hline $3 a$ & NHGRI & 21297633 \\
\hline$*$ & NHGRI & 21297633 \\
\hline$*$ & NHGRI & 21297633 \\
\hline$*$ & NHGRI & 19915572 \\
\hline$*$ & NHGRI & 20228798 \\
\hline$*$ & NHGRI & 21297633 \\
\hline$*$ & NHGRI & 19915573 \\
\hline$*$ & NHGRI & 21297633 \\
\hline$*$ & NHGRI & 21297633 \\
\hline$*$ & NHGRI & 19915572 \\
\hline$*$ & NHGRI & 19915573 \\
\hline$*$ & NHGRI & 18836448 \\
\hline$*$ & NHGRI & 19122664 \\
\hline$*$ & NHGRI & 20228798 \\
\hline$*$ & NHGRI & 21297633 \\
\hline$*$ & NHGRI & 19915572 \\
\hline$*$ & NHGRI & 21297633 \\
\hline$*$ & NHGRI & 21297633 \\
\hline$*$ & NHGRI & 19915573 \\
\hline$*$ & NHGRI & 21297633 \\
\hline $1 f$ & NHGRI & 21297633 \\
\hline $2 a$ & NHGRI & 21297633 \\
\hline$*$ & NHGRI & 20228799 \\
\hline$*$ & NHGRI & 21297633 \\
\hline$*$ & NHGRI & 21297633 \\
\hline$*$ & NHGRI & 21297633 \\
\hline$*$ & NHGRI & 20228799 \\
\hline
\end{tabular}




\begin{tabular}{|c|c|c|c|c|c|c|c|c|}
\hline 79 & rs6426833 & intergenic & RNF186, OTUD3 & $1: 20,171,860$ & $4.000 \times 10-35$ & $*$ & NHGRI & 21297633 \\
\hline 80 & rs6426833 & intergenic & RNF186, OTUD3 & $1: 20,171,860$ & $2.000 \times 10-21$ & $*$ & NHGRI & 20228799 \\
\hline 81 & rs6426833 & intergenic & RNF186, OTUD3 & $1: 20,171,860$ & $5.000 \times 10-13$ & $*$ & NHGRI & 19122664 \\
\hline 82 & rs6426833 & intergenic & RNF186, OTUD3 & $1: 20,171,860$ & $2.000 \times 10-11$ & $*$ & NHGRI & 19915572 \\
\hline 83 & rs6911490 & intergenic & RPL35P3, PRDM1 & $6: 106,522,027$ & $1.000 \times 10-8$ & $*$ & NHGRI & 21297633 \\
\hline 84 & rs7134599 & intergenic & RPL39P28, IFNG & $12: 68,500,075$ & $1.000 \times 10-16$ & $*$ & NHGRI & 21297633 \\
\hline 85 & rs1558744 & intergenic & RPL39P28, IFNG & $12: 68,504,592$ & $3.000 \times 10-12$ & $*$ & NHGRI & 19122664 \\
\hline 86 & rs1558744 & intergenic & RPL39P28, IFNG & $12: 68,504,592$ & $4.000 \times 10-12$ & $*$ & NHGRI & 20228799 \\
\hline 87 & rs3024505 & intergenic & RPS14P1, IL10 & $1: 206,939,904$ & $6.000 \times 10-17$ & $2 b$ & NHGRI & 21297633 \\
\hline 88 & rs3024505 & intergenic & RPS14P1, IL10 & $1: 206,939,904$ & $1.000 \times 10-12$ & $*$ & NHGRI & 18836448 \\
\hline 89 & rs3024505 & intergenic & RPS14P1, IL10 & $1: 206,939,904$ & $1.000 \times 10-8$ & $*$ & NHGRI & 20228799 \\
\hline 90 & rs668853 & intergenic & RPS2P34, RPS6P12 & $9: 85,311,147$ & $2.000 \times 10-6$ & $*$ & NHGRI & 19122664 \\
\hline 91 & rs4510766 & intergenic & SLC26A3, DLD & $7: 107,492,789$ & $2.000 \times 10-16$ & $*$ & NHGRI & 21297633 \\
\hline 92 & rs886774 & intergenic & SLC26A3, DLD & $7: 107,495,434$ & $3.000 \times 10-8$ & $*$ & NHGRI & 19915572 \\
\hline 93 & rs4598195 & intergenic & SLC26A3, DLD & $7: 107,503,441$ & $8.000 \times 10-8$ & $*$ & NHGRI & 20228799 \\
\hline 94 & rs2108225 & intergenic & SLC26A3, DLD & $7: 107,453,103$ & $1.000 \times 10-7$ & $*$ & NHGRI & 19915573 \\
\hline 95 & rs4598195 & intergenic & SLC26A3, DLD & $7: 107,503,441$ & $1.000 \times 10-7$ & $*$ & NHGRI & 19122664 \\
\hline 96 & rs4730273 & intergenic & SLC26A3, DLD & $7: 107,479,519$ & $5.000 \times 10-7$ & $*$ & NHGRI & 19122664 \\
\hline 97 & rs4730276 & intergenic & SLC26A3, DLD & $7: 107,484,437$ & $9.000 \times 10-7$ & $*$ & NHGRI & 19122664 \\
\hline 98 & rs4957048 & intergenic & SLC9A3, CEP72 & $5: 583,442$ & $1.000 \times 10-9$ & $*$ & NHGRI & 20228799 \\
\hline 99 & rs11739663 & intergenic & SLC9A3, CEP72 & $5: 594,083$ & $3.000 \times 10-8$ & $*$ & NHGRI & 21297633 \\
\hline 100 & rs7809799 & intergenic & SMURF1, KPNA7 & $7: 98,760,504$ & $9.000 \times 10-11$ & $*$ & NHGRI & 20228798 \\
\hline 101 & rs734999 & intergenic & TNFRSF14, C1orf93 & $1: 2,513,216$ & $3.000 \times 10-9$ & $1 f$ & NHGRI & 21297633 \\
\hline 102 & rs943072 & intergenic & VEGFA, C6orf223 & $6: 43,795,968$ & $2.000 \times 10-10$ & $*$ & NHGRI & 21297633 \\
\hline 103 & rs7524102 & intergenic & WNT4, ZBTB40 & $1: 22,698,447$ & $2.000 \times 10-13$ & $*$ & NHGRI & 21297633 \\
\hline 104 & rs7524102 & intergenic & WNT4, ZBTB40 & $1: 22,698,447$ & $3.000 \times 10-7$ & $*$ & NHGRI & 19915572 \\
\hline 105 & rs6499188 & intergenic & ZFP90, CDH3 & $16: 68,674,788$ & $4.000 \times 10-8$ & $*$ & NHGRI & 21297633 \\
\hline 106 & rs2872507 & intergenic & ZPBP2, GSDMB & $17: 38,040,763$ & $5.000 \times 10-11$ & $*$ & NHGRI & 21297633 \\
\hline 107 & rs8067378 & intergenic & ZPBP2, GSDMB & $17: 38,051,348$ & $1.000 \times 10-7$ & $1 f$ & NHGRI & 20228799 \\
\hline
\end{tabular}

Psoriasis, PS (data only from NHGRI)

\begin{tabular}{|c|c|c|c|c|c|c|c|c|}
\hline$\#$ & rs \# & Context & Gene & Location & P-value & $\begin{array}{l}\text { RegulomeDB } \\
\text { score }\end{array}$ & Source & PubMed \\
\hline 1 & rs2395029 & UTR-3 & HCP5 & $6: 31,431,780$ & $2.000 \times 10-26$ & $*$ & NHGRI & 18369459 \\
\hline 2 & rs33980500 & missense & TRAF3IP2 & $6: 111,913,262$ & $1.000 \times 10-16$ & $*$ & NHGRI & 20953188 \\
\hline 3 & rs20541 & missense & IL13 & $5: 131,995,964$ & $5.000 \times 10-15$ & $*$ & NHGRI & 19169254 \\
\hline 4 & rs12720356 & missense & TYK2 & $19: 10,469,975$ & $4.000 \times 10-11$ & $*$ & NHGRI & 20953190 \\
\hline 5 & rs11209026 & missense & IL23R & $1: 67,705,958$ & $7.000 \times 10-7$ & $*$ & NHGRI & 20953190 \\
\hline 6 & rs3213094 & intron & IL12B & $5: 158,750,769$ & $3.000 \times 10-26$ & $*$ & NHGRI & 19169255 \\
\hline 7 & rs240993 & intron & REV3L & $6: 111,673,714$ & $5.000 \times 10-20$ & $*$ & NHGRI & 20953190 \\
\hline 8 & rs17716942 & intron & $\mathrm{KCNH} 7$ & $2: 163,260,691$ & $1.000 \times 10-13$ & $*$ & NHGRI & 20953190 \\
\hline 9 & rs610604 & intron & TNFAIP3 & $6: 138,199,417$ & $9.000 \times 10-12$ & $*$ & NHGRI & 19169254 \\
\hline 10 & rs27524 & intron & CAST & $5: 96,101,944$ & $3.000 \times 10-11$ & $*$ & NHGRI & 20953190 \\
\hline 11 & rs27524 & intron & ERAP1 & $5: 96,101,944$ & $3.000 \times 10-11$ & $*$ & NHGRI & 20953190 \\
\hline 12 & rs4795067 & intron & NOS2 & $17: 26,106,675$ & $4.000 \times 10-11$ & $*$ & NHGRI & 20953189 \\
\hline 13 & rs3213094 & intron & IL12B & $5: 158,750,769$ & $5.000 \times 10-11$ & $*$ & NHGRI & 20953190 \\
\hline 14 & rs10782001 & intron & FBXL19 & $16: 30,942,625$ & $9.000 \times 10-10$ & $*$ & NHGRI & 20953189 \\
\hline 15 & rs2066808 & intron & STAT2 & $12: 56,737,973$ & $1.000 \times 10-9$ & $*$ & NHGRI & 19169254 \\
\hline 16 & rs280519 & intron & TYK2 & $19: 10,472,933$ & $4.000 \times 10-9$ & $*$ & NHGRI & 20953190 \\
\hline 17 & rs12586317 & intron & KIAA0391 & $14: 35,682,172$ & $2.000 \times 10-8$ & $*$ & NHGRI & 20953189 \\
\hline 18 & rs2201841 & intron & IL23R & $1: 67,694,202$ & $3.000 \times 10-8$ & $*$ & NHGRI & 19169254 \\
\hline 19 & rs2066808 & intron & STAT2 & $12: 56,737,973$ & $2.000 \times 10-7$ & $*$ & NHGRI & 20953190 \\
\hline 20 & rs610604 & intron & TNFAIP3 & $6: 138,199,417$ & $7.000 \times 10-7$ & $*$ & NHGRI & 20953190 \\
\hline 21 & rs495337 & cds-synon & SPATA2 & $20: 48,522,330$ & $1.000 \times 10-8$ & $*$ & NHGRI & 18364390 \\
\hline 22 & rs495337 & cds-synon & SPATA2 & $20: 48,522,330$ & $2.000 \times 10-7$ & $*$ & NHGRI & 20953189 \\
\hline 23 & rs10484554 & intergenic & WASF5P, HLA-B & $6: 31,274,555$ & $4.000 \times 10-214$ & $*$ & NHGRI & 20953190 \\
\hline 24 & rs12191877 & intergenic & RPL3P2, WASF5P & $6: 31,252,925$ & $1.000 \times 10-100$ & $*$ & NHGRI & 19169254 \\
\hline
\end{tabular}




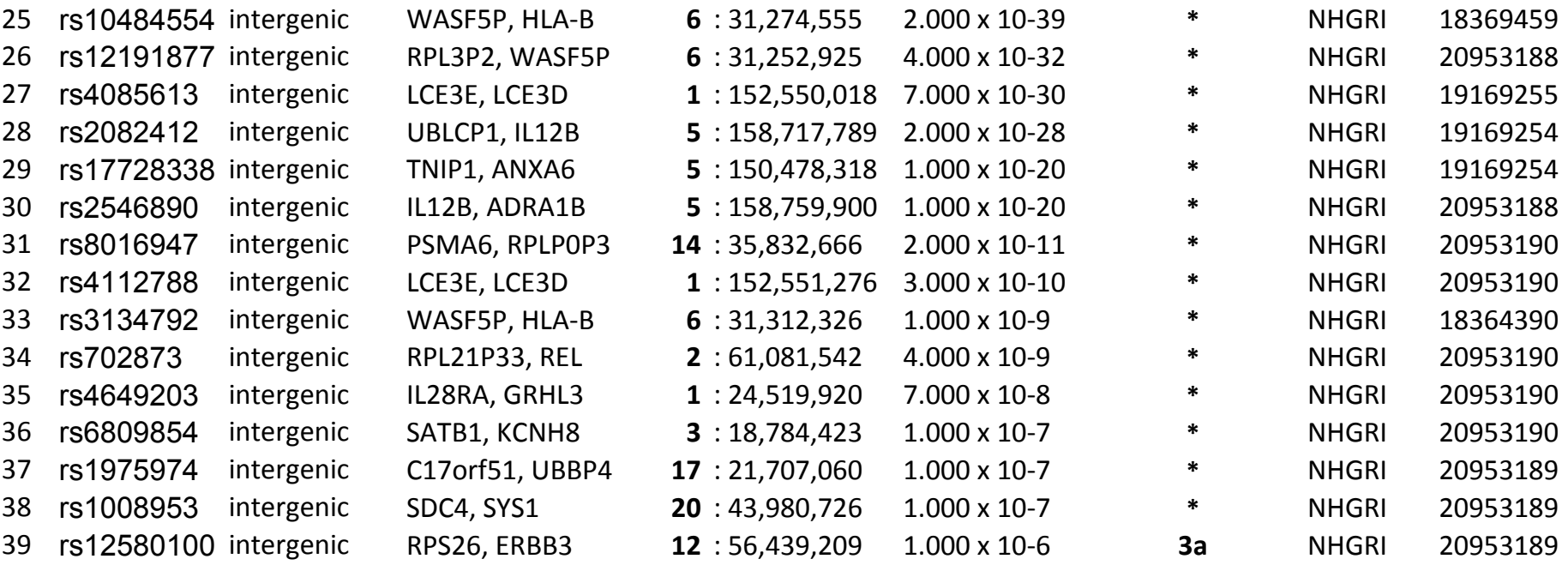

* all SNP are scored 4, 5 or 6 if not stated otherwise

AID associated GWAS SNPs retrieved from the NHGRI GWAS catalogue using PhenGenl software tool on the November 30th, 2014. Each SNP is identified by its dbSNP rs identifier along with its genomic context, associated genes with NCBI Gene identifier, location on a chromosome and PubMed ID.

Note: Regulome score is not retrieved from the Catalog; it is calculated as a part of our research additionally and will be discussed in the Section 3.3.3. 


\section{Supplemental Table 2. RegulomeDB scoring results for GWAS AID SNPs}

\section{Arthritis, Rheumatoid}

\begin{tabular}{|c|c|c|c|c|c|}
\hline$\#$ & rs \# & Context & Gene & P-value & $\begin{array}{l}\text { RegulomeDB } \\
\text { score }\end{array}$ \\
\hline 1 & rs4750316 & UTR-3 & DKFZp667F0711 & $2.000 \times 10-6$ & \\
\hline 2 & rs4750316 & UTR-3 & DKFZp667F0711 & $4.000 \times 10-6$ & \\
\hline 3 & rs1329568 & UTR-3 & LOC100130458 & $8.000 \times 10-7$ & \\
\hline 4 & rs805297 & nearGene-5 & APOM & $3.000 \times 10-10$ & $1 f$ \\
\hline 5 & rs2812378 & nearGene-5 & CCL21 & $3.000 \times 10-8$ & \\
\hline 6 & rs2841277 & nearGene-5 & PLD4 & $2.000 \times 10-14$ & \\
\hline 7 & rs6496667 & nearGene-5 & ZNF774 & $1.000 \times 10-6$ & \\
\hline 8 & rs3087243 & nearGene-3 & CTLA4 & $1.000 \times 10-8$ & $3 a$ \\
\hline 9 & rs10488631 & nearGene-3 & TNPO3 & $4.000 \times 10-11$ & $3 a$ \\
\hline 10 & rs2233434 & missense & NFKBIE & $6.000 \times 10-19$ & \\
\hline 11 & rs2476601 & missense & PTPN22 & $9.000 \times 10-74$ & $2 b$ \\
\hline 12 & rs2476601 & missense & PTPN22 & $2.000 \times 10-21$ & $2 b$ \\
\hline 13 & rs2476601 & missense & PTPN22 & $2.000 \times 10-11$ & $2 b$ \\
\hline 14 & rs3184504 & missense & $\mathrm{SH} 2 \mathrm{~B} 3$ & $6.000 \times 10-6$ & \\
\hline 15 & rs2230926 & missense & TNFAIP3 & $2.000 \times 10-6$ & \\
\hline 16 & rs2075876 & intron & AIRE & $4.000 \times 10-9$ & \\
\hline 17 & rs3816587 & intron & ANAPC4 & $9.000 \times 10-6$ & \\
\hline 18 & rs6859219 & intron & ANKRD55 & $1.000 \times 10-11$ & $2 b$ \\
\hline 19 & rs2867461 & intron & ANXA3 & $1.000 \times 10-12$ & \\
\hline 20 & rs2062583 & intron & ARHGEF3 & $2.000 \times 10-6$ & \\
\hline 21 & rs10821944 & intron & ARID5B & $6.000 \times 10-18$ & \\
\hline 22 & rs1600249 & intron & BLK & $5.000 \times 10-6$ & \\
\hline 23 & rs26232 & intron & C5orf30 & $4.000 \times 10-8$ & $2 b$ \\
\hline 24 & rs6910071 & intron & C6orf10 & $1.000 \times 10-299$ & \\
\hline 25 & rs2395148 & intron & C6orf10 & $2.000 \times 10-10$ & \\
\hline 26 & rs3093024 & intron & CCR6 & $8.000 \times 10-19$ & 6 \\
\hline 27 & rs3093023 & intron & CCR6 & $2.000 \times 10-11$ & If \\
\hline 28 & rs840016 & intron & CD247 & $2.000 \times 10-6$ & \\
\hline 29 & rs4810485 & intron & CD40 & $3.000 \times 10-9$ & $1 f$ \\
\hline 30 & rs4810485 & intron & CD40 & $8.000 \times 10-9$ & If \\
\hline 31 & rs42041 & intron & CDK6 & $4.000 \times 10-6$ & \\
\hline 32 & rs4942242 & intron & ENOX1 & $2.000 \times 10-7$ & \\
\hline 33 & rs1914816 & intron & ETFA & $7.000 \times 10-7$ & \\
\hline 34 & rs13315591 & intron & FAM107A & $5.000 \times 10-8$ & \\
\hline 35 & rs7940423 & intron & GALNTL4 & $1.000 \times 10-7$ & \\
\hline 36 & rs3783637 & intron & $\mathrm{GCH} 1$ & $2.000 \times 10-6$ & $2 b$ \\
\hline 37 & rs706778 & intron & IL2RA & $1.000 \times 10-11$ & \\
\hline 38 & rs13119723 & intron & KIAA1109 & $7.000 \times 10-7$ & \\
\hline 39 & rs1678542 & intron & KIF5A & $9.000 \times 10-8$ & \\
\hline
\end{tabular}




\begin{tabular}{|c|c|c|c|c|c|}
\hline 40 & rs13393173 & intron & LASS6 & $4.000 \times 10-6$ & \\
\hline 41 & rs17118552 & intron & MDGA2 & $2.000 \times 10-7$ & \\
\hline 42 & rs3890745 & intron & MMEL1 & $1.000 \times 10-7$ & \\
\hline 43 & rs3890745 & intron & MMEL1 & $4.000 \times 10-6$ & \\
\hline 44 & rs7046653 & intron & MOBKL2B & $5.000 \times 10-7$ & \\
\hline 45 & rs6500395 & intron & N4BP1 & $6.000 \times 10-7$ & \\
\hline 46 & rs3781913 & intron & PDE2A & $6.000 \times 10-10$ & $1 f$ \\
\hline 47 & rs2075876 & intron & PFKL & $4.000 \times 10-9$ & \\
\hline 48 & rs6026990 & intron & PHACTR3 & $6.000 \times 10-7$ & \\
\hline 49 & rs854555 & intron & PON1 & $2.000 \times 10-6$ & \\
\hline 50 & rs7404928 & intron & PRKCB & $4.000 \times 10-6$ & \\
\hline 51 & rs1957895 & intron & PRKCH & $4.000 \times 10-7$ & \\
\hline 52 & rs12901682 & intron & PSMA4 & $4.000 \times 10-8$ & \\
\hline 53 & rs2847297 & intron & PTPN2 & $2.000 \times 10-8$ & \\
\hline 54 & rs13137105 & intron & RCHY1 & $9.000 \times 10-7$ & \\
\hline 55 & rs13031237 & intron & REL & $8.000 \times 10-7$ & \\
\hline 56 & rs16977065 & intron & RIT2 & $1.000 \times 10-7$ & \\
\hline 57 & rs1809529 & intron & SLC6A11 & $3.000 \times 10-7$ & \\
\hline 58 & rs934734 & intron & SPRED2 & $5.000 \times 10-10$ & \\
\hline 59 & rs11121380 & intron & SPSB1 & $5.000 \times 10-8$ & \\
\hline 60 & rs7574865 & intron & STAT4 & $3.000 \times 10-7$ & \\
\hline 61 & rs7574865 & intron & STAT4 & $2.000 \times 10-6$ & \\
\hline 62 & rs3761847 & intron & TRAF1 & $4.000 \times 10-14$ & \\
\hline 63 & rs3761847 & intron & TRAF1 & $2.000 \times 10-7$ & \\
\hline 64 & rs12831974 & intron & TRHDE & $6.000 \times 10-6$ & $2 b$ \\
\hline 65 & rs11203203 & intron & UBASH3A & $4.000 \times 10-6$ & \\
\hline 66 & rs12046117 & intron & VTCN1 & $1.000 \times 10-6$ & \\
\hline 67 & rs1543922 & intron & ZNF175 & $3.000 \times 10-7$ & \\
\hline 68 & rs2240335 & cds-synon & PADI4 & $2.000 \times 10-8$ & \\
\hline 69 & rs2240335 & cds-synon & PADI4 & $2.000 \times 10-8$ & \\
\hline 70 & rs11676922 & intergenic & AFF3, LONRF2 & $1.000 \times 10-14$ & \\
\hline 71 & rs10865035 & intergenic & AFF3, LONRF2 & $2.000 \times 10-6$ & \\
\hline 72 & rs1898036 & intergenic & ATPBD4, COX6CP4 & $2.000 \times 10-7$ & \\
\hline 73 & rs11900673 & intergenic & B3GNT2, TMEM17 & $1.000 \times 10-8$ & \\
\hline 74 & rs2002842 & intergenic & BDP1P, SALL3 & $6.000 \times 10-6$ & \\
\hline 75 & rs11051970 & intergenic & BICD1, FGD4 & $1.000 \times 10-6$ & \\
\hline 76 & rs12565755 & intergenic & C1orf87, NFIA & $5.000 \times 10-8$ & \\
\hline 77 & rs874040 & intergenic & C4orf52, RBPJ & $1.000 \times 10-16$ & $2 b$ \\
\hline 78 & rs951005 & intergenic & C9orf144B, C9orf144 & $4.000 \times 10-10$ & \\
\hline 79 & rs11937061 & intergenic & CCNG2, CXCL13 & $2.000 \times 10-7$ & \\
\hline 80 & rs657075 & intergenic & CSF2, P4HA2 & $3.000 \times 10-10$ & \\
\hline 81 & rs6138150 & intergenic & CST2, CST5 & $3.000 \times 10-6$ & \\
\hline 82 & rs1273516 & intergenic & CYP4F22, RPL23AP2 & $9.000 \times 10-7$ & \\
\hline 83 & rs2837960 & intergenic & DSCAM, C21orf130 & $2.000 \times 10-6$ & \\
\hline 84 & rs6138892 & intergenic & EBF4, RPL19P1 & $3.000 \times 10-7$ & \\
\hline
\end{tabular}




\begin{tabular}{|c|c|c|c|c|c|}
\hline 85 & rs4937362 & intergenic & ETS1, FLI1 & $8.000 \times 10-7$ & \\
\hline 86 & rs2736340 & intergenic & FAM167A, BLK & $6.000 \times 10-9$ & \\
\hline 87 & rs9604529 & intergenic & FLJ44054 & $7.000 \times 10-7$ & \\
\hline 88 & rs16938910 & intergenic & GDAP1, PCBP2P2 & $4.000 \times 10-7$ & \\
\hline 89 & rs12109285 & intergenic & GUSBP1, CDH12 & $1.000 \times 10-7$ & \\
\hline 90 & rs1610677 & intergenic & HCP5P12, HLA-G & $4.000 \times 10-15$ & \\
\hline 91 & rs6457620 & intergenic & HLA-DQB1, HLA-DQA2 & $4.000 \times 10-186$ & \\
\hline 92 & rs6457617 & intergenic & HLA-DQB1, HLA-DQA2 & $5.000 \times 10-75$ & \\
\hline 93 & rs13192471 & intergenic & HLA-DQB1, HLA-DQA2 & $2.000 \times 10-58$ & \\
\hline 94 & rs7765379 & intergenic & HLA-DQB1, HLA-DQA2 & $5.000 \times 10-23$ & \\
\hline 95 & rs6457617 & intergenic & HLA-DQB1, HLA-DQA2 & $1.000 \times 10-9$ & \\
\hline 96 & rs660895 & intergenic & HLA-DRB1, HLA-DQA1 & $1.000 \times 10-108$ & If \\
\hline 97 & rs9272219 & intergenic & HLA-DRB1, HLA-DQA1 & $1.000 \times 10-45$ & \\
\hline 98 & rs615672 & intergenic & HLA-DRB1, HLA-DQA1 & $8.000 \times 10-27$ & \\
\hline 99 & rs9268853 & intergenic & HLA-DRB9, HLA-DRB5 & $5.000 \times 10-109$ & \\
\hline 100 & rs6028945 & intergenic & HSPE1P1, MAFB & $2.000 \times 10-7$ & \\
\hline 101 & rs743777 & intergenic & IL2RB, C1QTNF6 & $1.000 \times 10-6$ & \\
\hline 102 & rs743777 & intergenic & IL2RB, C1QTNF6 & $2.000 \times 10-6$ & \\
\hline 103 & rs10488631 & intergenic & IRF5, TNPO3 & $4.000 \times 10-11$ & $3 a$ \\
\hline 104 & rs2280381 & intergenic & IRF8, FOXF1 & $2.000 \times 10-6$ & \\
\hline 105 & rs7155603 & intergenic & JDP2, BATF & $1.000 \times 10-7$ & \\
\hline 106 & rs231735 & intergenic & KRT18P39, CTLA4 & $6.000 \times 10-9$ & \\
\hline 107 & rs983332 & intergenic & LMO4, RPL36AP10 & $5.000 \times 10-6$ & \\
\hline 108 & rs6774280 & intergenic & MRPS35P1, MRPS36P1 & $9.000 \times 10-7$ & \\
\hline 109 & rs9296015 & intergenic & NOTCH4, C6orf10 & $2.000 \times 10-38$ & \\
\hline 110 & rs1406428 & intergenic & NRXN1, CRYGGP & $2.000 \times 10-7$ & \\
\hline 111 & rs6920220 & intergenic & OLIG3, TNFAIP3 & $9.000 \times 10-13$ & \\
\hline 112 & rs10499194 & intergenic & OLIG3, TNFAIP3 & $1.000 \times 10-9$ & \\
\hline 113 & rs6920220 & intergenic & OLIG3, TNFAIP3 & $2.000 \times 10-9$ & \\
\hline 114 & rs6920220 & intergenic & OLIG3, TNFAIP3 & $1.000 \times 10-7$ & \\
\hline 115 & rs1329568 & intergenic & PAX5, RPL32P21 & $8.000 \times 10-7$ & \\
\hline 116 & rs16906916 & intergenic & PCDH15, GAPDHP21 & $8.000 \times 10-7$ & $3 b$ \\
\hline 117 & rs881375 & intergenic & PHF19, TRAF1 & $4.000 \times 10-8$ & \\
\hline 118 & rs12131057 & intergenic & POU3F1, RRAGC & $4.000 \times 10-7$ & \\
\hline 119 & rs10945919 & intergenic & QKI, C6orf118 & $3.000 \times 10-7$ & \\
\hline 120 & rs13017599 & intergenic & REL, RPS12P3 & $2.000 \times 10-12$ & \\
\hline 121 & rs12529514 & intergenic & RNF182, CD83 & $2.000 \times 10-8$ & \\
\hline 122 & rs437943 & intergenic & RPL31P31, ARAP2 & $4.000 \times 10-6$ & \\
\hline 123 & rs17374222 & intergenic & RPLP1, GEMIN8P1 & $2.000 \times 10-6$ & \\
\hline 124 & rs11761231 & intergenic & RPS14P10, RPS15AP22 & $4.000 \times 10-7$ & \\
\hline 125 & rs6679677 & intergenic & RPS2P14, RSBN1 & $6.000 \times 10-42$ & \\
\hline 126 & rs6679677 & intergenic & RPS2P14, RSBN1 & $6.000 \times 10-25$ & \\
\hline 127 & rs72991 & intergenic & SC5DL, SORL1 & $5.000 \times 10-7$ & \\
\hline 128 & rs7164176 & intergenic & SV2B, TRNAY16P & $5.000 \times 10-7$ & $3 a$ \\
\hline 129 & rs800586 & intergenic & TRPS1, EIF3H & $2.000 \times 10-7$ & \\
\hline
\end{tabular}


Arthritis, Psoriatic

\begin{tabular}{|c|c|c|c|c|c|}
\hline$\#$ & rs \# & Context & Gene & P-value & $\begin{array}{l}\text { RegulomeDB } \\
\text { score }\end{array}$ \\
\hline 1 & rs13191343 & nearGene-5 & HLA-C & $2.000 \times 10-72$ & \\
\hline 2 & rs33980500 & missense & TRAF3IP2 & $1.000 \times 10-20$ & \\
\hline 3 & rs12188300 & intergenic & IL12B, ADRA1B & $7.000 \times 10-17$ & \\
\hline 4 & rs13017599 & intergenic & REL, RPS12P3 & $1.000 \times 10-8$ & \\
\hline 5 & rs702873 & intergenic & RPL21P33, REL & $2.000 \times 10-7$ & \\
\hline
\end{tabular}

Spondylitis, Ankylosing

\begin{tabular}{|c|c|c|c|c|c|}
\hline$\#$ & rs \# & Context & Gene & P-value & $\begin{array}{l}\text { RegulomeDB } \\
\text { score }\end{array}$ \\
\hline 1 & rs10781500 & nearGene-5 & CARD9 & $1.000 \times 10-6$ & If \\
\hline 2 & rs30187 & missense & ERAP1 & $2.000 \times 10-27$ & \\
\hline 3 & rs11209026 & missense & IL23R & $2.000 \times 10-17$ & \\
\hline 4 & rs11209026 & missense & IL23R & $9.000 \times 10-14$ & \\
\hline 5 & rs17095830 & intron & ANO6 & $2.000 \times 10-8$ & \\
\hline 6 & rs4389526 & intron & ANTXR2 & $9.000 \times 10-8$ & \\
\hline 7 & rs4333130 & intron & ANTXR2 & $9.000 \times 10-8$ & \\
\hline 8 & rs1326986 & intron & C10orf112 & $4.000 \times 10-6$ & \\
\hline 9 & rs2075726 & intron & CSF2RB & $9.000 \times 10-6$ & \\
\hline 10 & rs13210693 & intron & FLJ37396 & $9.000 \times 10-7$ & \\
\hline 11 & rs2297909 & intron & KIF21B & $5.000 \times 10-12$ & \\
\hline 12 & rs27434 & cds-synon & ERAP1 & $5.000 \times 10-12$ & \\
\hline 13 & rs10865331 & intergenic & B3GNT2, TMEM17 & $7.000 \times 10-34$ & \\
\hline 14 & rs10865331 & intergenic & B3GNT2, TMEM17 & $2.000 \times 10-19$ & \\
\hline 15 & rs10440635 & intergenic & DAB2, PTGER4 & $3.000 \times 10-7$ & \\
\hline 16 & rs7743761 & intergenic & DHFRP2, HLA-S & $5.000 \times 10-304$ & \\
\hline 17 & rs13210693 & intergenic & FLJ37396, CCDC162 & $9.000 \times 10-7$ & $2 a$ \\
\hline 18 & rs2242944 & intergenic & FLJ45139, RPL23AP12 & $8.000 \times 10-20$ & \\
\hline 19 & rs378108 & intergenic & FLJ45139, RPL23AP12 & $2.000 \times 10-11$ & \\
\hline 20 & rs4349859 & intergenic & HLA-S, MICA & $1.000 \times 10-200$ & \\
\hline 21 & rs6556416 & intergenic & IL12B, ADRA1B & $2.000 \times 10-8$ & \\
\hline 22 & rs2310173 & intergenic & IL1R2, IL1R1 & $5.000 \times 10-7$ & \\
\hline 23 & rs8070463 & intergenic & KPNB1, TBKBP1 & $5.000 \times 10-8$ & \\
\hline 24 & rs11616188 & intergenic & LTBR, RPL31P10 & $4.000 \times 10-12$ & \\
\hline 25 & rs12146962 & intergenic & MTCO1P2, NPAS3 & $9.000 \times 10-6$ & \\
\hline 26 & rs4552569 & intergenic & RPL13AP14, EDIL3 & $9.000 \times 10-10$ & \\
\hline 27 & rs11249215 & intergenic & RUNX3, SYF2 & $9.000 \times 10-11$ & \\
\hline 28 & rs1018326 & intergenic & UBE2E3, ITGA4 & $2.000 \times 10-6$ & \\
\hline
\end{tabular}




\begin{tabular}{|c|c|c|c|c|c|}
\hline$\#$ & rs \# & Context & Gene & P-value & $\begin{array}{l}\text { RegulomeDB } \\
\text { score }\end{array}$ \\
\hline 1 & rs8192583 & UTR-5 & GPSM3 & $2.250 \times 10-20$ & $1 f$ \\
\hline 2 & rs3094187 & UTR-5 & TCF19 & $3.725 \times 10-13$ & $1 f$ \\
\hline 3 & rs2240803 & UTR-3 & DPCR1 & $8.193 \times 10-13$ & \\
\hline 4 & rs2395029 & UTR-3 & HCP5 & $2.000 \times 10-26$ & \\
\hline 5 & rs8365 & UTR-3 & RNF5 & $1.094 \times 10-17$ & $1 f$ \\
\hline 6 & rs3130453 & STOP-GAIN & CCHCR1 & $2.057 \times 10-12$ & \\
\hline 7 & rs3132965 & nearGene-5 & AGPAT1 & $8.389 \times 10-16$ & \\
\hline 8 & rs3094187 & nearGene-5 & CCHCR1 & $3.725 \times 10-13$ & \\
\hline 9 & rs7773175 & nearGene-5 & HLA-C & $4.772 \times 10-30$ & \\
\hline 10 & rs2249742 & nearGene-5 & HLA-C & $6.346 \times 10-18$ & $1 f$ \\
\hline 11 & rs2395471 & nearGene-5 & HLA-C & $8.836 \times 10-18$ & $1 f$ \\
\hline 12 & rs2249741 & nearGene-5 & HLA-C & $3.340 \times 10-12$ & \\
\hline 13 & rs2524082 & nearGene-5 & HLA-C & $2.215 \times 10-11$ & \\
\hline 14 & rs9267502 & nearGene-5 & LST1 & $3.786 \times 10-19$ & \\
\hline 15 & rs2734573 & nearGene-5 & MCCD1 & $1.499 \times 10-12$ & \\
\hline 16 & rs176095 & nearGene-5 & PBX2 & $2.183 \times 10-12$ & $1 f$ \\
\hline 17 & rs3130453 & nearGene-5 & TCF19 & $2.057 \times 10-12$ & \\
\hline 18 & rs2021723 & nearGene-5 & TRIM40 & $8.690 \times 10-15$ & \\
\hline 19 & rs8365 & nearGene-3 & AGER & $1.094 \times 10-17$ & \\
\hline 20 & rs1265086 & nearGene-3 & CCHCR1 & $2.689 \times 10-11$ & $1 f$ \\
\hline 21 & rs9468843 & nearGene-3 & DDR1 & $9.009 \times 10-14$ & \\
\hline 22 & rs176095 & nearGene-3 & GPSM3 & $2.183 \times 10-12$ & \\
\hline 23 & rs2853950 & nearGene-3 & HLA-C & 1.756 x 10-14 & \\
\hline 24 & rs11575907 & missense & HLA-DOB & $5.960 \times 10-19$ & \\
\hline 25 & rs20541 & missense & IL13 & $5.000 \times 10-15$ & \\
\hline 26 & rs11209026 & missense & IL23R & $7.000 \times 10-7$ & \\
\hline 27 & rs8192591 & missense & NOTCH4 & $3.403 \times 10-24$ & \\
\hline 28 & rs33980500 & missense & TRAF3IP2 & $1.000 \times 10-16$ & \\
\hline 29 & rs12720356 & missense & TYK2 & $4.000 \times 10-11$ & \\
\hline 30 & rs2295663 & intron & BAT5 & $8.396 \times 10-24$ & \\
\hline 31 & rs9267673 & intron & $\mathrm{C} 2$ & $2.300 \times 10-33$ & If \\
\hline 32 & rs6906662 & intron & C6orf10 & $2.109 \times 10-14$ & \\
\hline 33 & rs27524 & intron & CAST & $3.000 \times 10-11$ & \\
\hline 34 & rs1265078 & intron & CCHCR1 & $2.039 \times 10-21$ & \\
\hline 36 & rs2239518 & intron & DDR1 & $3.454 \times 10-12$ & $2 b$ \\
\hline 37 & rs27524 & intron & ERAP1 & $3.000 \times 10-11$ & \\
\hline 38 & rs10782001 & intron & FBXL19 & $9.000 \times 10-10$ & \\
\hline 39 & rs3213094 & intron & IL12B & $3.000 \times 10-26$ & \\
\hline 40 & rs3213094 & intron & IL12B & $5.000 \times 10-11$ & \\
\hline 41 & rs2201841 & intron & IL23R & $3.000 \times 10-8$ & \\
\hline 42 & rs17716942 & intron & $\mathrm{KCNH7}$ & $1.000 \times 10-13$ & \\
\hline 43 & rs12586317 & intron & KIAA0391 & $2.000 \times 10-8$ & \\
\hline
\end{tabular}




\begin{tabular}{|c|c|c|c|c|c|}
\hline 44 & rs9295938 & intron & MUC21 & $9.163 \times 10-18$ & \\
\hline 45 & rs4795067 & intron & NOS2 & $4.000 \times 10-11$ & \\
\hline 46 & rs3823418 & intron & PSORS1C1 & $1.169 \times 10-33$ & \\
\hline 47 & rs3094205 & intron & PSORS1C1 & $1.733 \times 10-15$ & \\
\hline 48 & rs3130573 & intron & PSORS1C2 & $6.212 \times 10-15$ & $1 f$ \\
\hline 49 & rs3130573 & intron & PSORS1C2 & $6.212 \times 10-15$ & $1 f$ \\
\hline 50 & rs240993 & intron & REV3L & $5.000 \times 10-20$ & \\
\hline 51 & rs3132965 & intron & RNF5 & $8.389 \times 10-16$ & \\
\hline 52 & rs2066808 & intron & STAT2 & $1.000 \times 10-9$ & \\
\hline 53 & rs2066808 & intron & STAT2 & $2.000 \times 10-7$ & \\
\hline 54 & rs3093662 & intron & TNF & $4.752 \times 10-21$ & \\
\hline 55 & rs610604 & intron & TNFAIP3 & $9.000 \times 10-12$ & \\
\hline 56 & rs610604 & intron & TNFAIP3 & $7.000 \times 10-7$ & \\
\hline 57 & rs2077580 & intron & TNXB & $7.445 \times 10-32$ & \\
\hline 58 & rs2107195 & intron & TRIM15 & $1.675 \times 10-17$ & \\
\hline 60 & rs280519 & intron & TYK2 & $4.000 \times 10-9$ & \\
\hline 61 & rs8192583 & cds-synon & NOTCH4 & $2.250 \times 10-20$ & \\
\hline 62 & rs443198 & intron & NOTCH4 & $1.526 \times 10-11$ & $1 f$ \\
\hline 63 & rs495337 & cds-synon & SPATA2 & $1.000 \times 10-8$ & \\
\hline 64 & rs495337 & cds-synon & SPATA2 & $2.000 \times 10-7$ & \\
\hline 65 & rs7762370 & intergenic & BTNL2, HLA-DRA & $5.130 \times 10-19$ & \\
\hline 66 & rs9501624 & intergenic & BTNL2, HLA-DRA & $2.357 \times 10-12$ & \\
\hline 67 & rs1975974 & intergenic & C17orf51, UBBP4 & $1.000 \times 10-7$ & \\
\hline 68 & rs3130955 & intergenic & HCG22, C6orf15 & $1.312 \times 10-20$ & If \\
\hline 69 & rs2844627 & intergenic & HCG27, HLA-C & $2.307 \times 10-36$ & \\
\hline 70 & rs2394895 & intergenic & HCG27, HLA-C & $2.593 \times 10-29$ & \\
\hline 71 & rs3130517 & intergenic & HCG27, HLA-C & $1.430 \times 10-28$ & \\
\hline 72 & rs3130713 & intergenic & HCG27, HLA-C & $9.808 \times 10-28$ & \\
\hline 73 & rs3130467 & intergenic & HCG27, HLA-C & $1.339 \times 10-27$ & \\
\hline 74 & rs9263967 & intergenic & HCG27, HLA-C & $7.296 \times 10-15$ & \\
\hline 75 & rs3130685 & intergenic & HCG27, HLA-C & $8.583 \times 10-14$ & \\
\hline 76 & rs3130425 & intergenic & HCG27, HLA-C & $1.808 \times 10-12$ & \\
\hline 77 & rs3095250 & intergenic & HCG27, HLA-C & $2.892 \times 10-12$ & \\
\hline 78 & rs3132496 & intergenic & HCG27, HLA-C & $2.898 \times 10-12$ & \\
\hline 79 & rs3132486 & intergenic & HLA-C, RPL3P2 & $6.660 \times 10-13$ & \\
\hline 80 & rs3132485 & intergenic & HLA-C, RPL3P2 & $2.623 \times 10-12$ & \\
\hline 81 & rs2647087 & intergenic & HLA-DQB1, HLA-DQA2 & $1.865 \times 10-16$ & \\
\hline 82 & rs2858333 & intergenic & HLA-DQB1, HLA-DQA2 & $8.551 \times 10-16$ & \\
\hline 83 & rs2856726 & intergenic & HLA-DQB1, HLA-DQA2 & $5.086 \times 10-12$ & \\
\hline 84 & rs12203586 & intergenic & HLA-DQB1, HLA-DQA2 & $5.743 \times 10-12$ & If \\
\hline 85 & rs9268853 & intergenic & HLA-DRB9, HLA-DRB5 & $8.870 \times 10-13$ & \\
\hline 86 & rs10484552 & intergenic & HLA-E, GNL1 & $7.487 \times 10-19$ & \\
\hline 87 & rs13437088 & intergenic & HLA-S, MICA & $2.539 \times 10-13$ & \\
\hline 88 & rs10947208 & intergenic & HLA-S, MICA & $2.182 \times 10-12$ & \\
\hline 89 & rs7756521 & intergenic & IER3, DDR1 & $7.581 \times 10-19$ & \\
\hline
\end{tabular}




\begin{tabular}{|c|c|c|c|c|c|}
\hline 90 & rs4711229 & intergenic & IER3, DDR1 & $5.431 \times 10-14$ & \\
\hline 91 & rs9295917 & intergenic & IER3, DDR1 & $5.091 \times 10-13$ & \\
\hline 92 & rs4713380 & intergenic & IER3, DDR1 & $6.508 \times 10-13$ & $2 b$ \\
\hline 93 & rs12526186 & intergenic & IER3, DDR1 & $1.272 \times 10-12$ & \\
\hline 94 & rs13198118 & intergenic & IER3, DDR1 & $2.074 \times 10-12$ & $2 b$ \\
\hline 95 & rs7749924 & intergenic & IER3, DDR1 & $2.249 \times 10-12$ & \\
\hline 96 & rs9295924 & intergenic & IER3, DDR1 & $2.685 \times 10-12$ & \\
\hline 97 & rs3131043 & intergenic & IER3, DDR1 & $1.814 \times 10-11$ & If \\
\hline 98 & rs2546890 & intergenic & IL12B, ADRA1B & $1.000 \times 10-20$ & \\
\hline 99 & rs4649203 & intergenic & IL28RA, GRHL3 & $7.000 \times 10-8$ & \\
\hline 100 & rs4085613 & intergenic & LCE3E, LCE3D & $7.000 \times 10-30$ & \\
\hline 101 & rs4112788 & intergenic & LCE3E, LCE3D & $3.000 \times 10-10$ & \\
\hline 102 & rs9501106 & intergenic & MICA, HLA-X & $3.527 \times 10-16$ & \\
\hline 103 & rs9295993 & intergenic & MICA, HLA-X & $1.344 \times 10-15$ & \\
\hline 104 & rs9266844 & intergenic & MICA, HLA-X & $1.022 \times 10-11$ & \\
\hline 105 & rs7772549 & intergenic & MICA, HLA-X & $1.032 \times 10-11$ & \\
\hline 106 & rs9266846 & intergenic & MICA, HLA-X & $2.441 \times 10-11$ & \\
\hline 107 & rs17476793 & intergenic & MICC, SUCLA2P1 & $1.261 \times 10-17$ & \\
\hline 108 & rs13191258 & intergenic & MUC21, HCG22 & $3.951 \times 10-33$ & \\
\hline 109 & rs2844645 & intergenic & MUC21, HCG22 & $7.292 \times 10-19$ & \\
\hline 110 & rs9366764 & intergenic & MUC21, HCG22 & $8.095 \times 10-18$ & \\
\hline 111 & rs9394031 & intergenic & MUC21, HCG22 & $8.254 \times 10-18$ & \\
\hline 112 & rs3871466 & intergenic & MUC21, HCG22 & 7.904 x 10-17 & \\
\hline 113 & rs2523870 & intergenic & MUC21, HCG22 & $2.725 \times 10-12$ & \\
\hline 114 & rs2517552 & intergenic & MUC21, HCG22 & $8.159 \times 10-12$ & \\
\hline 115 & rs2894176 & intergenic & MUC21, HCG22 & $9.713 \times 10-12$ & \\
\hline 116 & rs2517527 & intergenic & MUC21, HCG22 & $1.831 \times 10-11$ & \\
\hline 117 & rs9262492 & intergenic & MUC21, HCG22 & $2.993 \times 10-11$ & $2 b$ \\
\hline 118 & rs6916062 & intergenic & NOTCH4, C6orf10 & $2.524 \times 10-22$ & \\
\hline 119 & rs9267463 & intergenic & PPIAP9, RPL15P4 & $1.104 \times 10-23$ & \\
\hline 120 & rs9267464 & intergenic & PPIAP9, RPL15P4 & $1.806 \times 10-22$ & \\
\hline 121 & rs2734573 & intergenic & PPIAP9, RPL15P4 & $1.499 \times 10-12$ & \\
\hline 122 & rs8016947 & intergenic & PSMA6, RPLPOP3 & $2.000 \times 10-11$ & \\
\hline 123 & rs702873 & intergenic & RPL21P33, REL & $4.000 \times 10-9$ & \\
\hline 124 & rs842636 & intergenic & RPL21P33, REL & $6.000 \times 10-6$ & \\
\hline 125 & rs12191877 & intergenic & RPL3P2, WASF5P & $1.000 \times 10-100$ & \\
\hline 126 & rs12191877 & intergenic & RPL3P2, WASF5P & $2.024 \times 10-51$ & \\
\hline 127 & rs12191877 & intergenic & RPL3P2, WASF5P & $4.000 \times 10-32$ & \\
\hline 128 & rs12580100 & intergenic & RPS26, ERBB3 & $1.000 \times 10-7$ & $3 a$ \\
\hline 129 & rs6809854 & intergenic & SATB1, KCNH8 & $1.000 \times 10-7$ & \\
\hline 130 & rs1008953 & intergenic & SDC4, SYS1 & $1.000 \times 10-7$ & \\
\hline 131 & rs17728338 & intergenic & TNIP1, ANXA6 & $1.000 \times 10-20$ & \\
\hline 132 & rs1015465 & intergenic & TRIM31, TRIM40 & $3.685 \times 10-15$ & \\
\hline 133 & rs2082412 & intergenic & UBLCP1, IL12B & $2.000 \times 10-28$ & \\
\hline 134 & rs10484554 & intergenic & WASF5P, HLA-B & $4.000 \times 10-214$ & \\
\hline
\end{tabular}




\begin{tabular}{|c|c|c|c|c|c|}
\hline 135 & rs9468933 & intergenic & WASF5P, HLA-B & $1.621 \times 10-46$ & \\
\hline 136 & rs10484554 & intergenic & WASF5P, HLA-B & $2.000 \times 10-39$ & \\
\hline 137 & rs2894207 & intergenic & WASF5P, HLA-B & $3.766 \times 10-38$ & \\
\hline 138 & rs9380237 & intergenic & WASF5P, HLA-B & $2.576 \times 10-28$ & \\
\hline 139 & rs2524163 & intergenic & WASF5P, HLA-B & $5.329 \times 10-20$ & \\
\hline 140 & rs2243868 & intergenic & WASF5P, HLA-B & $2.798 \times 10-19$ & $1 f$ \\
\hline 141 & rs2853923 & intergenic & WASF5P, HLA-B & $2.701 \times 10-15$ & \\
\hline 142 & rs9380240 & intergenic & WASF5P, HLA-B & $4.412 \times 10-14$ & \\
\hline 143 & rs2442719 & intergenic & WASF5P, HLA-B & $2.809 \times 10-13$ & \\
\hline 144 & rs3873386 & intergenic & WASF5P, HLA-B & $1.173 \times 10-12$ & $1 f$ \\
\hline 145 & rs2156875 & intergenic & WASF5P, HLA-B & $3.707 \times 10-12$ & If \\
\hline 146 & rs9468937 & intergenic & WASF5P, HLA-B & $4.828 \times 10-12$ & \\
\hline 147 & rs3134792 & intergenic & WASF5P, HLA-B & $1.000 \times 10-9$ & \\
\hline 148 & rs9267673 & intergenic & ZBTB12, C2 & $2.300 \times 10-33$ & \\
\hline
\end{tabular}

\section{Crohn Disease}

\begin{tabular}{|c|c|c|c|c|c|}
\hline$\#$ & rs \# & Context & Gene & P-value & $\begin{array}{l}\text { RegulomeDB } \\
\text { score }\end{array}$ \\
\hline 1 & rs504963 & UTR-3 & FUT2 & $2.000 \times 10-8$ & \\
\hline 2 & rs10210302 & nearGene-5 & ATG16L1 & $5.000 \times 10-14$ & \\
\hline 3 & rs12677663 & nearGene-5 & C8orf84 & $2.000 \times 10-8$ & \\
\hline 4 & rs11190140 & nearGene-5 & $N K X 2-3$ & $3.000 \times 10-16$ & \\
\hline 5 & rs11190141 & nearGene-5 & NKX2-3 & $5.000 \times 10-7$ & \\
\hline 6 & rs11574514 & nearGene-5 & PSMB10 & $2.000 \times 10-7$ & \\
\hline 7 & rs2241880 & missense & ATG16L1 & $1.000 \times 10-13$ & \\
\hline 8 & rs2241880 & missense & ATG16L1 & $1.000 \times 10-12$ & \\
\hline 9 & rs2241880 & missense & ATG16L1 & $3.000 \times 10-6$ & \\
\hline 10 & rs3764147 & missense & C13orf31 (LACC1) & $2.000 \times 10-13$ & $1 f$ \\
\hline 11 & rs4077515 & missense & CARD9 & $1.000 \times 10-36$ & 1f \\
\hline 12 & rs11209026 & missense & IL23R & $1.000 \times 10-64$ & \\
\hline 13 & rs11209026 & missense & IL23R & $4.000 \times 10-21$ & \\
\hline 14 & rs11209026 & missense & IL23R & $1.000 \times 10-18$ & \\
\hline 15 & rs11209026 & missense & IL23R & $2.000 \times 10-18$ & \\
\hline 16 & rs3197999 & missense & MST1 & $6.000 \times 10-17$ & \\
\hline 17 & rs3197999 & missense & MST1 & $1.000 \times 10-12$ & \\
\hline 18 & rs2476601 & missense & PTPN22 & $1.000 \times 10-8$ & $2 b$ \\
\hline 19 & rs12720356 & missense & TYK2 & $1.000 \times 10-12$ & \\
\hline 20 & rs3792109 & intron & ATG16L1 & $7.000 \times 10-41$ & \\
\hline 21 & rs3828309 & intron & ATG16L1 & $2.000 \times 10-32$ & \\
\hline 22 & rs1847472 & intron & $\mathrm{BACH} 2$ & $5.000 \times 10-9$ & \\
\hline 23 & rs102275 & intron & C11orf10 & $2.000 \times 10-11$ & \\
\hline 24 & rs12521868 & intron & C5orf56 & $1.000 \times 10-20$ & \\
\hline 25 & rs2188962 & intron & C5orf56 & $2.000 \times 10-18$ & $2 a$ \\
\hline 26 & rs2188962 & intron & C5orf56 & $1.000 \times 10-7$ & \\
\hline 27 & rs6908425 & intron & CDKAL1 & $9.000 \times 10-10$ & \\
\hline
\end{tabular}




\begin{tabular}{|c|c|c|c|c|c|}
\hline 28 & rs151181 & intron & CLN3 & $2.000 \times 10-11$ & \\
\hline 29 & rs1998598 & intron & DENND1B & $9.000 \times 10-9$ & \\
\hline 30 & rs13428812 & intron & DNMT3A & $9.000 \times 10-10$ & \\
\hline 31 & rs2549794 & intron & ERAP2 & $1.000 \times 10-10$ & $1 f$ \\
\hline 32 & rs2301436 & intron & FGFR1OP & $1.000 \times 10-12$ & \\
\hline 33 & rs2301436 & intron & FGFR1OP & $6.000 \times 10-8$ & \\
\hline 34 & rs780093 & intron & GCKR & $5.000 \times 10-11$ & \\
\hline 35 & rs8005161 & intron & GPR65 & $4.000 \times 10-18$ & \\
\hline 36 & rs2058660 & intron & IL18RAP & $2.000 \times 10-12$ & \\
\hline 37 & rs11465804 & intron & IL23R & $7.000 \times 10-63$ & \\
\hline 38 & rs7517847 & intron & IL23R & $3.000 \times 10-12$ & \\
\hline 39 & rs11805303 & intron & IL23R & $6.000 \times 10-12$ & \\
\hline 40 & rs11465804 & intron & IL23R & $1.000 \times 10-6$ & \\
\hline 41 & rs12722489 & intron & IL2RA & $3.000 \times 10-9$ & \\
\hline 42 & rs2274910 & intron & ITLN1 & $1.000 \times 10-9$ & \\
\hline 43 & rs1793004 & intron & NELL1 & $3.000 \times 10-6$ & \\
\hline 44 & rs2076756 & intron & NOD2 & $4.000 \times 10-69$ & \\
\hline 45 & rs2076756 & intron & NOD2 & $1.000 \times 10-37$ & \\
\hline 46 & rs2076756 & intron & NOD2 & $1.000 \times 10-21$ & \\
\hline 47 & rs5743289 & intron & NOD2 & $6.000 \times 10-17$ & \\
\hline 48 & rs2076756 & intron & NOD2 & $7.000 \times 10-14$ & \\
\hline 49 & rs17221417 & intron & NOD2 & $4.000 \times 10-11$ & \\
\hline 50 & rs5743289 & intron & NOD2 & $1.000 \times 10-6$ & \\
\hline 51 & rs2797685 & intron & PER3 & $7.000 \times 10-9$ & \\
\hline 52 & rs6738825 & intron & PLCL1 & $4.000 \times 10-9$ & \\
\hline 53 & rs13003464 & intron & PUS10 & $5.000 \times 10-9$ & \\
\hline 54 & rs10181042 & intron & PUS10 & $7.000 \times 10-9$ & \\
\hline 55 & rs17309827 & intron & SLC22A23 & $7.000 \times 10-9$ & \\
\hline 56 & rs17293632 & intron & SMAD3 & $3.000 \times 10-19$ & $2 a$ \\
\hline 57 & rs7423615 & intron & SP140 & $3.000 \times 10-13$ & \\
\hline 58 & rs744166 & intron & STAT3 & $7.000 \times 10-12$ & \\
\hline 59 & rs10495903 & intron & THADA & $2.000 \times 10-14$ & \\
\hline 60 & rs4263839 & intron & TNFSF15 & $3.000 \times 10-10$ & $2 b$ \\
\hline 61 & rs181359 & intron & UBE2L3 & $5.000 \times 10-16$ & $1 f$ \\
\hline 62 & rs4809330 & intron & ZGPAT & $3.000 \times 10-15$ & \\
\hline 63 & rs1250550 & intron & ZMIZ1 & $1.000 \times 10-30$ & \\
\hline 64 & rs7076156 & intron & ZNF365 & $7.000 \times 10-9$ & \\
\hline 65 & rs2066847 & frameshift & NOD2 & $3.000 \times 10-24$ & \\
\hline 66 & rs2066847 & frameshift & NOD2 & $2.000 \times 10-15$ & \\
\hline 67 & rs9858542 & cds-synon & BSN & $4.000 \times 10-8$ & $1 f$ \\
\hline 68 & rs9858542 & cds-synon & BSN & $5.000 \times 10-8$ & \\
\hline 69 & rs1142287 & cds-synon & SCAMP3 & $2.000 \times 10-13$ & \\
\hline 70 & rs3810936 & cds-synon & TNFSF15 & $1.000 \times 10-15$ & \\
\hline 71 & rs6545946 & intergenic & B3GNT2, TMEM17 & $7.000 \times 10-9$ & \\
\hline 72 & rs359457 & intergenic & BOD1, CPEB4 & $3.000 \times 10-12$ & \\
\hline
\end{tabular}




\begin{tabular}{|c|c|c|c|c|c|}
\hline 73 & rs1398024 & intergenic & C10orf67, OTUD1 & $4.000 \times 10-6$ & \\
\hline 74 & rs7927894 & intergenic & C11orf30, LRRC32 & $1.000 \times 10-9$ & \\
\hline 75 & rs11584383 & intergenic & C1orf81, KIF21B & $1.000 \times 10-11$ & \\
\hline 76 & rs762421 & intergenic & C21orf33, ICOSLG & $1.000 \times 10-9$ & \\
\hline 77 & rs13361189 & intergenic & C5orf62, IRGM & $2.000 \times 10-10$ & \\
\hline 78 & rs1456893 & intergenic & C7orf72, IKZF1 & $5.000 \times 10-9$ & \\
\hline 79 & rs3091315 & intergenic & $\mathrm{CCL} 2, \mathrm{CCL} 7$ & $2.000 \times 10-13$ & \\
\hline 80 & rs3091316 & intergenic & $\mathrm{CCL} 2, \mathrm{CCL} 7$ & $4.000 \times 10-8$ & \\
\hline 81 & rs7807268 & intergenic & CNTNAP2, RPL32P17 & $4.000 \times 10-6$ & \\
\hline 82 & rs11742570 & intergenic & DAB2, PTGER4 & $7.000 \times 10-36$ & \\
\hline 83 & rs4613763 & intergenic & DAB2, PTGER4 & $7.000 \times 10-27$ & \\
\hline 84 & rs9292777 & intergenic & DAB2, PTGER4 & $3.000 \times 10-18$ & \\
\hline 85 & rs17234657 & intergenic & DAB2, PTGER4 & $2.000 \times 10-12$ & \\
\hline 86 & rs1373692 & intergenic & DAB2, PTGER4 & $2.000 \times 10-12$ & \\
\hline 87 & rs9292777 & intergenic & DAB2, PTGER4 & $2.000 \times 10-11$ & \\
\hline 88 & rs1992660 & intergenic & DAB2, PTGER4 & $4.000 \times 10-7$ & \\
\hline 89 & rs2062305 & intergenic & FABP3P2, TNFSF11 & $5.000 \times 10-10$ & \\
\hline 90 & rs10801047 & intergenic & FAM5C, RGS18 & $3.000 \times 10-8$ & \\
\hline 91 & rs9286879 & intergenic & FASLG, TNFSF18 & $2.000 \times 10-9$ & \\
\hline 92 & rs12035082 & intergenic & FASLG, TNFSF18 & $2.000 \times 10-7$ & \\
\hline 93 & rs2836754 & intergenic & FL45139, RPL23AP12 & $5.000 \times 10-7$ & \\
\hline 94 & rs281379 & intergenic & FUT2, MAMSTR & $7.000 \times 10-12$ & \\
\hline 95 & rs4409764 & intergenic & GOT1, NKX2-3 & $2.000 \times 10-20$ & $3 a$ \\
\hline 96 & rs10883365 & intergenic & GOT1, NKX2-3 & $4.000 \times 10-10$ & \\
\hline 97 & rs10883365 & intergenic & GOT1, NKX2-3 & $6.000 \times 10-8$ & \\
\hline 98 & rs9469220 & intergenic & HLA-DQB1, HLA-DQA2 & $2.000 \times 10-6$ & $1 f$ \\
\hline 99 & rs9258260 & intergenic & IFITM4P, 3.8-1.5 & $2.000 \times 10-10$ & $1 f$ \\
\hline 100 & rs10045431 & intergenic & IL12B, ADRA1B & $4.000 \times 10-13$ & \\
\hline 101 & rs10045431 & intergenic & IL12B, ADRA1B & $7.000 \times 10-8$ & \\
\hline 102 & rs6887695 & intergenic & IL12B, ADRA1B & $9.000 \times 10-6$ & \\
\hline 103 & rs3091338 & intergenic & IL3, CSF2 & $4.000 \times 10-8$ & \\
\hline 104 & rs7714584 & intergenic & IRGM, ZNF300 & $8.000 \times 10-19$ & \\
\hline 105 & rs11747270 & intergenic & IRGM, ZNF300 & $3.000 \times 10-16$ & \\
\hline 106 & rs1000113 & intergenic & IRGM, ZNF300 & $3.000 \times 10-7$ & \\
\hline 107 & rs6601764 & intergenic & KLF6, AKR1E2 & $9.000 \times 10-6$ & \\
\hline 108 & rs11167764 & intergenic & MRPL11P2, NDFIP1 & $2.000 \times 10-9$ & \\
\hline 109 & rs1819658 & intergenic & MRPS35P3, IPMK & $9.000 \times 10-17$ & \\
\hline 110 & rs1736135 & intergenic & NRIP1, CYCSP42 & $7.000 \times 10-9$ & \\
\hline 111 & rs2413583 & intergenic & PDGFB, RPL3 & $1.000 \times 10-26$ & \\
\hline 112 & rs694739 & intergenic & PRDX5, CCDC88B & $6.000 \times 10-10$ & $1 f$ \\
\hline 113 & rs2542151 & intergenic & PSMG2, PTPN2 & $5.000 \times 10-17$ & \\
\hline 114 & rs2542151 & intergenic & PSMG2, PTPN2 & $3.000 \times 10-8$ & \\
\hline 115 & rs2542151 & intergenic & PSMG2, PTPN2 & $2.000 \times 10-7$ & \\
\hline 116 & rs6651252 & intergenic & PVT1, GSDMC & $4.000 \times 10-18$ & \\
\hline 117 & rs10758669 & intergenic & RCL1, JAK2 & $3.000 \times 10-9$ & \\
\hline
\end{tabular}




$\begin{array}{llllll}118 & \text { rs4902642 } & \text { intergenic } & \text { RPL12P7, ZFP36L1 } & 2.000 \times 10-10 \\ 119 & \text { rs11175593 } & \text { intergenic } & \text { RPL30P13, LRRK2 } & 3.000 \times 10-10 & \\ 120 & \text { rs7746082 } & \text { intergenic } & \text { RPL35P3, PRDM1 } & 2.000 \times 10-10 & \\ 121 & \text { rs17582416 } & \text { intergenic } & \text { RPS12P16, CUL2 } & 2.000 \times 10-9 & \text { 2b } \\ 122 & \text { rs3024505 } & \text { intergenic } & \text { RPS14P1, IL10 } & 2.000 \times 10-14 & \mathbf{1 f} \\ 123 & \text { rs713875 } & \text { intergenic } & \text { RPS3AP51, LIF } & 7.000 \times 10-12 & \mathbf{1 f} \\ 124 & \text { rs13073817 } & \text { intergenic } & \text { SATB1, KCNH8 } & 7.000 \times 10-9 & \\ 125 & \text { rs6596075 } & \text { intergenic } & \text { SLC22A5, C5orf56 } & 3.000 \times 10-6 & \\ 126 & \text { rs11229030 } & \text { intergenic } & \text { SLC43A3, RTN4RL2 } & 8.000 \times 10-9 & \\ 127 & \text { rs736289 } & \text { intergenic } & \text { SLC7A10, CEBPA } & 9.000 \times 10-9 \\ 128 & \text { rs7705924 } & \text { intergenic } & \text { SLCO6A1, PAM } & 2.000 \times 10-8 \\ 129 & \text { rs212388 } & \text { intergenic } & \text { TAGAP, FNDC1 } & 2.000 \times 10-11 \\ 130 & \text { rs10734105 } & \text { intergenic } & \text { TCERG1L, FLJ46300 } & 3.000 \times 10-8 \\ 131 & \text { rs7702331 } & \text { intergenic } & \text { TMEM174, FOXD1 } & 6.000 \times 10-12 \\ 132 & \text { rs1551398 } & \text { intergenic } & \text { TRIB1, FAM84B } & 5.000 \times 10-9 \\ 133 & \text { rs1906493 } & \text { intergenic } & \text { TRIB1, FAM84B } & 3.000 \times 10-6 \\ 134 & \text { rs10761659 } & \text { intergenic } & \text { ZNF365, ALDH7A1P4 } & 4.000 \times 10-22 \\ 135 & \text { rs10995271 } & \text { intergenic } & \text { ZNF365, ALDH7A1P4 } & 4.000 \times 10-20 \\ 136 & \text { rs224136 } & \text { intergenic } & \text { ZNF365, ALDH7A1P4 } & 1.000 \times 10-10 \\ 137 & \text { rs10761659 } & \text { intergenic } & \text { ZNF365, ALDH7A1P4 } & 2.000 \times 10-6 \\ 138 & \text { rs2872507 } & \text { intergenic } & \text { ZPBP2, GSDMB } & 5.000 \times 10-9\end{array}$

Colitis, Ulcerative

\begin{tabular}{|c|c|c|c|c|c|}
\hline \# & rs \# & Context & Gene & P-value & $\begin{array}{l}\text { RegulomeDB } \\
\text { score }\end{array}$ \\
\hline 1 & rs10889677 & UTR-3 & IL23R & $1.000 \times 10-8$ & \\
\hline 2 & rs2297441 & UTR-3 & RTEL1 & $2.000 \times 10-10$ & \\
\hline 3 & rs10781500 & nearGene-5 & CARD9 & $7.000 \times 10-6$ & $1 f$ \\
\hline 4 & rs678170 & nearGene-5 & FAM55A & $5.000 \times 10-14$ & \\
\hline 5 & rs907611 & nearGene-5 & LSP1 & $1.000 \times 10-10$ & $2 a$ \\
\hline 6 & rs11190140 & nearGene-5 & NKX2-3 & $1.000 \times 10-8$ & \\
\hline 7 & rs3806308 & nearGene-5 & RNF186 & $7.000 \times 10-9$ & $2 b$ \\
\hline 8 & rs2297441 & nearGene-5 & $\begin{array}{l}\text { TNFRSF6B (it is acually } \\
\text { RTEL1) }\end{array}$ & $2.000 \times 10-10$ & \\
\hline 9 & rs1317209 & nearGene-3 & RNF186 & $2.000 \times 10-10$ & \\
\hline 10 & rs4077515 & missense & CARD9 & $5.000 \times 10-8$ & $1 f$ \\
\hline 11 & rs1801274 & missense & FCGR2A & $2.000 \times 10-20$ & \\
\hline 12 & rs1801274 & missense & FCGR2A & $2.000 \times 10-12$ & \\
\hline 13 & rs2305480 & missense & GSDMB & $3.000 \times 10-8$ & \\
\hline 14 & rs5771069 & missense & IL17REL & $4.000 \times 10-8$ & \\
\hline 15 & rs5771069 & missense & IL17REL & $2.000 \times 10-7$ & \\
\hline 16 & rs11209026 & missense & IL23R & $5.000 \times 10-28$ & \\
\hline 17 & rs11209026 & missense & IL23R & $3.000 \times 10-10$ & \\
\hline 18 & rs11209026 & missense & IL23R & $1.000 \times 10-8$ & \\
\hline
\end{tabular}




\begin{tabular}{|c|c|c|c|c|c|}
\hline 19 & rs3194051 & missense & IL7R & $4.000 \times 10-8$ & \\
\hline 20 & rs3197999 & missense & MST1 & $4.000 \times 10-9$ & \\
\hline 21 & rs17388568 & intron & ADAD1 & $9.000 \times 10-7$ & \\
\hline 22 & rs9822268 & intron & APEH & $2.000 \times 10-17$ & \\
\hline 23 & rs7554511 & intron & C1orf106 & $2.000 \times 10-13$ & \\
\hline 24 & rs7554511 & intron & C1orf106 & $1.000 \times 10-6$ & \\
\hline 25 & rs9263739 & intron & CCHCR1 & $4.000 \times 10-67$ & $1 f$ \\
\hline 26 & rs12261843 & intron & CCNY & $7.000 \times 10-10$ & \\
\hline 27 & rs4781011 & intron & CIITA & $3.000 \times 10-6$ & $2 b$ \\
\hline 28 & rs267939 & intron & DAP & $6.000 \times 10-12$ & \\
\hline 29 & rs798502 & intron & GNA12 & $3.000 \times 10-15$ & 1b \\
\hline 30 & rs3024493 & intron & IL10 & $1.000 \times 10-12$ & $2 b$ \\
\hline 31 & rs3024493 & intron & IL10 & $8.000 \times 10-8$ & $2 b$ \\
\hline 32 & rs2201841 & intron & IL23R & $1.000 \times 10-13$ & \\
\hline 33 & rs2870946 & intron & IL26 & $5.000 \times 10-7$ & \\
\hline 34 & rs2158836 & intron & LAMB1 & $7.000 \times 10-6$ & \\
\hline 35 & rs4654925 & intron & OTUD3 & $9.000 \times 10-22$ & \\
\hline 36 & rs35675666 & intron & PARK7 & $5.000 \times 10-9$ & \\
\hline 37 & rs7608910 & intron & PUS10 & $2.000 \times 10-14$ & \\
\hline 38 & rs13003464 & intron & PUS10 & $7.000 \times 10-9$ & \\
\hline 39 & rs1992950 & intron & SATB2 & $5.000 \times 10-6$ & \\
\hline 40 & rs4246905 & intron & TNFSF15 & $6.000 \times 10-12$ & \\
\hline 41 & rs1728785 & intron & ZFP90 & $3.000 \times 10-8$ & \\
\hline 42 & rs9858542 & cds-synon & BSN & $7.000 \times 10-9$ & $1 f$ \\
\hline 43 & rs9268480 & cds-synon & BTNL2 & $3.000 \times 10-6$ & \\
\hline 44 & rs10781499 & cds-synon & CARD9 & $3.000 \times 10-19$ & $1 f$ \\
\hline 45 & rs2155219 & intergenic & C11orf30, LRRC32 & $5.000 \times 10-16$ & \\
\hline 46 & rs10800309 & intergenic & C1orf192, FCGR2A & $3.000 \times 10-9$ & \\
\hline 47 & rs11584383 & intergenic & C1orf81, KIF21B & $2.000 \times 10-7$ & \\
\hline 48 & rs2838519 & intergenic & C21orf33, ICOSLG & $6.000 \times 10-11$ & \\
\hline 49 & rs941823 & intergenic & COG6, FOXO1 & $4.000 \times 10-12$ & \\
\hline 50 & rs9548988 & intergenic & COG6, FOXO1 & $3.000 \times 10-7$ & \\
\hline 51 & rs11676348 & intergenic & CXCR2, CXCR1 & $1.000 \times 10-10$ & $1 f$ \\
\hline 52 & rs6451493 & intergenic & DAB2, PTGER4 & $3.000 \times 10-9$ & $3 a$ \\
\hline 53 & rs2836878 & intergenic & FLJ45139, RPL23AP12 & $2.000 \times 10-22$ & \\
\hline 54 & rs6584283 & intergenic & GOT1, NKX2-3 & $8.000 \times 10-21$ & \\
\hline 55 & rs6584283 & intergenic & GOT1, NKX2-3 & $2.000 \times 10-7$ & \\
\hline 56 & rs6584283 & intergenic & GOT1, NKX2-3 & $2.000 \times 10-6$ & \\
\hline 57 & rs17085007 & intergenic & GPR12, RPS20P32 & $1.000 \times 10-16$ & \\
\hline 58 & rs17085007 & intergenic & GPR12, RPS20P32 & $7.000 \times 10-8$ & \\
\hline 59 & rs4676406 & intergenic & GPR35, AQP12B & $8.000 \times 10-11$ & \\
\hline 60 & rs9268853 & intergenic & HLA-DRB9, HLA-DRB5 & $1.000 \times 10-55$ & \\
\hline 61 & rs9268877 & intergenic & HLA-DRB9, HLA-DRB5 & $4.000 \times 10-23$ & \\
\hline
\end{tabular}




\begin{tabular}{|c|c|c|c|c|c|}
\hline 62 & rs2395185 & intergenic & HLA-DRB9, HLA-DRB5 & $5.000 \times 10-22$ & \\
\hline 63 & rs9268877 & intergenic & HLA-DRB9, HLA-DRB5 & $6.000 \times 10-18$ & \\
\hline 64 & rs2395185 & intergenic & HLA-DRB9, HLA-DRB5 & $1.000 \times 10-16$ & \\
\hline 65 & rs9268923 & intergenic & HLA-DRB9, HLA-DRB5 & $4.000 \times 10-15$ & \\
\hline 66 & rs6017342 & intergenic & HNF4A, RPL37AP1 & $1.000 \times 10-20$ & \\
\hline 67 & rs6017342 & intergenic & HNF4A, RPL37AP1 & $9.000 \times 10-17$ & \\
\hline 68 & rs6871626 & intergenic & IL12B, ADRA1B & $1.000 \times 10-21$ & \\
\hline 69 & rs2310173 & intergenic & IL1R2, IL1R1 & $3.000 \times 10-12$ & \\
\hline 70 & rs10975003 & intergenic & INSL6, INSL4 & $1.000 \times 10-6$ & \\
\hline 71 & rs16940202 & intergenic & IRF8, FOXF1 & $6.000 \times 10-19$ & \\
\hline 72 & rs4728142 & intergenic & KCP, IRF5 & $2.000 \times 10-8$ & If \\
\hline 73 & rs1297265 & intergenic & NRIP1, CYCSP42 & $7.000 \times 10-13$ & $2 a$ \\
\hline 74 & rs1736135 & intergenic & NRIP1, CYCSP42 & $2.000 \times 10-7$ & \\
\hline 75 & rs6920220 & intergenic & OLIG3, TNFAIP3 & $8.000 \times 10-17$ & \\
\hline 76 & rs254560 & intergenic & PITX1, H2AFY & $1.000 \times 10-9$ & \\
\hline 77 & rs10758669 & intergenic & RCL1, JAK2 & $2.000 \times 10-25$ & \\
\hline 78 & rs10758669 & intergenic & RCL1, JAK2 & $1.000 \times 10-6$ & \\
\hline 79 & rs6426833 & intergenic & RNF186, OTUD3 & $4.000 \times 10-35$ & \\
\hline 80 & rs6426833 & intergenic & RNF186, OTUD3 & $2.000 \times 10-21$ & \\
\hline 81 & rs6426833 & intergenic & RNF186, OTUD3 & $5.000 \times 10-13$ & \\
\hline 82 & rs6426833 & intergenic & RNF186, OTUD3 & $2.000 \times 10-11$ & \\
\hline 83 & rs6911490 & intergenic & RPL35P3, PRDM1 & $1.000 \times 10-8$ & \\
\hline 84 & rs7134599 & intergenic & RPL39P28, IFNG & $1.000 \times 10-16$ & \\
\hline 85 & rs1558744 & intergenic & RPL39P28, IFNG & $3.000 \times 10-12$ & \\
\hline 86 & rs1558744 & intergenic & RPL39P28, IFNG & $4.000 \times 10-12$ & \\
\hline 87 & rs3024505 & intergenic & RPS14P1, IL10 & $6.000 \times 10-17$ & $2 b$ \\
\hline 88 & rs3024505 & intergenic & RPS14P1, IL10 & $1.000 \times 10-12$ & \\
\hline 89 & rs3024505 & intergenic & RPS14P1, IL10 & $1.000 \times 10-8$ & \\
\hline 90 & rs668853 & intergenic & RPS2P34, RPS6P12 & $2.000 \times 10-6$ & \\
\hline 91 & rs4510766 & intergenic & SLC26A3, DLD & $2.000 \times 10-16$ & \\
\hline 92 & rs886774 & intergenic & SLC26A3, DLD & $3.000 \times 10-8$ & \\
\hline 93 & rs4598195 & intergenic & SLC26A3, DLD & $8.000 \times 10-8$ & \\
\hline 94 & rs2108225 & intergenic & SLC26A3, DLD & $1.000 \times 10-7$ & \\
\hline 95 & rs4598195 & intergenic & SLC26A3, DLD & $1.000 \times 10-6$ & \\
\hline 96 & rs4730273 & intergenic & SLC26A3, DLD & $5.000 \times 10-6$ & \\
\hline 97 & rs4730276 & intergenic & SLC26A3, DLD & $9.000 \times 10-6$ & \\
\hline 98 & rs4957048 & intergenic & SLC9A3, CEP72 & $1.000 \times 10-9$ & \\
\hline 99 & rs11739663 & intergenic & SLC9A3, CEP72 & $3.000 \times 10-8$ & \\
\hline 100 & rs7809799 & intergenic & SMURF1, KPNA7 & $9.000 \times 10-11$ & \\
\hline 101 & rs734999 & intergenic & TNFRSF14, C1orf93 & $3.000 \times 10-9$ & If \\
\hline 102 & rs943072 & intergenic & VEGFA, C6orf223 & $2.000 \times 10-10$ & \\
\hline 103 & rs7524102 & intergenic & WNT4, ZBTB40 & $2.000 \times 10-13$ & \\
\hline 104 & rs7524102 & intergenic & WNT4, ZBTB40 & $3.000 \times 10-7$ & \\
\hline
\end{tabular}




$\begin{array}{lllll}105 & \text { rs6499188 } & \text { intergenic } & \text { ZFP90, CDH3 } & 4.000 \times 10-8 \\ 106 & \text { rs2872507 } & \text { intergenic } & \text { ZPBP2, GSDMB } & 5.000 \times 10-11 \\ 107 & \text { rs8067378 } & \text { intergenic } & \text { ZPBP2, GSDMB } & 1.000 \times 10-7\end{array}$

$1 f$

\section{Inflammatory Bowel Diseases}

\begin{tabular}{|c|c|c|c|c|c|}
\hline$\#$ & rs \# & Context & Gene & P-value & RegulomeDB score \\
\hline 1 & rs10889677 & UTR-3 & IL23R & $9.037 \times 10-11$ & \\
\hline 2 & rs11209026 & missense & IL23R & $4.000 \times 10-11$ & \\
\hline 3 & rs11209026 & missense & IL23R & $4.592 \times 10-11$ & \\
\hline 4 & rs11209026 & missense & IL23R & $7.000 \times 10-11$ & \\
\hline 5 & rs2315008 & intron & ZGPAT & $9.000 \times 10-15$ & \\
\hline 6 & rs2076756 & intron & NOD2 & $1.262 \times 10-14$ & 5 \\
\hline 7 & rs7517847 & intron & IL23R & $2.991 \times 10-13$ & \\
\hline 8 & rs7517847 & intron & IL23R & $4.000 \times 10-13$ & \\
\hline 9 & rs1343151 & intron & IL23R & $1.628 \times 10-11$ & \\
\hline 10 & rs10489629 & intron & IL23R & $6.790 \times 10-11$ & \\
\hline 11 & rs2201841 & intron & IL23R & $3.574 \times 10-10$ & \\
\hline 12 & rs11465804 & intron & IL23R & $3.737 \times 10-10$ & \\
\hline 13 & rs5743289 & intron & NOD2 & $4.000 \times 10-10$ & \\
\hline 14 & rs2076756 & intron & NOD2 & $5.000 \times 10-10$ & \\
\hline 15 & rs1004819 & intron & IL23R & $1.504 \times 10-9$ & \\
\hline 16 & rs8049439 & intron & ATXN2L & $2.000 \times 10-9$ & $1 b$ \\
\hline 17 & rs2412973 & intron & HORMAD2 & $2.000 \times 10-9$ & \\
\hline 18 & rs1250550 & intron & ZMIZ1 & $6.000 \times 10-9$ & \\
\hline 19 & rs2066843 & cds-synon & NOD2 & $7.869 \times 10-13$ & \\
\hline 20 & rs9271366 & intergenic & HLA-DRB1, HLA-DQA1 & $2.000 \times 10-70$ & \\
\hline 21 & rs9271366 & intergenic & HLA-DRB1, HLA-DQA1 & $3.000 \times 10-31$ & \\
\hline 22 & rs2006996 & intergenic & TNFSF15, TNFSF8 & $4.000 \times 10-16$ & \\
\hline 23 & rs2006996 & intergenic & TNFSF15, TNFSF8 & $4.000 \times 10-13$ & \\
\hline 24 & rs2836878 & intergenic & FLJ45139, RPL23AP12 & $4.000 \times 10-12$ & \\
\hline 25 & rs9271366 & intergenic & HLA-DRB1, HLA-DQA1 & $8.000 \times 10-11$ & \\
\hline 26 & rs10500264 & intergenic & SLC7A10, CEBPA & $4.000 \times 10-10$ & \\
\hline 27 & rs11209032 & intergenic & IL23R, IL12RB2 & $8.645 \times 10-10$ & \\
\hline 28 & rs477515 & intergenic & HLA-DRB1, HLA-DQA1 & $1.000 \times 10-8$ & \\
\hline
\end{tabular}

Note:

prvided are only the highest scores, and two additional interesting SNPs; otherwise all SNPs without numbers have scores higher than 3: 4, 5, 6 or no data existed at the time of data retreival

AID associated GWAS SNPs, labeled with its rs identifier, are associated with genes; for each SNP its RegulomeDB score is given. Only highest scored SNPs are further analysed. ncSNPs are sorted by the associated AID. 


\section{Supplemetal Table 3. \\ Non-coding AID GWAS SNPs might influence the following genes and their corresponding KEGG pathways:}

Gene GenelD

1 ANKRD55 79722 none

2 APOM 55937 none

3 ATXN2L 11273 none

4 BAG6 7017 none

5 BSN 8927 none

$6 \quad \mathrm{C} 2$

717

hsa04610

hsa05133

hsa05150

hsa05322

7 CCDC88B 283234 none

8 CCR6

\section{5 hsa04060}

9 CTLA4

1493

$10 \quad \mathrm{CD} 40$

\section{KEGG pathway}

Gene function, association or other
Directly involved in Immune System (and not present ubiquitously) ankyrin repeat domain 55 ;linked to MS, insulin

no resistence and cancer

apolipoprotein $\mathrm{M}$ involved in lipid transport; associated with RA

unknown function;associated with a complex group of neurodegenerative disorders.

BCL2-associated athanogene 6; located within HLA class III;implicated in the control of apoptosis

bassoon presynaptic cytomatrix protein; involved in the organization of the cytomatrix at the nerve terminals active zone (CAZ) which regulates neurotransmitter release and in the formation of the retinal photoreceptor ribbon synapse; regulates neurotransmitter release from a subset of brain glutamatergic synapses.

complement component 2; serine endopeptidase; associated with Psoriasis, T1D, Macular degeneration, SLE, carcinoma
Pertussis

Staphylococcus aureus infection Systemic lupus erythematosus coiled-coil domain containing 88B; function not known no

yes important for B-lineage maturation and and antigen-driven B-cell differentiation, and it may regulate the migration and recruitment of dentritic and $T$ cells during inflammatory and immunological responses. hsa04062

hsa04514

hsa04660

hsa05320

hsa05323

H00081

H00082

$\mathrm{H} 00083$

H00408

Chemokine signaling pathway

Cell adhesion molecules (CAMs)

T cell receptor signaling pathway Autoimmune thyroid disease Rheumatoid arthritis Hashimoto's thyroiditis Graves' disease Allograft rejection Type I diabetes mellitus

hsa04064

hsa04514

hsa04620

hsa04672

hsa05144

hsa05145

hsa05166

hsa05169

\footnotetext{
NF-kappa B signaling pathway (noncanonical)

Cell adhesion molecules (CAMs)

Toll-like receptor signaling pathway

Intestinal immune network for IgA production

Malaria

Toxoplasmosis

HTLV-I infection

Epstein-Barr virus infection
}

immunoglobilin superfamily, encodes a protein which transmits an inhibitory signal to T cells associated with RA, T1D, Graves disease, Hashimoto thyroiditis, celiac disease, SLE, thyroid-associated orbitopathy, alopecia and other autoimmune diseases
CD40 molecule, TNF receptor superfamily member 5; mainly is NFkB and MARK dependant, very small involvment in Jak-STAT for INF autokrine activiation loop; mainly is NFkB and MARK dependant, very small involvment in Jak-STAT for INF autokrine activiation loop; GWAS associated RA, MS etc., liked with ALS, Alzheimer and hyper IgM immunodeficiency 
Transcriptional misregulation in cancer Asthma

Autoimmune thyroid disease

Systemic lupus erythematosus Allograft rejection

Primary immunodeficiency Viral myocarditis Immunodef, Hyper IgM syndrom

Cytokine-cytokine receptor interaction Chemokine signaling pathway Endocytosis

Epithelial cell signaling in Helicobacter pylori infection a receptor for interleukin 8; linked with melanoma developemnt

Antigen processing and presentation endoplasmic reticulum aminopeptidase 2; hydrolyzes $\mathrm{N}$-terminal aa of peptide substrates: (MHC) class I molecules rely on aminopeptidases (ERAP1,2 etc.) to trim precursors to antigenic peptides in the endoplasmic reticulum (ER) following cleavage in the cytoplasm

13 GNA12 $2768 \quad$ hsa04010

hsa04022

hsa04270

hsa04730

hsa04810

hsa05200

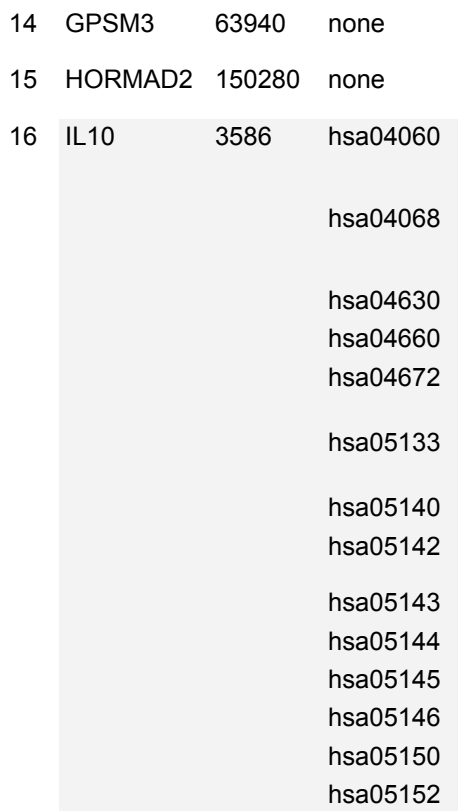

hsa05169

hsa05310

hsa05320

hsa05321
MAPK signaling pathway

no

cGMP-PKG signaling pathway

Vascular smooth muscle contraction

Long-term depression

Regulation of actin cytoskeleton

Pathways in cancer

G-protein signaling modulator 3; associated with Ps no and SLE

HORMA domain containing 2; assiciated with $C D$ IBD, nephropathy

Cytokine-cytokine receptor interaction IL10 is often anti-immflamatory as IL6 and it is acting via Jak-STAT, no NFkB; has pleiotropic effects in immunoregulation and inflammation

FoxO signaling pathway

Jak-STAT signaling pathway

$T$ cell receptor signaling pathway Intestinal immune network for IgA production

via Jak-STAT first and then to Foxo via STAT3 (same as IL6); an essential immunoregulator linked with infection and RA

Pertussis

IL10 is a product of this pathway

Act on B cell same as IL6 (IL6 is not IL10 family but hematopoetins); not included in TNFpathways;

IL10 and TNF are only products of this pathway

Leishmaniasis

product of this pathway

Chagas disease (American trypanosomiasis)

African trypanosomiasis Malaria

Toxoplasmosis

Amoebiasis

Staphylococcus aureus infection Tuberculosis

Epstein-Barr virus infection

Asthma

Autoimmune thyroid disease

Inflammatory bowel disease (IBD) real signaling to INF via Jak-STAT and product as well as immuno modulator 
17 IRF1 3659

hsa04917

hsa05133

hsa05160

hsa04620

18

IRF5

3663

hsa04620

19 LSP1

4046

hsa05152

Tuberculosis a member of the interferon regulatory transcription

$$
\text { Pertussis }
$$

Hepatitis C (engaged Toll-like R)

Toll-like receptor signaling pathway

factor (IRF) family; play roles in regulating

apoptosis and tumor-suppressoion a member of the interferon regulatory transcription

yes factor (IRF) family;

lymphocute specific protein 1 encodes an intracellular F-actin binding protein, lymphocute specific protein 1; extressed on lymphocytes, neutrophils, macrophages; regulate motility of lymphocytes, neutrophils, macrophages

NK2 homeobox 3, a member of the NKX family of homeodomain transcription factors; linked with cellular differentiation; associated with UC, CD

phosphodiesterase $2 \mathrm{~A}$ cGMP-PKG signaling pathway

Morphine addiction

hsa04022

hsa05032

Viral carcinogenesis no annotions

pre-B-cell leukemia homeobox 2 immunoglobulin kappa J region; plays a central role in Notch signaling, a signaling pathway involved in cell-cell communication; represses or activates transcription via the recruitment of chromatin activator that activates transcription of Notch target genes. remodeling complexes and acts as a transcriptional
25 RNF5

26

SLC22A4

6583

hsa04141

hsa05231

$\mathrm{H} 00286$

26

SLC22A5 $6584 \quad$ hsa05231

H00286

$\mathrm{H} 00525$

27 SMAD3 hsa04068

hsa04110

hsa04144

hsa04310

hsa04350

hsa04390

hsa04520

hsa04550

hsa05142

hsa05161

hsa05166

hsa05200

hsa05210

hsa05212
Protein processing in endoplasmic reticulum

Choline metabolism in cancer

Crohn's disease

Choline metabolism in cancer

Crohn's disease

Disorders of fatty-acid oxidation

FoxO signaling pathway

Cell cycle

Endocytosis

Wnt signaling pathway

TGF-beta signaling pathway

Hippo signaling pathway

Adherens junction

Signaling pathways regulating pluripotency of stem cells

Chagas disease (American

trypanosomiasis)

Hepatitis B

HTLV-I infection

Pathways in cancer

Colorectal cancer

Pancreatic cancer

\section{E3 ubiquitin-protein ligase RNF5}

solute carrier family 22 (organic cation/zwitterion transporter), member 4; choline receptor transporter; an organic cation transporter and plasma integral membrane protein partially ATP dependent; associated with neuroblastoma.

solute carrier family 22 (organic cation/zwitterion transporter), member 5; choline receptor transporter; involved in the active cellular uptake of carnitine; associated with asthma and CD

a transcriptional modulator activated by transforming growth factor-beta and has a role in the regulation of carcinogenesis; involved in the active cellular uptake of carnitine; associated with $\mathrm{CD}$, Asthma, hearth diseases; 
Cytokine-cytokine receptor interaction a member of the tumor necrosis factor (TNF) ligand family; a herpesvirus entry mediator, it enables
entry of HSV into cells; associated with UC; show entry of HSV into cells; associated with UC; shown
to stimulate the proliferation of T cells, and triggers

hsa05168

Herpes simplex infection apoptosis of various tumor cells; also prevent TNFA mediated apoptosis

30 TNPO3

23534

hsa04060 transportin 3; associated with RA, Sclerosis, Cirrhosis, Scleroderma, linked to muscular dystrophy

tumor necrosis factor (ligand) superfamily, member 15; not expressed in either B or T cells; is inducible by TNF and IL-1 alpha, can activate NF-kappaB and MAP kinases, and acts as an autocrine factor to induce apoptosis in endothelial cells and may function as an angiogenesis inhibitor. no

yes

TNF associated factor 1 is required with TRAF2 for TNF-alpha-mediated activation of MAPK8/JNK and NF-kappaB; mediates the anti-apoptotic signals from TNF receptors; can be induced by EBV. associated only with RA, liked with canacers and infection

$\begin{array}{cc}\text { hsa04668 } & \text { TNF signaling pathway } \\ \text { hsa05168 } & \text { Herpes simplex infection } \\ \text { hsa05169 } & \text { Epstein-Barr virus infection } \\ \text { hsa05200 } & \text { Pathways in cancer } \\ \text { hsa05202 } & \text { Transcriptional misregulation in } \\ \text { cancer }\end{array}$

Ubiquitin mediated proteolysis

Parkinson's disease ubiquitin-conjugating enzyme, ubiquitin-protein ligase; The modification of proteins with ubiquitin is an important cellular mechanism for targeting

hsa05012 abnormal or short-lived proteins for degradation.
Protein processing in endoplasmic reticulum
34 RNF5

35 hsa04141
E3 ubiquitin-protein ligase RNF5

zona pellucida binding protein 2 no

yes

\footnotetext{
Note: shaded are genes with Immune system participation

Provided are gene official symbols and IDs, names and IDs of KEGG pathways, gene function, association with immune/inflammatory diseases, or other relevant gene info, and evaluation whether the gene is considered linked directly to the immune system or not.
} 
Supplemental Table 4. Comparison between all pathways: missSNP gene pathways, ncSNP gene pathways and TNF containing pathways

ncSNP pathways

hsa04062

hsa04064

hsa04068

ha04144

hsa04330

hsa04350

hsa04514

hsa0461

hsa04612

hsa 04620

hsa04630

hsa04660

hsa04668

hsa04672

hsa04917

hsa05012

hsa05120

hsa05133

hsa05140

sa05142

hsa0514

hsa05144

hsa05145

hsa0514

sa05150

hsa05152

hsa05160

hsa0516

sa05168

hsa05169

hsa05200

hsa05202

sa05203

hsa05222

hsa05310

hsa05320

hsa0532

hsa05322

hsa0532

hsa05330

hsa05416

Cytokine-cytokine receptor interaction

Chemokine signaling pathway

NF-kappa B signaling pathway

FoxO signaling pathway

Endocytosis

Notch signaling pathway

TGF-beta signaling pathway - Homo sapiens (human)

Cell adhesion molecules (CAMs)

Complement and coagulation cascade

Antigen processing and presentation

Toll-like receptor signaling pathway

Jak-STAT signaling pathway

cell receptor signaling pathway

TNF signaling pathway

Intestinal immune network for IgA production

Prolactin signaling pathway

Parkinson's disease

Ppithelial cell signaling in Helicobacter pylori infection

Pertussis

Leishmaniasis

Chagas disease (American trypanosomiasis)

African trypanosomiasis

Malaria

Toxoplasmosis

Amoebiasis

Staphylococcus aureus infection

Tuberculosis

Hepatitis C (engaged Toll-like R)

HTLV-I infection

Herpes simplex infection

pstein-Barr virus infection

Pathways in cancer

Transcriptional misregulation in cance

Viral carcinogenesis

Small cell lung cancer

Asthma

Autoimmune thyroid disease

Inflammatory bowel disease (IBD)

Systemic lupus erythematosus

Rheumatoid arthritis

Allograft rejection

Primary immunodeficiency

Viral myocarditis
missSNP pathways

hsa04060 Cytokine-cytokine receptor interaction

hsa04064 NF-kappa B signaling pathway

hsa04066 HIF-1 signaling pathway

hsa04140 Regulation of autophagy

hsa04145 Phagosome

hsa04151 PI3K-Akt signaling pathway

hsa04330

hsa04611

hsa04612

hsa04621

hsa04630

hsa04640

hsa04660

hsa04662

hsa04664

hsa04666

hsa04668

hsa04722

hsa04919

hsa04920

hsa04932

hsa05131

hsa05140

hsa05145

hsa05150

hsa05152

hsa05160

hsa05162

hsa05164

hsa05168

hsa05169

hsa05206

hsa05321

has05340

Notch signaling pathway

Osteoclast differentiation

Platelet activation

Antigen processing and presentation

NOD-like receptor signaling pathway

Jak-STAT signaling pathway

Hematopoietic cell lineage

T cell receptor signaling pathway

B cell receptor signaling pathway

Fc epsilon RI signaling pathway

Fc gamma R-mediated phagocytosis

TNF signaling pathway

Neurotrophin signaling pathway

Thyroid hormone signaling pathway

Adipocytokine signaling pathway

Non-alcoholic fatty liver disease (NAFLD)

Shigellosis

Leishmaniasis

Toxoplasmosis

aphylococcus aureus infection

Tuberculosis

Measles

erpes simplex infection

Epstein-Barr virus infection

MicroRNAs in cance

Asthma

Inflammatory bowel disease (IBD)

Primary immunodeficiency
TNF pathways

hsa04060 Cytokine-cytokine receptor interaction

hsa04064 NF-kappa B signaling pathway

hsa04150 mTOR signaling pathway - Homo sapiens (human)

hsa04210 Apoptosis

hsa04350 TGF-beta signaling pathway - only probable, not confirmed in KEGG

hsa04380 Osteoclast differentiation

hsa04612 Antigen processing and presentation

hsa04620 Toll-like receptor signaling pathway

hsa04621 NOD-like receptor signaling pathway

hsa04622 RIG-I-like receptor signaling pathway - Homo sapiens (human)

hsa04640 Hematopoietic cell lineage

hsa04650 Natural killer cell mediated cytotoxicity - Homo sapiens (human)

hsa04660 T cell receptor signaling pathway

hsa04664 Fc epsilon RI signaling pathway

hsa04668 TNF signaling pathway

hsa04920 Adipocytokine signaling pathway

hsa04930 Type Il diabetes mellitus - Homo sapiens (human)

hsa04932 Non-alcoholic fatty liver disease (NAFLD)

hsa04940 Type I diabetes mellitus - Homo sapiens (human)

hsa05010 Alzheimer's disease - Homo sapiens (human)

hsa05014 Amyotrophic lateral sclerosis (ALS) - Homo sapiens (human)

hsa05133 Pertussis

hsa05134 Legionellosis - Homo sapiens (human)

hsa05140 Leishmaniasis

hsa05142 Chagas disease (American trypanosomiasis)

African trypanosomiasis

hsa05144 Malaria

hsa05145 Toxoplasmosis

hsa05146 Amoebiasis

hsa05152 Tuberculosis

hsa05160 Hepatitis $C$

hsa05161 Hepatitis B

hsa05164 Influenza A

hsa05166 HTLV-I infection

hsa05168 Herpes simplex infection

hsa05205 Proteoglycans in cancer

hsa05310 Asthma

hsa05321 Inflammatory bowel disease (IBD)

hsa05322 Systemic lupus erythematosus

hsa05323 Rheumatoid arthritis

hsa05330 Allograft rejection

hsa05332 Graft-versus-host disease

hsa05410 Hypertrophic cardiomyopathy (HCM)

hsa05414 Dilated cardiomyopathy

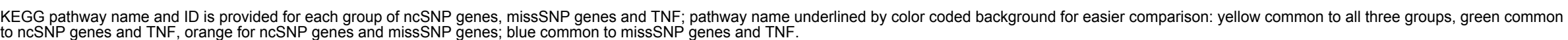


Intersections between TNF signaling pathway and AID GWAS SNP gene pathways AID GWAS SNP analysed by Cytoscape

\begin{tabular}{|c|c|c|c|c|c|c|c|c|c|c|}
\hline & Pathways & TNF signaling & $\begin{array}{l}\text { NF-kB } \\
\text { signaling } \\
\text { pathway }\end{array}$ & $\begin{array}{l}\text { NOD2 like } \\
\text { signaling }\end{array}$ & $\begin{array}{l}\text { Jak-STAT } \\
\text { signaling }\end{array}$ & $\begin{array}{l}\text { Antigen } \\
\text { procesing } \\
\text { and } \\
\text { presenting }\end{array}$ & $\begin{array}{l}\text { B cell R } \\
\text { signalin } \\
\mathbf{g}\end{array}$ & $\begin{array}{l}\text { T cell } R \\
\text { signaling }\end{array}$ & $\begin{array}{l}\text { Cytokine- } \\
\text { cytokine R } \\
\text { interaction }\end{array}$ & $\begin{array}{l}\text { Intestinal } \\
\text { immune network } \\
\text { for IgA } \\
\text { production }\end{array}$ \\
\hline 1 & TNF signaling & & & & & & & & & \\
\hline 2 & NF-kB signaling & \begin{tabular}{|l|} 
Ubuquitin mediated \\
proteolysis, Apoptosis; \\
TNFAIP3 among 17 members, \\
including NFKBIA, TNF, IKBKG, \\
RIPK1, TRAF1,2, CHUK etc
\end{tabular} & & & & & & & & \\
\hline 3 & NOD like signaling & $\begin{array}{l}\text { Apopotisis, Ubiquitin, MAPK } \\
\text { and NFKB signaling; NOD2, } \\
\text { TNFAIP3, NFKBIA-NFKB1, TNF } \\
\text { etc. } 15 \text { genes and } 4 \text { processes }\end{array}$ & $\begin{array}{l}\text { TNF, TNFAIP3 TRAF6 } \\
\text { NFKBIA, ubiquitin, } \\
\text { apoptosis CXCL2 and } \\
\text { L8 }\end{array}$ & & & & & & & \\
\hline 4 & $\begin{array}{l}\text { Jak-STAT signaling } \\
\text { pathway }\end{array}$ & $\begin{array}{l}\text { AKT3-PIK3R5, CSF5, bacially } \\
\text { only AKT and PI3K; Ubuquitin } \\
\text { mediated proteolysis, } \\
\text { Apoptosis, MAPK signaling, } \\
\text { Pl3K-Akt signaling pathway are } \\
\text { all downstream. }\end{array}$ & $\begin{array}{l}\text { basically NONE! } \\
\text { All are downstream } \\
\text { of action: BCL2L1 ( a } \\
\text { product after DNA } \\
\text { is a apopotosis } \\
\text { regulator); } \\
\text { Ubuquitin mediated } \\
\text { proteolysis, } \\
\text { Apoptosis, Cytok- } \\
\text { CytokR }\end{array}$ & $\begin{array}{l}\text { none; } \\
\text { apopotosis, MAPK } \\
\text { signaling, } \\
\text { Ubiquiting } \\
\text { mediated } \\
\text { proteolysis all } \\
\text { downstream }\end{array}$ & & & & & & \\
\hline 5 & $\begin{array}{l}\text { Antigen procesing } \\
\text { and presenting }\end{array}$ & TNF & $\begin{array}{l}\text { TNF, proteosome, T } \\
\text { cell R signaling, } \\
\text { K10784 }\end{array}$ & TNF & none & & & & & \\
\hline 6 & B cell signaling & $\begin{array}{l}\text { NFKBIA-NFKB1, AKT3-PIK3R5, } \\
\text { MAPK2K1-MARK1; FOS; PI3K- } \\
\text { Akt signaling pathway, NF-kB } \\
\text { signaling pathway, Ubiquitin } \\
\text { mediated proteolysis. }\end{array}$ & $\begin{array}{l}\text { NFKBIA SYK LYN BTK } \\
\text { ubiqutin mediated } \\
\text { proteolysis, calcium } \\
\text { signaling }\end{array}$ & $\begin{array}{l}\text { NFKB1- NFKBIA, } \\
\text { Ubuquitin } \\
\text { proteolysis, MAPK } \\
\text { signaling, NFkB } \\
\text { signaling }\end{array}$ & none & none & & & & \\
\hline 7 & $\mathrm{~T}$ cell signaling & $\begin{array}{l}\text { MAPK1-MAP2K1, AKT3- } \\
\text { PIK3R5, NFKB1-NFKBIA; } \\
\text { MAPK14, MAP3K8, MAP2K7, } \\
\text { MAP3K7, MAP3K14, CSF2, } \\
\text { FOS, TNF; Ubiquitin med } \\
\text { proteolysis, NFkB signaling, } \\
\text { PI3K-Akt signaling, MAPK } \\
\text { signaling }\end{array}$ & $\begin{array}{l}\text { TNF NFKBIA AKT PI3K } \\
\text { Mapk FOS and many } \\
\text { others (20+) }\end{array}$ & \begin{tabular}{|l|} 
TNF NFKBA NFKB1 \\
MAPK signaling, \\
NFKB signaling, \\
Ubiquitin mediated \\
proteolysis
\end{tabular} & none & $\begin{array}{l}\text { TNF, CD4 and } \\
\text { IFNG }\end{array}$ & $\begin{array}{l}\text { many } \\
\text { common } \\
\text { genes }\end{array}$ & & & \\
\hline 8 & $\begin{array}{l}\text { Cytokine-cytokine R } \\
\text { interaction }^{\star}\end{array}$ & $\begin{array}{l}\text { LTA-TNFRSF1B, TNF- } \\
\text { TNFRSF1A, LIF, IL6, IL15, IL1B, } \\
\text { CSF1, CXCL1, CXCL5, CX3CL1, } \\
\text { CXC10, CLL5, CCL2, CCL20, } \\
\text { FAS, CSF2 (18 total) }\end{array}$ & $\begin{array}{l}\text { CD40, CD40L TNF } \\
\text { TNFRSF1A and a few } \\
\text { TNF-TNFR family }\end{array}$ & $\begin{array}{l}\text { TNF and IL6 IL18 } \\
\text { IL1R CXCL1,2,8 and } \\
\text { CCL2 and } 5\end{array}$ & $\begin{array}{l}\text { IL24 IL22R CSF2 } \\
\text { (granulocyte- } \\
\text { macrophage } \\
\text { colony- } \\
\text { stimulating } \\
\text { factor) }\end{array}$ & TNF INFG & none? & none? & & \\
\hline 9 & $\begin{array}{l}\text { Intestinal immune } \\
\text { network for IgA } \\
\text { production }\end{array}$ & IL15, MAP3K14 (NIK), IL6 & $\begin{array}{l}\text { TNFRSF } 13 \text { CD } 40 \text { BCR } \\
\text { TCR signaling etc. }\end{array}$ & none & none & NA & many genes & many genes & many genes & \\
\hline 10 & $\begin{array}{l}\text { Osteoclast } \\
\text { differenciation }\end{array}$ & TNF, NFkB signaling & $\begin{array}{l}\text { TNF MAP3K14 (NIK) } \\
\text { NFKBIA TRAF2 NFKB2 } \\
\text { CHUK IKBKG SYK } \\
\text { IL1R1 TNFRSF11 } \\
\text { TNFRSF1A }\end{array}$ & $\begin{array}{l}\text { TNF NFKBIA NFKB1 } \\
\text { MAPK1,8,11 NFkB } \\
\text { signaling, MAPK } \\
\text { signaling }\end{array}$ & $\begin{array}{l}\text { MAPK signaling } \\
\text { PI3K-AKT } \\
\text { signaling Jak-STAT } \\
\text { signaling PIK3R5 } \\
\text { IRFS GRB2 AKT3 } \\
\text { JAK1 etc. } \\
\end{array}$ & TNF INFG CREB1 & NA & NA & $\begin{array}{l}\text { TNF TNFSF11 } \\
\text { TNFRSF11A } \\
\text { TNFRSF1A IL1A } \\
\text { IFNG }\end{array}$ & \\
\hline 11 & $\begin{array}{l}\text { Notch signaling } \\
\text { pathway }\end{array}$ & none & none & none & none & none & none & none & none & none \\
\hline
\end{tabular}

* not a real a pathway, but the coplex collection of cytokines and cytokines receptors 
Supplemental Table 6.

Enriched pathway-based set for missSNP geneset by ConsensusPathDB

uploaded list:

mapped entities:

23

22

enriched pathway-based sets:
78

17 genes $(77.3 \%)$ from the input list are present in at least one pathway.

\begin{tabular}{|c|c|c|c|c|c|}
\hline pathway name & set size & $\begin{array}{l}\text { candidates } \\
\text { contained }\end{array}$ & p-value & q-value & $\begin{array}{c}\text { pathway } \\
\text { source }\end{array}$ \\
\hline JAK-STAT-Core & 104 & $5(5.0 \%)$ & 3.53E-07 & 3.99E-05 & Signalink \\
\hline Jak-STAT signaling pathway - Homo sapiens (human) & 156 & $5(3.4 \%)$ & $2.40 \mathrm{E}-06$ & 0.000135 & KEGG \\
\hline Immune System & 1069 & $9(0.9 \%)$ & $5.74 \mathrm{E}-06$ & 0.000216 & Reactome \\
\hline Signaling by Interleukins & 117 & $4(3.8 \%)$ & 1.84E-05 & 0.000428 & Reactome \\
\hline NOD1/2 Signaling Pathway & 35 & $3(8.8 \%)$ & $1.90 \mathrm{E}-05$ & 0.000428 & Reactome \\
\hline $\begin{array}{l}\text { Nucleotide-binding domain, leucine rich repeat containing } \\
\text { receptor (NLR) signaling pathways }\end{array}$ & 49 & $3(6.2 \%)$ & $5.40 \mathrm{E}-05$ & 0.00102 & Reactome \\
\hline MAP kinase activation in TLR cascade & 59 & $3(5.5 \%)$ & 8.14E-05 & 0.00108 & Reactome \\
\hline ERK2 activation & 10 & $2(22.2 \%)$ & $8.38 \mathrm{E}-05$ & 0.00108 & Reactome \\
\hline NOD-like receptor signaling pathway & 57 & $3(5.3 \%)$ & $9.06 \mathrm{E}-05$ & 0.00108 & KEGG \\
\hline ERK1 activation & 10 & $2(20.0 \%)$ & 0.000105 & 0.00108 & Reactome \\
\hline Interleukin-6 signaling & 11 & $2(20.0 \%)$ & 0.000105 & 0.00108 & Reactome \\
\hline Inflammatory bowel disease (IBD) & 67 & $3(4.8 \%)$ & 0.000122 & 0.00115 & KEGG \\
\hline ERK activation & 13 & $2(16.7 \%)$ & 0.000153 & 0.00123 & Reactome \\
\hline Cytokine Signaling in Immune system & 198 & $4(2.2 \%)$ & 0.000158 & 0.00123 & Reactome \\
\hline TRAF6 Mediated Induction of proinflammatory cytokines & 74 & $3(4.3 \%)$ & 0.000167 & 0.00123 & Reactome \\
\hline Innate Immune System & 607 & $6(1.0 \%)$ & 0.000175 & 0.00123 & Reactome \\
\hline MyD88 cascade initiated on plasma membrane & 87 & $3(3.6 \%)$ & 0.000277 & 0.00147 & Reactome \\
\hline Toll Like Receptor 10 (TLR10) Cascade & 87 & $3(3.6 \%)$ & 0.000277 & 0.00147 & Reactome \\
\hline Toll Like Receptor 5 (TLR5) Cascade & 87 & $3(3.6 \%)$ & 0.000277 & 0.00147 & Reactome \\
\hline $\begin{array}{l}\text { TRAF6 mediated induction of NFkB and MAP kinases upon } \\
\text { TLR7/8 or } 9 \text { activation }\end{array}$ & 88 & $3(3.6 \%)$ & 0.000287 & 0.00147 & Reactome \\
\hline MyD88 dependent cascade initiated on endosome & 90 & $3(3.5 \%)$ & 0.000308 & 0.00147 & Reactome \\
\hline Toll Like Receptor 7/8 (TLR7/8) Cascade & 90 & $3(3.5 \%)$ & 0.000308 & 0.00147 & Reactome \\
\hline RAF/MAP kinase cascade & 19 & $2(11.8 \%)$ & 0.000314 & 0.00147 & Reactome \\
\hline Toll Like Receptor 9 (TLR9) Cascade & 94 & $3(3.3 \%)$ & 0.000352 & 0.00147 & Reactome \\
\hline Toll Like Receptor TLR1:TLR2 Cascade & 97 & $3(3.3 \%)$ & 0.000364 & 0.00147 & Reactome \\
\hline Toll Like Receptor 2 (TLR2) Cascade & 97 & $3(3.3 \%)$ & 0.000364 & 0.00147 & Reactome \\
\hline MyD88:Mal cascade initiated on plasma membrane & 97 & $3(3.3 \%)$ & 0.000364 & 0.00147 & Reactome \\
\hline Toll Like Receptor TLR6:TLR2 Cascade & 97 & $3(3.3 \%)$ & 0.000364 & 0.00147 & Reactome \\
\hline GRB2 events in EGFR signaling & 23 & $2(10.0 \%)$ & 0.000438 & 0.00156 & Reactome \\
\hline SOS-mediated signalling & 23 & $2(10.0 \%)$ & 0.000438 & 0.00156 & Reactome \\
\hline TRIF-mediated TLR3/TLR4 signaling & 105 & $3(3.0 \%)$ & 0.00048 & 0.00156 & Reactome \\
\hline MyD88-independent cascade & 105 & $3(3.0 \%)$ & 0.00048 & 0.00156 & Reactome \\
\hline Toll Like Receptor 3 (TLR3) Cascade & 105 & $3(3.0 \%)$ & 0.00048 & 0.00156 & Reactome \\
\hline SHC-mediated signalling & 24 & $2(9.5 \%)$ & 0.000484 & 0.00156 & Reactome \\
\hline SHC1 events in EGFR signaling & 24 & $2(9.5 \%)$ & 0.000484 & 0.00156 & Reactome \\
\hline Cytokine-cytokine receptor interaction & 265 & $4(1.6 \%)$ & 0.000515 & 0.00162 & KEGG \\
\hline Signalling to p38 via RIT and RIN & 24 & $2(9.1 \%)$ & 0.000531 & 0.00162 & Reactome \\
\hline ARMS-mediated activation & 25 & $2(8.7 \%)$ & 0.000582 & 0.00164 & Reactome \\
\hline SHC-related events & 27 & $2(8.7 \%)$ & 0.000582 & 0.00164 & Reactome \\
\hline Canonical NF-kappaB pathway & 24 & $2(8.7 \%)$ & 0.000582 & 0.00164 & PID \\
\hline SHC-related events triggered by IGF1R & 27 & $2(8.3 \%)$ & 0.000634 & 0.00175 & Reactome \\
\hline Activated TLR4 signalling & 120 & $3(2.7 \%)$ & 0.000686 & 0.00177 & Reactome \\
\hline Frs2-mediated activation & 27 & $2(8.0 \%)$ & 0.000688 & 0.00177 & Reactome \\
\hline Signaling by Leptin & 29 & $2(8.0 \%)$ & 0.000688 & 0.00177 & Reactome \\
\hline Prolonged ERK activation events & 29 & $2(7.4 \%)$ & 0.000804 & 0.00197 & Reactome \\
\hline SHC1 events in ERBB4 signaling & 30 & $2(7.4 \%)$ & 0.000804 & 0.00197 & Reactome \\
\hline Toll Like Receptor 4 (TLR4) Cascade & 129 & $3(2.5 \%)$ & 0.000858 & 0.00206 & Reactome \\
\hline Canonical NF-kappaB pathway & 23 & $2(8.7 \%)$ & 0.000532 & 0.00207 & PID \\
\hline GRB2 events in ERBB2 signaling & 32 & $2(6.9 \%)$ & 0.000928 & 0.00218 & Reactome \\
\hline Measles - Homo sapiens (human) & 134 & $3(2.3 \%)$ & 0.00103 & 0.00235 & KEGG \\
\hline
\end{tabular}


SHC1 events in ERBB2 signaling

Signalling to RAS

Signaling by SCF-KIT

VEGFR2 mediated cell proliferation

Toll-Like Receptors Cascades

IL23-mediated signaling events

NOD pathway

IL-6 signaling pathway

Signalling to ERKs

FRS2-mediated cascade

Interleukin-2 signaling

IL6-mediated signaling events

Tuberculosis - Homo sapiens (human)

Signaling events mediated by PTP1B

Epstein-Barr virus infection - Homo sapiens (human)

IL4

NCAM signaling for neurite out-growth

IL6

Allograft Rejection

Hematopoietic cell lineage - Homo sapiens (human)

FCERI mediated MAPK activation

IRS-mediated signalling

IRS-related events

TNF alpha Signaling Pathway

NF-kappa B signaling pathway - Homo sapiens (human)

IRS-related events triggered by IGF1R

Insulin receptor signalling cascade

IGF1R signaling cascade

$\begin{array}{rllll}34 & 2(6.5 \%) & 0.00106 & 0.00235 & \text { Reactome } \\ 34 & 2(6.5 \%) & 0.00106 & 0.00235 & \text { Reactome } \\ 149 & 3(2.2 \%) & 0.00125 & 0.00272 & \text { Reactome } \\ 37 & 2(5.9 \%) & 0.00128 & 0.00272 & \text { Reactome } \\ 149 & 3(2.1 \%) & 0.00133 & 0.00278 & \text { Reactome } \\ 38 & 2(5.7 \%) & 0.00135 & 0.00278 & \text { PID } \\ 39 & 2(5.1 \%) & 0.00168 & 0.00339 & \text { Wikipathways } \\ 43 & 2(4.9 \%) & 0.00185 & 0.00361 & \text { Wikipathways } \\ 44 & 2(4.9 \%) & 0.00185 & 0.00361 & \text { Reactome } \\ 46 & 2(4.7 \%) & 0.00204 & 0.00384 & \text { Reactome } \\ 51 & 2(4.7 \%) & 0.00204 & 0.00384 & \text { Reactome } \\ 49 & 2(4.4 \%) & 0.00223 & 0.00413 & \text { PID } \\ 179 & 3(1.7 \%) & 0.00234 & 0.00427 & \text { KEGG } \\ 55 & 2(4.1 \%) & 0.00264 & 0.00473 & \text { PID } \\ 202 & 3(1.5 \%) & 0.00344 & 0.00607 & \text { KEGG } \\ 64 & 2(3.3 \%) & 0.00393 & 0.00683 & \text { NetPath } \\ 75 & 2(3.0 \%) & 0.00488 & 0.00835 & \text { Reactome } \\ 77 & 2(2.8 \%) & 0.00561 & 0.00947 & \text { NetPath } \\ 80 & 2(2.5 \%) & 0.00689 & 0.0114 & \text { Wikipathways } \\ 87 & 2(2.4 \%) & 0.00723 & 0.0117 & \text { KEGG } \\ 88 & 2(2.4 \%) & 0.00723 & 0.0117 & \text { Reactome } \\ 89 & 2(2.4 \%) & 0.0074 & 0.0118 & \text { Reactome } \\ 92 & 2(2.4 \%) & 0.00775 & 0.0118 & \text { Reactome } \\ 87 & 2(2.4 \%) & 0.00775 & 0.0118 & \text { Wikipathways } \\ 91 & 2(2.3 \%) & 0.00828 & 0.0119 & \text { KEGG } \\ 93 & 2(2.3 \%) & 0.0081 & 0.0122 & \text { Reactome } \\ 96 & 2(2.2 \%) & 0.00847 & 0.0125 & \text { Reactome } \\ 96 & 2(2.2 \%) & 0.00865 & 0.0125 & \text { Reactome }\end{array}$

Number of queried genes entered for enrichment analyses and number of genes mapped in pathways; number of enriched pathway sets; pathways names with the number of genes in each pathway and percentage of queried genes for each pathway along with number of genes in a gene set for each pathway; $p$ values, after fdr and Bonferroni corrections. 


\section{Supplemental Table 7. Enriched pathway-based set for ncSNP geneset by ConsensusPath DB}

uploaded list:

mapped entities:

enriched pathway-based sets:
23

23

26

14 genes $(60.9 \%)$ from the input list are present in at least one pathway.

\begin{tabular}{|c|c|c|c|c|c|}
\hline pathway name & set size & $\begin{array}{l}\text { candidates } \\
\text { contained }\end{array}$ & p-value & q-value & pathway source \\
\hline Chemokine receptors bind chemokines & 60 & $3(5.3 \%)$ & 4.91E-05 & 0.00101 & Reactome \\
\hline Peptide GPCRs & 72 & $3(5.3 \%)$ & 4.91E-05 & 0.00101 & Wikipathways \\
\hline Pertussis - Homo sapiens (human) & 75 & $3(4.0 \%)$ & 0.000112 & 0.00153 & KEGG \\
\hline Cytokine-cytokine receptor interaction - Homo sapiens (human) & 265 & $4(1.6 \%)$ & 0.000229 & 0.00234 & KEGG \\
\hline Senescence and Autophagy & 105 & $3(2.9 \%)$ & 0.000303 & 0.00248 & Wikipathways \\
\hline the information processing pathway at the ifn beta enhancer & 30 & $2(6.9 \%)$ & 0.000624 & 0.00426 & BioCarta \\
\hline Chemokine signaling pathway - Homo sapiens (human) & 189 & $3(1.6 \%)$ & 0.00153 & 0.00784 & KEGG \\
\hline Peptide ligand-binding receptors & 198 & $3(1.6 \%)$ & 0.00153 & 0.00784 & Reactome \\
\hline Autoimmune thyroid disease - Homo sapiens (human) & 54 & $2(3.8 \%)$ & 0.002 & 0.00827 & KEGG \\
\hline Staphylococcus aureus infection - Homo sapiens (human) & 57 & $2(3.8 \%)$ & 0.00208 & 0.00827 & KEGG \\
\hline Endocytosis - Homo sapiens (human) & 213 & $3(1.4 \%)$ & 0.00227 & 0.00827 & KEGG \\
\hline G alpha (i) signalling events & 242 & $3(1.4 \%)$ & 0.00259 & 0.00827 & Reactome \\
\hline GPCRs, Class A Rhodopsin-like & 259 & $3(1.4 \%)$ & 0.00262 & 0.00827 & Wikipathways \\
\hline Inflammatory bowel disease (IBD) - Homo sapiens (human) & 67 & $2(3.2 \%)$ & 0.00293 & 0.00857 & KEGG \\
\hline $\begin{array}{l}\text { Epithelial cell signaling in Helicobacter pylori infection - Homo } \\
\text { sapiens (human) }\end{array}$ & 68 & $2(3.0 \%)$ & 0.0033 & 0.00872 & KEGG \\
\hline Regulation of Telomerase & 71 & $2(2.9 \%)$ & 0.0034 & 0.00872 & PID \\
\hline Regulation of nuclear SMAD2/3 signaling & 79 & $2(2.6 \%)$ & 0.00423 & 0.01 & PID \\
\hline Allograft Rejection & 80 & $2(2.5 \%)$ & 0.00468 & 0.01 & Wikipathways \\
\hline Apoptosis & 84 & $2(2.5 \%)$ & 0.00479 & 0.01 & Wikipathways \\
\hline GPCR downstream signaling & 986 & $5(0.5 \%)$ & 0.0049 & 0.01 & Reactome \\
\hline GPCRs, Other & 98 & $2(2.3 \%)$ & 0.00539 & 0.0105 & Wikipathways \\
\hline Class A/1 (Rhodopsin-like receptors) & 325 & $3(1.0 \%)$ & 0.00569 & 0.0106 & Reactome \\
\hline Signal Transduction & 2077 & $7(0.4 \%)$ & 0.0069 & 0.0123 & Reactome \\
\hline T cell receptor signaling pathway - Homo sapiens (human) & 104 & $2(2.0 \%)$ & 0.00736 & 0.0125 & KEGG \\
\hline $\begin{array}{l}\text { Chagas disease (American trypanosomiasis) - Homo sapiens } \\
\text { (human) }\end{array}$ & 104 & $2(1.9 \%)$ & 0.00764 & 0.0125 & KEGG \\
\hline Signaling by GPCR & 1108 & $5(0.5 \%)$ & 0.00811 & 0.0128 & Reactome \\
\hline
\end{tabular}

Number of queried genes entered for enrichment analyses and number of genes mapped in pathways; number of enriched pathway sets; pathways names with the number of genes in each pathway and percentage of queried genes for each pathway along with number of genes in a gene set for each pathway; $p$ values, after fdr and Bonferroni corrections. 


\section{Supplemental Table 8. Enriched pathway-based sets for allSNP set}

$\begin{array}{ll}\text { uploaded list: } & 56 \\ \text { mapped entities: } & 54 \\ \text { enriched pathway-based sets: } & 85\end{array}$

31 genes (68.9\%) from the input list are present in at least one pathway

pathway name

JAK-STAT-Core

Cytokine-cytokine receptor interaction - Homo sapiens (human)

Inflammatory bowel disease (IBD) - Homo sapiens (human)

Immune System

Jak-STAT signaling pathway - Homo sapiens (human)

Senescence and Autophagy

Allograft Rejection

NOD1/2 Signaling Pathway

Innate Immune System

IL-6 signaling pathway

Signaling by Interleukins

Epstein-Barr virus infection - Homo sapiens (human)

IL6-mediated signaling events

ERK2 activation

Nucleotide-binding domain, leucine rich repeat containing receptor (NLR) sign

ERK1 activation

Interleukin-6 signaling

Staphylococcus aureus infection - Homo sapiens (human)

MAP kinase activation in TLR cascade

ERK activation

Chemokine receptors bind chemokines

Peptide GPCRs

NOD-like receptor signaling pathway - Homo sapiens (human)

Notch-HLH transcription pathway

NICD traffics to nucleus

TRAF6 Mediated Induction of proinflammatory cytokines

RAF/MAP kinase cascade

Pertussis - Homo sapiens (human)

Tuberculosis - Homo sapiens (human)

GRB2 events in EGFR signaling

SOS-mediated signalling

Apoptosis

SHC-mediated signalling

SHC1 events in EGFR signaling

MyD88 cascade initiated on plasma membrane

Toll Like Receptor 10 (TLR10) Cascade

Toll Like Receptor 5 (TLR5) Cascade

Cytokine Signaling in Immune system

TRAF6 mediated induction of NFkB and MAP kinases upon TLR7/8 or 9 activa

Signalling to $\mathrm{p} 38$ via RIT and RIN

MyD88 dependent cascade initiated on endosome

Toll Like Receptor 7/8 (TLR7/8) Cascade

ARMS-mediated activation

SHC-related events

Canonical NF-kappaB pathway

SHC-related events triggered by IGF1R

Toll Like Receptor 9 (TLR9) Cascade

Toll Like Receptor TLR1:TLR2 Cascade

Toll Like Receptor 2 (TLR2) Cascade

MyD88:Mal cascade initiated on plasma membrane

Toll Like Receptor TLR6:TLR2 Cascade

\begin{tabular}{|c|c|c|c|c|}
\hline set size & $\begin{array}{c}\text { candidates } \\
\text { contained }\end{array}$ & p-value & q-value & pathway source \\
\hline 104 & $6(6.0 \%)$ & $3.36 \mathrm{E}-07$ & 2.87E-05 & Signalink \\
\hline 265 & $8(3.2 \%)$ & $3.52 \mathrm{E}-07$ & 2.87E-05 & KEGG \\
\hline 67 & $5(7.9 \%)$ & $8.80 \mathrm{E}-07$ & 4.78E-05 & KEGG \\
\hline 1069 & $13(1.3 \%)$ & $1.38 \mathrm{E}-06$ & 5.63E-05 & Reactome \\
\hline 156 & $6(4.1 \%)$ & $3.24 \mathrm{E}-06$ & 0.000106 & KEGG \\
\hline 105 & $5(4.8 \%)$ & $1.11 \mathrm{E}-05$ & 0.000302 & Wikipathways \\
\hline 80 & $4(5.0 \%)$ & 7.62E-05 & 0.00177 & Wikipathways \\
\hline 35 & $3(8.8 \%)$ & 0.000122 & 0.00248 & Reactome \\
\hline 607 & $8(1.4 \%)$ & 0.000174 & 0.00312 & Reactome \\
\hline 43 & $3(7.3 \%)$ & 0.000214 & 0.00312 & Wikipathways \\
\hline 117 & $4(3.8 \%)$ & 0.000219 & 0.00312 & Reactome \\
\hline 202 & $5(2.5 \%)$ & 0.00023 & 0.00312 & KEGG \\
\hline 49 & $3(6.7 \%)$ & 0.000282 & 0.00332 & PID \\
\hline 10 & $2(22.2 \%)$ & 0.000285 & 0.00332 & Reactome \\
\hline 49 & $3(6.2 \%)$ & 0.000342 & 0.00341 & Reactome \\
\hline 10 & $2(20.0 \%)$ & 0.000355 & 0.00341 & Reactome \\
\hline 11 & $2(20.0 \%)$ & 0.000355 & 0.00341 & Reactome \\
\hline 57 & $3(5.7 \%)$ & 0.000459 & 0.004 & KEGG \\
\hline 59 & $3(5.5 \%)$ & 0.000512 & 0.004 & Reactome \\
\hline 13 & $2(16.7 \%)$ & 0.00052 & 0.004 & Reactome \\
\hline 60 & $3(5.3 \%)$ & 0.000568 & 0.004 & Reactome \\
\hline 72 & $3(5.3 \%)$ & 0.000568 & 0.004 & Wikipathways \\
\hline 57 & $3(5.3 \%)$ & 0.000568 & 0.004 & KEGG \\
\hline 13 & $2(15.4 \%)$ & 0.000613 & 0.004 & Reactome \\
\hline 13 & $2(15.4 \%)$ & 0.000613 & 0.004 & Reactome \\
\hline 74 & $3(4.3 \%)$ & 0.00104 & 0.00641 & Reactome \\
\hline 19 & $2(11.8 \%)$ & 0.00106 & 0.00641 & Reactome \\
\hline 75 & $3(4.0 \%)$ & 0.00127 & 0.00706 & KEGG \\
\hline 179 & $4(2.3 \%)$ & 0.00144 & 0.00706 & KEGG \\
\hline 23 & $2(10.0 \%)$ & 0.00147 & 0.00706 & Reactome \\
\hline 23 & $2(10.0 \%)$ & 0.00147 & 0.00706 & Reactome \\
\hline 84 & $3(3.7 \%)$ & 0.00158 & 0.00706 & Wikipathways \\
\hline 24 & $2(9.5 \%)$ & 0.00163 & 0.00706 & Reactome \\
\hline 24 & $2(9.5 \%)$ & 0.00163 & 0.00706 & Reactome \\
\hline 87 & $3(3.6 \%)$ & 0.0017 & 0.00706 & Reactome \\
\hline 87 & $3(3.6 \%)$ & 0.0017 & 0.00706 & Reactome \\
\hline 87 & $3(3.6 \%)$ & 0.0017 & 0.00706 & Reactome \\
\hline 198 & $4(2.2 \%)$ & 0.00174 & 0.00706 & Reactome \\
\hline 88 & $3(3.6 \%)$ & 0.00176 & 0.00706 & Reactome \\
\hline 24 & $2(9.1 \%)$ & 0.00179 & 0.00706 & Reactome \\
\hline 90 & $3(3.5 \%)$ & 0.00188 & 0.00706 & Reactome \\
\hline 90 & $3(3.5 \%)$ & 0.00188 & 0.00706 & Reactome \\
\hline 25 & $2(8.7 \%)$ & 0.00195 & 0.00706 & Reactome \\
\hline 27 & $2(8.7 \%)$ & 0.00195 & 0.00706 & Reactome \\
\hline 24 & $2(8.7 \%)$ & 0.00195 & 0.00706 & PID \\
\hline 27 & $2(8.3 \%)$ & 0.00213 & 0.00706 & Reactome \\
\hline 94 & $3(3.3 \%)$ & 0.00214 & 0.00706 & Reactome \\
\hline 97 & $3(3.3 \%)$ & 0.00221 & 0.00706 & Reactome \\
\hline 97 & $3(3.3 \%)$ & 0.00221 & 0.00706 & Reactome \\
\hline 97 & $3(3.3 \%)$ & 0.00221 & 0.00706 & Reactome \\
\hline 97 & $3(3.3 \%)$ & 0.00221 & 0.00706 & Reactome \\
\hline
\end{tabular}




\begin{tabular}{|c|c|c|c|c|c|}
\hline Frs2-mediated activation & 27 & $2(8.0 \%)$ & 0.00231 & 0.0071 & Reactome \\
\hline Signaling by Leptin & 29 & $2(8.0 \%)$ & 0.00231 & 0.0071 & Reactome \\
\hline Prolonged ERK activation events & 29 & $2(7.4 \%)$ & 0.00269 & 0.00783 & Reactome \\
\hline SHC1 events in ERBB4 signaling & 30 & $2(7.4 \%)$ & 0.00269 & 0.00783 & Reactome \\
\hline Cytokines and Inflammatory Response & 29 & $2(7.4 \%)$ & 0.00269 & 0.00783 & Wikipathways \\
\hline TRIF-mediated TLR3/TLR4 signaling & 105 & $3(3.0 \%)$ & 0.00289 & 0.00798 & Reactome \\
\hline MyD88-independent cascade & 105 & $3(3.0 \%)$ & 0.00289 & 0.00798 & Reactome \\
\hline Toll Like Receptor 3 (TLR3) Cascade & 105 & $3(3.0 \%)$ & 0.00289 & 0.00798 & Reactome \\
\hline T cell receptor signaling pathway - Homo sapiens (human) & 104 & $3(3.0 \%)$ & 0.00297 & 0.00807 & KEGG \\
\hline GRB2 events in ERBB2 signaling & 32 & $2(6.9 \%)$ & 0.0031 & 0.00815 & Reactome \\
\hline the information processing pathway at the ifn beta enhancer & 30 & $2(6.9 \%)$ & 0.0031 & 0.00815 & BioCarta \\
\hline Asthma - Homo sapiens (human) & 32 & $2(6.7 \%)$ & 0.00332 & 0.00858 & KEGG \\
\hline SHC1 events in ERBB2 signaling & 34 & $2(6.5 \%)$ & 0.00354 & 0.00887 & Reactome \\
\hline Signalling to RAS & 34 & $2(6.5 \%)$ & 0.00354 & 0.00887 & Reactome \\
\hline IL12 signaling mediated by STAT4 & 33 & $2(6.2 \%)$ & 0.00377 & 0.0093 & PID \\
\hline Activated TLR4 signalling & 120 & $3(2.7 \%)$ & 0.00408 & 0.00992 & Reactome \\
\hline VEGFR2 mediated cell proliferation & 37 & $2(5.9 \%)$ & 0.00425 & 0.0102 & Reactome \\
\hline IL23-mediated signaling events & 38 & $2(5.7 \%)$ & 0.00449 & 0.0106 & PID \\
\hline Toll Like Receptor 4 (TLR4) Cascade & 129 & $3(2.5 \%)$ & 0.00505 & 0.0118 & Reactome \\
\hline NOD pathway & 39 & $2(5.1 \%)$ & 0.00556 & 0.0128 & Wikipathways \\
\hline FoxO signaling pathway - Homo sapiens (human) & 134 & $3(2.4 \%)$ & 0.00565 & 0.0128 & KEGG \\
\hline Measles - Homo sapiens (human) & 134 & $3(2.3 \%)$ & 0.00603 & 0.0135 & KEGG \\
\hline Signalling to ERKs & 44 & $2(4.9 \%)$ & 0.00613 & 0.0135 & Reactome \\
\hline Systemic lupus erythematosus - Homo sapiens (human) & 136 & $3(2.3 \%)$ & 0.00642 & 0.014 & KEGG \\
\hline FRS2-mediated cascade & 46 & $2(4.7 \%)$ & 0.00672 & 0.0142 & Reactome \\
\hline Interleukin-2 signaling & 51 & $2(4.7 \%)$ & 0.00672 & 0.0142 & Reactome \\
\hline Differentiation Pathway & 44 & $2(4.5 \%)$ & 0.00703 & 0.0147 & Wikipathways \\
\hline Signaling by SCF-KIT & 149 & $3(2.2 \%)$ & 0.00726 & 0.015 & Reactome \\
\hline Notch Signaling Pathway & 45 & $2(4.4 \%)$ & 0.00735 & 0.015 & Wikipathways \\
\hline Toll-Like Receptors Cascades & 149 & $3(2.1 \%)$ & 0.00769 & 0.0155 & Reactome \\
\hline Notch signaling pathway - Homo sapiens (human) & 48 & $2(4.2 \%)$ & 0.00833 & 0.0165 & KEGG \\
\hline Signaling events mediated by PTP1B & 55 & $2(4.1 \%)$ & 0.00866 & 0.017 & PID \\
\hline SIDS Susceptibility Pathways & 156 & $3(1.9 \%)$ & 0.0096 & 0.0186 & Wikipathways \\
\hline Autoimmune thyroid disease - Homo sapiens (human) & 54 & $2(3.8 \%)$ & 0.00972 & 0.0186 & KEGG \\
\hline
\end{tabular}

Number of queried genes entered for enrichment analyses and number of genes mapped in pathways; number of enriched pathway sets; pathways names with the number of genes in each pathway and percentage of queried genes for each pathway along with number of genes in a gene set for each pathway; $p$ values, after fdr and Bonferroni corrections. 


\section{Supplemental Figure 1. Genomic context distribution of AID GWAS SNPS}

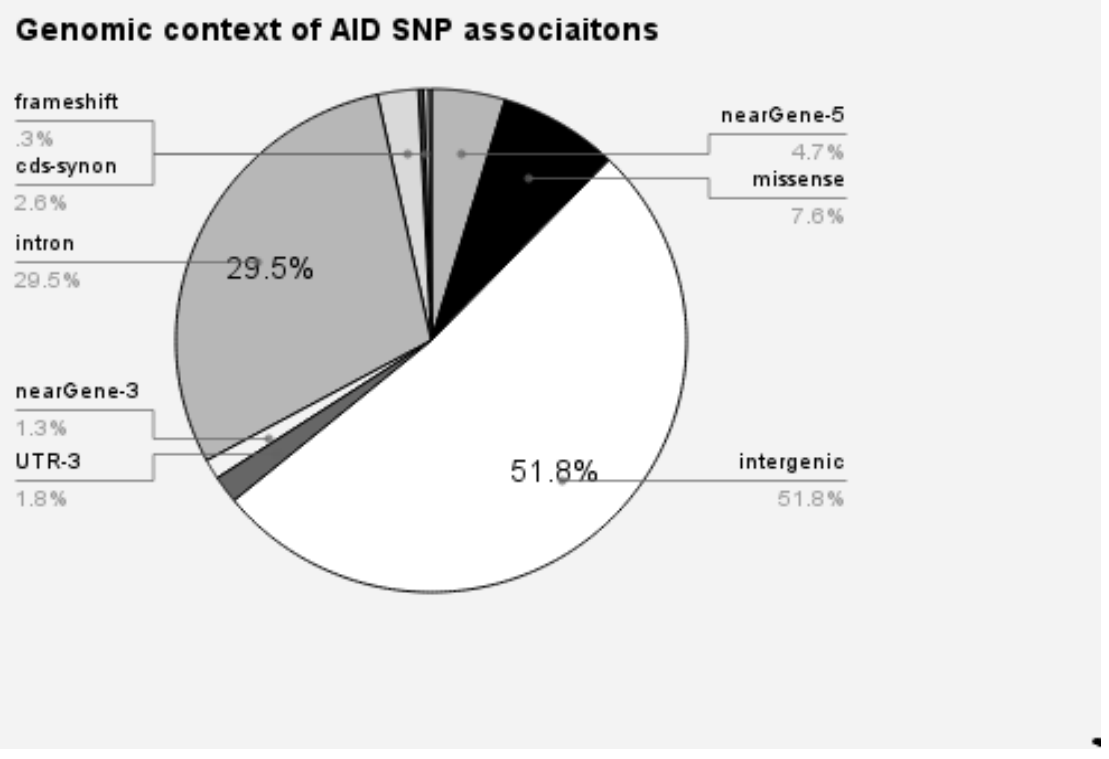

\section{Caption:}

AID SNPs were retrieved from NHGRI Catalog by the end of November 2014.

Over $50 \%$ of all AID SNPs are intergenic, $30 \%$ are intronic and only $7,5 \%$ are missense; $3 \%$ are UTR-3 and UTR-5 SNPs, 5\% are nearGene-5 and 1\% are nearGene-3; coding synonymous SNPs represent only $2,5 \%$. There are only 2 frameshift SNP among the retrieved AID GWAS associations. 
Ideogram of the SNP associations published since the beginning of the GWAS era for the seven autoimmune diseases under our study (AID)

383 SNPs and over 350 genes over 22 chromosomes from all association results.

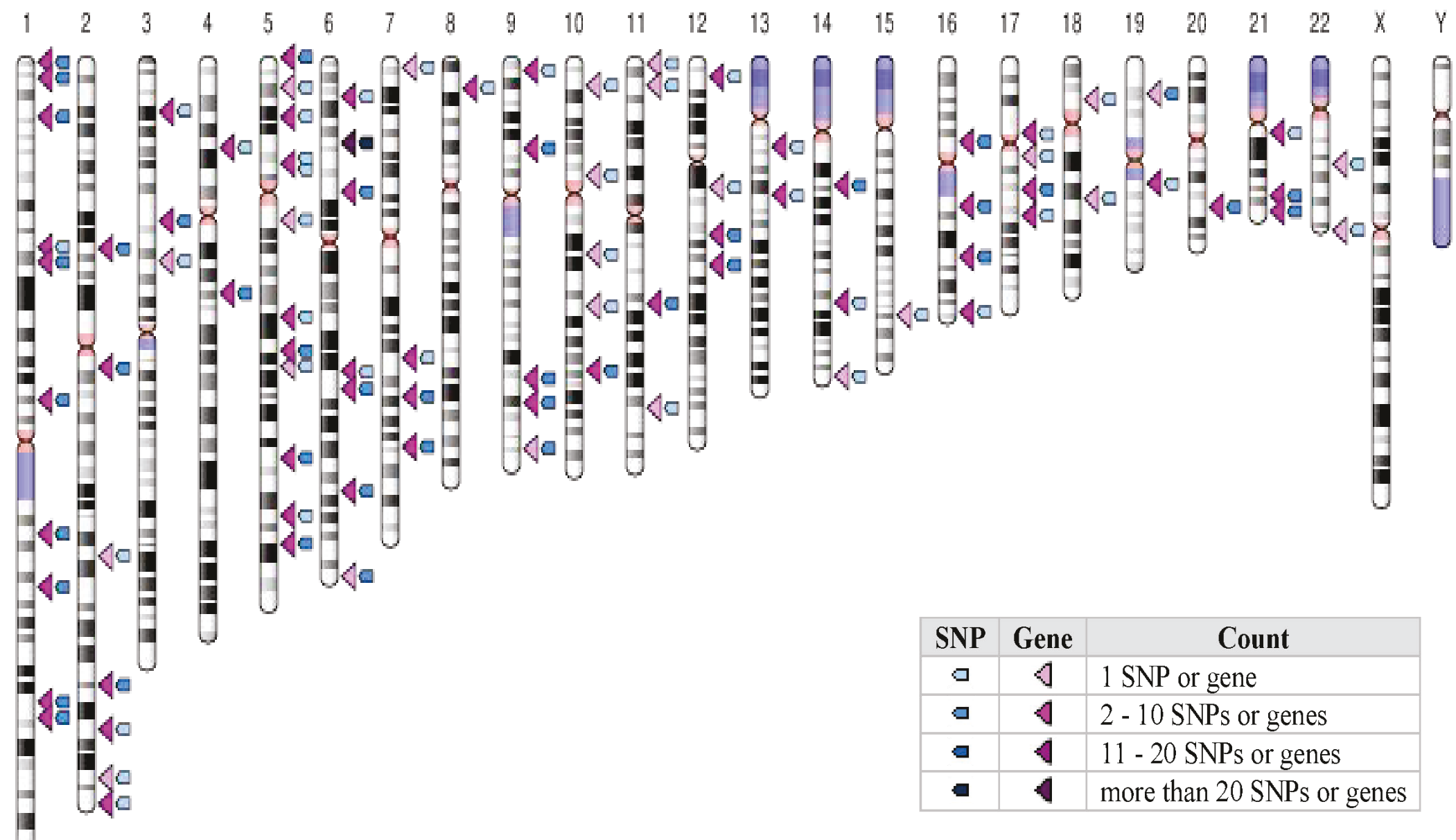




\section{Supplemental Figure 3. Network images for missSNP harboring genes/proteins and TNF}

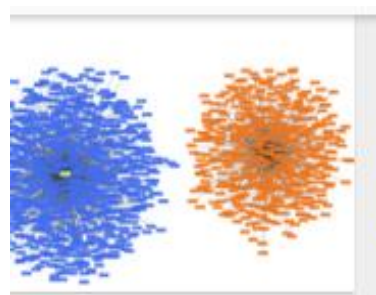

๑ TNF network im.

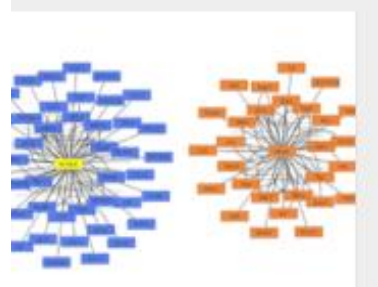

॥ NFKBIE networ..

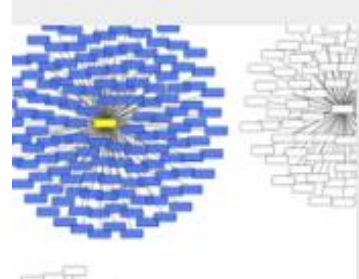

- MICA network i..

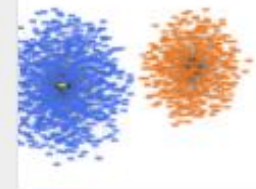

• IL6R networki..

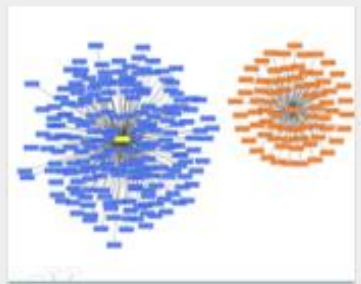

— TNFAIP3 netwo...

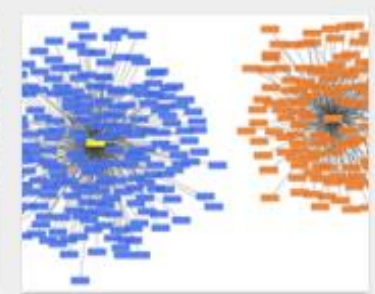

• TYK2 network i...

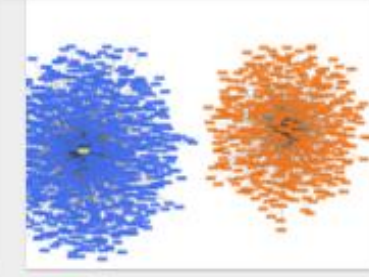

• TNF network im...

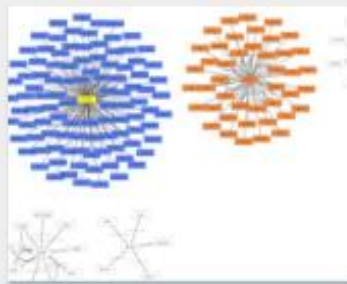

- SH2B3 network..

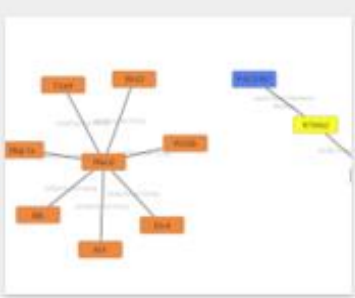

- RTKN2 network..

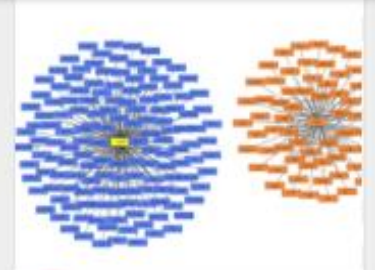

- IL23R network i...

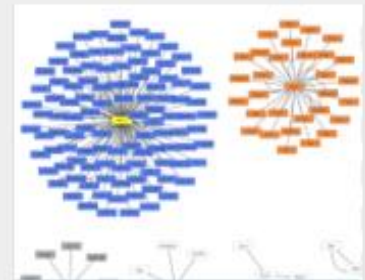

- MST1 network i...

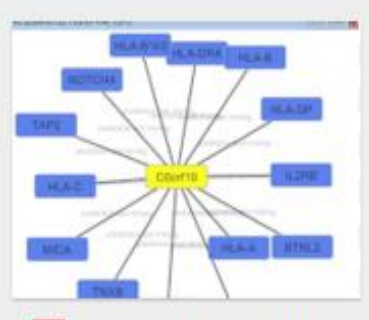

- C6orf10 networ..

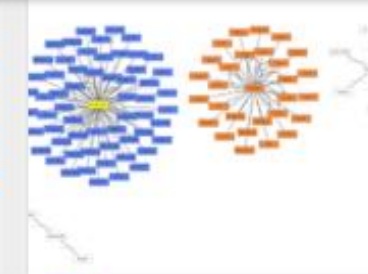

- TRAF3IP2 netw...

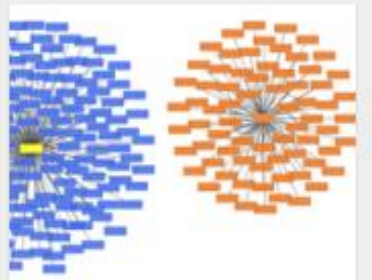

— NOD2 network i...

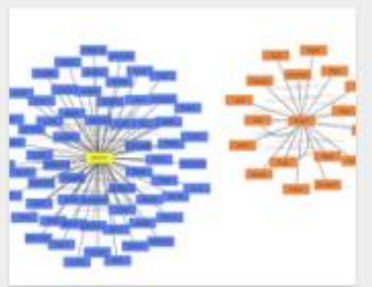

- ERAP1 network...

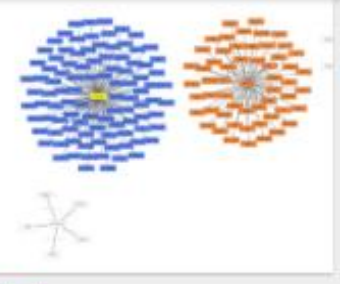

- ATG16L1 netw...

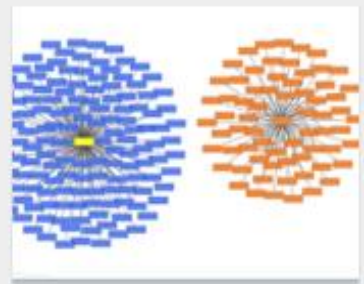

- CARD9 newtwo...

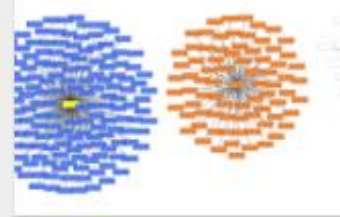

• PTPN22 networ...

The networks constructed using Cytoscape for the missSNP genes and TNF are presented as yellow colored nodes: all other nodes are color coded for a species (blue for human, red for mouse); nodes represent genes/proteins; edges represent any type of interactions between two proteins as detected by Cytoscape. 